\title{
Rheological behavior of dense assemblies of granular materials
}

\author{
Final technical report
}

Report Period:

Start date: 1 April 2007

End date: 31 March 2011

Principal Investigator:

Prof. Sankaran Sundaresan (Princeton University)

Co-principal Investigators:

Prof. Gabriel I. Tardos (The City College of the City University of New York)

Prof. Shankar Subramaniam (Iowa State University)

Report issued on:

30 April 2011

Submitting organization:

Princeton University, Princeton, NJ 08544

DOE Topic/Area of Interest: 2 - Computational Energy Sciences

A. Dense Multiphase Flow Simulation

2. Frictional Flow Regime

DE-PS26-06NT42751-2A2

DOE-UCR Grant No.:

DE-FG26-07NT43070 


\section{Contents}

Disclaimer

$\begin{array}{ll}\text { Abstract } & \text { ii }\end{array}$

1 PROJECT MILESTONES AND STATUS 1

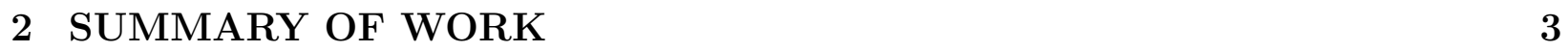

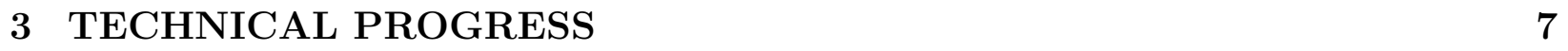

3.1 Work performed at City College . . . . . . . . . . . . . . 7

3.1.1 Transmission of stresses in static and sheared granular beds . . . . . 7

3.1.2 Shearing of a granular material in a Couette cell . . . . . . . . . . . . 13

3.1.3 Measurement of solid fraction using a capacitance probe . . . . . . . 24

3.2 Work performed at Princeton University . . . . . . . . . . . . . . . . 28

3.2.1 Validation of the DEM approach . . . . . . . . . . . . . . 28

3.2.2 Constitutive modeling of quasi-static granular flows . . . . . . . . . . 28

3.2.3 Constitutive modeling of intermediate granular flows . . . . . . . . . 32

3.3 Work performed at Iowa State University . . . . . . . . . . . . . . 36

3.3 .1 Key Findings . . . . . . . . . . . . . . . . . . 36

3.3.2 Assessment of continuum model performance in silo discharge using DEM simulations . . . . . . . . . . . . . . . . . . . 37

3.3.3 Validation of rheological regime transition phenomenon through DEM simulations and experiments . . . . . . . . . . . . . 41

3.3.4 Constitutive modeling framework to capture granular 'phase' transition 42

3.3.5 Constitutive model for contact stress based on relative-acceleration (RA) concept . . . . . . . . . . . . . . . 51

4 PROJECT PARTICIPANTS AND COLLABORATION 57

4.1 Participants . . . . . . . . . . . . . . . . . . 57

4.2 Collaboration Among the Project Participants . . . . . . . . . . . . . 57

5 PUBLICATIONS AND PRESENTATIONS 59

5.1 Publications . . . . . . . . . . . . . . . . . . . . . . . . . . . . . . . . . 59

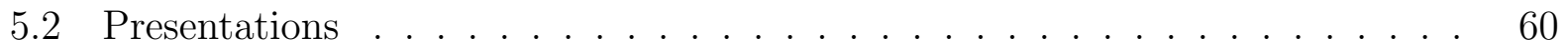


Appendices

$\begin{array}{ll}\text { A Relative acceleration concept } & 67\end{array}$

$\begin{array}{ll}\text { B Bridging the rheology of granular flows in three regimes } & 72\end{array}$

C A constitutive model with microstructure evolution for flow of rate-independent granular materials

D A plasticity model with microstructure evolution for quasi-static granular flows

E Granular flow in Silo discharge: DEM simulations and model assessment 116

F Experimental and computational studies of dense granular flow: transition from quasi-static to intermediate regime in a Couette shear device

G Granular rheology and 'phase' transition through order parameter: DEM simulations and constitutive modeling

H Shear flow of assemblies of cohesive granular materials under constant applied normal stress

I Dynamic shear of cohesive homogeneous assemblies of dense granular materials

J An investigation of frictional and collisional powder flows using a unified constitutive equation

$\mathrm{K}$ Transmission of normal stresses in un-sheared and sheared granular beds: the influence of particle size, shape, stiffness and cohesion

L Effect of Material Properties, Boundary Conditions and Flow Fields on the Rheology of Dense Granular Matter

M Importance of Solid Fraction and Its Fluctuations on the Rheology and Flow Characteristics of Compressible Powders

N Study of Powder Flow Patterns in a Couette Cell with Axial Flow using Tracers and Solid Fraction Measurements 


\section{Disclaimer}

This report was prepared as an account of work sponsored by an agency of the United States Government. Neither the United States Government nor any agency thereof, nor any of their employees, makes any warranty, express or implied, or assumes any legal liability or responsibility for the accuracy, completeness, or usefulness of any information, apparatus, product, or process disclosed, or represents that its use would not infringe privately owned rights. Reference herein to any specific commercial product, process, or service by trade name, trademark, manufacturer, or otherwise does not necessarily constitute or imply its endorsement, recommendation, or favoring by the United States Government or any agency thereof. The views and opinions of authors expressed herein do not necessarily state or reflect those of the United States Government or any agency thereof. 


\section{Abstract}

\section{Context of the Research Project}

Assemblies of granular materials behave differently when they are flowing rapidly, from when they are slowly deforming. The behavior of rapidly flowing granular materials, where the particle-particle interactions occur largely through binary collisions, is commonly related to the properties of the constituent particles through the kinetic theory of granular materials. The same cannot be said for slowly moving or static assemblies of granular materials, where enduring contacts between particles are prevalent. For instance, a continuum description of the yield characteristics of dense assemblies of particles in the quasistatic flow regime cannot be written explicitly on the basis of particle properties, even for cohesionless particles. Continuum models for this regime have been proposed and applied, but these models typically assume that the assembly is at incipient yield and they are expressed in terms of the yield function, which we do not yet know how to express in terms of particle-level properties. The description of the continuum rheology in the intermediate regime is even less understood. Yet, many practically important flows in nature and in a wide range of technological applications occur in the dense flow regime and at the transition between dilute and dense regimes; the lack of validated continuum rheological models for particle assemblies in these regimes limits predictive modeling of such flows. This research project is aimed at developing such rheological models.

\section{Project Overview}

The overall objective of this project was to construct: (i) validated continuum models for frictional flow of granular materials in the quasi-static and intermediate regimes, including transitions from stagnant-to-shearing, quasistatic-to-intermediate and intermediate-toinertial regimes; and (ii) closures for the parameters appearing in the models in terms of particle scale properties. Towards this end, we first sought a better physical understanding of the mean stress levels and fluctuations accompanying particle flows in the presence of enduring contact between particles. We then developed constitutive models for (a) frictional, quasi-static flow that capture the essential features of the elastic and plastic regimes and the transition between them and (b) the intermediate regime that smoothly bridge the quasistatic and inertial flow regimes. The project involved a combination of experiments, Discrete 
Element Method (DEM) simulations, theory and continuum model analysis. Experimental data on mean stresses and stress fluctuations have been gathered in three different geometries. Most data were generated in a cylindrical aerated Couette cell, for different particles, fluidizing gas flow rates and overburdens. Additional experimental data were collected by shearing powders in the Jenike 2D geometry and a circular flat-bottom bin in which both flow rates and stresses were measured. DEM simulations of particle assemblies subjected to various model flows have been conducted to quantify stress and strain-rate fluctuations and establish links between particle scale properties and macroscopic rheological behavior over the entire range of flow conditions where enduring contact between particles persist. Theoretical work focused on developing continuum models that bridge the regimes of flow. The theoretical models and DEM simulation results have been validated against experimental data.

\section{Significant Accomplishments}

We experimentally probed the rheology of a variety of granular materials in the quasi-static and intermediate regimes using Jenike-type and Couette shear cells. We found the rheological characteristics to be robust, with all materials exhibiting an intermediate regime with similar shear rate dependence of the stresses. A simple stress model capturing this rate dependence was developed. DEM simulations of intermediate regime flows supported a model of this form and furthermore gave rise to a model bridging all three regimes. Based on DEM simulations of quasi-static flows, a dynamic stress model was developed that is capable of describing complex flow behaviors under general deformations. An order parameter model for dense inertial flows is also developed. Finally, hopper flow simulations reveal that the quasi-static, intermediate, and inertial regimes can coexist, underscoring the value of developing and bridging constitutive models in the three regimes. 


\section{Chapter 1}

\section{PROJECT MILESTONES AND STATUS}

The eight milestones for this project are listed in the Table below. Each has been completed in a timely manner. The results of the work are presented in the following chapter, with additional information found in the appendices at the end of the report. 


\begin{tabular}{|c|c|c|c|}
\hline $\begin{array}{l}\text { Milestone } \\
\quad \#\end{array}$ & Description & $\begin{array}{l}\text { Completion } \\
\text { Date }\end{array}$ & $\begin{array}{l}\text { Revised } \\
\text { Date }\end{array}$ \\
\hline 1 & $\begin{array}{l}\text { Submission of the Project management plan } \\
\text { Status: Milestone achieved in a timely manner }\end{array}$ & $4 / 30 / 07$ & \\
\hline 2 & $\begin{array}{l}\text { Complete critical analysis of DEM models and boundary } \\
\text { effect through comparison of DEM results and experimental } \\
\text { data under constant volume conditions } \\
\text { Status: Milestone achieved in a timely manner }\end{array}$ & $10 / 31 / 07$ & \\
\hline 3 & $\begin{array}{l}\text { Complete critical analysis of DEM models and boundary } \\
\text { effect through comparison of DEM results and experimental } \\
\text { data under constant applied normal stress conditions } \\
\text { Status: Milestone achieved in a timely manner }\end{array}$ & $3 / 31 / 08$ & \\
\hline 4 & $\begin{array}{l}\text { Complete assessment of existing hypoplastic and order- } \\
\text { parameter based continum models using data from DEM } \\
\text { simulations of uniaxial and triaxial tests, plane shear com- } \\
\text { putational data on stress evolution and experimental data } \\
\text { Status: Milestone achieved in a timely manner }\end{array}$ & $10 / 31 / 08$ & \\
\hline 5 & $\begin{array}{l}\text { Complete work on refinement of hypoplastic and order- } \\
\text { parameter based rheological model for intermediate regime, } \\
\text { using data generated through experiments and simulations } \\
\text { or define the essential elements of a new and more funda- } \\
\text { mental model } \\
\text { Status: Milestone achieved in a timely manner }\end{array}$ & $3 / 31 / 09$ & \\
\hline 6 & $\begin{array}{l}\text { Complete MFIX simulations of flow in the Couette cell and } \\
\text { compare against experimental data } \\
\text { Status: Milestone achieved in a timely manner }\end{array}$ & $10 / 31 / 09$ & $3 / 31 / 10$ \\
\hline 7 & $\begin{array}{l}\text { Complete MFIX simulations of discharge from flat-bottomed } \\
\text { silo and compare against experimental data. } \\
\text { Status: Milestone achieved in a timely manner }\end{array}$ & $3 / 31 / 10$ & $6 / 31 / 10$ \\
\hline 8 & $\begin{array}{l}\text { Complete Final Report } \\
\text { Status: Milestone achieved in a timely manner }\end{array}$ & $4 / 30 / 10$ & $4 / 30 / 11$ \\
\hline
\end{tabular}




\section{Chapter 2}

\section{SUMMARY OF WORK}

The experimental part of our research entails two main geometries (i) shearing of a granular material in a Jenike-type cell and (ii) experiments in a Couette device where the material is sheared in a narrow gap between two concentric cylinders. We measured normal stresses directly on stationary and moving surfaces using a modified version of a stress transducer equipped with a radio transmitter, average shear stresses from the knowledge of the total torque and solid fraction distributions using a capacitance probe. The ultimate goal was to model the same geometries and to compare measured and computed values.

In the Jenike-type experimental apparatus we measured stress transmission through a granular layer. We were able to reproduce some results from DEM simulations, validating the DEM approach used in other parts of this project. The net result is that, unlike in fluids, stresses are transmitted only partially when the layer of granules is not sheared; shearing of the layer results in an increase in the transmitted stress and in significant spatial and temporal fluctuations that depend on particle size, shape and the overall thickness of the layer. This is an important finding when one compares theoretical predictions and experimental results as one can expect only approximate fit and only when conditions are identical.

We further studied the flow of dry, frictional powders in the quasi-static and intermediate regimes using the Couette geometry. In order to obtain reproducible results, it was necessary to allow the material an additional degree of freedom by superimposing a very slow axial motion on the radial shearing. We measured normal and shear stresses and extracted a constitutive equation valid in both regimes by calculating the ratio of shear-to-normal stresses. We found that this ratio reproduced the static angle of internal friction only at very low shear rates. At increasing shearing rates, the ratio of the stresses increases due to collisions between particles that sustain loads. We showed that a modified Couette device with slow axial flow superimposed on the shearing motion can be used as a powder "rheometer" to characterize its flow properties. The stress ratio obtained from the above measurements contains a term characterizing "solid"-like behavior and an additional term that captures some "fluid"-like properties at higher shear rates. Finally, we used the experimentally obtained constitutive equation (stress ratio) in a continuum model using Cauchy's equations of momentum to predict flow in the Couette device. 
Solid volume fraction was measured using the capacitance probe available commercially from Capacitec, Inc. The commercial instrument is made to measure small distances with great precision but it was modified for the present experiment to detect solid fraction. The knowledge of the porosity in the shearing bed and its variation is crucial to understanding the behavior of the material. Mounted on the stationary wall, the probe measures the change in solid fraction in a semi-spherical volume of about $3 \mathrm{~mm}$ diameter. To reach inside the shearing gap, the probe is physically pushed inside, parallel to itself in equal steps of $1 \mathrm{~mm}$ using a caliper. There is a significant difference in the behavior of the shearing layer in the batch and the continuous-flow Couette cells. To investigate the reason for such a change we measured the solid fraction in both batch and continuous modes and found that the different behavior is due to slight, localized, decreases in solid fraction that permit particles to move relative to one another. We also found that changes in solid fraction are different in stagnant layers where they increase as a function of shear rate and in sheared layers where they decrease as a function of increasing shear. Based on this difference, we developed a method to measure the thickness of the active layer in a flowing powder.

To ensure the suitability of DEM simulations for investigating granular rheology in the quasistatic and intermediate regimes, we simulated flow in a Jenike cell and compared the results with those of the experiments described in the previous section. We found good agreement in the measurements of stress and stress fluctuations between the two; the frequency of the fluctuations did differ slightly, likely due to the sampling frequency or asphericity of particles in the experiments. Furthermore, the simulations were able to capture the temporal fluctuations and spatial heterogeneity observed in the experiments. These findings supported the use of DEM for the computational part of the rheological study.

We performed both steady simple shear and dynamic simple shear DEM simulations in the quasi-static regime. Pressure and shear stress were correlated with two microstructural variables - the coordination number and the fabric tensor of contacts. The evolution of the microstructure over time was then described by a pair of ordinary differential equations. The stress-microstructure relations along with the microstructure evolution equations constitute a dynamic rheological model for quasi-static granular flows. This simple model is capable of describing complex flow behaviors for which most other models fail. We showed, for example, that this model is capable of predicting compaction of a granular bed during constant-pressure, oscillatory shear.

We next performed steady simple shear DEM simulations over a wide range of volume fractions and shear rates - extending into all three flow regimes - for a variety of particle friction coefficients. We identified a jamming point (or critical volume fraction) that depends on the friction coefficient and that plays a major role in governing rheological behavior. We then found a universal scaling for collapsing stress data with respect to the distance to this jamming point. We used this collapse to propose a steady-state stress model that spans over all three regimes. This model compares favorably with the recent and successful inertial number models.

We performed DEM simulations of wall bounded shear flow in the presence of axial discharge that approximately model the CCNY Couette cell experiment and found that the simulations capture the correct qualitative trends of shear stress with shear rate corresponding to 
transition from quasi-static to intermediate regime behavior. The ratio of shear to normal stress also manifests the same transition and intermediate behavior, and it remains almost constant (approximately equal to the apparent friction coefficient of the glass beads) for lower shear rates. In the absence of axial flow (in batch mode operation) the constitutive behavior corresponding to quasi-static regime was reproduced. In summary this regime transition (from quasi-static to intermediate regime) is induced due to the secondary axial flow in the continuous mode of operation of Couette device.

In order to characterize the extent of the intermediate regime, we performed a comprehensive set of DEM simulations of homogeneously sheared granular flow and established a detailed regime map. We also characterized the granular 'phase' transition in dense granular flows by extracting the order parameter (OP) from DEM and discovered a third stable granular 'phase' that is neither completely fluidlike nor completely solidlike. We proposed a refined order parameter model, which has the capability to accurately predict the granular stress up to a solid volume fraction of 0.57 . However, this model based on kinetic theory fails to capture the correct stress-strain rate scaling in the intermediate regime. We tested the performance of four other constitutive models in the intermediate regime and found that none of these models captures the correct scaling of stress with strain rate.

We performed DEM simulations of discharge from silos and characterized the spatial extent of different flow regimes. We found that the intermediate regime spans a significant spatial region of the granular flow in silos. Our study also revealed that the spatial extent of the intermediate regime is directly correlated with errors in the stress predicted by existing constitutive models in the MFIX two-fluid code, which were compared with DEM stress values. The difficulty in accurately predicting stresses in the intermediate regime directly contributes to differences in the discharge velocity profile and discharge rate.

Analysis of DEM simulations of homogeneously sheared granular flow in the intermediate regime reveals that the dominant contact stress is responsible for the characteristic stressstrain scaling in the intermediate regime, and modeling this contribution is the main challenge. To address this shortcoming we propose a new model for the contact stress based on the 'relative acceleration' concept. The average contact stress is related to the (i) PDF (probability density function) of contact force, (ii) average coordination number, (iii) the pair correlation function and (iv) fabric tensor. Preliminary results show that this RA-based contact stress model successfully captures the stress-strain scaling even in the intermediate regime of the granular flows.

In summary, we have employed a combined experimental and computational approach to the investigation of the rheology of dense granular materials. We experimentally probed the rheology of a variety of granular materials in the quasi-static and intermediate regimes using Jenike-type and Couette shear cells. We found the rheological characteristics to be robust, with all materials exhibiting an intermediate regime with similar shear rate dependence of the stresses. A simple stress model capturing this rate dependence was developed. DEM simulations of intermediate regime flows supported a model of this form and furthermore gave rise to a model bridging all three regimes. Based on DEM simulations of quasi-static flows, a dynamic stress model was developed that is capable of describing complex flow behaviors under general deformations. An order parameter model for dense inertial flows is also 
developed. Finally, hopper flow simulations reveal that the quasi-static, intermediate, and inertial regimes can coexist, underscoring the value of developing and bridging constitutive models in the three regimes. 


\section{Chapter 3}

\section{TECHNICAL PROGRESS}

This section highlights the technical accomplishments of the research performed during the period 1 April 2007 - 31 March 2011. Additional details can be found in the manuscripts resulting from this research, which are attached to this document as appendices.

\subsection{Work performed at City College}

We describe in this section the experimental part of our research. It entails two main geometries (i) shearing a granular material in a Jenike-type cell at different shearing rates and (ii) experiments in a Couette device where the material is sheared in a narrow gap between two concentric cylinders. We measure normal stresses directly on stationary and moving surfaces, shear stresses from the knowledge of the total torque and solid fraction distributions using a capacitance probe. The ultimate goal is to model the same geometries by simulation and to compare measured and computed values for all parameters.

\subsubsection{Transmission of stresses in static and sheared granular beds}

The main objective is to probe through a combination of experiments and discrete element method (DEM) simulations stresses transmitted across a sheared granular layer. Towards this end, we employed the classical Jenike cell (commonly used to measure internal and wall friction coefficients), where we added normal force (stress) transducers on the shearing bottom wall and applied known normal stresses to the upper surface of the granular layer. Using this experimental technique we compared the normal stress applied to the top surface of the particle assembly with stresses recorded by the normal stress sensors on the bottom. Particles of different sizes were tested in layers of different thicknesses and at different shearing rates and the transmitted stresses were recorded at several locations on the bottom surface.

While the selection of a sensor system appears to be a simple undertaking, this is not 


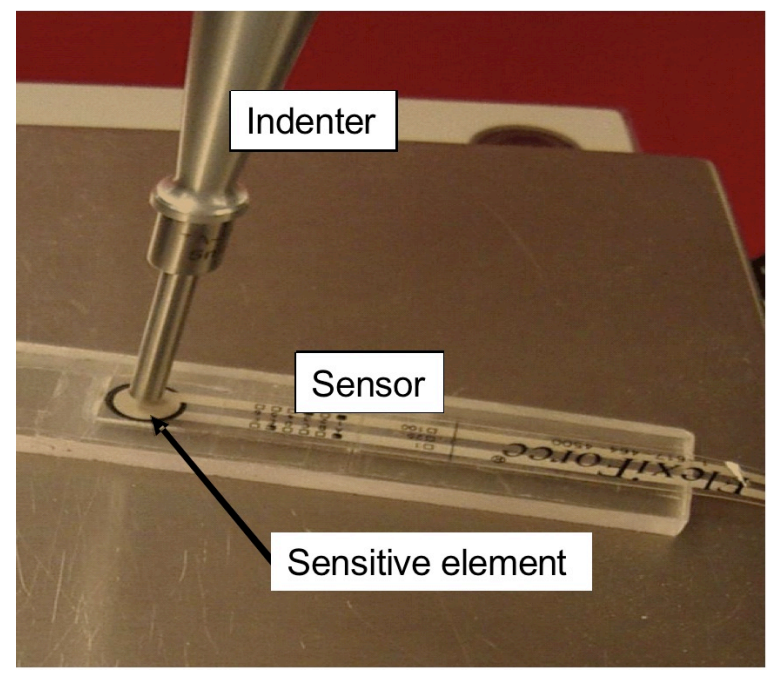

Figure 3.1: Normal stress sensor and mode of calibration

the case for stress sensors used in granular systems. Such instrumentation is not available commercially and there are two ways of solving the problem: either construct the system "in house" or re-engineer an existing system developed for a different application. We employed the second route after failing to develop our own sensors and being unable to use several other systems developed for different purposes by other vendors. Two systems, manufactured by Tekscan, Inc., one with and the other without remote sensing, were selected; a picture of the sensor is shown in Figure 3.1. Since these sensors were developed for a different application (namely, sensing forces in athletes shoes and on car seats) and are made of very fine, interwoven plastic and conductive layers, it was necessary to apply a protective film and to re-calibrate the unit for each experiment. To perform the calibration, the sensor was mounted on a removable Lucite substrate to enable the transport of the calibrated device from calibration to the experimental unit.

Calibration was performed in the instrument depicted in Figure 3.1 where the calibration pressure was applied on top by an Instron-type indenter (Texture Analyzer) and the sensor was situated on the Lucite strip, as shown. The force applied by the indenter was measured by the Texture Analyzer and divided by the active area of the sensor (area delimited by the black circle, approximately $1.1 \mathrm{~cm}^{2}$ ) to yield the appropriate applied normal stress. The indenter was chosen to match the size of the sensitive element and the calibration procedure was repeated several times before and during experiments. In this way, the normal stress applied on the sensor was transformed into a DC voltage that could be simply monitored by a data acquisition system controlled by a computer. Following calibration, the stress sensor and the Lucite strip were introduced into the powder flow by cutting an appropriate groove into the boundary (wall) so as to keep the sensor flush with the surface. Since the thickness of the sensor is less the $0.1 \mathrm{~mm}$ (see Figure 3.1) there is practically no interference from the sensor to the moving powder layer.

A first set of experiments were carried out in a conventional Jenike shear cell. A schematic representation of the granular layer with a normal stress applied to the top and a sensor 


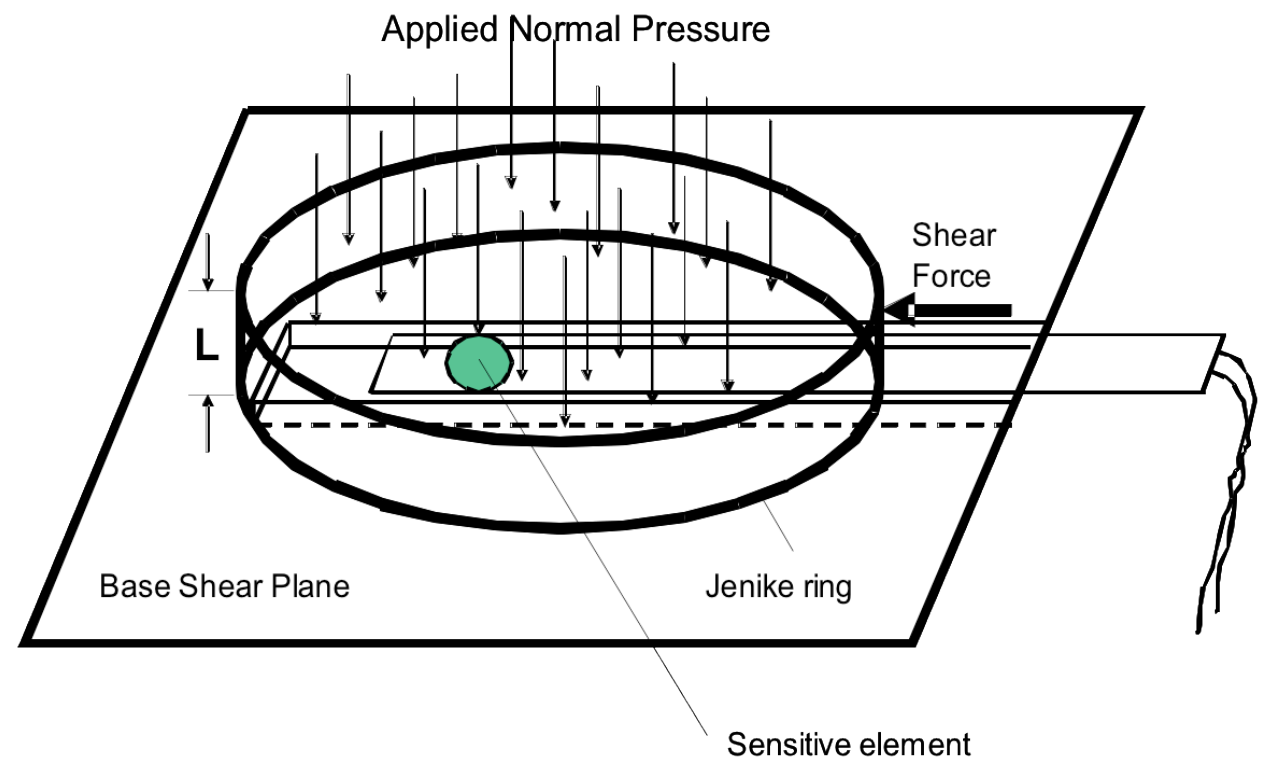

Figure 3.2: Schematic of the Jenike-cell tester

mounted at the bottom is shown in Figure 3.2. The load on the top was applied using the classical Jenike "frame" and the circular cover with a raised point in the center to assure that the stress is evenly distributed as shown in Figure 3.3a. Figures 3.3b and 3.3c show the empty cell with the Lucite strip and the pressure sensor mounted on it. Two smooth (aluminum and Lucite) walls and several "rough" walls were used by gluing different grainsize sand paper on the wall of the device as seen in Figure 3.3b. One or more rings of the device were used in order to increase the thickness of the granular layer. The sensor was located $7 \mathrm{~mm}$ off the central cord of the cell perpendicular to the direction of shearing at zero displacement as shown in Figure 3.3c. During a typical experiment, the cell moved at $3.2 \mathrm{~mm} /$ minute over a distance of about $2 \mathrm{~cm}$.

A variety of particles, spherical and odd-shaped, cohesive and free flowing were tested; a sample is given in Table 3.1. The specific examples of particles chosen for discussion in this report were imposed by the limitations of the DEM simulation that require very long times, of the order of weeks, for small particles. The overall behavior of the transmitted stress for the other particles is qualitatively similar to the examples presented below.

Figure 3.4 gives a typical response curve, i.e., transmitted normal stress (in Psi) vs. time (in seconds), for a bed of $2 \mathrm{~mm}$ glass beads in a $2.6 \mathrm{~cm}$ thick layer. There are several stages in applying the load: in the empty cell, the measured stress was zero as it should be "no load" in the figure). A normal stress of one Psi was then applied to the static bed and, as seen in the figure, the sensor only "recorded" approximately 0.2 Psi. As the bed was sheared in the subsequent phase, the stress increased and started to fluctuate around the 1 Psi average value. As the normal stress on the top cover was increased to 2 psi, the average value measured by the sensor also increased and fluctuated around two Psi. as long as shearing was applied. The fluctuations are clearly large ( $\sim 50 \%$ of the average stress). At the point where the shearing stopped, the bed "froze" at about 2 Psi and the stress decreased to zero 

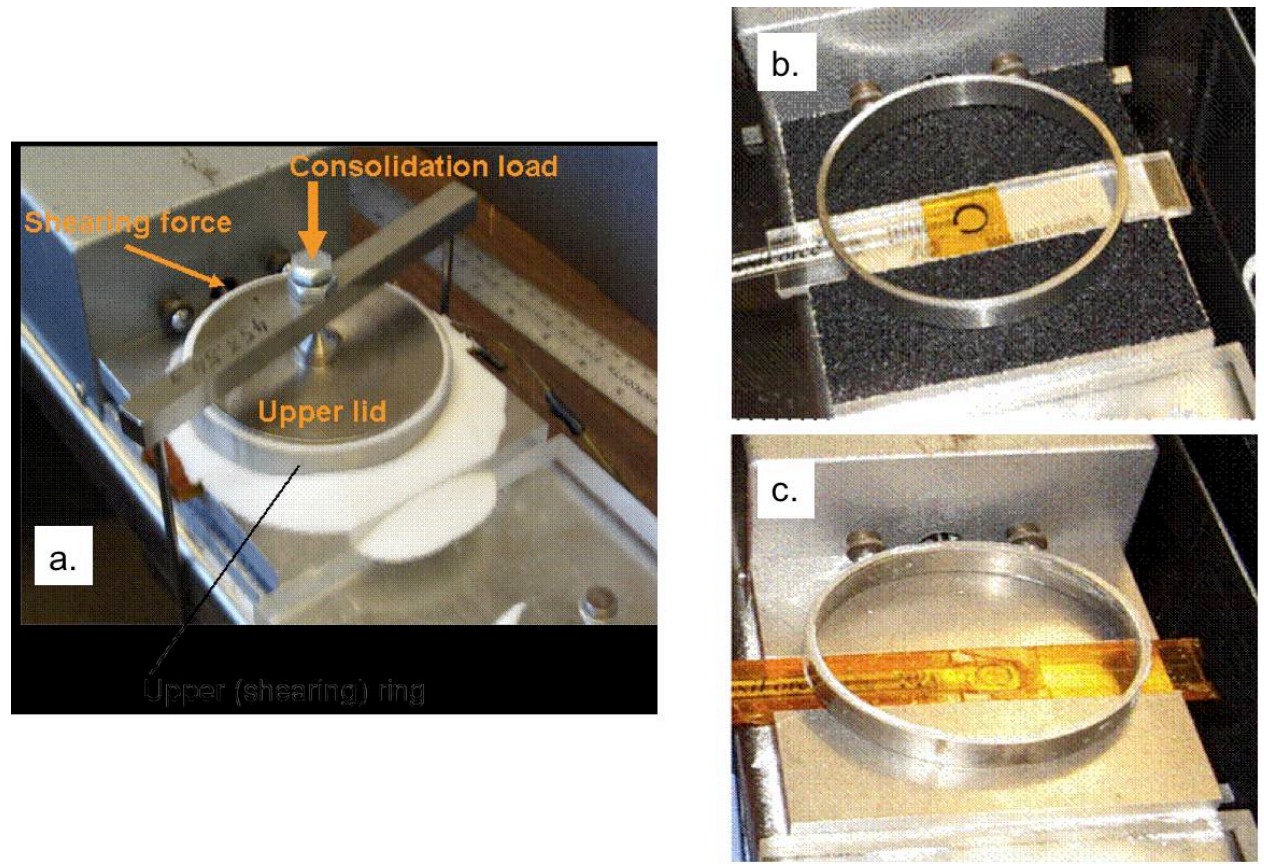

Figure 3.3: Picture of the sensor arrangement in the Jenike cell experiment

Table 3.1: Materials studied in the Jenike cell

\begin{tabular}{|c|c|c|c|c|}
\hline $\begin{array}{c}\text { Particle } \\
\text { size }[\mathbf{m m}]\end{array}$ & Material & $\begin{array}{c}\text { Applied normal } \\
\text { stress }[\mathbf{k P a}]\end{array}$ & $\begin{array}{c}\text { Layer thickness } \\
L[\mathbf{c m}]\end{array}$ & Remarks \\
\hline 5.0 & glass & $9.6,19.2$ & $1.6,2.6,3.0,6.0$ & spherical \\
\hline 3.0 & glass & $9.6,19.2,28.8$ & $1.6,2.6$ & spherical \\
\hline \multirow{2}{*}{4.0} & polyethylene & 9.6 & 6.0 & aspherical \\
& polyethylene & 9.6 & 6.0 & aspherical and rough \\
\hline \multirow{2}{*}{2.0} & glass & $9.6,19.2$ & $1.6,2.6,3.0,4.7$ & spherical \\
& cast steel & $9.6,19.2$ & $1.6,3.0$ & \\
\hline \multirow{2}{*}{1.5} & glass & 19.2 & 1.5 & \\
& glass & 9.6 & $1.6,2.6$ & oddly shaped \\
& glass & 9.6 & $1.6,3.0,4.7,6.4$ & aspherical \\
& crushed glass & 9.6 & $1.6,3.0,4.7,6.4$ & shredded tire \\
\hline
\end{tabular}




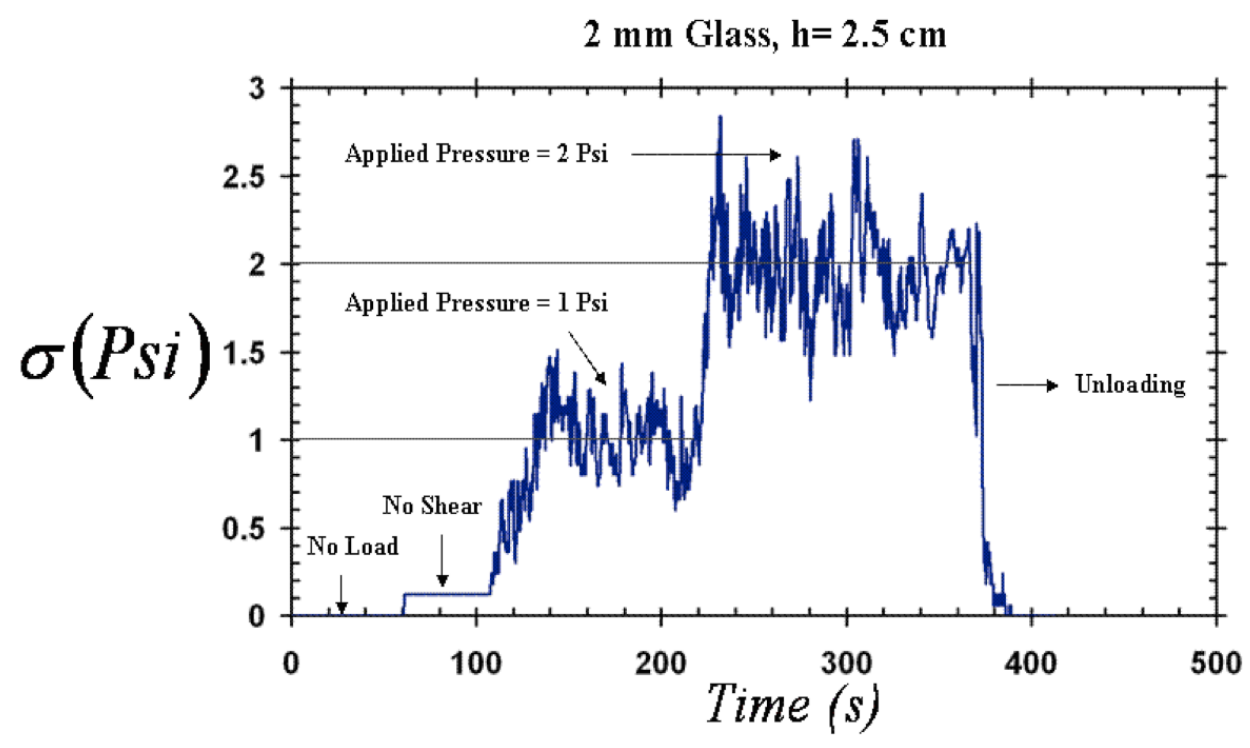

Figure 3.4: Stress response to an applied pressure on the sheared layer

as the layer was unloaded.

Figure 3.5 presents additional results using larger particles: $5 \mathrm{~mm}$ glass beads and an applied normal stress of 1 Psi. While the average stress during shearing was only $5 \%$ higher than the applied normal stress, stress fluctuations were larger compared to the smaller particles (see Figure 3.4), about $70 \%$ of the average value. Results in Figures 3.4 and 3.5 are somewhat similar in that the measured stress is always smaller then the applied stress under static conditions and we always observe "freezing" of the material once the shearing stops. While shearing is in progress, the applied and measured stresses become more or less equal. We note that the measured stress fluctuates, sometimes significantly, depending on particle size. This is mainly due to the fact that, since the cell has a constant volume, the number of larger particles is significantly smaller then the number of smaller particles $(5 \mathrm{~mm}$ in diameter as compared to $2 \mathrm{~mm}$ in diameter). Consequently when the same load is applied, each larger particle carries a larger portion of the load. This larger portion is then transmitted to the transducer that either senses the presence of the particle (when the particles roles over it) or not (when the particle rolls off the sensor), giving rise to fluctuations that are smaller with smaller particles and correspondingly larger with large particles. Care was taken to use "small enough" particles so that the sensor can "see" a sufficiently large number of them at any given time: about 25 in the case of $2 \mathrm{~mm}$ in diameter spheres. Results with $5 \mathrm{~mm}$ particles are less reliable since the sensor can "see" only about 5-7 particles at one time. This may have also resulted in larger fluctuations of the measured stress.

In summary, we found that the normal stress was transmitted to the sensor mounted on the base mostly unaltered through thin layers of non-cohesive powders when the layers were in shear. The normal stress was not transmitted to the base uniformly in static layers as the material "froze" depending on its history: an initially unstressed layer transmitted to the base only a portion of the normal stress while a previously stressed material maintained its 


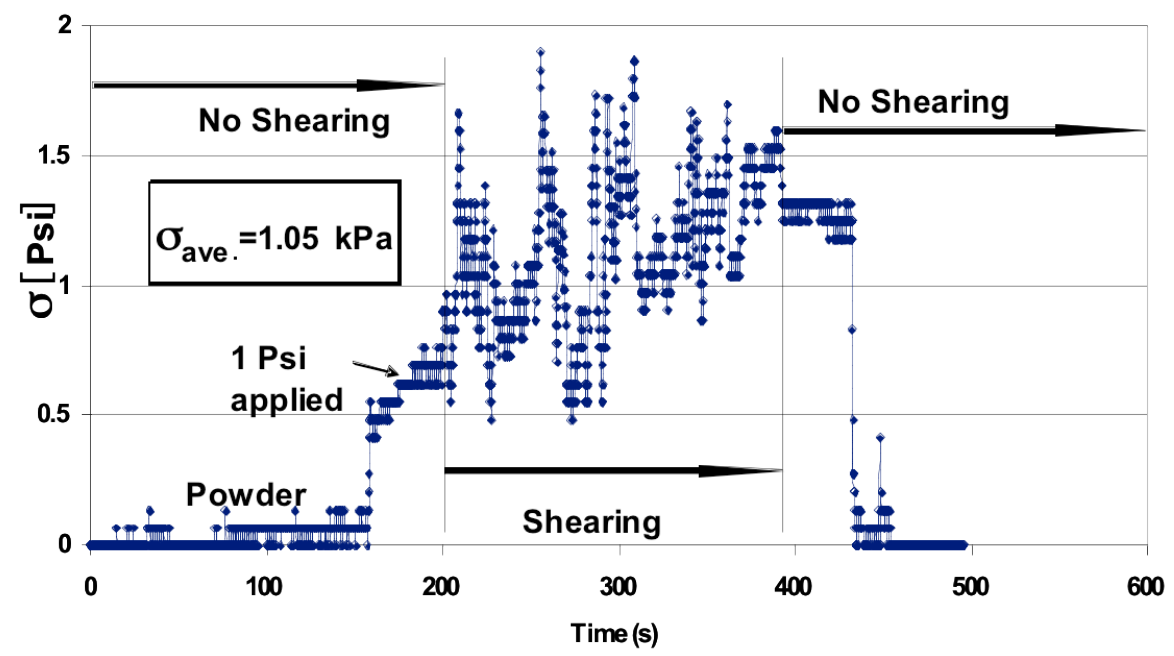

Figure 3.5: Constant pressure applied to $5 \mathrm{~mm}$ diameter glass beads

internal stress levels until it was unloaded.

The advantage of the above experiments is that they were performed in a known geometry that of the classical Jenike cell where all parameters are standard. The great disadvantage is that there is only one, pre-set, velocity of the split cell and therefore only one shear rate could be applied. To overcome this limitation, an instrument (henceforth referred to as the "fast" Jenike cell since the only difference compared to a conventional shear cell is its capability of faster shearing) was specially built to enable shearing of particles in the Jenike geometry at higher rates. The instrument was constructed as an addition to the moving arm of a tableting machine. A picture of the cell with the lower part of the driving mechanism and the detail of the cell itself with the stress sensor mounted on the bottom are shown in Figure 3.6. The dimensions of the cell and the stress sensor are identical to those used in the traditional Jenike test. The loading of the cell with the normal force is also similar except that the weight is added at the top of the device instead of using the Jenike "frame". The cell executes a back-and-forth movement from the initial position shown in the detail in Figure 3.6. After a short pause, the back-and-forth movement is repeated. Usually, up to 4-5 cycles were performed during one experiment. The unit has adjustable speed from approximately $1.5 \mathrm{~mm} / \mathrm{s}(\sim 90 \mathrm{~mm} / \mathrm{min}$ as compared to the traditional Jenike cell at 3.2 $\mathrm{mm} / \mathrm{min}$ ) to approximately $150 \mathrm{~mm} / \mathrm{s}$. Several experiments were performed at different speeds and with different particles, employing a single sensor in some and multiple sensors in the rest of the experiments. Several examples are given below for a cell depth of 60 $\mathrm{mm}$.

A typical experimental result is shown in Figure 3.7. A normal stress of 1 psi was applied, resulting in a jump in the sensed stress to about 0.4 psi. This behavior is similar to the one seen in the (conventional) slow Jenike cell (see Figures 3.4 and 3.5). Upon shearing at 


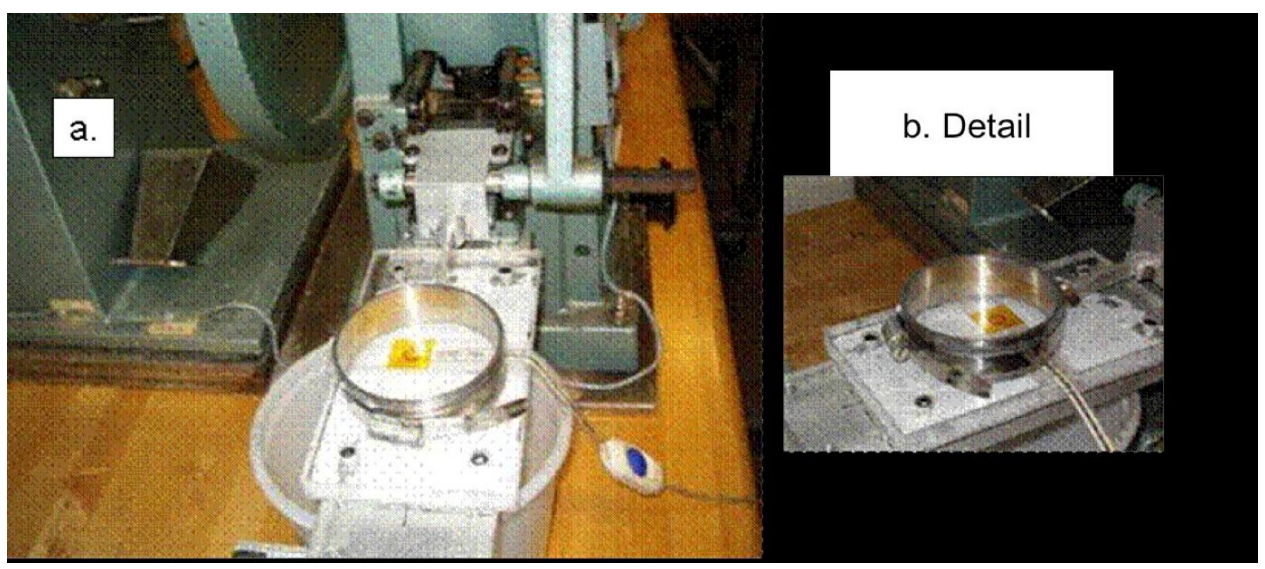

Figure 3.6: Picture and detail of the "fast" Jenike cell

$15 \mathrm{~mm} / \mathrm{s}$, the sensed stress fluctuated significantly with an average of about $41 \%$ above the applied load. This additional load is probably due to the inertia of the material as it moves at relatively high speed inside the cell.

The spatial non-uniformity of stress transmitted to the base was studied through experiments where two sensor locations were used: (a) identical to the position in the conventional slow Jenike cell, i.e., $7 \mathrm{~mm}$ off-center (as shown in Figure 3.6) and (b) two sensors mounted as depicted in Figure 3.8. The starting and ending positions of the sensors are $2.35 \mathrm{~cm}$ and 1.9 $\mathrm{cm}$ from the walls of the cell while the stroke of the movement is $5.25 \mathrm{~cm}$.

Figure 3.9 depict sample results obtained with the two sensor assembly shown in Figure 3.8. The stress fluctuations were clearly pronounced and were much larger than what was observed earlier for the case of slow shear (see Figure 3.5). It appears reasonable to conclude that the fluctuations in dense assemblies increased with increasing shear rate (compare Figures 3.5 and 3.9). Note that during the time when the cell is not under shear between cycles, the stresses recorded by the two sensors were essentially frozen; this stress level at a given location on the base may be above or below the average value. It also appears that certain locations may record mostly lower stress levels than others. It is also apparent from Figure 3.9 that the stress transmitted to the base was spatially inhomogeneous as the stresses measured at different locations were quite different and also showed different fluctuations in time.

\subsubsection{Shearing of a granular material in a Couette cell}

In this section, we present experiments to study the transition of powder from slow to moderately fast flows in a Couette cell where a dry powder is sheared between two concentric cylinders. A schematic representation of the Couette device used during the present investigation is shown in Figure 3.10 with details of the sensors and the rotating cylinder. Dimensions in the figure are given in inches.

The vertical shear gap forms between the rotating and the stationary cylinders and both its width and height can be adjusted by appropriate choice of the radius and height of the 


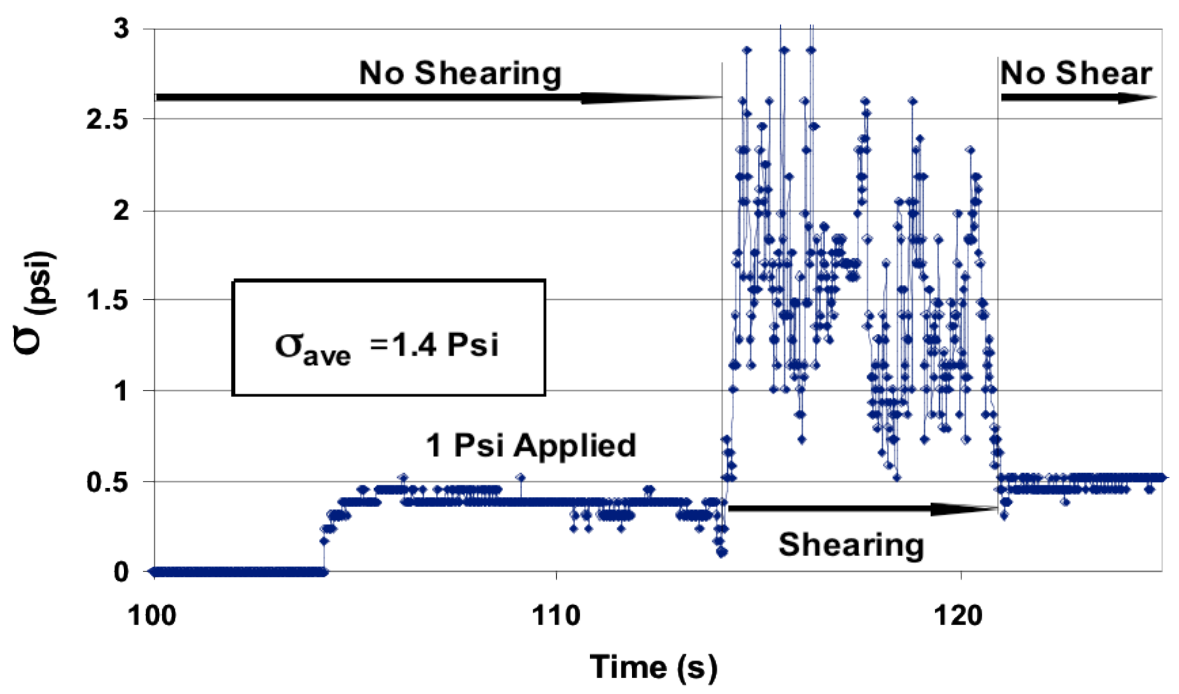

Figure 3.7: Typical stress transmission experiment at high shear rate in the "fast" Jenike cell

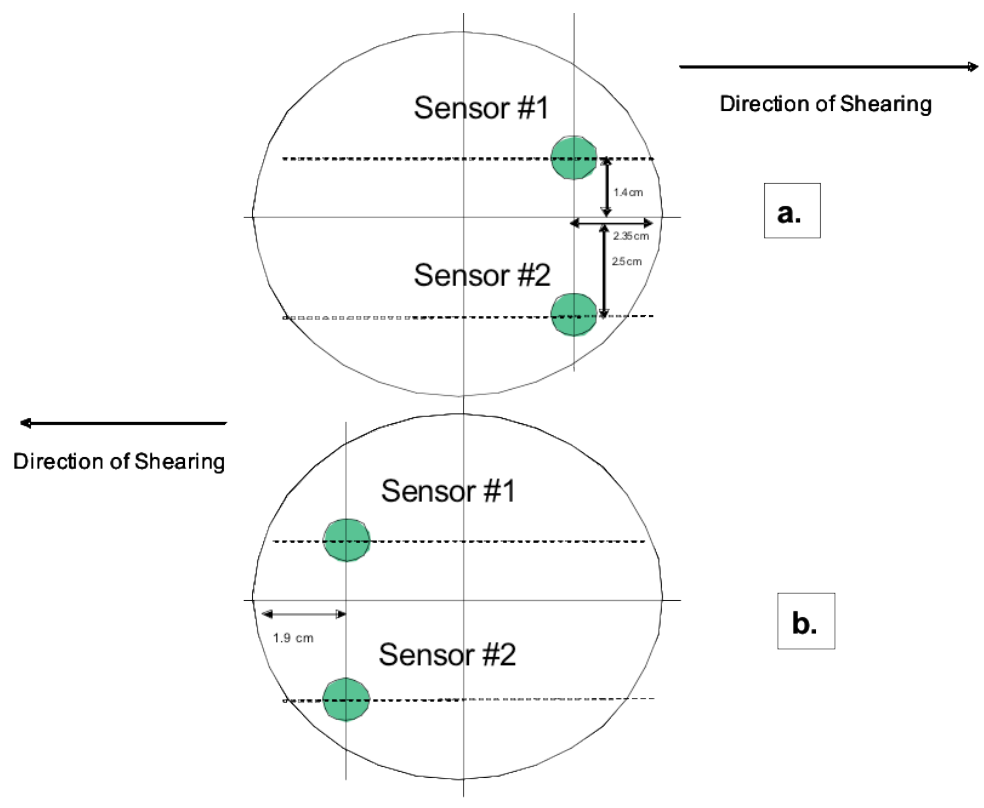

Figure 3.8: Position of sensors in "fast Jenike" cell 


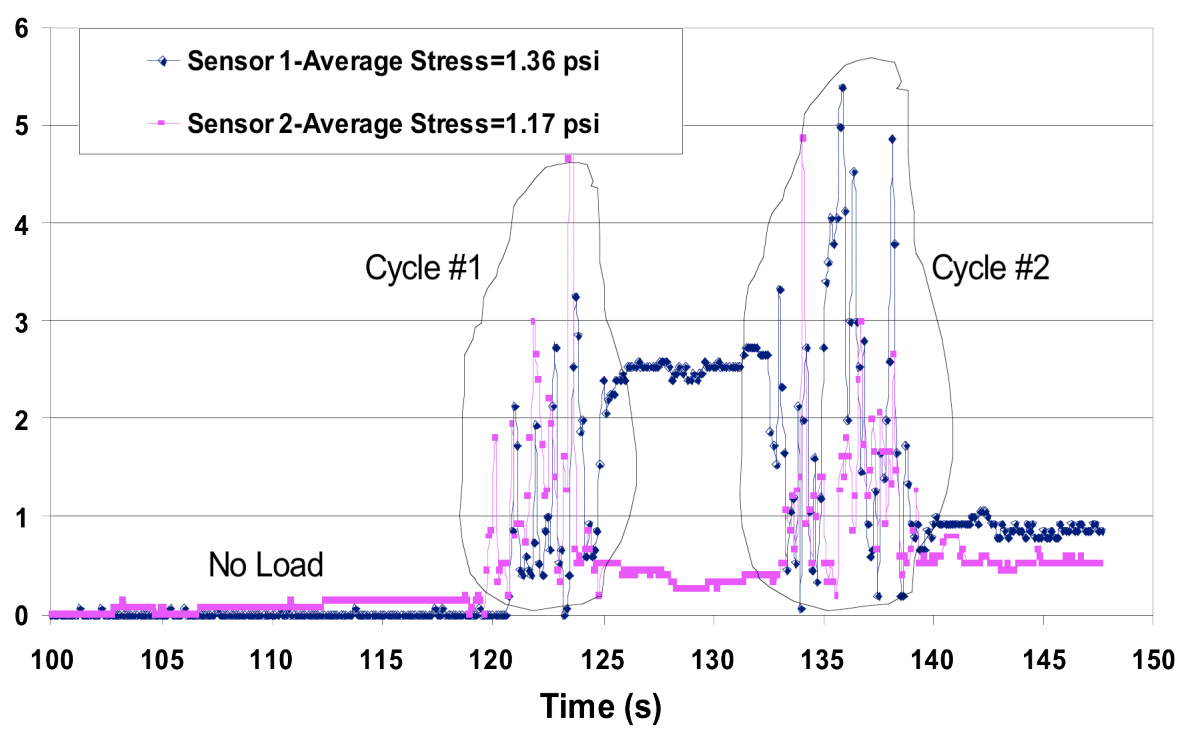

Figure 3.9: Measurement in the "fast Jenike cell" using two sensors: sensor position shown in Figure 3.8. One Psi applied to a bed of $5 \mathrm{~mm}$ diameter glass beads, sheared at $16 \mathrm{~mm} / \mathrm{sec}$.

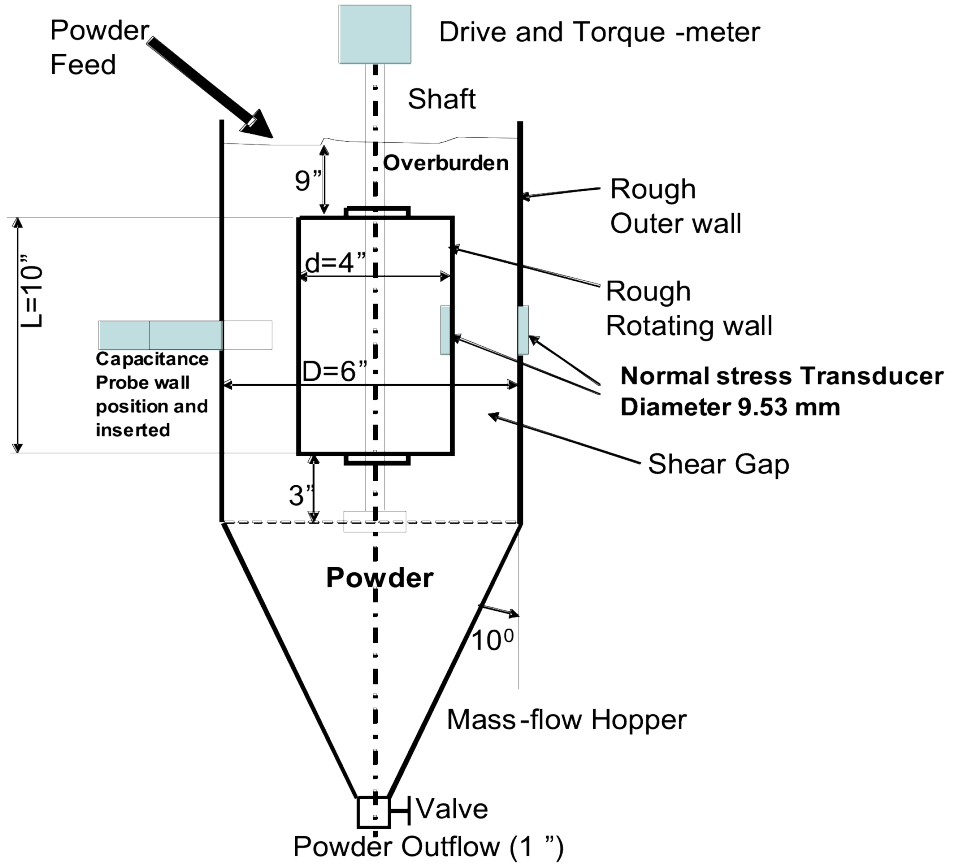

Figure 3.10: Schematic representation of the axial-flow Couette device used during experimentation 
rotating cylinder. The material is fed from above using a vibratory feeder (not shown in the figure) and is discharged by a screw-in-cylinder metering device (also not shown) that transfers the material to a precision balance for flow rate measurement. The walls of the Couette are made rough by gluing sand paper on the shearing surfaces as shown in the figure. The roughness of the walls is chosen to match or exceed the coefficient of internal friction of the material thereby attempting to assure a non-slip boundary condition. By closing the discharge valve at the bottom of the device, the Couette device can be operated in batch mode or, by feeding and removing material to achieve a steady state vertical flow, one can operate the device continuously. The material above the rotating cylinder (denoted overburden in the figure) is stationary and only provides dead weight to the shearing layer.

Experiments in the Couette device were performed without (batch) and with axial flow (continuous), and several depths of overburden to control the pressure in the shearing gap. Normal stresses were measured on both the outer, stationary wall as well as the inner, rotating (shearing) wall of the device as shown in Figure 3.10. Shear stresses were measured indirectly and recalculated from the torque on the rotating cylinder. The sketch in Figure 3.10 also shows the capacitance probe to measure solid fraction but results from these measurements are discussed in the next section. One should note that all measurements, stresses and solid fraction are performed at the same height in the granular bed, approximately in the middle of the shearing gap.

Experiments were performed with an inner cylinder of 4 in. diameter and a stationary cylinder of 6 in. so that the shearing gap was approximately 1 in. as shown in Figure 3.10. Three different curves of the same kind are reproduced in Figure 3.11a for small $(0.1 \mathrm{~mm})$, medium $(0.5 \mathrm{~mm})$ and large $(1.0 \mathrm{~mm})$ diameter glass particles. One can easily see the different rates at which these particles pack at a constant shearing rate (15 RPM of the rotating cylinder): larger particles pack at a much higher rate due to their enhanced capability of bridging the relatively small shear gap. It appears from Figure 3.11a that the trends shown would continue but in reality, the range of the torque-meter is exceeded and the experiment has to be stopped. Figure 3.11b depicts similar behavior for the $0.1 \mathrm{~mm}$ in diameter glass beads but for a much wider shear gap (2.25 in.). Here, we calculated the average shear stress using Equation (1) and the linear speed is calculated from the angular velocity of the cylinder.

$$
\tau_{\text {ave }}=T / 2 \pi R^{2} L
$$

where $T$ is the total torque, and $D=2 R$ and $L$ are the dimensions of the rotating cylinder. As seen in the figure, the torque increases significantly during the packing phase but does not exceed the range of the torque-measuring device. This is mainly due to the shorter cylinder used and by the relatively large shear gap that allows some additional movement of the granular layer in the radial direction. It appears from the figure that the torque has reached its steady state value at the lowest shear rate (blue curve) and we use this experiment to show how the shear stress depends on shear rate when the Couette is operated in batch mode (without vertical flow) at constant height. As seen in Figure 3.11b, increasing the shear rate (angular velocity of the inner cylinder, $v$ ) more than five-fold, does not result in any significant change in the shear stress: the material is in the frictional, quasi-static regime and the average shear stress is practically independent of shearing rate. 
a

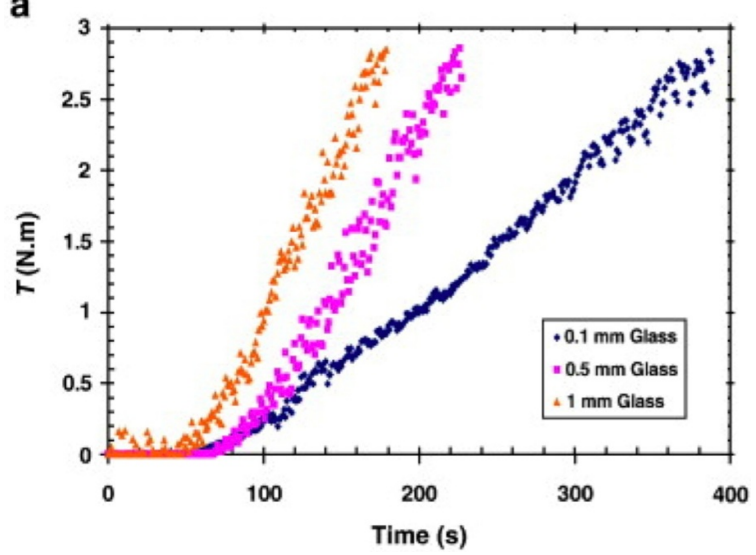

b

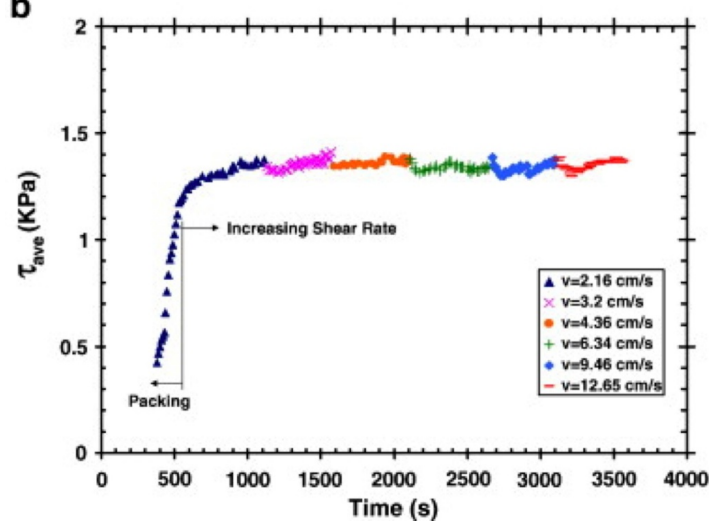

Figure 3.11: Batch Couette experiments: (a) torque vs. time for different particles; (b) shear stress vs. time and rotational speed

We reproduced in Figure 3.12a similar experiment to that shown in Figure 3.11b but allowing the material to flow at a small rate vertically in the shear gap by letting some material out through the valve at the bottom and feeding the same amount to the top. The torque in this case decreases significantly due to a minute change in solid fraction that allows chains of particles to break as the inner cylinder rotates (see next section for a more detailed discussion). Using Equation (1), the average shear stress can be calculated as a function of shear rate as shown in Figure 3.12. As seen, the shear stress exhibits two very distinct regimes: a quasi-static regime where the shear stress is mostly independent of the shear rate (at low shear rates) and an additional regime (intermediate" as denoted in the figure) where the dependence takes the form of an increasing function. We assume that the behavior resembles a power-law with an index $n$. In fact, the power-law index appears to be variable, increasing at higher shear rates. One would assume that continuing to increase the shear rate, would yield even higher values of the index $n$ as the flow approaches the inertial regime (where the coefficient should be $n=2$ ) but such experiments are outside the capability of our Couette device since centrifugal forces take over and the material is pushed radially outward and a slip condition sets in near the rotating cylinder.

The purpose of these experiments was to show the significant difference in behavior of the shearing layer in the batch, no-flow and the continuous-flow device. While in the batch mode, the material continues to pack increasing its solid fraction as a function of time, in the flowing system a steady state sets in at an approximately constant solid fraction. This solid fraction is below a certain critical value, characteristic for each powder, where the material can "dilate" sufficiently and "flow" under shear. In this expanded, mobilized (sometimes also called "fluidized", even though there is no gas present to actually fluidize the powder) condition, the powder can transit from the quasi-static to the intermediate regime as the shearing rate is increased. We showed in separate experiments that the influence of the vertical flow rate is negligible if it can be kept small, just enough to prevent packing. The rest of this section is mainly concerned with this second case where there is a minute flow of material in the vertical direction and where a quasi-static, shear-rate-independent and an intermediate, dense-phase, shear-rate-dependent shear stress (torque) develops. The 


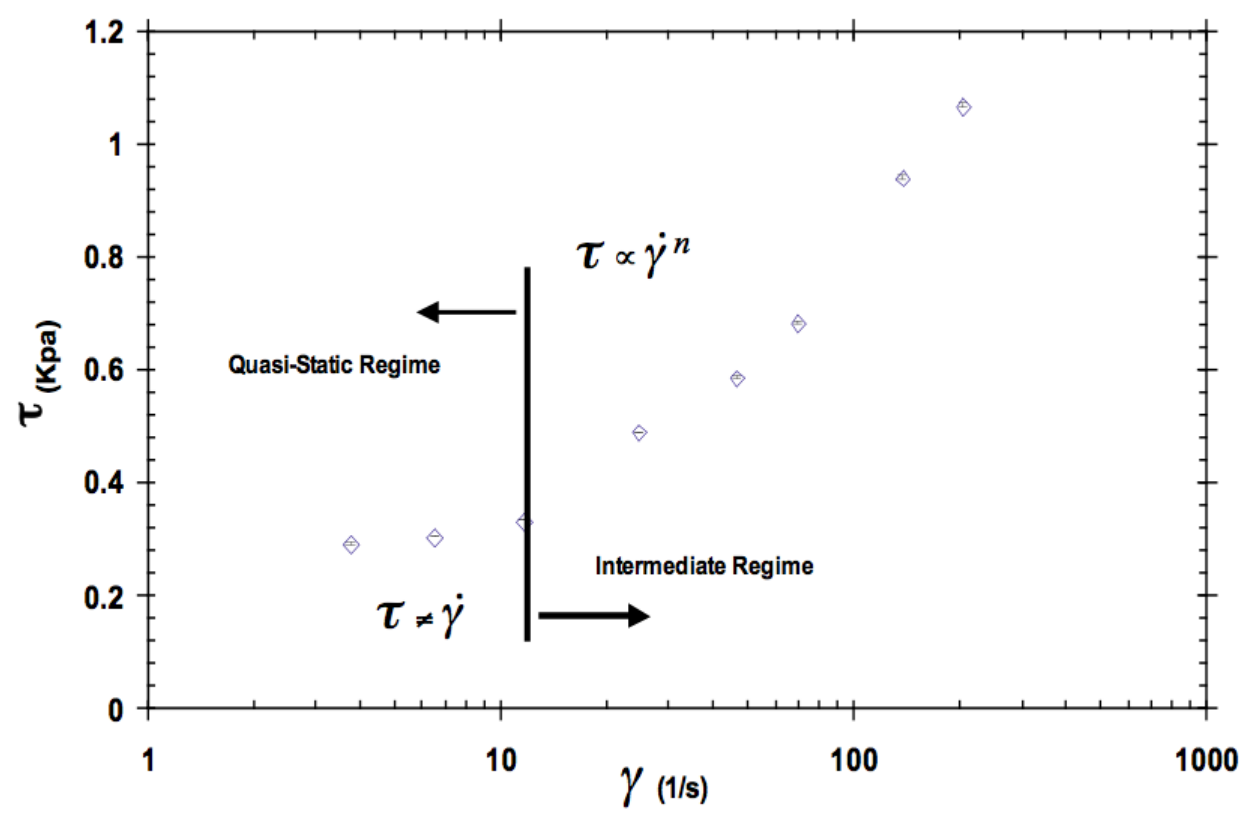

Figure 3.12: Axial-flow experiment in the Couette device: spherical glass beads, $0.1 \mathrm{~mm}$ in diameter

main goal of the experiments is to measure the shear and normal stresses on the shearing wall, their location and their ratio.

Since there are no direct shear stress measuring sensors commercially available that can be employed in a dense granular bed, the shear stress on the rotating cylinder was measured indirectly using the torque on the shaft exerted by the granular medium on the entire length, $L$ of the cylinder as in Equation (1). To explore the dependence of the shear stress on the depth of the granular layer, we employed cylinders of different lengths, $L=4,8,12$ and 16 in. (and diameter of 4 in.) and calculated the average shear stress in each case. The experiments were performed with no overburden (material on top of the rotating cylinder) using $0.5 \mathrm{~mm}$ in diameter glass beads. Results are given in Figure 3.13 where the average shear stress is given as a function of cylinder height, $L$ for different shear rates by rotating the inner cylinder from 5 to 45 RPM. As seen, the shear stress is approximately linear as a function of height and depends strongly on shear rate as already shown above.

Another interesting observation is that, by forcing the trend of the experimental measurements to go to zero stress at zero height, data from the shortest cylinder $(L=4$ in. in length) fall above the line at a somewhat higher shear stress. This behavior is due to friction between the lower cover of the cylinder and the granular material (in addition to friction in the shearing layer between the cylinders) which is not a linear function of height. When the length, $L$ (height) of the shearing layer is small, the influence of this portion of the cylinder is significant and hence the torque is higher. At larger length (height), the influence of the bottom cover becomes less significant and the dependence is almost perfectly linear. The main conclusion from this measurement is that using cylinders longer (taller) than about 8 in. will mostly eliminate the influence of the end effect (lower cover) and that the value of 


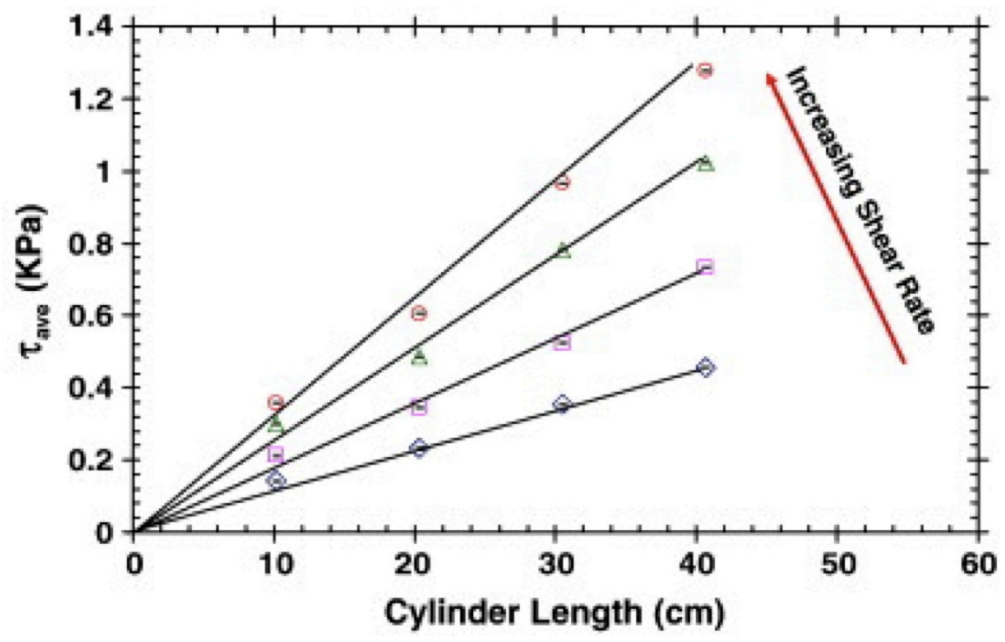

Figure 3.13: Average shear stress measurements in continuous Couette device using different overall lengths, $L$, cylinders at different shear rates

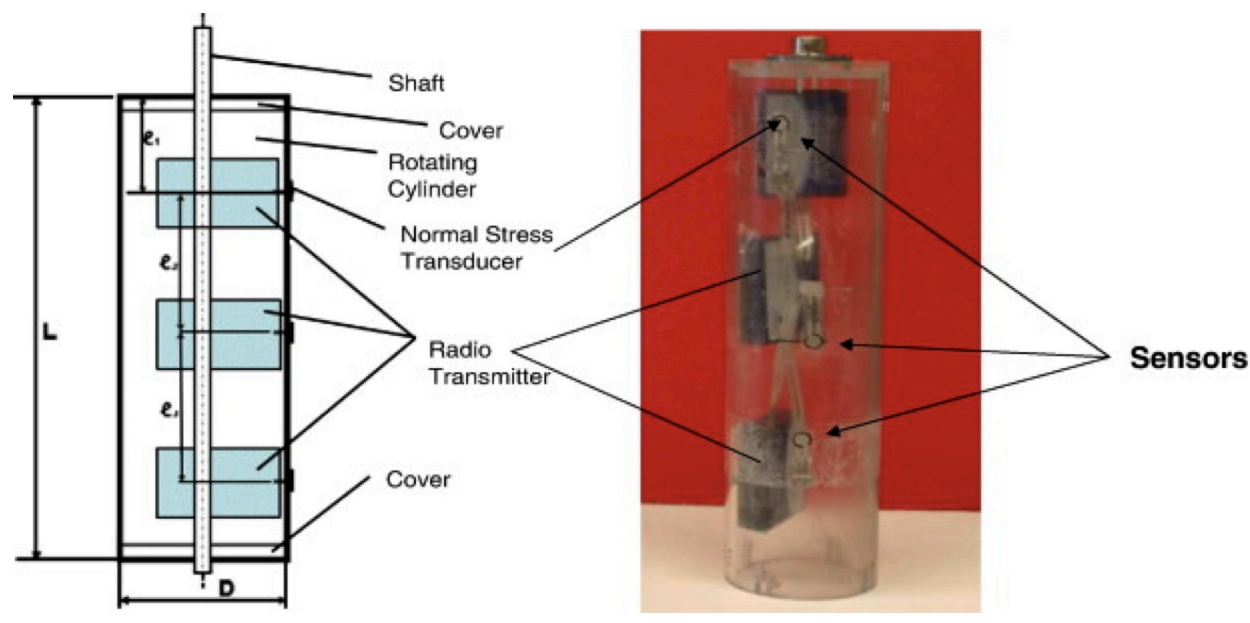

Figure 3.14: Schematic and picture of the Couette device with three remote normal stress sensors

the average shear stress will most likely occur at the middle point over the length, $L$.

Figure 3.14 shows a schematic representation and a picture of the instrumentation to measure normal stresses inside the bulk material on the rotating cylinder at three depths (11, 12 and 13). The stress sensor is the same as depicted in Figure 3.1. Since the cylinder is rotating inside the granular layer, the signal cannot be hard-wired but is instead transmitted by radio to a receiver connected directly to the data acquisition system. The cylinder used is 4 in. in diameter (this dimension is imposed by the size of the radio transmitter) and $20 \mathrm{in.} \mathrm{long} \mathrm{(to}$ accommodate the three radio transmitters). The outside diameter of the stationary cylinder that forms the Couette device (not shown in the figure) is 6 in. in diameter and thus defines a granular layer of $1 \mathrm{in}$. in thickness that surrounds the sensors.

These particular experiments were performed with an overburden of approximately 4 in. so 


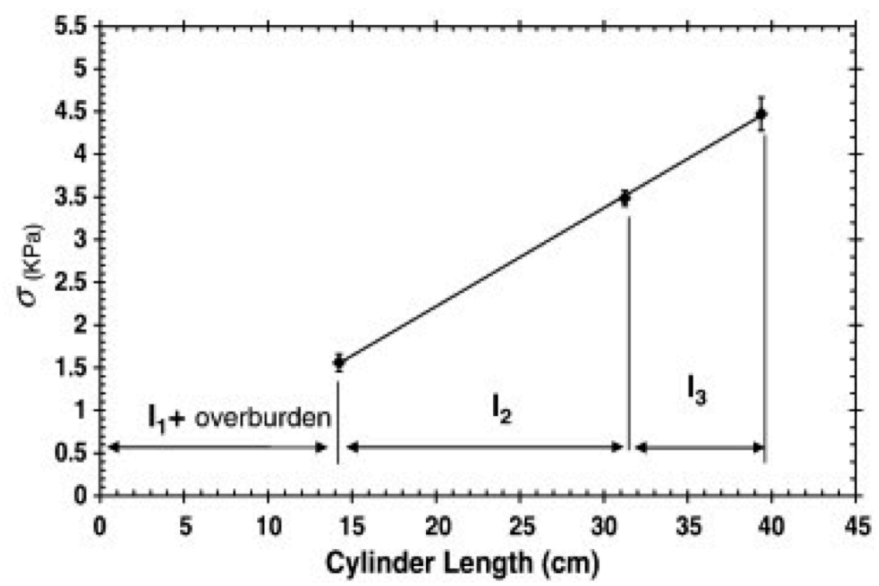

Figure 3.15: Normal stress as a function of height as measured on the shearing cylinder surface by remote sensing

that the depth of the three sensors was $I_{1}-I_{3}$ plus the overburden. Different rotation rates from 5 to $60 \mathrm{RPM}$ and glass beads of $0.5 \mathrm{~mm}$ in diameter were used. The experiment was performed with a very small vertical flow rate; this results in a very slow axial velocity in the Couette gap of less than $0.05 \mathrm{~mm} / \mathrm{s}$. Figure 3.15 depicts the results of the stress measurements as a function of depth. The distribution appears to be linear with the error bars representing the fluctuation of stress and the dependence on the applied shear. As seen, the normal stress in the sheared layer is not shear-rate-dependent even at the highest shear rate (at $60 \mathrm{RPM}$ ), within the error of the measurement and the presence of fluctuations. This allows the calculation of an average normal stress on the rotating cylinder that appears, according to these results, to reside in the middle of the cylinder $(L / 2)$.

The most interesting result from the above measurements is that one can calculate the ratio of the average shear to normal stresses as a function of shear rate. A typical result is shown in Figure 3.16 where the ratio of the average shear stress and local normal stress measured in the middle of the rotating cylinder $(L / 2)$ are shown as a function of shear rate. The particles used are $0.1 \mathrm{~mm}$ in diameter spherical glass beads. The shear stress is the same as the one depicted in Figure 3.12 and the stress is practically constant at low shear rates and increases significantly as soon as the material reaches the intermediate regime of flow. This behavior is mainly due to the superposition of collisions between particles on the sliding friction of surfaces so that the overall shear stress and "friction coefficient" also increase.

We conclude that it is possible to measure normal stresses on the sharing wall of the Couette device inside the granular layer and calculate the ratio of the average shear to normal stress as a function of shear rate. It appears, as suggested by previous theoretical simulations, that the dynamic angle of friction is reproduced by this ratio only at very low shear rates. As the shearing rate increases, the ratio of the stresses also increases due to collisions between particles that sustain loads in addition to dry friction that is prevalent at low shear rates. Our experiment could not probe the higher limit of the "friction coefficient" in the rapid granular flow regime (when only collisions are important) because of equipment limitations and the inherent presence of gravity that makes at least some enduring contacts between 


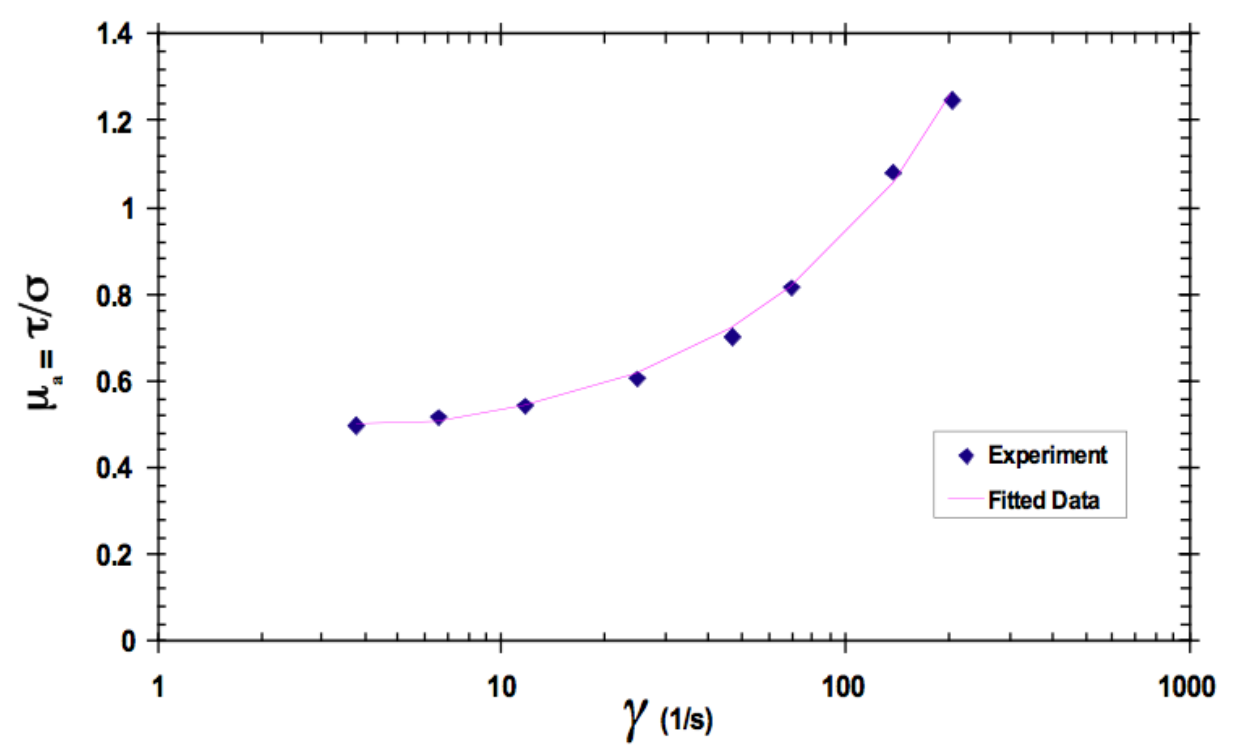

Figure 3.16: Measured ratio of shear to normal stresses (apparent friction coefficient) and fitted curve as a function of shearing rate for $0.1 \mathrm{~mm}$ in diameter round glass

particles unavoidable.

We try to generalize the above findings for the case when the powder transitions from the quasi-static to the intermediate regime of flow and propose a yield condition that holds at low and higher shear rates. We show below how the experimental yield condition obtained from experiments in the Couette device and given in Figure 3.16 can fit the following equation:

$$
\tau / \sigma=a+b|\dot{\gamma}|^{n}
$$

where $\tau$ and $\sigma$ are the shear and normal stresses and $a, b$ and $n$ are coefficients characterized by the experimental curve. We use the notation for the modulus (absolute value) of the shear rate to designate that only its magnitude is of relevance and not its direction. A slight generalization can be obtained by replacing the constant coefficient $a$ by $\tan \phi$ to obtain:

$$
\tau / \sigma=\tan (\phi)+b|\dot{\gamma}|^{n}
$$

This equation has the advantage that it reduces to the Coulomb yield condition at zero shearing rate.

We repeated the above measurements and calculations for several particles and we show in Figure 3.17 an example where we change the friction coefficient of smooth $\left(\phi=26^{\circ}\right)$ and rough $\left(\phi=36^{\circ}\right)$ Polyethylene particles (4 mm in size and cylindrical shape) at different shear rates, the rheological parameters for each case and a picture of the particles. The coefficient $b$ is higher for the rough material due to higher surface friction, as one would expect. In fact, the experiment shows that our Couette device is sensitive enough to distinguish between different surface roughnesses.

In order to generalize the rheological model obtained from our Couette cell we tested a large 


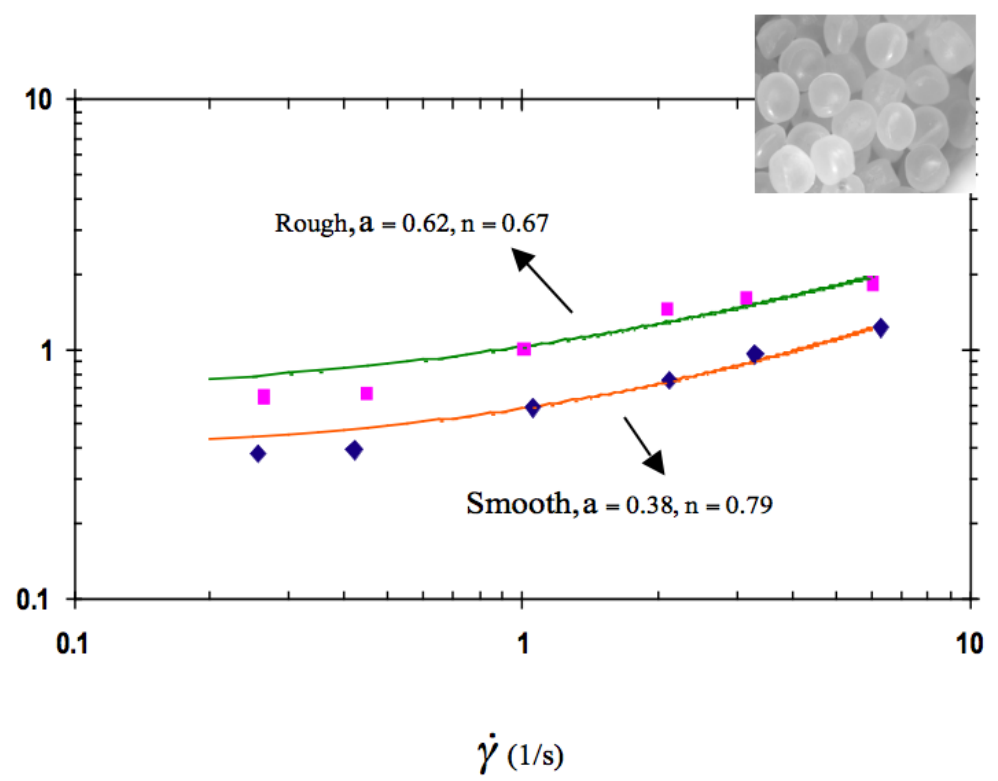

Figure 3.17: Apparent friction coefficient for $4 \mathrm{~mm}$ in diameter rough and smooth Polyethylene particles

number of particles with different physical properties such as size, size distribution, shape, surface roughness, rigidity etc. and these are summarized in Table 3.2.

As seen in Table 3.2, the coefficient $\mathrm{n}$ varies for different materials from about 0.3 to approximately 1.3 as a function of particle shape, surface roughness and particle softness and elasticity, etc., and cannot be taken as a constant. Simple addition of the stresses from the quasi-static and the intermediate regime i.e., taking $n=1$ only applies for specific cases. We also see that the coefficient $b$ reaches approximately $\tan \left(\phi_{w}\right) \sim \tan (\phi)$ when the shear rate tends to zero (quasi-static regime of flow) and this is when the plasticity rule (Coulomb criteria) applies. Results with spherical glass particles of different sizes reveal that by just increasing size the power law index remains almost unchanged. Comparison between round and crushed glass $(0.5$ and $1 \mathrm{~mm})$ shows that the shape has an important role on the rheology of the powder bed. The power law index $n$ is much higher $(\sim 1)$ for crushed glass than that for round glass beads $(\sim 0.7)$. This shows that collisional effects are dominant for an odd shaped particle, as $n$ is a characteristic for viscous behavior. Irregular-shape and broader size distribution increase the contact areas between particles so that the viscous term of the constitutive equation becomes dominant. The effect of surface roughness, as described above, was considered using smooth and rough Polyethylene. We found that rough and smooth materials of the same size and shape behave differently. The coefficients $b$ is higher due to higher surface friction. Smooth particles exhibit a larger coefficient $n$ (0.79 as compared to 0.67 for rough particles) which means that collisions can occur more frequently and this effect dissipates more energy by friction.

We studied in this section, flow of dry, frictional powders in the quasi-static and intermediate regimes using the simple geometry of the Couette. We measure normal and shear stresses on the shearing surface and propose a constitutive equation valid in both regimes. We found 
Table 3.2: Physical properties and rheological model obtained in the Couette cell for different particles; $\phi^{\circ}$ and $\phi_{W}^{\circ}$ are internal and wall angles of friction, $b$ and $n$ are from the fitted model to Equation (3).

\begin{tabular}{|c|c|c|c|c|c|c|c|}
\hline Material & $\begin{array}{c}\text { MPS } \\
{[\mathbf{m m}]}\end{array}$ & Shape & Remarks & $\boldsymbol{\phi}^{\circ}$ & $\boldsymbol{\phi}_{W}^{\circ}$ & $\begin{array}{c}\mathbf{b} \\
\text { (model) }\end{array}$ & $\begin{array}{c}\mathbf{n} \\
\text { (model) }\end{array}$ \\
\hline Glass & 0.1 & Round & Rigid & 27.0 & 25.3 & 0.45 & 0.73 \\
\hline Glass & 0.5 & Round & Rigid & 27.1 & 25.7 & 0.45 & 0.70 \\
\hline Glass & 0.5 & Odd & Rigid/Crushed & 26.0 & 24.1 & 0.43 & 0.94 \\
\hline Glass & 1 & Round & Rigid & 27.4 & 24.2 & 0.39 & 0.72 \\
\hline Glass & 1 & Odd & Rigid/Crushed & 34.6 & 30.4 & 0.48 & 1.03 \\
\hline Glass & 2 & Round & Rigid & 28.2 & 25.5 & 0.44 & 0.78 \\
\hline Glass & 3 & Round & Rigid & 28.4 & 24.6 & 0.42 & 0.75 \\
\hline Polyethylene & 4 & Odd & Cylindrical/Smooth & 35.7 & 33.7 & 0.62 & 0.67 \\
\hline Polyethylene & 4 & Odd & Cylindrical/Smooth & 25.6 & 22.4 & 0.38 & 0.79 \\
\hline Polyethylene & 0.3 & Odd & Soft/Compressible & 25.3 & 23.9 & 0.43 & 0.45 \\
\hline Polyethylene & 0.5 & Odd & Soft/Compressible & 27.9 & 25.5 & 0.45 & 0.68 \\
\hline EPDM & 1.5 & Odd & Soft/Elastic & 31.1 & 24.2 & 0.39 & 1.11 \\
\hline Detergent/HP & 0.45 & Odd & Soft/Deformable & 30.5 & 29.8 & 0.57 & 1.21 \\
\hline FCC Catalyst & 0.07 & Odd & Rigid/Broad & 37.1 & 36.3 & 0.70 & 0.69 \\
& & & Size Distribution & & & & \\
\hline
\end{tabular}

that it is possible to measure normal stresses on the shearing wall of the Couette device inside the granular layer and calculate the ratio of the average shear to normal stress as a function of shear rate. It appears that the dynamic angle of friction of the powder is reproduced by this ratio only at very low shear rates. As the shearing rate increases, the ratio of the stresses also increases due to collisions between particles that sustain some load. We show that a modified Couette device with slow axial flow superimposed on the shearing motion can be used to determine the constants ( $b$ and $n$ ) of a yield condition for any material that is somewhat free flowing and thus can be used as a "powder rheometer". The yield condition is valid in both the quasi-static as well as the "intermediate" regimes of flow and contains a term characterizing "solid"-like behavior and an additional term that captures some "fluid"-like properties at higher shear rates.

We found that while the sizes of particles does not have a major impact on the rheological parameters, size distribution, shape, surface roughness, ability to dilate or pack, deformability and elasticity strongly affect the rheology of the bulk. Odd-shaped particles with the same average size as spherical particles yield a higher power-law index. Rough and smooth materials of the same size and shape behave differently: the rough material shows a higher constant coefficients $b$ due a larger friction coefficient while the smooth particles have a higher exponent $n$ because of less force chains (due to smaller friction) and more freedom of movement. Particles with more ability to dilate i.e., exhibit larger inter-particle spaces, show less dependency of shear stress to shear rate while deformation and elasticity increase the rheological index $n$ as more shear stress is required for the same shearing rate for elastic and/or deformable powders. 


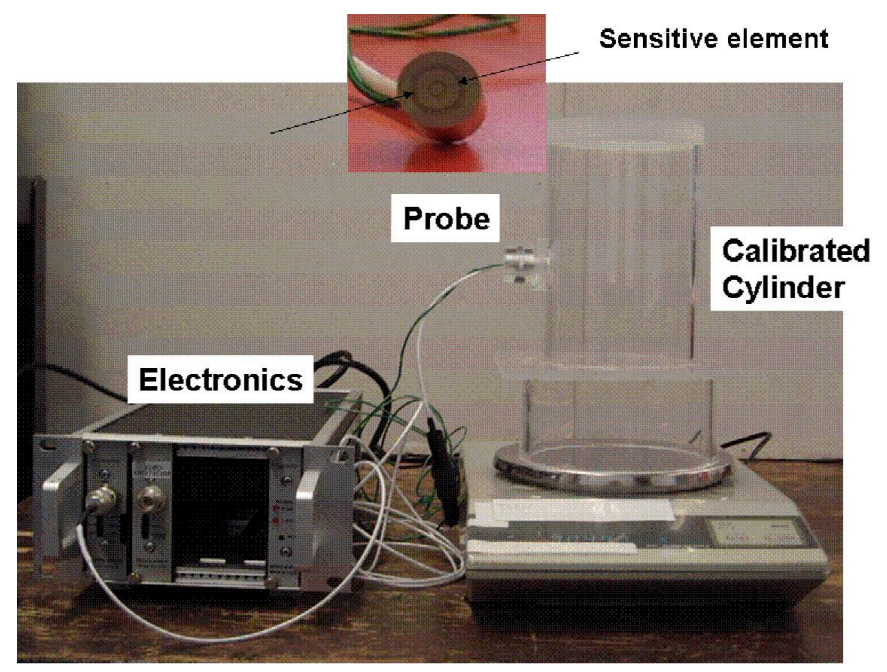

Figure 3.18: Picture of Capacitance probe and calibration device

\subsubsection{Measurement of solid fraction using a capacitance probe}

Solid volume fraction can be measured using the capacitance probe available commercially from Capacitec, Inc. The commercial instrument is made to measure small distances with great precision but it was modified for the present experiment to detect solid fraction. This is achieved by filling the "detection volume" of the probe with powder and performing a thorough calibration of the powders dielectric constant at different porosities. The knowledge of the porosity in the shearing bed and its variation is crucial to understanding the behavior of the material. There is a significant difference in the behavior of the shearing layer in the batch, no-flow and the continuous-flow Couette cells. We showed previously that while in the batch mode the torque increases continuously, by draining a small amount of material in the axial direction (continuous Couette), the value of the torque decreases and it reaches a steady state. To investigate the reason for such a change we measured the solid fraction in both batch and continuous modes. Mounted on the stationary wall, the probe measures the change in solid fraction in a semispherical volume of about $3 \mathrm{~mm}$ in diameter. To reach inside the shearing gap, the probe is physically pushed inside, parallel to itself in equal steps of $1 \mathrm{~mm}$ using a caliper.

A picture of the capacitance probe used in this study, the electronic circuit together with the calibrating cylinder is given in Figure 3.18. The probe is mounted flush to the outer wall of the calibrating cylinder, as shown. The solid fraction measurement requires a very thorough calibration since the capacitance of the powder varies with the amount of gas (air) trapped in the system. The system depicted in Figure 3.18 achieves this by filling the cylinder with the appropriate powder and tapping it continuously so that densities from free filling to highly packed can be achieved. The conditioned signal is then connected to a data acquisition computer specially designated for this measurement. In a real experiment, the probe is mounted onto the outer wall of the Couette device in a position opposite to the normal stress sensor so as to measure the solid fraction at the same height in the bed. 


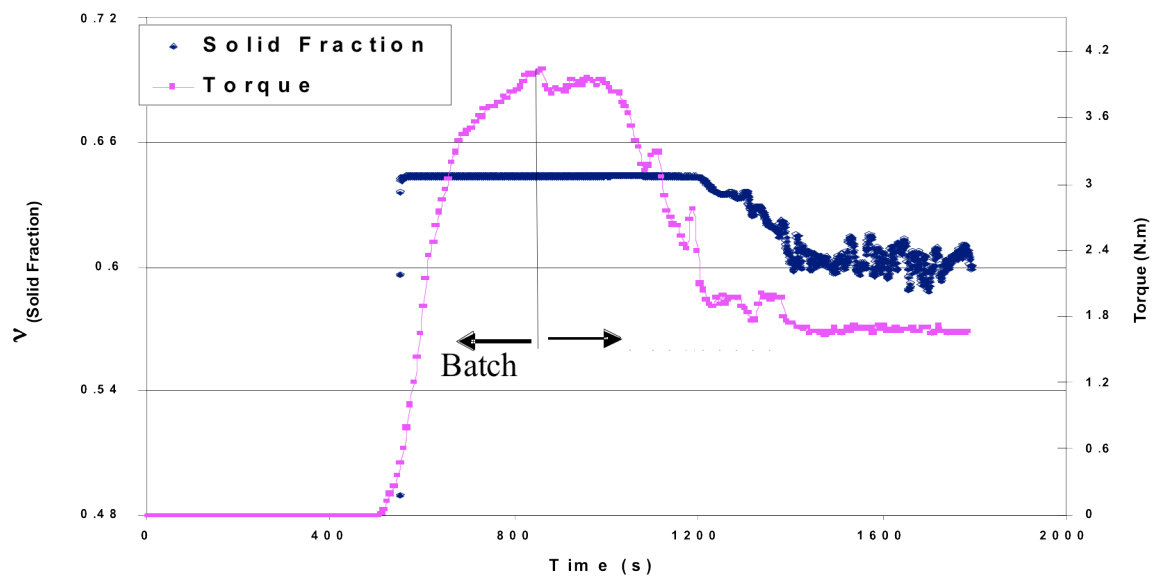

Figure 3.19: Torque vs. solid fraction measured on the stationary wall in batch and continuous Couette for $0.5 \mathrm{~mm}$ crushed glass

We show several examples of solid volume fraction measurements in both batch and continuous modes at two different positions. Figures 3.19 and 3.20 show simultaneous results of torque and solid fraction in the two modes of operation, batch and continuous, on the outer stationary wall (Figure 3.19) and in the shearing zone (Figure 3.20) for $0.5 \mathrm{~mm}$ crushed glass as a function of time. The experiment entails the filling of the bed with particles to an overburden height of approximately $20 \mathrm{~cm}$ and measuring the torque and solid fraction in the batch mode. Subsequently, a constant axial flow is established and the torque and solid fraction are again monitored as a function of time.

As seen in Figures 3.19 and 3.20, the torque increases when the bed is in batch mode; the solid fraction also increases and reaches its maximum value as the granular bed is constrained from further dilation. Upon continuous removal of particles ("continuous" in the figure), the torque reaches a maximum and then starts to decrease to reach a steady value. The solid fraction exhibits similar behavior except that it takes somewhat longer for the axial flow to affect the decrease in solid fraction. In both cases, one can see that after starting the axial flow (denoted by "continuous" in the figure), both torque and solid fraction decrease but the response of the torque is faster. The decrease in solid fraction indicates that the change of regime happens because particles gain freedom of movement in the space around them so that they can actually move freely past one another and may also collide. During a similar experiment with finer and more deformable powder, $0.3 \mathrm{~mm}$ ground (odd shape) Polyethylene (Figure 3.21), we again see that the axial flow prevents the bed from further packing and increase in torque.

Furthermore, both the torque and solid fraction respond faster to the onset of axial flow (denoted "continuous" in the figure) than in the case of the more rigid (glass) powder and they decrease together almost simultaneously. Change in solid fraction by imposing the axial flow is higher for the fine, odd-shaped (more compressible) particles as compared to the rigid (incompressible) powder. Table 3.3 shows the average value and standard deviation of solid fraction for different particles in batch and continuous modes at a constant angular velocity 


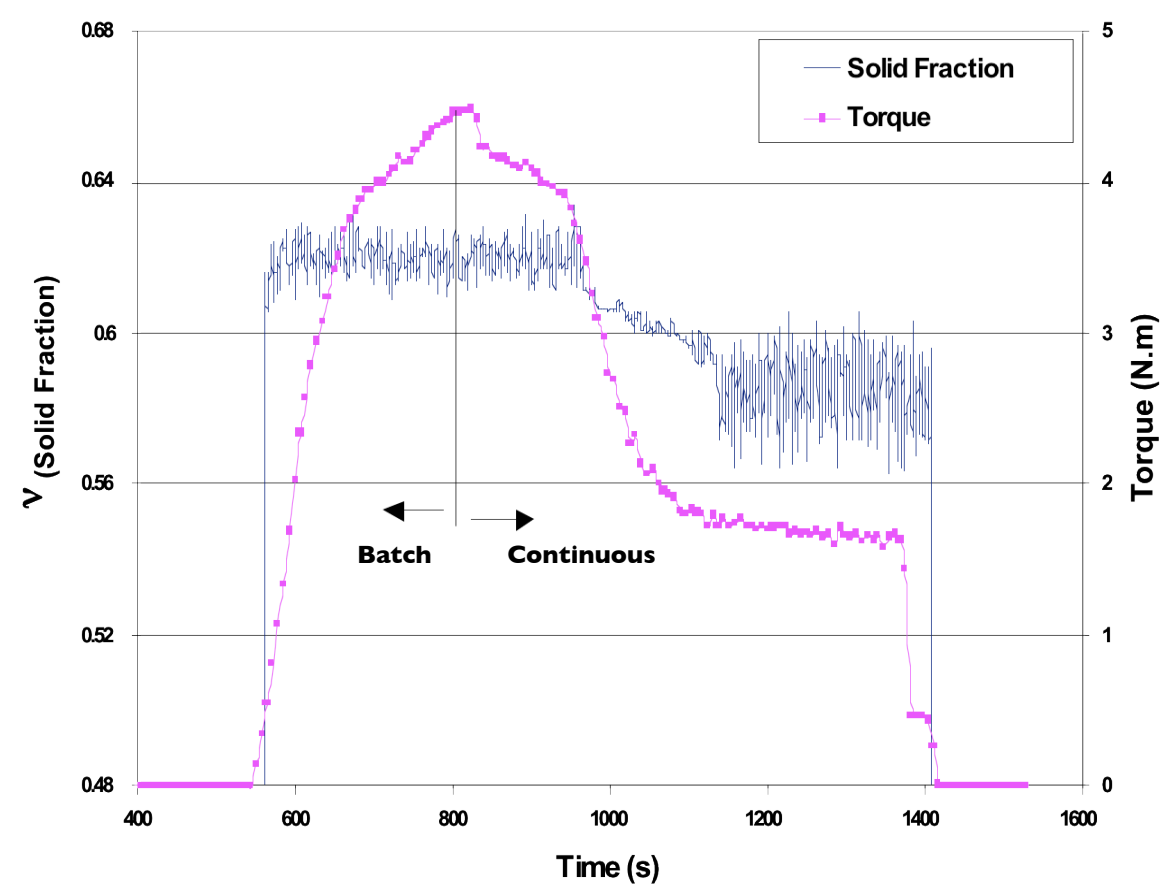

Figure 3.20: Torque vs. solid fraction measured on the stationary wall in batch and continuous Couette for $0.5 \mathrm{~mm}$ crushed glass

Table 3.3: Solid fraction in batch and continuous Couette for different materials

\begin{tabular}{|c|c|c|c|c|c|}
\hline Material & $\begin{array}{c}\boldsymbol{\nu} \\
\text { (Batch) }\end{array}$ & $\begin{array}{c}\text { Std. } \\
\text { Dev. }\end{array}$ & $\begin{array}{c}\boldsymbol{\nu} \\
\text { (Continuous) }\end{array}$ & $\begin{array}{c}\text { Std. } \\
\text { Dev. }\end{array}$ & $\begin{array}{c}\text { Change } \\
\text { in } \boldsymbol{\nu}(\boldsymbol{\%})\end{array}$ \\
\hline 1 mm Round Glass & 0.6405 & 0.00253 & 0.6161 & 0.00635 & 2.44 \\
0.5 mm Round Glass & 0.6356 & 0.00021 & 0.6046 & 0.00565 & 3.10 \\
0.5 mm Crushed Glass & 0.6441 & 0.00011 & 0.6133 & 0.00552 & 3.07 \\
$0.3 \mathrm{~mm}$ Crushed PE & 0.4832 & 0.00568 & 0.4484 & 0.00931 & $>3.48$ \\
\hline
\end{tabular}

of 15 RPM. 


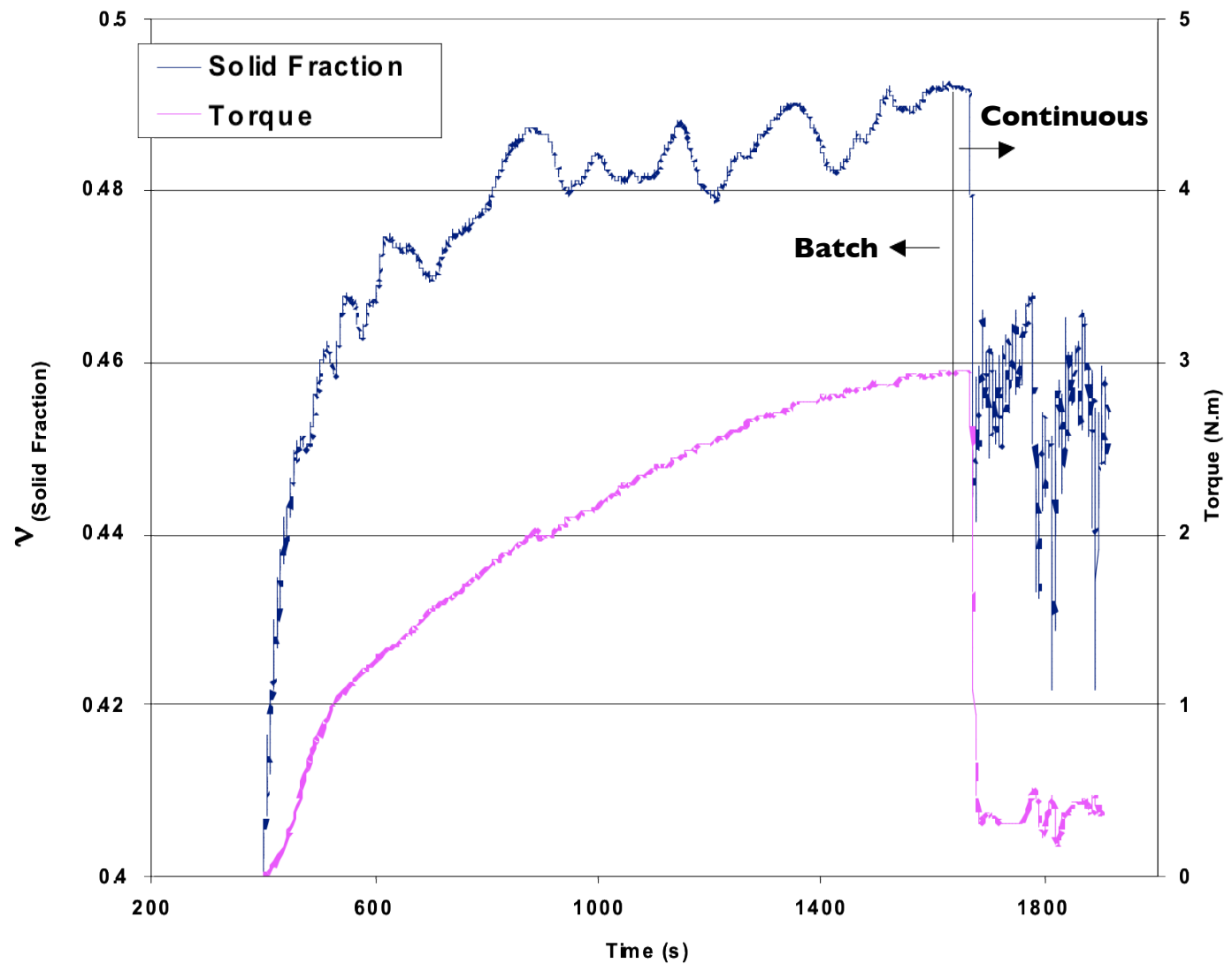

Figure 3.21: Solid fraction and torque in the Couette with $0.3 \mathrm{~mm}$ Polyethylene chips 


\subsection{Work performed at Princeton University}

The work at Princeton was performed in three phases. The first phase aimed to validate the DEM approach by comparing its results with those from the experiments performed at CCNY. The second phase focused on modeling dynamic behaviors of quasi-static granular flows. In the third phase, attention turned to the poorly understood intermediate regime. The highlights of this work are presented below, with details located in the manuscripts attached as appendices to this report.

\subsubsection{Validation of the DEM approach}

To ensure the suitability of DEM simulations for investigating granular rheology in the quasistatic and intermediate regimes, we simulated flow in a Jenike cell and compared the results with those of the experiments described in the previous section. We found good agreement in the measurements of stress and stress fluctuations between the two; the frequency of the fluctuations did differ slightly, likely due to the sampling frequency or asphericity of particles in the experiments. Furthermore, the simulations were able to capture the temporal fluctuations and spatial heterogeneity observed in the experiments. Additional details can be found in Appendix K. These findings supported the use of DEM for the computational part of the rheological study.

\subsubsection{Constitutive modeling of quasi-static granular flows}

Plasticity and hypoplasticity models are often used for modeling slowly deformed granular materials and usually predict rheology by evolving a (micro)structural variable during the shearing process $[1,2]$. However, these evolution equations are generally based on little or no micromechanical data. We therefore investigated the relation between microstructure and macroscopic stresses via DEM simulations and developed a simple hypoplasticity model capable of predicting complex dynamic flow behavior of granular materials in the quasi-static regime. This work is summarized below; please refer to Appendix $\mathrm{C}$ for a more thorough discussion.

We performed DEM simulations of homogeneous simple shear flow under both constantvolume and constant-pressure conditions for various volume fractions, with the shear rate kept low to ensure flow in the quasi-static regime. An additional set of isotropic compression (IC) simulations was also performed for comparison. Data were then extracted for the stresses, the coordination number (excluding rattlers) $Z_{2}$, and the fabric of contacts A. As seen in Figure 3.22a, the pressure increases with the coordination number for both the simple shear and IC deformations. However, a difference in the pressure between the two cases is observed, and this difference is correlated with anisotropy of the fabric that develops due to deviatoric deformation. If we write the stress tensor $\boldsymbol{\sigma}$ as

$$
\boldsymbol{\sigma}=p \mathbf{I}-p \eta \hat{\mathrm{S}}
$$




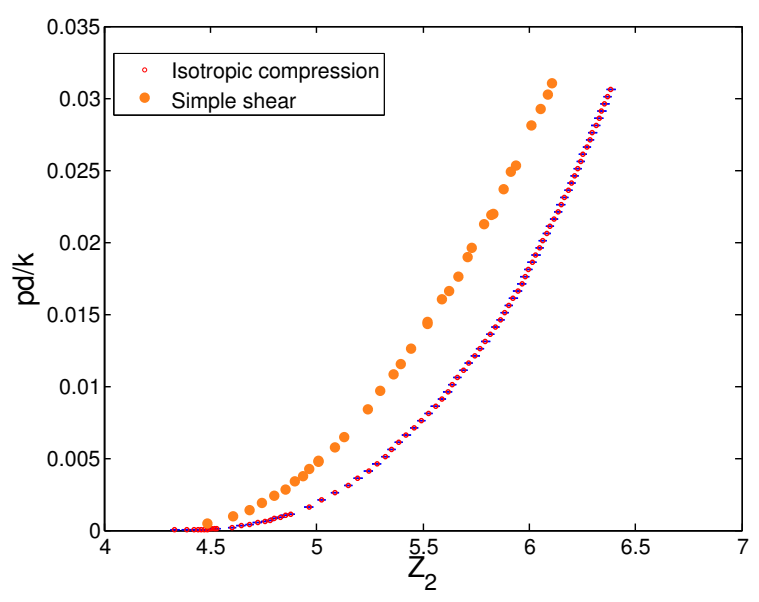

(a)

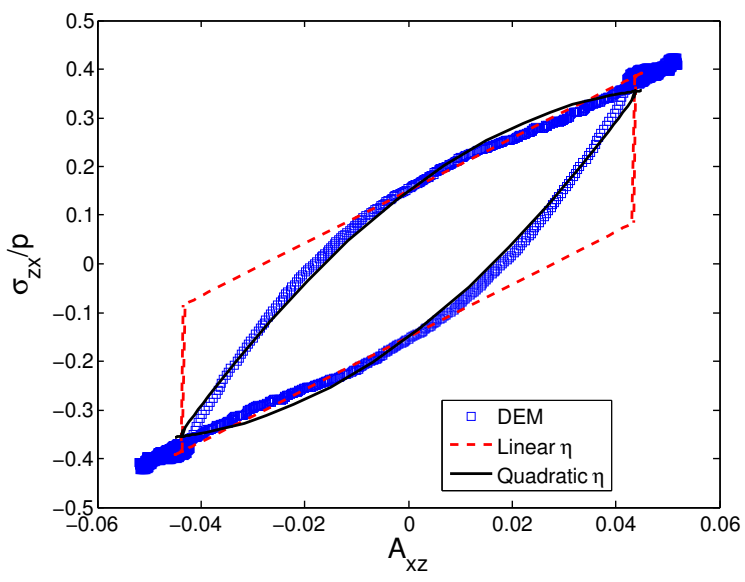

(b)

Figure 3.22: Dependence of stresses on microstructure. (a) Scaled pressure $p d / k$ is observed to be a function of the coordination number $Z_{2}$. (b) Shear stress ratio $\sigma_{z x} / p=\eta \mathrm{S}_{z x} /|\mathrm{D}|$ varies with the shear component $A_{x z}$ of the fabric tensor. Fits to Eqn. 3.6 are shown, both with the full quadratic form (solid black line) and the truncated linear form $\left(b_{3}=0\right.$, red dashed line).

we find that the pressure $p$ and shear stress ratio $\eta$ obey

$$
p d / k=\left(a_{1}+a_{2}|\mathrm{~A}|\right)\left(Z_{2}-Z_{c}\right)^{2}
$$

and

$$
\eta=b_{1}+b_{2} \mathrm{~A}: \hat{\mathrm{S}}+b_{3}(\mathrm{~A}: \hat{\mathrm{S}})^{2},
$$

where $\mathrm{I}$ is the identity tensor, $\hat{\mathrm{S}}=\mathrm{S} /|\mathrm{D}|$ is the deviatoric part $\mathrm{S}$ of the deformation rate tensor $\boldsymbol{D}$ scaled by $|\mathrm{D}|, k$ is the particle stiffness, $d$ is the particle diameter, and $|\boldsymbol{\theta}|=\sqrt{\frac{1}{2} \boldsymbol{\theta}: \boldsymbol{\theta}}$ is the magnitude of a tensor $\boldsymbol{\theta}$. The parameters $a_{i}, b_{i}$, and $Z_{c}$ are model constants. As seen in Figure 3.22b, Eq. 3.6 is capable of capturing the dependence of the stress ratio on the fabric in an unsteady simple shear flow; even a truncated, linear form (i.e. with $\left.b_{3}=0\right)$ shows reasonable agreement. Eqs. 3.5-3.6 thus connect the macroscopic stresses to the microstructural state of the particle assembly.

This microstructure, as stated earlier, evolves with the strain in the system. To capture the dynamics of microstructure (and hence stresses), we developed expressions for the rate of change of the coordination number and fabric tensor. Specifically, we write the evolution equation for the fabric tensor as

$$
\stackrel{\AA}{\AA}=c_{1} \mathrm{~S}+c_{2}|\mathrm{D}| \mathrm{A}+c_{3}(\mathrm{~A}: \mathrm{S}) \mathrm{A} .
$$

and that of the coordination number as

$$
\dot{Z}_{2}=d_{1}(\mathrm{~A}: \mathrm{S}-\chi|\mathrm{S}|)+d_{2}|\mathrm{D}|\left(f(\phi)-Z_{2}\right)+d_{3} \operatorname{tr}(\mathrm{D}),
$$




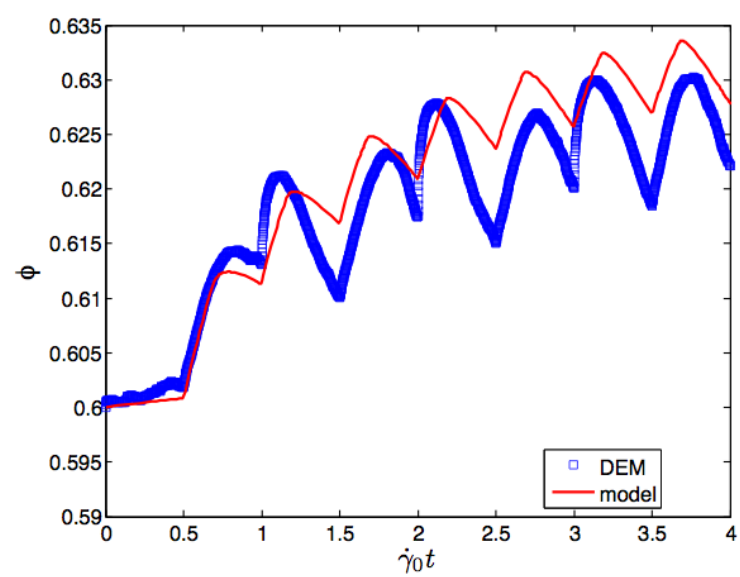

(a)

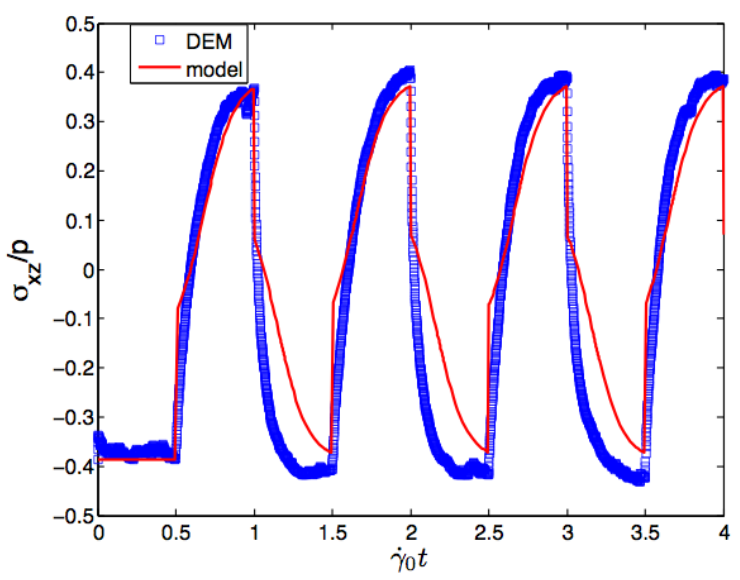

(b)

Figure 3.23: Evolution of (a) shear stress ratio and (b) volume fraction with accumulated strain for an assembly subjected to small-amplitude oscillatory shear under the constant pressure condition with $\mu=0.5$.

where $\AA$ is the Jaumann derivative, $\left.\chi=-\left(c_{2}+\sqrt{c_{2}^{2}-8 c_{1} c_{3}}\right) / 2 c_{3}\right)$ is a solution of $\mathrm{A}: \hat{\mathrm{S}}$ using Eq. 3.7 for steady simple shear flow, $f(\phi)=d_{4}\left(\phi-\phi_{c}\right)^{1 / 2}$ relates the coordination number and volume fraction under steady shear, and $c_{i}$ and $d_{i}$ are material constants.

The microstructural basis of the model, along with the microstructural evolution equations, allow it to describe complex quasi-static flow behaviors for which other models often fail. One example is that of oscillatory simple shear of granular particle assembly under constant pressure. The performance of the model for this scenario can be assessed from Figure 3.23. The model is capable of capturing not only the dynamic stress evolution during and after each shear reversal but moreover the gradual compaction of the bed over time.

Further model validation was performed by using the model to solve for the flow in a conical hopper, a simple one-dimensional problem. We assume incompressible flow and radial gravity for small half angle and apply boundary conditions of smooth side wall and zero stresses at inlet and outlet. The simplified fabric evolution equation can be presented in a nondimensional form as

$$
\frac{d A_{r r}}{d \hat{r}}=-\frac{1}{\hat{r}}\left(2 c_{1}+\sqrt{3} c_{2} A_{r r}+3 c_{3} A_{r r}^{2}\right),
$$

where $\hat{r}=\frac{r}{R_{\text {out }}}$ is the radial distance scaled by that at the outlet and $A_{r r}$ is the fabric tensor component in the radial direction. The momentum balance equation is

$$
\frac{d \hat{\sigma}}{d \hat{r}}=\frac{2 \phi \hat{V}^{2}}{\hat{r}^{5}}+\frac{2(\kappa-1) \hat{\sigma}}{\hat{r}}-\phi
$$

where $\hat{V}=\frac{V}{\sqrt{R_{\text {out }}^{5} g}}$ and $\hat{\sigma}=\frac{\sigma}{\rho_{s} g R_{\text {out }}}$ are scaled volumetric flow rate and stress, respectively. This equation poses the same form as the traditional hour-glass theory for conical hopper flows. However, the parameter $\kappa$ here is a function of the macroscopic friction $\eta$, which is in turn linked to the fabric tensor component, in contrast to being a constant in the 


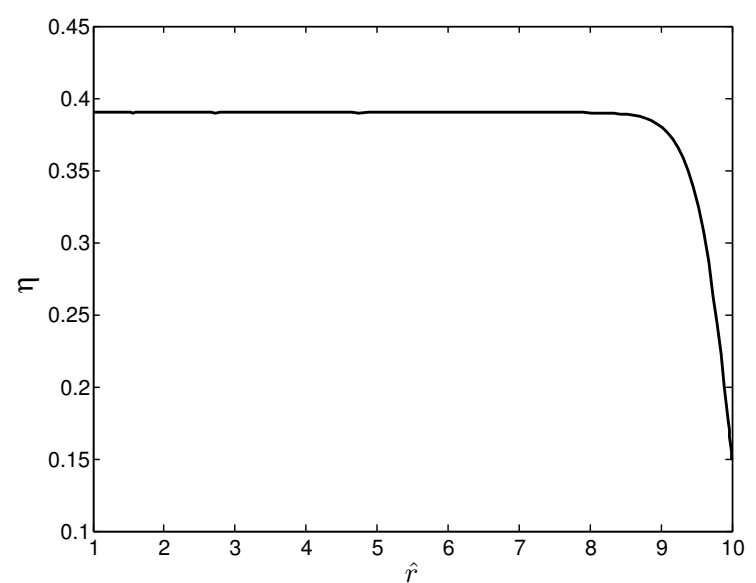

(a)

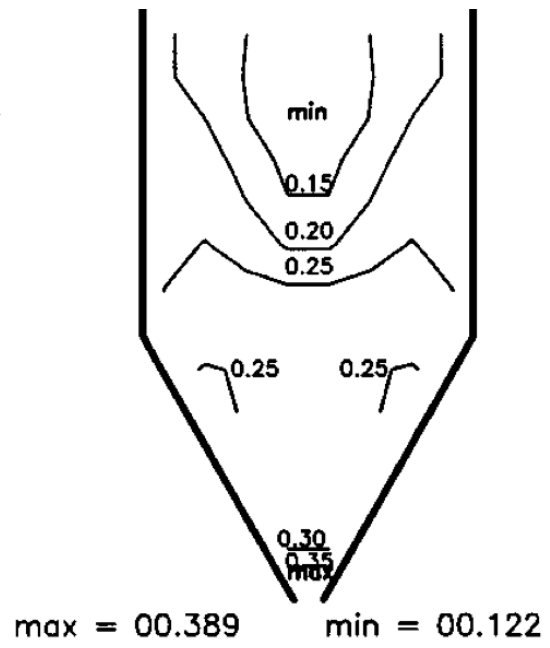

(b)

Figure 3.24: Comparison of (a) model predictions and (b) DEM results [3] for the evolution of the macroscopic friction along the flow direction.

latter. As shown in Figure 3.24, the model predicts a similar internal stress profile as in the DEM simulations of Potapov and Campbell [3], with the macroscopic friction increasing from the top to the outlet. This behavior is not captured by conventional plasticity models and supports the use of microstructurally based models such as that developed here.

Finally, the model was refined to describe deformations other than simple shear. We performed DEM simulations of triaxial compression (TC), triaxial extension (TE), and biaxial compression (BC) and compared the stress data with model predictions. The resulting differences were effectively captured by augmenting Eq. 3.6 with the term $b_{4} \mathrm{~A} \cdot \mathrm{A}: \hat{\mathrm{S}}$. This refined model produces distinct von Mises-type yield surfaces for each deformation type, with the differences tied to the microstructure. Figure 3.25 shows the model yield surfaces along with DEM data and a Lade-Duncan yield surface [4], which is known to capture the stress ratio for all deformation types. The agreement among the three further supports the use of a microstructural model, as path-dependent yield behavior is dictated by microscopic state of the granular material. 


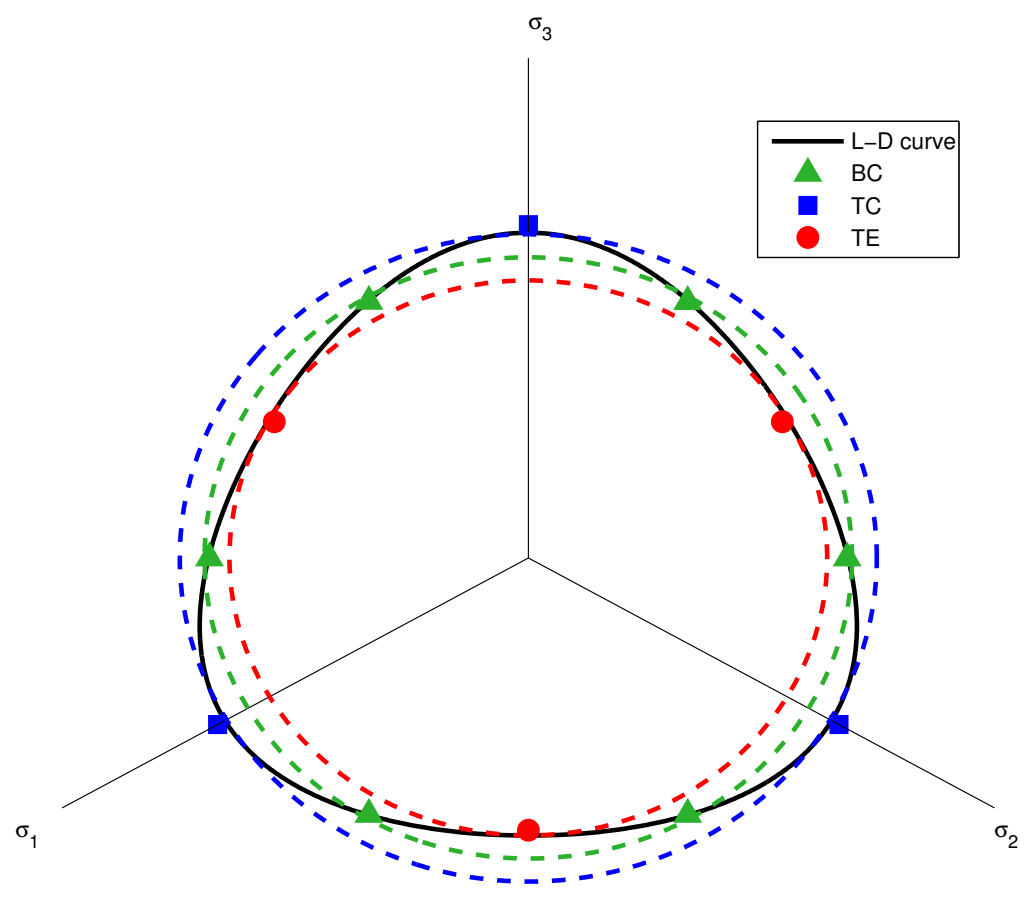

Figure 3.25: Yield surfaces for TC, TE, and BC deformations as predicted by our model (dashed lines) and by a Lade-Duncan model [4] (solid black line) with $\mu=0.5$. DEM data (symbols) are seen to lie on both surface types.

\subsubsection{Constitutive modeling of intermediate granular flows}

It has been known for some time that the rheology of granular materials depends primarily on the solids volume fraction of the material and the rate of deformation in the flow $[5,6]$. However, until recently there had been little work done on probing the rheology in all flow regimes, particularly in the context of the jamming transition. We therefore investigated the rheology of frictional granular matter about the jamming transition and constructed a rheological model for flows in the quasi-static, inertial, and intermediate regimes. A summary of this work is provided here, with the details reserved for Appendix B.

A series of constant-volume, homogeneous, steady, simple-shear DEM simulations were performed for a variety of cases of the shear rate $\dot{\gamma}$, volume fraction $\phi$, and interparticle friction coefficient $\mu$. Results for $\mu=0.5$ are shown in Figure 3.26, in which pressure $p$ and shear stress $\tau$ are plotted versus shear rate for various $\phi$. Quantities are made dimensionless by scaling them with respect to particle stiffness $k$, diameter $d$, and density $\rho_{s}$. Three regimes emerge clearly: a quasi-static regime flow slowly sheared, dense flows; an inertial regime for slowly-sheared, dilute flows; and an intermediate regime for very rapidly sheared flows. A critical volume fraction $\phi_{c}$ is observed to separate the quasi-static and inertial regimes at 
low shear rates, with the critical $p$-vs. $-\dot{\gamma}$ asymptote governing the flow at all volume fractions for sufficiently high shear rates. Interestingly, this intermediate asymptote is independent of the $\mu$ despite $\phi_{c}$ being a function of $\mu$. This $\phi_{c}$ plays an important role in the scaling of the stresses with respect to $\phi$ and $\dot{\gamma}[7,8,9]$; in particular, we find

$$
\begin{array}{ll}
p^{*}=p /\left|\phi-\phi_{c}\right| & \dot{\gamma}_{p}^{*}=\dot{\gamma} /\left|\phi-\phi_{c}\right|^{1.5} \\
\tau^{*}=\tau /\left|\phi-\phi_{c}\right| & \dot{\gamma}_{\tau}^{*}=\dot{\gamma} /\left|\phi-\phi_{c}\right|^{1.4},
\end{array}
$$

which produce a collapse of the stress data, as shown in Figure 3.27. We then apply a powerlaw rheological model to each regime and use a blending function of the form $B\left(y_{1}, y_{2}\right)=$ $\left(y_{1}^{w}+y_{2}^{w}\right)^{1 / w}$ to capture regime transitions. The resulting global rheological model is

$$
\begin{aligned}
& p= \begin{cases}p_{\mathrm{QS}}+p_{\text {Int }} & \text { for } \phi \geq \phi_{c} \\
\left(p_{\text {Inert }}^{-1}+p_{\text {Int }}^{-1}\right)^{-1} & \text { for } \phi<\phi_{c}\end{cases} \\
& \tau= \begin{cases}\tau_{\mathrm{QS}}+\tau_{\text {Int }} & \text { for } \phi \geq \phi_{c} \\
\left(\tau_{\text {Inert }}^{-1}+\tau_{\text {Int }}^{-1}\right)^{-1} & \text { for } \phi<\phi_{c}\end{cases}
\end{aligned}
$$

with the individual regime contributions defined as

$$
\begin{aligned}
p_{\mathrm{QS}} & =\alpha_{\mathrm{QS}}\left|\phi-\phi_{c}\right| & \tau_{\mathrm{QS}} & =\beta_{\mathrm{QS}}\left|\phi-\phi_{c}\right| \\
p_{\text {Int }} & =\alpha_{\text {Int }} \hat{\dot{\gamma}}^{2 / 3} & \tau_{\text {Int }} & =\beta_{\text {Int }} \hat{\dot{\gamma}}^{5 / 7} \\
p_{\text {Inert }} & =\frac{\alpha_{\text {Inert }} \hat{\dot{\gamma}}^{2}}{\left|\phi-\phi_{c}\right|^{2}} & \tau_{\text {Inert }} & =\frac{\beta_{\text {Inert }} \hat{\dot{\gamma}}^{2}}{\left|\phi-\phi_{c}\right|^{9 / 5}} .
\end{aligned}
$$

As shown in Figure 3.27, the blended model predicts stresses continuously in shear rate for all three regimes and the transitions between them. The constitutive parameters $\alpha_{Q S}$, $\beta_{Q S}, \alpha_{\text {Inert }}$, and $\beta_{\text {Inert }}$ are functions of $\mu$, while $\alpha_{\text {Int }}$ and $\beta_{\text {Int }}$ are $\mu$-independent as noted earlier.

A model was also developed for the normal stress differences (NSDs), defined as $N_{1}=$ $\sigma_{x x}-\sigma_{z z}$ and $N_{2}=\sigma_{z z}-\sigma_{y y}$. DEM data for these NSDs are shown in Figure 3.28. Because $N_{1} / p$ and $N_{2} / p$ are observed to vary little with respect to $\dot{\gamma}$ for all but the highest shear rates investigated, we chose to focus on low- $\dot{\gamma}$ behavior. Two qualitative trends emerge from Figure 3.28. The first is that $N_{1} / p$ is positive at low volume fractions and negative at high volume fractions; the second is that $N_{2} / p$ is a decreasing function of $\phi$ for low $\phi$ but an increasing one at high $\phi$. In each case, the change in behavior occurs at a specific volume fraction. The sign change in $N_{1} / p$ occurs at a volume fraction $\phi^{*}$ that is distinctly lower than the known jamming point $\phi_{c}$ [10], while the minimum of $N_{2} / p$ occurs at about $\phi_{c}$. Values of $\phi_{c}$ and $\phi^{*}$ are shown in Table 3.4. Since the variations in the NSDs about these critical volume fractions are roughly linear, we can model them as

$$
N_{1} / p=\alpha_{1}\left(\phi-\phi^{*}\right)
$$



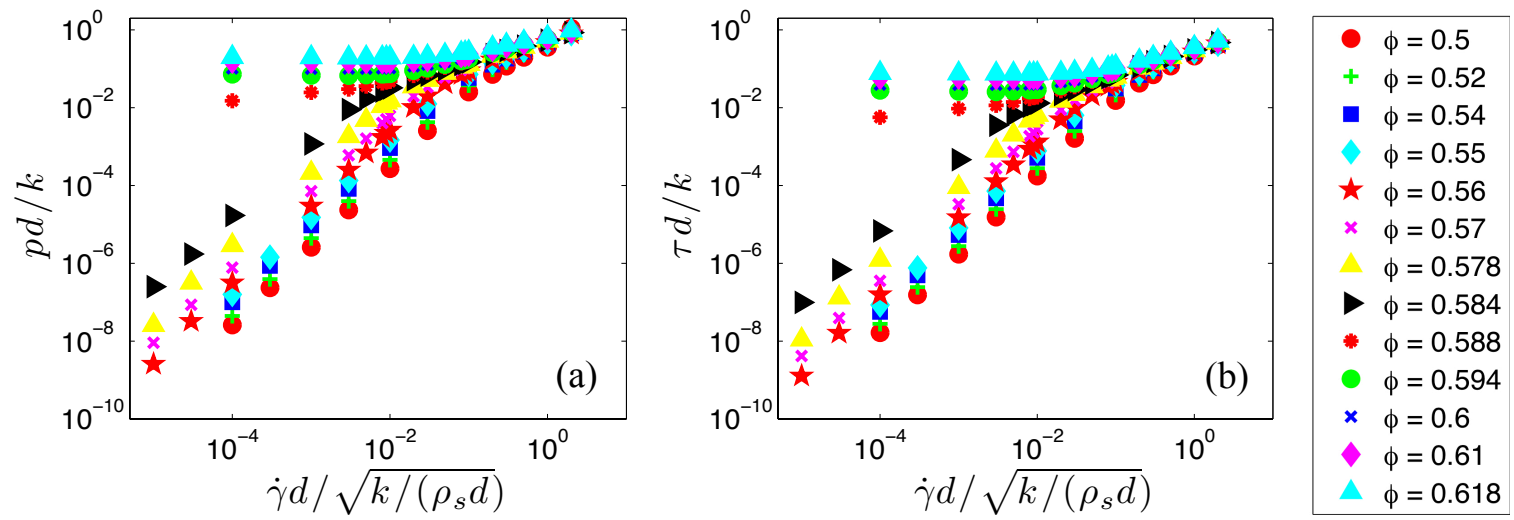

Figure 3.26: Dimensionless (a) pressure and (b) shear stress vs. dimensionless shear rate for various volume fractions with $\mu=0.5$. Three flow regimes are observed, each with the scalings $p \sim \dot{\gamma}^{m}$ and $\tau \sim \dot{\gamma}^{n}$ : a quasi-static regime with $m=n=0$, an inertial regime with $m=n=2$, and an intermediate regime with $m \approx 2 / 3$ and $n \approx 5 / 7$.
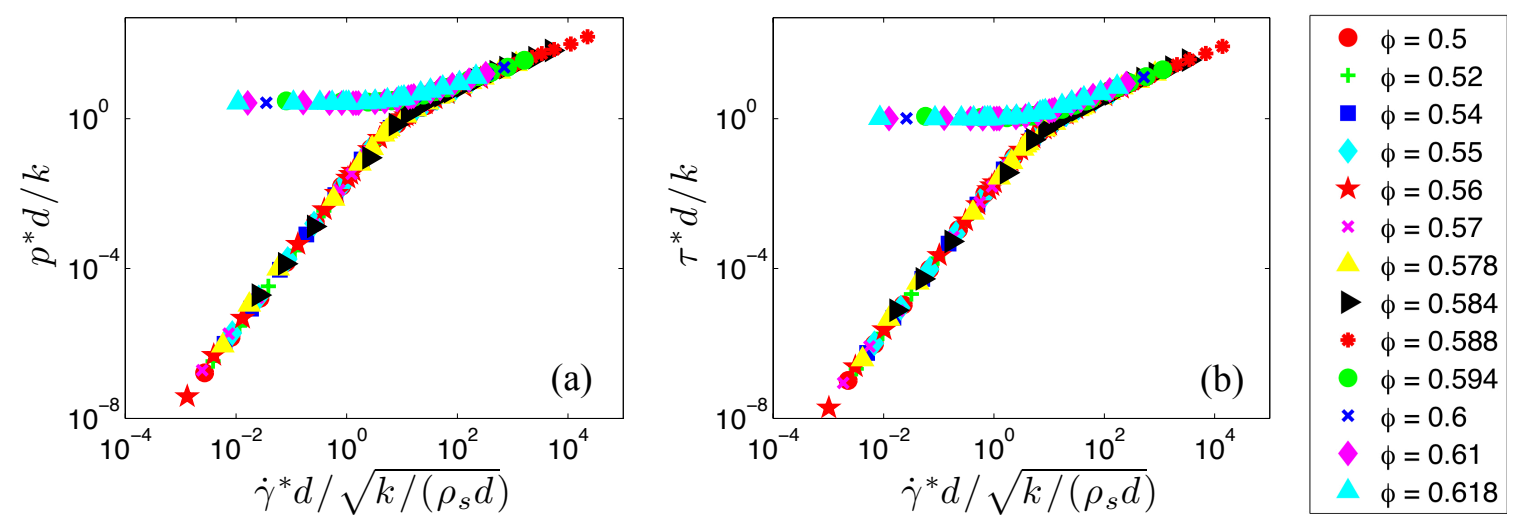

Figure 3.27: Collapse of stress vs. shear rate curves from Figure 3.26 The pressure is scaled as $p^{*}=p /\left|\phi-\phi_{c}\right|$, the shear stress as $\tau^{*}=\tau /\left|\phi-\phi_{c}\right|$, and the shear rate as $\dot{\gamma}_{p}^{*}=\dot{\gamma} /\left|\phi-\phi_{c}\right|^{1.5}$ or $\dot{\gamma}_{\tau}^{*}=\dot{\gamma} /\left|\phi-\phi_{c}\right|^{1.4}$. A simple blending function (solid lines) captures regime asymptotes and transitions. 
Table 3.4: Critical volume fraction estimates for different cases of the interparticle friction coefficient

\begin{tabular}{c|c|c}
$\mu$ & $\phi_{c}$ & $\phi^{*}$ \\
\hline 0.1 & 0.613 & 0.597 \\
0.3 & 0.5955 & 0.581 \\
0.5 & 0.587 & 0.573 \\
1.0 & 0.5805 & 0.566
\end{tabular}

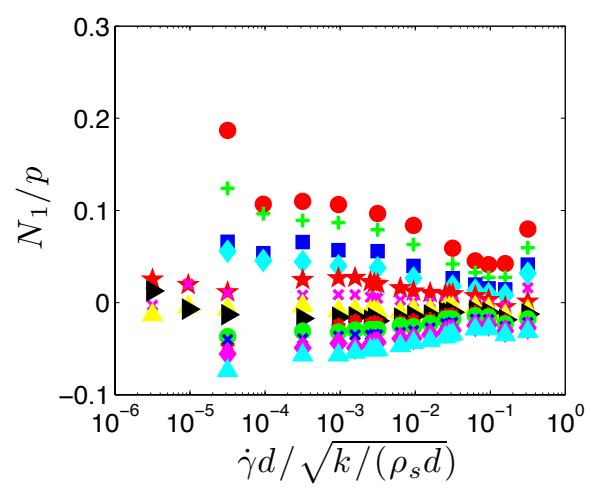

(a)

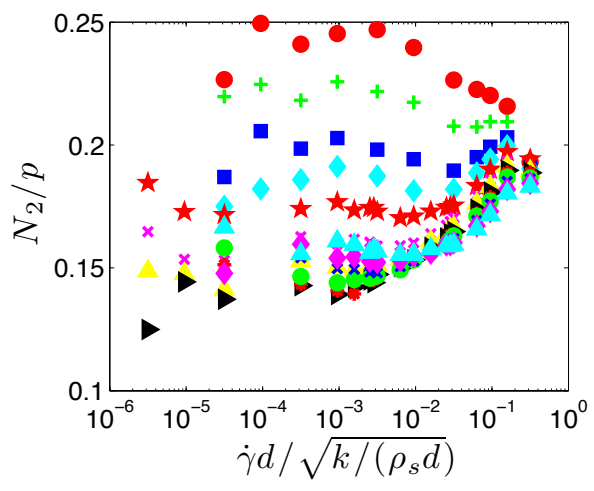

(b)

Figure 3.28: Normal stress differences versus shear rate for various volume fractions with $\mu=0.5$.

and

$$
N_{2} / p= \begin{cases}\alpha_{2}\left(\phi-\phi_{c}\right)+\beta & \text { for } \phi \geq \phi_{c} \\ \alpha_{3}\left(\phi-\phi_{c}\right)+\beta & \text { for } \phi<\phi_{c}\end{cases}
$$

where $\alpha_{i}$ and $\beta$ are in general a function of $\mu$; for $\mu=0.5$ we set $\alpha_{1}=-1.5, \alpha_{2}=0.67$, $\alpha_{3}=-1.28$, and $\beta=0.14$. Individual stress components can be calculated from the pressure and normal stress differences as

$$
\begin{aligned}
\sigma_{x x} & =p+\frac{2}{3} N_{1}+\frac{1}{3} N_{2} \\
\sigma_{y y} & =p-\frac{1}{3} N_{1}-\frac{2}{3} N_{2} \\
\sigma_{z z} & =p-\frac{1}{3} N_{1}+\frac{1}{3} N_{2} .
\end{aligned}
$$




\subsection{Work performed at Iowa State University}

Rheology of dense granular flow has been studied using a combination of (a) Discrete element method (DEM) simulations (b) Constitutive modeling and (c) Continuum simulations using MFIX two-fluid model [11]. Table 3.5 summarizes the tasks/outcomes under each of these categories.

Table 3.5: Summary of ISU tasks and outcomes.

\begin{tabular}{|c|l|}
\hline \multicolumn{1}{|c|}{ Methods } & Tasks/outcomes \\
\hline DEM & (1) Performed a comprehensive characterization of the \\
intermediate regime of granular flow. \\
(2) Quantified the spatial extent of different regimes, \\
e.g., quasi-static, intermediate, inertial, in silo discharge \\
problem. \\
(3) Validated regime transition observed in Couette cell \\
experiments at CNNY (Collaborative work with CCNY). \\
(4) Probed granular 'phase' transition by calculating the \\
order parameter (OP) using DEM and discovered a third \\
stable phase corresponding to a third steady state \\
for the OP in dense granular flows.
\end{tabular}

\subsubsection{Key Findings}

We performed continuum simulations of silo discharge using the MFIX two-fluid code [11] and quantified the error in the granular stress predicted by different constitutive models (Schaeffer [15] and Princeton models [16]) by comparing these stresses to those obtained from DEM simulations of the same silo. We found that the errors in the predicted stresses can be fairly large (up to 56\%). Through a comprehensive set of DEM simulations of homogeneously sheared granular flow, we characterized the extent of the intermediate regime of granular flow in the parameter space of solid volume fraction, particle friction and shear rate. We then quantified the spatial extent of the intermediate regime in the DEM simulation of the silo discharge problem, and found that the intermediate regime spans a significant part of the granular flow in the silo. Also the spatial extent of the intermediate regime 
is directly correlated with discrepancy in stress predicted by existing continuum models in the MFIX two-fluid code [11], leading to differences from the DEM data in predicted discharge velocity at exit, and discharge rate. The continuum predictions of discharge rate also differed from the Beverloo correlation [17], whereas the DEM prediction is closer to the Beverloo correlation.

We also compared DEM simulations of a sheared Couette cell with experiments (performed at CCNY) to validate the regime transition phenomenon, and found that DEM reliably reproduces qualitative features of the regime transition although the stress levels in DEM differed from the experiment. We also characterized the granular 'phase' transition in dense granular flows by extracting the order parameter (OP) from DEM and discovered a third stable granular 'phase' which is neither completely fluidlike nor completely solidlike. The OP model of Aranson and Tsimring [18] has been refined and developed further as an alternative constitutive model for dense granular flow. We also assessed the performance of different constitutive models (due to Jop et al. [12], Losert et al. [13], Savage [14]) with DEM data on homogeneously sheared granular flow in the intermediate regime, and found that none of these models capture the correct behavior of stress with strain rate in the intermediate regime. We are now developing a new contact stress model based on the relative-acceleration concept that gives promising results even in the intermediate regime.

\subsubsection{Assessment of continuum model performance in silo dis- charge using DEM simulations}

Granular material exhibit different constitutive behaviors in different regimes of flow, depending up on particle (particle-particle friction coefficient, particle inelasticity) and flow (shear rate) properties. In general these regimes are classified based on scaling of shear stress with strain-rate [5], and developing accurate constitutive models that work in all regimes is an active area of research. Due to these regime transition and limitations of current constitutive models, even a seemingly simple practical device for the storage and discharge of granular material such a flat-bottomed silo poses a surprisingly difficult challenge for continuum models [16, 19]. Figure 3.29(b) shows that the discharge rate predicted using 3D continuum simulations deviates considerably (more than 80\%) from the Beverloo correlation [17] and 3D DEM simulation data.

A previous study performed by Benyahia [19] also revealed this discrepancy in discharge rate between 2D continuum simulations and Beverloo correlation [17]. The advantage of having the 3D DEM data in our study is that we could also directly quantify the error in stress predicted by the constitutive models. In order to perform a quantitative assessment of different continuum models, we directly compare the granular stress predicted using continuum models with that from DEM data for a 3D silo geometry. Figures 3.30(a) and 3.30(b) are the contour plots of error in solid stress prediction using Schaeffer [15] and Princeton [16] model, respectively (details about this error quantification study are given in Sec. 3.2 of Appendix E). These figures show that the maximum error incurred in solid stress prediction (when compared with the stresses obtained from DEM simulations) is around $42 \%$ and $56 \%$ 


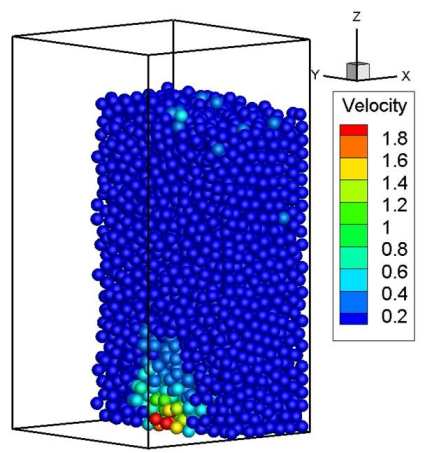

(a)

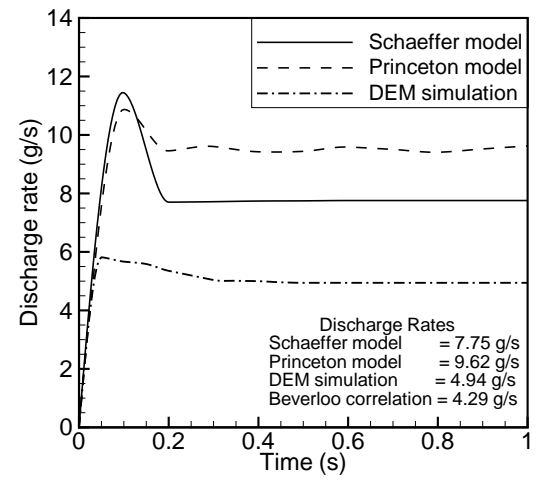

(b)

Figure 3.29: (a) DEM simulation of granular discharge from a flat-bottomed silo with a circular orifice $\left(d_{\text {orifice }} / d_{p}=6\right)$, and (b) Dependence of discharge rate of granular material from a flat-bottomed silo on the constitutive model for the solid-phase stress tensor.

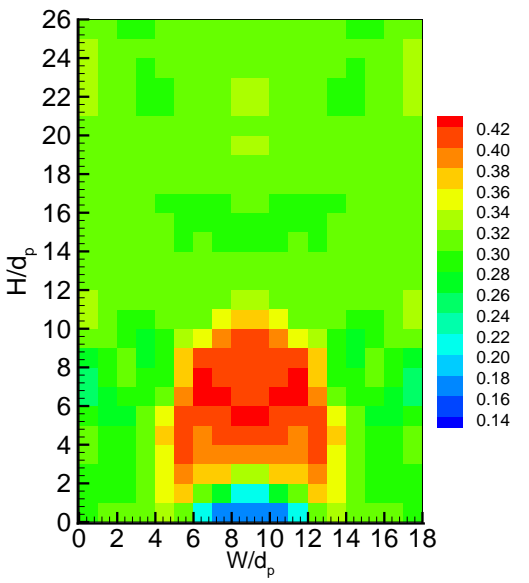

(a)

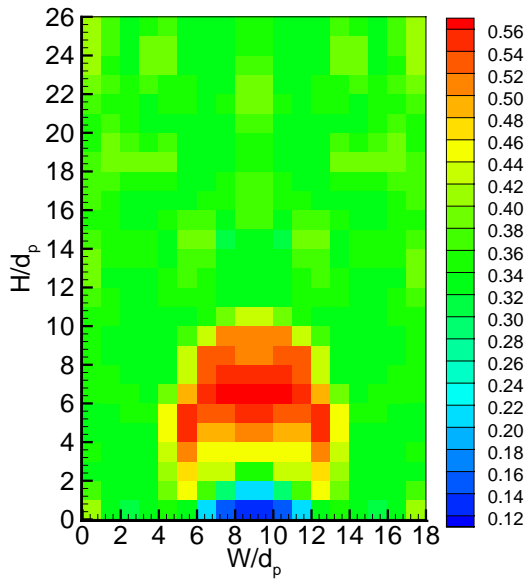

(b)

Figure 3.30: Error in stress prediction (error quantified using vector norm of relative error). (a) For Schaeffer frictional model, and (b) For Princeton frictional model. Simulation parameters: $D=6 d_{p}, \mu_{p}=0.5, e=0.91$. 
for Schaeffer and Princeton models, respectively.

In these silo simulations DEM predicts the highest stress levels, whereas the Princeton frictional model predicts the lowest values for stress. Since frictional effects are present at a lower value of solid volume fraction in the Princeton model, whereas in the Schaeffer model friction starts at maximum packing, hence the computed stresses using Princeton model are lower than that of the Schaeffer model. Jalali et al. [20] performed a similar study, in which they compared the solid phase momentum transfer between these two continuum models and DEM simulations, and showed that the DEM calculations can be about three times higher than that calculated from continuum models.

\section{Characterization of different regimes using DEM simulations}

In order to further understand this discrepancy in silo discharge we quantified the spatial extent of different regimes in the silo. This is achieved by constructing a regime map for granular rheology using DEM simulation data obtained from homogeneously sheared assembly of granular particles over a range of solid volume fractions (0.45 to 0.64), shear rates $\left(k^{*}=2.5 \times 10^{4}\right.$ to $\left.k^{*}=10^{9}\right)$ and particle friction coefficients (0.1 to 1.0) (details about these DEM simulations are given in Sec. 3 of Appendix G). In a previous study Tardos et al. [21] provided a regime map from data obtained from their experiments, however there is no comprehensive study that characterizes the different regimes of granular flow over a wide range of simulation parameters. DEM simulations were performed over a range of solid volume fractions, shear rates and particle friction coefficient (details given in Sec. 3 of Appendix G). Figures 3.31(a) and 3.31(b) show such regime maps obtained from these DEM simulations of homogeneously sheared granular flow in space of solid volume fraction $\nu$, particle friction coefficient $\mu_{p}$ for non-dimensional shear rate $k^{*}\left(k_{n} / \rho_{s} d_{0}^{3} \dot{\gamma}^{2}\right)$ of $2.5 \times 10^{4}$ and $10^{9}$, respectively. In Figs. 3.31(a) and 3.31(b) the inertial regime $\left(\sigma \propto \dot{\gamma}^{2}\right)$ is represented with red color, whereas the blue color shows the presence of quasi-static regime $(\sigma \neq f(\dot{\gamma}))$. In between both these regimes, there exists an intermediate regime $\left(\sigma \propto \dot{\gamma}^{n}, 0<n<2\right)$ which is represented with green color in Figs. 3.31(a) and 3.31(b).

\section{Quantification of spatial extent of different regimes in silo discharge}

The spatial extent of different regimes inside silo geometry is characterized with the help of regime map established in Figs. 3.31(a) and 3.31(b). We extracted values of local solid volume fraction and mean strain rate in each cell from the DEM simulation of the flatbottomed silo and assigned them different regimes (inertial, intermediate and quasi-static) following the above established regime map. Figures 3.32(a) and 3.32(b) show the spatial extent of different regimes in a flat-bottomed silo for a particle friction coefficient of 0.50 and 0.25 , respectively (details of this quantification of spatial extent using DEM simulations of silo discharge are given in Sec. 4.2 of Appendix E). In Figs. 3.32(a) and 3.32(b) the red color represents the presence of inertial regime (which found to be exist near the discharge orifice), the blue color in the same figure represents the presence of quasi-static regime, any other color represent the presence of intermediate regime. 


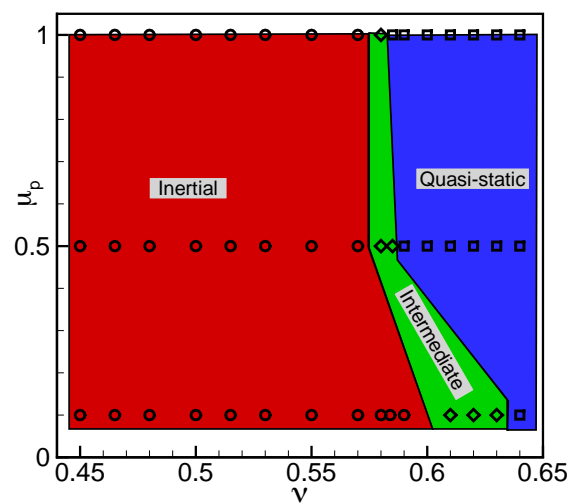

(a)

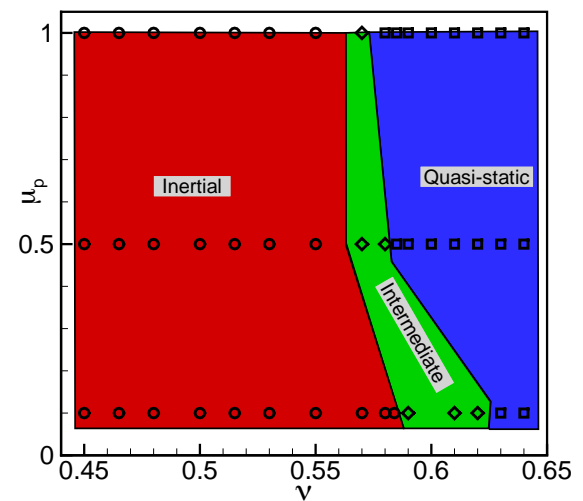

(b)

Figure 3.31: Regime map for granular flows, constructed from data of 3D DEM simulations of homogenously sheared granular flow (a) $k^{*}=k_{n} / \rho_{s} d_{0}^{3} \dot{\gamma}^{2}=2.5 \times 10^{4}$ and (b) $k^{*}=k_{n} / \rho_{s} d_{0}^{3} \dot{\gamma}^{2}=$ $10^{9}$.

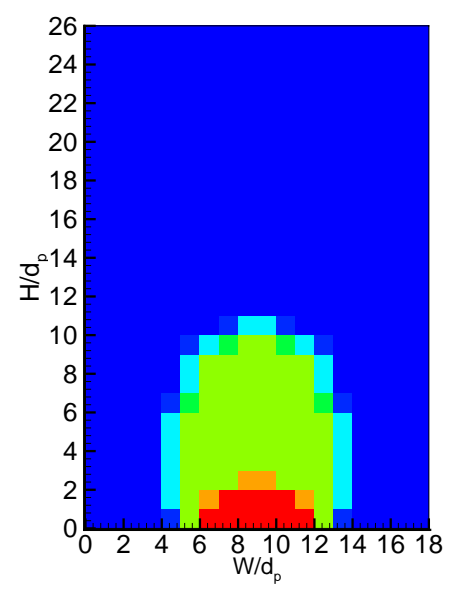

(a)

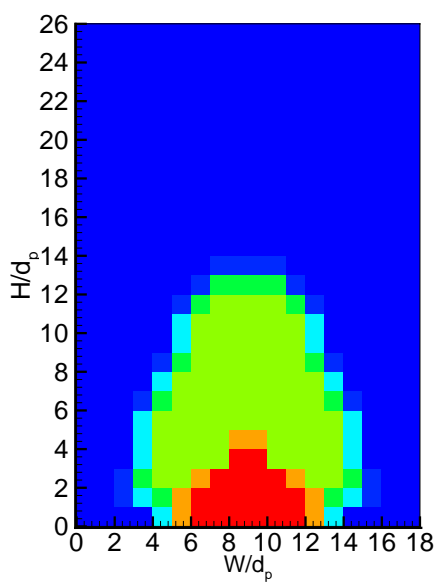

(b)

Figure 3.32: Characterization of spatial extent of different regimes of granular rheology in a flat-bottomed silo based on local solid volume fraction, mean strain rate and particle friction coefficient. The Blue color represents quasi-static regime, the Red color represents the inertial (rapid flow) regime, whereas presence of any other color indicates the spatial extent of the intermediate regime. (a) Simulation parameters: $D=6 d_{p}, \mu_{p}=0.5, e=0.91$, and (b) Simulation parameters: $D=6 d_{p}, \mu_{p}=0.25, e=0.91$. 
From this study it is evident that all three different regimes (inertial, intermediate, and quasi-static) co-exist even for a simple problem such as the discharge of granular particles from a silo. It is also interesting to notice that, the intermediate regime spans a considerable spatial region inside silo. A careful study of Figs. 3.32(a) and 3.32(b) reveal that the spatial extent of intermediate regime expands as the particle friction coefficient decreases from 0.50 to 0.25 , these values of friction coefficient represent the general behavior of granular material such as glass beads. A close examination of Figs. 3.32(a), 3.32(b), 3.30(a) and 3.30(b) also reveal that the maximum error incurred in the stress prediction spreads around the spatial location where intermediate regime is present. Hence it can be concluded that the widely used constitutive models (Schaeffer [15] and Princeton [16] model) perform poorly in the intermediate (transitional) regime of granular flows, where both collisional and frictional interactions between the particles are important.

In the next section we describe an experimental and computational study performed to understand the phenomenon of the regime transition in dense granular flows using a modified Couette shear cell. This work was performed in collaboration with the CCNY team.

\subsubsection{Validation of rheological regime transition phenomenon through DEM simulations and experiments}

In order to understand the regime transition from quasi-static to intermediate behavior we study the rheology of dense granular material in an annular Couette cell by experimentation (performed at CCNY) and simulations (additional details about these experiments and simulations are given in Sec. 2 of Appendix F). In Fig. 3.33 the average shear stress on the inner wall is plotted with time for the batch mode operation of Couette device. Figure 3.33 shows that changing the shear rate more than fivefold in the DEM simulations does not result in any significant change in the shear stress, which is a feature of the quasi-static regime. Similar quasi-static behavior was also observed in the experiments, however the magnitude of the measured shear stress is about 2.5 times higher than that predicted in the simulations.

The variation of shear stress with shear rate in the continuous mode with a vertical speed, $V_{z}=3.2 \times 10^{-3} \sqrt{g d_{0}}$ corresponding to the flow rate in experiments is shown in Fig. 3.34(a) (the left vertical axis corresponds to the DEM simulation results and the right vertical axis corresponds to the experimental data). The experimental and DEM results in Fig. 3.34(a) show that the flow exhibits two distinct regimes: a quasi-static regime, where the shear stress is independent of the shear rate (at very low shear rate), and an intermediate regime, where the dependence takes the form of a power-law. The transition starts at a non-dimensional shear rate $\hat{\gamma}$, of about 0.1 , whereas the flow in the batch-mode at the same $\hat{\gamma}$ does not undergo transition (see the quasi-static behavior at even higher shear rates in Fig. 3.33). This transition behavior indicates that the axial flow in continuous mode facilitates regime transition. The DEM shear stress, however, is about 4.5 times lower in magnitude than its experimental counterpart, consistent with a factor-of-two difference found in a similar study performed by Ji et al. [22]. This difference could be due to differences in particle properties, 


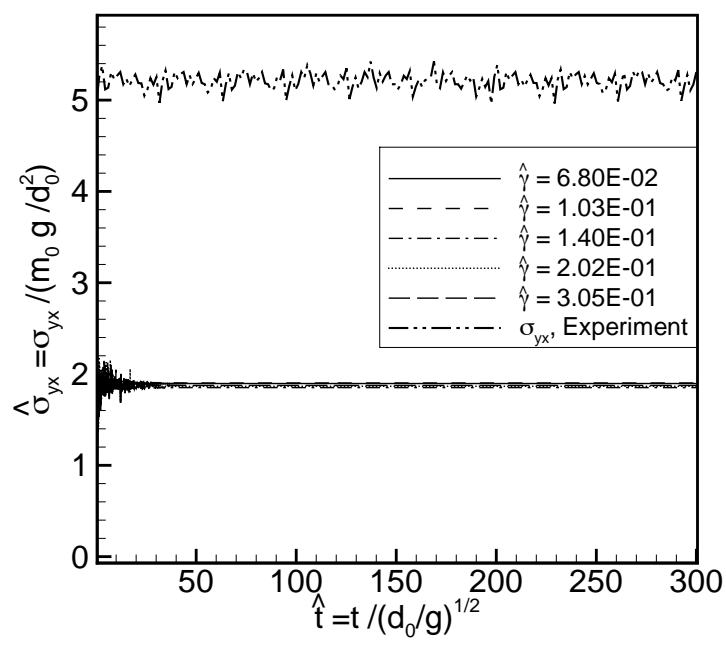

Figure 3.33: Temporal evolution of the average non-dimensional shear stress on the Couette cell wall during the batch-mode operation.

domain geometry, system setup and solid volume fraction.

The ratio of shear to normal stress $\left(\sigma_{y x} / \sigma_{y y}\right)$ is plotted in Fig. 3.34(b) as a function of the non-dimensional shear rate for the continuous model of operation. The variation of this ratios also manifests the same transition and intermediate regime behavior: it remains almost constant approximately equal to the particle friction coefficient for lower shear rates; it has a power-law dependence as the shear rate increases beyond a certain critical value. The experimental ratios are close to, albeit slightly lower than, the DEM result. The better agreement again confirms that the large differences in stress magnitude is mostly due to the different confinements (with/without overburden, etc.) and that the simulations reveal essentially the same rheology as in the experiment.

\subsubsection{Constitutive modeling framework to capture granular 'phase' transition}

\section{Challenges in constitutive modeling of granular flows}

A quantitative description of the macroscale behavior of granular flow in industrial devices requires a reliable constitutive model. However, development of a constitutive model that can perform accurately in all the regimes of granular flow is a challenging task. The stress tensor in the granular material is a function of both particle and flow properties that correspond to different scales of the problem, e.g., macroscale, meso-scale and microscale. The solid volume fraction and shear rate correspond to macroscale properties, whereas interparticle friction coefficient and coefficient of restitution are microscale parameters. Quantities such as the order parameter $(\mathrm{OP})$ and the pair correlation function, characterize meso-scale structure 


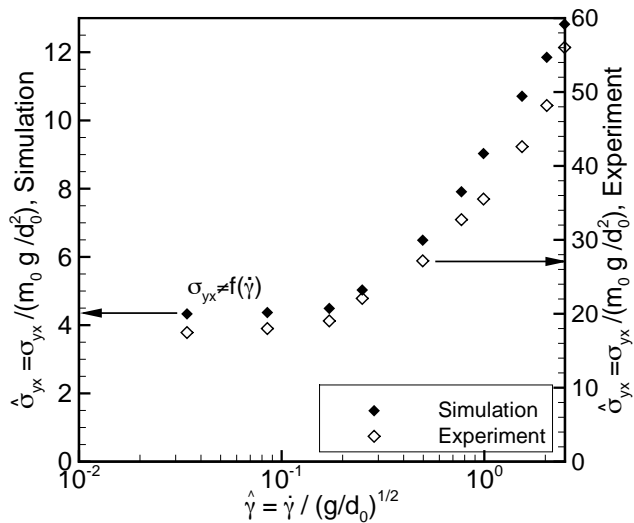

(a)

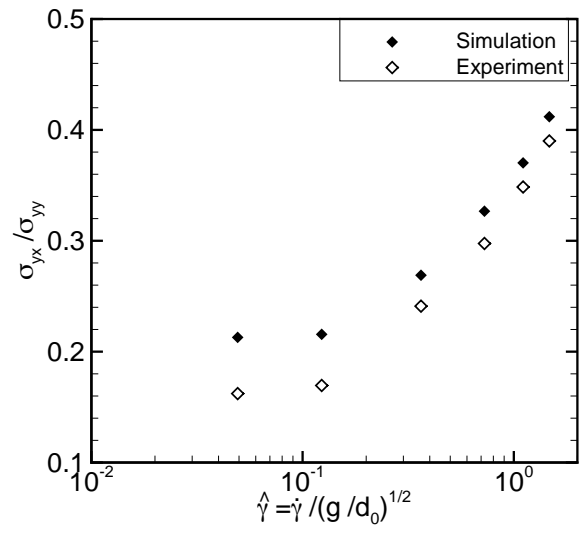

(b)

Figure 3.34: (a) Variation of the average shear stress with the non-dimensional shear rate for the continuous-mode operation. The filled symbols denote the DEM simulation results, while the open symbols are for data obtained from experiments. Different scales are used on the left and right axes for the DEM and experimental data, respectively, to emphasize the similarity in their trends and (b) Ratio of shear to normal stress as a function of shear rate for the continuous mode of operation. Filled symbols correspond to DEM simulation data whereas the open symbols are for experimental data.

in the granular flow. Hence, the granular stress tensor can be represented as,

$$
\sigma_{i j}=f(\text { macroscale parameters, meso-scale parameters, microscale parameter }) \text {. }
$$

Most constitutive models $[23,16]$ used to predict the behavior of granular flows are based on an additive decomposition of the total granular stress as a weighted sum of kinetic and frictional contributions $\left(\sigma_{i j}=\sigma_{i j}^{k}+\sigma_{i j}^{f}\right)$, with the weight factor specified solely as a function of the solid volume fraction. Both experiments in a 2D granular shear cell (GSC) [24, 25] and DEM simulations [26] reveal that grain contacts in the transitional regime are characterized by a mix of enduring solidlike and fluidlike contacts that is indicative of a granular 'phase' transition. In particular, these grain interactions are not determined by the solid volume fraction alone, but are dependent on particle properties (such as particle friction coefficient, inelasticity) and flow properties (such as shear rate). The 'phase' of granular matter can be characterized by the order parameter, which is a meso-scale quantity. The simple additive models are not capable of capturing this complex constitutive behavior in different regimes of granular flows, in part because they do not account for meso-scale flow features. Since most constitutive models in use are phenomenological, this observation motivates development of a constitutive model for the granular flows that reflects the 'phase' transition based on microscale physical interaction between the grains. 


\section{The OP framework for constitutive modeling}

In this work we selected the order parameter (OP) approach because the OP captures the granular 'phase' transition from fluidlike to solidlike behavior through its dependence on the particle and flow properties. The OP model also takes into account the intermediate scale (meso-scale) of the problem in the framework of a constitutive model. In a homogeneous granular flow, the OP is defined [27] as the ratio of the number of space-time averaged "enduring" (solidlike) contacts $\overline{\left\langle Z_{s}\right\rangle}$ to all contacts $\overline{\langle Z\rangle}$ within a sampling volume,

$$
\rho \equiv \frac{\overline{\left\langle Z_{s}\right\rangle}}{\overline{\langle Z\rangle}} .
$$

The OP is useful in characterizing two limiting cases: when the granulate is in a state of "enduring" contacts, and when it is strongly agitated, i.e., completely fluidlike. In the solid state all contacts are enduring and hence $\rho=1$. In the fluid limit $\overline{\left\langle Z_{s}\right\rangle}$ is zero and $\overline{\langle Z\rangle}$ is small but finite, and therefore $\rho=0$. In order to understand the granular 'phase' transition through the OP we extract this quantity from 3D DEM simulations of sheared granular flow over a range of solid volume fractions, particle friction coefficient and shear rates. Details of these DEM simulations are provided in Sec. 3 of Appendix G.

\section{OP dynamics from homogeneous shear simulations}

The OP characterizes the 'phase' or state of the granular material. Aranson and Tsimring [18] in their original work related the OP to the derivative of the free energy density function $F(\rho, \delta)$ :

$$
F(\rho, \delta)=\int^{\rho} \rho(\rho-1)(\rho-\delta) d \rho
$$

through the Ginzburg-Landau equation:

$$
\frac{D \rho}{D t}=D_{c} \nabla^{2} \rho-F(\rho, \delta)
$$

Further they postulated that this free energy density function $F(\rho, \delta)$ (see Eq. 3.25) has two local minima at $\rho=1$ (solidlike) and $\rho=0$ (fluidlike) to account for the bistability near the solid-fluid transition. The relative stability of the two phases is controlled by the parameter $\delta$, which is a function of the stress tensor. For small $\delta$ the solidlike state is more favorable, and vice versa. A typical profile of the free energy density function postulated by Aranson and Tsimring [18], is shown in Fig. 3.35, for different values of $\delta$. With the formulation of the free energy density function $F(\rho, \delta)$ in Eq. 3.25, the solution of the Ginzburg-Landau equation (Eq. 3.26) always results in a steady state value of the OP which is either 1 or 0 , depending on the value of parameter $\delta$. The form of free energy density function has not been comprehensively validated against 3D DEM data in order to verify this postulated form of the function $F(\rho, \delta)$. Hence, we extracted the $\mathrm{OP}$ values from our 3D DEM simulations to understand the OP dynamics. 


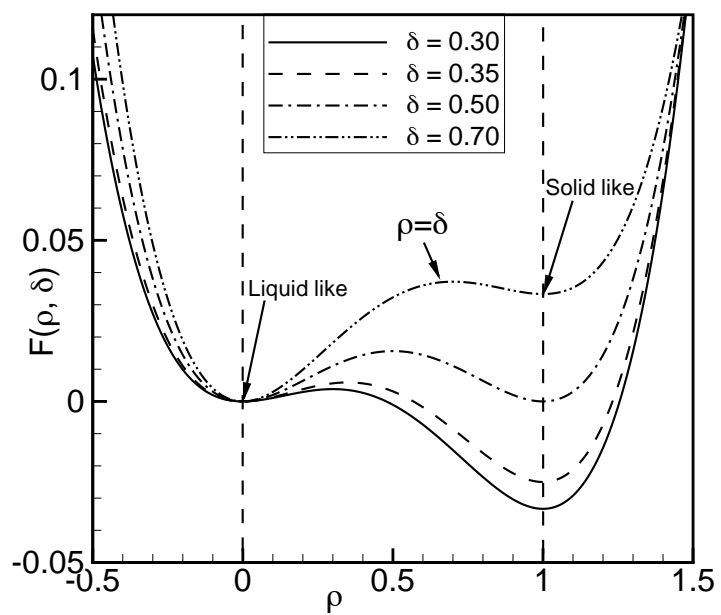

Figure 3.35: Typical profile of the free energy density function $F(\rho, \delta)$ postulated by Aranson and Tsimring [18].

In Fig. 3.36(a) the steady state values of the OP are plotted with solid volume fraction with three different values of particle friction coefficients. Figure 3.36(a) shows that the OP is indeed a strong function of the particle friction coefficient. An increase of about $300 \%$ in the value of the OP is seen when particle--particle coefficient of friction increases from 0.1 to 1.0 at the same solid volume fraction. This result is expected because at higher interparticle friction the particles are prevented from sliding over each other, resulting in solidlike contacts. A sudden jump in the value of the OP is seen near the maximum packing limit, this sudden increase in the value of the OP is ascribed to the presence of strong force chains near the packing limit. Figures 3.36(b), 3.36(c), and 3.36(d) are the similar plots of the OP with solid volume fraction as described in Fig. 3.36(a), but for different values of shear rates. These plots (Figs. 3.36(a), 3.36(b), 3.36(c), 3.36(d)) show that the particle friction coefficient is the most important parameter which affects the OP, whereas the shear rate has a least impact on the OP values.

A careful examination of these plots (Figs. 3.36(a), 3.36(b), 3.36(c), 3.36(d)) reveals that as the non-dimensional shear rate $k^{*}\left(k_{n} / \rho_{s} d_{0}^{3} \dot{\gamma}^{2}\right)$ increases (going from Fig. 3.36(a) to Fig. 3.36(d)), the sudden jump in the OP value (near the maximum packing limit) becomes increasingly sharper and sharper. There is an another interesting point to note that, as the particle friction coefficient increases this sudden jump in the OP values occur at lower and lower values of solid volume fraction. This finding is consistent with the results of Song et al. [28], who studied the jamming of packed spheres through a phase diagram and showed that the minimum solid volume fraction required for jamming decreases with the increase in the particle friction coefficient. It is also noteworthy that at the highest value of $k^{*}$ (which corresponds to lowest shear rate, see Fig. 3.36(d)) the OP attains its limiting value of 1 near the maximum packing limit, whereas the OP approaches to its other limiting value of 0 , when both solid volume fraction and friction coefficient tends towards zero. 


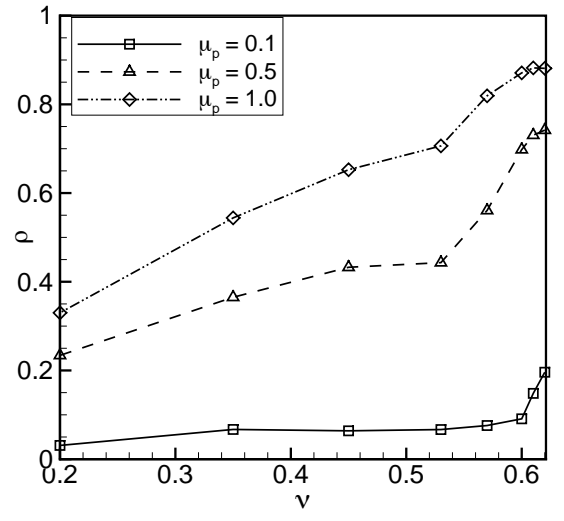

(a)

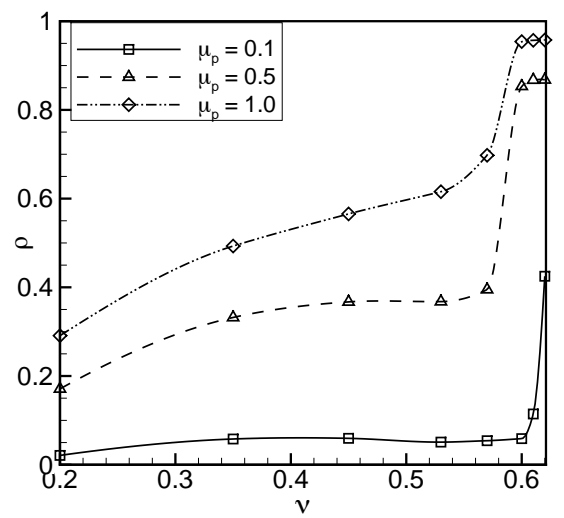

(c)

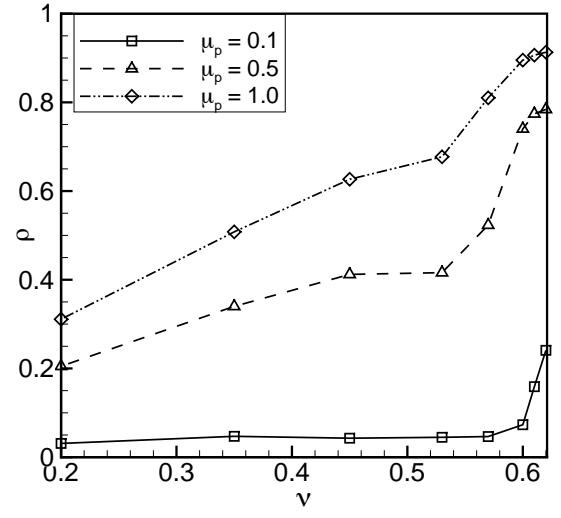

(b)

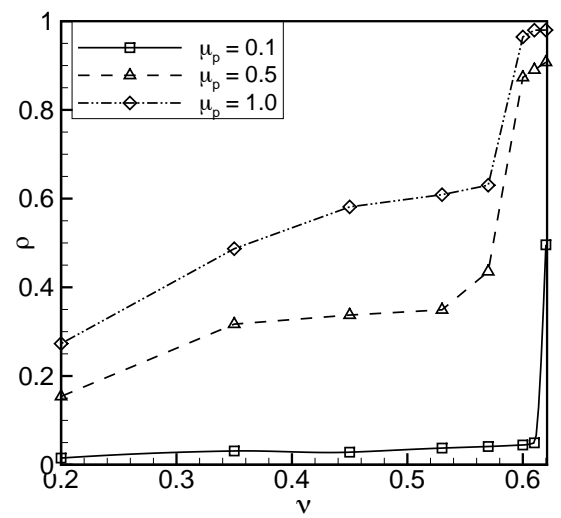

(d)

Figure 3.36: The OP (at steady state) plotted with solid volume fraction (a) for $k^{*}=2.5 \times 10^{4}$ and $e=0.7$, (b) for $k^{*}=10^{5}$ and $e=0.7$, (c) for $k^{*}=10^{7}$ and $e=0.7$ and (d) for $k^{*}=10^{9}$ and $e=0.7$. 
The DEM results (Figs. 3.36(a), 3.36(b), 3.36(c), 3.36(d)) indicate that, there should be one more intermediate local minimum of this free energy density function at $\rho=\rho_{s s}$, which causes the OP to attain a steady state stable value which is neither zero (fluidlike) nor one (solidlike). Hence, a modification in the form of free energy density function is proposed (see Eq. 3.27), to account for this third stable granular 'phase' at $\rho=\rho_{s s}$. Figure 3.37 shows a typical profile of the proposed free energy density function $F^{*}(\rho, \delta)$. In Eq. 3.27, $\rho_{s s}$ and $\delta_{s s}$ can be specified using DEM data.

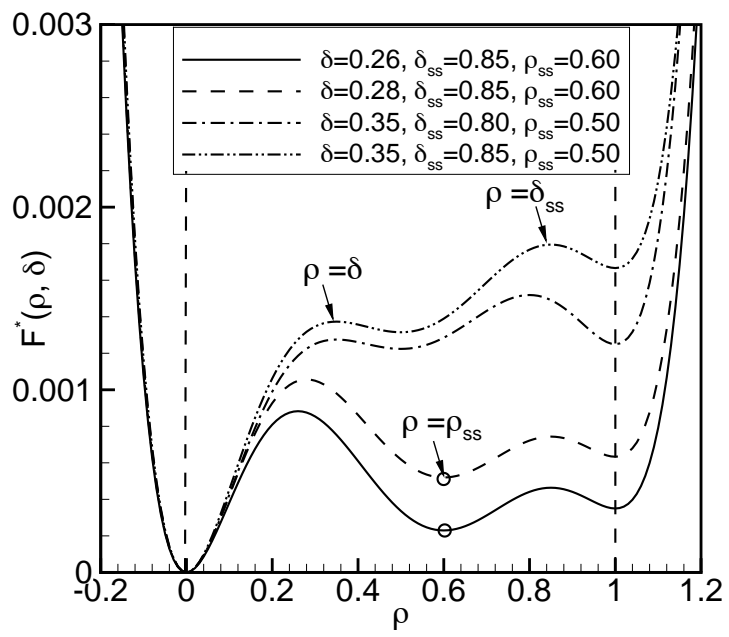

Figure 3.37: Typical profile of proposed free energy density function $F^{*}(\rho, \delta)$ with third intermediate local minima at $\rho=\rho_{s s}$.

$$
F^{*}(\rho, \delta)=\int^{\rho} \rho(\rho-1)\left(\rho-\rho_{s s}\right)(\rho-\delta)\left(\rho-\delta_{s s}\right) d \rho
$$

After understanding the OP dynamics through the results from DEM simulations, we further refined and improved the objective OP model [29] in order to make it tractable. Details of the OP model description and refinement is provided in Sec. 4 of Appendix G. After refining the OP based model the next logical step is to assess the performance of proposed ROP (refined order parameter) model which is presented in the following section.

\section{Assessment of the ROP model}

The predictive capability of the ROP-KT model (constitutive relation for the fluidlike stress contribution obtained from kinetic theory of granular flows) is assessed in different regimes by comparing predictions for the total granular stress to those from DEM simulations of homogeneously sheared granular flow. Here we will only present the model predictions in the near transitional and deep intermediate regimes (for detailed model assessment reader should refer to Sec. 6 of Appendix G). 


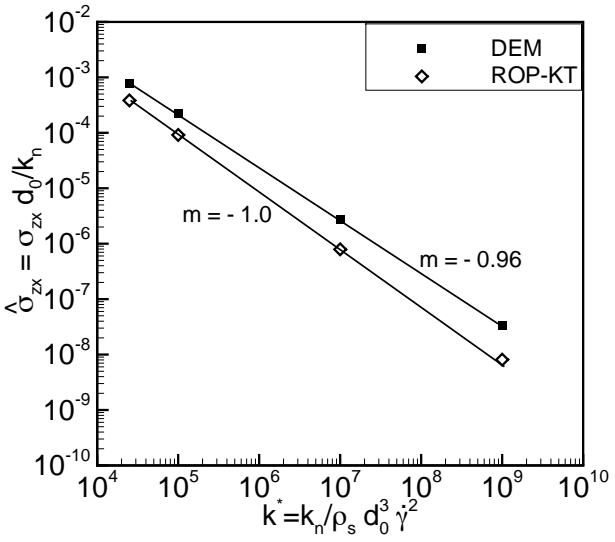

(a)

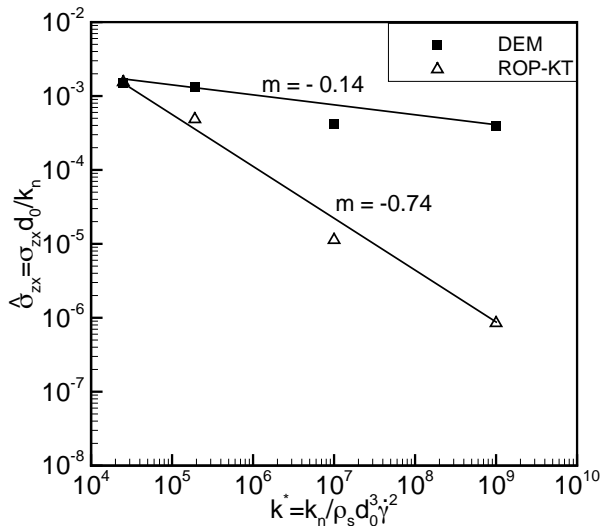

(b)

Figure 3.38: (a) The total granular stress as a function of shear rate $k^{*}$. Simulation parameters: $\nu=0.57, \mu_{p}=0.5, e=0.7$. and (b) The total granular stress as a function of shear rate $k^{*}$. Simulation parameters: $\nu=0.62, \mu_{p}=0.1, e=0.7$.

In order to quantify the performance of the ROP-KT model near the transition from inertial to intermediate regime, we considered a solid volume fraction of 0.57. Figure 3.38(a) compares the total granular stress predicted by the ROP-KT model with data from the DEM simulations. In the transitional regime $(\nu=0.57)$ the $\mathrm{ROP}-\mathrm{KT}$ model predicts the total granular stress reasonably well (see Fig. 3.38(a)), with an error of $15 \%$ when compared with the DEM data. It is interesting to note that at this solid volume fraction there are significant multiparticle contacts as indicated by the mean coordination number value of 2.2 obtained from DEM simulations (result not shown here). Nevertheless, the kinetic theory based ROP model performs reasonably well. As shown in Sec. 6 of Appendix G, the ROP-KT model performs well for all the solid volume fraction lower than 0.57 .

To assess the performance of the ROP-KT model in the deep intermediate regime, we selected a case with solid volume fraction of 0.62 and interparticle friction coefficient of 0.1 . At this solid volume fraction the ROP-KT model does not predict either the magnitude or the scaling of the total granular stress correctly, as evidenced by the mismatch in the slope in Fig. 3.38(b). Although the ROP-KT model decomposes the total granular stress into solidlike and fluidlike parts, unlike other models $[14,23,16]$ the weighting factors for these contributions depends on the shear rate through the OP. Note that as a consequence the total granular stress predicted by the ROP-KT model actually shows an intermediate scaling $\left(\sigma \propto \dot{\gamma}^{2}, n=-2 m=1.48\right)$ with the shear rate, even though the fluidlike stress follows a inertial scaling.

Based on this assessment study, it can be concluded that the ROP-KT model has the capability to accurately predict granular stress up to a solid volume fraction of 0.57 . As the solid volume fraction exceeds 0.57 the flow transitions to the intermediate regime and the ROP-KT model fails to capture the correct trend of shear stress with shear rate. The differences in the magnitude of the stress prediction in the intermediate regime is attributed 
to the fact that the ROP-KT model assumes that the fluidlike stress contribution follows the kinetic theory closure even in the dense regime. However, this assumption does not hold in the deep intermediate regime where both collision and frictional interactions between the particles are important. Although the ROP model coefficients include a dependence on the shear rate, this dependence is not able to accurately predict the stress-strain scaling in the deep intermediate regime of flow. This study motivates the development of a contact stress model that can capture the intermediate regime scaling of the granular stress.

\section{Performance evaluation of different constitutive models in the intermediate regime}

The performance of different constitutive models is assessed in the intermediate regime of granular flow. In Fig. 3.39, the shear component of the total granular stress is plotted with shear rate for a solid volume fraction of 0.62 with interparticle friction coefficient of 0.1 (this combination of solid volume fraction and particle friction coefficient corresponds to the intermediate regime). Details of this assessment study are given in Sec. 7 of Appendix G. In

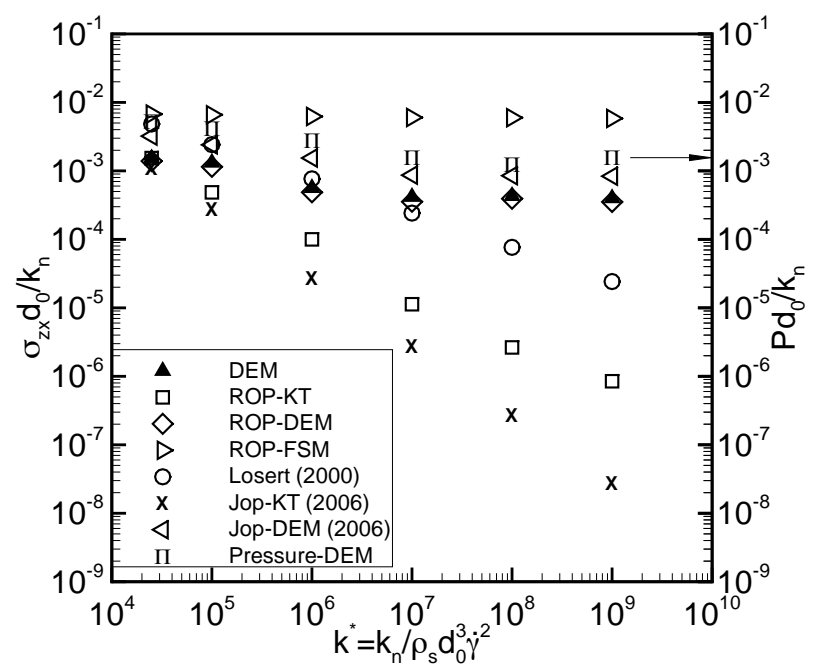

Figure 3.39: Shear component of the total granular stress (different symbols represent result obtained with different constitutive models) plotted with shear rate. Simulation parameters: $\nu=0.62, \mu_{p}=0.1, e=0.7$.

summary, this assessment study performed with different constitutive models in the intermediate regime reveals that none of the existing models capture the correct scaling of shear stress with shear rate in this regime. However, the ROP model and the model proposed by Jop et al. [12] capture the correct scaling of shear stress with shear rate, provided one of the inputs to the model is supplied from the DEM data. Figure 3.39 also shows that the pressure follows the same scaling with shear rate as the shear stress in the intermediate regime. Hence, it is important for a constitutive model to predict the correct behavior of the pressure with the strain rate. 
In order to better understand the scaling of the granular stress in the intermediate regime, the total granular stress obtained from DEM simulations of homogeneously shear granular flow is first decomposed into contact (virial) and streaming (dynamics) contributions. In

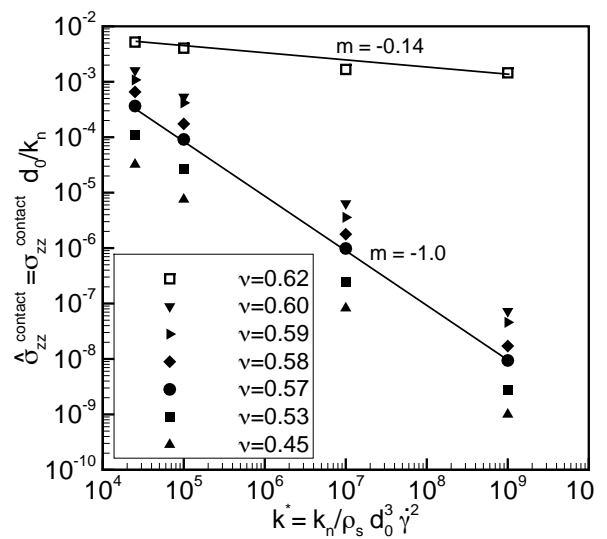

(a)

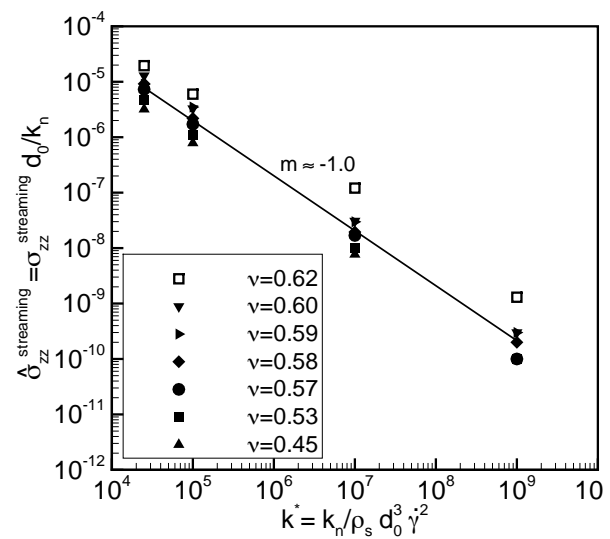

(b)

Figure 3.40: (a) Contact (virial) contribution of the total granular stress as a function of shear rate and (b) Streaming (dynamic) contribution of the total granular stress as a function of shear rate. Simulation parameters: $\mu_{p}=0.1, e=0.7$. The data points correspond to the intermediate regime is shown with blank square symbols.

Fig. 3.40(a) the contact contribution to the normal component of the total granular stress is plotted with shear rate for a range of solid volume fractions. Figure 3.40(a) shows that the contact contribution of the total granular stress follows the same scaling $\left(\sigma \propto \dot{\gamma}^{n}, n=\right.$ $-2 m=0.28$ ) with shear rate as the total granular stress in the intermediate regime (data points correspond to the intermediate regime are shown with blank squares). However, the streaming contribution (shown in Fig. 3.40(b)) of the total granular stress in the intermediate regime still follows the inertial scaling of the stress $\left(\sigma \propto \dot{\gamma}^{2}\right)$ with the shear rate. As expected, at this high solid volume fraction the contact part of the stress contributes more than $95 \%$ to the total granular stress. Hence it is critical for the performance of any constitutive model in dense regime to accurately capture the behavior of the contact stress.

In order to evaluate the ROP model directly, the total granular stress obtained from the DEM simulations is decomposed into solidlike (stress arise from solidlike contacts) and fluidlike (stress arise from fluidlike contacts plus streaming stress) contributions. These contributions to the total granular stress are plotted with shear rate $k^{*}$, in Figs. 3.41(a) and 3.41(b), respectively. It is noteworthy that both the solidlike and fluidlike stress follow intermediate scaling of the stress $\left(\sigma \propto \dot{\gamma}^{n}, n_{\text {solidlike }}=0.20, n_{\text {fluidlike }}=0.44\right)$, because both of them carry portions of streaming and contact stress as well; the only difference is whether the contacts are enduring or not. A large number of the constitutive models available in the literature are based on additive decomposition of the total granular stress. However, this analysis of the stress decomposition (into solidlike and fluidlike contributions) reveals that a constitutive model can capture the intermediate behavior of the granular stress only when the weighting factors for the solidlike and fluidlike stress contributions are themselves a functions of shear 


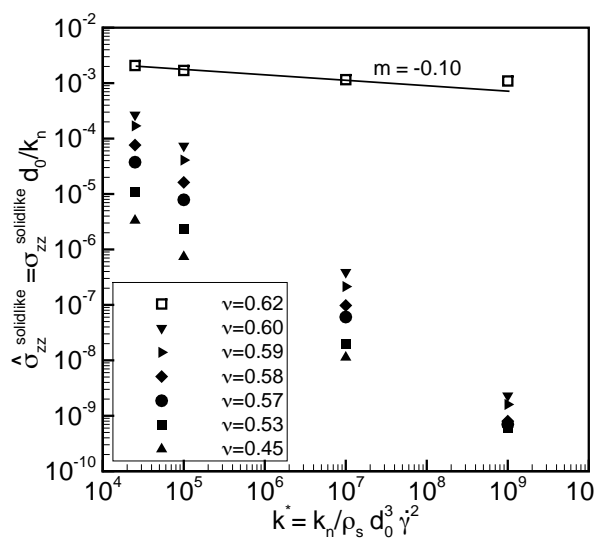

(a)

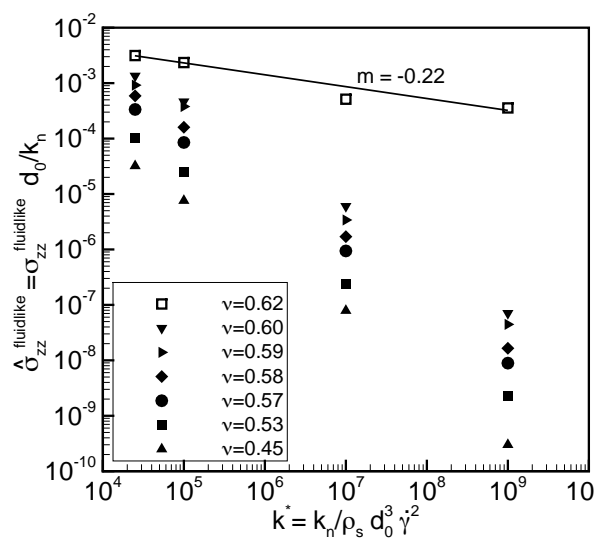

(b)

Figure 3.41: (a) Solidlike contribution of the total granular stress as a function of shear rate and (b) Fluidlike contribution of the total granular stress as a function of shear rate. Simulation parameters: $\mu_{p}=0.1, e=0.7$. The data point correspond to the intermediate regime is shown with blank square symbols.

rate. The ROP model has this feature because the model coefficients of the ROP model $(\alpha$ and $\beta$ ) are indeed functions of shear rate through the order parameter. However, this dependence is not able to accurately capture the scaling of the granular stress in the deep intermediate regime, where flow dynamics is dominated by the long-lasting, multiparticle contacts.

This study shows that the deep intermediate regime still poses a significant challenge for constitutive models to capture the correct scaling of the shear stress with the strain rate. In the following section we propose a relative-acceleration (RA) based constitutive model for this intermediate regime of the granular flow, and also present some of the preliminary results obtained using this model.

\subsubsection{Constitutive model for contact stress based on relative- acceleration (RA) concept}

The stress tensor in DEM simulations of the granular media can be decomposed in two parts. One part arises from momentum flux and is called the streaming contribution, a kinetic energy density. The second contribution arises from the static stress due to particle contact, a potential energy density. In the general case, the streaming stress in a sample volume $V$ is $\boldsymbol{\sigma}^{\mathbf{d}}=1 / V \sum_{i}^{N} m^{(i)} \mathbf{v}^{(i)} \mathbf{v}^{(i)}$, which is a dyadic product of the fluctuating velocity giving a second-order tensor with trace $\operatorname{tr}\left(\boldsymbol{\sigma}^{\mathbf{d}}\right) / D=n T$ being the kinetic energy density in $D$ dimensions. The additional contribution to the stress is due to collisions and contacts, which can be derived from the principle of virtual displacement for soft interaction potential and can be modified for hard sphere system. The static (contact) stress is given as $\boldsymbol{\sigma}^{s}=$ $1 / V \sum_{i}^{N} \sum_{j, j \neq i} \frac{1}{2} \mathbf{r}^{(i)(j)} \mathbf{f}^{(i)(j)}$, where $\mathbf{r}^{(i)(j)}$ is the vector pointing from the center of particle $j$ 
to the center of particle $i$, and $\mathbf{f}^{(i)(j)}$ is the contact force acting on particle $i$ by particle $j$ (see Fig 3.42). Combining the streaming (dynamic) and contact (static) contributions to the stress tensors, one has for smooth, soft spheres:

$$
\sigma_{\alpha \beta}^{t o t}=\frac{1}{V}\left[\sum_{i} m^{(i)} v_{\alpha}^{(i)} v_{\beta}^{(i)}+\sum_{i} \sum_{j \neq i} \frac{1}{2} r_{\alpha}^{(i)(j)} f_{\beta}^{(i)(j)}\right],
$$

where the first summation runs over all the particles in volume $V$ and the second summation runs over all the contacts in the averaging volume $V$.

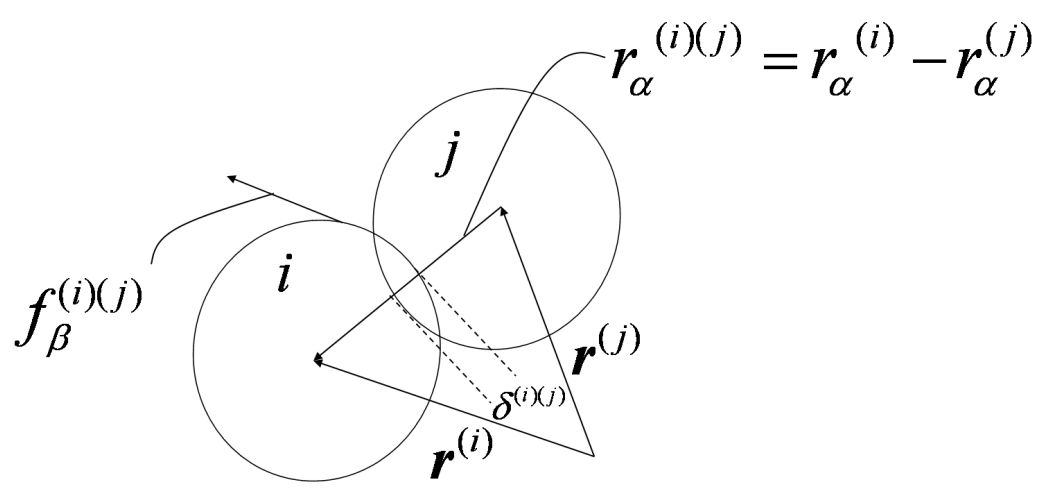

Figure 3.42: Schematic of two particles $i$ and $j$ contact with overlap $\delta^{(i)(j)}$ and position vectors $\mathbf{r}^{(i)}, \mathbf{r}^{(j)}$.

\section{Relative-acceleration concept}

The relative-acceleration based coarse graining approach $[30,31]$ based on transport equation for two-particle density has been successfully used to model the solute interactions in the presence of solvent, leading to accurate prediction of nanoparticle aggregation using Brownian dynamics simulations. This concept can be used to develop a microscale physics-based model for the contact part of the granular stress in the intermediate and dense regime. In a recent work [32], it is shown that the relative contribution from streaming stress is negligible compared to the contact (virial) part in the intermediate and dense regime. This result reveals that accurate modeling of the contact (virial) contribution of the granular stress is 
critical. This contact contribution of the granular stress is written as:

$$
\sigma_{\alpha \beta}=\frac{1}{V}\left[\sum_{i} \sum_{j>i} \frac{1}{2} r_{\alpha}^{(i)(j)} f_{\beta}^{(i)(j)}\right] .
$$

The Eq. 3.29 used in DEM is essentially a stress estimator. We propose a statistical model for this stress estimator that is,

$$
\sum_{i} \sum_{j>i} r_{\alpha}^{(i)(j)} f_{\beta}^{(i)(j)} \approx m\left\langle N_{c}\right\rangle(V)\left\langle r_{\alpha}^{(i)(j)} \Delta A_{\beta}^{(i)(j)}\right\rangle
$$

In Eq. 3.30, $\left\langle N_{c}\right\rangle(V)$ is the mean number of contacts in sampling volume $V$, whereas $\Delta \mathbf{A}^{(i)(j)}$ is the relative acceleration between particle $i$ and $j$. In order to completely specify the model on the right hand side of Eq. 3.30, specification of following quantities are needed:

1. The mean number of contacts $\left(\left\langle N_{c}\right\rangle(V)\right)$ in volume $V$, which can be written in terms of average coordination number as follows [33]:

$$
\left\langle N_{c}\right\rangle(V)=\frac{N_{C N}}{2}\langle N(V)\rangle,
$$

where $N_{C N}$ is the average or bulk coordination number and $\langle N(V)\rangle$ is the average number of particles in volume $V$. The average coordination number $N_{C N}$ is the average number of contacts per particle, which is given as

$$
N_{C N}=\frac{\sum_{i} N_{c}^{(i)}}{N-N_{1}}
$$

where $N_{c}$ is number of contacts for $i^{\text {th }}$ particle, $N$ is the total number of particles in volume $V$, whereas $N_{1}$ is the number of floaters (particles with less than one contact in non-gravity environment).

2. The second required quantity is $\left\langle r_{\alpha}^{(i)(j)} \Delta A_{\beta}^{(i)(j)}\right\rangle$ for a contact.

The detailed derivation of this RA-based constitutive model for contact stress is provided in Appendix A. After following the sequence of mathematical steps (as given in Appendix A), for very small overlaps, one can write the contact contribution of the granular stress as follows:

$$
\sigma_{\alpha \beta}=\frac{1}{2 V} N_{C N}\langle N(V)\rangle k_{n} R_{\alpha \beta} d \int_{0}^{r_{c}} \delta^{(i)(j)} g(\mathbf{r}) d \mathbf{r} .
$$

Equation 3.33 will be used to compute contact stress for a granular assembly. Figure 3.43 illustrates the concept of the proposed relative acceleration based contact stress model for granular flows in the intermediate and dense regime. 


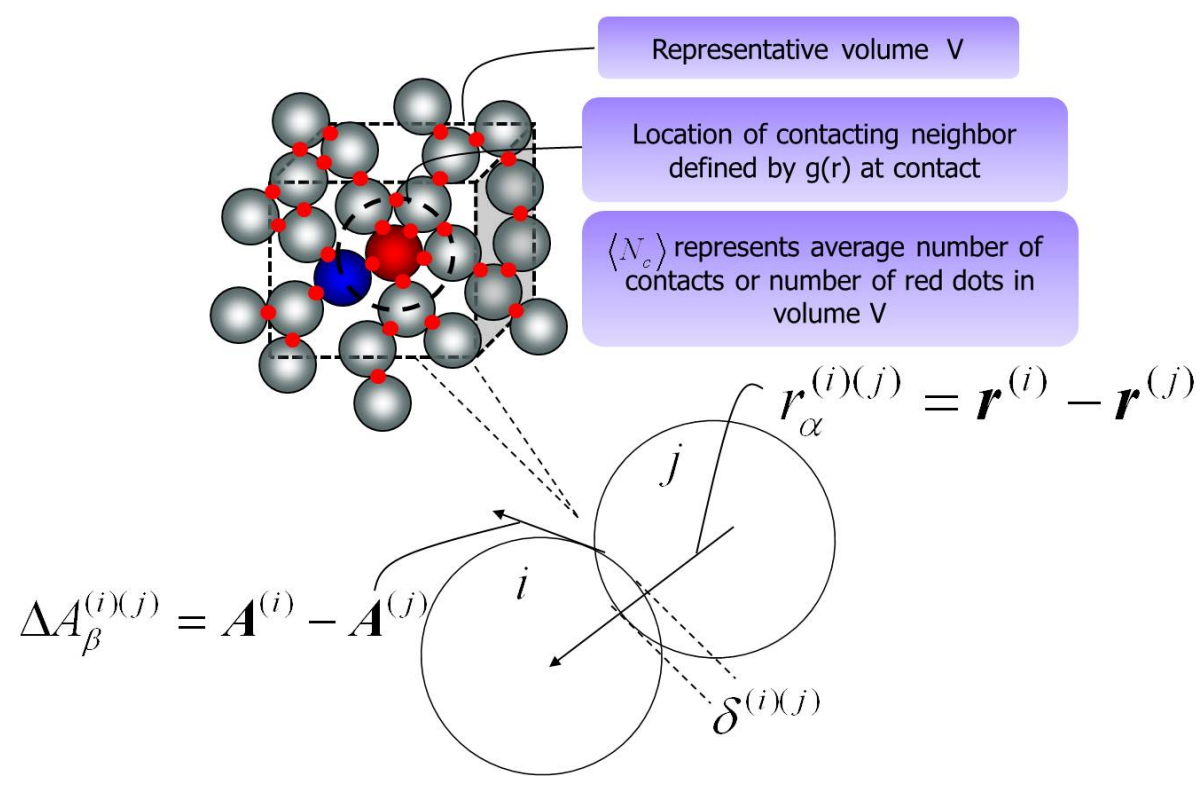

Figure 3.43: Illustration of proposed relative acceleration (RA) based contact model for granular stress. 


\section{Inputs to the contact stress model}

There are four important inputs required to compute the contact stress (see Eq. 3.33), which are listed below:

1. $N_{C N}$ (Average coordination number): We propose to extract the average coordination number from DEM simulations. A fit for coordination number with solid volume fraction (for different values of particle friction coefficient) can be obtained by using DEM data. Alternatively the model equation provided by Sun and Sundaresan [34] can be used to obtained coordination number.

2. $\delta^{(i)(j)}$ (Normal overlap): The pdf (probability density function) of normal overlap between contacting particles can be specified using data obtained from DEM simulations. This pdf of normal overlap will be same as the force pdf, which has been already extracted from the DEM simulations.

3. $R_{\alpha \beta}$ (Fabric tensor): Fabric tensor represents the anisotropy present in the granular media. In order to give a model for the fabric tensor we plan to follow work of Sun and Sundaresan [34].

4. $g(\mathbf{r})$ (Pair correlation function): Analytical fit for pair correlation function incorporating dependence on solid volume fraction, friction coefficient and shear rate will be specified by using DEM simulations.

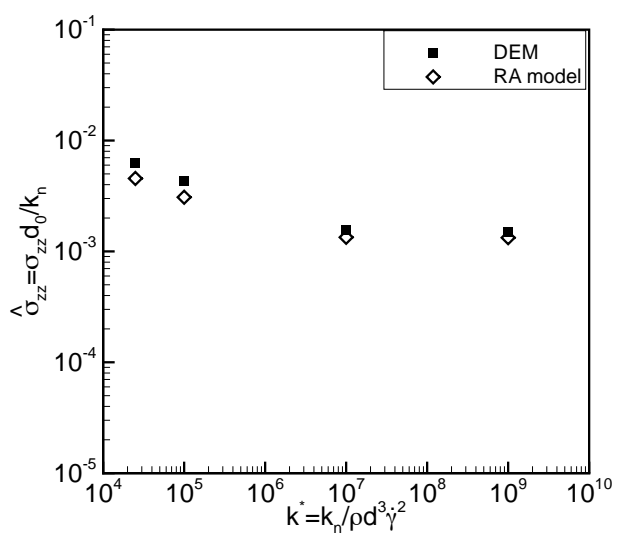

(a)

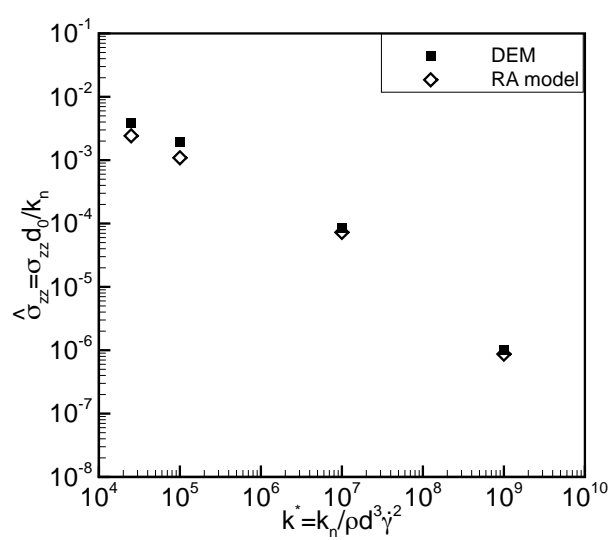

(b)

Figure 3.44: (a) The normal component of the total granular stress as a function of shear rate $k^{*}$. Simulation parameters: $\nu=0.62, \mu_{p}=0.1, e=0.7$ and (b) The normal component of the total granular stress as a function of shear rate $k^{*}$. Simulation parameters: $\nu=0.61$, $\mu_{p}=0.1, e=0.7$.

Figures 3.44(a) and 3.44(b) show the preliminary results obtained using the proposed RAbased contact stress model (the model input are taken from DEM simulation data for preliminary assessment purpose) for a solid volume fraction of 0.62 and 0.61 , respectively. Preliminary results show that this RA-based contact stress model successfully captures the 
stress-strain scaling even in the intermediate regime of the granular flows. 


\section{Chapter 4}

\section{PROJECT PARTICIPANTS AND COLLABORATION}

\subsection{Participants}

In addition to the three principal investigators of the project, the following individuals participated in this project.

\section{City College of the City University of New York}

- Mehrdad Kheiripour Langroudi: Graduate student in Chemical Engineering

\section{Princeton University}

- Sebastian Chialvo: graduate student in Chemical Engineering

- Dr. Jin Sun: Post-doctoral fellow

\section{Iowa State University}

- Vidyapati, graduate student

\subsection{Collaboration Among the Project Participants}

The PIs have interacted actively through emails, sharing of data, and visits.

1. The PIs and some other project participants had a meeting during NETL 2009 Workshop on Multiphase Flow Science at Morgantown, WV. They discussed the project progresses and exchanged ideas on how to compliment each other's work with their own data or model.

2. Professor Subramaniam and Mr. Vidyapati visited professor Tardos' granular mechanics laboratory at CCNY. They exchanged ideas on coordinating their simulation and 
experimental work and made plans for simulating the Couette cell experiments.

3. Professor Tardos performed a series of Couette cell experiments and supplied the results to the collaborators. This part of work has been simulated by the ISU team and comparisons are documented in this report.

4. Professor Tardos performed a series of shear experiments in a Jenike cell (described in this report) and supplied the results to the collaborators. Dr. Jin Sun in Sundaresan's group set up a simulation of this experiment. As the simulation time was exceedingly long, faster shear experiments were suggested to Professor Tardos. He and his student constructed a new Jenike-type tester to perform experiments at a faster rate and provided the data on normal stresses recorded by the sensors. Dr. Jin Sun simulated these experiments and found that fluctuations of the same magnitude can indeed be captured by the DEM simulations and also explained microscopically. This exercise validated the DEM simulations.

5. Dr. Jin Sun at Princeton University has assisted the new student at Iowa State University (Mr. Vidyapati) in using the LAMMPS code to perform shear flow simulations.

6. Sundaresan and Sun have shared their thoughts on fruitful simulations of Couette flow for Vidyapati to perform to complement the work being done at Princeton. 


\section{Chapter 5}

\section{PUBLICATIONS AND PRESENTATIONS}

The following publications and presentations were made during the course of this research project.

\subsection{Publications}

1. S. Chialvo, J. Sun, and S. Sundaresan. Bridging the rheology of granular flows in three regimes. Under review for Physical Review Letters. See Appendix B.

2. M. K. Langroudi, M. Shahnam, and G. Tardos. A CFD approach to model high shearing dry particulate flows with a free surfaces: Flow in a spheronizer. In preparation.

3. Vidyapati and Subramaniam, S. Granular rheology and 'phase' transition through order parameter: DEM simulations and constitutive modeling. Preprint. See Appendix F.

4. Vidyapati and Subramaniam, S. Granular flow in Silo discharge: DEM simulations and model assessment. Preprint. See Appendix E.

5. S. Chialvo, J. Sun and S. Sundaresan. A plasticity model with microstructure evolution for quasi-static triaxial deformations of dense granular media. In preparation.

6. J. Sun and S. Sundaresan. A constitutive model with microstructure evolution for flow of rate-independent granular materials. Under review for Journal of Fluid Mechanics. See Appendix C.

7. Vidyapati, M. K. Langroudi, S. Subramaniam, G. Tardos, and S. Sundaresan. Experimental and computational studies of dense granular flow: transition from quasi-static to intermediate regime in a Couette device. In press, Powder Technology, (2011). See Appendix G. 
8. M. K. Langroudi and G. Tardos. Importance of Solid Fraction and Its Fluctuations on the Rheology and Flow Characteristics of Compressible Powders. Under review, Mechanics Research Communications (2011). See Appendix M.

9. M. K. Langroudi, P. Mort, and G. Tardos. Study of powder flow patterns in the Couette cell with axial flow using Tracers and Solid Fraction Measurements. In press, Granular Matter (2011). See Appendix N.

10. J. Sun and S. Sundaresan. A plasticity model with microstructure evolution for quasistatic granular flows. AIP Conference Proceedings 1227, (2010), 280-289. See Appendix D.

11. L. R. Aarons, L., J. Sun and S. Sundaresan. Dynamic shear of cohesive homogeneous assemblies of dense granular materials. Industrial \& Engineering Chemistry Research, DOI: 10.1021/ie901187w. June, 2010. See Appendix I.

12. M. K. Langroudi, G. Tardos, J. Sun, and S. Sundaresan. Transmission of normal stresses in un-sheared and sheared granular beds: the influence of particle size, shape, stiffness and cohesion. Powder Technology, 203 (2010), 23-32. See Appendix K.

13. M. K. Langroudi, S. Turek, A. Ouazzi, and G. Tardos. An investigation of frictional and collisional powder flows using a unified constitutive equation. Powder Technology, 197 (2010), 91-101. See Appendix J.

14. M. K. Langroudi, G. Tardos, J. Michaels, and P. Mort. Effect of material properties, boundary conditions and flow fields on the rheology of dense granular matter. AIP Conference Proceedings 1145 (2009), 587-590. See Appendix L.

15. L. Aarons and S. Sundaresan. Shear flow of assemblies of cohesive granular materials under constant applied normal stress. Powder Technology, 183(3):340-355, 2008. See Appendix H.

\subsection{Presentations}

1. Vidyapati and Subramaniam, S. Discrete element simulations and constitutive modeling of dense granular flows. Computational Fluid Dynamics Seminar Series. Iowa State University, Ames, IA, March 2011.

2. S. Chialvo, J. Sun, and S. Sundaresan. Bridging across quasi-static, intermediate, and inertial regimes of granular flow. International Symposium on Recent and Emergent Advances in Chemical Engineering. Chennai, India, December 2-4, 2010.

3. S. Chialvo, J. Sun, and S. Sundaresan. Rheology of simple shear flows of dense granular assemblies in different regimes. 63rd Annual Meeting of the APS Division of Fluid Dynamics. Long Beach, CA, November 21-23, 2010.

4. S. Chialvo, J. Sun, and S. Sundaresan. Bridging across quasi-static, intermediate, and inertial regimes of granular flow. AIChE Annual Conference. Salt Lake City, UT, 
November 7-12, 2010.

5. M. K. Langroudi. A New Technique to Measure Shear Band Thickness: The Influence of Solid Volume Fraction on Powder Rheology in a Couette cell with Secondary Axial Flow. AIChE Annual Conference. Salt Lake City, UT, November 7-12, 2010.

6. Vidyapati and Subramaniam, S. Refined order parameter model and its performance in homogeneous shear flows. $47^{\text {th }}$ Annual Technical Meeting of the Society of Engineering Science. Ames, IA, October 2010.

7. M. K. Langroudi. Importance of Solid Fraction in Rheology of Granular Matter - Use of a Capacitance Probe to indirectly find the Shear Band Thickness in a Couette cell with Axial Flow. Gordon Research Conference (GRC) on Granular and Granular-Fluid Flow. Waterville, ME, June 2010.

8. S. Sundaresan. Towards a Comprehensive Rheological Model for Granular Flows: from Quasi-static to Inertial Regimes. IMF Toulouse, France, June, 2010.

9. G. I. Tardos. Rheological behavior of dense assemblies of granular materials: Experiments and Simulations. DoE Workshop on Granular Matter. Pittsburgh, May 6, 2010 .

10. Subramaniam S., Vidyapati, Sun, J., Tardos, G.I., and Sundaresan, S. Discrete element simulation and modeling of granular flow in Silos and Hoppers. NETL 2010 workshop on Multiphase Flow Science. Morgantown, WV, May 2010.

11. G. I. Tardos. Industrially Relevant Powder Flows studied using Constitutive Equations and Measured Powder Characteristics. DARPA - Granular Dynamics Workshop, February 17, 2010.

12. Vidyapati, M. K. Langroudi., J. Sun, S. Subramaniam, G.I. Tardos, and S. Sundaresan. DEM simulation of granular flow in a Couette device. 62nd Annual Meeting of the APS Division of Fluid Dynamics, Minneapolis, MN, November 22-24, 2009.

13. J. Sun and S. Sundaresan. Constitutive modeling of slow flows of dense granular assemblies. AIChE Annual Conference. Nashville, TN, November 8-13, 2009.

14. M. K. Langroudi and G. I. Tardos. Measurement of Solid Fraction and Its Fluctuations in Dense Granular Flows Using a Capacitance Probe. AIChE Annual Conference. Nashville, TN, November 8-13, 2009.

15. M. K. Langroudi and G. I. Tardos. Rheological Behavior of Dense Granular Matter. AIChE Annual Conference. Nashville, TN, November 8-13, 2009.

16. Gabriel I. Tardos, M. K. Langroudi, and Paul R. Mort. A Study of Powder Flow Patterns (Dispersion vs. Convection) in a Continuous Annular Couette Cell Using Tracers. AIChE Annual Conference. Nashville, TN, November 8-13, 2009.

17. S. Chialvo, J. Sun and S. Sundaresan. Yielding of dense assemblies of frictional particles. Joint IUTAM-ISIMM Symposium on Mathematical Modeling and Physical Instances of Granular Flows. Reggio Calabria, Italy, September 14-18, 2009. 
18. J. Sun and S. Sundaresan. A plasticity model with microstructure evolution for dense granular flows. Joint IUTAM-ISIMM Symposium on Mathematical Modeling and Physical Instances of Granular Flows. Reggio Calabria, Italy, September 14-18, 2009.

19. M. K. Langroudi. Effect of Material Properties, Boundary Conditions and Flow Fields on the Rheology of Dense Granular Matter. The 6th International Conference on Micromechanics of Granular Media. Golden, CO, July 2009.

20. G. I. Tardos. Toward a Grand Challenge in Powder Flows: The effect of material properties, boundary conditions and shear rate on flow and stress fields in flowing powders. Invited lecture, IFPRI, Spa, Belgium, June 27, 2010 and Ann Arbor, June 29, 2009.

21. G. Tardos. An Experimental Study of the Rheological Behavior of Dense Assemblies of Granular Materials. NETL 2009 Workshop on Multiphase Flow Science, April 22-23, 2009. Morgantown, WV.

22. S. Subramaniam and Vidyapati. Rheological Behavior of Dense Granular Materials: DEM Simulations and Order Parameter Model. NETL 2009 Workshop on Multiphase Flow Science, April 22-23, 2009. Morgantown, WV.

23. J. Sun and S. Sundaresan. Frictional Flow of Dense Granular Materials. NETL 2009 Workshop on Multiphase Flow Science, April 22-23, 2009. Morgantown, WV.

24. J. Sun and S. Sundaresan. Rheological behaviors of dense granular flows. Granular and Multiphase Flows Colloquium Series. Department of Mechanical \& Industrial Engineering, New Jersey Institute of Technology, Newark, NJ, April 15, 2009.

25. M. K. Langroudi and G. I. Tardos. Development of a Visco-Plastic Constitutive Law for Dense Flow of Granular Matter. AIChE Annual Conference. Philadelphia, November 2008.

26. J. Sun, L. Aarons and S. Sundaresan. Memory and microstructure of quasi-static granular flows under unsteady simple shear. Poster at Gordon Research Conference on Granular 86 Granular-fluid Flow, ME, June, 2008.

27. M. K. Langroudi, J. N. Michaels and G. I. Tardos. Stress measurements in dry, dense powder flows using a Couette device: proposed constitutive equations for continuous modeling of powder flows. Poster at Gordon Research Conference on Granular \& Granular-fluid Flow, ME, June, (2008).

28. M. K. Langroudi and G. I. Tardos. Solid to Liquid Transition in Dry, Dense Powder flows: Development of a Visco-Plastic Model based on Local Stress Measurements. IFPRI Spring Technical Meeting. New York, April, 04, 2008.

29. L. Aarons \& S. Sundaresan. Shear flow of assemblies of cohesive materials. AIChE Annual Conference. November 4-9, 2007, Salt Lake City, UT.

30. L. Aarons \& S. Sundaresan. Unsteady shear flow of assemblies of cohesive materials. AIChE Annual Conference. November 4-9, 2007, Salt Lake City, UT. 
31. M. K. Langroudi \& G. I. Tardos. Rheological behavior of free flowing dense powders: use of a couette cell to validate constitutive equations for different regimes. AIChE Annual Conference. November 4-9, 2007, Salt Lake City, UT. 


\section{Bibliography}

[1] R. Hill. The Mathematical Theory of Plasticity. Oxford University Press, 1950.

[2] Andrew Schofield and Peter Wroth. Critical State Soil Mechanics. McGraw-Hill Inc., 1968.

[3] Alexander V. Potapov and Charles S. Campbell. Computer simulation of hopper flow. Physics of Fluids, 8(11):2884-2894, 1996.

[4] P. V. Lade and J. M. Duncan. Elastoplastic stress-strain theory for cohesionless soil. Journal of the Geotechnical Engineering Division, 101(10):1037-1053, 1975.

[5] Charles Campbell. Granular shear flows at the elastic limit. J. Fluid Mech., 465:261-291, 2002.

[6] Cheng-lung Chen and Chi-Hai Ling. Granular-flow rheology: Role of shear-rate number in transition regime. Journal of Engineering Mechanics, 122(5):469-480, 1996.

[7] Peter Olsson and S. Teitel. Critical scaling of shear viscosity at the jamming transition. Phys. Rev. Lett., 99(17):178001, Oct 2007.

[8] Takahiro Hatano. Scaling properties of granular rheology near the jamming transition. J. Phys. Soc. Jpn., 77(12):123002, 2008.

[9] Michio Otsuki and Hisao Hayakawa. Universal scaling for the jamming transition. Prog. Theor. Phys., 121(3):647-655, 2009.

[10] Meheboob Alam and Stefan Luding. First normal stress difference and crystallization in a dense sheared granular fluid. Physics of Fluids, 15(8):2298-2312, 2003.

[11] M. Syamlal, W. Rogers, and T. J. O'Brien. Mfix documentation: Theory guide. Technical Report DOE/METC-95/1013, NTIS/DE95000031, National Energy Technology Laboratory, Department of Energy, 1993. See also URL http://www.mfix.org.

[12] P. Jop, Y. Forterre, and O. Pouliquen. A constitutive law for dense granular flows. Nature, 441(8):727-730, 2006.

[13] W. Losert, L. Bocquet, T.C. Lubensky, and J.P. Gollub. Particle dynamics in sheared granular matter. Phys. Rev. Lett., 85(7):1428-1431, 2000.

[14] S.B. Savage. Analyses of slow high-concentration flows of granular materials. J. Fluid Mech., 377:1-77, 1998. 
[15] D.G. Schaeffer. Instability in the evolution equations describing incompressible granular flow. Journal of Differential Equations, 66:19-50, 1987.

[16] A. Srivastava and S. Sundaresan. Analysis of a frictional-kinetic model for gas-particle flow. Powder Technology, 129:72-85, 2003.

[17] W.A. Beverloo, H.A. Leniger, and J. van de Velde. The flow of granular solids through orifices. Chemical Engineering Science, 15:243-250, 1961.

[18] I.S. Aranson and L.S. Tsimring. Continuum theory of partially fluidized granular flows. Phys. Rev. E, 65:061303-1-061303-20, 2001.

[19] Sofiane Benyahia. Validation study of two continuum granular frictional flow theories. Ind. Eng. Chem. Res., 47:8926-8932, 2008.

[20] P. Jalali and T. Hyppanen. Verification of continuum models for solids momentum transfer by means of discrete element method. Ind. Eng. Chem. Res., 49:5270-5278, 2010 .

[21] G.I. Tardos, S. McNamara, and I. Talu. Slow and intermediate flow of a frictional bulk powder in the couette geometry. Powder Technology, 131:23-39, 2003.

[22] S. Ji, D. M. Hanes, and H. H. Shen. Comparisons of physical experiment and discrete element simulatuions of sheared granular material in an annular shear cell. Mechanics of Materials, 41:764-776, 2009.

[23] P.C. Johnson and R. Jackson. Frictional collisional constitutive relations for granular materials, with application to plane shearing. J. Fluid Mech., 176:67-98, 1987.

[24] J.J. McCarthy, V. Jasti, M. Marinack, and C.F. Higgs. Quantitative validation of the discrete element methid using an annular shear cell. Powder Technology, 230:70-77, 2010.

[25] V. Jasti and C.F. Higgs. Experimental study of granular flows in a rough annular shear cell. Phys. Rev. E, 78(041306):1-8, 2008.

[26] D. Volfson, L.S. Tsimring, and I.S. Aranson. Order parameter description of stationary partially fluidized shear granular flows. Phys. Rev. E, 90:254301-1-254301-4, 2003.

[27] D. Volfson, L.S. Tsimring, and I.S. Aranson. Partially fluidized shear granular flows: Continuum theory and molecular dynamics simulations. Phys. Rev. E, 68:021301-1021301-15, 2003.

[28] C. Song, P. Wang, and H.A. Makse. A phase diagram for jammed matter. Nature, 453:629-632, 2008.

[29] D. Gao, S. Subramaniam, R.O. Fox, and D.K. Hoffman. Objective decomposition of the stress tesnor in granular flows. Phys. Rev. E, 71:021302-1-021302-5, 2005.

[30] S. Markutsya. Modeling and simulation of nanoparticle aggregation in colloidal systems. PhD thesis, Iowa State University, November 2010. 
[31] S. Markutsya and S. Subramaniam. Coarse graining approach to infer mesoscale interaction potentials from atomisitic interactions for aggragating systems. In review with Journal of Colloid and Interface Science, 2011.

[32] Vidyapati, M. Kheiripour, J. Sun, S. Sundaresan, G. I. Tardos, and S. Subramaniam. Experimental and computational studies of dense granular flow: transition from quasistatic to intermediate regime in a Couette shear device. In review with Powder Technology, 2011.

[33] H. P. Zhang and H.A. Makse. Jamming transition in emulsions and granular materials. Phys. Rev. E, 72(011301):1-12, 2005.

[34] J. Sun and S. Sundaresan. A plasticity model with microstructure evolution for quasistatic granular flows. Iutam-Isimm Symposium on Mathematical Modeling and Physical Instances of Granular flows, 1227:280-289, 2010. 
Appendix A

Relative acceleration concept 


\section{Relative acceleration concept}

The relative acceleration based coarse graining approach $[1,2]$ based on transport equation for two-particle density has been successfully used to model the solute interactions in the presence of solvent, leading to accurate prediction of nanoparticle aggregation using Brownian dynamics simulations. This concept can be used to develop a microscale physics-based model for the contact part of the granular stress in the intermediate and dense regime. In a recent work [3], it is shown that the relative contribution from streaming stress is negligible compared to the contact (virial) part in the intermediate and dense regime. This result reveals that accurate modeling of the contact (virial) contribution of the granular stress is critical. This contact contribution of the granular stress is written as:

$$
\sigma_{\alpha \beta}=\frac{1}{V}\left[\sum_{i} \sum_{j>i} \frac{1}{2} r_{\alpha}^{(i)(j)} f_{\beta}^{(i)(j)}\right] .
$$

The Eq. 1 used in DEM is essentially a stress estimator. We propose a statistical model for this stress estimator that is,

$$
\sum_{i} \sum_{j>i} r_{\alpha}^{(i)(j)} f_{\beta}^{(i)(j)} \approx m\left\langle N_{c}\right\rangle(V)\left\langle r_{\alpha}^{(i)(j)} \Delta A_{\beta}^{(i)(j)}\right\rangle .
$$

In Eq. 2, $\left\langle N_{c}\right\rangle(V)$ is the mean number of contacts in sampling volume $V$, whereas $\Delta \mathbf{A}^{(i)(j)}$ is the relative acceleration between particle $i$ and $j$. In order to compute $\left\langle r_{\alpha}^{(i)(j)} \Delta A_{\beta}^{(i)(j)}\right\rangle$, the concept of relative acceleration is used. The average relative acceleration between a pair of particles each located at $\mathbf{x}_{1}$ and $\mathbf{x}_{2}$ with velocities $\mathbf{v}_{1}$ and $\mathbf{v}_{2}$ respectively, is $\langle\Delta \mathbf{A} \mid \mathbf{r}, \mathbf{w}\rangle$, where $\mathbf{r}=\mathbf{x}_{2}-\mathbf{x}_{1}$ is the pair relative separation and $\mathbf{w}=\mathbf{v}_{2}-\mathbf{v}_{1}$ is the pair relative velocity. At steady state the conditional average relative acceleration $\langle\Delta \mathbf{A} \mid \mathbf{r}, \mathbf{w}\rangle$, can be decomposed as follows:

$$
\langle\Delta \mathbf{A} \mid \mathbf{r}, \mathbf{w}\rangle=\langle\Delta \mathbf{A} \mid \mathbf{r}\rangle+\langle\Delta \mathbf{A} \mid \mathbf{w}\rangle,
$$

where $\langle\Delta \mathbf{A} \mid \mathbf{r}\rangle$ is the average relative acceleration conditional on pair relative separation and $\langle\Delta \mathbf{A} \mid \mathbf{w}\rangle$ is the average relative acceleration conditional on pair relative velocity. In LD (Langevin dynamics), the term $\langle\Delta \mathbf{A} \mid \mathbf{w}\rangle$ can be modeled in terms of inelasticity and damping. In dense regime the contribution from this term $(\langle\Delta \mathbf{A} \mid \mathbf{w}\rangle)$ is expected to be very small and hence can be neglected. We focus on other term $\langle\Delta \mathbf{A} \mid \mathbf{r}\rangle$, the average pair-relative acceleration conditioned on pair separation. The unconditional average relative acceleration between the particles can be written in terms of conditional average relative acceleration as follows:

$$
\langle\Delta \mathbf{A}\rangle=\frac{1}{n^{2}} \int\langle\Delta \mathbf{A} \mid \mathbf{r}\rangle \rho^{(2)}(\mathbf{r}) d \mathbf{r},
$$


where $\rho^{(2)}(\mathbf{r})$ is the two particle density and $n$ is the number density. With the assumption of homogeneous point field, Eq. 4 can be written as:

$$
\langle\Delta \mathbf{A}\rangle=\int\langle\Delta \mathbf{A} \mid \mathbf{r}\rangle g(\mathbf{r}) d \mathbf{r}
$$

where $g(\mathbf{r})$ is the pair correlation function at contact. The average relative acceleration between two particles can be written as (assuming Newton's third law holds for a contacting pair),

$$
\langle\Delta \mathbf{A} \mid \mathbf{r}\rangle=\frac{\mathbf{f}^{(i)(j)}}{m^{(i)}}-\frac{\mathbf{f}^{(j)(i)}}{m^{(j)}}=2 \frac{\mathbf{f}^{(i)(j)}}{m},
$$

where $m$ is the mass of the particle. Now using Eqs. 5 and 6, one can derive following:

$$
\langle\Delta \mathbf{A}\rangle=\int_{0}^{r_{c}} \mathbf{f}^{(i)(j)} \frac{2}{m} g(\mathbf{r}) d \mathbf{r},
$$

where $\mathbf{f}^{(i)(j)}$ is the force on particle $i$ due to particle $j$. This force between contacting particles can be written as follows (As a starting point only normal component of the force is considered, tangential component can be added into the model by invoking Coulomb criterion at slippage $\left.\left(\left|f_{t}^{(i)(j)}\right|=\mu\left|f_{n}^{(i)(j)}\right|\right)\right)$ :

$$
\mathbf{f}^{(i)(j)}=f\left(\delta^{(i)(j)} / d\right) k_{n} \delta^{(i)(j)} \mathbf{n}^{(i)(j)},
$$

where $\delta^{(i)(j)}$ is the normal overlap between the contacting particle $i$ and $j$, $k_{n}$ is the normal spring stiffness and $d$ is the particle diameter. For a linear spring-dashpot model (Hookean model) $f\left(\delta^{(i)(j)} / d\right)=1$, hence by using Eqs. 7 and 8 , one can arrive at following:

$$
\langle\Delta \mathbf{A}\rangle=\int_{0}^{r_{c}} k_{n} \delta^{(i)(j)} \mathbf{n}^{(i)(j)} \frac{2}{m} g(\mathbf{r}) d \mathbf{r} .
$$

However, to have a model for contact stress one needs to compute $\left\langle r_{\alpha}^{(i)(j)} \Delta A_{\beta}^{(i)(j)}\right\rangle$, which can be obtained from Eq. 9 as follows

$$
\left\langle r_{\alpha}^{(i)(j)} \Delta A_{\beta}^{(i)(j)}\right\rangle=\frac{2 k_{n}}{m} \int_{0}^{r_{c}} r_{\alpha}^{(i)(j)} \delta^{(i)(j)} n_{\beta}^{(i)(j)} g(\mathbf{r}) d \mathbf{r}
$$

The unit normal vector $n_{\alpha}^{(i)(j)}$, in the direction of line joining the centers of contacting particles can be written as

$$
n_{\alpha}^{(i)(j)}=\frac{r_{\alpha}^{(i)(j)}}{r^{(i)(j)}}
$$

Hence using Eqs. 10 and 11, one can write following:

$$
\left\langle r_{\alpha}^{(i)(j)} \Delta A_{\beta}^{(i)(j)}\right\rangle=\frac{2 k_{n}}{m} \int_{0}^{r_{c}} r^{(i)(j)} n_{\alpha}^{(i)(j)} n_{\beta}^{(i)(j)} \delta^{(i)(j)} g(\mathbf{r}) d \mathbf{r} .
$$


The normalized distribution of contacts in granular media is generally described by three-dimensional second order fabric tensor [4]

$$
R_{\alpha \beta}=n_{\alpha}^{(i)(j)} n_{\beta}^{(i)(j)}
$$

From Eqs. 13 and 12 one can arrive at following

$$
\left\langle r_{\alpha}^{(i)(j)} \Delta A_{\beta}^{(i)(j)}\right\rangle=\frac{2 k_{n}}{m} R_{\alpha \beta} \int_{0}^{r_{c}} r^{(i)(j)} \delta^{(i)(j)} g(\mathbf{r}) d \mathbf{r}
$$

Now using Eqs. 1, 2, and 14, the contact stress due to normal contact forces can be written as:

$$
\sigma_{\alpha \beta}=\frac{1}{2 V} N_{C N}\langle N(V)\rangle k_{n} R_{\alpha \beta} \int_{0}^{r_{c}} r^{(i)(j)} \delta^{(i)(j)} g(\mathbf{r}) d \mathbf{r},
$$

where $r^{(i)(j)}$ is defined as

$$
r^{(i)(j)}=d-\delta^{(i)(j)} .
$$

Hence the contact stress can be further written as

$$
\sigma_{\alpha \beta}=\frac{1}{2 V} N_{C N}\langle N(V)\rangle k_{n} R_{\alpha \beta} \int_{0}^{r_{c}}\left(d-\delta^{(i)(j)}\right) \delta^{(i)(j)} g(\mathbf{r}) d \mathbf{r},
$$

which can further be simplified as

$$
\sigma_{\alpha \beta}=\frac{1}{2 V} N_{C N}\langle N(V)\rangle k_{n} R_{\alpha \beta} \int_{0}^{r_{c}}\left(d \delta^{(i)(j)}-\delta^{2(i)(j)}\right) g(\mathbf{r}) d \mathbf{r} .
$$

For very small overlaps, one can write Eq. 18 as follows:

$$
\sigma_{\alpha \beta}=\frac{1}{2 V} N_{C N}\langle N(V)\rangle k_{n} R_{\alpha \beta} d \int_{0}^{r_{c}} \delta^{(i)(j)} g(\mathbf{r}) d \mathbf{r} .
$$

Equation 19 will be used to compute contact stress for a granular assembly. 


\section{References}

[1] S. Markutsya, Modeling and simulation of nanoparticle aggregation in colloidal systems. PhD thesis, Iowa State University, November 2010.

[2] S. Markutsya and S. Subramaniam, "Coarse graining approach to infer mesoscale interaction potentials from atomisitic interactions for aggragating systems.," In review with Journal of Colloid and Interface Science, 2011.

[3] Vidyapati, M. Kheiripour, J. Sun, S. Sundaresan, G. I. Tardos, and S. Subramaniam, "Experimental and computational studies of dense granular flow: transition from quasi-static to intermediate regime in a Couette shear device.," In review with Powder Technology, 2011.

[4] R. J. Bathurst and L. Rothenburg, "Observation on stress-force-fabric relationships in idealized granular material.," Mechanics of Materials, vol. 9, pp. $65-80,1990$. 
Appendix B

Bridging the rheology of granular flows in three regimes 


\title{
Bridging the rheology of granular flows in three regimes
}

\author{
Sebastian Chialvo, ${ }^{1}$ Jin Sun, ${ }^{2}$ and Sankaran Sundaresan ${ }^{1}$ \\ ${ }^{1}$ Chemical and Biological Engineering Department, \\ Princeton University, Princeton, NJ 08540, USA \\ ${ }^{2}$ Institute for Infrastructure and Environment, University of Edinburgh, Edinburgh EH9 3JL, Scotland, UK
}

(Dated: April 15, 2011)

\begin{abstract}
We investigate the rheology of granular materials via molecular dynamics simulations of homogeneous, simple shear flows of soft, frictional, noncohesive spheres. As others have for frictionless particles, we observe three flow regimes existing in different domains of particle volume fraction and shear rate, with all stress data collapsing upon scaling by powers of the distance to the jamming point. Though this jamming point is a function of the interparticle friction coefficient, the relation between stress and strain rate at this point is found to be independent of friction. We also propose a rheological model that blends the asymptotic relations in each regime to obtain a general description for these flows. Finally, we note some similarities between our model and those based on the inertial number.
\end{abstract}

PACS numbers: 45.70.-n, 47.57.Gc, 64.60.F-, 64.70.ps, 83.10.Gr, 83.80.Fg

Flows of granular matter occur in numerous geophysical and industrial processes and, as such, have garnered the attention of researchers for many years. Early efforts to describe these flows focused on either dilute flows (or inertial flows, where kinetic theories [1-4] apply) or very dense flows (or quasi-static flows, for which plasticity models $[5,6]$ can be used). However, attention has turned recently to the interface between these two regimes in the context of a jamming transition, proposed to occur in granular and other soft matter [7]. Of particular interest are several works that find a critical rheology around this transition in flows of frictionless, soft spheres [8-10] and disks [11, 12]. Furthermore, they find scalings for the mean normal and shear stresses with respect to volume fraction that apply over a wide range of volume fractions and shear rates. Granular materials, though, are typically considered stiff, frictional materials, and to date there has been little work on identifying a critical rheology $[13,14]$ for such matter despite significant progress in understanding their static jamming behavior [15-18]. In this Letter, we investigate the rheology of frictional granular matter about the jamming transition and discuss the construction of a rheological model for flows in the quasi-static, inertial, and intermediate (i.e. critical) regimes.

Simulation Methods. - We perform computer simulations using a package of the discrete element method (DEM) [19] implemented in the molecular dynamics package LAMMPS [20]. In DEM, particles interact only via repulsive, finite-range contact forces. We employ a linear spring-dashpot (LSD) model, for which the normal and tangential forces on a spherical particle $i$ resulting from the contact of two identical spheres $i$ and $j$ are

$$
\begin{aligned}
& \mathbf{F}_{i j}^{n}=k_{n} \delta_{i j} \mathbf{n}_{i j}-\gamma_{n} m_{\mathrm{eff}} \mathbf{v}_{i j}^{n} \\
& \mathbf{F}_{i j}^{t}=-k_{t} \mathbf{u}_{i j}^{t} \mathbf{n}_{i j}-\gamma_{t} m_{\mathrm{eff}} \mathbf{v}_{i j}^{t},
\end{aligned}
$$

for overlap distance $\delta_{i j}$, particle diameter $d$, spring stiff- ness constants $k_{n}$ and $k_{t}$, viscous damping constants $\gamma_{n}$ and $\gamma_{t}$, effective mass $m_{\mathrm{eff}}=m_{i} m_{j} /\left(m_{i}+m_{j}\right)$ for particle masses $m_{i}$ and $m_{j}$, relative particle velocity components $\mathbf{v}_{i j}^{n}$ and $\mathbf{v}_{i j}^{t}$, and elastic shear displacement $\mathbf{u}_{i j}^{t}$. By Newton's Third Law, particle $j$ experiences the force $\mathbf{F}_{j i}=-\mathbf{F}_{i j}$. Particle sliding occurs when the Coulomb criterion $\left|\mathbf{F}_{i j}^{t}\right|<\mu\left|\mathbf{F}_{i j}^{n}\right|$ is not satisfied for particle friction coefficient $\mu$. Additionally, after setting $k_{t} / k_{n}=2 / 7$ and $\gamma_{t}=0$, we set $\gamma_{n}$ such that the restitution coefficient $e=\exp \left(-\gamma_{n} \pi / \sqrt{4 k_{n} / m_{\text {eff }}-\gamma_{n}^{2}}\right)=0.7$.

Using the above contact model, assemblies of about 2000 particles in a periodic box are subjected to homogeneous steady simple shear at a shear rate $\dot{\gamma}$ via the Lees-Edwards boundary condition [21]. The box size, and hence the solids volume fraction $\phi$, are kept constant for each simulation. The macroscopic stress tensor is calculated as

$$
\boldsymbol{\sigma}=\frac{1}{V} \sum_{i}\left[\sum_{j \neq i} \frac{1}{2} \mathbf{r}_{i j} \mathbf{F}_{i j}+m_{i}\left(\mathbf{v}_{i}^{\prime}\right)\left(\mathbf{v}_{i}^{\prime}\right)\right],
$$

where $V$ is the box volume, $\mathbf{r}_{i j}$ is the center-to-center contact vector from particle $j$ to particle $i$, and $\mathbf{v}_{i}^{\prime}$ is the particle velocity relative to its mean streaming velocity; from this result, an ensemble-averaged pressure $p$ and shear stress $\tau$ can be extracted. All macroscopic quantities will be presented in dimensionless form, scaled by some combination of the particle diameter $d$, stiffness $k=k_{n}$, and solid density $\rho_{s}$. Since particles are assumed to overlap without deformation, we ensure that the average overlap is small (i.e. $\delta / d \sim p d / k \lesssim 0.07$ ).

Flow regimes. - We performed a series of simple shear simulations over a range of shear rates and volume fractions reaching into all three flow regimes and for particle friction coefficients between 0 and 1 . Figure 1 shows the scaled pressure $p d / k$ and shear stress $\tau d / k$ versus the scaled shear rate $\dot{\gamma}=\dot{\gamma} d / \sqrt{k /\left(\rho_{s} d\right)}$ at various volume 
TABLE I. Critical volume fraction estimates for different cases of the interparticle friction coefficient. The value of $\phi_{c}$ for the frictionless case agrees with the experimentally determined result of Nordstrom [10].

\begin{tabular}{c|ccccc}
$\mu$ & 0.0 & 0.1 & 0.3 & 0.5 & 1.0 \\
\hline$\phi_{c}$ & 0.636 & 0.613 & 0.596 & 0.587 & 0.581
\end{tabular}

fractions for (a) $\mu=0.5$ and (b) $\mu=0.1$. At low shear rates, there is an observed separatrix occurring at a critical volume fraction $\phi_{c}$, which we identify as the jamming point; stresses scale quadratically with shear rate below $\phi_{c}$ but show no rate dependence above it. These two bands correspond to the inertial and quasi-static regimes, respectively. As shear rate increases, the quasi-static and inertial isochores approach a shared asymptote characteristic of the critical point in which dependence on the volume fraction vanishes; this region corresponds to the intermediate regime. Interestingly, the intermediate asymptote appears to be independent of the friction coefficient, in contrast to results at lower shear rates and despite the fact that $\phi_{c}=\phi_{c}(\mu)$. Values of $\phi_{c}$ for different cases of $\mu$ are presented in Table I. It should be noted that these critical values inferred from dynamical behavior of sheared systems may differ from the jamming points of static packings. Additionally, we observe a propensity for assemblies near $\phi_{c}$ to form and break force chains intermittently during the shearing process, resulting in stress fluctuations of several orders of magnitude. This phenomenon limits the precision of the $\phi_{c}$ estimates to within about \pm 0.001 .

A better understanding of the regime transitions can be gained by constructing a regime map, or "phase diagram," from the slopes of the curves in Figure 1. Such a map is shown in Figure 2. The intermediate regime is observed to lie in a window centered around $\phi=\phi_{c}$, and the width of this window is dependent on the value of the dimensionless shear rate. This feature has important implications for the modeling of dense granular flows. The large stiffness of granular materials such as sand or glass beads has been shown to prevent the development of multiple contacts per particle in gravity-driven, inclinedplane flows of monodisperse spheres [22, 23]; binary collisions are said to dominate and give rise to the traditional Bagnold scaling of the stresses (i.e. $p, \tau \sim \dot{\gamma}^{2}$ ). The scenario of large $k$ (and hence small $\hat{\gamma}$ ) is indeed consistent with a narrow intermediate regime window. However, recent experimental results support the existence of an intermediate regime for granular flows [24, 25]. Therefore, in the context of building a general rheological model for granular flows, it is preferable to choose a framework that includes the intermediate regime.

Another important observation from Figure 2 is the smoothness of the transitions between the regimes. This feature suggests that "true" quasi-static, inertial, or intermediate flow is achieved only in certain limits. As

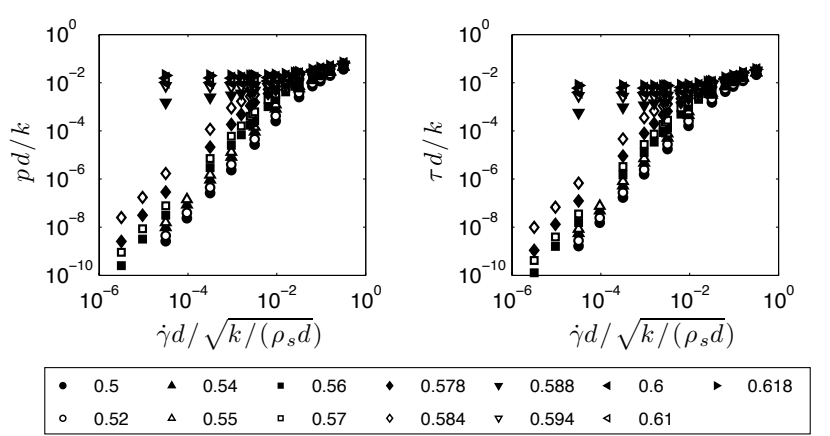

(a)

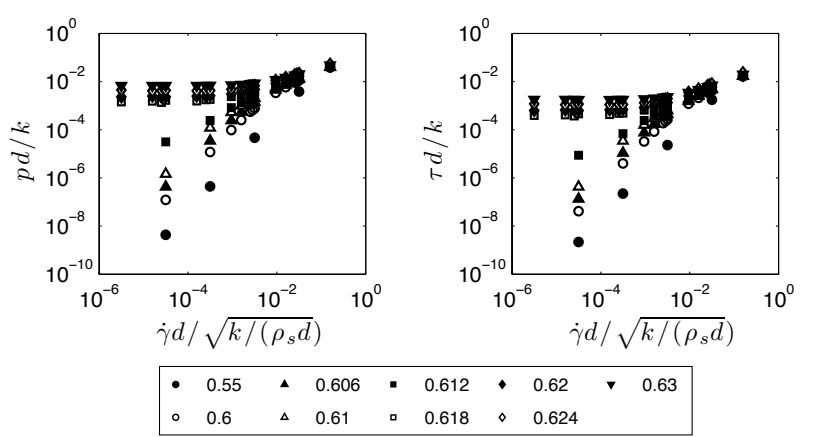

(b)

FIG. 1. Dimensionless pressure and shear stress vs. dimensionless shear rate for various volume fractions with (a) $\mu=0.5$ and (b) $\mu=0.1$. In both cases, three flow regimes are observed, each with the scalings $p \sim \dot{\gamma}^{m}$ and $\tau \sim \dot{\gamma}^{n}$ : a quasi-static regime with $m=n=0$, an inertial regime with $m=n=2$, and an intermediate regime with $m \approx 2 / 3$ and $n \approx 5 / 7$. Values of $\phi_{c}(\mu)$ are given in Table I.

$\hat{\dot{\gamma}} \rightarrow 0$, we see quasi-static flow for $\phi>\phi_{c}$, inertial flow for $\phi<\phi_{c}$, and intermediate flow at $\phi=\phi_{c}$. We also see intermediate flow as $\hat{\dot{\gamma}} \rightarrow \infty$ for all volume fractions over the wide range examined in this study. The smooth transitions also suggest that the rheology at a particular $(\dot{\gamma}, \phi)$ is a composite of contributions from low- $\hat{\dot{\gamma}}$ and high- $\hat{\dot{\gamma}}$ behaviors, and this notion will play a large role in our construction of a rheological model.

Stress scalings. - It has been demonstrated in experimental [10] and computational [8, 9, 11] studies of frictionless particles that stress data will collapse onto two curves (one above $\phi_{c}$ and one below) upon scaling the stresses and shear rate by powers of $\left|\phi-\phi_{c}\right|$, the distance to jamming. This idea is consistent with several models of the radial distribution function, used in kinetic theories for the inertial regime, that diverge at close packing $[26,27]$. Such a collapse can be achieved for frictional particles as well, as shown in Figure 3, with

$$
\begin{array}{rlrl}
p^{*} & =p /\left|\phi-\phi_{c}\right|^{a_{1}} & & \dot{\gamma}_{p}^{*}=\dot{\gamma} /\left|\phi-\phi_{c}\right|^{b_{1}} \\
\tau^{*}=\tau /\left|\phi-\phi_{c}\right|^{a_{2}} & & \dot{\gamma}_{\tau}^{*}=\dot{\gamma} /\left|\phi-\phi_{c}\right|^{b_{2}}
\end{array}
$$

and constitutive exponents $a_{i}$ and $b_{i}$. This result for frictional spheres was also found independently in Ref. [14]. 


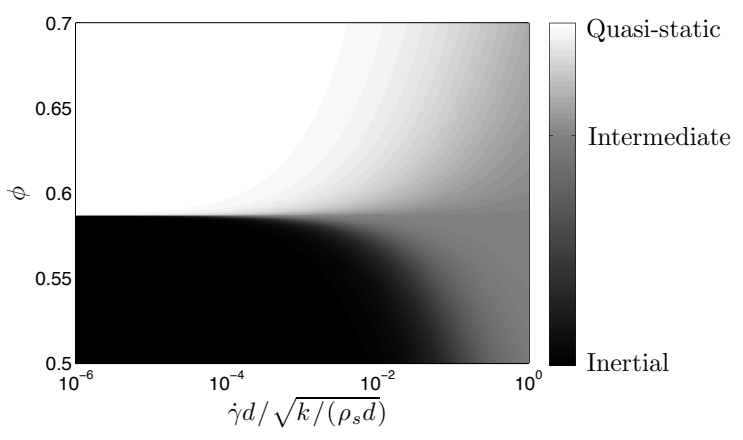

FIG. 2. Regime map for $\mu=0.5$, with volume fraction vs. dimensionless shear rate. The intermediate regime occurs only at $\phi_{c}$ in the limit $\hat{\dot{\gamma}} \rightarrow 0$ but emerges from this point to encompass all volume fractions as $\hat{\dot{\gamma}} \rightarrow \infty$.

From the collapse it is clear that an asymptotic power-law relationship between stress and shear rate exists for each flow regime, and we can write the form of each asymptote as

$$
\begin{aligned}
& \frac{p}{\left|\phi-\phi_{c}\right|^{a_{1}}} \sim\left(\frac{\dot{\gamma}}{\left|\phi-\phi_{c}\right|^{b_{1}}}\right)^{m} \\
& \frac{\tau}{\left|\phi-\phi_{c}\right|^{a_{2}}} \sim\left(\frac{\dot{\gamma}}{\left|\phi-\phi_{c}\right|^{b_{2}}}\right)^{n}
\end{aligned}
$$

where $m=n=0$ in the quasi-static regime, $m=n=2$ in the inertial regime, and $m=m^{*}$ and $n=n^{*}$ in the intermediate regime. The exponents $a_{i}$ and $b_{i}$ can be fitted from the DEM data, but the values are sensitive to the choice of $\phi_{c}$ used [9] and hence should be chosen with care. Our quasi-static regime data suggest $a_{1}=a_{2}=1$; inertial regime data reveal that $\tau^{2} / p \dot{\gamma} \sim\left|\phi-\phi_{c}\right|^{-1.6}$ and $\tau / p \sim\left|\phi-\phi_{c}\right|^{0.2}$, leading us to set $b_{1}=1.5$ and $b_{2}=1.4$. The values of $m^{*}=2 / 3$ and $n^{*}=5 / 7$ are then set by requiring volume fraction dependence to disappear in the intermediate limit. We choose not to fit these exponents from simulation data, as there is disagreement in the literature as to their exact values $[8,9,12]$. We do note, though, that our values are similar to those already proposed for frictionless particles using the linear springdashpot model. We also point out that all of the above scaling exponents depend on the contact model used $[8$, 9]. Based on a small set of simple shear simulations with a Hertzian contact model, we observe the values of $a_{1}$, $a_{2}, m^{*}, n^{*}$ to be larger than in the LSD case by a factor of about $3 / 2$, which is consistent with previous results for static, jammed systems [15].

Regime blending. - Though the individual regime limits can be described using Eqs. 6-7, the transitions between them have yet to be modeled. To this end, we employ a blending function $B$ of the form

$$
B\left(y_{1}, y_{2}\right)=\left(y_{1}^{w}+y_{2}^{w}\right)^{1 / w}
$$

with $w>0$ yielding an additive blend for the quasi-
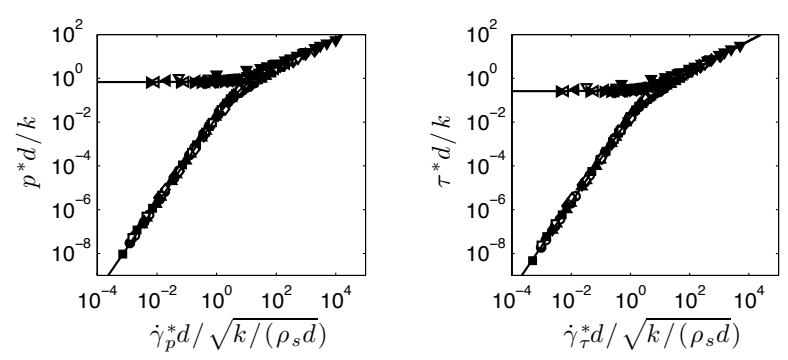

(a)
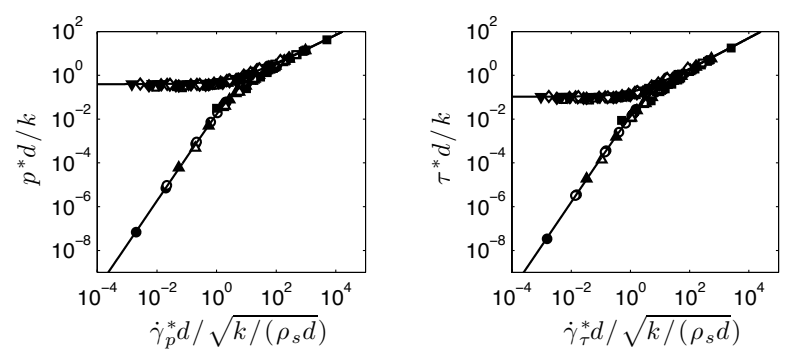

(b)

FIG. 3. Collapse of stress vs. shear rate curves from Figure 1 for (a) $\mu=0.5$ and (b) $\mu=0.1$. In both cases, the pressure is scaled as $p^{*}=p /\left|\phi-\phi_{c}\right|$, the shear stress as $\tau^{*}=\tau /\left|\phi-\phi_{c}\right|$, and the shear rate as $\dot{\gamma}_{p}^{*}=\dot{\gamma} /\left|\phi-\phi_{c}\right|^{1.5}$ or $\dot{\gamma}_{\tau}^{*}=\dot{\gamma} /\left|\phi-\phi_{c}\right|^{1.4}$. A simple blending function (solid lines) captures regime asymptotes and transitions.

static-to-intermediate transition and $w<0$ providing a harmonic blend for the inertial-to-intermediate transition. Figure 3 demonstrates the use of Eq. 8 with the asymptotic forms of Eqs. 6-7 and $w= \pm 1$. The blended model is able to capture the behavior continuously in shear rate for all three regime limits as well as the transitions; moreover, it does so without defining the stresses in piecewise fashion over arbitrary shear rate domains. Notably, it also predicts the narrowing intermediate window around $\phi=\phi_{c}$ in the limit of zero shear rate, as the quasi-static and inertial contributions to the stress become small near the jamming point. The general form of the complete model can hence be written as

$$
\begin{aligned}
& p= \begin{cases}p_{\mathrm{QS}}+p_{\text {Int }} & \text { for } \phi \geq \phi_{c} \\
\left(p_{\text {Inert }}^{-1}+p_{\text {Int }}^{-1}\right)^{-1} & \text { for } \phi<\phi_{c}\end{cases} \\
& \tau= \begin{cases}\tau_{\mathrm{QS}}+\tau_{\text {Int }} & \text { for } \phi \geq \phi_{c} \\
\left(\tau_{\text {Inert }}^{-1}+\tau_{\text {Int }}^{-1}\right)^{-1} & \text { for } \phi<\phi_{c}\end{cases}
\end{aligned}
$$

with the individual regime contributions defined as

$$
\begin{aligned}
p_{\mathrm{QS}} & =\alpha_{\mathrm{QS}}\left|\phi-\phi_{c}\right| & \tau_{\mathrm{QS}} & =\beta_{\mathrm{QS}}\left|\phi-\phi_{c}\right| \\
p_{\text {Int }} & =\alpha_{\text {Int }} \hat{\dot{\gamma}}^{2 / 3} & \tau_{\text {Int }} & =\beta_{\text {Int }} \hat{\dot{\gamma}}^{5 / 7} \\
p_{\text {Inert }} & =\frac{\alpha_{\text {Inert }} \hat{\dot{\gamma}}^{2}}{\left|\phi-\phi_{c}\right|^{2}} & \tau_{\text {Inert }} & =\frac{\beta_{\text {Inert }} \hat{\dot{\gamma}}^{2}}{\left|\phi-\phi_{c}\right|^{9 / 5}}
\end{aligned}
$$

The stresses at $\phi=\phi_{c}$ can be calculated using either blend, since Eqs. 11 and 13 yield $p_{Q S}\left(\phi=\phi_{c}\right)=0$ and 


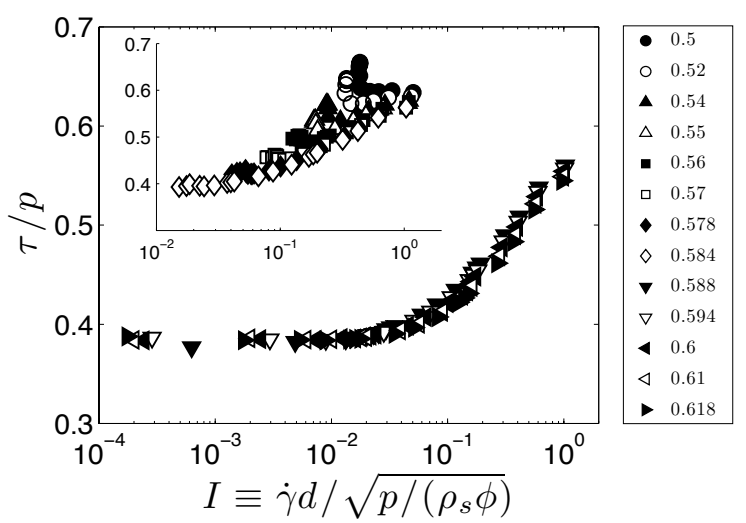

FIG. 4. Shear stress ratio vs. inertial number for $\mu=0.5$. A reasonable collapse is achieved for volume fractions near or above the jamming point (main figure). Inset: Significant scatter is observed when $\phi$ is extended below $\phi_{c}$, particularly at low shear rates (i.e. in the inertial regime).

$p_{\text {Inert }}\left(\phi=\phi_{c}\right)=\infty$, which both yield $p=p_{\text {Int }}$ upon substitution into Eq. 9; this case is included with the quasi-static blending solely for the sake of simplicity. The constitutive parameters $\alpha_{Q S}, \beta_{Q S}, \alpha_{\text {Inert }}$, and $\beta_{\text {Inert }}$ are functions of $\mu$, while $\alpha_{\text {Int }}$ and $\beta_{\text {Int }}$ are $\mu$-independent as noted earlier.

Inertial number. - Some recent, successful rheological models for dense granular flows employ a dimensionless parameter called the inertial number as the basis for achieving stress collapses over a range of volume fractions and shear rates [24, 28]. This inertial number $I \equiv \dot{\gamma} d / \sqrt{p /\left(\rho_{s} \phi\right)}$ is a ratio of the macroscopic shear timescale to the microscopic timescale for particle rearrangement, and the physics of a granular flow is said to be determined by the competition of these two mechanisms. For comparison of our simulation results with these models, we plot in Figure 4 the shear stress ratio $\eta \equiv \tau / p$ versus $I$ for $\mu=0.5$. Data from the quasistatic and intermediate regimes indeed cluster together; however, the inertial regime tends to give rise to larger stress ratios than do the other regimes at a given value of $I$. A possible reason for this discrepancy is that these $\eta(I)$ models are constructed based on a relatively narrow set of volume fractions near $\phi_{c}$. As such, they treat granular flows as incompressible [24] or only slightly compressible [28]. While this treatment is reasonable for the densest flows, our data suggest that it is less appropriate for less dense flows in which compressibility becomes important. If, however, we restrict our analysis to intermediate and quasi-static regime data, we see a collapse consistent with that of Refs. [24] and [28].

Summary. - We have investigated shear flows of frictional granular materials in all three flow regimes in order to gain a better understanding of the scalings within each regime and the transitions between them. We find scaling relations for stresses with respect to both shear rate and the distance to the jamming point and, for the intermediate regime, observed identical power-law behavior for particles with different friction coefficients. Furthermore, we propose a simple blending function for patching each regime's asymptotic form in order to predict stresses in between regimes. These findings establish a framework for a global model for steady-state simple shear flows.

We gratefully acknowledge the support of DOE/NETL Grant No. DE-FG26-07NT43070.

[1] V. Garzó and J. W. Dufty, Phys. Rev. E 59, 5895 (1999).

[2] C. K. K. Lun, S. B. Savage, D. J. Jeffrey, and N. Chepurniy, J. Fluid Mech. 140, 223 (1984).

[3] J. T. Jenkins and M. W. Richman, Phys. Fluids 28, 3485 (1985).

[4] P. C. Johnson and R. Jackson, J. Fluid Mech. 176, 67 (1987).

[5] D. G. Schaeffer, J. Differ. Equations 66, 19 (1987).

[6] J. H. Prevost, Soil Dyn. Earthq. Eng. 4, 9 (1985).

[7] A. J. Liu and S. R. Nagel, Nature 396, 21 (1998).

[8] T. Hatano, J. Phys. Soc. Jpn. 77, 123002 (2008).

[9] M. Otsuki and H. Hayakawa, Prog. Theor. Phys. 121, 647 (2009).

[10] K. N. Nordstrom, E. Verneuil, P. E. Arratia, A. Basu, Z. Zhang, A. G. Yodh, J. P. Gollub, and D. J. Durian, Phys. Rev. Lett. 105, 175701 (2010).

[11] P. Olsson and S. Teitel, Phys. Rev. Lett. 99, 178001 (2007)

[12] B. P. Tighe, E. Woldhuis, J. J. C. Remmers, W. van Saarloos, and M. van Hecke, Phys. Rev. Lett. 105, 088303 (2010).

[13] C. S. Campbell, J. Fluid Mech. 465, 261 (2002).

[14] M. Otsuki and H. Hayakawa, arXiv:1006.3597 (2010).

[15] H. P. Zhang and H. A. Makse, Phys. Rev. E 72, 011301 (2005).

[16] C. Song, P. Wang, and H. A. Makse, Nature 453, 629 (2008).

[17] T. S. Majmudar, M. Sperl, S. Luding, and R. P. Behringer, Phys. Rev. Lett. 98, 058001 (2007).

[18] M. van Hecke, J. Phys. Condens. Mat. 22, 033101 (2010).

[19] P. A. Cundall and O. D. L. Strack, Geotechnique 29, 47 (1979).

[20] S. Plimpton, J. Comput. Phys. 117, 1 (1995).

[21] A. W. Lees and S. F. Edwards, J. Phys. C 5, 1921 (1972).

[22] K. A. Reddy and V. Kumaran, Phys. Rev. E 76, 061305 (2007).

[23] L. E. Silbert, G. S. Grest, R. Brewster, and A. J. Levine, Phys. Rev. Lett. 99, 068002 (2007).

[24] P. Jop, Y. Forterre, and O. Pouliquen, Nature 441, 727 (2006).

[25] M. K. Langroudi, S. Turek, A. Ouazzi, and G. Tardos, Powder Tech. 197, 91 (2010).

[26] S. Torquato, Phys. Rev. E 51, 3170 (1995).

[27] S. Ogawa, A. Umemura, and N. Oshima, Z. Angew. Math. Phys. 31, 483 (1980).

[28] F. da Cruz, S. Emam, M. Prochnow, J.-N. Roux, and F. Chevoir, Phys. Rev. E 72, 021309 (2005). 


\section{Appendix $\mathrm{C}$}

\section{A constitutive model with microstructure evolution for flow of rate-independent granular materials}




\title{
A constitutive model with microstructure evolution for flow of rate-independent granular materials
}

\author{
By JIN SUN AND SANKARAN SUNDARESA N ${ }^{1}$ \\ ${ }^{1}$ Department of Chemical Engineering, Princeton University, Princeton, NJ 08544, USA
}

(Received ?? 2010 and in revised form ?? 2010)

A constitutive model is developed for the complex rheology of rate-independent granular materials. The closures for the pressure and the macroscopic friction coefficient are linked to microstructure through evolution equations for coordination number and fabric. The material constants in the model are functions of particle-level properties and are calibrated using data generated through simulations of steady and unsteady simple shear using the discrete element method (DEM). This model is verified against DEM simulations at complex loading conditions.

\section{Introduction}

Dense granular materials are ubiquitous in nature and are widely encountered in industrial processes, such as hopper discharge, chute flow, moving beds, standpipe flow, etc. Constituent grains (referred to as particles hereafter) in these materials touch multiple neighbors for finite durations and the stress is largely transmitted through force chains. We focus our attention on one important aspect of dense flow behavior, namely the rateindependent response, where the inertial effects associated with the individual particle interactions are negligible and the stress is largely independent of the deformation rate.

Many constitutive models have been developed in the literature for rate-independent granular materials. Starting from the basic notion of an "ideal Coulomb material", where the granular materials are treated as rigid under increasing load until the load reaches a yield point, the models have progressed in terms of incorporating more and more underlying physics. Under a broad category of so-called plastic-potential models (Hill 1950; Harris 2006), work hardening, non-associated flow rules (Lade 1977) and critical state concept (Schofield \& Wroth 1968) have been introduced for either rigid-plastic or elasticplastic materials. Under another category of double-shearing model, models have been extended from incompressible flows (Spencer 1964) to including dilatancy (Mehrabadi \& Cowin 1978), work-hardening (Anand \& Gu 2000), and fabric anisotropy (Nemat-Nasser 2000; Zhu et al. 2006), which was motivated by the relations between stress and fabric as observed in experiments using photoelastic particles (Oda et al. 1980; Subhash et al. 1991).

More recent experiments (Utter \& Behringer 2004; Majmudar \& Behringer 2005) and DEM simulations (Luding 2004, 2005; Aarons et al. 2009) further revealed that stress evolution is correlated to microstructure rearrangement during unsteady flow. The jamming transition in dense granular systems profoundly modifies the dynamics and rheology close to the transition (Hébraud \& Lequeux 1998; Keys et al. 2007; Majmudar et al. 2007) 
and may be related to the onset of rate-independent response. The jamming transition has also been shown to depend on particle friction coefficients (Song et al. 2008).

In this paper, we probe the relation between the rate-independent rheology and the microstructure of a granular material under homogeneous simple shear using DEM simulations, and construct a constitutive model. The main text describes a simple model that neglects normal stress differences, and the extension to include normal stress differences is presented in an appendix. In this model, the pressure and macroscopic friction coefficient are expressed in terms of microstructural internal variables. Evolution equations are proposed for these internal variables. We correlate the material constants in the model to particle-level properties, such as particle elasticity and friction coefficient. We note at the outset that the present study focuses exclusively on simple shear flows and that the application of this model to completely constrained deformations, such as uniaxial or triaxial compression will be reported in future publications.

After we describe the DEM and simulation details in section 2, we present simulation results for the evolution of the stress and the internal variables during unsteady shear in section 3. We then detail the development and verification of the constitutive model in sections 4 and 5, respectively. We summarize the attributes of this model in section 6 .

As for notation, we employ lightface italics for scalars, the boldface regular font for vectors and the San Serif font for the second order tensors.

\section{Computational methods and simulation details}

The DEM simulations were carried out using the large-scale atomic/molecular massive parallel simulator (LAMMPS) developed at Sandia National Laboratories (Plimpton 1995). In the simulations, particles interact only at contact. Two spherical particles with position vectors $\left\{\mathbf{r}_{i}, \mathbf{r}_{j}\right\}$ and radii $\left\{R_{i}, R_{j}\right\}$, experience a force, $\mathbf{F}_{i j}=\mathbf{F}_{\mathrm{n}_{i j}}+\mathbf{F}_{\mathrm{t}_{i j}}$, when $\delta_{i j}=d-r_{i j}>0$, where $d=R_{i}+R_{j}$ and $r_{i j}=\left|\mathbf{r}_{i j}\right|=\left|\mathbf{r}_{i}-\mathbf{r}_{j}\right|, \mathbf{n}_{i j}$ is the contact normal unit vector pointing from the center of particle $j$ to that of particle $i$, and $\mathbf{t}_{i j}$ is a unit vector in the tangential plane. The force is calculated using a spring-dashpot model, which has been tested and used in many other studies (Cundall \& Strack 1979; Silbert et al. 2001, 2007; Campbell 2002; Rycroft et al. 2009). The normal and tangential components of the interaction force acting on particle $i$ for the Hookean contact model are

$$
\begin{aligned}
& \mathbf{F}_{\mathrm{n}_{i j}}=k_{\mathrm{n}} \delta_{i j} \mathbf{n}_{i j}-\gamma_{\mathrm{n}} m^{*} \mathbf{v}_{\mathrm{n}_{i j}}, \\
& \mathbf{F}_{\mathrm{t}_{i j}}=-k_{\mathrm{t}} \mathbf{u}_{\mathrm{t}_{i j}}-\gamma_{\mathrm{t}} m^{*} \mathbf{v}_{\mathrm{t}_{i j}},
\end{aligned}
$$

where $k_{\mathrm{n}, \mathrm{t}}$ and $\gamma_{\mathrm{n}, \mathrm{t}}$ are the spring elastic and viscous damping constants, $\mathbf{v}_{\mathbf{n}_{i j}}$ and $\mathbf{v}_{\mathrm{t}_{i j}}$ are the normal and tangential component of particle relative velocity, respectively, and $m^{*}=\frac{m_{i} m_{j}}{m_{i}+m_{j}}$ is the effective mass of spheres with masses $m_{i}$ and $m_{j}$. The corresponding contact force on particle $j$ is simply given by Newton's third law, i.e., $\mathbf{F}_{j i}=-\mathbf{F}_{i j}$. The tangential force at each contact is computed by keeping track of the elastic shear displacement, $\mathbf{u}_{\mathrm{t}_{i j}}$, throughout the lifetime of a contact. The elastic shear displacement is set to zero at the initiation of a contact and its rate is given by

$$
\frac{\mathrm{d} \mathbf{u}_{\mathrm{t}_{i j}}}{\mathrm{~d} t}=\mathbf{v}_{\mathrm{t}_{i j}}-\frac{\left(\mathbf{u}_{\mathrm{t}_{i j}} \cdot \mathbf{v}_{i j}\right) \mathbf{r}_{i j}}{r_{i j}^{2}} .
$$

The last term in Eq. 2.2 arises from the rigid body rotation around the contact point and ensures that $\mathbf{u}_{\mathrm{t}_{i j}}$ always lies in the local tangent plane of contact. As the shear displacement increases, the tangential force reaches the limit imposed by a static yield 
criterion, $\left|\mathbf{F}_{\mathrm{t}_{i j}}\right| \leqslant \mu\left|\mathbf{F}_{\mathrm{n}_{i j}}\right|$, characterized by a local particle friction coefficient, $\mu$. The tangential force is then set to the limit value by truncating the magnitude of $\mathbf{u}_{\mathrm{t}_{i j}}$.

The contact force according to the Hertzian model is related to that of the Hookean model as

$$
\mathbf{F}_{i j \mathrm{HZ}}=\sqrt{\delta_{i j} R^{*}} \mathbf{F}_{i j \mathrm{HK}},
$$

where $\mathbf{F}_{i j \mathrm{HK}}$ is given in Eq. 2.1 and $R^{*}=\frac{R_{i} R_{j}}{R_{i}+R_{j}}$ is the effective radius for two particles with radii $R_{i}$ and $R_{j}$, respectively. The normal and tangential elastic constants in the Hertzian model are related to the particle material properties, Young's modulus, $E$, and Poisson ratio, $\nu$, as

$$
k_{\mathrm{nHZ}}=\frac{2 E}{3\left(1-\nu^{2}\right)}, k_{\mathrm{tHZ}}=\frac{4 E}{(1+\nu)(2-\nu)},
$$

respectively, which gives the ratio between them $k_{\mathrm{tHZ}} / k_{\mathrm{nHZ}}=\frac{6(1-\nu)}{2-\nu}$ (Zhang \& Makse 2005). The elastic constants in the Hookean model $k_{\mathrm{n}}$ and $k_{\mathrm{t}}$ scale to those of the Hertzian model by the factor $\sqrt{\delta_{i j} R^{*}}$ for each contact. The value of the linear spring elastic constant is chosen to be large enough to minimize particle overlap, yet not so large as to require an unreasonably small simulation time step. In the simulations, we set $k_{\mathrm{t}}=$ $2 / 7 k_{\mathrm{n}}$ (Shäfer et al. 1996) and $\gamma_{\mathrm{t}}=\frac{1}{2} \gamma_{\mathrm{n}}$. In the Hookean model simulations, $\gamma_{\mathrm{n}}$ is chosen to yield a value of 0.7 for the normal restitution coefficient $e=\exp \left(-\frac{\gamma_{\mathrm{n}} \pi}{\sqrt{4 k_{\mathrm{n}} / m_{\mathrm{eff}}-\gamma_{\mathrm{n}}^{2}}}\right)$. In the Hertzian model simulations, the restitution coefficient depends on the initial approaching velocity. Hookean model is the default model for the results presented except where Hertzian model is explicitly indicated.

In order to maintain homogeneous deformation over large strain scales, we performed the simulations in three-dimensional (3D) periodic domains without gravity. Simple shear flow was induced via the Lees-Edwards boundary conditions (Lees \& Edwards 1972). Throughout this study the $x, y$ and $z$ directions will refer to the flow, neutral (vorticity) and gradient directions, respectively. The shearing motion induced by this boundary driven algorithm takes time to develop so that the flow would not be homogeneous immediately after a shear rate change, which raises questions about the suitability of the algorithm to study homogeneous time-dependent flows. This disadvantage can be greatly alleviated through the use of the SLLOD algorithm (Evans \& Morriss 1990). The SLLOD algorithm implies that a change in shear rate is not achieved by simply moving the boundaries of the system faster or slower, but by applying a force to the entire system. Thus the SLLOD algorithm was applied to all the simulations presented in this paper. However, we note that the SLLOD algorithm only affected dynamical behavior at very small strain scales (typically less than $10^{-3}$ ) after the deformation rate change and did not alter the large strain scale dynamical behavior in unsteady shear shown in the next section. Thus, we would have obtained essentially the same model even if the SLLOD algorithm was not employed.

Isochoric and dilatant simple shear have been simulated to probe the rheology of the assembly. The isochoric flow was studied under a constant volume (CV) condition particular to the simulation scheme described above. Experiments of shearing dense granular materials under CV are less common due to dilatancy - a densely packed assembly will expand in volume under initial shear. A pertinent experiment would be the undrained shear test on water-saturated sand, where the volume of the whole specimen can be kept constant within experimental error range (Schofield \& Wroth 1968). Since a good rheological model must capture the flow characteristics under all kinds of tests, irrespective of whether such tests are easily implemented in the laboratory or not, we avail the CV sim- 
ple shear simulations in this study. Dilatant simple shear was examined under constant pressure (CP) condition, where each normal stress component was controlled individually to match that observed in a steady CV simulation. This procedure produces rheological response that can easily be compared to the CV simulations and is essentially the same as that in shear experiments with either free surfaces (Mueth et al. 2000; Bocquet et al. 2001; Tardos et al. 2003; Toiya et al. 2004) or controlled normal loads (Nedderman 1992; Lu et al. 2007; Kheiripour Langroudi et al. 2010) in Couette cells or Jenike shear cells.

Steady state is reached after an assembly is sheared over a large strain (of order unity at least) and the stress and microstructural quantities cease to evolve. The macroscopic rate of deformation tensor at the steady state then follows $D=\frac{1}{2} \dot{\gamma}\left(\mathbf{e}_{(x)} \mathbf{e}_{(z)}+\mathbf{e}_{(z)} \mathbf{e}_{(x)}\right)$, where $\mathbf{e}_{(x)}$ and $\mathbf{e}_{(z)}$ are unit vectors in $x$ and $z$ directions, respectively, and $\dot{\gamma}$ is the flow velocity gradient (the shear rate). The type of unsteady flows studied here is shear reversal flow, where the flow direction is reversed after the steady state is reached and maintained for a certain strain. In this case, the $\dot{\gamma}$ changes its sign after reversal. The shear rate is maintained low in the sense that the inertia number (MiDi 2004) (or Weissenberg number (Goddard 1986)), $I=\frac{\dot{\gamma} d}{\sqrt{p / \rho_{s} \phi}}$, where $p$ is a confining pressure, $\rho_{s}$ is particle density and $\phi$ is particle volume fraction, is smaller than $10^{-3}$, so that the rateindependent response is guaranteed according to previous experimental and simulation data from our and other researchers' work (Campbell 2002; MiDi 2004; da Cruz et al. 2005; Aarons et al. 2009).

Homogeneous stress and strain can be extracted from this type of flows, which facilitates constitutive modeling. The macroscopic stress is calculated as

$$
\boldsymbol{\sigma}=\frac{1}{V} \sum_{i}\left[\sum_{j \neq i} \frac{1}{2} \mathbf{r}_{i j} \mathbf{F}_{i j}+m_{i}\left(\mathbf{v}_{i}^{\prime}\right)\left(\mathbf{v}_{i}^{\prime}\right)\right],
$$

where $V$ is the total volume of the simulation domain and $\mathbf{v}_{i}^{\prime}$ is the fluctuating velocity of a particle relative to its mean streaming velocity in the shear flow. The first and the second terms on the right of Eq. 2.5 represent the contact force contribution and the inertial effect associated with particle streaming, respectively. In all the results presented, the second term was at least four orders of magnitude smaller than the first one, consistent with the rate-independent response.

\section{Unsteady shear rheology}

In this paper we present and analyze the results obtained in simulations of an assembly of 2000 mono-disperse spheres in a 3D periodic domain. The dependence on system sizes, i.e., particle numbers, was tested using assemblies with particle numbers varying from 1000 to 10000 and it was found that a system size of 2000 particles was sufficient to get stress and microstructural data that are independent of the system size. All the results presented here are ensemble averages of 10-20 realizations with different initial configurations.

An assembly with particle volume fraction $\phi$ of 0.6 was first sheared at a shear rate of $\dot{\gamma}_{0}=2\left|\mathrm{D}_{0}\right|$, where $\left|\mathrm{D}_{0}\right|$ is the modulus of the initial strain rate tensor $\left(:=\sqrt{\frac{1}{2} \mathrm{D}_{0}^{\mathrm{T}}: \mathrm{D}_{0}}\right)$, to reach a statistical steady state under the CV condition and the time $t=0$ was chosen as an arbitrary state in this statistical steady state. The assembly was then subjected to a specific shear pattern as described below. Fig. 1 shows the variation of various statistical quantities with accumulated shear strain $\dot{\gamma}_{0} t$. The shearing was stopped at $\dot{\gamma}_{0} t=1$, and the region $1<\dot{\gamma}_{0} t<2$ should be interpreted as a dimensionless time measure. The 


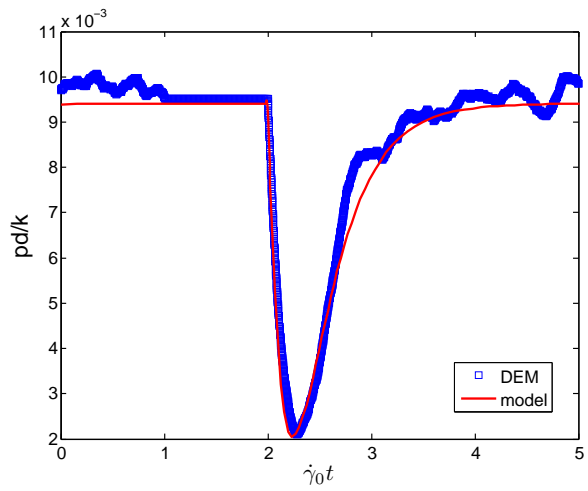

(a)

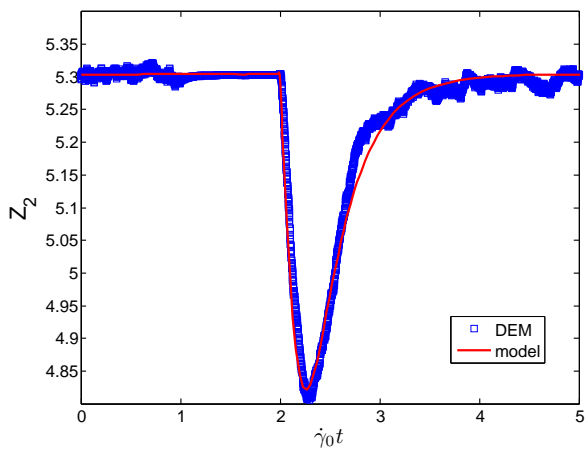

(c)

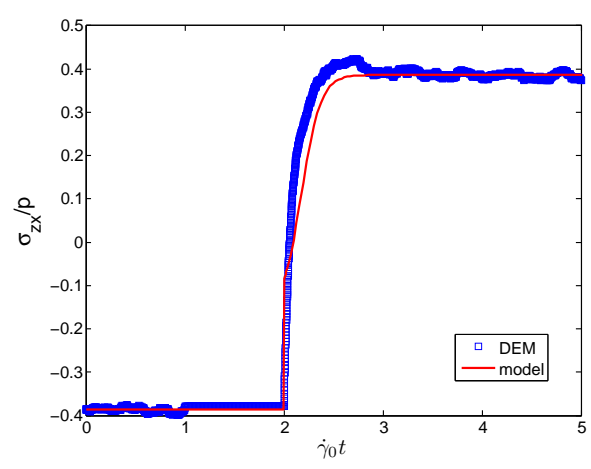

(b)

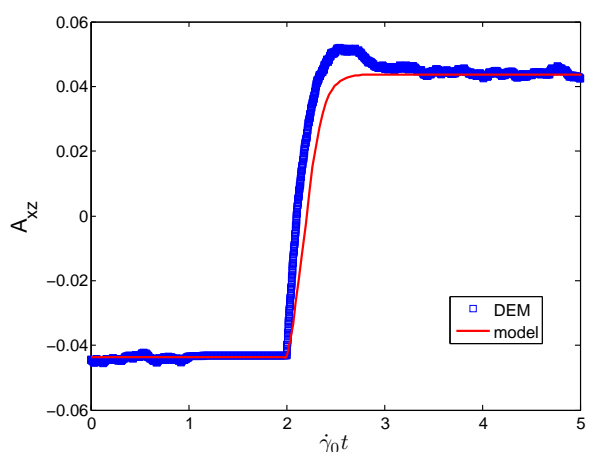

(d)

FigURE 1. Evolutions of (a) scaled pressure $p d / k$ (where $k=k_{\mathrm{n}}$ ), (b) stress ratio, (c) coordination number and (d) $A_{x z}$ for an assembly subjected to unsteady shear under constant volume condition with $\phi=0.60$. Blue square symbols denote the data from DEM simulations and the red solid curves are the constitutive model results. The shearing was stopped during $1<\dot{\gamma}_{0} t<2$. The inter-particle friction coefficient $\mu$ is 0.5 and the inertia number $I \approx 0.0003$ at steady state.

pressure scaled by particle diameter and elastic spring constant, and the shear stress-topressure ratio are shown in Figs. 1(a) and (b), respectively. They clearly exhibited rateindependent response as the stress level was retained during the no-shear period $(1<$ $\left.\dot{\gamma}_{0} t<2\right)$ with little change from the steady state. The rate-independent characteristic has also been verified by collapsing the stress data from simulations at various of shear rates; those results add no significant information and are not shown in the figures. At $\dot{\gamma}_{0} t=2$, the flow direction was reversed and the shear rate became $\dot{\gamma}=-\dot{\gamma}_{0}$. The pressure and stress ratio had significant transitions spanning a strain larger than unity after the reversal. The pressure dropped to a lower value and slowly returned to the steady state. As the volumetric strain was kept at zero, this behavior rules out volume fraction change as a necessary cause to the pressure transition. The steady state magnitude of the stress ratio is about 0.4 , which was close to the measured quasi-static value in a shear cell experiment using polystyrene beads (Savage \& Sayed 1984). Upon shear reversal, the stress ratio slowly evolved to a new steady state value with the same magnitude as the initial state, but with an opposite sign. Similar stress transition trend after shear reversal has also been observed in experiments with dense granular materials (Toiya et al. 2004; Utter \& Behringer 2004) and with dense suspensions (Gadala-Maria \& Acrivos 1980). 


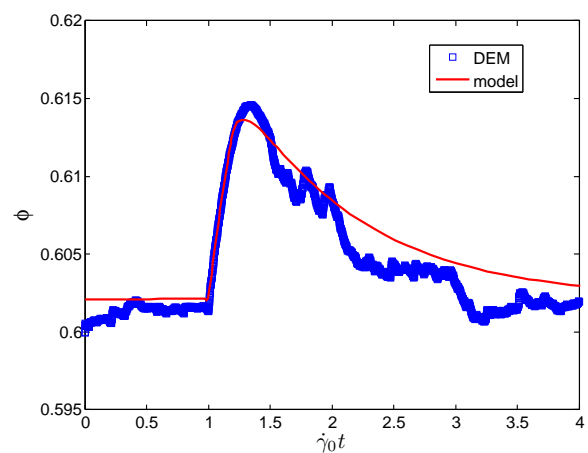

(a)

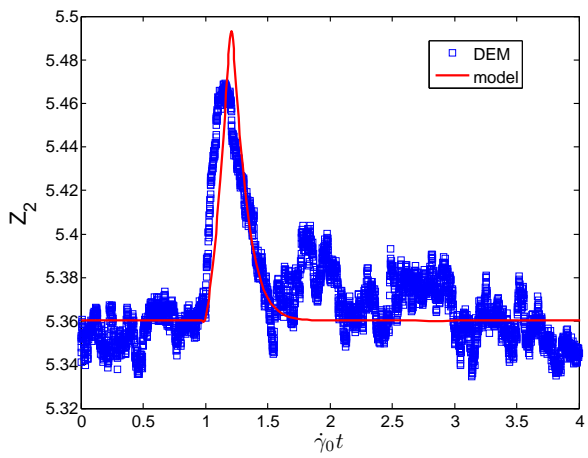

(c)

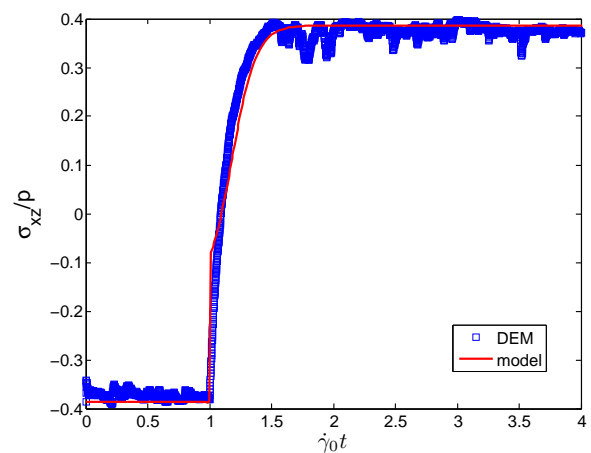

(b)

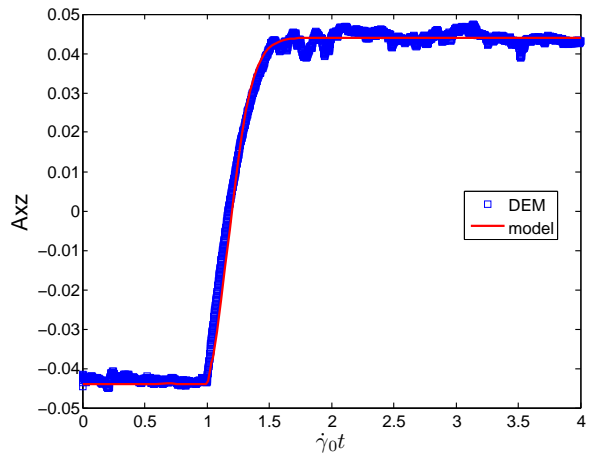

(d)

Figure 2. Evolutions of (a) volume fraction, (b) stress ratio, (c) coordination number and (d) $A_{x z}$ for an assembly subjected to unsteady shear under constant pressure condition. Blue square symbols denote the data from DEM simulations and the red solid curves are the constitutive model results. The inter-particle friction coefficient $\mu$ is 0.5 and the inertia number $I \approx 0.0003$ at steady state.

For the case where the assembly was sheared under CP condition, the volume fraction and stress ratio (see Figs. 2(a) and (b), respectively) manifested transitions after shear reversal at $\dot{\gamma}_{0} t=1$. The volume fraction increased at first (the assembly compacted) and then decreased (the assembly dilated) back to the steady state after a shear strain of about 0.5. The compaction behavior was also reported in the Couette shear cell experiment after shear reversal (Toiya et al. 2004). The stress ratio evolution had a similar trend as that in the constant volume case.

To further investigate the mechanism of this transition due to shear reversal, we studied the microstructure evolution during this unsteady shear. We quantified the microstructure using two variables, the average (mechanical) coordination number $Z$ and the fabric tensor A. The average (mechanical) coordination number is defined as the mean contacts per particle in the contact network, $Z=2 N_{\mathrm{c}} / N$, where $N_{\mathrm{c}}$ is the total number of contacts (with non-zero contact forces) and $N$ is the total number of particles in the contact network (Zhang \& Makse 2005). It characterizes the connectivity of a granular assembly. When the coordination number is equal to a critical value $Z_{c}$, the granular assembly is at an isostatic state, where the number of degrees of freedom is matched by number of constraints between particles. $Z_{c}$ varies from 4 to 6 as particle friction coefficient changes from infinity to zero in three dimensions (Song et al. 2008). $Z_{c}$ is a microstructural sig- 
nature of the jamming transition (O'Hern et al. 2003; Song et al. 2008), and can also be identified as a critical point, above which rate-independent behavior is found. In the computation of $Z$ from our DEM data, we neglect particles with zero (floaters) or one contact (rattlers) as they do not participate in the contact network, consistent with the practice of other researchers (Shundyak et al. 2007). In any case, floaters and rattlers make up only a very small portion of total particles in our simulations. We henceforth use the symbol $Z_{2}$ to distinguish from those including floaters and rattlers and we only used $Z_{2}$ to calibrate our model constants.

The fabric tensor was used to characterize microstructure anisotropy. It can be understood as a statistical moment with respect to the probability distribution function of an orientational vector $\mathbf{n}$. With different choices of the vector $\mathbf{n}$ and weighting factors, there have been various definitions (Oda et al. 1980; Bathurst \& Rothenburg 1990; Radjai et al. 1998; Goddard 1998; AzÈma et al. 2009). In this paper we identified $\mathbf{n}$ as the unit contact normal vector pointing from center to center of two spherical particles in contact. We defined the fabric tensor as the symmetric traceless second rank tensor

$$
\mathrm{A}=\frac{1}{N_{c}} \sum_{\alpha=1}^{N_{c}} \mathbf{n}^{\alpha} \mathbf{n}^{\alpha}-\frac{\mathbf{1}}{\mathbf{3}} \mathrm{I}
$$

where I is the unitary tensor. Its eigenvectors give the principal directions of the mean contact orientations. The eigenvalues, in turn, provide a measure of the extent, to which contacts are oriented in the principal directions. We may use the difference between the largest (major) and smallest (minor) eigenvalues or the second invariant as a measure of the anisotropy intensity. That being said, the structural anisotropy can easily be related to the shear $(x z)$ component of the fabric tensor for simple shear flows; our DEM data shows that the numerical values of $A_{x x}$ and $A_{z z}$ are small in magnitude compared to $A_{x z}$, while $A_{y y}, A_{x y}$ and $A_{y z}$ are essentially zero. The off-diagonal terms $A_{x z}$ and $A_{z x}$ are larger than the diagonal terms by at least an order of magnitude, and so the difference between the major and minor eigenvalues is very close to $2 A_{x z}$ and the corresponding eigenvectors lie in the $x-z$ plane at an angle of $\pm 45^{\circ}$ to the $x$-axis. Thus we present below the magnitude of $A_{x z}$ as a measure of the intensity of the anisotropy and the sign of $A_{x z}$ for the orientation of the principal direction in simple shear flows.

The evolutions of $Z_{2}$ and $A_{x z}$ are plotted in Figs. 1(c) and (d) for the CV case. It can immediately be recognized that the $Z_{2}$ and $A_{x z}$ have similar evolution patterns as the pressure and stress ratio, respectively. The $Z_{2}$ values held up during the no-shear phase $\left.\left(1<\dot{\gamma_{0}} t<2\right)\right)$, decreased significantly after the shear reversal and returned to the steady-state value after the same strain scale as the pressure did. The transition of the coordination number after shear reversal indicates that the contacts were broken at first and then built up. The $A_{x z}$ variation shows that the microstructure lost its anisotropy and then slowly reestablished the anisotropy with a principal direction rotated by $90^{\circ}$ over a comparable strain scale as the shear stress-to-pressure ratio did. The evolutions of the two variables together revealed the microstructural rearrangement after shear reversal, which involved both connectivity and anisotropy changes and had a strain scale of $\sim 1$. During the CP shear, large scale microstructural rearrangement was also indicated by the evolutions of $Z_{2}$ and $A_{x z}$ in Figs. 2(c) and (d). The $Z_{2}$ increased a small amount after the shear reversal. Since the pressure was maintained constant for the entire shearing process, this increase suggests that pressure is influenced by the anisotropy in addition to the coordination number. The evolution of $A_{x z}$ (see Fig. 2(d)) again mirrors that of the stress ratio. 


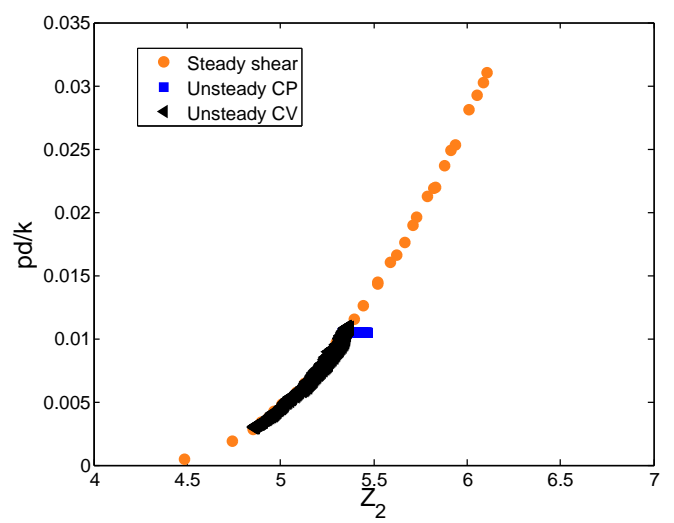

FiguRE 3. Scaled pressure variations against coordination number for simulations of steady and unsteady shear under both constant volume (CV) and constant pressure (CP) conditions. The inter-particle friction coefficient $\mu$ is 0.5 .

\section{Constitutive model formulation and calibration}

We will demonstrate that a simple model of the form

$$
\boldsymbol{\sigma}=p \mathbf{l}-p \eta \hat{\mathrm{S}}
$$

where $\hat{S}:=S /|D|$ and $S$ is the deviatoric strain rate tensor $\left(S=D-\frac{1}{3} \operatorname{tr}(D) I\right)$ and $\eta$ is a scalar macroscopic friction coefficient, is enough to capture the dynamic shear results, provided $p$ and $\eta$ are expressed in terms of $Z_{2}$ and $A$, and suitable evolution equations are postulated for these microstructural quantities. This stress equation can be considered as a simplified version of a rate-independent rheological model (Goddard 1984, 2006). It can also be derived from a plasticity model assuming a pressure-dependent yield function and a co-axial flow rule (Schaeffer 1987). We demonstrate in appendix B how the model can be extended to include normal stress differences present in shear flows.

\subsection{The pressure and $\eta$ relations}

Granular pressure has been modeled as a function of particle volume fraction in elasticity (Makse et al. 2000; O'Hern et al. 2003), or at the critical state of plastic flow (Schofield \& Wroth 1968). However, neither the elastic pressure nor the critical state pressure relations can be generalized to unsteady flow, as is evident from the pressure variation under CV or volume fraction variation under CP in Figs. 1 and 2, respectively. By using coordination number as a state variable, we are able to collapse the pressure data for steady and unsteady shear quite well with smooth functional dependence as shown in Fig. 3. The small discrepancy between the unsteady and steady shear data is attributed to the anisotropy variation during shear reversal.

According to these features, we model the pressure (attributed to contact force) as a function of $Z_{2}$ and $\mathrm{A}$ :

$$
p d / k=\left(a_{1}+a_{2}|\mathrm{~A}|\right)\left(Z_{2}-Z_{c}\right)^{\alpha},
$$

where $a_{1}$ and $a_{2}$ are material parameters and $\alpha=2$. The pressure in an isotropic state, modeled by the $a_{1}\left(Z_{2}-Z_{c}\right)^{\alpha}$ term, was examined using stress-controlled isotropic compression. $\dagger$ The values of pressure in both isotropic compression (at the infinite compactiv-

$\dagger$ Details of the simulation protocol are presented in appendix A. Under isotropic compression the pressure manifested a dependence on the packing preparation history, e.g., on the initial 


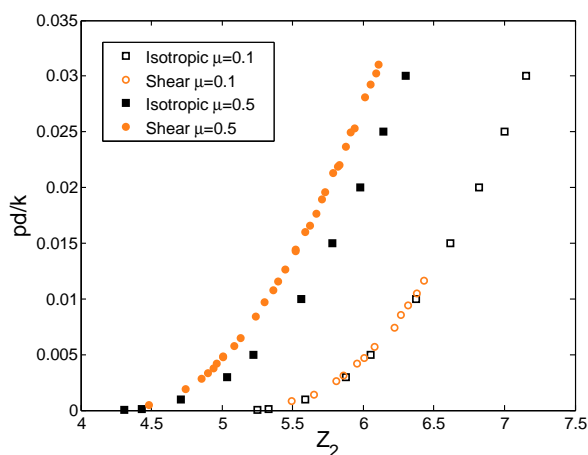

(a)

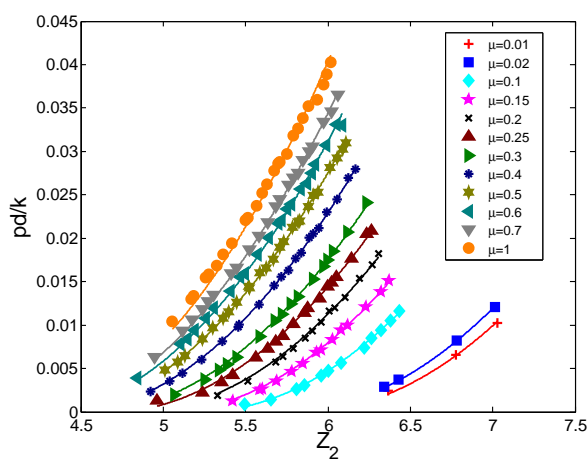

(c)

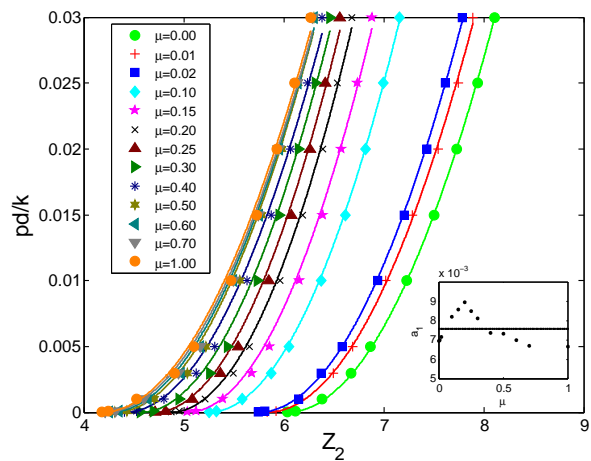

(b)

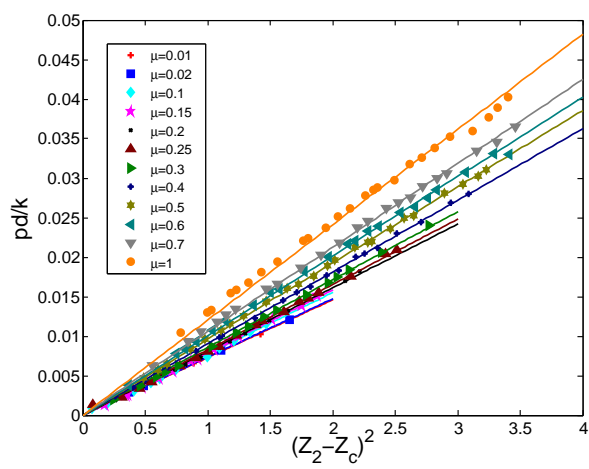

(d)

FIGURE 4. (a) Scaled pressure variations against coordination number for isotropic compression and steady-state simple shear with open and filled symbols used for assemblies with inter-particle friction coefficient $\mu=0.1$ and $\mu=0.5$, respectively; the same variation for (b) isotropic compression and (c) steady-state simple shear for assemblies with inter-particle friction coefficient $\mu$ ranging from 0 to 1 ; the data in (c) are re-plotted against $\left(Z_{2}-Z_{c}\right)^{2}$ in (d). The symbols in the figures denote the DEM data; the solid lines in (b) and show the fits using $p d / k=a_{1}\left(Z_{2}-Z_{c}\right)^{2}$; the solid lines in (c) and (d) indicate results from the model of $p d / k=\left(0.0073+a_{2}|\mathrm{~A}|\right)\left(Z_{2}-Z_{c}\right)^{2}$. The inset in (b) shows the variation of fitting parameter $a_{1}$ versus $\mu$.

ity limit (Song et al. 2008)) and steady state simple shear are plotted as functions of $Z_{2}$ for $\mu=0.1$ and $\mu=0.5$ in Fig. 4 (a). It can be seen that the pressure starts to be measurable from the same coordination number, $Z_{c}$, for both isotropic compression and simple shear. Thus $Z_{c}$ is identified as the transition point above which granular systems exhibit dynamics dominated by their jammed structures, and manifest rate independence when subjected to simple shear flows. The pressure in simple shear flow is, however, higher than that in the isotropic state at the same coordination number, which is reflected in Eq. 4.2.

We calibrate the $a_{1}$ term on the right hand side in Eq. 4.2 using the isotropic compression data and the $a_{2}$ term using the simple shear data. The variation of pressure under isotropic compression with the coordination number is shown in Fig. 4(b) for assemblies with $\mu$ ranging from 0 to 1 . We determined the $Z_{c}$ values by first extrapolating these

volume fractions before compression (Song et al. 2008). Details on our approach of modeling this history dependence and the rationale of simplifying the relation are discussed in appendix A. 
DEM data to the zero pressure limits and then fine-tuning them as a fitting parameter in the equation of $p d / k=a_{1}\left(Z_{2}-Z_{c}\right)^{2}$, which has the same form as the critical scaling shown for frictionless particles near jamming transition (O'Hern et al. 2003). The goodness of the fit in Fig. 4(b) indicates that this scaling is essentially universal for frictional particles with fitting parameter $a_{1}$ fluctuating in a small range as shown in the inset of Fig. 4(b). The weak dependence of $a_{1}$ on $\mu$ was also observed in 2D simulations of ploydisperse particles (Somfai et al. 2007). In favor of simplicity of the model, $a_{1}$ is approximated as a constant independent of $\mu$. Particle friction plays the role of shifting $Z_{c}$, but does not affect the scaling in any significant way. The critical coordination number $Z_{c}$ monotonically decreases from 6 toward 4 for increasing finite $\mu$ values as indicated by our DEM data (see Fig. 5(a)). We note that $Z_{c}$ should have numerical values of 6 and 4 at $\mu=0$ and $\mu=+\infty$, respectively; these values are determined by the isostatic state at jamming transition (Song et al. 2008). However, a fit with a plateau value slightly higher than 4 is used to better represent our DEM data for $\mu$ in the range of 0 to 1.

The material parameter $a_{2}$ was calibrated using the data from steady simple shear DEM simulations, shown by symbols in Fig. 4(c). Fits of pressure against $Z_{2}$ and $\left(Z_{2}-\right.$ $\left.Z_{c}\right)^{2}$ using Eq. 4.2 with a fixed $a_{1}$ are illustrated by solid lines in Figs. $4(\mathrm{c})$ and (d), respectively. The fitting parameter $a_{2}$ increases monotonically with $\mu$ as indicated by the increasing slopes in Fig. 4(d). Simple shear data for $\mu=0$ had a low confidence level due to the large fluctuations of coordination numbers observed in the simulations of high volume fractions and are not shown in this figure. However, the variation of $a_{2}$ versus $\mu$ is clearly monotonic and can be fitted using a linear function $a_{2}=x_{1} \mu$ as shown in Fig. 5(b). The vanishing of $a_{2}$ at the $\mu=0$ limit is also consistent with the physics that the anisotropy contribution to pressure vanishes at the frictionless limit, which can be inferred from the absence of dilatancy for frictionless spheres observed in our simulations and also in the 3D simulations of Peyneau \& Roux (2008). The inference will become clear after we introduce the microstructure evolution equation. The numerical values of $a_{1}$ and of the parameter $x_{1}$ for $a_{2}$ are listed in Tab. 1

The scaling in Eq. 4.2 did depend on force models used in simulations. Correspondingly for a physical particle assembly, it means that the elasticity property of particles affects the pressure scaling of the assembly. This can be seen from a micromechanical equation

$$
\boldsymbol{\sigma} \approx=\frac{3 \phi Z}{2 \pi d^{3}} \bar{r}\left(k_{\mathrm{n}} \bar{\delta}_{\mathrm{n}} \frac{1}{N_{c}} \sum_{i} \sum_{j \neq i} \mathbf{n n}-k_{\mathrm{t}} \bar{\delta}_{\mathrm{t}} \frac{1}{N_{c}} \sum_{i} \sum_{j \neq i} \mathbf{n t}\right),
$$

where $\bar{r}, \overline{\delta_{\mathrm{n}}}$ and $\bar{\delta}_{\mathrm{t}}$ are mean quantities. The pressure for particles with Hertzian contact can then be shown to have a $\frac{3}{2}$ power relation with that for particles with Hookean contact, i.e.,

$$
\frac{p}{k_{\mathrm{Hz}}} \sim\left(\frac{p d}{k}\right)^{\frac{3}{2}},
$$

where $k_{\mathrm{Hz}}$ and $k$ are the normal elastic constants for Hertzian and Hookean contact models, respectively. This power relation was confirmed by collapsing our DEM data of pressure from the two models using this relation, as illustrated in Fig. 6.

The shear stress-to-pressure ratio in Figs. 1 and 2 was shown to have a similar evolution pattern as the shear component of the fabric tensor $A_{x z}$. Fig. 7 shows a plot of the stress ratio during a shear reversal under constant volume condition (the same data as in Fig. 1(b)) against $A_{x z}$. The data for unsteady shear under constant pressure would collapse essentially onto the same curve and is thus not plotted for clarity. The two branches show how the stress ratio evolved from one steady state to the other and back. 


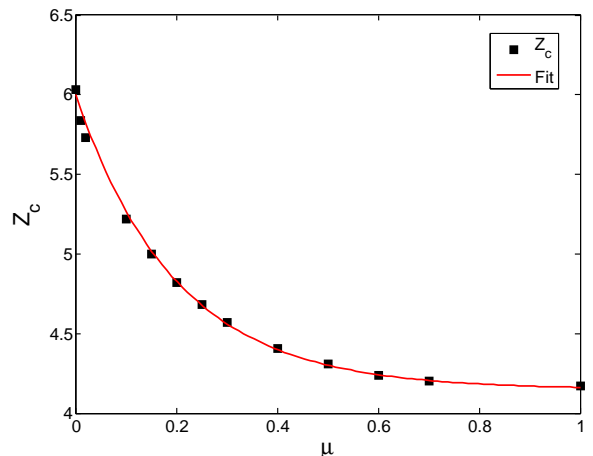

(a)

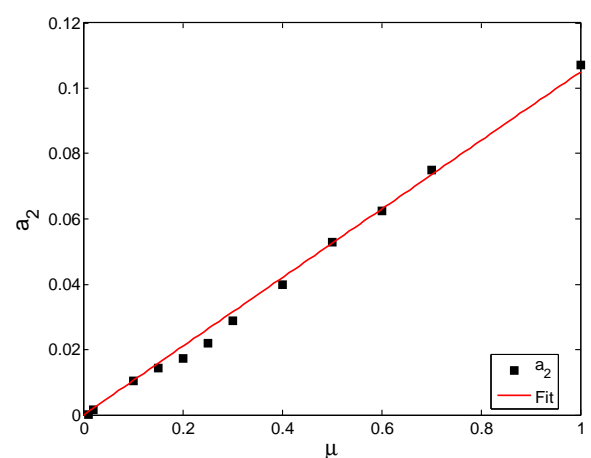

(b)

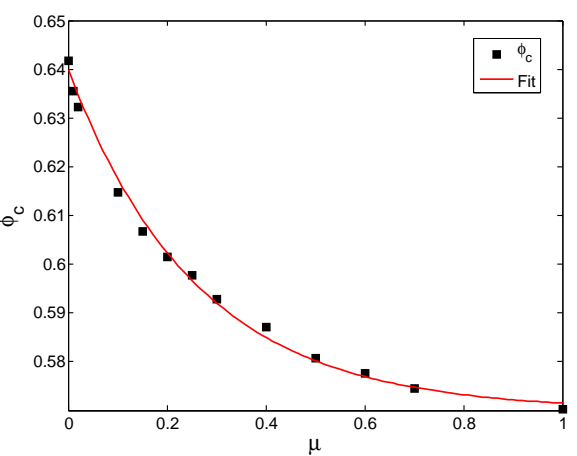

(c)

FIGURE 5. Variations of (a) the critical coordination number $Z_{c}$, (b) the material parameter, $a_{2}$, in the pressure equation and (c) the critical volume fraction $\phi_{c}$ with respect to inter-particle friction coefficient, $\mu$.

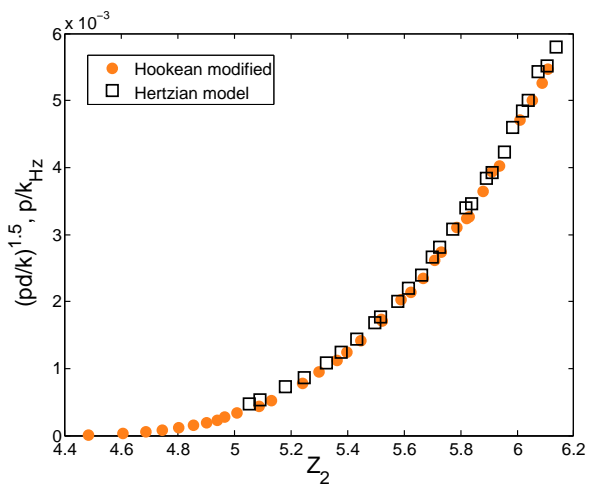

FIgURE 6. Pressure versus coordination number for simulations using Hookean and Hertzian contact models in steady-state simple shear. The Hookean model data collapses with the Hertzian model data after using a $\frac{3}{2}$ power scaling. The inter-particle friction coefficient is 0.5 . 


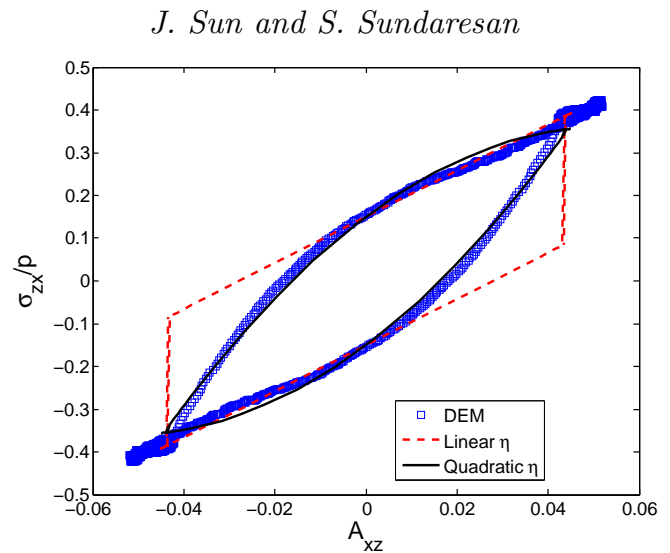

FiguRE 7. Stress ratio variations against the shear component of fabric tensor for unsteady simple shear. Symbols, dashed lines and solid lines in the figure denote the DEM results, model results using the linear and quadratic $\eta$ equations, respectively. The assembly is subjected to unsteady shear under constant volume condition with $\phi=0.60$ and $\mu=0.5$ and the inertia number $I \approx 0.0003$ at steady state.

Figure 7 suggested a model for $\eta$

$$
\eta=b_{1}+b_{2} \mathrm{~A}: \hat{\mathrm{S}}
$$

where the $b$ 's are are material constants. The stress ratios calculated from Eq. 4.5 are plotted in (red) dashed lines and compared with the DEM data in Fig. 7. This equation yields only linear approximation of the DEM results but captures most of the stress ratio variation except immediately after shear reversal. A quadratic relation,

$$
\eta=\hat{b}_{1}+\hat{b}_{2} \mathrm{~A}: \hat{\mathrm{S}}+\hat{b}_{3}(\mathrm{~A}: \hat{\mathrm{S}})^{2},
$$

shown in (black) solid lines in Fig. 7 better approximated the stress ratio variation. However, the strain extents where Eqs. 4.5 and 4.6 differed significantly were very small. We therefore employed Eq. 4.5 for the constitutive model in this paper as it has one less fitting parameter.

The value of the steady shear $\eta$ was found to increase with $\mu$ and reach a plateau value at large $\mu$ values as shown in Fig. 8 (a). The steady-shear stress ratio $\eta$ does not vanish for frictionless particles $(\mu=0)$, does have a non-zero macroscopic friction. This is consistent with the findings by Peyneau \& Roux (2008); the internal friction angle $\varphi=\arctan (\eta)$ found in our study is about $5.7^{\circ}$, which is close to the value $5.76^{\circ} \pm 0.02^{\circ}$ reported by them (Peyneau \& Roux 2008). For $\mu=0.5$ or higher, $\eta$ is about 0.4 (see Fig. 8 (a)), which is very close to the ratio of shear to normal stress for polystyrene beads in the shear cell experiment reported in (Savage \& Sayed 1984). The $\eta$ versus $\mu$ relation is consistent with data from other experimental or computational studies (Thornton 2000; Suiker \& Fleck 2004; Ketterhagen et al. 2009).

The correlations of the material parameters, $b_{1}$ and $b_{2}$, with the friction coefficient $\mu$ were established using the simulation data (see Fig. 8 (b)). A functional form $x_{1} e^{x_{2} \mu}+x_{3}$ was employed for these correlations, the numerical values for which are listed in Tab. 1. Coefficient $b_{1}$ was found to be essentially zero at $\mu=0$ within the numerical errors of the DEM simulations. The vanishing of $b_{1}$ can also be understood from the micromechanical stress Eq. 4.3: without particle friction, the tangential force contribution to stress (the second term on the right hand side of the equation) disappears and the deviatoric stress is then proportional to the fabric (in the first term on the right hand side of the equation), 


$\begin{array}{cccccccccc} & \alpha & a_{1} & a_{2} & a_{3} & a_{4} & b_{1} & b_{2} & Z_{c} & \phi_{c} \\ x_{1} & 2 & 0.0073 & -0.1 & 1.57 & -6 & -0.16 & 1.6 & 1.85 & 0.058 \\ x_{2} & & & & -4.5 & -2 & -6 & -6 & -5 & -5 \\ x_{3} & & & & -1.7 & 6 & 0.16 & -2.9 & 4.15 & 0.582\end{array}$

TABLE 1. Numerical values of $\alpha, a_{1}$ and $x_{1}-x_{3}$ in the fitting expressions, $x_{1} \mu$ and $x_{1} e^{x_{2} \mu}+x_{3}$, for the material parameters in the pressure and $\eta$ equations, and the $Z_{2}$ evolution equation.

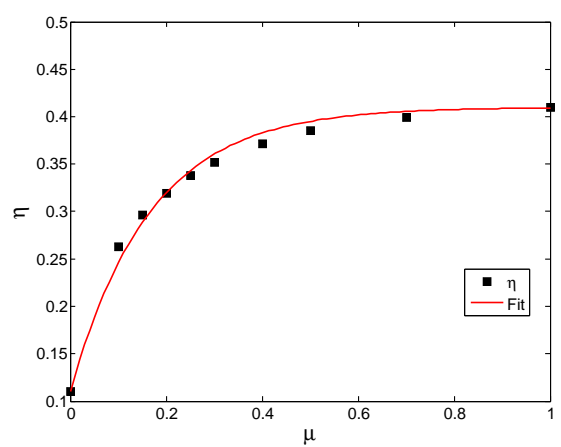

(a)

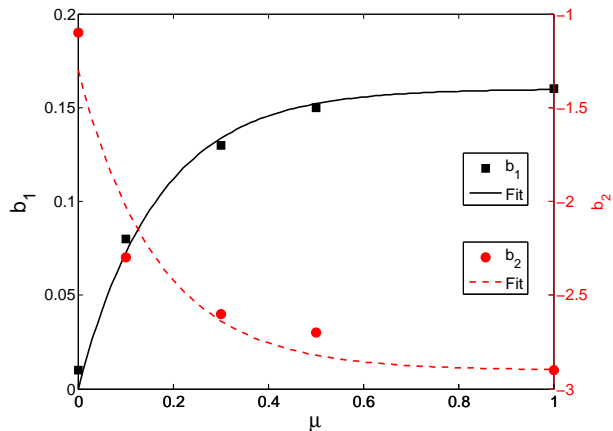

(b)

Figure 8. (a) Variation of $\eta$ versus $\mu$ during steady-state shear. (b) Correlations of $b_{1}$ and $b_{2}$ with $\mu$ are shown using the left and right $y$-axes, respectively. The equations used for the fitting curves in the (a) and (b) are $\eta=-0.3 e^{-6 \mu}+0.41, b_{1}=-0.16 e^{-6 \mu}+0.16$ and $b_{2}=1.6 e^{-6 \mu}-2.9$, respectively.

to satisfy which $b_{1}$ has to be zero in Eq. 4.5. Physically, this means that anisotropy is the sole contribution to macroscopic friction at the frictionless particle limit. In contrast, $b_{1}$ is non-zero for frictional particles (see Fig. 8 (b)), which indicates the macroscopic friction exists even when anisotropy is zero for frictional particle assemblies, where the tangential forces contribute to the deviatoric stress.

\subsection{Microstructure evolution equations}

To complete the constitutive model, appropriate evolution equations for the microstructural variables are required. Stress can then be evolved through microstructural evolution for unsteady flow. Based on the DEM results for microstructure evolution, we model the rate of change of both $\mathrm{A}$ and $Z_{2}$ as functions of $\mathrm{A}$ and $\mathrm{D}$. We postulate the following evolution equation for the fabric:

$$
\AA=c_{1} \mathrm{~S}+c_{2}|\mathrm{D}| \mathrm{A}+c_{3}(\mathrm{~A}: \mathrm{S}) \mathrm{A} .
$$

Here, $\stackrel{\AA}{\mathrm{A}}=\dot{\mathrm{A}}+\mathrm{A} \cdot \mathrm{W}-\mathrm{W} \cdot \mathrm{A}$, where $\mathrm{W}$ is the spin tensor, $\mathrm{W}=\frac{1}{2}\left(\nabla \mathbf{v}-(\nabla \mathbf{v})^{\mathrm{T}}\right)$, and $\dot{A}$ denotes its material time derivative. It can readily be ascertained that this evolution equation preserves rate independence and that the fabric evolves with strain extent.

An inspection of the simple shear flow results revealed a small (about 10\%), but systematic decrease in the modulus of fabric, $|A|=\sqrt{\frac{1}{2} A^{T}: A}$, with increasing volume fraction for each $\mu$ value studied. For the purpose of the present modeling effort, this variation was deemed unimportant and so we approximated the fabric as being essentially independent of volume fraction. The values of $|\mathrm{A}|$ obtained at various volume fractions were averaged to obtain a typical value for steady shear flow. We have also assumed that $|A|$ in the 
$\begin{array}{cccccccc}c_{1} & c_{2} & c_{3} & d_{1} & d_{2} & d_{3} & \beta_{1} & \beta_{2} \\ -0.52 & -2.8 & 100 & -45 & 5.6 & -40 & 7.5 & 0.5\end{array}$

TABLE 2. Numerical values of the material parameters in the microstructure evolution equations.

model is independent of $\mu$ as well, which was confirmed to hold for $\mu>0.1$. Thus $c_{1}-c_{3}$ in Eq. 4.7 are simply constants; their numerical values deduced from the shear reversal data are listed in Tab. 2. (This $\mu$-independent A was used while determining the parameters $a_{2}$ and $b_{2}$ described earlier for self-consistency.)

It should be noted that this evolution equation was not derived from first principles; instead, it was constructed to yield the desired response characteristics. The first term on the right hand side was introduced to recognize that shear drives the system to anisotropic state; the second term (with $c_{2}<0$ ) is stabilizing and allows a steady state to be established at large strain extents. The third term plays a mixed role; following shear reversal, it is destabilizing initially and plays a stabilizing role at later stages. This dual role permits a degree of control over the response characteristics. Throughout this study we tried to restrict the model to include only $A,|A|, \hat{D},|\hat{D}|$ and $A \hat{D}$; this was achieved for all the equations except the $A$ evolution equation where we added the third term on the right hand side to give an extra flexibility in capturing the transition. We solved Eq. 4.7 for the homogeneous simple shear and compared to the DEM results in Figs. 1(d) and 2(d). It can be seen that Eq. 4.7 is able to capture quite well the steady state and the evolution of $A_{x z}$. Such comparisons were ascertained at several different values of $\mu$ and $\phi$.

The need to evolve the coordination number has been recognized in the literature before. For example, Gera et al. (2004) proposed a heuristic evolution equation for $Z$ in the context of two-fluid modeling of fluidized beds, while Rothenburg \& Kruyt (2004) postulated an evolution equation for coordination number for planar granular materials under loading processes. We postulate the following rate-independent evolution equation for the coordination number $Z_{2}$

$$
\dot{Z}_{2}=d_{1}(\mathrm{~A}: \mathrm{S}-\chi|\mathrm{S}|)+d_{2}|\mathrm{D}|\left(f(\phi)-Z_{2}\right)+d_{3} \operatorname{tr}(\mathrm{D})
$$

where $\left.\chi=-\left(c_{2}+\sqrt{c_{2}^{2}-8 c_{1} c_{3}}\right) / 2 c_{3}\right)$ is a solution of $\mathrm{A}: \hat{\mathrm{S}}$ using Eq. 4.7 for steady simple shear flow and $d_{1}-d_{3}$ are material constants. (As discussed in appendix $\mathrm{A}$ this equation is limited to deformations with $|\operatorname{tr}(\hat{\mathrm{D}})| \ll 1$.) The first two terms and the fourth term vanish at steady simple shear and the relation between $\phi$ and $Z_{2}$ at steady-state shear in the third term drives coordination number to a steady value. The function $f(\phi)$ dictates how the coordination number varies with volume fraction during the steady shear. We found the scaling between $Z_{2}$ and $\phi$ for frictional spheres under simple shear to be essentially the same as the critical scaling near jamming transition for frictionless (O'Hern et al. 2003) and frictional (Zhang \& Makse 2005) particles, i.e., $Z_{2}-Z_{c} \sim\left(\phi-\phi_{c}\right)^{\frac{1}{2}}$. This is confirmed by the good fits to the DEM simple shear data using $Z_{2}-Z_{c}=\beta_{1}\left(\phi-\phi_{c}\right)^{\frac{1}{2}}$ for all the $\mu$ values studied as shown in Fig. 9(a), where symbols denote DEM data and solid lines are used for the fits. The inset of Fig. $9(\mathrm{a})$ shows that the fitting parameter $\beta_{1}$ fluctuates in a small range about its mean value of 7.5 . Plotting $Z_{2}-Z_{c}$ against $\left(\phi-\phi_{c}\right)^{\frac{1}{2}}$ all DEM data collapse onto a band centered around the line of $Z_{2}-Z_{c}=7.5\left(\phi-\phi_{c}\right)^{\frac{1}{2}}$ shown in Fig. 9(b), which supports the use of a constant $\beta_{1}$ to represent the variations 


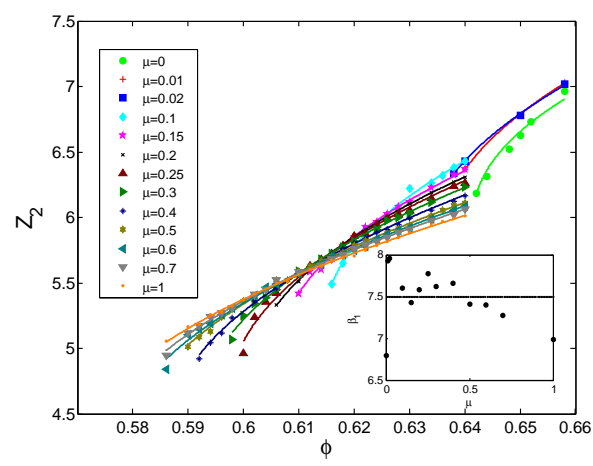

(a)

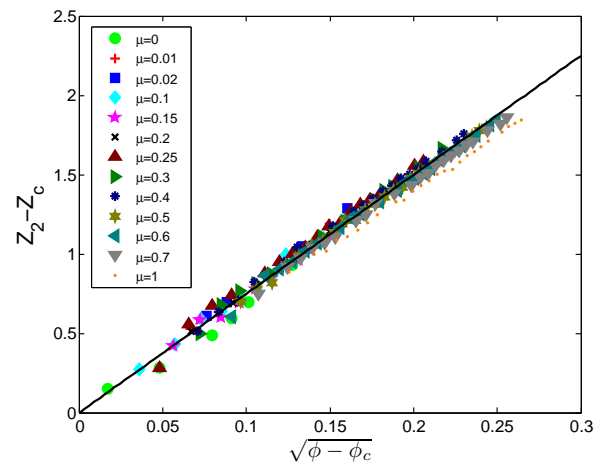

(b)

FiguRE 9. Coordination number variations are plotted as (a) $Z_{2}$ versus $\phi$ and (b) $Z_{2}-Z_{c}$ versus $\left(\phi-\phi_{c}\right)^{\frac{1}{2}}$ from steady simple shear DEM data (symbols) and fits (solid lines). The equations used for the fits are $Z_{2}-Z_{c}=\beta_{1}\left(\phi-\phi_{c}\right)^{\frac{1}{2}}$ and $Z_{2}-Z_{c}=7.5\left(\phi-\phi_{c}\right)^{\frac{1}{2}}$ in (a) and (b), respectively. The inset in (a) shows the variation of $\beta_{1}$ against $\mu$ with a line indicating its mean.

for all $\mu$ values. Therefore the equation for $f(\phi)$ in our model takes the following form

$$
f(\phi)=Z_{c}+\beta_{1}\left(\phi-\phi_{c}\right)^{\beta_{2}},
$$

where material constants $\beta_{1}=7.5$ and $\beta_{2}=0.5$. Fig. $5(\mathrm{c})$ displays $\phi_{c}$ determined through the curve-fit above, and its correlation is listed in Tab. 1. Constant numerical values were found to be adequate for $d_{1}-d_{3}$ (to capture the evolution of $Z_{2}$ through Eq. 4.8 reasonably well) and listed in Tab. 2. The $\operatorname{tr}(\mathrm{D})$ term is used to take account of the effects of compaction and dilation on increasing and decreasing the coordination number. Thus the Reynolds dilatancy constraint is implicitly incorporated in Eq. 4.8, which can be rewritten in an explicit form proposed by Goddard $(2010), \operatorname{tr}(\mathrm{D})=\alpha|\mathrm{S}|$, where the coefficient of dilatancy $\alpha=\frac{1}{d_{3}}\left[d_{1}(\mathrm{~A}: \hat{\mathrm{S}}+\chi)+d_{2}\left(f(\phi)-Z_{2}\right)-\frac{\dot{Z}_{2}}{|\mathrm{~S}|}\right]$, is dynamically changing according to the evolutions of $Z_{2}$ and $A$. The computational results from Eq. 4.8 are compared to the DEM data in Figs. 1(c) and 2(c). For both conditions, the $Z_{2}$ magnitudes and its evolution strain scales after shear reversal were correctly produced. The small quantitative discrepancies evident in these comparisons do not affect the pressure calculation significantly as will be shown next.

\section{Model verification}

Equations 4.1, 4.2, 4.5, 4.7 and 4.8 compose a constitutive model which neglects normal stress difference. Admittedly, the model involves a number of adjustable parameters; nevertheless, it is emphasized that the number of parameters is not that many, considering that the model tracks the evolution of pressure, shear stress, fabric, coordination number and volume fraction under both steady and dynamic conditions for a range of particle friction coefficients. The present study is aimed at demonstrating that the microscopic physics of jamming and anisotropy can indeed be incorporated into a continuum constitutive model, at least at a semi-empirical level. A desirable resulting attribute is that all the model parameters are linked to particle scale properties. 


\subsection{Model self-consistency}

The evolutions of pressure and stress ratio predicted by the model for shear flows under the CV condition are shown in Figs. 1(a) and (b) and compared with the DEM data. Although there are small quantitative differences between the model and the DEM results, the model reproduces all the key features correctly. To simulate simple shear under the $\mathrm{CP}$ condition, we allow $\operatorname{tr}(\mathrm{D})$ (in the $Z_{2}$ evolution equation) to vary while insisting that $p$ remained invariant. The modeled evolution of the volume fraction and stress ratio, as shown in Figs. 2(a) and (b), matched the DEM data well. These comparisons reveal that the constitutive model is self-consistent and is able to correctly describe the pressure and stress ratio evolutions during steady and unsteady flows of rate-independent granular materials. Similar model verification was done at several values (details not shown).

\subsection{Model predictions}

We now demonstrate the model's predictive capability by applying it to new unsteady shear flow situations without re-calibrating any material constants and comparing the predicted dynamic response with DEM data.

We subjected the assembly to a cyclic simple shear with small strain amplitude in the first test. The shear rate was a square-wave function with a magnitude of $\dot{\gamma}_{0}$ and the maximum strain in each flow direction followed $\gamma_{\mathrm{A}}$. Experimental studies on cyclic shear of dense granular materials (Youd 1971; Wood \& Budhu 1980; Toiya et al. 2004) have found that the assemblies compacted when $\gamma_{\mathrm{A}}$ was small and a large portion of the volume reduction occurred immediately after the shear reversal. The DEM simulation illustrated in Fig. 10 was performed with $\gamma_{\mathrm{A}}=0.5$ and started from a steadily sheared state where $\phi=0.6$. The volume fraction evolution is plotted using (blue) squares in Fig. 10(a) against the accumulated strain $\dot{\gamma}_{0} t$. The $\phi$ increased from 0.6 to about 0.61 after the first shear reversal at $\dot{\gamma}_{0} t=0.5$ and then slowly increased to about 0.62 at $\dot{\gamma}_{0} t=4$. Our model, without adjusting any of the model constants, predicts a similar compaction behavior as shown by the (red) solid line in Fig. 10(a), but the final volume fraction is slightly higher than that of the DEM data. Fig. 10(b) shows that the model can also predict the shear stress ratio well. The microstructural variables $Z_{2}$ and $A_{x z}$ are shown in Figs. 10(c) and (d), respectively. The predicted $Z_{2}$ values have slightly larger discrepancies than the $A_{x z}$ values do.

For the second test, we studied the Reynolds dilatancy, i.e., the tendency of a dense granular assembly to expand in volume upon initial shearing, which was first revealed by O. Reynolds (Reynolds 1885). The Reynolds dilatancy is an essential constraint for dense granular flow and has important influence on granular plasticity. As shown in Fig. 11, we shear an initially isotropic assembly at $\dot{\gamma}_{0}$ to a steady state under a constant pressure. The DEM data indicates the dilatancy, i.e., $\phi$ decreased from about 0.612 to 0.602 (see Fig. 11(a)). We note that there was an initial densification before the dilation, which was also observed in experiments with sand (Okada \& Nemat-Nasser 1994). The shear stress ratio quickly reached the steady state in Fig. 11(b), as did the $Z_{2}$ and $A_{x z}$ (see Fig. 11(c) and (d)). Our model results denoted by the (red) solid lines in Fig. 11 predicted the correct dilatancy behavior and also the correct dynamic trends for stress ratio and microstructural variables. The model also provides a microscopic explanation for the Reynolds dilatancy. Since the shear flow increases the anisotropy magnitude of the assembly, the coordination number has to decrease as dictated by the pressure equation (Eq. 4.2) to keep a constant pressure. The coordination number evolution equation in turn requires a dilation to achieve this decrease in $Z_{2}$. Therefore, shearing an initially isotropic assembly of frictional particles leads to the Reynolds dilatancy. In contrast, shearing an initially isotropic assembly of frictionless (spherical) particles does not lead 


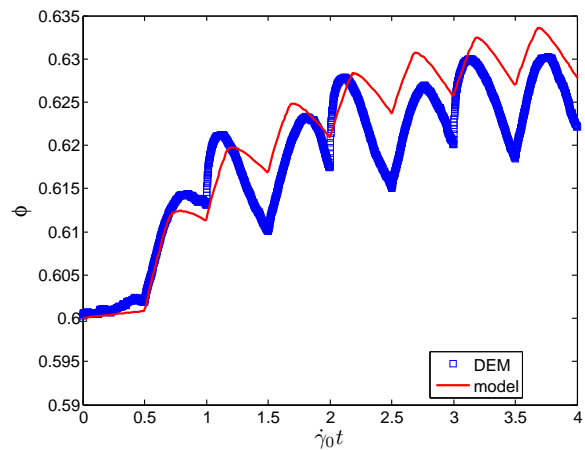

(a)

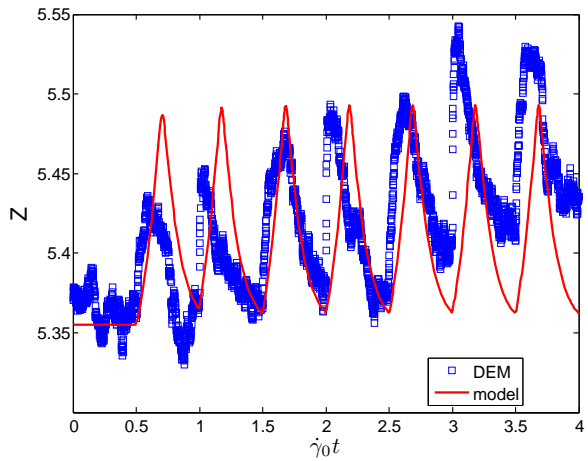

(c)

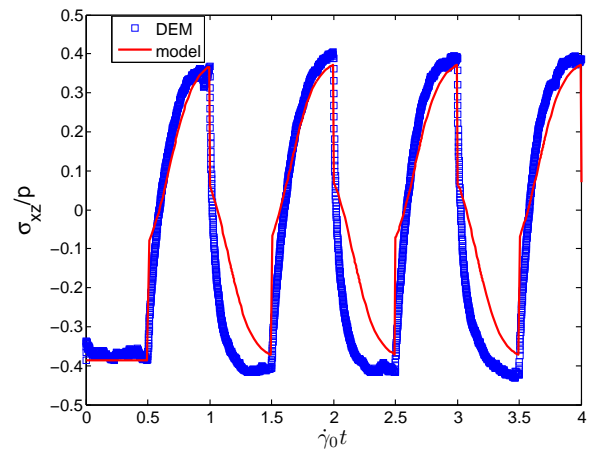

(b)

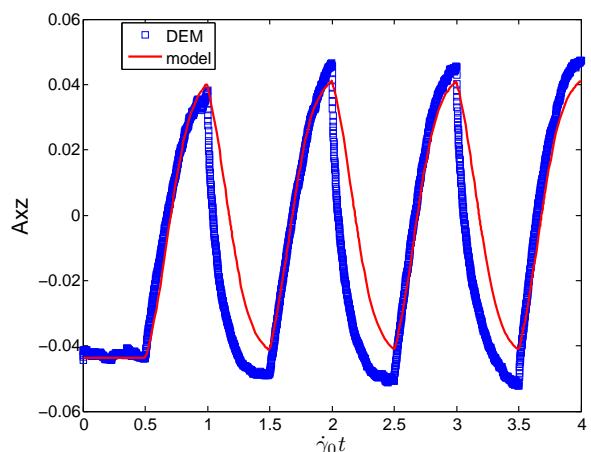

(d)

FIGURE 10. Evolution of (a) volume fraction, (b) stress ratio, (c) coordination number and (d) $A_{x z}$ for an assembly subjected to small-amplitude oscillatory shear under the constant pressure condition with $p d / k \approx 0.01$. Blue square symbols denote the data from DEM simulations and the red solid curves are the constitutive model results. The inter-particle friction coefficient $\mu$ is 0.5 and the inertia number $I \approx 0.0003$ at steady state.

to measurable dilatancy, as the anisotropy does not affect the pressure for frictionless particles (according to Eq. 4.2).

\section{Summary}

We have proposed a constitutive model for the rate-independent rheology of dense granular materials subjected to simple shear. It is suitable for flows where $|\operatorname{tr}(\hat{\mathrm{D}})| \ll 1$ (but need not to be zero), which includes a wide range of practical flow problems. The model for the stress (Eq. 4.1) consists of a pressure term and a deviatoric term with a macroscopic friction coefficient. The closures for the pressure and the friction coefficient (Eqs. 4.2 and 4.5) are linked to two microstructural variables, namely the fabric and the average coordination number (excluding floaters and rattlers), whose evolutions are modeled by Eqs. 4.7 and 4.8. For the Hookean model, the pressure is given by Eq. 4.2. The analogous form for a Hertzian model can be deduced from Eq. 4.4 as $\frac{p}{k_{\mathrm{Hz}}} \approx\left(a_{1}+a_{2}|\mathrm{~A}|\right)^{\frac{3}{2}}\left(Z_{2}-Z_{c}\right)^{3}$. The material constants in the model are functions of particle-level properties and were calibrated using shear flow DEM data. We have verified the model's self-consistency by reproducing the steady and unsteady DEM data used in 


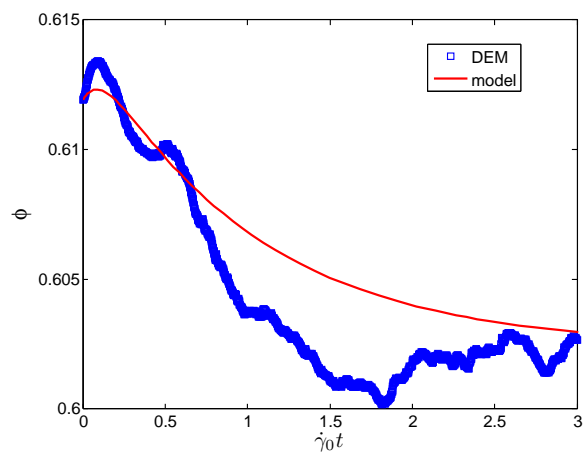

(a)

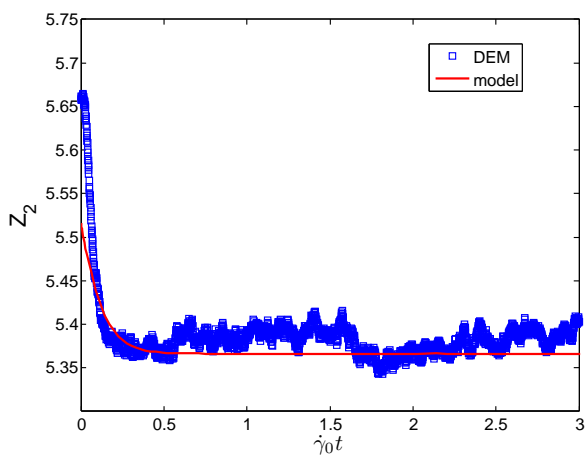

(c)

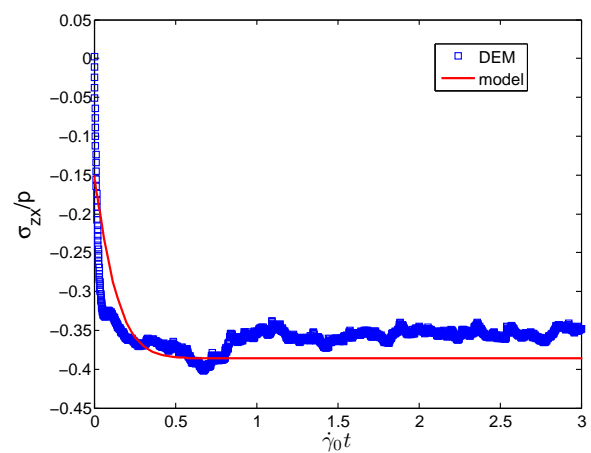

(b)

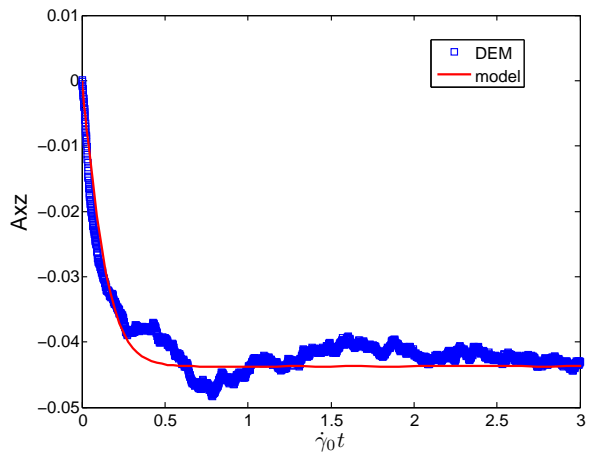

(d)

FiguRE 11. Evolution of (a) volume fraction, (b) stress ratio, (c) coordination number and (d) $A_{x z}$ for an assembly subjected to shear start from an isotropic condition under the constant pressure condition with $p d / k \approx 0.01$. Blue square symbols denote the data from DEM simulations and the red solid curves are the constitutive model results. The inter-particle friction coefficient $\mu$ is 0.5 and the inertia number $I \approx 0.0003$ at steady state.

the calibration. We have also demonstrated its predictive capability through cyclic shear and Reynolds dilatancy tests.

The authors are grateful to Sebastian Chialvo for generating some of the DEM data. This work is supported by a DOE-UCR grant DE-FG26-07NT43070.

\section{Appendix A. Isotropic compression pressure data and model}

We used the following protocol to prepare isotropic assemblies under different levels of confining pressure. Particles were first treated as elastic, frictionless spheres and thermalized and equilibrated over a broad range of volume fractions up to $\phi_{i}=0.63$ in a cubic periodic domain. The particles were then assigned the desired friction coefficient and inelasticity, and compressed isotropically at a very slow rate to a finite pressure level, at which mechanical equilibrium was maintained for a long period of time (usually $>10^{7}$ DEM time steps). At this stage, jamming transition, where static pressure is zero, was crossed. Thus the jamming transition can be approached by performing simulations for lower and lower final pressure. This protocol is analogous to the one used by Song et al. (2008), where the volume fraction after compression was controlled instead of pressure. 


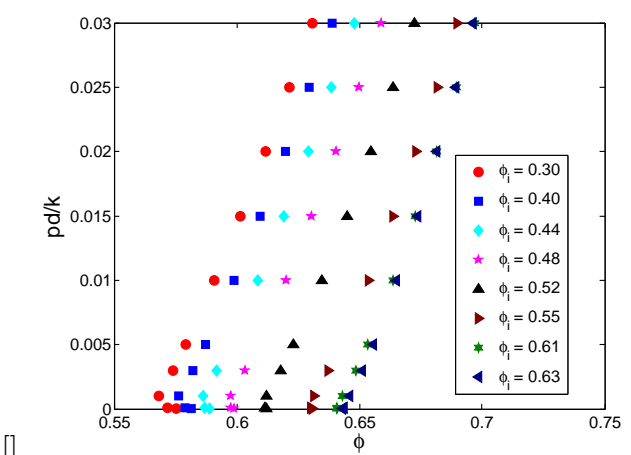

(a)

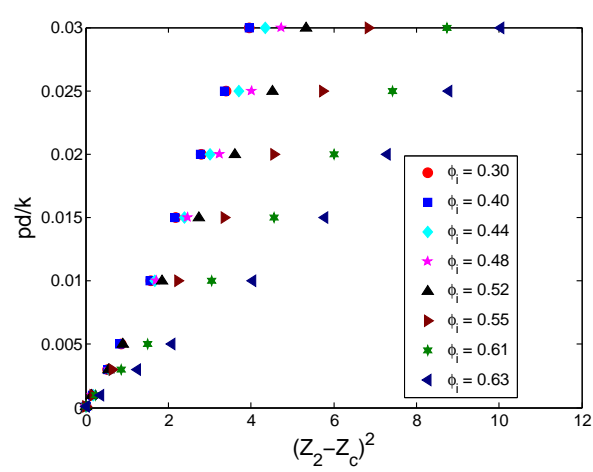

(c)

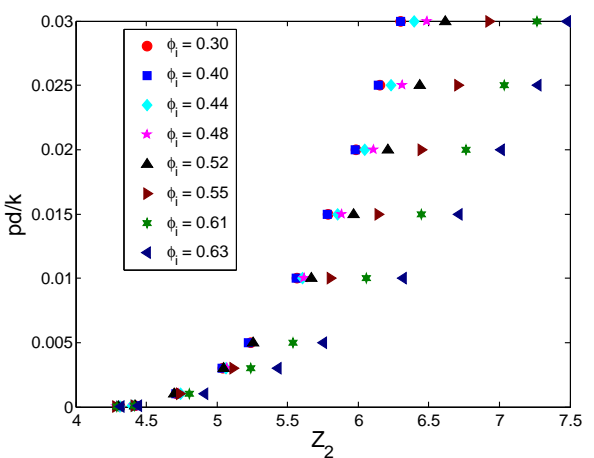

(b)

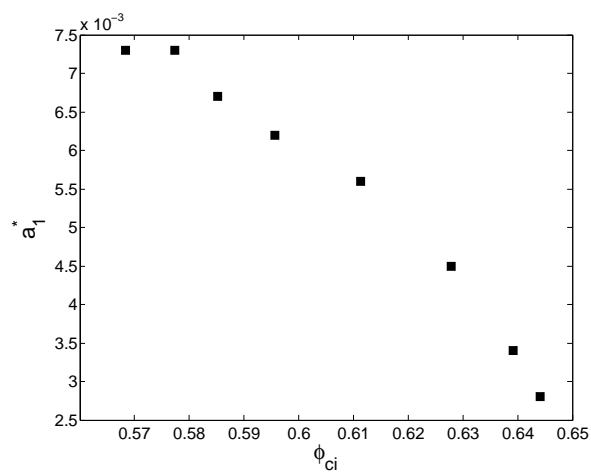

(d)

FiguRE 12. Scaled pressure varies with (a) volume fraction $\phi$ and (b) coordination number $Z_{2}$ during isotropic compression simulations starting from different initial volume fractions $\phi_{i}$. The same data in (b) are re-plotted against $\left(Z_{2}-Z_{c}\right)^{2}$ in (c). Variation of the pre-factor $a_{1}^{*}$ in the $(p d / k)-\left(Z_{2}-Z_{c}\right)^{2}$ relation is plotted against jamming volume fractions in (d). The error bars for the standard deviations of $\phi$ and $Z_{2}$ values resulted from the three realizations performed are comparable to the symbol size and thus not plotted in the figures. Particles in the assemblies have inter-particle friction coefficient $\mu=0.5$.

Many realizations with different disordered initial configurations were performed for each $\phi_{i}$ value and each pressure level to achieve good statistics.

Volume fractions and coordination numbers during the equilibrium states at pressure levels ranging from $p d / k=10^{-5}$ to $p d / k=3 \times 10^{-2}$ are reported in Figs. 12 (a) and (b), respectively for particle assembly with $\mu=0.5$. The jamming points, $\phi_{c i}$ and $Z_{c}$ can be determined by extrapolating the results to the zero pressure limit using data in these two figures. It can be seen that pressure has a nearly linear scaling with $\left(\phi-\phi_{c i}\right)$ and a quadratic scaling with $\left(Z_{2}-Z_{c}\right)$, which is consistent with previous findings (using the Hookean contact model) by O'Hern et al. (2003). The quadratic scaling is also shown by the linearity between $p d / k$ and $\left(Z_{2}-Z_{c}\right)^{2}$ in Fig. 12 (c). Comparison of the results of simulations starting from eight $\phi_{i}$ values $\left(\phi_{i}=0.3,0.4,0.44,0.48,0.52,0.55,0.61\right.$ and 0.63 ) reveals that the critical volume fraction for jamming, $\phi_{c i}$, is not unique; however the critical coordination number for jamming, $Z_{c}$, is, which has also been reported in other studies (Song et al. 2008; Chaudhuri et al. 2010). The uniqueness of $Z_{c}$ is one of the reasons for modeling the pressure as a function of $Z_{2}$ instead of $\phi$. Although the $p d / k$ versus $Z_{2}$ curves with different $\phi_{i}$ values appear to collapse onto a master curve at the 
vicinity of $Z_{c}$ as reported in (Chaudhuri et al. 2010), they diverge at higher pressure or larger $Z_{2}$ values. Simulations for different $\mu$ values were repeated with similar findings.

To take account of this history dependence on packing preparation, an additional variable, compactivity $X$, needs to be introduced into the model. It may be interpreted as a measure of the number of different ways of arranging the grains in the system into a volume with certain degree of disorder. The two limits, $X=0$ and $+\infty$, correspond to the most and least compact stable arrangements. Compactivity can be shown to be a function of $Z$ and $\phi$ and can be calculated using the statistical theory developed in (Song et al. 2008; Briscoe et al. 2009). Using the compactivity and the scaling observed in Fig. 12 (c), the isotropic compression pressure can be modeled as

$$
p d / k=\left(\left.a_{1}\right|_{\left(X_{c} \rightarrow+\infty\right)}+g\left(X_{c}\right) \operatorname{tr}(\hat{\mathrm{D}})\right) *\left(Z_{2}-Z_{c}\right)^{2}:=a_{1}^{*}\left(Z_{2}-Z_{c}\right)^{2},
$$

where $g\left(X_{c}\right)$ is a function of $X_{c}$ at jamming transition and $\operatorname{tr}(\hat{\mathrm{D}})=-\sqrt{6}$ for isotropic compression. The $\operatorname{tr}(\hat{\mathrm{D}})$ term is zero in simple shear under constant volume condition and its order of magnitude is much less than 1 for most dynamical systems involving significant deviatoric deformations. This term reflects the fact that the dependence on packing preparation vanishes after shearing for a substantial strain as shown in the main text. To illustrate the functional dependence of $a_{1}^{*}$ on $X_{c}$ without an elaborate effort of calculating $X_{c}$, we plot $a_{1}^{*}$ against $\phi_{c i}$ in Fig. 12 (d) as $X_{c}$ only depends on $\phi_{c i}$ at a fixed $Z_{c}$. It can be seen that there is a smooth functional dependence and $a_{1}^{*} \rightarrow a_{1}$ as $X_{c} \rightarrow+\infty$ at the lowest two $\phi_{c i}$ values (corresponding to $\phi_{i}=0.4$ and $\phi_{i}=0.3$ ).

Although the isotropic compression pressure can be represented formally using Eq. A 1, for the purpose of modeling of large-strain-scale behavior of dynamical flows, we simplify the equation by retaining only the $a_{1}$ term, which is the leading and asymptotic term. This simplification is applicable to assemblies constructed with $|\operatorname{tr}(\hat{\mathrm{D}})| \ll 1$ and is also consistent with the configurations of most granular assemblies in engineering practice that are usually constructed from low volume fractions. This simplified equation is used in the pressure Eq. 4.2 in the main text.

\section{Appendix B. Normal stress differences}

We first identify the basic characteristics of a stress tensor during steady-state simple shear by plotting the stress components scaled by the pressure against volume fraction in Fig. 13. It can be seen that scaled stress magnitudes are not very sensitive to the volume fraction variation. Normal stress components are different from each other with deviation of $5-10 \%$ from the mean pressure (see Fig. 13(a)). It is also seen that the magnitude of the first normal stress difference (Barnes et al. 1989), $N_{1}=\sigma_{x x}-\sigma_{z z}$, is about one third of that of the second normal stress difference, $N_{2}=\sigma_{z z}-\sigma_{y y}$. Only the $\sigma_{z x}\left(=\sigma_{x z}\right)$ shear stress component is non-trivial while others are very close to zero (see Fig. 13(b)). The parity plot of the shear stress components in the Fig. 13(b) inset shows that the stress tensor is approximately symmetric for these homogeneous shear flows, although it could be asymmetric near walls or in shear bands (Mohan et al. 2002).

According to Eq. 4.1 in the basic model the normal stress components are equal under simple shear, i.e., $\sigma_{x x}=\sigma_{y y}=\sigma_{z z}=p$. This is not consistent with the normal stress difference observed from our DEM simulation results (e.g., see Fig. 13) and experimental data (for example, reported by Savage (1979) for neutrally buoyant suspensions). We now extend the basic model in two stages to capture the normal stress differences: first, to capture the normal stress component with the largest deviation from the mean pressure, i.e., $\sigma_{y y}$ and represent $\sigma_{x x}$ and $\sigma_{z z}$ by their mean value, $\bar{\sigma}_{13}=\frac{1}{2}\left(\sigma_{x x}+\sigma_{z z}\right)$; second, 


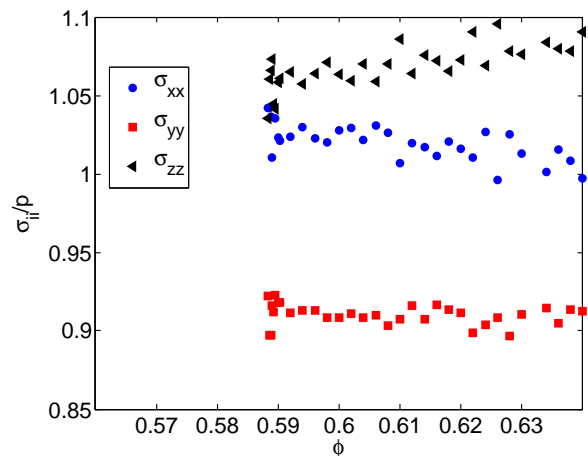

(a)

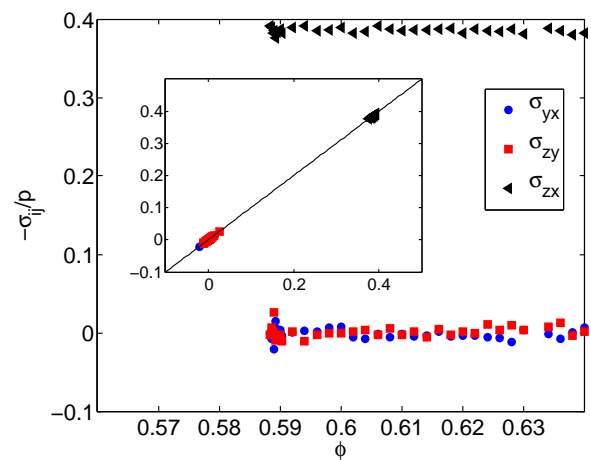

(b)

FiguRE 13. (a) Normal and (b) shear stress components scaled by the pressure are plotted against volume fraction $\phi$ during steady-state simple shear under both constant volume and constant pressure conditions. The inset figure in (b) is the parity plot of the symmetric parts of the shear stress components, such as $\sigma_{x z}$ versus $\sigma_{z x}$. The inter-particle friction coefficient $\mu$ is 0.5 and the inertia number $I<0.001$.

to further capture the difference between $\sigma_{x x}$ and $\sigma_{z z}$. In terms of the first and second normal stress differences, $N_{1}$ and $N_{2}$, we first to model $\frac{1}{2} N_{1}+N_{2}$ (since $\bar{\sigma}_{13}-\sigma_{y y}=$ $\frac{1}{2} N_{1}+N_{2}$ ) and then to model $N_{1}$.

We first model $\bar{\sigma}_{13}$ and $\sigma_{y y}$ components by augmenting the stress equation as

$$
\boldsymbol{\sigma}=p \mathbf{l}-p \eta \hat{\mathrm{S}}+a_{3} p\left(\mathrm{~A} \hat{\mathrm{S}}+\hat{\mathrm{S} A}-\frac{2}{3}(\mathrm{~A}: \hat{\mathrm{S}}) \mathrm{I}\right) .
$$

The augmented Eq. B 1 has the property that the pressure and the deviatoric stress components derived from it are not altered from those in the basic Eq. 4.1 as this extra term is traceless and the deviatoric parts in $A \hat{S}$ and $\hat{S} A$ cancel each other for simple shear flows. Therefore the material parameters determined in the basic model need not be re-calibrated for this augmented stress equation. For steady simple shear flows, the normal stress components take the following forms

$$
\begin{aligned}
\sigma_{x x}=\sigma_{z z}=\bar{\sigma}_{13} & =p\left(1+\frac{2}{3} a_{3} A_{x z} \operatorname{sgn}(\dot{\gamma})\right) \\
\sigma_{y y} & =p\left(1-\frac{4}{3} a_{3} A_{x z} \operatorname{sgn}(\dot{\gamma})\right),
\end{aligned}
$$

where $\operatorname{sgn}(\dot{\gamma})$ is the sign of shear rate. Therefore,

$$
\frac{1}{2} N_{1}+N_{2}=2 a_{3} A_{x z} \operatorname{sgn}(\dot{\gamma})
$$

According to Eq. B 1 the difference between $\sigma_{y y}$ and the mean pressure $p$ is exactly twice of the difference between $\bar{\sigma}_{13}$ and $p$, which will be shown to be supported by our DEM data.

To calibrate the parameter $a_{3}$, we used the steady shear DEM data. Fig. 14 (a) compares the predictions of the augmented model of $\bar{\sigma}_{13}$ and $\sigma_{y y}$ scaled by pressure with the steady shear DEM data for assemblies of particles with $\mu=0.5$ and $\mu=0.1$. When applied to unsteady shear, the augmented model agrees with most of the dynamical data although the instantaneous values at shear reversal are over-predicted as shown in Fig. 14 (b) for a shear reversal flow under constant volume condition. The over-prediction is caused by the constant $a_{3}$ used in the model. The dynamical behavior of the model 


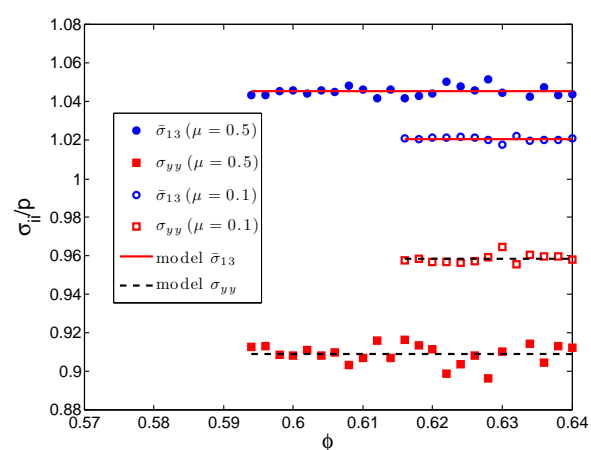

(a)

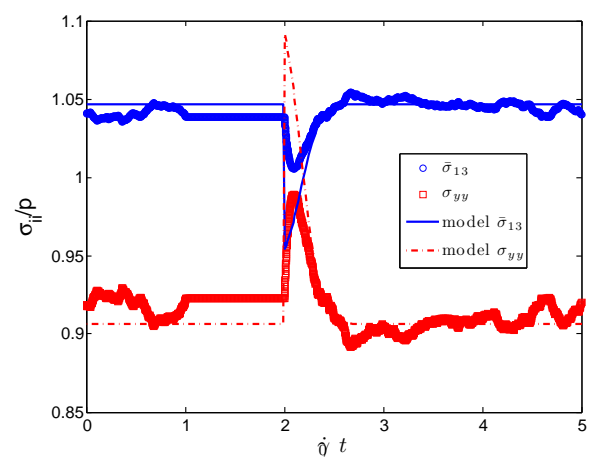

(b)

FiguRE 14. Normal stress components $\bar{\sigma}_{13}$ and $\sigma_{y y}$ scaled by pressure are plotted against volume fraction and shear strain for an assembly in (a) steady-state simple shear and (b) unsteady shear under the constant volume condition, respectively. Symbols denote data from DEM simulations and curves are the constitutive model results. In (a) solid symbols are for particles with $\mu=0.5$ and open symbols for $\mu=0.1$. In (b) particle friction coefficient is 0.5 and volume fraction is 0.6 .

can be refined by making $a_{3}$ vary with microstructure evolution, which was not pursued in this paper.

The second stage of the extensionaimed at modeling the first normal stress difference $N_{1}$ leads to

$$
\boldsymbol{\sigma}=p \mathbf{I}-p \eta \hat{\mathrm{S}}+a_{3} p\left(\mathrm{~A} \hat{\mathrm{S}}+\hat{\mathrm{S}} \mathrm{A}-\frac{2}{3}(\mathrm{~A}: \hat{\mathrm{S}}) \mathrm{I}\right)+a_{4} p\left(\mathrm{~A}-\frac{1}{2}(\mathrm{~A}: \hat{\mathrm{S}}) \hat{\mathrm{S}}\right) .
$$

The normal stress components take the following forms for simple shear flows

$$
\begin{aligned}
\sigma_{x x} & =p\left(1+\frac{2}{3} a_{3} A_{x z} \operatorname{sgn}(\dot{\gamma})+a_{4} A_{x x}\right) \\
\sigma_{z z} & =p\left(1+\frac{2}{3} a_{3} A_{x z} \operatorname{sgn}(\dot{\gamma})+a_{4} A_{z z}\right) \\
\sigma_{y y} & =p\left(1-\frac{4}{3} a_{3} A_{x z} \operatorname{sgn}(\dot{\gamma})\right),
\end{aligned}
$$

where $A_{x x}=-A_{z z}$ and $A_{y y}=0$ as prescribed in the fabric evolution Eq. 4.7 and supported by our DEM data (Aarons et al. 2009). The first and second normal stress differences are thus fully resolved as

$$
\begin{aligned}
& N_{1}=\sigma_{x x}-\sigma_{z z}=a_{4} p\left(A_{x x}-A_{z z}\right) \\
& N_{2}=\sigma_{z z}-\sigma_{y y}=a_{3} p A_{x z} \operatorname{sgn}(\dot{\gamma})+a_{4} p A_{z z},
\end{aligned}
$$

which implies that the first normal stress difference is caused by the corresponding fabric normal component difference. As both $A_{x x}$ and $A_{z z}$ are small in magnitude, the magnitude of $N_{1}$ is also small, which is supported by our DEM data shown below.

We used the DEM normal stress and fabric data averaged over a range of volume fractions during steady-state simple shear to calibrate $a_{4}$. We then compared the predictions of the normal stress components scaled by pressure to the corresponding DEM data for assemblies of particles with friction coefficients $\mu=0.5$ and $\mu=0.1$ in Fig. 15 (a) and (b), respectively. It can be seen that both the first and second normal stress differences are captured and agree well with the DEM data for both different $\mu$ values. When applied to a shear reversal flow under constant volume condition, the further augmented 


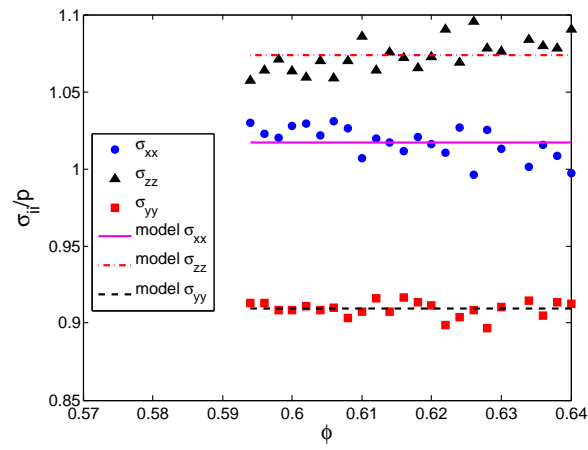

(a)

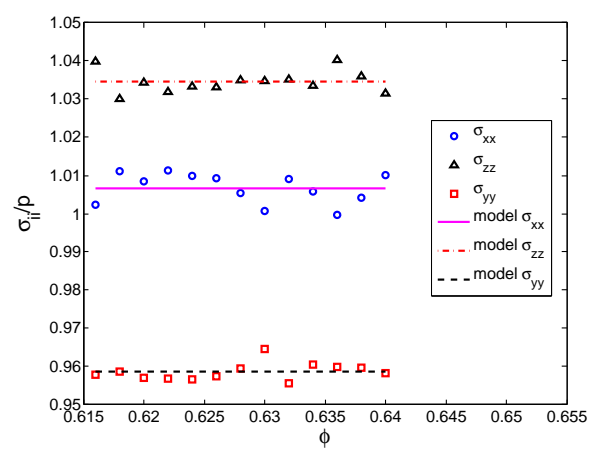

(b)

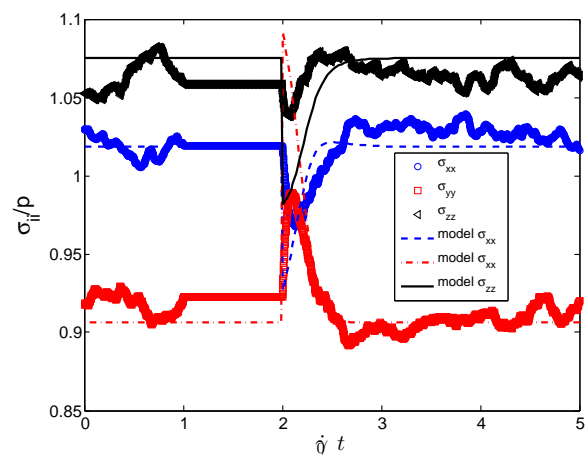

(c)

FIGURE 15. Normal stress components $\sigma_{x x}, \sigma_{y y}$ and $\sigma_{z z}$ scaled by pressure are plotted against volume fraction and shear strain for an assembly in (a) steady-state simple shear with particle friction coefficient $\mu=0.5$, (b) steady-state simple shear with particle friction coefficient $\mu=0.1$ and (c) unsteady shear under the constant volume condition, respectively. Symbols denote data from DEM simulations and curves are the constitutive model results. In (c) particle friction coefficient is 0.5 and volume fraction is 0.6 .

model captured most of the trends, but some quantitative differences remain as shown in Fig. 15 (c). The main difference is again the over-prediction at shear reversal caused by the constant $a_{4}$ used in the model.

The normal stress difference increases monotonically with increasing particle friction coefficient $\mu$. This trend is reflected by parameter $a_{3}$ and $a_{4}$ increasing in magnitude as reported in Fig. 16 (a) and (b), which can be fitted using the equations $a_{3}=1.57 e^{-4.5 \mu}$ 1.7 and $a_{4}=-6.5 e^{-2 \mu}+6.5$, respectively.

This dynamic model may not be necessary for some applications. In fact, the full model can be greatly simplified for steady-state simple shear, which is given in appendix C.

\section{Appendix C. Simplified model for steady simple shear}

The full model, consisting of Eq. B 4 for stress, Eq. 4.2 for pressure, Eq. 4.5 for macroscopic friction coefficient, Eq. 4.7 for fabric evolution and Eq. 4.8 for coordination number evolution, can be simplified for steady simple shear flows as follows. For these flows, the strain rate tensor $\hat{\mathrm{D}}$ takes the form $\operatorname{sgn}(\dot{\gamma})\left(\mathbf{e}_{(x)} \mathbf{e}_{(z)}+\mathbf{e}_{(z)} \mathbf{e}_{(x)}\right)$ and $\hat{\mathrm{S}}=\hat{\mathrm{D}}$. The expressions for steady-state $A: \hat{S},|A|$ and $A \cdot \hat{S}$, deduced by taking the corresponding scalar products 


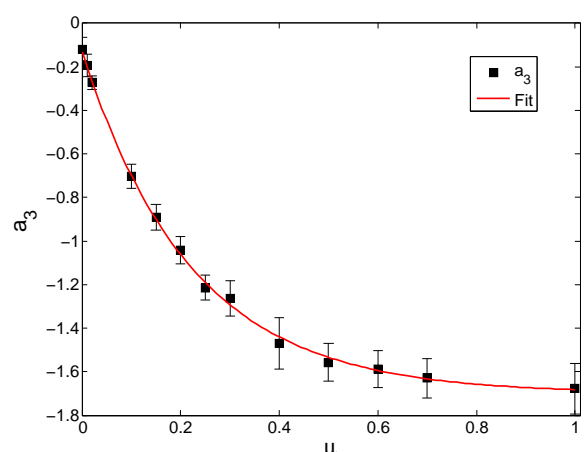

(a)

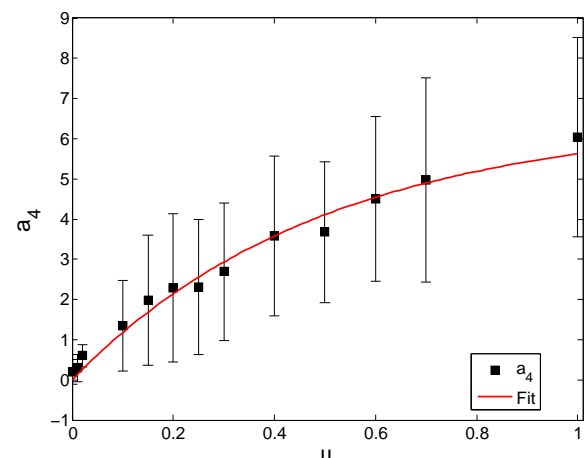

(b)

FiguRE 16. Variation of parameters (a) $a_{3}$ and (b) $a_{4}$ versus friction coefficient $\mu$. The equations used for the fitting curve in the (a) and (b) are $a_{3}=1.57 e^{-4.5 \mu}-1.7$ and $a_{4}=-3.7 e^{-1.2 \mu}+4$, respectively.

and dot product of the steady solution of the fabric evolution Eq. 4.7, are

$$
\begin{gathered}
\mathrm{A}: \hat{\mathrm{S}}=-\frac{c_{2}+\sqrt{c_{2}^{2}-8 c_{1} c_{3}}}{2 c_{3}}:=\chi, \\
|\mathrm{A}|=\sqrt{\frac{-c_{1} \chi}{2\left(c_{2}+c_{3} \chi\right)}}:=\kappa,
\end{gathered}
$$

and

$$
\mathrm{A} \cdot \hat{\mathrm{S}}=-\frac{c_{1}}{c_{2}+c_{3} \chi} \hat{\mathrm{S}} \cdot \hat{\mathrm{S}}:=\lambda \hat{\mathrm{S}} \cdot \hat{\mathrm{S}}
$$

respectively. No simple analytical solution could be found for $A$. If the rotational contribution in the Jaumann derivative, i.e., A.W - W $A$, was neglected, an analytical solution could be obtained; however, this approximate solution would predict zero first normal stress difference. For applications where the first normal stress difference is not of serious concern, one can employ the following simplified version of the model. Substituting Eq. C 3 to the stress Eq. B 4, Eqs. 4.9 and C 2 to the pressure Eq. 4.2 and Eq. C 1 to the macroscopic friction coefficient Eq. 4.5, we obtain the simplified stress, pressure and friction coefficient and equations,

$$
\begin{gathered}
\boldsymbol{\sigma}=p \mathbf{I}-p \eta \hat{\mathbf{S}}+2 a_{3} p\left(\lambda \hat{\mathrm{S}} \cdot \hat{\mathrm{S}}-\frac{1}{3} \chi \mathbf{l}\right), \\
p d / k=\left(a_{1}+a_{2} \kappa\right) \beta_{1}^{2}\left(\phi-\phi_{c}\right),
\end{gathered}
$$

and

$$
\eta=b_{1}+b_{2} \chi
$$

respectively. The microstructural variables do not appear directly in this model as there is no need to evolve them. One can further truncate this model, if adequate for specific applications, by only retaining the first two terms on the right hand side of Eq. C 4. This highly simplified model has a very familiar form (Savage 1983; Jackson 1986), but all the parameters are now linked to particle scale properties.

\section{REFERENCES}


Aarons, Lee R., Sun, Jin \& Sundaresan, Sankaran 2009 Unsteady shear of dense assemblies of cohesive granular materials under constant volume conditions. Industrial \& Engineering Chemistry Research 49 (11), 5153-5165.

AnAND, L. \& Gu, C. 2000 Granular materials: constitutive equations and strain localization. Journal of the Mechanics and Physics of Solids 48 (8), 1701-1733.

AzÈma, E., Radjai, F. \& Saussine, G. 2009 Quasistatic rheology, force transmission and fabric properties of a packing of irregular polyhedral particles. Mechanics of Materials 41 (6), 729-741.

Barnes, H. A., Hutton, J. F. \& Walters, K. 1989 An Introduction to Rheology. Elsevier Ltd.

Bathurst, Richard J. \& Rothenburg, Leo 1990 Observations on stress-force-fabric relationships in idealized granular materials. Mechanics of Materials 9 (1), 65-80.

Bocquet, L., Losert, W., Schalk, D., Lubensky, T. C. \& Gollub, J. P. 2001 Granular shear flow dynamics and forces: Experiment and continuum theory. Phys. Rev. E 65 (1), 011307.

Briscoe, Christopher, Song, Chaoming, Wang, Ping \& Makse, Hernán A. 2009 Jamming III: characterizing randomness via the entropy of jammed matter. arXiv:0909.4324v1

Campbell, Charles S. 2002 Granular shear flows at the elastic limit. Journal of Fluid Mechanics 465, 261-291.

Chaudhuri, Pinaki, Berthier, Ludovic \& Sastry, Srikanth 2010 Jamming transitions in amorphous packings of frictionless spheres occur over a continuous range of volume fractions. Physical Review Letters 104 (16).

da Cruz, Frederic, Emam, Sacha, Prochnow, Michael, Roux, Jean-Noel \& Chevoir, FrANCOIS 2005 Rheophysics of dense granular materials: Discrete simulation of plane shear flows. Physical Review E (Statistical, Nonlinear, and Soft Matter Physics) 72 (2), 02130917

Cundall, P. A. \& Strack, D. L. 1979 A discrete numerical model for granular assemblies. Géotechnique 29, 47-65.

Evans, Denis J. \& Morriss, Gray P. 1990 Statistical Mechanics of Nonequilibrium Liquids. Theoretical chemistry 1. San Diego: Academic Press.

Gadala-Maria, F. \& Acrivos, Andreas 1980 Shear-induced structure in a concentrated suspension of solid spheres. Journal of Rheology 24 (6), 799-814.

Gera, Dinesh, Syamlal, Madhava \& O'Brien, Thomas J. 2004 Transportn equation for modeling particle contacts. In Fluidization XI, 11th International Conference on Fluidization (ed. U. Arena, R. Chirone, M. Miccio \& P. Salatino). Engineering Conferences International, Naples, Italy.

GodDARD, J. 1986 Dissipative materials as constitutive models for granular media. Acta Mechanica 63 (1), 3-13.

GODDARD, J. 2010 Parametric hypoplasticity as continuum model for granular media: from stokesium to mohr-coulombium and beyond. Granular Matter 12 (2), 145-150.

Goddard, J. D. 1984 Dissipative materials as models of thixotropy and plasticity. Journal of Non-Newtonian Fluid Mechanics 14, 141-160.

GoddARD, J. D. 1998 Continuum modeling of granular assemblies. In Physics of dry granular media (ed. H. J. Herrmann), chap. 1, pp. 1-24. Kluwer.

GodDARD, J. D. 2006 A dissipative anisotropic fluid model for non-colloidal particle dispersions. Journal of Fluid Mechanics 568 (-1), 1-17.

HARRIS, DAVID 2006 Some properties of a new model for slow flow of granular materials. Meccanica 41 (3), 351-362.

Hébraud, P. \& Lequeux, F. 1998 Mode-coupling theory for the pasty rheology of soft glassy materials. Physical Review Letters 81 (14).

Hill, R. 1950 The Mathematical Theory of plasticity. Oxford: Oxford University Press.

JACKSON, RoY 1986 Some features of the flow of granular materials and aerated granular materials. Journal of Rheology 30 (5), 907-930.

Ketterhagen, William R., Curtis, Jennifer S., Wassgren, Carl R. \& Hancock, BRuno C. 2009 Predicting the flow mode from hoppers using the discrete element method. Powder Technology 195 (1), 1-10. 
Keys, Aaron S., Abate, Adam R., Glotzer, Sharon C. \& Durian, Douglas J. 2007 Measurement of growing dynamical length scales and prediction of the jamming transition in a granular material. Nat Phys 3 (4), 260-264.

Kheiripour Langroudi, M., Sun, J., Sundaresan, S. \& Tardos, G. I. 2010 Transmission of stresses in static and sheared granular beds: The influence of particle size, shearing rate, layer thickness and sensor size. Powder Technology 203 (1), 23-32.

LAde, Poul V. 1977 Elasto-plastic stress-strain theory for cohesionless soil with curved yield surfaces. International Journal of Solids and Structures 13 (11), 1019-1035.

LeEs, A. W. \& Edwards, S. F. 1972 The computer study of transport processes under extreme conditions. Journal of Physics C: Solid State Physics 5 (15), 1921-1928.

Lu, Kevin, Brodsky, E. E. \& Kavehpour, H. P. 2007 Shear-weakening of the transitional regime for granular flow. Journal of Fluid Mechanics 587 (-1), 347-372.

Luding, Stefan 2004 Micro-macro transition for anisotropic, frictional granular packings. International Journal of Solids and Structures 41 (21), 5821 - 5836, fabric tensor;Stiffness tensor;Discrete element model;Isotropic contact;.

Luding, Stefan 2005 Anisotropy in cohesive, frictional granular media. Journal of Physics Condensed Matter 17 (24), 2623-2640 -, friction coefficients;Particle molecular dynamics;Granular media;Flow behavior;

Majmudar, T. S. \& Behringer, R. P. 2005 Contact force measurements and stress-induced anisotropy in granular materials. Nature 435 (7045), 1079-1082.

Majmudar, T. S., Sperl, M., Luding, S. \& Behringer, R. P. 2007 Jamming transition in granular systems. Physical Review Letters 98 (5), 058001-4.

Makse, Hernán A., Johnson, David L. \& Schwartz, Lawrence M. 2000 Packing of compressible granular materials. Phys. Rev. Lett. 84 (18), 4160-4163.

Menrabadi, M. M. \& Cowin, S. C. 1978 Initial planar deformation of dilatant granular materials. Journal of the Mechanics and Physics of Solids 26 (4), 269-284.

MiDi, G. D. R. 2004 On dense granular flows. European Physical Journal E - Soft Matter 14 (4), 341-305.

Mohan, L. Srinvasa, Rao, K. Kesava \& Nott, Prabhu R. 2002 A frictional cosserat model for the slow shearing of granular materials. Journal of Fluid Mechanics 457 (-1), 377-409.

Mueth, Daniel M., Debregeas, Georges F., Karczmar, Greg S., Eng, Peter J., Nagel, Sidney R. \& JAEger, HeinRICh M. 2000 Signatures of granular microstructure in dense shear flows. Nature 406 (6794), 385-389.

Nedderman, R. M. 1992 Statics and Kinematics of Granular Materials. Cambridge University Press.

Nemat-Nasser, Sia 2000 A micromechanically-based constitutive model for frictional deformation of granular materials. Journal of the Mechanics and Physics of Solids 48 (6-7), $1541-1563$

Oda, M., Konishi, J. \& Nemat-Nasser, S. 1980 Some experimentally based fundamental results on the mechanical behaviour of granular materials. Géotechnique.

O’Hern, Corey S., Silbert, Leonardo E., Liu, Andrea J. \& Nagel, Sidney R. 2003 Jamming at zero temperature and zero applied stress: The epitome of disorder. Physical Review E 68 (1), 011306-1-19.

OkAdA, N. \& Nemat-NAsser, S. 1994 Energy dissipation in inelastic flow of saturated cohesionless granular media. Géotechnique 44, 1-19.

Peyneau, Pierre-Emmanuel \& Roux, Jean-Noel 2008 Frictionless bead packs have macroscopic friction, but no dilatancy. Physical Review E (Statistical, Nonlinear, and Soft Matter Physics) $\mathbf{7 8}$ (1), 011307-17.

Plimpton, Steve 1995 Fast parallel algorithms for short-range molecular dynamics. Journal of Computational Physics 117 (1), 1-19.

Radjai, Farhang, Wolf, Dietrich E., Jean, Michel \& Moreau, Jean-Jacques 1998 Bimodal character of stress transmission in granular packings. Physical Review Letters 80 (1), 61-64.

REYNOLDS, OsBoRne 1885 On the dilatancy of media composed of rigid particles. with experimental illustrations. Philosophical Magazine 20 (127), 469-481.

Rothenburg, L. \& Kruyt, N. P. 2004 Critical state and evolution of coordination number 
in simulated granular materials. International Journal of Solids and Structures 41 (21), $5763-5774$.

Rycroft, Chris H., Kamrin, Ken \& Bazant, Martin Z. 2009 Assessing continuum postulates in simulations of granular flow. Journal of the Mechanics and Physics of Solids $\mathbf{5 7}$ (5), 828-839.

SAVAGe, StuART B. 1979 Gravity flow of cohesionless granular materials in chutes and channels. Journal Fluid Mechanics 92, 53-96.

SAVAGE, S. B. 1983 Granular flows down rough inclines - review and extension. In Mechanics of granular materials: new models and constitutive relations (ed. J. T. Jenkins \& M. Satake), pp. 261-282. Elsevier Ltd.

Savage, S. B. \& SAYed, M. 1984 Stresses developed by dry cohesionless granular materials sheared in an annular shear cell. Journal of Fluid Mechanics 142 (-1), 391-430.

SCHAEFFER, D. G. 1987 Instability in the evolution equations describing incompressible granular flow. Journal of Differential Equations 66, 19-50.

Schofield, Andrew \& Wroth, Peter 1968 Critical State Soil Mechanics. McGraw-Hill Inc.

ShäFER, J., Dippel, S. \& Wolf, D. E. 1996 Force schemes in simulations of granular materials. Journal de Physique I 6 (1), 5-20.

Shundyak, Kostya, van Hecke, Martin \& van SAarloos, Wim 2007 Force mobilization and generalized isostaticity in jammed packings of frictional grains. Physical Review E $\mathbf{7 5}$ (1).

Silbert, Leonardo E., Ertas, Deniz, Grest, Gary S., Halsey, Thomas C., Levine, Dov \& Plimpton, Steven J. 2001 Granular flow down an inclined plane: Bagnold scaling and rheology. Physical Review E 64 (5), 051302.

Silbert, Leonardo E., Grest, Gary S., Brewster, Robert \& Levine, Alex J. 2007 Rheology and contact lifetimes in dense granular flows. Physical Review Letters 99 (6), $068002-4$.

Somfai, Ellak, van Hecke, Martin, Ellenbroek, Wouter G., Shundyak, Kostya \& VAN SAarloos, Wim 2007 Critical and noncritical jamming of frictional grains. Physical Review E (Statistical, Nonlinear, and Soft Matter Physics) 75 (2), 020301-4.

Song, Chaoming, Wang, Ping \& Makse, Hernan A. 2008 A phase diagram for jammed matter. Nature 453 (7195), 629-632.

Spencer, A. J. M. 1964 A theory of the kinematics of ideal soils under plane strain conditions. Journal of the Mechanics and Physics of Solids 12 (5), 337-351.

Subhash, G., Nemat-Nasser, S., Mehrabadi, M. M. \& Shodj, H. M. 1991 Experimental investigation of fabric-stress relations in granular materials. Mechanics of Materials 11 (2), 87-106.

Suiker, Akke S. J. \& Fleck, Norman A. 2004 Frictional collapse of granular assemblies. Journal of Applied Mechanics 71 (3), 350-358.

Tardos, Gabriel I., McNamara, Sean \& Talu, Ilkay 2003 Slow and intermediate flow of a frictional bulk powder in the couette geometry. Powder Technology 131 (1), 23-39.

Thornton, C. 2000 Numerical simulations of deviatoric shear deformation of granular media. Géotechnique $\mathbf{5 0}$ (1), 43-53.

Toiya, Masahiro, Stambaugh, Justin \& Losert, Wolfgang 2004 Transient and oscillatory granular shear flow. Physical Review Letters 93 (8), 088001-4.

Utter, B. \& Behringer, R. P. 2004 Transients in sheared granular matter. The European Physical Journal E - Soft Matter 14 (4), 373-380.

Wood, D.M. \& Budhu, M. 1980 The behaviour of leighton buzzard sand in cyclic simple shear tests. In Soilds under Cyclic and Transient Loading (ed. G. N. Pande \& O.C. Zienkiewicz), p. 9. Rotterdam, The Netherlands: Balkema.

Youd, T. L. 1971 Maximum density of sand by repeated straining in simple shear. Highway Research Record 374, 1-6.

Zhang, H. P. \& MAKse, H. A. 2005 Jamming transition in emulsions and granular materials. Physical Review E (Statistical, Nonlinear, and Soft Matter Physics) 72 (1), 011301-12.

Zhu, Huaning, Mehrabadi, Morteza M. \& Massoudi, Mehrdad 2006 Incorporating the effects of fabric in the dilatant double shearing model for planar deformation of granular materials. International Journal of Plasticity 22 (4), 628-653. 


\section{Appendix D}

\section{A plasticity model with microstructure evolution for quasi-static granular flows}




\title{
A plasticity model with microstructure evolution for quasi-static granular flows
}

\author{
Jin Sun and Sankaran Sundaresan \\ Department of Chemical Engineering, Princeton University, Princeton, New Jersey 08544 USA
}

\begin{abstract}
We develop a plasticity model to predict complex rheological behaviors of quasi-static granular flows. The stress is decomposed to a pressure term and a deviatoric term with a macroscopic friction coefficient. The closures to the pressure and the friction coefficient are linked to the microstructure evolution, which is modeled by the coordination number and fabric evolution equations. The material constants in the model are functions of particle-level properties and are calibrated using the data from simulations of steady and unsteady simple shear using the discrete element method (DEM).
\end{abstract}

Keywords: Dense granular flows, constitutive modeling, discrete element method

PACS: $83.10 . \mathrm{Gr}, 83.10 . \mathrm{Rs}, 83.50 . \mathrm{Ax}, 45.70-\mathrm{n}, 46.35+\mathrm{z}, 62.20 . \mathrm{fq}$

\section{INTRODUCTION}

Continuum models play an important role in scientific understanding and design of processes handling dense granular materials, e.g., hopper discharge or dense-phase pneumatic conveying [1]. However, developing an accurate constitutive model for dense flows remains an open problem. Dense flow characteristics change as the deformation rate varies. We focus our attention to the quasi-static regime, where granular materials flow so slowly that their stresses approach constant values and thus exhibit the characteristic of rate-independence.

In the quasi-static regime, particles make enduring contacts with each other and often deform irreversibly under external loads. Due to these physical features, rate-independent plasticity models for metals have been adapted for granular materials. In plasticity theory, concepts of a yield function, a plastic potential and a flow rule are used to construct constitutive models [2,3]. For example, a Mohr-Coulomb yield criterion has been used to derive a rigid plasticity model for flow down a rough inclined plane [4]. A von Mises-type yield function was used in derivation of another rigid-perfectly plastic model for analysis of instability of hopper flows [5]. These model were applicable only to incompressible flows. Critical state soil mechanics models were formulated to predict volume fraction changes associated with loading histories [6]. The models, including both rigid plastic and elastic-plastic models, employ a volume fraction dependent elliptical yield function and the associate flow rule. They have been shown to be very useful to illuminate soil behaviors. However, they do have their limitations, mainly that they are not applicable to cyclic loading conditions and that they do not adequately model stress-induced anisotropy.

Experiments have shown that volume fraction is insufficient in describing granular flow behaviors and structural quantities such as coordination number and fabric tensor have been measured [7, 8] using photoelastic particles. These experiments of dense assemblies deformed under biaxial loads and in simple shear, have shown that particle contacts distributed non-uniformly and a fabric tensor can be defined to characterize the anisotropy. Analogous microstructure anisotropy and rearrangement have also been speculated by Gadala-Maria and Acrivos [9] in their experiments of unsteady shear of non-colloidal suspensions. Stress measurements in these experiments have all shown significant transitions when loading conditions changed even though they did not depend on the magnitudes of deformation rates.

To account for the anisotropy and these complex behaviors, description of microstructure has been introduced to the constitutive model. For example, an elastic-plastic model has been proposed for soil by incorporating a structural tensor in a von Mises-type yield function [10]. Fabric tensor has been employed for an elastic-plastic model based on the double-sliding mechanism $[11,12]$. While these types of models had a certain degree of success in predicting complex rheological behaviors, they also substantially increased the model complexity. The more serious deficiencies are that their microstructure evolution equations are based on no [10] or very little [11, 12] micromechanical data and that their material constants are not directly connected to particle-level properties, such as friction coefficients. Recent theoretical and computational results have indicated the importance of particle friction coefficients on granular jamming transitions [13]. These shortcomings limit the applicability and accuracy of the models.

In this paper, we present the development of a simple plasticity model based on the micromechanical data obtained 
from extensive simulations using the discrete element method (DEM). We attempt to use the simplest form to capture the most of complex rheological behaviors of interest, which emphasizes applications to quasi-static shear flows. The very small elastic strain [14] will be neglected in the model due to the focus on continuous flows instated of statics. We will show the macroscopic rheological responses and the microstructure characteristics and evolutions under both steady and unsteady shear. The correspondence between the macroscopic responses and the microstructure variations will be demonstrated. Based on these physical insights, We will then present how the constitutive model can be closed by relations involving the microstructural internal variables. The evolution equations will also be developed based on the DEM data to form a complete constitutive model. As particle-level properties, such as particle elasticity and friction coefficient, are known in the DEM simulations, we correlate the material constants in the model to these properties. The correctness of the model will be demonstrated. We note that similar modeling effort have been reported recently for dense suspension flows $[15,16]$.

After we describe the DEM method and simulation setups in the next section, we will present simulation results of the rheological responses and microstructural evolutions during unsteady shear. In the next section, we will construct our model within proper mathematical constraints using the physical insights gained from the DEM simulations. We will summarize the attributes of our model in the conclusion section.

As for notation, we employ the regular Roman font for scalars, the Roman boldface for vectors and the San Serif font for the second order tensors.

\section{COMPUTATIONAL METHODS AND SIMULATION DETAILS}

In this paper, we present the results of DEM simulations carried out using the large-scale atomic/molecular massive parallel simulator (LAMMPS) developed at Sandia National Laboratories [17]. In the simulations, particles interact only at contact. For two particles $\{i, j\}$ with radii $\left\{a_{i}, a_{j}\right\}$ separated by $r_{i j}$, they experience a force, $\mathbf{F}_{i j}=\mathbf{F}_{\mathrm{n}_{i j}}+\mathbf{F}_{\mathrm{t}_{i j}}$, when $\delta_{i j}=d-r_{i j}>0$, where $d=a_{i}+a_{j}, \mathbf{n}_{i j}$ is the contact normal unit vector pointing from the center of particle $j$ to that of particle $i$, and $\mathbf{t}_{i j}$ is the tangential unit vector. The force is calculated using a spring-dashpot model, which has been well tested and used in many other studies $[18,19,20]$. The normal and tangential components of the interact force acting on particle $i$ are

$$
\begin{gathered}
\mathbf{F}_{\mathrm{n}_{i j}}=f\left(\delta_{i j} / d\right)\left(k_{\mathrm{n}} \delta_{i j} \mathbf{n}_{i j}-\gamma_{\mathrm{n}} m_{\mathrm{eff}} \mathbf{v}_{\mathbf{n}_{i j}}\right), \\
\mathbf{F}_{\mathrm{t}_{i j}}=f\left(\delta_{i j} / d\right)\left(-k_{\mathrm{t}} \mathbf{u}_{\mathbf{t}_{i j}}-\gamma_{\mathrm{t}} m_{\mathrm{eff}} \mathbf{v}_{\mathbf{t}_{i j}}\right),
\end{gathered}
$$

where $k_{\mathrm{n}, \mathrm{t}}$ and $\gamma_{\mathrm{n}, \mathrm{t}}$ are the spring stiffness and viscous damping constants, respectively, and $m_{\mathrm{eff}}=m_{i} m_{j} /\left(m_{i}+m_{j}\right)$ is the effective mass of spheres with masses $m_{i}$ and $m_{j}$. The corresponding contact force on particle $j$ is simply given by Newton's third law, i.e., $\mathbf{F}_{j i}=-\mathbf{F}_{i j}$. The function $f\left(\delta_{i j} / d\right)=1$ is for the Hookean (linear spring-dashpot) model, and $f\left(\delta_{i j} / d\right)=\sqrt{\delta_{i j} / d}$ is for Hertzian contacts with viscoelastic damping between spheres. In the simulations using the linear spring-dashpot model, we set $k_{\mathrm{t}}=2 / 7 k_{\mathrm{n}}, \gamma_{\mathrm{t}}=0$, and $\gamma_{\mathrm{n}}$ is chosen to satisfy the normal restitution coefficient $e=\exp \left(-\frac{\gamma_{\mathrm{n}} \pi}{\sqrt{4 k_{\mathrm{n}} / m_{\text {eff }}-\gamma_{\mathrm{n}}^{2}}}\right)=0.7$. In the simulations using the Hertzian model, the restitution coefficient depends on the initial approaching velocity. For the results presented here, only Hookean model was employed.

The value of the spring stiffness constant is chosen to be large enough to minimize particle interpenetration, yet not so large as to require an unreasonably small simulation time step. The tangential force at each contact is computed by keeping track of the elastic shear displacement, $\mathbf{u}_{\mathrm{t}_{i j}}$, throughout the lifetime of a contact. The rate of change of the elastic shear displacement is set to zero at the initiation of a contact and given by

$$
\frac{\mathrm{d} \mathbf{u}_{\mathrm{t}_{i j}}}{\mathrm{~d} t}=\mathbf{v}_{\mathrm{t}_{i j}}-\frac{\left(\mathbf{u}_{\mathrm{t}_{i j}} \cdot \mathbf{v}_{i j}\right) \mathbf{r}_{i j}}{r_{i j}^{2}} .
$$

The last term in Eq. 3 arises from the rigid body rotation around the contact point and ensures that $\mathbf{u}_{\mathbf{t}_{i j}}$ always lies in the local tangent plane of contact. As the shear displacement increases, the tangential force reaches the limit imposed by a static yield criterion, characterized by a local particle friction coefficient. The tangential force is limited by truncating the magnitude of $\mathbf{u}_{\mathbf{t}_{i j}}$ to satisfy the Coulomb criterion $\left|\mathbf{F}_{\mathbf{t}_{i j}}\right|<\mu\left|\mathbf{F}_{\mathbf{n}_{i j}}\right|$.

In order to maintain homogeneous deformations over large strain scales, we performed the simulations in threedimensional (3D) periodic domains without gravity. Simple shear flow was induced via the Lees-Edwards boundary conditions [21], with the stream velocity in the $x$-direction and the velocity gradient in the $z$-direction. The shearing motion induced by this boundary driven algorithm takes time to occur so that the flow would not be homogeneous 
immediately after a shear rate change, which renders the algorithm not suitable to study time dependent flows. This disadvantage can be greatly alleviated through the use of the SLLOD algorithm [22]. The SLLOD algorithm implies that a change in shear rate is not achieved by simply moving the boundaries of the system faster or slower, but by applying a force to the entire system. Thus the SLLOD algorithm was applied to all the simulations presented in this paper. Homogeneous stress and strain can be extracted from this type of flows, which facilitates the constitutive modeling. The macroscopic stress is calculated by

$$
\sigma=\frac{1}{V} \sum_{i}\left[\sum_{j \neq i} \frac{1}{2} \mathbf{r}_{\mathbf{i j}} \mathbf{F}_{\mathbf{i j}}+m_{i}\left(\mathbf{v}_{i}^{\prime}\right)\left(\mathbf{v}_{i}^{\prime}\right)\right],
$$

where $V$ is the total volume of the simulation domain and $\mathbf{v}_{i}^{\prime}$ is the fluctuating velocity of a particle relative to its mean streaming velocity in the shear flow.

Steady state is reached after an assembly is sheared over a large strain (of order unity at least) and the stress and microstructural quantities cease to evolve. The macroscopic strain rate tensor at the steady state then follows

$$
\mathrm{D}=\frac{1}{2} \dot{\gamma}\left(\begin{array}{ccc}
0 & 1 & 0 \\
1 & 0 & 0 \\
0 & 0 & 0
\end{array}\right)
$$

where the $\dot{\gamma}$ is the flow velocity gradient and also referred to as the shear rate. The type of unsteady flows studied here is the shear reversal flow, where the flow direction is reversed after the steady state is reached and maintained for a certain strain. In this case, the $\dot{\gamma}$ changes its sign after reversal. The shear rate is maintained low in the sense that the inertia number [23] (or Weissenberg number [24]), $I=\frac{\dot{\gamma} d}{\sqrt{p / \rho}}$, is smaller than $10^{-3}$. So that the quasi-static response is guaranteed according to previous experimental and simulation data from our and other researchers' work [23, 25, 26].

Rheological responses of the assembly may be probed by examining either the stress responses under strain constraints or the strain responses under stress constraints. Simulations were performed in these two ways using both constant volume and constant confining pressure conditions. Under constant volume condition, the volumetric strain is kept zero so that dilation of the assembly is avoided and the strain rate tensor will be exact as in Eq. 5 . Under constant confining pressure condition, the difference between normal stress components is taken into account and the volumetric strain is non-zero during transient states. The caveat of constant pressure condition is that this type of control is difficult to achieve in physical experiments. However, the common constant-loading type of shearing experiments produce essentially the same rheological responses as the constant pressure simulations performed in this paper. The rheological behaviors of these shear flows serve as the physical basis to the constitutive modeling. The data from both steady and unsteady shear simulations are used to calibrate the coefficients in the model.

\section{UNSTEADY SHEAR RHEOLOGICAL BEHAVIORS}

In the following, simulations of an assembly of 2000 mono-disperse spheres under the different flow conditions will be presented and analyzed. The dependence on system sizes, i.e., particle numbers, has been tested using assemblies with particle number varying from 1000 to 10000 particles. It has been found that the variations of variables decrease with increasing number of particles and have little difference after 2000 particles. In all cases, particle friction coefficient $\mu$ equals 0.5 and the inertial number $I \approx 0.0003$ at steady state. The Hookean contact model is used by default in these simulations unless otherwise indicated. All the results are ensemble averages of 10-20 realizations with different initial configurations.

In the case presented in Fig. 1, an assembly with particle volume fraction $\phi$ of 0.6 was sheared at $\dot{\gamma}_{0}$ to reach a steady state under the constant volume condition. The data were plotted against accumulated shear strain $\dot{\gamma}_{0} t$ and started with the steady state. The shearing was stopped at $\dot{\gamma}_{0} t=1$, i.e., the shear strain equals one. The pressure scaled by particle diameter and stiffness, and the shear stress to pressure ratio are shown in Figs. 1(a) and (b) respectively. They clearly exhibited the rate-independent response as the stress level was retaining during the no-shear period when $1<\dot{\gamma}_{0} t<2$ with little change from the steady state. The rate-independent characteristic has also been verified by collapsing the stress data from simulations with a variety of shear rates. Those results add no significantly more information and are not shown in the figures. At $\dot{\gamma}_{0} t=2$, the flow direction was reversed and the shear rate became $\dot{\gamma}=-\dot{\gamma}_{0}$. The pressure and stress ratio had significant transitions spanning a strain larger than unity after the reversal. The pressure dropped to 

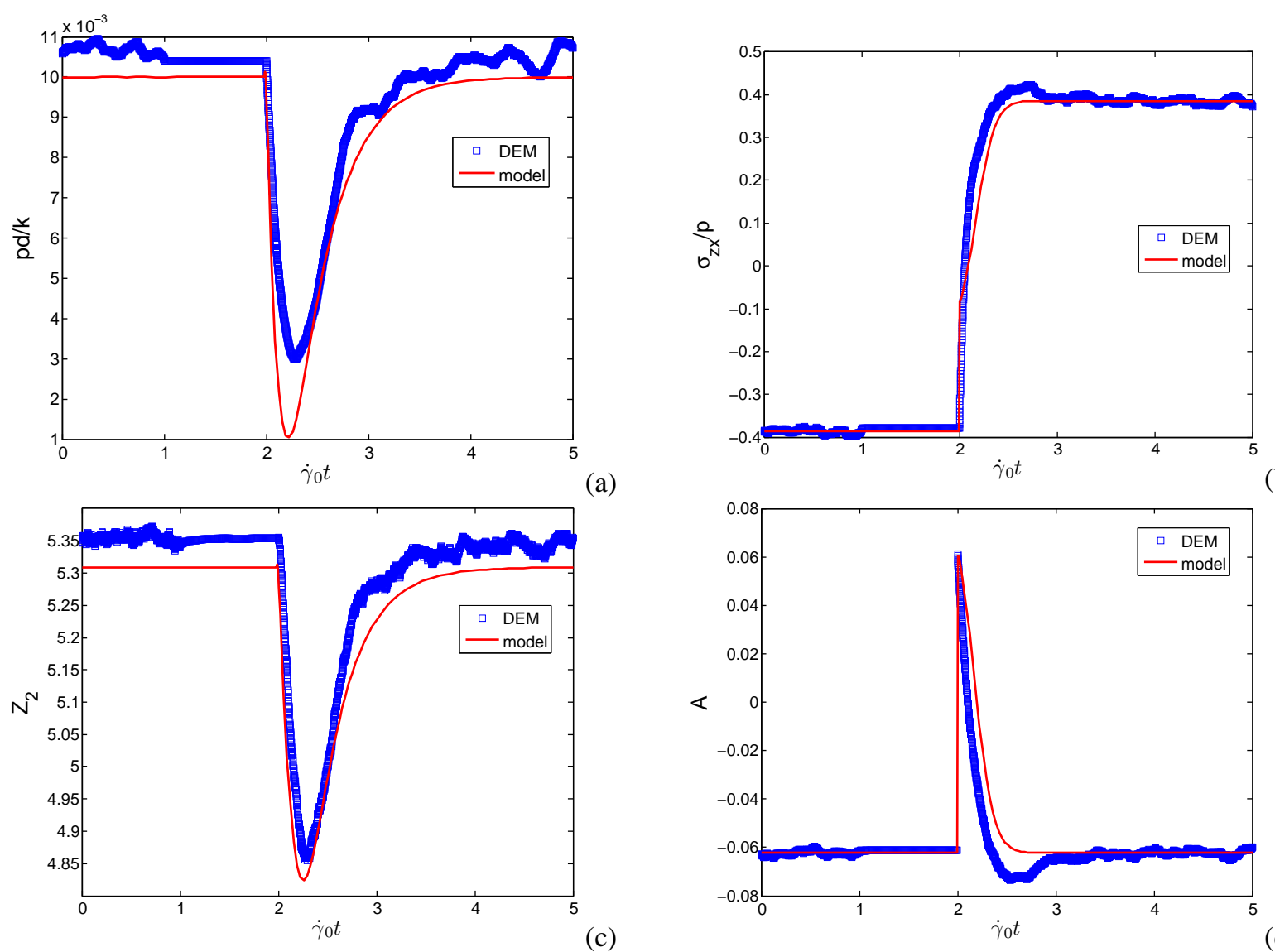

(a)

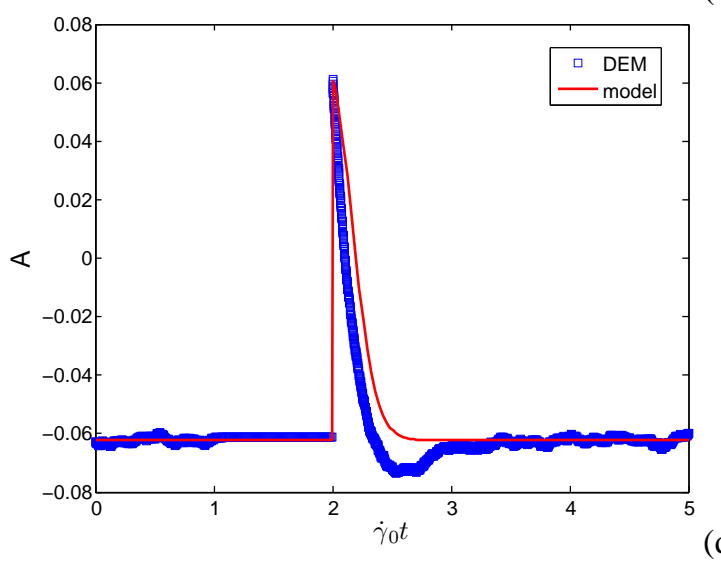

(b)

(c)

FIGURE 1. Evolutions of (a) pressure, (b) stress ratio, (c) coordination number and (d) anisotropy for an assembly subjected to unsteady shear under constant volume condition with $\phi=0.60$. Blue square symbols denote the data from DEM simulations and the red solid curves are the constitutive model results.

a lower value and slowly returned to the steady state. As the volumetric strain was kept at zero, this behavior rules out the volume fraction change as a necessary cause to the pressure transition. The steady state magnitude of the stress ratio is about 0.4, which is close to the measured quasi-static value in a shear cell experiment using polystyrene beads [4]. Upon shear reversal, the stress ratio slowly evolved to the new steady state value, which was same in magnitude as the initial state, but had an opposite sign. Similar stress transitions after shear reversal has also been observed in experiments with dense granular materials [27] and with dense suspensions [9].

For the case where the assembly was sheared under constant pressure condition, we observe that the volume fraction and stress ratio (see Figs. 2(a) and (b) respectively) also experienced transitions after shear reversal at $\dot{\gamma}_{0} t=1$. The volume fraction increased at first (the assembly compacted) and then decreased (the assembly dilated) after a shear strain of about 0.5 back to the steady state. The compaction behavior was also reported in the Couette shear cell experiment after shear reversal [27]. The volume fraction evolution under constant pressure is another evidence that the pressure is not a unique function of volume function. The stress ratio evolution had a similar trend as that in the constant volume case. At this point, we conclude that the macroscopic rheological responses have a significant transition with a strain scale of order unity after shear reversal, under both constant volume and constant pressure constraints. We note that a constitutive model without history effect would predict an instantaneous recovery to the steady state and not be able to capture this gradual transition.

To further investigate the mechanism of this transition due to shear reversal, we study the microstructure evolution during this unsteady shear. We quantify the microstructure using two variables, the average coordination number $Z$ and the fabric tensor $A$. The average coordination number is defined as the mean contacts per particle in the contact network, $Z=2 N_{\mathrm{c}} / N$, where $N_{\mathrm{c}}$ is the total number of contacts and $N$ is the total number of particles in the contact network [28]. It characterizes the connectivity of a granular assembly. When the coordination number is equal to a 

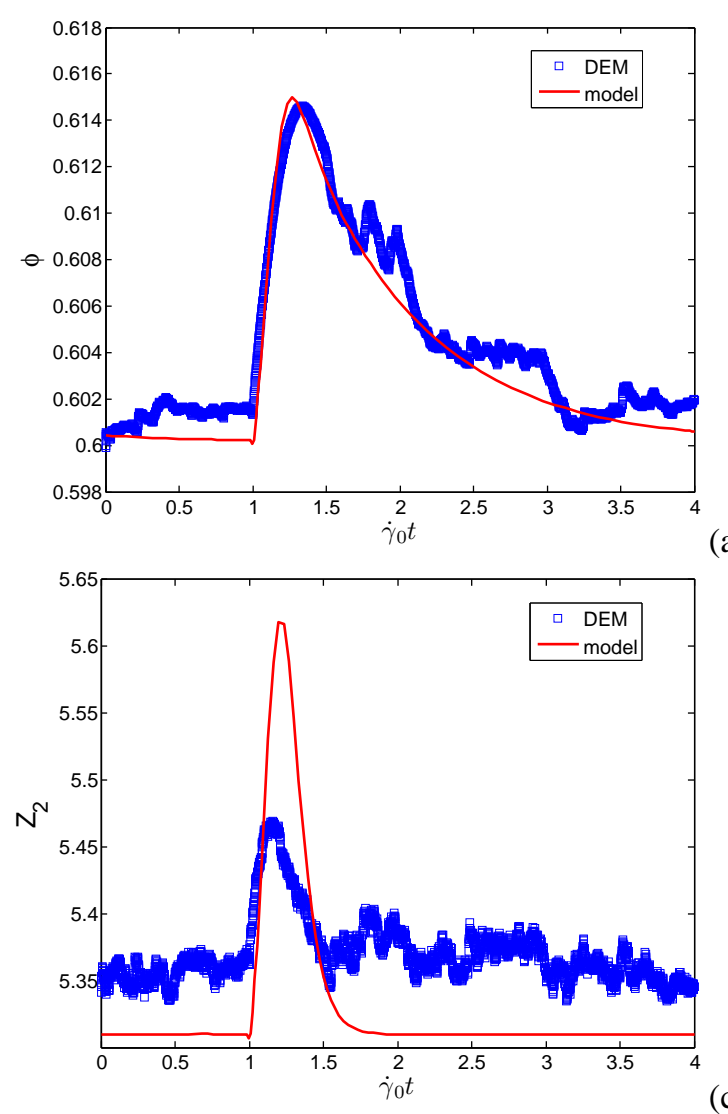

(a)

(c)

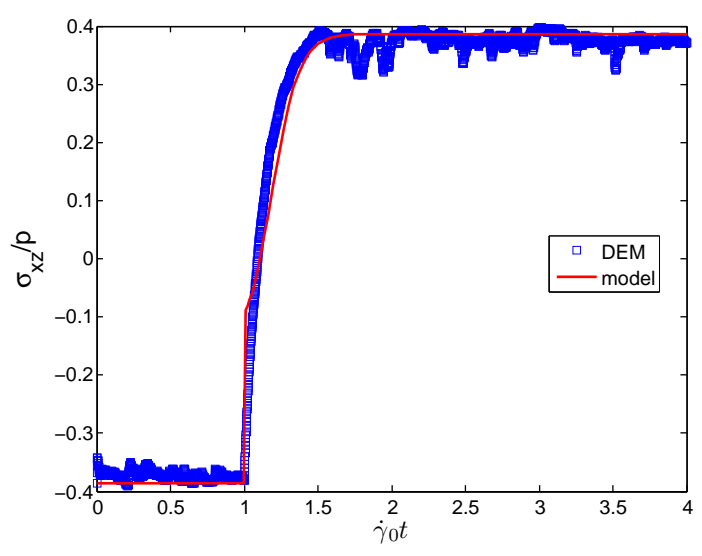

(b)

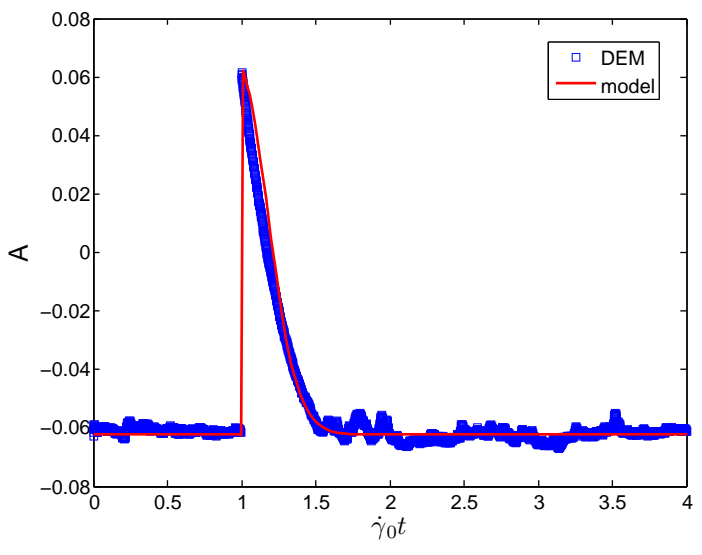

FIGURE 2. Evolutions of (a) pressure, (b) stress ratio, (c) coordination number and (d) anisotropy for an assembly subjected to unsteady shear under constant pressure condition. Blue square symbols denote the data from DEM simulations and the red solid curves are the constitutive model results.

critical value $Z_{c}$, the granular assembly is at an isostatic state, where the number of degrees of freedom is matched by number of constraints between particles. $Z_{c}$ varies from 4 to 6 as particle friction coefficient changes from infinite to zero in three dimensions (3D) [13]. $Z_{c}$ is also identified with the jamming point, above which granular flows can transit to the quasi-static regime $[29,13]$. The value of $Z_{c}$ will be determined for the granular systems as shown later in the paper. In the computation of $Z$ from our DEM particle data, we neglect any particle with one or zero contact, i.e., do not count the floaters or rattlers. The rationale is that only particles with two and more contacts participate in the contact network. The fact is that the floaters and rattlers make up only a very small portion of the total particles in our simulations. When presenting our data, we use the symbol $Z_{2}$ to distinguish from those including floaters and rattlers; in the text, we drop the subscript 2 with the understanding that we only use $Z_{2}$ to calibrate our model constants.

Fabric tensor is used to characterize the anisotropy of microstructure. It can be understood as a statistical moment with respect to the probability distribution function of an orientational vector $\mathbf{n}$. With different choices of the vector $\mathbf{n}$ and weighting factors, there have been various definitions [7, 30, 31, 32]. In this paper we identify $\mathbf{n}$ as the unit contact normal vector pointing from center to center of two particles in contact. We define the fabric tensor as

$$
\mathrm{A}=\frac{1}{N_{\mathrm{c}}} \sum_{\alpha=1}^{N_{c}} \mathbf{n}^{\alpha} \mathbf{n}^{\alpha}-\frac{\mathbf{1}}{\mathbf{3}} \mathrm{l},
$$

where $\mathrm{I}$ is the unitary tensor. By this definition, $\mathrm{A}$ is a symmetric traceless second rank tensor. The eigenvectors of the fabric tensor give the principal directions of the mean contact orientations. The eigenvalues, in turn, provide a measure of the extent of contact orientations along the principal directions. We may use the difference between the largest (major) and smallest (minor) eigenvalues or the second invariant as a measure of the anisotropy intensity. That being said, the structural anisotropy can easily be related to the shear $(x z)$ component of the fabric tensor for simple 
shear flows. Our DEM data shows that the numerical values of the diagonal components of $\mathrm{A}$ are close to zero and so are the $A_{x y}$ and $A_{y z}$ off-diagonal components. Only the $A_{x z}$ component is non-trivial. Approximately, the difference between the major and minor eigenvalues is $2 A_{x z}$ and the corresponding eigenvectors lie in the $x-z$ plan at an angle of $\pm 45^{\circ}$ to the $x$-axis. Thus we study the magnitude of $A_{x z}$ for the intensity of the anisotropy and the sign of $A_{x z}$ for the orientation of the principle direction in simple shear flows.

The evolutions of $Z$ and $A_{x z}$ are plotted in Figs. 1(c) and (d) for the constant-volume case. It can be immediately recognized that the $Z$ and $A_{x z}$ have similar evolution patterns as the pressure and stress ratio, respectively. The $Z$ values held up during the shear-stop phase, decreased significantly after the shear reversal and returned to the steady state value after the same strain scale as the pressure did. The transition of the coordination number after shear reversal indicates that the contacts were broken at first and then built up. The $A_{x z}$ variation shows that the microstructure lost its anisotropy and then regained slowly the anisotropy with an opposite principle direction over a comparable strain scale as the shear stress ratio did. The evolutions of the two variables together revealed the microstructural rearrangement after shear reversal, which involves both connectivity and anisotropy changes and has a large strain scale of order unity. During the constant-pressure shear, large scale microstructural rearrangement was also indicated by the evolutions of $Z$ and $A_{x z}$ in Figs. 2(c) and (d). The $Z$ increased after the shear reversal, whose magnitude is non-negligible, albeit small. Since the pressure was maintained constant for the entire shearing process, this increase suggests that pressure may be influence by the anisotropy in addition to the coordination number. The anisotropy effect on pressure will be further specified in the next section. The evolution of $A_{x z}$ (see Fig. 2(d)) again mirrors that of the stress ratio.

To summarize our findings from the unsteady shear simulations, we have demonstrated the rate-independent pressure and stress ratio have a significant transition after shear reversal. This transition is well correlated to the microstructure rearrangement caused by the reversal, which suggests that the microstructural variables, $Z$ and $A$ can be used as internal variables for constitutive models.

\section{PLASTICITY MODEL FORMULATION AND CALIBRATION}

The micromechanical analyses of the DEM results in section show the microstructural rearrangement during unsteady flows. This dissipative change of structure calls for a plasticity model to describe the quasi-static rheological behaviors. A constitutive framework for purely dissipative materials $[33,34]$ is compatible to the physical instances revealed by our simulations and thus applicable to the current modeling work. For such material, the local Cauchy stress $\sigma(t)$ is given by

$$
\sigma(t)=\eta: \mathrm{D}(t) \quad\left(\text { i.e., } \sigma_{i j}=\eta_{i j k l} D_{k l}(t)\right),
$$

where $\eta$ is a positive-definite viscosity depending generally on $\mathrm{D}(t)$ and on local deformation history. For plastic material response at the quasi-static regime, $\mathrm{D} \rightarrow 0$, we have

$$
\eta=\mathscr{O}\left(|\mathrm{D}|^{-1}\right), \text { for }|\mathrm{D}| \rightarrow 0,
$$

where $|D|=\sqrt{\frac{1}{2} D^{T}: D}$ denotes the modulus of the strain rate tensor. The stress can be re-written as

$$
\sigma(t)=\mu_{\mathrm{p}}: \hat{\mathrm{D}}(t)
$$

where $\mu_{\mathrm{p}}=\lim _{|\mathrm{D}| \rightarrow 0}(|\mathrm{D}| \eta)$ is a plastic modulus and $\hat{\mathrm{D}}=\mathrm{D} /|\mathrm{D}|$ denotes a versor of a second rank tensor. With this general framework, the rate-independent plastic response of dense granular materials can be described.

The fabric evolutions were shown in section to be able to effectively represent the deformation history and clearly established the fabric tensor as an internal variable. The Eq. 9 can be simplified by assuming $\mu_{\mathrm{p}}$ is given uniquely as an isotropic function of the fabric tensor $A$ as derived in [35]. The stress can then be expressed as an isotropic function of $A$ and $\hat{D}$, given in a general expression obeying the representation theorem [43]

$$
\begin{aligned}
\sigma & =\psi_{0} \mathrm{I}+\psi_{1} \hat{\mathrm{D}}+\psi_{2} \mathrm{~A}+\psi_{3} \hat{\mathrm{D}}^{2}+\psi_{4} \mathrm{~A}^{2}+\psi_{5}(\mathrm{~A} \hat{\mathrm{D}}+\hat{\mathrm{D} A}) \\
& +\psi_{6}\left(\mathrm{~A}^{2} \hat{\mathrm{D}}+\hat{\mathrm{D}} \mathrm{A}^{2}\right)+\psi_{7}\left(\mathrm{~A} \hat{\mathrm{D}}^{2}+\hat{\mathrm{D}}^{2} \mathrm{~A}\right)+\psi_{8}\left(\mathrm{~A}^{2} \hat{D}^{2}+\hat{D}^{2} \mathrm{~A}^{2}\right),
\end{aligned}
$$

where the $\psi$ coefficients are scalar polynomials of joint invariants of $\mathrm{A}$ and $\hat{\mathrm{D}}$ and $\mathrm{I}$ is the unit tensor. It should be noted that this equation is in the same form as the Hand anisotropic fluid model [36]. 


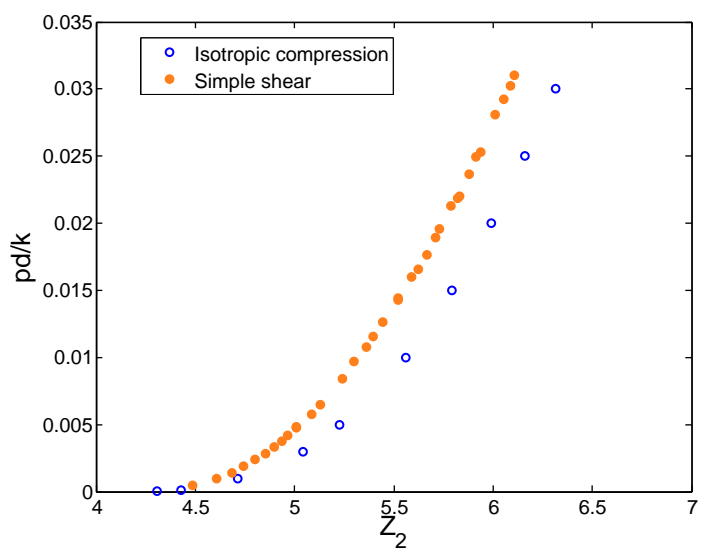

FIGURE 3. Pressure variations against coordination number from simple shear and isotropic compression simulations. The inter-particle friction coefficient is 0.5 .

There are numerous combinations that may be used for the coefficients in this general expression, which renders it very complicated to determine and calibrate them. We attempt to determine a simple stress expression within this framework that is able to describe the essential physical features of the steady and unsteady quasi-static shear flows as probed in the last section. For this purpose, we retain only the constant and linear terms in D, i.e., the $\psi_{0}$ and $\psi_{1}$ terms in equation 10. We identify the $\psi_{0}$ with the confining pressure $p$ and make the $\psi_{1}$ dependent on $p$ too. Our simplified stress equation reads

$$
\sigma=p \mathrm{I}-p \eta \frac{\mathrm{S}}{|\mathrm{D}|}
$$

where $S$ is the deviatoric strain rate tensor and $\eta$ is a scalar macroscopic friction coefficient. This stress equation is consistent with the granular plasticity proposed by Goddard [34], whose friction tensor is reduced to the current scaler friction coefficient. It also bears a similar form as previous rigid-plastic models [4, 5] and a recent visco-plastic model [37] but has an important distinction that $\eta$ has history dependence built in using the fabric tensor A. This attribute will become clear as we detail the equations for pressure and the friction coefficient $\eta$ in the following section.

\section{The pressure and $\eta$ relations}

Granular pressure is conventionally modeled as a function of particle volume fraction. The elastic contribution can be understood from DEM simulation to have a power law relation [38], i.e., $p \sim\left(\phi-\phi_{\mathrm{c}}\right)^{\alpha}$, where $\phi_{\mathrm{c}}$ is a critical volume fraction of transition to jamming or quasi-static state. The pressure in plastic flow at critical state is usually proposed according to the critical state soil mechanics [6] as a logarithmic relation with the volume fraction: $\frac{1}{\phi} \sim \log p$. Similar pressure-volume fraction relations have been widely used in plastic flows [39, 40, 41, 42]. However, both the elastic pressure and the critical state pressure relations neglect the microstructural rearrangements brought by the disturbance in flow conditions. As shown in Figs. 1 and 2, the microstructural rearrangements as shown by the coordination number and anisotropy evolutions after shear reversal decoupled the apparent correspondence between the pressure and the volume fraction variations. To take account of the pressure variation due to this microstructural rearrangement, we model the pressure as a function of $Z$ and $A$ consistent with our stress equation (see Eq. 10). We model the anisotropy effect on pressure by decomposing the pressure into one part at the isotropic state and the other due to the anisotropy

$$
p d / k=a_{1}\left(Z-Z_{c}\right)^{a_{2}}+a_{3}(\mathrm{~A}: \mathrm{A})\left(Z-Z_{c}\right)^{a_{4}},
$$

where $Z_{c}$ is the critical coordination number for the transition to quasi-static regime and the $a$ 's are material constants. The pressure data at the isotropic state was generated from stress-controlled isotropic compression of the assembly. The pressure is plotted as a function of $Z$ in Fig. 3 for both isotropic compression and steady state simple shear. We are able to calibrate the first term on the right hand side in Eq. 12 using the isotropic compression data and the second term using the pressure and A : A difference from the simple shear data. 


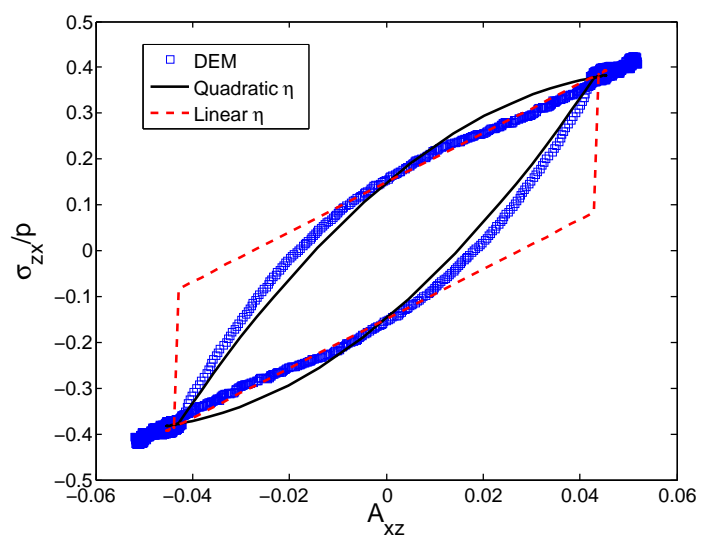

FIGURE 4. Stress ratio variations against the shear component of fabric tensor for unsteady simple shear. The assembly is subjected to unsteady shear under constant volume condition with $\phi=0.60$ and $\mu=0.5$.

The shear stress to pressure ratio has been shown in Figs. 1 and 2 to have a similar evolution pattern as the shear component of the fabric tensor $A_{x z}$. In Fig. 4, we further look at the functional dependence of the stress ratio. The stress ratios during a shear reversal under constant volume condition (the same data as in Fig. 1(b)) were plotted against $A_{x z}$ in Fig. 4. The data for unsteady shear under constant pressure would collapse essentially into the same curve and is thus not plotted for clarity. The two branches in Fig. 4 show that the stress ratio evolves from one steady state to the other and back. Figure 4 clearly reveals a functional dependence of the stress ratio on $A_{x z}$. We therefore model $\eta$ using the following expression

$$
\eta=b_{1}+b_{2} \frac{\mathrm{A}: \mathrm{S}}{|\mathrm{D}|}
$$

where the $b$ 's are are material constants. The stress ratios calculated from Eq. 13 are plotted in the dashed line and compared with the DEM data in Fig. 4. This equation yields only linear approximation of the DEM results but captures most of the stress ratio variation except immediately after the stress ratio starts to change. A quadratic relation,

$$
\eta=b_{1}+b_{2} \frac{\mathrm{A}: \mathrm{S}}{|\mathrm{D}|}+b_{3} \frac{(\mathrm{A}: \mathrm{S})^{2}}{|\mathrm{D}|^{2}}
$$

shown in the solid lines in Fig. 4 better approximates the stress ratio variation. However, the strain, during which the differences resulted from Eqs. 13 and 14 are most significant, is very small. We therefore employ Eq. 13 for the constitutive model in this paper.

\section{Microstructure evolution equations}

To complete the constitutive model, appropriate evolution equations for the microstructural variables are required. The stress can then be evolved through the microstructural evolutions during unsteady flows. Based on our DEM data of the microstructure evolutions, we model the rate of change of both $A$ and $Z$ as functions of $A$ and $D$. The reason for this choice will become evident when a comparison is made with the DEM data.

In order to be frame indifferent, the time derivative of a tensor must take account of the frame rotation [43]. We use the Jaumann derivative here, i.e., $\AA=\dot{A}+A \cdot W-W \cdot A$, where $W$ is the spin tensor and $\dot{A}$ denotes the total time derivative. The most general expression for $\AA$ has been shown according to the representation theorem to be [36]

$$
\begin{aligned}
\AA= & \alpha_{0} \mathrm{I}+\alpha_{1} \mathrm{D}+\alpha_{2} \mathrm{~A}+\alpha_{3} \mathrm{D}^{2}+\alpha_{4} \mathrm{~A}^{2}+\alpha_{5}(\mathrm{AD}+\mathrm{DA})+ \\
& \alpha_{6}\left(\mathrm{~A}^{2} \mathrm{D}+\mathrm{DA}^{2}\right)+\alpha_{7}\left(\mathrm{AD}^{2}+\mathrm{D}^{2} \mathrm{~A}\right)+\alpha_{8}\left(\mathrm{~A}^{2} \mathrm{D}^{2}+\mathrm{D}^{2} \mathrm{~A}^{2}\right),
\end{aligned}
$$

where the $\alpha$ coefficients are scalar polynomials of joint invariants of $A$ and D. We again simply this expression according to the physical behaviors observed in our DEM simulations shown in Figs. 1 and 2. The expression obtained 
reads

$$
\AA=c_{1} \mathrm{~S}+c_{2}|\mathrm{D}| \mathrm{A}+c_{3}(\mathrm{~A}: \mathrm{D}) \mathrm{A},
$$

which only retains the $\alpha_{1}$ and $\alpha_{2}$ terms from Eq. 15 with linearity in D. We performed linear stability analysis of this equation and found this form to be able to return to steady state correctly. We solved Eq. 16 for the homogeneous simple shear and compared to the DEM results in Figs. 1(d) and 2(d). It can be seen that Eq. 16 is able to capture the steady state and the evolution of $A_{x z}$ to a good degree.

The evolution equation for the coordination number $Z$ follows the same general form as Eq. 15. Since $Z$ is a scalar, the equation will reduce to a scalar form. In fact, $Z$ can be treated as the trace of a generalized structure tensor with deviatoric part as $\mathrm{A}$. The evolution equation after simplification takes the following form

$$
\dot{Z}=d_{1}\left(\mathrm{~A}: \mathrm{D}+d_{2}|\mathrm{D}|\right)+d_{3}|\mathrm{D}|(f(\phi)-Z)+d_{4} \operatorname{tr}(D),
$$

where $d_{1}-d_{4}$ are material constants and $\operatorname{tr}(D)$ is the trace of $\mathrm{D}$. The function $f(\phi)$ dictates how the coordination number varies with volume fraction during the steady shear. The equation for $f(\phi)$, established by fitting the $Z$ variation against $\phi$ at steady state, is

$$
f(\phi)=Z_{c}+\beta_{1}\left(\phi-\phi_{c}\right)^{\beta_{2}} .
$$

The $\beta$ coefficients are material constants calibrated using the DEM data. $\operatorname{The} \operatorname{tr}(D)$ term is used to take account of the effects of compaction and dilation on increasing and decreasing the coordination number. Therefore the Reynolds dilatancy constraint is implicitly incorporated in Eq. 17, which can be re-written in an explicit form proposed by Goddard and Didwania [44]

$$
\operatorname{tr}(D)=\alpha|\mathrm{D}|
$$

where the coefficient of dilatancy $\alpha=\frac{1}{d_{4}}\left[d_{1}\left(\frac{\mathrm{A}: \mathrm{S}}{|\mathrm{D}|}+d_{2}\right)+d_{3}(f(\phi)-Z)-\frac{\dot{Z}}{|\mathrm{D}|}\right]$, is dynamically changing according to the evolutions of $Z$ and A. The computational results from Eq. 17 were compared to the DEM data in Figs. 1(c) and 2(c). For both conditions, the evolution strains scales after shear reversal were correctly produced. There were inaccuracies in the steady state and minimum and maximum values calculations, which do not affect the pressure calculation significantly as will be shown next.

Equations 11, 12,13,16 and 17 compose the complete constitutive model, which takes a simple form of plasticity model for stress equation but incorporates the microstructure evolution. We now study if the macroscopic quantities can be correctly described by this model. With the $Z$ and components of $A$ solved from Eqs. 12 and 16, we feed these quantities to Eqs. 12 and 13 to calculate the pressure and stress ratio, respectively for shear flows under the constant volume condition. The results are shown in Figs. 1(a) and (b) to compare with the DEM data. The rate-independence characteristic of the model is obvious as the calculated pressure and stress ratio did not change when the shearing motion was stopped at $\dot{\gamma} t=1$. The large-strain evolutions of the pressure and stress ratio after the shear reversal at $\dot{\gamma} t=2$ were correctly captured. There were small quantitative differences between the model and the DEM results. For the shear under constant pressure condition, we adjusted $Z$ to match the target pressure value, which was constant and not shown in the figure. The modeled evolution of the volume fraction and stress ratio, as shown in Figs. 2(a) and (b), matched the DEM data well. It can be concluded from these comparisons that the constitutive model is self-consistent and able to correctly describe the rheological behaviors of the steady and unsteady quasi-static shear flows.

\section{CONCLUSIONS}

We have developed a plasticity model that is able to predict complex rheological behaviors of quasi-static granular flows. The stress equation simply contains a pressure term and a deviatoric term with a macroscopic friction coefficient. The closures to the pressure and the friction coefficient are linked to the microstructure evolution, which is modeled by the coordination number and fabric evolution equations. The material constants in the model are functions of particlelevel properties and can be calibrated using the shear flow data from the DEM simulations.

We note that the current model is intended to capture the most salient rheological features of the quasi-static granular flows using the simplest model. Therefore, some rheological features are neglected in favor of the model simplicity. For example, the normal stress differences, which are observed in our DEM simulations, are not captured in this model. However, the model can be extended to include such subtle features by using additional terms in Eq. 10. The model can be refined in a similar way when applied to triaxial deformations. These types of extension work will be interesting to pursue in the future. 


\section{ACKNOWLEDGMENTS}

The authors are grateful to professors Joe Goddard, Prabhu Nott and Stefan Lunding for their stimulating discussions on continuum modeling of dense granular materials. We also thank Sebastian Chialvo for providing some DEM data. This work is supported by a DOE-UCR grant DE-FG26-07NT43070.

\section{REFERENCES}

1. S. Sundaresan, Powder Technology 115, 2-7 (2001).

2. R. Hill, The Mathematical Theory of plasticity, Oxford University Press, Oxford, 1950.

3. R. M. Nedderman, Statics and Kinematics of Granular Materials, Cambridge University Press, 1992.

4. S. B. Savage, Mechanics of granular materials: new models and constitutive relations, Elsevier Ltd, 1983, chap. Granular flow down rough inclines-review and extension.

5. D. G. Schaeffer, Journal of Differential Equations 66, 19-50 (1987).

6. A. Schofield, and P. Wroth, Critical State Soil Mechanics, McGraw-Hill Inc., 1968.

7. M. Oda, J. Konishi, and S. Nemat-Nasser, Géotechnique (1980).

8. G. Subhash, S. Nemat-Nasser, M. M. Mehrabadi, and H. M. Shodj, Mechanics of Materials 11, 87-106 (1991).

9. F. Gadala-Maria, and A. Acrivos, Journal of Rheology 24, 799-814 (1980).

10. J. H. Prevost, Soil Dynamics and Earthquake Engineering 4, 9-17 (1985).

11. S. Nemat-Nasser, Journal of the Mechanics and Physics of Solids 48, 1541-1563 (2000).

12. S. Nemat-Nasser, and J. Zhang, International Journal of Plasticity 18, 531-547 (2002).

13. C. Song, P. Wang, and H. A. Makse, Nature 453, 629-632 (2008).

14. R. Kuwano, and R. J. Jardine, Géotechnique 52, 727-749 (2002).

15. J. J. Stickel, R. J. Phillips, and R. L. Powell, Journal of Rheology 50, 379-413 (2006).

16. J. J. Stickel, R. J. Phillips, and R. L. Powell, Journal of Rheology 51, 1271-1302 (2007).

17. S. Plimpton, Journal of Computational Physics 117, 1-19 (1995/3/1).

18. L. E. Silbert, D. Ertas, G. S. Grest, T. C. Halsey, D. Levine, and S. J. Plimpton, Physical Review E 64, 051302 (2001).

19. L. E. Silbert, G. S. Grest, R. Brewster, and A. J. Levine, Physical Review Letters 99, 068002-4 (2007).

20. C. H. Rycroft, K. Kamrin, and M. Z. Bazant, Journal of the Mechanics and Physics of Solids 57, 828-839 (2009/5).

21. A. W. Lees, and S. F. Edwards, Journal of Physics C: Solid State Physics 5, 1921-1928 (1972).

22. D. J. Evans, and G. P. Morriss, Statistical Mechanics of Nonequilibrium Liquids, Academic Press, San Diego, 1990.

23. G. D. R. MiDi, European Physical Journal E - Soft Matter 14, 341-305 (2004).

24. J. Goddard, Acta Mechanica 63, 3-13 (1986).

25. F. da Cruz, S. Emam, M. Prochnow, J.-N. Roux, and F. Chevoir, Physical Review E (Statistical, Nonlinear, and Soft Matter Physics) 72, 021309-17 (2005).

26. L. Aarons, and S. Sundaresan, Powder Technology 169, 10-21 (2006).

27. M. Toiya, J. Stambaugh, and W. Losert, Physical Review Letters 93, 088001-4 (2004).

28. H. P. Zhang, and H. A. Makse, Physical Review E (Statistical, Nonlinear, and Soft Matter Physics) 72, 011301-12 (2005).

29. C. S. O'Hern, L. E. Silbert, A. J. Liu, and S. R. Nagel, Physical Review E 68 (2003).

30. R. J. Bathurst, and L. Rothenburg, Mechanics of Materials 9, 65-80 (1990).

31. F. Radjai, D. E. Wolf, M. Jean, and J.-J. Moreau, Physical Review Letters 80 (1998).

32. J. D. Goddard, Physics of dry granular media, Kluwer, 1998.

33. J. D. Goddard, Journal of Non-Newtonian Fluid Mechanics 14, 141-160 (1984).

34. J. D. Goddard, Journal of Fluid Mechanics 568, 1-17 (2006).

35. S. C. Cowin, Mechanics of Materials 4, 137-147 (1985).

36. G. L. Hand, Journal of Fluid Mechanics 13, 33-46 (1962).

37. P. Jop, Y. Forterre, and O. Pouliquen, Nature 441, 727-730 (2006).

38. H. A. Makse, D. L. Johnson, and L. M. Schwartz, Phys. Rev. Lett. 84, 4160-4163 (2000).

39. S. C. Cowin, Journal of Applied Mechanics 44, 409-412 (1977).

40. N. P. Kruyt, Journal of Applied Mechanics 57, 1032-1035 (1990).

41. S. B. Savage, Journal of Fluid Mechanics 377, 1-26 (1998).

42. A. Srivastava, and S. Sundaresan, Powder Technology 129, 72-85 (2003).

43. C. Truesdell, and W. Noll, The Non-Lindear Field Theories of Mechanics, Springer, 2004, 3rd edn.

44. J. D. Goddard, and A. K. Didwania, The Quarterly Journal of Mechanics \& Applied mathematics 51, 15-41 (1998).

45. T. L. Youd, Highway Research Record 374, 1-6 (1971).

46. D. Wood, and M. Budhu, Soilds under Cyclic and Transient Loading, Balkema, Rotterdam, The Netherlands, 1980, chap. The behaviour of Leighton Buzzard sand in cyclic simple shear tests, p. 9.

47. O. Reynolds, Philosophical Magazine 20, 469-481 (1885).

48. N. Okada, and N.-N. S., Géotechnique 44, 1-19 (1994). 
Appendix E

Granular flow in Silo discharge: DEM simulations and model assessment 


\title{
Granular flow in Silo discharge: DEM simulations and model assessment
}

\author{
Vidyapati and S. Subramaniam \\ Department of Mechanical Engineering, Center for Computational Thermal-Fluids \\ Research, Iowa State University, Ames, IA 50011, USA
}

\begin{abstract}
The granular frictional flow model is a critical component for accurate prediction of the discharge rate from silos and hoppers, which generally operates in dense regime of granular flow. In this study, both continuum and discrete approaches have been used to model the discharge of granular particles from a flat-bottomed silo. Two different continuum granular frictional flow models (Schaeffer and Princeton model) have been employed to study a two-dimensional (2D) bin discharge problem. The computed discharge rates from these two different continuum frictional flow models are compared with the discharge rate obtained from the well-known empirical correlation. Results indicate that both these frictional models over predict the discharge rate from the bin. A quantitative comparison between results of continuum models and DEM simulations is performed by comparing discharge rates, solid velocities and solid stresses for a three-dimensional (3D) flat-bottomed silo. It is reconfirmed with these simulations that, both these frictional models over-predict the discharge rate from silo. Discrete element method (DEM) simulations are also used to investigate and quantify the influence of different simulation parameters affecting the discharge rate from a silo. Finally spatial extent of different regimes is quantified inside a silo geometry, by using a regime map established from DEM data of homogeneously sheared granular flow. It is shown that all three different regimes of granular flows (inertial, intermediate, and quasi-static) co-exist in this silo discharge problem.
\end{abstract}

Key words: Granular flows; Silo discharge; Discrete element method; Frictional flow models

\section{Introduction}

Granular flows are the fundamental particle systems found in different solid processing industry and nature [1]. Understanding the behavior of these materials is a topic of active research that continues to yield exciting and often surprising results. Flat-bottomed silos are important devices that are widely 
used in the processing and handling of these granular materials in many industrial and agricultural applications. Accurate prediction of discharge rate is critical for the dependable design and optimum performance of these devices. In order to accurately predict the discharge rate from silo, a reliable and accurate continuum description of granular flows is needed. However, even in the applications that involves relatively dilute flow, it is possible to find regimes that are dense and frictional, this poses a significant challenge to formulate a comprehensive continuum theory that can be applied to different regimes of granular flows.

There are two approaches for modeling this particulate system: continuum and discrete. The first approach (continuum) is more relevant for industrial applications that use large amount of solids. The second approach (discrete) is a powerful numerical method, in which motion of individual particle is determined on the basis of all the force acting upon it [2]. Contrary to the continuum techniques, it simulates effect at particle level, but it is limited to relatively small number of particles because of computer time limitations. The assessment of different frictional flow models is critical for a silo geometry, where both dilute and dense regimes co-exist simultaneously. The inertial (rapid flows) regime has been successfully studied by means of corrections of the kinetic theory of gases [3, 4], whereas the quasi-static regime is generally described by plasticity theories $[5,6]$. However, no unified theory has been proposed for the intermediate (transitional) flow regime, in which energy is dissipated by inelastic collisions and interparticle friction $[7,8]$. The primary objective of this study is to quantify the performance of different continuum frictional models by comparing their prediction for discharge rate with the discrete element method (DEM) simulations and well-known empirical correlation [9].

Through continuum simulations of discharge of granular particles from a twodimensional (2D) bin, performance of two different frictional flow models is evaluated for dense granular flows. In dense regime the flow is dominated by long-lasting, frictional, and sliding contacts, hence assessment of frictional models are critical for overall performance of any continuum model. The first frictional flow model assessed in this work, is the model proposed by Schaeffer [5], which has been traditionally used in the MFIX computer code [10]. The second frictional flow model assessed, is the model developed by Srivastava and Sundaresan [11], which was further implemented in MFIX code. Srivastava and Sundaresan [11] also conducted validation study of their proposed frictional theory by comparing its results with the well-known Beverloo correlation [9]. Study performed by Benyahia [12] compares the discharge rate obtained using these two frictional theories for different values of orifice widths. However, we are not aware of any detailed study, which directly compares the results for discharge rate and solid velocities obtained using these two different continuum frictional models. In this work, we assess the performance of these two 
different frictional flow models using a two-dimensional (2D) bin discharge problem, which is same example studied by Srivastava and Sundaresan [11].

Discrete element method (DEM) simulations have been used in the past to model the discharge of granular particles from the silos and hoppers $[13,14$, 15, 16]. Anand et al. [15] studied the discharge from a rectangular hopper using DEM and quantified the effect of different simulation parameters on the discharge rate from hopper. Nevertheless, there has been no comprehensive study, which addresses the issue of direct quantitative comparison between the results of DEM and continuum simulations for a flat-bottomed silo. In this work, a 3D flat-bottomed silo is simulated using discrete element method (DEM), also effect of different simulation parameters on the discharge rate is quantified by probing the individual particle in the system. These kinds of information are difficult to get from experiments, for example there have been no detailed experimental studies, investigating the effect of particle friction coefficient or coefficient of restitution on silo discharge rate. There is an implicit assumption in most of the experimental correlation that these interaction parameters play very little or no role in predicting the discharge rate from silo [15]. DEM is a good tool which helps us answering these questions, and suggest possible ways to improve the overall design of silos and hoppers. DEM simulations are also used to assess the performance of continuum models and help in improving the model performance by revealing the complex flow dynamics involved in silo discharge problem. The model assessment is accomplished by comparing result for discharge rates, solid velocities and solid stresses obtained using continuum theories with that of DEM simulations. The discharge rates obtained using DEM simulations are also compared with the existing correlation [9]. In this way, the accuracy of the DEM simulations can be assessed and the sensitivity of the various parameters affecting the discharge rate can be quantified.

The next section describes the details of continuum simulations along with a brief description about two different friction flow models which are assessed in this work. In the following section, details of DEM simulations of silo discharge are discussed and results from a quantitative comparison study between DEM and continuum simulations are presented. Method used to characterize the spatial extent of different regimes in a silo geometry is discussed in Sec. 4. Finally conclusions are drawn in Sec. 5.

\section{Continuum simulations}

Continuum description of granular flows plays an important role in simulating problems at larger and industrial scale. Two different kinds of continuum simulations are performed in this study. In first phase of this study a two- 
dimensional bin (same example studied by Srivastava and Sundaresan [11]) is simulated and results are compared with the well-known correlation for discharge rate. In the later phase of this study, a three-dimensional flat-bottomed silo is simulated using continuum approach and results are compared with the data from DEM simulations.

\subsection{Simulation setup for a two-dimensional bin}

Simulations of particle discharge from a two-dimensional rectangular bin is performed using the averaged two-fluid (TF) continuum equations using MFIX computer code [10]. MFIX is an Eulerian-Eulerian computational fluid dynamics (CFD) model in which gas and granular solids are modeled as interspersed continua. However, the current study focuses only on dense granular flows, so the effect of interstitial fluid can be neglected (provided the particle diameters are relatively larger). Hence no effect of fluid is introduced in the model equations. The "dry" granular kinetic theory model used in MFIX code [10] is essentially the same as that derived by Lun et al. [3]. Conservation of mass for constant solid density is given as:

$$
\rho_{s}\left[\frac{\partial \nu}{\partial t}+\nabla \cdot\left(\nu \mathbf{v}_{s}\right)\right]=0
$$

where $\rho_{s}$ is the solid density, $\nu$ is the solid volume fraction, $\mathbf{v}_{s}$ is the velocity vector for solid phase. Conservation of linear momentum is given by:

$$
\rho_{s}\left[\frac{\partial \nu \mathbf{v}_{s}}{\partial t}+\nabla \cdot\left(\nu \mathbf{v}_{s} \mathbf{v}_{s}\right)\right]=\nabla \cdot\left(\boldsymbol{\tau}_{k}+\boldsymbol{\tau}_{f}\right)+\nu \rho_{s} \mathbf{g},
$$

where $\boldsymbol{\tau}_{k}$ and $\boldsymbol{\tau}_{f}$ are the kinetic and frictional part of the stress tensor respectively. The translational granular energy conservation equation is given by:

$$
\frac{3}{2} \rho_{s}\left[\frac{\partial \nu \Theta_{s}}{\partial t}+\nabla \cdot\left(\nu \Theta_{s} \mathbf{v}_{s}\right)\right]=-\nabla \cdot \mathbf{q}+\tau_{k}: \nabla \mathbf{v}_{s}-\rho_{s} J_{s}
$$

where $\Theta_{s}$ is the granular temperature, $\mathbf{q}$ is the flux of granular energy, and $J_{s}$ is the granular energy dissipation due to inelastic collisions. Solids kineticcollisional and frictional stress terms are given by:

$$
\begin{gathered}
\boldsymbol{\tau}_{k}=\left[-P_{s}+\eta \mu_{b} \nabla \cdot \mathbf{v}_{s}\right] \mathbf{I}+2 \mu_{s} \mathbf{S}_{S}, \\
\boldsymbol{\tau}_{f}=-P_{f} \mathbf{I}+2 \mu_{f} \mathbf{S}_{S}, \\
\mathbf{S}_{S}=\frac{1}{2}\left[\nabla \mathbf{v}_{s}+\left(\nabla \mathbf{v}_{s}\right)^{T}\right]-\frac{1}{3} \nabla \cdot \mathbf{v}_{s} \mathbf{I},
\end{gathered}
$$

where $P_{s}$ is the solid pressure, $\eta$ is a constant depending on particle restitution coefficient [11], $\mu_{b}$ is the bulk viscosity of the solid phase, $\mathbf{I}$ is the identity 
tensor, $\mu_{s}$ is the granular viscosity, and $\mathbf{S}_{S}$ is the strain-rate tensor as given in Eq. 6. The closures for different terms are taken by Lun et al. [3].

The problem studied in this work is the same example studied by Srivastava and Sundaresan [11]: a 2D rectangular bin $8 \mathrm{~cm}$ wide and $100 \mathrm{~cm}$ high with an open top and an orifice centered at the bottom. Figure 1(a) shows the schematic of simulation domain for bin discharge problem. Figures 1(b) shows the contour plot of the gas void fraction at time 0.1 sec. The width of the center orifice at the bottom is set to $1.4 \mathrm{~cm}$. A $5 \mathrm{~cm}$ high region below the bin is included in the domain so that a boundary condition is not required right at the exit of the bin. Simulations are performed with three different

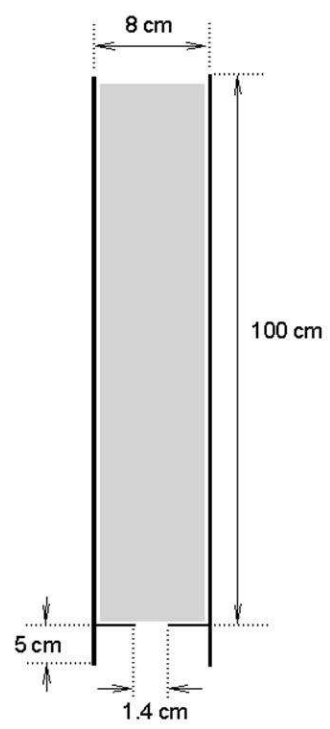

(a)

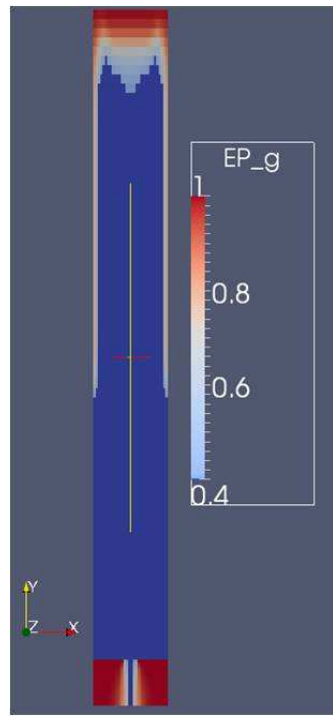

(b)

Fig. 1. (a) Schematic diagram showing the geometry of bin discharge, and (b) Gas void fraction after $0.1 \mathrm{sec}$ of simulation.

grid resolutions of $16 \times 160,32 \times 270$ and $64 \times 325$ in $x$ and $y$ direction, respectively. The solid density and velocity magnitude changes less than $4.0 \%$ by increasing the grid size from $32 \times 270$ to $64 \times 325$, hence a grid resolution of $32 \times 270$ is chosen for this study. The initial solid volume fraction in the bin is set to 0.60, whereas initial granular temperature is taken to be non-zero everywhere $\left(1 \mathrm{~cm}^{2} / \mathrm{s}^{2}\right)$. Table 1 lists the values of the model parameter used in the simulations, most of which taken from Srivastava and Sundaresan [11].

The boundary condition for momentum and pseudo-thermal energy (PTE) for the particulate phase at the walls of the bins are taken from Johnson and Jackson [17]. This can be written as:

$$
\mathbf{n} \cdot\left(\boldsymbol{\tau}_{k}+\boldsymbol{\tau}_{f}\right) \cdot \frac{\mathbf{v}_{s l}}{\left|\mathbf{v}_{s l}\right|}+\left(\mathbf{n} \cdot \boldsymbol{\tau}_{f} \cdot \mathbf{n}\right) \tan \delta+\frac{\pi \sqrt{3}}{6 \nu^{\max }} \phi^{\prime} \rho_{s} \nu g_{0} \Theta_{s}^{1 / 2} \mathbf{v}_{s l}=0
$$




\begin{tabular}{|c|c|}
\hline Parameter & Values \\
\hline gas density, $\rho_{g}$ & $1.3 \times 10^{-3} \mathrm{~g} / \mathrm{cm}^{3}$ \\
\hline gas viscosity, $\mu_{g}$ & $1.8 \times 10^{-4} \mathrm{~g} / \mathrm{cm}-\mathrm{s}$ \\
\hline solid density, $\rho_{s}$ & $2.9 \mathrm{~g} / \mathrm{cm}^{3}$ \\
\hline particle diameter, $d_{p}$ & $1 \mathrm{~mm}$ \\
\hline angle of internal friction, $\phi$ & 28.5 \\
\hline angle of wall friction, $\delta$ & 12.3 \\
\hline specularity coefficient, $\phi^{\prime}$ & 0.25 \\
\hline particle-particle coefficient of restitution, $e$ & 0.91 \\
\hline coefficient of restitution at wall, $e_{w}$ & 0.91 \\
\hline maximum solid packing, $\nu^{\max }$ & 0.65 \\
\hline
\end{tabular}

Table 1

Values of model parameters used in simulations.

$$
\mathbf{n} \cdot \mathbf{q}=\frac{\pi \sqrt{3}}{6 \nu^{\text {max }}} \phi^{\prime} \rho_{s} \nu g_{0} \Theta_{s}^{1 / 2}\left|\mathbf{v}_{s l}\right|^{2}-\frac{\pi \sqrt{3}}{4 \nu^{\text {max }}}\left(1-e_{w}^{2}\right) \rho_{s} \nu g_{0} \Theta_{s}^{3 / 2}
$$

where $\mathbf{n}$ is the unit normal from the boundary into the particle assembly, $\boldsymbol{\tau}_{k}$, $\boldsymbol{\tau}_{f}$ are kinetic and frictional stress tensor respectively, $\nu^{\max }$ maximum solid volume fraction, $\Theta_{s}$ is the granular temperature, $\mathbf{q}$ is the flux of granular energy, $\delta$ is the angle of wall friction for the material, $\phi^{\prime}$ is the specularity coefficient, $\rho_{s}$ is the solid density, $\nu$ is the solid volume fraction, $e_{w}$ is the coefficient of restitution at the wall and $\mathbf{v}_{s l}$ is $\mathbf{v}-\mathbf{v}_{\text {wall }}$, the slip velocity of the particle assembly at the wall. The gas phase is allowed to slip freely at the wall. At the open boundaries of the domain, the gas pressure is set to be atmospheric. For all other dependent variables, the usual continuation condition (i.e. zero gradient in the direction normal to the boundary) is applied. The bin is initialized with particles at initial void fraction of 0.40 with particles at rest.

\subsection{Frictional stress models}

In dense granular flows the frictional contribution of the stress tensor dominates the kinetic contribution of the stress tensor. Hence frictional part of the stress tensor plays a critical role in predicting the overall behavior of these flows. Two different frictional flow models (Schaeffer [5] and Princeton model [11]) are used to simulate the dense granular flow in this work. A brief description of these models are presented below,

(1) Schaeffer model [5]: This frictional model has been traditionally used in 
the MFIX code [10]. This model is used at the critical state when the solid volume fraction exceeds the maximum packing limit. In this model, $I_{2 D}$ represent the second invariant of the deviator of the strain rate tensor, which is related to the norm of the square of the strain rate tensor used by Schaeffer [5] simply by $\sqrt{I_{2 D}}=\sqrt{\left(\mathbf{S}_{S}: \mathbf{S}_{S}\right)} / 2$. This model expresses the frictional stresses by the following equations:

$$
\begin{gathered}
P_{f}=P_{c}= \begin{cases}10^{25}\left(\nu-\nu^{\max }\right)^{10} & \text { if } \nu>\nu^{\max } \\
0 & \text { if } \nu \leq \nu^{\text {max }}\end{cases} \\
\mu_{f}= \begin{cases}\frac{P_{c} \sin (\phi)}{2 \sqrt{I_{2 D}}} & \text { if } \nu>\nu^{\max } \\
0 & \text { if } \nu \leq \nu^{\max }\end{cases}
\end{gathered}
$$

where $P_{c}$ is the critical state pressure, $\mathbf{S}_{S}$ is the strain rate tensor, $\nu$ and $\nu^{\max }$ is the solid volume fraction and its value at maximum packing limit respectively.

(2) Princeton model [11]: This frictional model is proposed by Srivastava and Sundaresan [11], who gave expression of the frictional stresses for a compressible granular assembly. This model is a modification of Savage model [18] that accounts for strain-rate fluctuations even in the dense regime of granular flow. The frictional stresses start influencing the granular flow at a minimum frictional solids volume fraction $\left(\nu^{\text {min }}\right)$, which is below the maximum packing $\left(\nu^{\max }\right)$ as proposed by Johnson and Jackson [17]. In this study, the critical state theory applies only when the granular assembly is incompressible (i.e., above maximum packing). The Srivastava-Sundaresan [11] model is expressed by the following equations:

$$
P_{c}= \begin{cases}10^{25}\left(\nu-\nu^{\max }\right)^{10} & \text { if } \nu>\nu^{\max } \\ F r \frac{\left(\nu-\nu^{\min }\right)^{r}}{\left(\nu^{\max }-\nu\right)^{s}} & \text { if } \nu^{\max } \geq \nu>\nu^{\text {min }} \\ 0 & \text { if } \nu \leq \nu^{\text {min }}\end{cases}
$$

where $F r=0.5$ dynes $/ \mathrm{cm}^{2}, r=2$, and $s=5$ are constants in this model [11].

$$
\begin{gathered}
\frac{P_{f}}{P_{c}}=\left(1-\frac{\nabla \cdot \mathbf{v}_{s}}{n \sqrt{2} \sin (\phi) \sqrt{\mathbf{S}_{S}: \mathbf{S}_{S}+\Theta_{s} / d_{p}^{2}}}\right)^{n-1} \\
\mu_{f}=\frac{\sin (\phi)}{\sqrt{2}} \frac{P_{f}}{\sqrt{\mathbf{S}_{S}: \mathbf{S}_{S}+\Theta_{s} / d_{p}^{2}}}\left(n-(n-1)\left(\frac{P_{f}}{P_{c}}\right)^{\frac{1}{n-1}}\right)
\end{gathered}
$$

The coefficient $n$ has different values depending on whether the granular assembly is experiencing a dilation or compaction:

$$
n= \begin{cases}\frac{\sqrt{3}}{2} \sin (\phi) & \text { if } \nabla \cdot \mathbf{v}_{s} \geq 0 \\ 1.03 & \text { if } \nabla \cdot \mathbf{v}_{s} \leq 0\end{cases}
$$


In Eq. $14 n$ is a parameter for shape of yield surface.

\subsection{Discharge from a two-dimensional bin using continuum approach}

Discharge rate is the most important quantity measured in these simulations. In Fig. 2(a) the temporal variation of the discharge rates obtained from two different frictional models are compared. This result shows that, at early times there is a rapid increase in the discharge rate, which is then followed by a plateau region where the discharge rate did not vary appreciably with time. As Fig. 2(a) shows, that the steady discharge predicted by Princeton frictional model (shown with dashed line) is about $20 \%$ higher than the steady discharge obtained using Schaeffer frictional model (shown with solid line). This difference in the prediction of discharge rate is attributed to the fact that, in Princeton model the friction starts at a lower solid volume fraction (in these simulations it is set to 0.50) than the Schaeffer frictional model, where friction starts at maximum packing [12]. During the period of steady discharge, the depth of the material in the bin varied considerably. The discharge rate is, therefore, roughly independent of the height of the material in the bin. Experimentally, it has long been known that the flow rate of Geldart type B granular material (the same type of particles simulated in this study) from bins and hoppers is independent of the surcharge level [19]. The discharge

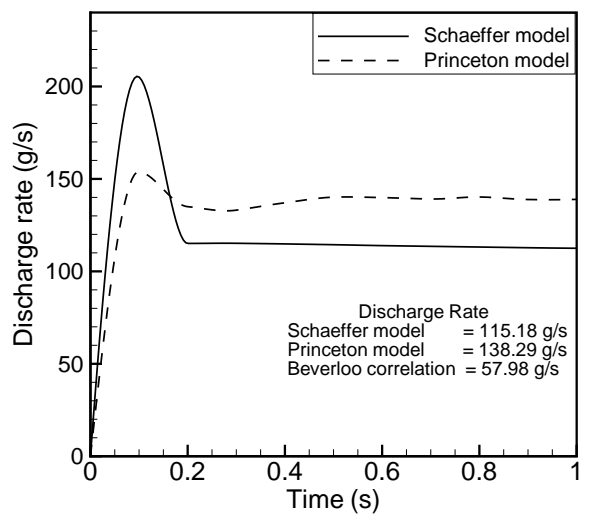

(a)

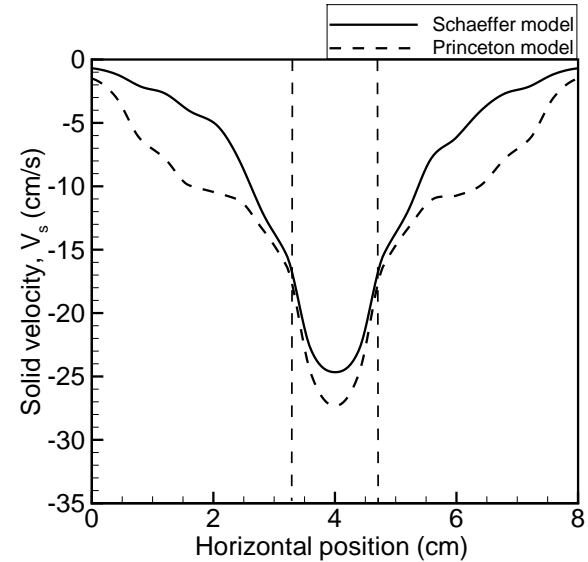

(b)

Fig. 2. (a) Temporal variation of discharge rate using two different frictional flow model. The solid line shows the discharge profile obtained from Schaeffer model, whereas the dashed line is the prediction for the discharge rate obtained using Princeton model, and (b) Transverse velocity profile during steady discharge at time $t=4.0 \mathrm{sec}$, at $1 \mathrm{~cm}$ above the discharge orifice. The solid line is the predicted profile of solid velocity by Schaeffer frictional model, whereas the dashed line show the prediction obtained using Princeton model. The dashed vertical lines indicate the location of edge of the orifice. 
rates obtained using both these frictional theories are compared with the Beverloo correlation [9], which is written by Srivastava and Sundaresan [11] for a $2 \mathrm{D}$ bin as:

$$
\dot{m}=C \rho_{b} g^{0.5} D^{1.5} H_{0} .
$$

Equation 15 gives the discharge rate $\dot{m}$ as function of the width of the orifice $D$. Here $C$ is an empirical dimensionless constant in the range of $0.55<C<$ 0.65. The initial solid bulk density is $\rho_{b}=\rho_{s} \nu$, and $g$ is the gravitational acceleration. The depth of the bin $H_{0}$ is taken as $1 \mathrm{~cm}$ in this study. The empirical constant $C$ is computed based on the steady discharge obtained from these two different frictional flow models. The computed value of $C$ (the empirical constant in the Beverloo correlation) comes out to be 1.24 and 1.54 for the Schaeffer and Princeton frictional models, respectively. In their original work Srivastava and Sundaresan [11] reported $C \approx 1.6$ based on steady discharge rates obtained in their simulations. It is noteworthy that, $C$ value computed from both these frictional models are significantly larger than typical experimental values [9] (which ranges between 0.55 to 0.65 ).

The discharge rate from the silo is closely related with the discharge velocities of solids near the orifice. Figure 2(b) compares the instantaneous transverse velocity profile at $1 \mathrm{~cm}$ above orifice for two different frictional models. This profile is extracted during steady discharge at time $t=4.0 \mathrm{sec}$. The dashed vertical line shows the location of edges of the orifice. As Fig. 2(b) shows, the velocity of solid particles increases as it approaches towards orifice for both of these models. The solid velocity attains a highest value at the center of the bin. Near the wall the particles flow with very low velocity as seen in Fig. 2(b). As expected from the result of discharge rates (see Fig. 2(a)) the solid velocity near the orifice is larger for Princeton model than Schaeffer model, which leads to higher discharge rate prediction from the Princeton model. It is clear from Fig. 2(b) that, there is some flow even in the corners of the bin, regions which should truly be stagnant. Hence, it can be inferred that these frictional models are unable to predict the formation of the experimentally observed stagnant zones on either side of the orifice.

\section{Discrete element method (DEM) simulations}

These three-dimensional (3D) DEM simulations are performed to model the discharge of granular particles from a flat-bottomed silo. For all DEM simulations performed in this study the granular material is modeled as a particle assembly consisting of monodisperse, spherical, cohesionless particles of diameter $d_{p}$ and mass $m_{p}$. A soft sphere model is used, in which particles interact via contact laws and friction only on contact. Since the realistic modeling of particle deformation is complicated, a simplified contact force and the overlap relation [20], the spring-dashpot model, is used in this work. Details of 
the computational model used in the discrete element simulations are given in Appendix $A$, and additional details can be found in Silbert et al. [20], and Sun et al. [21]. For all the DEM simulations reported here, the mass and diameter of particles are set 1 , so the density of particles turns to be $6 / \pi$. The value of normal spring constant $k_{n}$ was set to be 191000 which capture the general behavior of intermediate to high $k_{n}$ system [20].

\subsection{DEM simulations of Silo discharge}

The movement of individual particles during the outflow caused by gravity, is studied using 3D discrete element method (DEM) simulations. All the cases are simulated using a molecular dynamics code LAMMPS [22]. The domain size selected for the simulations is $18 \times 18 \times 36$ particle diameter units in $x, y$ and $z$ direction respectively. The domain size is selected in such a manner that the discharge rate remains unchanged with any further change in the domain size. The only external force acting on the system is gravity, which acts in the negative $z$ direction. In all the simulations the discharge outlet is circular in shape with diameter $6 d_{p}$, where $d_{p}$ is the particle diameter (except for some simulations which are performed to quantify the effect of discharge outlet size on the discharge rate). The domain is bounded with flat-frictional wall in all the directions $(x, y$ and $z)$. The number of particles simulated in this study ranges from 11136 to 13340 depending on the solid volume fraction of specific simulation, for a given domain size.

To ensure a constant and domain size independent discharge rate from the silo, following design constraints are used [15],

(1) $H>D$, where $H$ is the fill height at centerline and $D$ is the size of discharge outlet.

(2) $W>2.5 D$, where $W$ is the silo width and $D$ is the size of discharge outlet.

(3) $D \geq 6 d_{p}$, where $D$ is the size of discharge outlet and $d_{p}$ is the particle diameter.

In order to ensure that the discharge rate from the silo remains unchanged with the domain size selected, we performed simulations with different domain sizes and study the discharge rate. Figure 3 shows the amount of mass discharged with time for a simulation with initial solid volume fraction of 0.55 for three domain sizes of $15 d_{p} \times 15 d_{p} \times 30 d_{p}, 16 d_{p} \times 16 d_{p} \times 30 d_{p}$ and $18 d_{p} \times 18 d_{p} \times 36 d_{p}$ respectively. The slope of the straight portion of the plot gives the discharge rate. It can be inferred from this plot that the discharge rate is almost independent of the domain size selected, provided it meets the minimum design constraints $(W>2.5 D)[15]$. This result is in good agreement with the find- 
ings of Brown and Richards [23], who reported that discharge rate remains constant as long as $W>2.5 \mathrm{D}$. Figures 4(a), 4(b) and 4(c) show the snap-

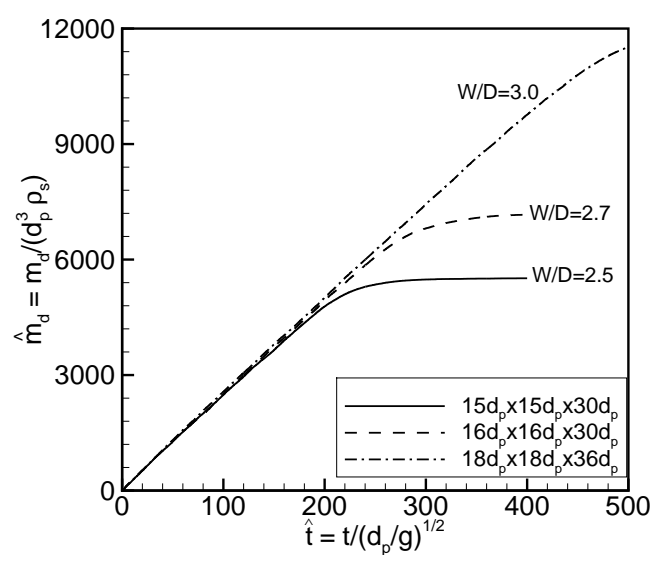

Fig. 3. Effect of domain size on discharge rate from flat-bottomed silo. Simulation parameters: $D=6 d_{p}, \mu_{p}=\mu_{w}=0.1, e=0.88$.

shots of the particle discharge from flat-bottomed silo at time $t=\frac{T_{d}}{40}, t=\frac{T_{d}}{2}$ and $t=\frac{T_{d}}{4}$ respectively, where $T_{d}$ is the discharge time scale (time required to discharge the complete mass from silo) extracted from the DEM simulations. These figures show that, as the simulation evolves with time, the number of particles in the domain decreases due to discharge from the bottom orifice.

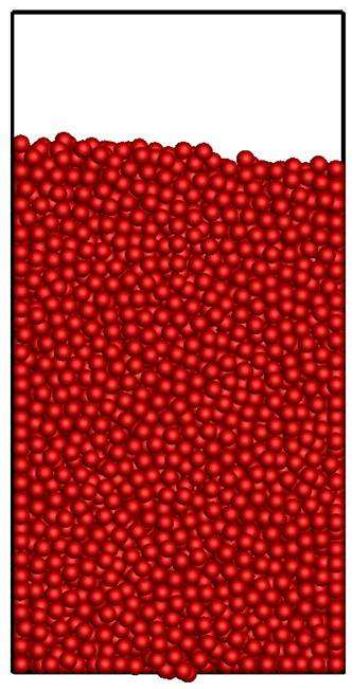

(a)

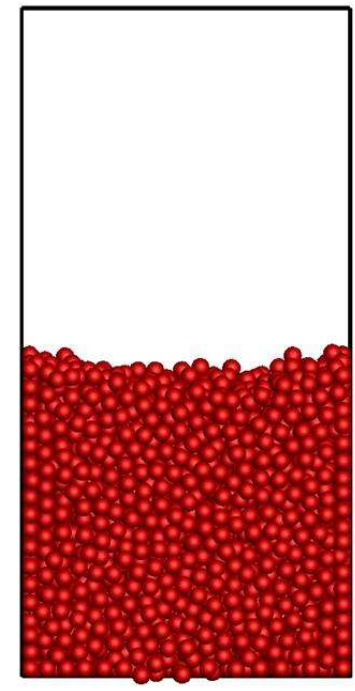

(b)

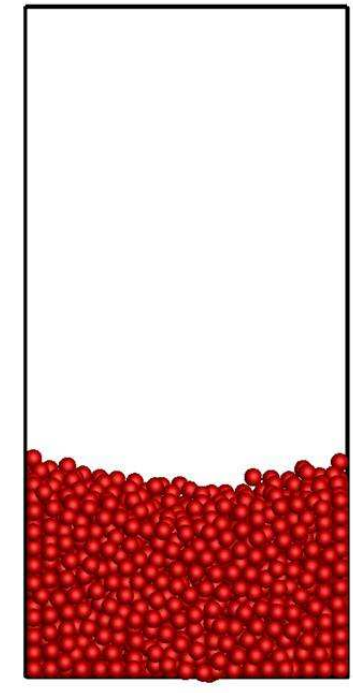

(c)

Fig. 4. (a) Snapshot of the particle discharge from silo at time $t=\frac{T_{d}}{40}$, (b) Snapshot of the particle discharge from silo at time $t=\frac{T_{d}}{2}$, and (c) Snapshot of the particle discharge from silo at time $t=\frac{T_{d}}{4}$. 


\subsection{Quantitative comparison between DEM and continuum simulations using discharge from a three-dimensional silo}

To perform a quantitative assessment of different continuum frictional models, we directly compare the results for the discharge rates, solid velocities and solid stresses obtained from DEM and continuum simulations for a same 3D silo geometry. The domain size selected is $18 \times 18 \times 36$ particle diameter in $x$, $y$ and $z$ direction. The discharge outlet is located at the center of the domain. The only difference in the both kinds of simulations is that, the continuum simulations has a square outlet, whereas the DEM simulations has a circular outlet. However, the effective diameter (hydraulic diameter) of both the discharge outlets are kept same as $6 d_{p}$. The boundary condition for the continuum simulations are same as the continuum simulations of two-dimensional bin (which is explained in Sec. 2.1). The particle properties (the solid density, and the particle diameter) are kept same as discussed in Sec. 2.1. The initial solid volume fraction for these simulations are set to 0.60 .

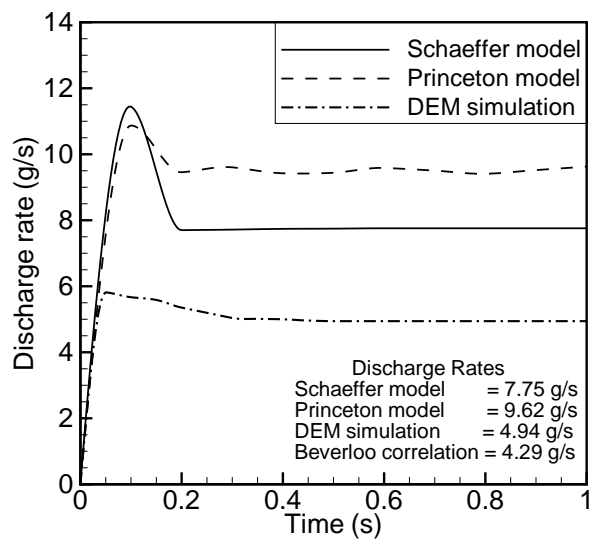

Fig. 5. Temporal variation of discharge rate using two different frictional flow models and DEM for a three-dimensional flat-bottomed silo. The solid line shows the discharge profile obtained from Schaeffer model, the dashed line is the prediction for the discharge rate obtained using Princeton model, whereas the dash dot line shows the temporal variation of discharge rate obtained from DEM simulation. Simulation parameters: $D=6 d_{p}, \mu_{p}=0.5, e=0.91$.

Figure 5 shows the temporal variation of the discharge rate for a 3D flatbottomed silo. The solid line shows the profile of discharge rate obtained with Schaeffer model, the dashed line shows the discharge rate obtained with the Princeton model and the discharge rate obtained from DEM simulation is shown with dash dot line in the same figure. As Fig. 5 shows, the steady discharge obtained from Schaeffer model is $7.75 \mathrm{~g} / \mathrm{s}$, whereas the steady discharge obtained from Princeton model is $9.62 \mathrm{~g} / \mathrm{s}$. We also compute the discharge rate from DEM simulation and Beverloo correlation and compare them with the 
discharge rate obtained from continuum simulations. These calculation are done for a particle with density of $2.9 \mathrm{~g} / \mathrm{cm}^{3}$, and the diameter of the particles are set to $0.1 \mathrm{~cm}$. For these particle properties, DEM predicts a steady discharge rate of $4.94 \mathrm{~g} / \mathrm{s}$ (as shown with dash dot line in Fig. 5). The Beverloo correlation, which is given by Eq. 16:

$$
\dot{m}=0.58 \rho_{b} g^{0.5}\left(D-k d_{p}\right)^{2.5}
$$

predicts a discharge rate of $4.29 \mathrm{~g} / \mathrm{s}$. In Eq. $16, \dot{m}$ is the discharge rate, $\rho_{b}$ is the bulk density, $g$ is the acceleration due to gravity, $D$ is the outlet discharge size, $k$ is the Beverloo constant and $d_{p}$ is the particle diameter. From this result it is clear that, both of these continuum frictional models for granular flow over predicts the discharge rate by more than $80 \%$, when compared with the discharge rate obtained using Beverloo correlation. However, there is a very good agreement between the discharge rate predicted using DEM simulations and Beverloo correlation, which also verifies the accuracy of our DEM simulations. We also compare the discharge velocities obtained using different frictional models to the discharge velocities obtained from DEM simulations, which is shown in Fig. 6. As expected the discharge velocities predicted by Princeton model are higher than the discharge velocity computed using Schaeffer frictional model. The discharge velocities predicted by the DEM simulations are lowest, which also verifies the lower discharge rate prediction from the DEM simulations.

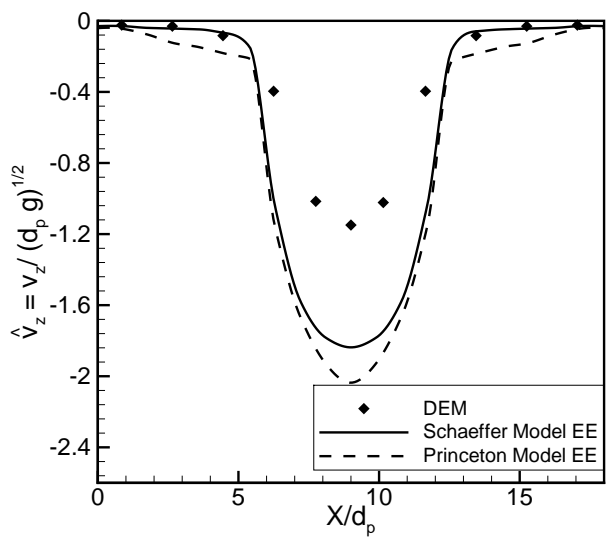

Fig. 6. Transverse solid velocity profile near the orifice during steady discharge. The solid line is the predicted profile of solid velocity by Schaeffer frictional model, whereas the dashed line shows the prediction obtained using Princeton model. The filled diamonds are the solid velocity data obtained from the DEM simulations. Simulation parameters: $D=6 d_{p}, \mu_{p}=0.5, e=0.91$.

Further, the error incurred in the stress prediction is quantified by comparing the predicted granular stress (using continuum models) with that of DEM data. We extract stresses from both continuum and DEM simulations in dif- 
ferent cells, and quantify the error using vector norm of the relative error (see equation 17) in each cell. These stresses are extracted in a slice of thickness $2 d_{p}$ (along $y$ direction), which is located at the center of the silo. In $x$ (along width of the silo) and $z$ (along height of the silo) a grid size of $2 d_{p}$ is used to perform this error analysis. Figures $7(\mathrm{a})$ and $7(\mathrm{~b})$ are the contour plots of error $(\hat{\epsilon})$ in solid stress prediction using Schaeffer and Princeton models, respectively. These figures show that the maximum error incurred in solid stress prediction (when compared with the stresses obtained from DEM simulations) is around $42 \%$ and $56 \%$ for Schaeffer and Princeton model, respectively.

$$
\hat{\epsilon}=\frac{\left\|\left(\sigma_{i j}\right)_{M o d e l}-\left(\sigma_{i j}\right)_{D E M}\right\|_{2}}{\left\|\left(\sigma_{i j}\right)_{D E M}\right\|_{2}}
$$

It is also interesting to note that, the stresses predicted by the DEM are high-

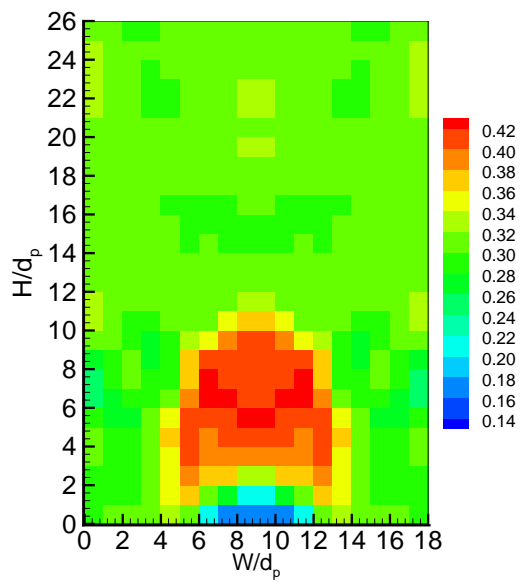

(a)

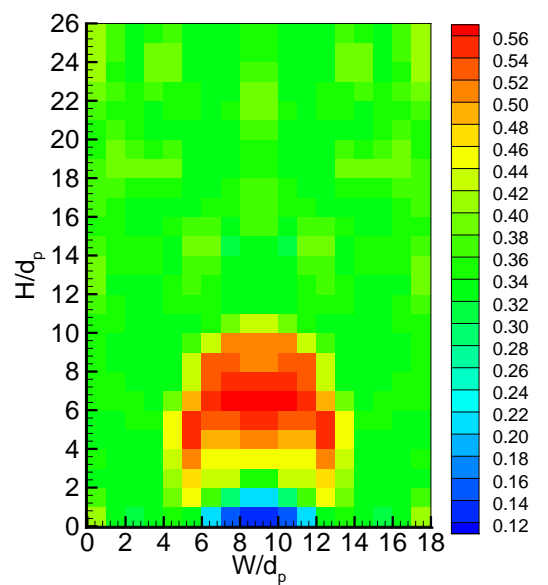

(b)

Fig. 7. Error in stress prediction (error quantified using vector norm of relative error, see equation 17). (a) For Schaeffer frictional model, and (b) For Princeton frictional model. Simulation parameters: $D=6 d_{p}, \mu_{p}=0.5, e=0.91$.

est in magnitude, whereas Princeton frictional model predicts lowest stresses. The reason for the lower stress prediction using Princeton model can be ascribed to the fact that, in Princeton model the friction starts at lower value of solid volume fraction than the Schaeffer frictional model (in which friction starts at maximum packing), hence the computed stresses are lower than that of Schaeffer frictional model. Jalali et al. [24] performed a similar study, where they compared the solid phase momentum transfer between continuum and DEM simulations, and showed that the DEM calculations can be about three times larger than that calculated from continuum models. 


\subsection{Influence of different physical parameters on silo discharge}

In order to quantify the influence of different physical parameters on the discharge rate from silo, we perform series of DEM simulations with different values of these parameters and study the discharge rate. The domain size selected for these simulations is $18 \times 18 \times 36$ particle diameter in $x, y$ and $z$ direction. The size of discharge outlet which is circular in size is set to be 6 particle diameter, other parameter are kept same as discussed in Sec. 3.1. Friction is expected to play an important role in determining the discharge rate from silo. However, in most of the experimental correlation it is an implicit assumption that friction coefficient (both particle-particle and particle-wall) plays little or no role in the determining the discharge rate from silos or hoppers. In order to quantify the effect of friction coefficient (both particle-particle and particle-wall), we performed simulations with different sets of particleparticle and particle-wall friction coefficient and compute the discharge rate.

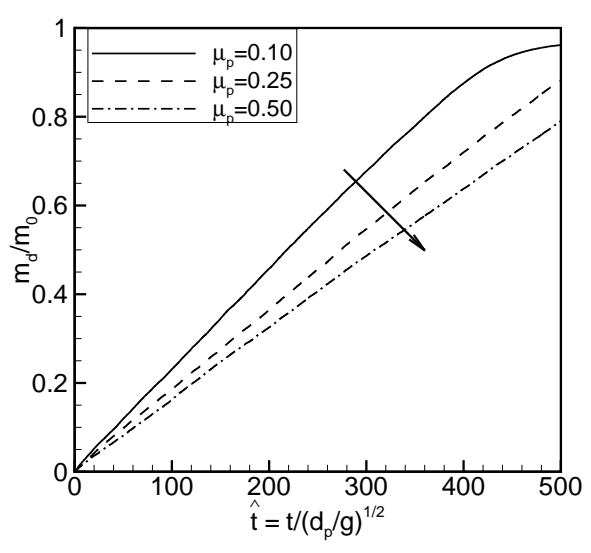

(a)

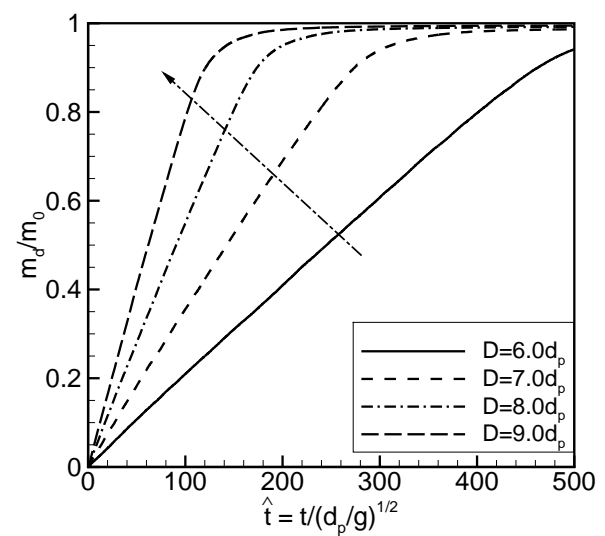

(b)

Fig. 8. (a) Amount of mass discharged (scaled with the initial mass in the silo) with time for 3 different values of particle-particle friction coefficient. Simulation parameters: $\mu_{w}=0.1, e=0.88$, and (b) The amount of mass discharged (scaled with the initial mass in the silo, $m_{0}$ ) plotted with time for different sizes of discharge outlet. Simulation parameters: $\mu_{p}=\mu_{w}=0.1, e=0.88$.

In Fig. 8(a) the amount of mass discharged (scaled with initial mass in the silo, $\left.m_{0}\right)$ is plotted with time for 3 different values of particle-particle friction coefficient of $0.10,0.25,0.50$ for a fixed wall friction coefficient of 0.10 . Figure 8 (a) shows that, as the particle-particle coefficient of friction increases the discharge rate decreases. The discharge rate decreases by about $30 \%$ when the particle-particle friction coefficient increases from 0.1 to 0.5 . Hence, it can be concluded that, the particle-particle friction coefficient is a primary parameter which governs the discharge rate from the silo, and hence cannot be neglected, 
as generally assumed in experimental correlations. However, our DEM simulations reveal (results not shown here) that, the wall friction coefficient has a negligible or no influence on the discharge rate from silo. Increasing the wall friction coefficient from 0.10 to 0.75 also does not lead to any significant change in the discharge rate. This result can be attributed to the fact that, for a broad silo $(W / D=3)$, the wall friction coefficient does not affects the flow near the orifice outlet and hence has a little effect on the discharge rate.

The coefficient of restitution is one such another parameter which has not been explored in the experimental studies. In order to understand its the influence on the discharge rate, we performed simulations with different values of particle restitution coefficient (ranging from 0.70 to 0.95 ). Nevertheless, we find almost no change in the discharge rate (results not shown here) when particle restitution coefficient is increased from 0.70 to 0.95 . In a study performed by Ristow [25] reported a change in discharge rate of $1.2 \%$ when the coefficient of restitution increased from 0.3 and 0.7 . This finding can be ascribed to the fact that, inside the flow through silo or hopper, the flow is dominated by long-lasting, frictional, multiparticle contacts, hence it is not surprising that the effect of coefficient of restitution will have a negligible influence on the flow dynamics.

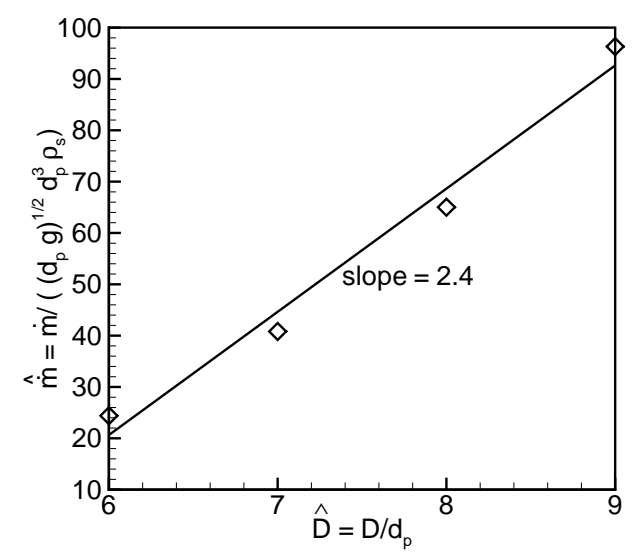

Fig. 9. Discharge rate with orifice outlet width. Simulation parameters: $\mu_{p}=\mu_{w}=0.1, e=0.88$.

Discharge outlet size is one of the other important parameter which affects the discharge rate from the silo. Figure 8(b) shows the amount of mass discharged (scaled with the initial mass in the silo, $m_{0}$ ) with time for 4 different outlet sizes of $6 d_{p}, 7 d_{p}, 8 d_{p}$ and $9 d_{p}$. It can be seen from Fig. 8(b) that, the discharge rate increases with increase in the discharge outlet size, and it is one of the most important parameter in the silo discharge problem. Discharge rate increases almost four times by increasing the discharge outlet size from $6.0 d_{p}$ to $9.0 d_{p}$. In Fig. 9 discharge rate is plotted with discharge outlet size $\hat{D}=D / d_{p}$ for a 
simulation with particle-particle and particle-wall friction coefficient of 0.10 . As Fig. 9 shows, the discharge rate is a function of outlet width raised to 2.4 power for circular orifices, which matches extremely well with the Beverloo correlation, which predicts the discharge rate as function of outlet width raised to 2.5 power for a three-dimensional silo (see Eq. 16).

\section{Characterization of different regimes in silo discharge}

Granular materials exhibit different constitutive behaviors in different regimes of flow, depending up on particle and flow properties. In general these regimes are classified based on scaling of shear stress with strain-rate [26]. In order to quantify the spatial extent of different regimes in a silo discharge problem, we first established a regime map for granular flows using DEM simulation data obtained from homogeneously sheared assembly of granular particles, for a range of solid volume fractions, shear rates and particle friction coefficients. Later, this regime map is used as a guideline to quantify the spatial extent of different regimes in a silo discharge problem.

\subsection{Homogeneous shear simulations}

A regime map for the granular flows is established using data obtained from the DEM simulations of homogeneously sheared granular flow. These constantvolume DEM simulations of homogeneously sheared granular flows are performed in a cubical domain of $14 \times 14 \times 14$ particle diameter units, for solid volume fraction ranging from 0.45 to 0.62 . The effect of the control volume size is tested by examining cubical volumes ranging in size from $7 d_{p} \times 7 d_{p} \times 7 d_{p}$ to $20 d_{p} \times 20 d_{p} \times 20 d_{p}$. No effect is found on the resultant stress as long as side of the cube selected is at least $10 d_{p}$. A similar study was performed by Campbell [26], where he showed that the resultant stress remains unchanged as long as the dimensions were at least $7 d_{p} \times 7 d_{p} \times 7 d_{p}$. These zero gravity, homogeneous shear simulations are performed with periodic boundary conditions in all directions $(x, y, z)$ and uniform shear is generated in the domain using the "SLLOD" algorithm [27]. The time step $\Delta t$ for the simulations is selected to be one fiftieth of the binary collision time $t_{c}$, which is small enough for a temporally converged numerical simulations [20]. Simulations are run to a nondimensional time of $\dot{\gamma} t=500$, which is long enough to attain a statically stationary solution [26]. After reaching the steady state, the quantities are averaged over nondimensional time of $\dot{\gamma} t=200$.

In order to establish a regime map, each of these simulations is assigned dif-

ferent regimes (inertial, intermediate or quasi-static) based on the scaling of 
shear stress with strain rate. In inertial regime the stress scales as square of strain rate $\left(\sigma \propto \dot{\gamma}^{2}\right)$ [28], whereas in the quasi-static regime stress remains independent of the strain rate $(\sigma \neq f(\dot{\gamma}))[26]$. In between these two extreme regime there exist an intermediate regime where stress is related with strain rate in form of power law $\left(\sigma \propto \dot{\gamma}^{n}\right)$, where $n$ takes values between 0 to 2 based on particle and flow properties [8]. Figures 10(a) and 10(b) show such regime maps obtained from the DEM simulations of homogeneously sheared granular flow in space of solid volume fraction $\nu$, particle friction coefficient $\mu_{p}$ for non-dimensional shear rate $k^{*}$ of $2.5 \times 10^{4}$ and $10^{9}$, respectively. In Figs. 10(a) and $10(\mathrm{~b})$ the inertial regime $\left(\sigma \propto \dot{\gamma}^{2}\right)$ is represented with red color, whereas the blue color shows the presence of quasi-static regime $(\sigma \neq f(\dot{\gamma}))$. In between both these regimes, there exists an intermediate regime $\left(\sigma \propto \dot{\gamma}^{n}, 0<n<2\right)$ which is represented with green color in Figs. 10(a) and 10(b).

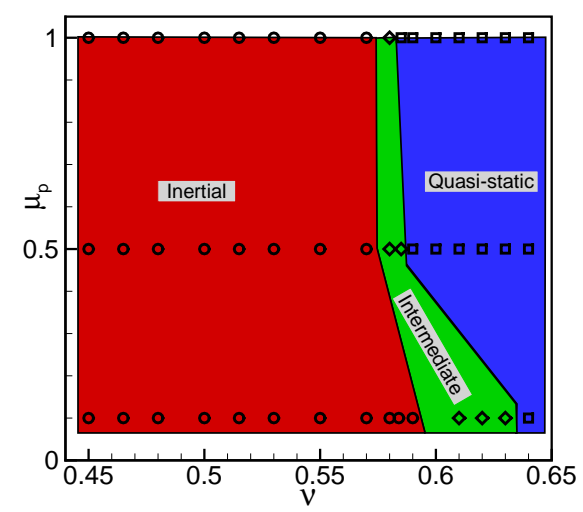

(a)

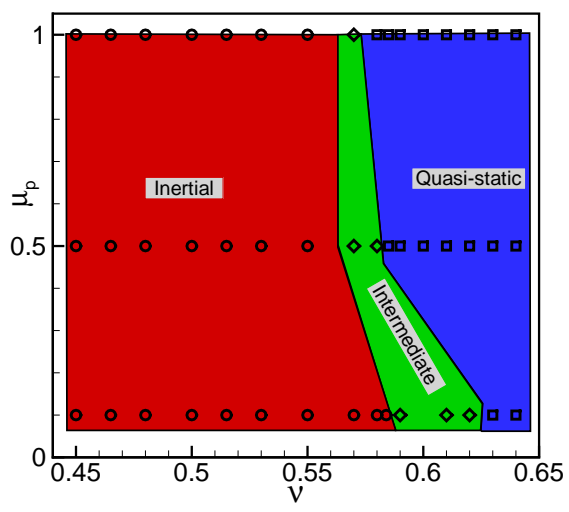

(b)

Fig. 10. Regime map for granular flows, constructed from data of 3D DEM simulations of homogenously sheared granular flow (a) $k^{*}=k_{n} / \rho_{s} d_{0}^{3} \dot{\gamma}^{2}=2.5 \times 10^{4}$ and (b) $k^{*}=k_{n} / \rho_{s} d_{0}^{3} \dot{\gamma}^{2}=10^{9}$.

\subsection{Spatial extent of different regimes in silo discharge}

The spatial extent of different regimes inside a silo geometry is characterized with the help of regime map established in Sec. 4.1 (see Figs. 10(a) and 10(b)). The idea is to extract values of local solid volume fraction, mean strain rate in each cell, from DEM simulation of flat-bottomed silo and assign them different regimes (inertial, intermediate and quasi-static) following regime map established in Figs. 10(a) and 10(b). These calculations are performed in a thin slice of thickness $2 d_{p}$ (in $y$ direction) which is located at the center of the silo. In the $x$ (along width of the silo) and $z$ direction (along the height of the silo) a grid size of $2 d_{p}$ is used to extract local quantities such as, solid

volume fraction and mean strain rate. Using this criterion, different cells are 
assigned an integer value of 0,2 and 1 for quasi-static, inertial (rapid flows) and intermediate regimes respectively. Steps followed to quantify the spatial extent of different regimes in a silo geometry is explained below:

(1) Solid volume fraction: At different spatial locations the local solid volume fraction is obtained from the DEM data of silo discharge.

(2) Mean strain rate: The strain rate tensor in 3D is given by,

$$
\dot{\gamma}_{i j}=\left[\begin{array}{ccc}
\dot{\gamma}_{x x} & \dot{\gamma}_{x y} & \dot{\gamma}_{x z} \\
\dot{\gamma}_{y x} & \dot{\gamma}_{y y} & \dot{\gamma}_{y z} \\
\dot{\gamma}_{z x} & \dot{\gamma}_{z y} & \dot{\gamma}_{z z}
\end{array}\right] .
$$

To compute the mean strain rate the second invariant of strain rate tensor $\left(I_{2 D}\right)$ is used,

$$
I_{2 D}=\frac{1}{2}\left[[\operatorname{tr}(\dot{\gamma})]^{2}-\operatorname{tr}\left(\dot{\gamma}^{2}\right)\right] .
$$

Using Eq. 18 and the definition of the strain rate tensor $\dot{\gamma}_{i j}$, the second invariant of the strain rate tensor can be written as,

$$
I_{2 D}=\left[\left(\dot{\gamma}_{x x} \dot{\gamma}_{y y}+\dot{\gamma}_{y y} \dot{\gamma}_{z z}+\dot{\gamma}_{x x} \dot{\gamma}_{z z}\right)-\left(\dot{\gamma}_{x y} \dot{\gamma}_{y x}+\dot{\gamma}_{x z} \dot{\gamma}_{z x}+\dot{\gamma}_{y z} \dot{\gamma}_{z y}\right)\right]
$$

(3) Particle friction coefficient: Two different values (0.50 and 0.25) of particle friction coefficient is used to characterize the spatial extent of different regimes in a silo geometry. These values of friction coefficient offer a reasonable representation of general granular material [20].

We extract these quantities (local solid volume fraction and mean strain rate) from DEM simulation of discharge from a flat-bottomed silo and assign each cell a value 0 (for quasi-static regime), 2 (for inertial regime), or 1 (for intermediate regime). Figures 11(a) and 11(b) show the spatial extent of different regimes in flat-bottomed silo obtained using above described method for a particle friction coefficient of 0.50 and 0.25 , respectively. In Figs. 11(a) and 11(b) the red color represents the presence of inertial regime (which found to be exist near the discharge orifice), the blue color in the same figure represent the presence of quasi-static regime, any other color represents the presence of intermediate regime. From this study, it is evident that all three different regimes (inertial, intermediate, and quasi-static) co-exist even for a simple problem like discharge of granular particles from the silo. It is also interesting to notice that, the intermediate regime spans a considerable spatial region in the silo. A careful study of these figures reveal that the spatial extent of intermediate regime expands as the particle friction coefficient decreases from 0.50 to 0.25 . It is interesting to correlate Figs. 7(a), 7(b), 11(a) and 11(b), close examination of these figures reveal that the maximum error incurred in the stress prediction spreads around the spatial location where intermediate regime is present. Hence, it can be concluded that the widely used continuum 


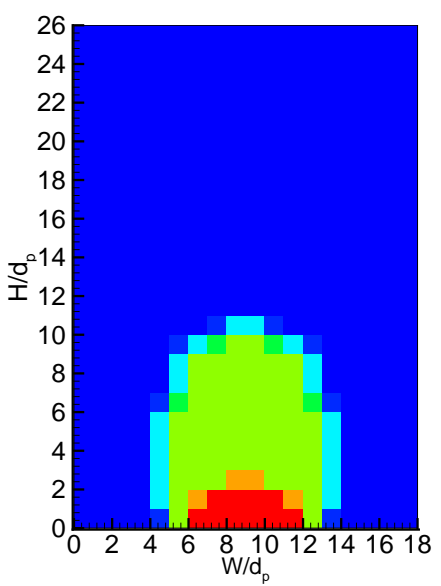

(a)

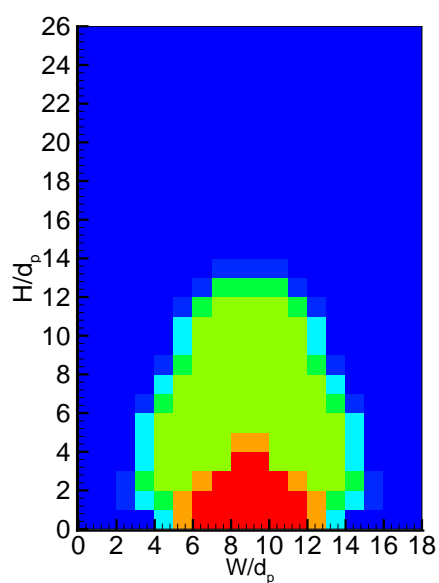

(b)

Fig. 11. Characterization of spatial extent of different regimes of granular rheology in a flat-bottomed silo based on local solid volume fraction, mean strain rate and particle friction coefficient. The Blue color represents quasi-static regime, the Red color represents the inertial (rapid flow) regime, whereas presence of any other color indicates the spatial extent of the intermediate regime. (a) Simulation parameters: $D=6 d_{p}, \mu_{p}=0.5, e=0.91$, and (b) Simulation parameters: $D=6 d_{p}, \mu_{p}=0.25$, $e=0.91$.

models perform poorly in the intermediate (transitional) regime of granular flows, where both collisional and frictional interaction between the particles are important.

\section{Conclusions}

In this work, we study the discharge dynamics of granular particles from a silo geometry using both continuum and discrete approaches. Performance of different continuum frictional models for granular flows is assessed in a silo geometry by comparing their prediction for discharge rate with well-known correlation [9] and DEM simulations. In first phase of this study, discharge of granular particles from a 2D rectangular bin is performed using two different continuum frictional models (Schaeffer [5] and Princeton model [11]) and results are compared with Beverloo correlation. It is found that both these continuum models predicts discharge rate which are much higher than the discharge rate obtained using Beverloo correlation. It is also found that both of these frictional models are unable to capture the formation of stagnant shoulders at the corners of the bin, which has been observed in the experimental studies. In the second phase of this study a quantitative assessment of these two different frictional models are performed by directly comparing their results for discharge rates, solid velocities and stresses with that of the DEM simulations for a same flat-bottomed silo geometry. It is reconfirmed with 
these simulations that, both these continuum models (Schaeffer and Princeton models) over-predict the discharge rate from the silo. The DEM prediction of the discharge rate is in very good agreement with the discharge rate predicted by the Beverloo correlation. This also validates the accuracy of DEM simulations used in this study. The error incurred in solid stress prediction is quantified using vector norm of relative error, which shows a maximum error of $42 \%$ and $56 \%$ in solid stress prediction using Schaeffer and Princeton models, respectively. The parametric study performed to understand the influence of different physical parameters on silo discharge reveals that, the interparticle friction coefficient and the discharge outlet size are the most important parameters which govern the discharge rate from silo. However, we found almost no or negligible influence of wall friction and particle restitution coefficient on the discharge rate. Spatial extent of different regimes is quantified using a regime map established from data obtained from DEM simulations of homogeneously sheared granular flow. This analysis shows that, all three different regimes of granular flow (inertial, intermediate and quasi-static) co-exist in a silo discharge problem. This study also reveals that, the maximum error in solid stress prediction is near the spatial location where intermediate regime spans. This result motivates the need for accurate continuum models for dense granular flow, which can be applied to different regimes of granular flow with better accuracy.

\section{Acknowledgements}

The authors wish to acknowledge the financial support from U.S. department of Energy National Energy Technology Laboratory (grant DE-FG2607NT43070), administered under advanced coal research at U.S. colleges and universities.

\section{A Contact model description}

For two contacting particle $\{i, j\}$, with radii $\left\{a_{i}, a_{j}\right\}$ at positions $\left\{\mathbf{r}_{i}, \mathbf{r}_{j}\right\}$, with velocities $\left\{\mathbf{v}_{i}, \mathbf{v}_{j}\right\}$ and angular velocities $\left\{\boldsymbol{\omega}_{i}, \boldsymbol{\omega}_{j}\right\}$, the normal compression $\delta_{i j}$, relative normal velocity $\mathbf{v}_{n_{i j}}$, and relative tangential velocity $\mathbf{v}_{t_{i j}}$ are [20]:

$$
\begin{aligned}
\delta_{i j} & =d_{p}-\mathbf{r}_{i j} \\
\mathbf{v}_{n_{i j}} & =\left(\mathbf{v}_{i j} \cdot \mathbf{n}_{i j}\right) \mathbf{n}_{i j} \\
\mathbf{v}_{t_{i j}} & =\mathbf{v}_{i j}-\mathbf{v}_{n_{i j}}-\left(a_{i} \boldsymbol{\omega}_{i}+a_{j} \boldsymbol{\omega}_{j}\right) \times \mathbf{n}_{i j},
\end{aligned}
$$

where $d=a_{i}+a_{j}, \mathbf{r}_{i j}=\mathbf{r}_{i}-\mathbf{r}_{j}, \mathbf{n}_{i j}=\mathbf{r}_{i j} / r_{i j}$, with $r_{i j}=\left|\mathbf{r}_{i j}\right|$ and $\mathbf{v}_{i j}=\mathbf{v}_{i}-\mathbf{v}_{j}$. 
The rate of change of the elastic tangential displacement $\mathbf{u}_{t_{i j}}$, set to zero at the initiation of a contact is,

$$
\frac{d \mathbf{u}_{t_{i j}}}{d t}=\mathbf{v}_{t_{i j}}-\frac{\left(\mathbf{u}_{t_{i j}} \cdot \mathbf{v}_{i j}\right) \mathbf{r}_{i j}}{r_{i j}^{2}}
$$

The last term in Eq. A.4 arises from the rigid body rotation around the contact point and ensures that $\mathbf{u}_{t_{i j}}$ always lies in the local tangent plane of contact. Normal and tangential forces acting on particle $i$ are:

$$
\begin{gathered}
\mathbf{F}_{n_{i j}}=f\left(\delta_{i j} / d_{p}\right)\left(k_{n} \delta_{i j} \mathbf{n}_{i j}-\gamma_{n} m_{e f f} \mathbf{v}_{n_{i j}}\right), \\
\mathbf{F}_{t_{i j}}=f\left(\delta_{i j} / d_{p}\right)\left(-k_{t} \mathbf{u}_{t_{i j}}-\gamma_{t} m_{e f f} \mathbf{v}_{t_{i j}}\right),
\end{gathered}
$$

where $k_{n, t}$ and $\gamma_{n, t}$ are the spring stiffness and viscoelastic constants, respectively, and $m_{e f f}=m_{i} m_{j} /\left(m_{i}+m_{j}\right)$ is the reduced mass of spheres with masses $m_{i}$ and $m_{j}$. The corresponding contact force on particle $j$ is simply given by Newton's third law, i.e., $\mathbf{F}_{j i}=-\mathbf{F}_{i j}$. The function $f\left(\delta_{i j} / d_{p}\right)=1$ is for the linear spring-dashpot model, and $f\left(\delta_{i j} / d_{p}\right)=\sqrt{\delta_{i j} / d_{p}}$ is for Hertzian contacts with viscoelastic damping between spheres.

Static friction is implemented by keeping track of the elastic shear displacement throughout the lifetime of a contact. The static yield criterion, characterized by a local particle friction coefficient $\mu$, is modeled by truncating the magnitude of $\mathbf{u}_{t_{i j}}$ as necessary to satisfy $\left|\mathbf{F}_{t_{i j}}\right|<\left|\mu \mathbf{F}_{n_{i j}}\right|$. Thus the contact surfaces are treated as "sticking" when $\left|\mathbf{F}_{t_{i j}}\right|<\left|\mu \mathbf{F}_{n_{i j}}\right|$, and as "slipping" when the yield criterion is satisfied.

The amount of energy lost in collisions is characterized by the value of the coefficient of restitution, which is defined as the negative ratio of the particle normal velocity after collision to the velocity before collision. For the linear spring-dashpot model, the coefficient of normal restitution and contact time can be analytically obtained:

$$
e_{n}=\exp \left(-\gamma_{n} t_{\mathrm{c}} / 2\right)
$$

where the contact time $t_{c}$ is given by

$$
t_{c}=\pi\left(k_{n} / m_{\mathrm{eff}}-\gamma_{n}^{2} / 4\right)^{-1 / 2}
$$

The value of the spring constant should be large enough to avoid particle interpenetration, yet not so large as to require an unreasonably small simulation time step $d t$, since an accurate simulation typically requires $d t \sim t_{c} / 50$ [26]. After the contact force is calculated, the equation of motion, which are ordinary differential equations, can be numerically integrated to get the particle trajectories. 


\section{References}

[1] D. Fenistein and M. van Hecke, "Kinematics-wide shear zones in granular bulk flow.," Nature, vol. 425, pp. 256-256, 2003.

[2] P. Cundall and O. Strack, "A discrete numerical model for granular assemblies.," Géotechnique, vol. 29, pp. 47-65, 1979.

[3] C. Lun, S. Savage, D. Jeffrey, and N. Chepuriny, "Kinetic theories for granular flow:inelastic particles in couette flow and slightly inelastic particles in general flow fields.," J. Fluid Mech., vol. 140, pp. 223-256, 1984.

[4] J. Jenkins and S. Savage, "A theory for the rapid flow of identical, smooth, nearly elastic, spherical particles.," J. Fluid Mech., vol. 130, pp. 187-202, 1983.

[5] D. Schaeffer, "Instability in the evolution equations describing incompressible granular flow.," Journal of Differential Equations, vol. 66, pp. 19-50, 1987.

[6] R. M. Nedderman, Static and kninematics of granular material. Cambridge University Press, 2nd. ed., 1992.

[7] G.D.R. MiDi, "On dense granular flows.," Eur. Phys. J. E, vol. 14, pp. 341-365, 2004.

[8] G. Tardos, S. McNamara, and I. Talu, "Slow and intermediate flow of a frictional bulk powder in the couette geometry.," Powder Technology, vol. 131, pp. 23-39, 2003.

[9] W. Beverloo, H. Leniger, and J. van de Velde, "The flow of granular solids through orifices.," Chemical Engineering Science, vol. 15, pp. 243-250, 1961.

[10] M. Syamlal, W. Rogers, and T. J. O'Brien, "Mfix documentation: Theory guide," Tech. Rep. DOE/METC-95/1013, NTIS/DE95000031, National Energy Technology Laboratory, Department of Energy, 1993. See also URL http://www.mfix.org.

[11] A. Srivastava and S. Sundaresan, "Analysis of a frictional-kinetic model for gas-particle flow.," Powder Technology, vol. 129, pp. 72-85, 2003.

[12] S. Benyahia, "Validation study of two continuum granular frictional flow theories.," Ind. Eng. Chem. Res., vol. 47, pp. 8926-8932, 2008.

[13] W. Ketterhagen, C. Jennifer, C. Wassgren, and B. Hancock, "Predicting the flow mode from hoppers using the discrete element method.," Powder Technology, vol. 195, pp. 1-10, 2009.

[14] T. Goda and F. Ebert, "Three-dimensional discrete element simulations in hoppers and silos.," Powder Technology, vol. 158, pp. 58-68, 2005.

[15] A. Anand, J. Curtis, C. Wassgren, B. Hancock, and W. Ketterhagen, "Predicting discharge dynamics from a rectangular hopper using the discrete element method.," Chemical Engineering Science, vol. 63, pp. 58215830, 2008.

[16] J. Landry, S. Gary, and S. Plimpton, "Discrete element simulations of stress distribution in silos: crossover from two to three dimensions.," Powder Technology, vol. 139, pp. 233-239, 2004. 
[17] P. Johnson and R. Jackson, "Frictional collisional constitutive relations for granular materials, with application to plane shearing.," J. Fluid Mech., vol. 176, pp. 67-98, 1987.

[18] S. Savage, "Analyses of slow high-concentration flows of granular materials.," J. Fluid Mech., vol. 377, pp. 1-77, 1998.

[19] R. M. Nedderman, U. Tuzun, S. Savage, and G. Houlsby, "The flow of granular materials I: Discharge rate from hoppers.," Chemical Engineering Science, vol. 37, pp. 1597-1609, 1982.

[20] L. Silbert, D. Ertas, G. Grest, T. Halsey, D. Levine, and S. J. Plimpton, "Granular flow down an inclined plane: Bagnold scaling and rheology," Phys. Rev. E, vol. 64, pp. 051302-1-051302-13, 2001.

[21] J. Sun, F. Battaglia, and S. Subramaniam, "Dynamics and structures of segregation in a dense, vibrating granular bed." Phys. Rev. E, vol. 74, no. 061307, pp. 1-13, 2006.

[22] S. Plimpton, "Fast parallel algorithms for short-range molecular dynamics," Journal of Computational Physics, vol. 117, pp. 1-19, 1995.

[23] R. Brown and J. Richards, "Profile of flow of granules through apertures.," Transactions of the Institution of Chemical Engineering, vol. 38, pp. 260-269, 1960.

[24] P. Jalali and T. Hyppanen, "Verification of continuum models for solids momentum transfer by means of discrete element method.," Ind. Eng. Chem. Res., vol. 49, pp. 5270-5278, 2010.

[25] G. Ristow, "Outflow rate and wall stress for two-dimensional hoppers.," Physica A, vol. 235, pp. 319-326, 1997.

[26] C. Campbell, "Granular shear flows at the elastic limit.," J. Fluid Mech., vol. 465, pp. 261-291, 2002.

[27] G. Lois, A. Lemaitre, and M. Carlson, "Numerical test of constitutive laws for dense granular flows.," Phys. Rev. E, vol. 72, pp. 051303-1-051303-16, 2005.

[28] R. Bagnolds, "Experiments on a gravity-free dispersion of large solid spheres in a newtonian fluid under shear.," Proceedings of the Royal Society of London Series A: Mathematical and Physical Sciences, vol. 225, pp. 49-63, 1954. 


\section{Appendix $\mathbf{F}$}

Experimental and computational studies of dense granular flow: transition from quasi-static to intermediate regime in a Couette shear device 


\title{
Granular rheology and 'phase' transition through order parameter: DEM simulations and constitutive modeling
}

\author{
Vidyapati and S. Subramaniam \\ Department of Mechanical Engineering, Center for Computational Thermal-Fluids \\ Research, Iowa State University, Ames, IA 50011, USA
}

\begin{abstract}
DEM (discrete element method) simulations are used to understand the granular rheology and 'phase' transition by studying the order parameter (OP) dynamics. These DEM simulations reveal existence of a third intermediate steady state stable value of the OP, which is neither zero (fluidlike) nor one (solidlike). Hence, a modified form of the free energy density function is proposed to accommodate this third intermediate steady state stable value of the OP which is observed in the DEM simulations. Further a constitutive model for granular flows is developed based on an objective version [Gao et al., Phys. Rev. E, 71(021302), 2005] of the original OP concept proposed by Volfson et al. [Phys. Rev. E, 68(021301), 2003] with the intention of capturing the transitional behavior in a continuum description of granular flows. This OP-based model is refined by extracting new model coefficients from 3D DEM simulations of the homogeneous shear granular flows. The proposed linear version of the objective OP model has the advantage that the total granular stress is a linear combination of the solidlike and fluidlike stresses, and it is denoted as the refined order parameter (ROP) model. The performance of this ROP model along with four different existing constitutive models is assessed and results are explained by analyzing the granular stress data from DEM simulations.
\end{abstract}

Key words: Granular flow; Order parameter; Discrete element method; Constitutive model; Transitional regime

\section{Introduction}

The difficulty in treating granular material is that the grains can exhibit constitutive behavior like a solid (in a sand pile), like a liquid (when poured from a hopper or silo), or like a gas (when it is strongly agitated) [1]. These different constitutive behaviors depend on both, the microscale properties (e.g., 
particle friction and coefficient of restitution) as well as on macroscale properties (e.g., solid volume fraction and shear rate). However, a quantitative description of the large scale behavior of granular flow in industrial devices, such as hopper discharge, chute flow, and dense-phase pneumatic conveying, rely on a continuum description of granular flows [2]. These different behaviors pose significant challenges in formulating a comprehensive constitutive theory that can describe all the regimes of granular flow. For the two extreme regimes - rapid and quasi-static - constitutive equations have been proposed based on the kinetic theory of rapid flows [3] and soil mechanics for quasistatic flow $[4,5]$, respectively. However, the transitional (intermediate) regime, where both collisional and frictional interactions between the particles are important, still lacks a predictive constitutive model and has motivated many studies over the past decade $[6,7]$. Nevertheless, these theories were unable to capture the transition between solidlike and fluidlike behavior of the granular material.

Most constitutive models $[8,9,10]$ used to predict the behavior of granular flows are based on an additive decomposition of the total granular stress as a weighted sum of kinetic and frictional contributions $\left(\sigma_{i j}=\sigma_{i j}^{k i n}+\sigma_{i j}^{f r i c}\right)$, with the weight factor specified solely as a function of the solid volume fraction. Experiments in a 2D granular shear cell (GSC) $[11,12]$ as well as DEM simulations [13] reveal that grain contacts in the transitional regime are characterized by a mix of enduring solidlike and fluidlike contacts that is indicative of a granular 'phase' transition. In particular, these grain interactions are not determined by the solid volume fraction alone, but are dependent on particle properties (such as particle friction coefficient, inelasticity) and flow properties (such as shear rate). Consequently, the simple additive models are not capable of capturing this complex constitutive behavior in the transitional regime of granular flows. Since most constitutive models in use are phenomenological, this observation motivates the development of a constitutive model for the transitional regime that reflects the 'phase' transition based on microscale physical interaction between the grains.

We briefly review four constitutive models that we evaluated over a range of solid volume fractions, particle friction coefficients and shear rates. A continuum theory for slow dense granular flows based on so-called associated flow rule is proposed by Savage [8]. This theory relates the strain rate and the shear stress in a plastic frictional system. Averaging strain-rate fluctuations yields a Bingham-like constitutive relation in which the shear stress has two contributions: a viscous part, and a strain-rate independent part. According to this theory the stress and strain rate tensors are always coaxial. Furthermore, the theory also postulates that the viscosity diverges as the density approaches the close packing limit. A similar hydrodynamic model based on a Newtonian stress-strain constitutive relation with density-dependent viscosity is proposed by Losert et al. [14]. In this model also the viscosity diverges 
when the density approaches the random close packing density of grains. Jop et al. [6] proposed a constitutive relation for dense granular flows inspired by the analogy between granular flows and visco-plastic fluids such as Bingham fluids. In the Jop's model [6] granular flow is described as an incompressible fluid with the stress tensor given as a function of the inertia number, $I=\dot{\gamma} /\left(P / \rho_{s}\right)^{0.5}$.

Volfson et al. [13] proposed a different approach based on the order parameter (OP) description of granular matter. The OP is defined as the ratio of number of solidlike (enduring) contacts to all contacts in a given sampling volume. The OP attains its maximum value of 1 when the granular matter is in a 'solid' state and takes its minimum value of 0 in the completely 'fluid' limit. They decomposed the total granular stress $\sigma_{i j}$ into the sum of a "solidlike" stress $\sigma_{i j}^{s}$ and a "fluidlike" stress $\sigma_{i j}^{f}$. The relative magnitude of the solidlike and fluidlike contributions is a function of the OP. Models are then proposed for the "solidlike" and "fluidlike" contributions, in terms of the total granular stress tensor $\sigma_{i j}$. Nevertheless, the postulated form of the free energy density function $F(\rho, \delta)$ in this work always renders a steady state value of the OP which is either 0 or 1 . The $\mathrm{OP}$ values obtained from this procedure was never validated against DEM data in order to verify this postulated form of the free energy density function. Also this model [13] does not satisfy the objectivity requirement.

The original OP model by Volfosn et al. [13] was generalized to an objective form by Gao et al. [15], which is independent of the coordinate system. The model coefficients of the objective OP model specified by Gao et al. [15] are obtained by fitting DEM data for 2D inhomogeneous Couette flow with wall boundary conditions. In the present work, new model coefficients for the objective OP model are extracted from data of 3D DEM simulations of homogenous shear flows. The objective OP model [15] is further linearized to allow the inversion of the total granular stress from fluidlike or solidlike stress relations. This linearized OP model with new coefficients is denoted as the refined order parameter (ROP) model. Following Aranson and Tsimring [16], it is assumed that the fluidlike contribution of the total granular stress can be computed using a constitutive relation from the kinetic theory of granular flows (KTGF) [17]. Then the performance of the ROP model is assessed by comparing the predicted granular stress with DEM data in different regimes of granular flows.

The following section describes the order parameter. In the next section, details of the DEM simulations of sheared granular flow are presented and the OP dynamics obtained from 3D DEM simulations is discussed. The description and refinement of the OP-based continuum model is discussed in Sec. 4. Section 5 describes the specification for the proposed ROP model. The performance of the ROP model in different regimes is presented in Sec. 6. In Sec. 7 
we assess performance of four different constitutive models in the intermediate regime, and the results are explained by analyzing the granular stress data from DEM. Finally conclusions are drawn in Sec. 8.

\section{Order parameter description of granular 'phase' transition}

In a homogeneous granular flow, the OP is defined [13] as the ratio of the number of space-time averaged "enduring" (solidlike) contacts $\overline{\left\langle Z_{s}\right\rangle}$ to all contacts $\overline{\langle Z\rangle}$ within a sampling volume,

$$
\rho \equiv \frac{\overline{\left\langle Z_{s}\right\rangle}}{\overline{\langle Z\rangle}} .
$$

The OP is useful in characterizing two limiting cases: when the granulate is in a state of "enduring" contacts, and when it is strongly agitated, i.e., completely fluidlike. In the solid state all contacts are enduring and hence $\rho=1$. In the fluid limit $\overline{\left\langle Z_{s}\right\rangle}$ is zero and $\overline{\langle Z\rangle}$ is small but finite, and therefore $\rho=0$. Since the OP distinguishes between "solidlike" contacts and "fluidlike" contacts among all contacts in the granular material, its estimation requires a precise definition of these two types of contacts. A contact is considered enduring (solidlike), if it is in stuck state $\left(F_{t}<\mu_{t} F_{n}\right)$ and its duration is longer than a typical time of collision $t^{*}$, which is generally taken as 1.1 times the binary collision time $t_{c}[13]$. The first requirement eliminates long-lasting sliding contacts and the second requirement excludes short-term collisions. When either of the requirements is not fulfilled, the contact is defined as "fluidlike".

In order to understand the granular 'phase' transition through the OP we extract this quantity from 3D DEM simulations of sheared granular flow over a range of solid volume fractions, particle friction coefficients and shear rates. On the following section we describe these 3D DEM simulations of sheared granular flow.

\section{DEM simulations of sheared granular flow}

The OP is extracted by performing three-dimensional (3D) discrete element method (DEM) simulations of sheared granular flow on monodisperse, noncohesive spheres of diameter $d_{0}$ and mass $m_{0}$ over a range of solid volume fractions, particle friction coefficients and shear rates. A soft-sphere model is used in which particles interact via contact laws and friction only on contact. Since the realistic modeling of particle deformation is complicated, a simplified contact force model based on a linear spring-dashpot combination is used in 
this work [18]. Details of the computational model used in the discrete element simulations are given in Appendix A.

These constant-volume DEM simulations of sheared granular flow are performed in a cubical domain of side length $L=14 d_{0}$, for solid volume fraction ranging from 0.20 to 0.62 . The effect of the system size is examined by varying the side length from $7 d_{0}$ to $20 d_{0}$. Asymptotic stress was found after the side length exceeded $10 d_{0}$, consistent with the finding by Campbell [19]. For all the simulations reported, the mass and diameter of the particles are set to 1 , so the density of the particles turns to be $6 / \pi$. The value of normal spring stiffness $k_{n}$, is set to be $2 \times 10^{5}$ which capture the general behavior of intermediate to high $k_{n}$ system [18]. The value of the coefficient of restitution $e$ is chosen to be 0.7 . All these simulations are performed with zero gravity. The integration time step $\Delta t$ for all the simulations is selected to be $t_{c} / 50$, where $t_{c}$ is the binary collision time. This time step is shown to be sufficiently small to ensure temporal convergence [18]. Simulations are run to a nondimensional time of $\dot{\gamma} t=500$, which is long enough to attain a statistically stationary solution [19]. After reaching steady state the quantities are time-averaged over a time window corresponding to $200 \dot{\gamma}^{-1}$. As a first obvious step we verified our OP calculations with previously published results of Volfson et al. [20] for an inhomogeneous wall-bounded shear simulations and also confirmed that the OP is capable of capturing granular 'phase' transition from solidlike to fluidlike behavior. Details of these simulations are given in Appendix B.

For purpose of understanding the physics of granular 'phase' transition and to generate benchmark data for the model assessment in different regimes, we performed homogeneous shear simulations (where the stress remains independent of position) over a wide range of solid volume fractions, shear rates and particle friction coefficients. These homogeneous shear simulations are performed with periodic boundary conditions in all directions $(x, y, z)$ and uniform shear is generated in the domain using the "SLLOD" algorithm [21]. Using data obtained from these homogeneous shear simulations we established a regime map by assigning each of these simulations different regimes (inertial, intermediate or quasi-static) based on the scaling of shear stress with strain rate. In the inertial regime the stress scales as square of the strain rate $\left(\sigma \propto \dot{\gamma}^{2}\right)[22]$, whereas in the quasi-static regime stress remains independent of the strain rate $(\sigma \neq f(\dot{\gamma}))[19]$. In between these two extreme regimes there exist an intermediate regime where stress is related with the strain rate in the form of power law $\left(\sigma \propto \dot{\gamma}^{n}\right)$, where $n$ takes values between 0 to 2 based on the particle and flow properties [23]. Figures 1(a) and 1(b) show such regime maps obtained from the DEM simulations of homogeneously sheared granular flow in space of solid volume fraction $\nu$, particle friction coefficient $\mu_{p}$ for a non-dimensional shear rate $k^{*}$ of $2.5 \times 10^{4}$ and $10^{9}$, respectively. In Figs. 1(a) and $1(\mathrm{~b})$ the inertial regime $\left(\sigma \propto \dot{\gamma}^{2}\right)$ is represented with red color, whereas the blue color shows the presence of quasi-static regime $(\sigma \neq f(\dot{\gamma}))$. In between 
both these regimes, there exists an intermediate regime $\left(\sigma \propto \dot{\gamma}^{n}, 0<n<2\right)$ which is represented with green color in Figs. 1(a) and 1(b).

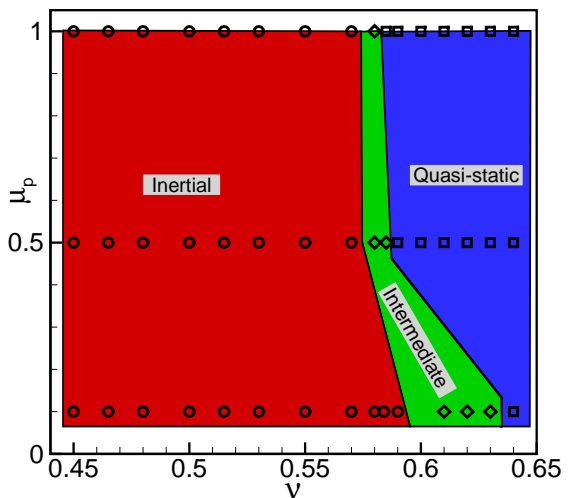

(a)

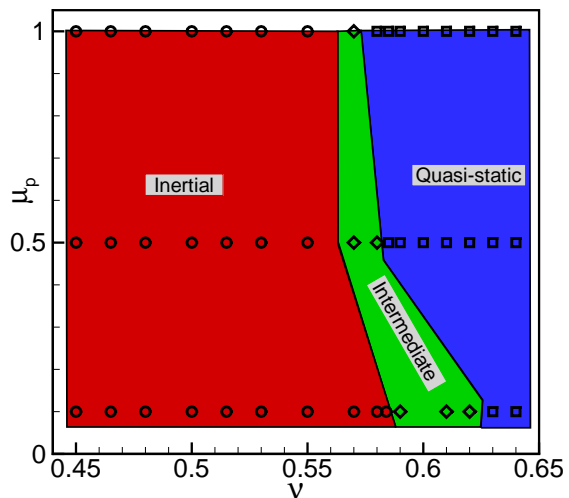

(b)

Fig. 1. Regime map for granular flows, constructed from data of 3D DEM simulations of homogenously sheared granular flow (a) $k^{*}=k_{n} / \rho_{s} d_{0}^{3} \dot{\gamma}^{2}=2.5 \times 10^{4}$ and (b) $k^{*}=k_{n} / \rho_{s} d_{0}^{3} \dot{\gamma}^{2}=10^{9}$.

\subsection{OP dynamics from homogeneous shear simulations}

The OP characterizes the 'phase' or state of the granular material. We extract the OP from 3D DEM simulations of homogeneously sheared granular flow. In Fig. 2(a) the steady state values of the OP are plotted with solid volume fraction with three different values of particle friction coefficients. Figure 2(a) shows that the OP is indeed a strong function of the particle friction coefficient. An increase of about $300 \%$ in the value of the OP is seen when particleparticle coefficient of friction increases from 0.1 to 1.0 at the same solid volume fraction. This result is expected because the larger interparticle friction is, the more particles are prevented from sliding over each other, hence more numbers of solid contacts. A sudden jump in the value of the OP is seen near the maximum packing limit, this sudden increase in the value of the $\mathrm{OP}$ is ascribed to the presence of strong force chains near the packing limit. Figures 2(b), 2(c), and 2(d) are the similar plots of the OP with solid volume fraction as described in Fig. 2(a), but for different values of shear rates. These plots (Figs. 2(a), 2(b), 2(c), 2(d)) show that the particle friction coefficient is the most important parameter which affects the OP, whereas the shear rate has a least impact on the $\mathrm{OP}$ values.

A careful examination of these plots (Figs. 2(a), 2(b), 2(c), 2(d)) reveal that as the non-dimensional shear rate $k^{*}\left(k_{n} / \rho_{s} d_{0}^{3} \dot{\gamma}^{2}\right)$ increases (going from Fig. 2(a) to Fig. 2(d)), the sudden jump in the OP value (near the maximum packing 


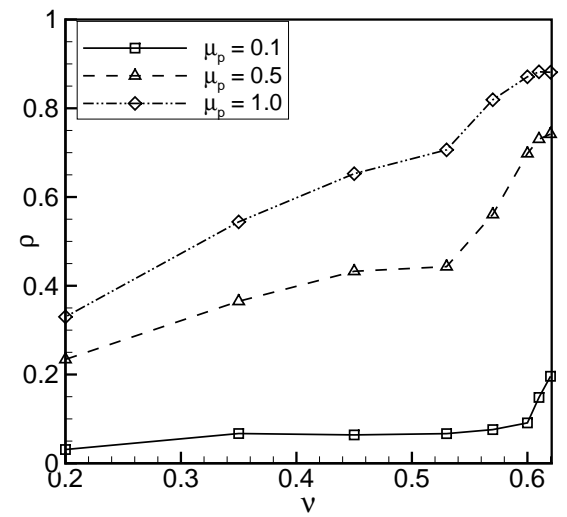

(a)

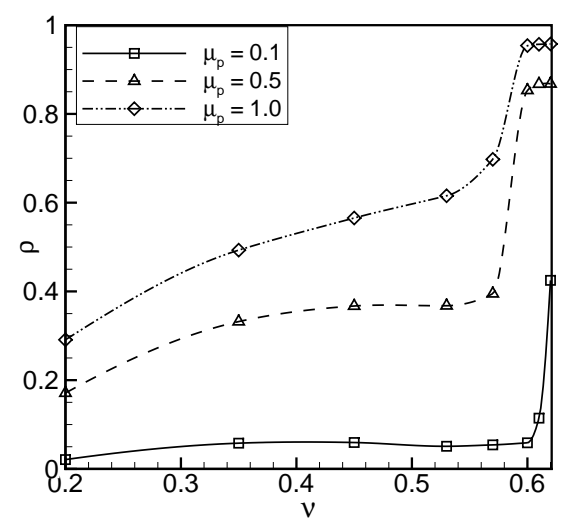

(c)

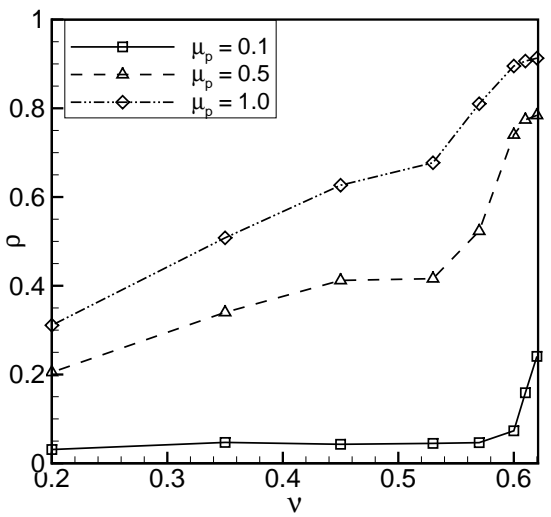

(b)

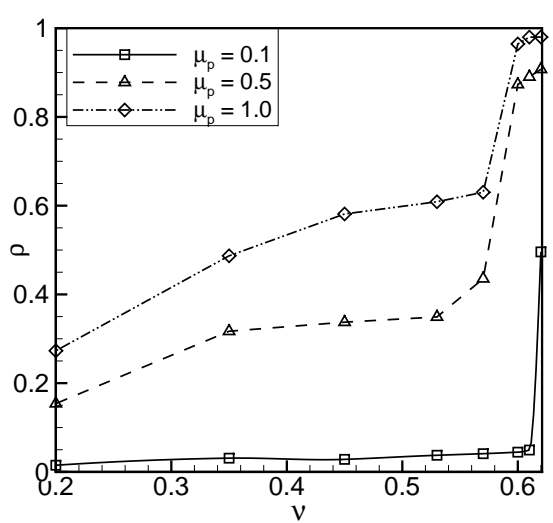

(d)

Fig. 2. The OP (at steady state) plotted with solid volume fraction (a) for $k^{*}=2.5 \times 10^{4}$ and $e=0.7,(\mathrm{~b})$ for $k^{*}=10^{5}$ and $e=0.7,(\mathrm{c})$ for $k^{*}=10^{7}$ and $e=0.7$ and (d) for $k^{*}=10^{9}$ and $e=0.7$.

limit) becomes increasingly sharper and sharper. There is an another interesting point to note that, as the particle friction coefficient increases this sudden jump in the OP values occur at lower and lower values of solid volume fraction. This finding is consistence with the results of Song et al. [24], who studied the jamming of packed spheres through a phase diagram and showed that the minimum solid volume fraction required for jamming decreases with the increase in the particle friction coefficient. It is also noteworthy that at the highest value of $k^{*}$ (which corresponds to lowest shear rate, see Fig. 2(d)) the OP attains its limiting value of 1 near the maximum packing limit, whereas the OP approaches to its other limiting value of 0 , when both solid volume fraction and friction coefficient tends towards zero.

It should be noted that Aranson and Tsimring [16] in their original work related the OP to the derivative of the free energy density function $F(\rho, \delta)$ through Ginzburg-Landau equation. Further they postulated that this free 


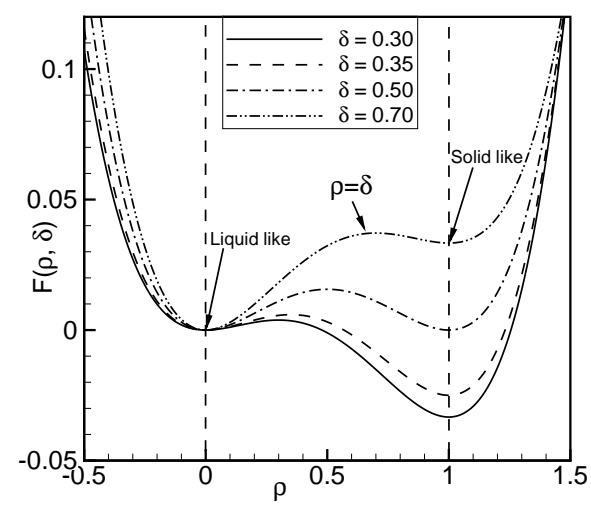

(a)

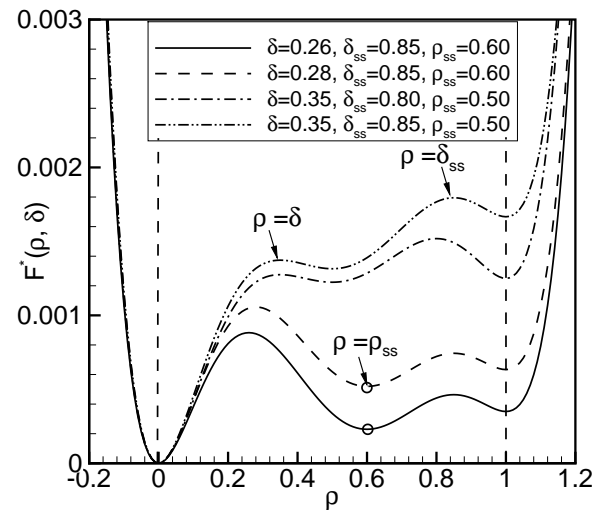

(b)

Fig. 3. (a) Typical profile of the free energy density function $F(\rho, \delta)$ postulated by Aranson and Tsimring [16], and (b) Typical profile of proposed free energy density function $F^{*}(\rho, \delta)$ with third intermediate local minima at $\rho=\rho_{s s}$.

energy density function $F(\rho, \delta)$ (see Eq. 2) has two local minima at $\rho=1$ (solidlike) and $\rho=0$ (fluidlike) to account for the bistability near the solidfluid transition. The relative stability of the two phases is controlled by the parameter $\delta$, which is a function of the stress tensor. For small $\delta$ the solidlike state is more favorable, and vice versa. A typical profile of the free energy density function postulated by Aranson and Tsimring [16], is shown in Fig. 3(a), for different values of $\delta$.

$$
F(\rho, \delta)=\int^{\rho} \rho(\rho-1)(\rho-\delta) d \rho
$$

With the above formulation of the free energy density function $F(\rho, \delta)$, the solution of the Ginzburg-Landau equation (see Eq. 3) always renders a steady state value of the OP which is either 1 or 0 , depending on the value of parameter $\delta$.

$$
\frac{D \rho}{D t}=D_{c} \nabla^{2} \rho-F(\rho, \delta)
$$

However, in the present work the OP is modeled as a function of solid volume fraction, shear rate and interparticle friction coefficient using data obtained from DEM simulations of homogeneously sheared granular flow. The DEM results (Figs. 2(a), 2(b), 2(c), 2(d)) indicate that, there should be one more intermediate local minima of this free energy function at $\rho=\rho_{s s}$, which causes the OP to attain a steady state stable value which is neither zero (fluidlike) nor one (solidlike). Hence, a modification in the form of free energy density function is proposed (see Eq. 4), to account for this third intermediate local minima at $\rho=\rho_{s s}$. Figure 3(b) shows a typical profile of the proposed free energy density function $F^{*}(\rho, \delta)$. In Eq. $4, \rho_{s s}$ and $\delta_{s s}$ can be specified using DEM data.

$$
F^{*}(\rho, \delta)=\int^{\rho} \rho(\rho-1)\left(\rho-\rho_{s s}\right)(\rho-\delta)\left(\rho-\delta_{s s}\right) d \rho
$$


After understanding the OP dynamics through the results from DEM simulations, the next step is to explore and improve the objective OP model [15] in order to make it tractable, which is presented in the following section.

\section{Order parameter model description and refinement}

\subsection{OP model description}

The original order parameter model was developed by Aranson, Tsimring and Volfson in a series of papers [16, 20, 13]. The fundamental premise of this model is that one can define an OP in granular flows similar to that used in the Landau theory of phase transitions [25]. This original OP model [20] decomposes the total granular stress tensor into "solidlike" and "fluidlike" contributions based on the OP. The OP model gives expression for "solidlike" and "fluidlike" stress tensors that are functions of the order parameter $\rho$ and the total granular stress tensor $\sigma_{i j}$. This original OP model by Volfson et al. [20] is generalized to an objective form by Gao et al. [15]. The objective expressions for $\sigma_{i j}^{f}$, the "fluidlike", and $\sigma_{i j}^{s}$, the "solidlike" contribution to the total granular stress, which are coordinate system independent, are [15]:

$$
\begin{gathered}
\sigma_{i j}^{f}=\sigma_{0}\left\{\alpha \delta_{i j}+\beta b_{i j}+\gamma\left[\left(b^{2}\right)_{i j}-\frac{1}{3}\left(b^{2}\right)_{l l} \delta_{i j}\right]\right\} \\
\sigma_{i j}^{s}=\sigma_{0}\left\{(1-\alpha) \delta_{i j}+(1-\beta) b_{i j}-\gamma\left[\left(b^{2}\right)_{i j}-\frac{1}{3}\left(b^{2}\right)_{l l} \delta_{i j}\right]\right\},
\end{gathered}
$$

where $\sigma_{0}=\sigma_{i i} / 3$ is the scale of stress (summation is implied over repeated indices). The components of the second-order tensor $b^{2}$ are defined as:

$$
\left(b^{2}\right)_{i j}=b_{i k} b_{k j}
$$

and $\left(b^{2}\right)_{l l}$ is a scalar that is defined as,

$$
\left(b^{2}\right)_{l l}=b_{l k} b_{k l}
$$

In Eqs. 5 and $6, b_{i j}$ is defined as,

$$
b_{i j}=\frac{\sigma_{i j}}{\sigma_{0}}-\delta_{i j}
$$

The scalar model coefficients $\alpha$ and $\beta$ are functions of the order parameter $\rho$, which are specified in Gao et al. [15]:

$$
\begin{aligned}
& \alpha=(1-\rho)^{1.8}, \\
& \beta=(1-\rho)^{2.5} .
\end{aligned}
$$


These model coefficients were obtained using DEM data from 2D inhomogeneous Couette flow with wall boundary conditions. In Aranson and Tsimring [16] original OP theory, the fluidlike stress in Eq. 5 was modeled using a constitutive relation from the KTGF (kinetic theory of granular flows), and the total granular stress and solidlike contribution were obtained through simple relations (that do not satisfy the objectivity requirement). However, the nonlinear form of Eqs. 5 and 6 does not easily permit solution for the total granular stress and solidlike stress from the fluidlike KTGF closure. In order to render the objective OP model tractable, and to obtained the model coefficients from 3D DEM data of homogeneously sheared granular flow, we performed refinement of the OP model which is presented in Sec. 4.2.

\subsection{Refinement of the OP model}

The model coefficients $\alpha, \beta$ and $\gamma$ for the objective OP model [15] that best fit the fluidlike stress tensor $\sigma_{i j}^{f}$ relation given by Eq. 5, are computed using data for the total granular stress $\sigma_{i j}$ and fluidlike stress $\sigma_{i j}^{f}$ obtained from 3D DEM simulations of homogeneous shear flow. In 3D there are six independent nonzero components of the fluidlike stress tensor (assuming the stress tensor to be symmetric). Therefore, one can specify three model coefficients $\alpha, \beta$ and $\gamma$ to match the six components of the "fluidlike" stress from DEM simulation data only in a least-squares sense (it should be noted that, later in the Sec. 6 the ROP model assessment is performed with the fluidlike stress contribution obtained from the kinetic theory). Figure 4(a) shows the model coefficients $\alpha$, $\beta$ and $\gamma$ with the OP for the nonlinear model given by Eq. 5. The symbols are obtained through least-squares solution to Eq. 5 for all the DEM cases performed in this study (simulation parameters for these cases are summarized in Table 1 and also shown in the form of a regime map in Fig. ??). The lines in Fig. 4(a) are a polynomial fit to the data. Note that the magnitude of the

\begin{tabular}{|c|c|c|}
\hline Solid volume fraction & Particle friction coefficient & Shear rate $\left(k^{*}\right)$ \\
\hline 0.45 & $0.1,0.5,1.0$ & $2.5 \times 10^{4}, 10^{5}, 10^{7}, 10^{9}$ \\
\hline 0.53 & $0.1,0.5,1.0$ & $2.5 \times 10^{4}, 10^{5}, 10^{7}, 10^{9}$ \\
\hline 0.57 & $0.1,0.5,1.0$ & $2.5 \times 10^{4}, 10^{5}, 10^{7}, 10^{9}$ \\
\hline 0.58 & $0.1,0.5,1.0$ & $2.5 \times 10^{4}, 10^{5}, 10^{7}, 10^{9}$ \\
\hline 0.59 & $0.1,0.5,1.0$ & $2.5 \times 10^{4}, 10^{5}, 10^{7}, 10^{9}$ \\
\hline 0.60 & $0.1,0.5,1.0$ & $2.5 \times 10^{4}, 10^{5}, 10^{7}, 10^{9}$ \\
\hline 0.62 & $0.1,0.5,1.0$ & $2.5 \times 10^{4}, 10^{5}, 10^{7}, 10^{9}$ \\
\hline
\end{tabular}

Table 1

Parameters for homogeneous shear simulations. 
third model coefficient $\gamma$ remains close to zero for complete range of the OP. Since the model coefficient $\gamma$ determines the magnitude of the nonlinear terms in Eq. 5, this indicates the possibility of forming a linear model. The model coefficients $\alpha$ and $\beta$ corresponding to a linear objective model are computed by dropping the term containing $\gamma$ in Eq. 5, and performing the least-squares solution of Eq. 12.

$$
\sigma_{i j}^{f}=\sigma_{0}\left\{\alpha \delta_{i j}+\beta b_{i j}\right\}
$$

Figure 4(b) shows model coefficients $\alpha$ and $\beta$ with the OP for the proposed

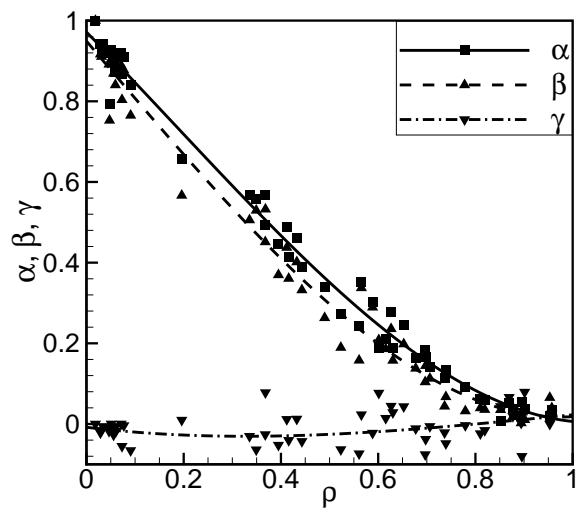

(a)

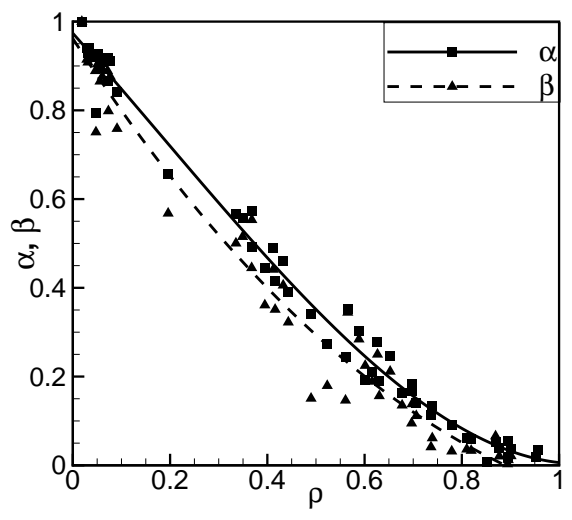

(b)

Fig. 4. The objective OP model coefficients as a function of the order parameter (a) for a nonlinear objective model and (b) for a linear objective model.

linear objective model. At $\rho=0$, the model coefficients $\alpha$ and $\beta$ are equal to 1 , which indicates that the total granular stress is solely due to fluidlike contribution. At $\rho=1$, the model coefficient $\alpha$ and $\beta$ are zero, which indicates that the total granular stress is due to only the solidlike contribution. The error incurred in both (nonlinear and linear) objective model is quantified by the vector norm of the relative error in the least-squares solution:

$$
\hat{\epsilon}=\frac{\|\mathbf{K} \mathbf{x}-\mathbf{y}\|_{2}}{\|\mathbf{y}\|_{2}},
$$

where $\mathbf{x}$ is the solution vector for the model coefficients, $\mathbf{K} \mathbf{x}$ is the total granular stress components given by the OP model and $\mathbf{y}$ is the total granular stress from DEM. The error incurred in terms of this vector norm is shown in Fig. 5 for complete range of the OP for both nonlinear and linear objective models. As Fig. 5 shows, the error incurred in both the objective models is less than $11 \%$. The percentage error incurred in the linear model is approximately same as the error incurred in the nonlinear model. Therefore, a linear version of the objective OP model with new model coefficient extracted from 3D DEM data of homogeneous shear flow is now proposed. This linear version of the objective OP model is referred to as the refined order parameter (ROP) model. In 


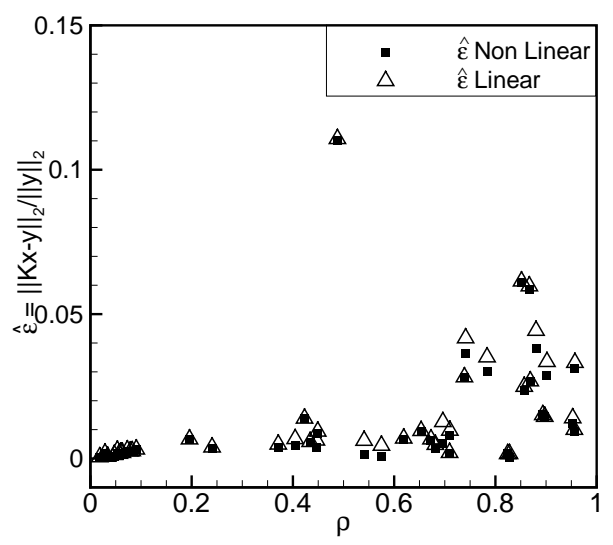

Fig. 5. Error in the total granular stress objective models as a function of the OP for both linear and nonlinear models.

the following section we will present the specification for the proposed ROP model.

\section{Specification of the ROP model}

The model equations for the proposed linear ROP model are:

$$
\begin{gathered}
\sigma_{i j}^{f}=\sigma_{0}\left\{\alpha \delta_{i j}+\beta b_{i j}\right\}, \\
\sigma_{i j}^{s}=\sigma_{0}\left\{(1-\alpha) \delta_{i j}+(1-\beta) b_{i j}\right\} .
\end{gathered}
$$

The model coefficients $(\alpha$ and $\beta$ ) of the linear ROP model are specified as polynomial fits to the data in Fig. 4(b) with

$$
\alpha=a+b \rho+c \rho^{2}+d \rho^{3}
$$

where $a=1.0, b=-1.23, c=-0.31$ and $d=0.54$, and

$$
\beta=A+B \rho+C \rho^{2}+D \rho^{3},
$$

with $A=1.0, B=-1.69, C=0.76$ and $D=-0.07$. The advantage of the linear ROP model is that now the total granular stress can be inverted from the solidlike and fluidlike stress relations.

Previously Aranson and Tsimring [16] showed that a constitutive relation from the kinetic theory for the fluidlike stress gave a good match for the kinematic variables in dense chute flow. Therefore, we follow Aranson and Tsimring [16] and model the "fluidlike" stress using a constitutive relation from the kinetic theory of granular flows (KTGF) even in the dense regime. Once the fluidlike contribution of the total granular stress is known, the total granular stress 
tensor $\sigma_{i j}$ can be expressed in terms of the "fluidlike" stress and the ROP model coefficients $(\alpha$ and $\beta$ ) using Eq. 18

$$
\sigma_{i j}=\frac{\sigma_{0}}{\beta}\left[\frac{\sigma_{i j}^{f}}{\sigma_{0}}+\delta_{i j}(\beta-\alpha)\right],
$$

where $\sigma_{0}=\sigma_{i i}^{f} /(3 \alpha)$. The kinetic theory closures are taken from Lun et al. [17] to compute the fluidlike contribution of the total granular stress tensor which are:

$$
\begin{gathered}
\sigma_{i j}^{f}=\left[\rho_{s} \nu\left(1+4 \eta_{k} \nu g_{0}\right) T-\eta_{k} \mu_{b} \nabla \cdot \mathbf{u}\right] \delta_{i j}-\left(\frac{2+\alpha_{k}}{3}\right) \\
\times\left\{\frac{2 \mu}{g_{0} \eta_{k}\left(2-\eta_{k}\right)}\left(1+\frac{8}{5} \nu \eta_{k} g_{0}\right)\left[1+\frac{8}{5} \eta_{k}\left(3 \eta_{k}-2\right) g_{0}\right]+\frac{6}{5} \eta_{k} \mu_{b}\right\} S_{i j} \\
S_{i j}=\frac{1}{2}\left(\frac{\partial u_{i}}{\partial x_{j}}+\frac{\partial u_{j}}{\partial x_{i}}\right)-\frac{1}{3} \frac{\partial u_{i}}{\partial x_{i}} \\
\mu=\frac{5 \rho_{s} d_{0}(\pi T)^{2}}{96} \\
\mu_{b}=\frac{256 \mu \nu^{2} g_{0}}{5 \pi} \\
g_{0}(\nu)=\frac{(1+e)}{1-\left(\nu / \nu_{\max }\right)^{1 / 3}} \\
\eta_{k}=\frac{(1.3)}{2}
\end{gathered}
$$

where $\sigma_{i j}^{f}$ is the fluidlike part of the stress tensor, $\rho_{s}$ is the density of the solid particle, $\nu$ is the solid volume fraction, $T$ is the granular temperature, $\mathbf{u}$ is the mean velocity vector, $S_{i j}$ is the strain rate tensor. For inhomogeneous granular flows the granular temperature $T$ is obtained as solution to a transport equation [17]. However the granular temperature can be obtained through simple relation in a homogeneous shear flows.

\subsection{Homogeneous shear case}

The kinetic theory model for the fluidlike stress requires granular temperature as input (see Eq. 19). For a steady homogeneously sheared granular flow (in absence of fluid phase) the granular temperature results from a balance of production and dissipation terms. This balance results in a algebraic equation 
for the granular temperature $T[26]$ :

$$
T=\left\{\frac{-K_{1} \nu S_{i i}+\sqrt{K_{1}^{2}\left(S_{i i}\right)^{2} \nu^{2}+4 K_{4} \nu\left[K_{2}\left(S_{i i}\right)^{2}+2 K_{3}\left(S_{i j} S_{i j}\right)\right]}}{2 \nu K_{4}}\right\}^{2}
$$

where constants $K_{1}, K_{2}, K_{3}$ and $K_{4}$ are:

$$
\begin{gathered}
K_{1}=2(1+e) \rho_{s} g_{0} \\
K_{2}=4 d_{0} \rho_{s}(1+e) \nu g_{0} /(3 \sqrt{\pi})-\frac{2}{3} K_{3} \\
K_{3}=\frac{d_{0} \rho_{s}}{2}\left[\frac{\sqrt{\pi}}{3(3-e)}[0.5(3 e+1)\right. \\
\left.\left.+0.4(1+e)(3 e-1) \nu g_{0}\right]+\frac{8 \nu g_{0}(1+e)}{5 \sqrt{\pi}}\right] \\
K_{4}=\frac{12\left(1-e^{2}\right) \rho_{s} g_{0}}{d_{0} \sqrt{\pi}} .
\end{gathered}
$$

We extract the granular temperature using Eq. 26, and compare those values

Table 2

\begin{tabular}{|c|c|c|}
\hline Solid volume fraction & Algebraic Equation & DEM \\
\hline 0.45 & 0.529 & 0.603 \\
\hline 0.53 & 0.514 & 0.570 \\
\hline 0.58 & 0.508 & 0.553 \\
\hline 0.60 & 0.505 & 0.550 \\
\hline 0.62 & 0.503 & 0.528 \\
\hline
\end{tabular}

Comparison of granular temperature $\hat{T}=T /\left(d_{0} \dot{\gamma}\right)^{2}$ obtained from algebraic equation and DEM. Simulation parameters: $\mu_{p}=0.5, e=0.7$ and $k^{*}=k_{n} / \rho_{s} d_{0}^{3} \dot{\gamma}^{2}=10^{5}$.

to the granular temperature obtained from DEM simulations in Table 2. The interparticle friction coefficient used for these simulations is 0.5 , with a coefficient of restitution of 0.7 . The non-dimensional shear rate $k^{*}\left(k_{n} / \rho_{s} d_{0}^{3} \dot{\gamma}^{2}\right)$ is set to be $10^{5}$ for this comparison. Table 2 shows that the maximum difference in the steady state granular temperature obtained from the algebraic equation (Eq. 26) and DEM simulation is less than $14 \%$ over a range of solid volume fractions considered.

With the specification of the fluidlike contribution to the total granular stress $\sigma_{i j}^{f}$ and the model coefficients ( $\alpha$ and $\beta$ ), one can solve the ROP model to 
obtain the total granular stress $\sigma_{i j}$ using Eq. 18. The next logical step is to assess the performance of proposed ROP model which is presented in following section.

\section{Assessment of the ROP model for homogeneous shear flows}

The predictive capability of the ROP-KT model (constitutive relation for the fluidlike stress contribution obtained from kinetic theory of granular flows) is assessed in different regimes (as shown in the form of a regime map in Fig. ??) by comparing predictions for the total granular stress to those from DEM simulations of homogeneously sheared granular flow. The validity of the kinetic theory closure for the fluidlike stress is also assessed in different regimes by comparing $\sigma_{i j}^{f}$ with the corresponding fluidlike stress tensor obtained from the DEM data.

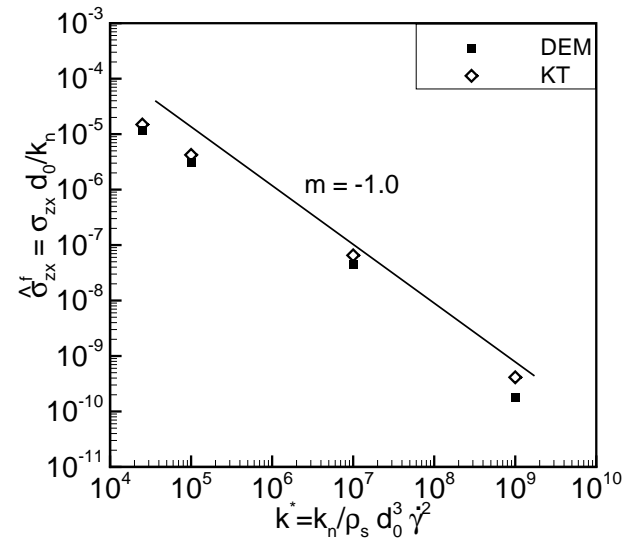

(a)

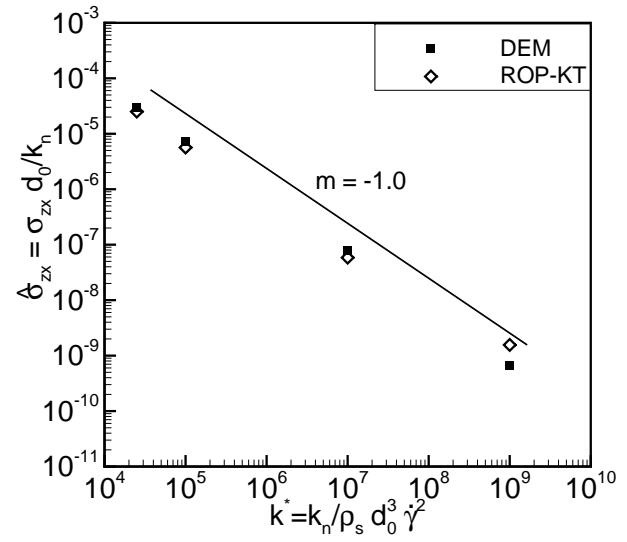

(b)

Fig. 6. (a) The fluidlike stress contribution to the total granular stress as a function of shear rate $k^{*}$ and (b) The total granular stress as a function of shear rate $k^{*}$. Simulation parameters: $\nu=0.45, \mu_{p}=0.5, e=0.7$.

\subsection{Inertial regime (solid volume fraction of 0.45 )}

Figure 6(a) shows a logarithmic plot of the elastic scaling of the fluidlike shear stress as a function of shear rate for a solid volume fraction of 0.45 . In this scaling, stress values in the inertial regime where $\sigma \propto \dot{\gamma}^{2}$ correspond to a line with slope -1 (in Fig. 6(a) the slope of the line is represented with letter $m$ ). The fluidlike contribution obtained from the kinetic theory is shown by blank diamonds, whereas the filled squares show the data directly obtained from 
the DEM simulations. Figure 6(a) shows that the fluidlike contribution to the total granular stress obtained using kinetic theory closely follows the fluidlike stress contribution obtained from DEM simulations. Both the DEM data and predictions obtained from kinetic theory follow inertial scaling $\left(\sigma \propto \dot{\gamma}^{2}\right)$ with the shear rate. This type of scaling of the shear stress with the applied shear rate has been previously reported by Campbell [19] in the inertial regime of the granular flows. In Fig. 6(b) the shear component of the total granular stress is plotted with shear rate $k^{*}$ for a solid volume fraction of 0.45 . The total granular stress predicted using ROP-KT model closely follows the data obtained from the DEM simulations. The ROP-KT model is able to predict the total granular stress in the inertial regime within 5\%. The total granular stress obtained from both, the model and DEM follows the inertial scaling $\left(\sigma \propto \dot{\gamma}^{2}\right)$ of stress with applied shear rate.

\subsection{Near transitional regime (solid volume fraction of 0.57 )}

In order to quantify the performance of the ROP-KT model near the transition from inertial to intermediate regime, we considered a higher solid volume fraction of 0.57. In Fig. 7(a) the variation of the fluidlike contribution of the stress (obtained from kinetic theory as well as from the DEM data) is shown with the shear rate. This plot shows that the kinetic theory closure performs reasonably well in predicting the fluidlike stress contribution when compared with the DEM data. The fluidlike contribution to the total granular stress ob-

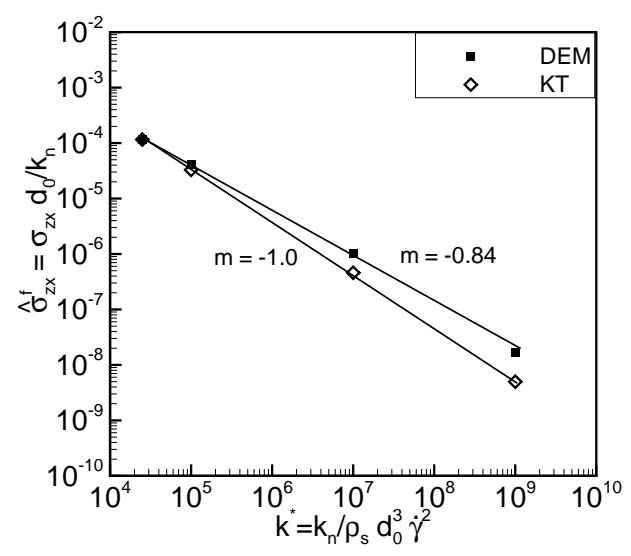

(a)

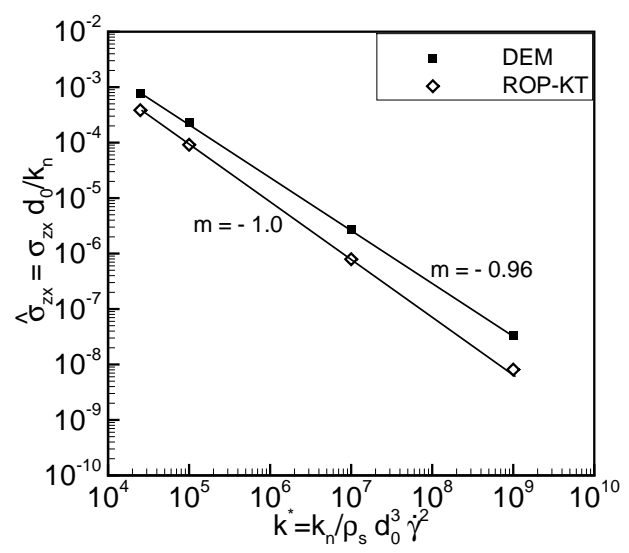

(b)

Fig. 7. (a) The fluidlike stress contribution to the total granular stress as a function of shear rate $k^{*}$ and (b) The total granular stress as a function of shear rate $k^{*}$. Simulation parameters: $\nu=0.57, \mu_{p}=0.5, e=0.7$.

tained from the kinetic theory follows the inertial scaling of the stress, whereas the data obtained from the DEM simulations start to deviate from this scaling and shows a different power law behavior of stress $\left(\sigma \propto \dot{\gamma}^{n}, n=-2 m=1.68\right)$ 
with the shear rate. Figure $7(\mathrm{~b})$ compares the total granular stress predicted by ROP-KT model with data from the DEM simulations. In the transitional regime $(\nu=0.57)$ the ROP-KT model predicts the total granular stress reasonably well (see Fig. $7(\mathrm{~b})$ ), with an error of $15 \%$ when compared with the DEM data. At this volume fraction there are significant multiparticle contacts as indicated by the mean coordination number value of 2.2 obtained from DEM simulations (result not shown here). Therefore, the KT (kinetic theory) closure for the fluidlike stress is not validated at this volume fraction, as evidenced by the mismatch in the slope in Fig. 7(b).

\subsection{Deep intermediate regime (solid volume fraction of 0.62 )}

To assess the performance of the ROP-KT model in the deep intermediate regime, we selected a case with solid volume fraction of 0.62 and interparticle friction coefficient of 0.1 . At this solid volume fraction the ROP-KT model does not predict either the magnitude or the scaling of the fluidlike stress (Fig.8(a)) and the total granular stress (Fig.8(b)) correctly. The fluidlike contribution obtained from DEM data clearly shows the intermediate scaling of the stress $\left(\sigma \propto \dot{\gamma}^{n}, n=-2 m=0.66\right)$ with shear rate, whereas the kinetic theory closure necessarily follows the inertial scaling of the stress $\left(\sigma \propto \dot{\gamma}^{2}\right)$ with applied shear rate. Although the ROP-KT model decomposes the total gran-

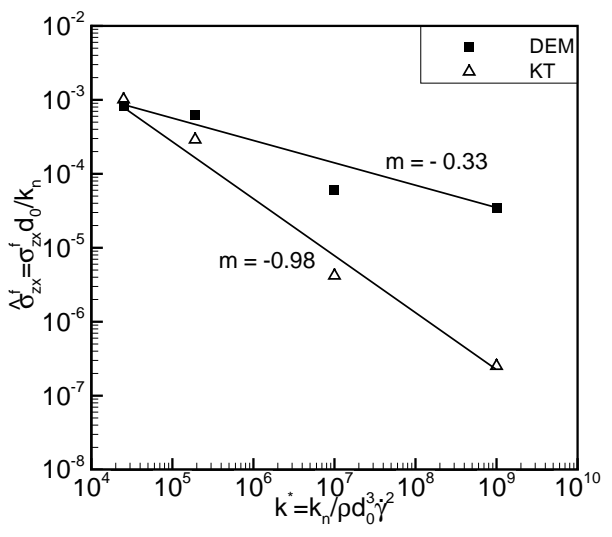

(a)

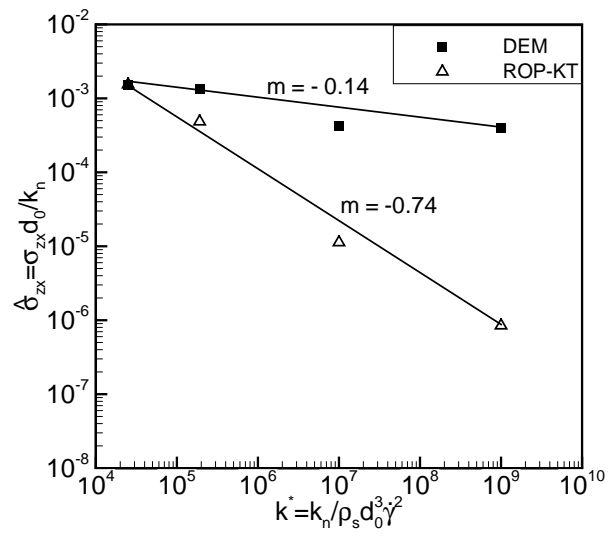

(b)

Fig. 8. (a) Fluidlike stress contribution to the total granular stress as a function of shear rate and (b) The total granular stress as a function of shear rate. Simulation parameters: $\nu=0.62, \mu_{p}=0.1, e=0.7$.

ular stress into solidlike and fluidlike parts, unlike other models $[8,9,10]$ the weighting factors for these contributions depends on the shear rate through the OP (see Eqs. 16 and 17). Note that as a consequence the total granular stress predicted by the ROP-KT model actually shows an intermediate scaling $\left(\sigma \propto \dot{\gamma}^{2}, n=-2 m=1.48\right)$ with the shear rate, even though the fluidlike 
stress follows a inertial scaling.

\subsection{Summary of ROP model assessment}

Based on this assessment study, it can be concluded that the ROP-KT model has the capability to accurately predict granular stress up to a solid volume fraction of 0.57 . As the solid volume fraction exceeds 0.57 the flow transitions to the intermediate regime and the ROP-KT model fails to capture the correct trend of shear stress with shear rate. The differences in the magnitude of the stress prediction in the intermediate regime is attributed to the fact that the ROP-KT model assumes that the fluidlike stress contribution follows the kinetic theory closure even in the dense regime. However, this assumption does not hold in the deep intermediate regime where both collision and frictional interactions between the particles are important. Although the ROP model coefficients include a dependence on the shear rate, but this is not able to accurately predict the stress-strain scaling in the deep intermediate regime of flow.

\section{Performance evaluation of different constitutive models in the intermediate regime}

The performance of different constitutive models is assessed in the intermediate regime of granular flow. In Fig. 9, the shear component of the total granular stress is plotted with shear rate for a solid volume fraction of 0.62 with interparticle friction coefficient of 0.1 (this combination of solid volume fraction and particle friction coefficient corresponds to the intermediate regime). The different constitutive models assessed are listed below:

(1) ROP-KT: This is the constitutive model proposed in present work, where the ROP model is coupled with the kinetic theory of granular flows (KTGF) [17] for the fluidlike stress contribution of the total granular stress. As discussed earlier, this model fails to capture the correct trends of shear stress with shear rate in the deep intermediate regime.

(2) ROP-DEM: In this constitutive model, the fluidlike contribution to the total granular stress is supplied from the DEM data and then the ROP model (see Eq. 18) is solved to compute the total granular stress. Figure 9 shows that, the ROP-DEM model is capable of predicting the total granular stress accurately (within 5\%) even in the intermediate regime, provided the fluidlike (or the solidlike) stress contribution is taken from the DEM data. This result shows that the limitation is not in the objective ROP model concept, but in the constitutive model for the fluidlike 


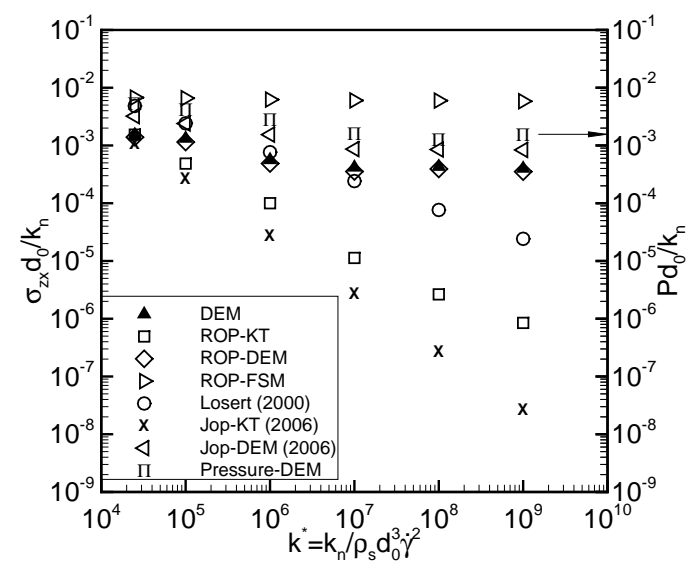

Fig. 9. Shear component of the total granular stress (different symbols represent result obtained with different constitutive models) plotted with shear rate. Simulation parameters: $\nu=0.62, \mu_{p}=0.1, e=0.7$.

(or solidlike) stress contributions in the intermediate regime.

(3) ROP-FSM: The FSM (Frictional Stress Model) is proposed by Srivastava and Sundaresan [10] for the frictional part of the total granular stress. The FSM model is used to compute the solidlike stress contribution, then the ROP model is solved to obtain the total granular stress. This frictional stress model is based on the critical state theory of soil mechanics. At the critical state the granular assembly deforms without any volume change and the frictional contribution of the stress is given by:

$$
\frac{\boldsymbol{\sigma}^{\text {fric }}}{p_{c}(\nu)}=\mathbf{I}-\sqrt{2} \sin \phi \frac{\mathbf{S}}{\sqrt{\mathbf{S}: \mathbf{S}}}
$$

where the form for $p_{c}(\nu)$ (critical state pressure) is taken from Johnson and Jackson [9]

$$
p_{c}(\nu)= \begin{cases}F \frac{\left(\nu-\nu_{\min }\right)^{r}}{\left(\nu_{\max }-\nu\right)^{s}} & \text { if } \nu>\nu_{\min } \\ 0 & \text { if } \nu \leq \nu_{\min }\end{cases}
$$

where $F, r$ and $s$ are constants, taken from Srivastava and Sundaresan [10]. As shown in Fig. 9 this model when coupled with the ROP model for the solidlike stress contribution, predicts stresses which are independent of the shear rate (a behavior similar to quasi-static regime). However, the data obtained from the DEM simulations show a dependency of shear stress on the shear rate in this regime.

(4) Losert (2000): Losert et al. [14] proposed a constitutive model with density dependent viscosity. The shear stress in this model is given as,

$$
\sigma_{x y}=\eta \dot{\gamma}
$$


where viscosity is related with the density as follows,

$$
\eta=\left(\nu_{\max }-\nu\right)^{-1.75} .
$$

Figure 9 shows that, the shear stress predicted using this model fails to capture the correct scaling of shear stress with shear rate in the intermediate regime.

(5) Jop (2006): Jop et al. [6] described the granular material as an incompressible fluid with the internal stress tensor given by the following relations,

$$
\begin{gathered}
\sigma_{i j}=-P \delta_{i j}+\tau_{i j} \\
\tau_{i j}=\eta(|\dot{\gamma}|, P) \dot{\gamma}_{i j}
\end{gathered}
$$

with, $\eta(|\dot{\gamma}|, P)=\mu(I) P /|\dot{\gamma}|$ and $I=|\dot{\gamma}| d /\left(P \rho_{s}\right)^{0.5}$, where $\dot{\gamma}_{i j}=\partial u_{i} / \partial x_{j}+$ $\partial u_{j} / \partial x_{i}$ is the strain rate tensor and $|\dot{\gamma}|=\left(0.5 \dot{\gamma}_{i j} \dot{\gamma}_{i j}\right)^{0.5}$ is the second invariant of $\dot{\gamma}_{i j}$. In this rheology, $P$ represents an isotropic pressure and $\eta(|\dot{\gamma}|, P)$ is an effective viscosity, which is related to the friction coefficient $\mu(I)$ as follows:

$$
\mu(I)=\mu_{s}+\left(\mu_{2}-\mu_{s}\right) /\left(I_{0} / I+1\right) .
$$

However, the closure for the isotropic pressure $P$ is not specified in this work. If the closure for $P$ is taken from kinetic theory [17], then the total granular stress is obtained using this model. As shown in Fig. 9 this model coupled with the kinetic theory (Jop-KT (2006)) for the isotropic pressure fails to capture the correct trends of shear stress with shear rate in the intermediate regime. However, when the isotropic pressure $P$ is supplied from the DEM data (Jop-DEM (2006)), the model captures the correct trend of shear stress with the shear rate in the intermediate regime.

Figure 9 also shows the variation of the isotropic pressure (right vertical axis) obtained from the DEM simulations with shear rate for a homogeneously sheared granular assembly. This plot shows that the pressure follows the same scaling with shear rate as the shear stress in the intermediate regime. Hence, it is important for a constitutive model to predict the correct behavior of the pressure with strain rate.

\subsection{Decomposition of the total granular stress from DEM}

In order to investigate the scaling of the granular stress in the intermediate regime, the total granular stress obtained from DEM simulations of homogeneously sheared granular flow is first decomposed into contact (virial) and streaming (dynamic) contributions. In Fig. 10(a) the contact contribution to the normal component of the total granular stress is plotted with 


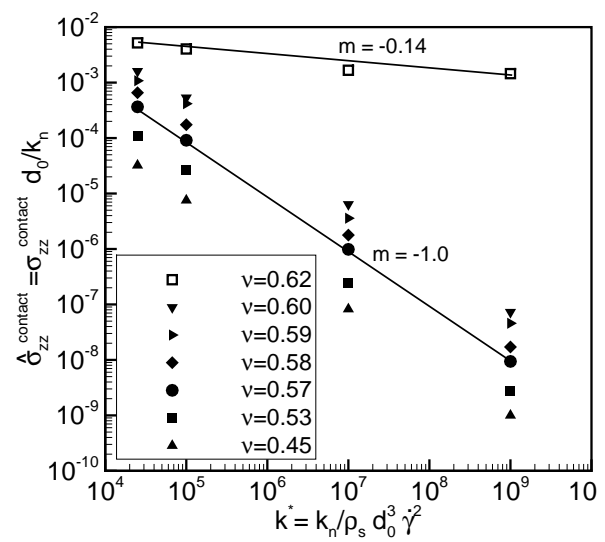

(a)

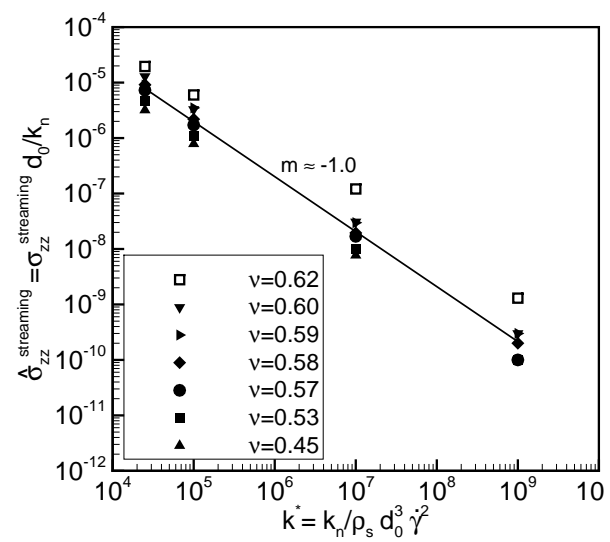

(b)

Fig. 10. (a) Contact (virial) contribution of the total granular stress as a function of shear rate and (b) Streaming (dynamic) contribution of the total granular stress as a function of shear rate. Simulation parameters: $\mu_{p}=0.1, e=0.7$. The data points correspond to the intermediate regime is shown with blank square symbols.

shear rate for a range of solid volume fractions. Figure 10(a) shows that the contact contribution of the total granular stress follows the same scaling $\left(\sigma \propto \dot{\gamma}^{n}, n=-2 m=0.28\right)$ with shear rate as the total granular stress in the intermediate regime (data points correspond to the intermediate regime are shown with blank squares). However, the streaming contribution (shown in Fig. 10(b)) of the total granular stress in the intermediate regime still follows the inertial scaling of the stress $\left(\sigma \propto \dot{\gamma}^{2}\right)$ with the shear rate. As expected, at this high solid volume fraction the contact part of the stress contributes more than $95 \%$ to the total granular stress. Hence it is critical for the performance of any constitutive model in dense regime to accurately capture the behavior of contact stress.

In order to evaluate the ROP model directly, the total granular stress obtained from the DEM simulations is decomposed into solidlike (stress arise from solidlike contacts) and fluidlike (stress arise from fluidlike contacts plus streaming stress) contributions. These contributions to the total granular stress are plotted with shear rate $k^{*}$, in Figs. 11(a) and 11(b), respectively. It is noteworthy that both the solidlike and fluidlike stress follow intermediate scaling of the stress $\left(\sigma \propto \dot{\gamma}^{n}, n_{\text {solidlike }}=0.20, n_{\text {fluidlike }}=0.44\right)$, because both of them carry portions of streaming and contact stress as well; the only difference is whether the contacts are enduring or not. A large number of the constitutive models available in the literature are based on additive decomposition of the total granular stress. However, this analysis of the stress decomposition (into solidlike and fluidlike contributions) reveals that a constitutive model can capture the intermediate behavior of the granular stress only when the weighting factors for the solidlike and fluidlike stress contributions are themselves a functions of shear rate. The ROP model has this feature because the model 


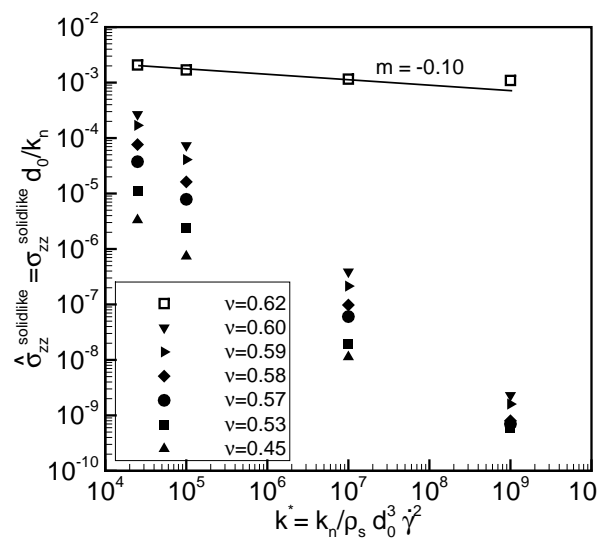

(a)

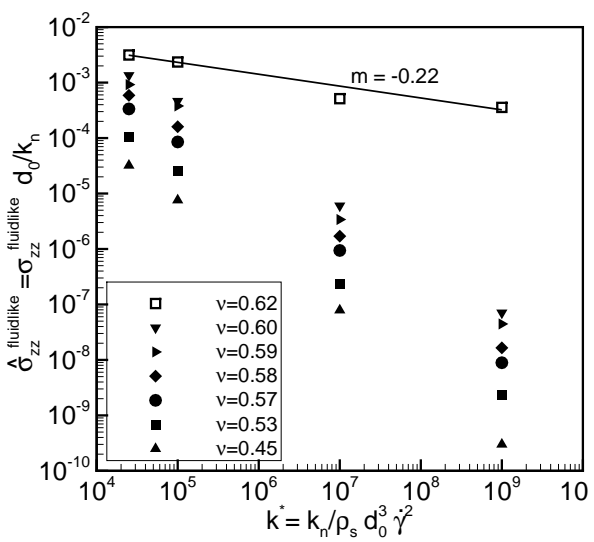

(b)

Fig. 11. (a) Solidlike contribution of the total granular stress as a function of shear rate and (b) Fluidlike contribution of the total granular stress as a function of shear rate. Simulation parameters: $\mu_{p}=0.1, e=0.7$. The data point correspond to the intermediate regime is shown with blank square symbols.

coefficients of the ROP model ( $\alpha$ and $\beta$ ) are indeed functions of shear rate through the order parameter (see Eqs. 16 and 17). However, this dependence is not able to accurately capture the scaling of the granular stress in the deep intermediate regime, where flow dynamics is dominated by the long-lasting, multiparticle contacts.

\section{Conclusions}

The DEM simulations performed in this study reveal that indeed the order parameter (OP) is capable of capturing the granular 'phase' transition from solidlike to fluidlike behavior through its dependence on particle properties (such as particle friction coefficient) and flow properties (such as shear rate). These DEM simulations also reveal that the particle friction coefficient is the most important parameter which affects the OP, whereas the shear rate has a least impact on the OP values. The OP values extracted from these simulations indicate a third intermediate steady state stable value of the OP which is neither zero (fluidlike) or one (solidlike). Hence, a modification in the form the free energy density function is proposed to represent this third intermediate local minima of the free energy density at $\rho=\rho_{\text {ss }}$. Further a constitutive model based on the objective version of the original order parameter model [13] is developed and tested in different regimes of granular flows. This OP-based model is refined by extracting new model coefficients from 3D DEM simulations of homogeneously sheared granular assembly. This refined model enables a linear implementation of the objective form (coordinate system independent) of the OP model [15] through a simplification that allows 
inversion of total granular stress from solidlike and fluidlike stress relations. The error incurred due to this simplification in the prediction of the total granular stress is shown to be less than $11 \%$ for the complete range of the OP values obtained in a homogeneous shear flow. To complete the ROP model specification, it was first assumed that the fluidlike contribution follows a constitutive relation from the kinetic theory of the granular flows (KTGF) even in the dense regime [16]. The performance of the ROP-KT model is assessed by comparing the predicted granular stress with 3D DEM data of homogeneously sheared granular flow over a range of solid volume fractions and shear rates. The ROP-KT model accurately (within 15\%) predicts the granular stress up to solid volume fraction of 0.57 . Beyond a solid volume fraction of 0.57 , the flow transitions to the intermediate regime and the ROP-KT model fails to capture even the correct trends of the shear stress with shear rate. The assumption in the ROP-KT model that the fluidlike contribution is adequately modeled by KTGF (kinetic theory of granular flows) clearly breaks down in the intermediate regime, although it appears to be adequate even for solid volume fraction greater than 0.53 where multiparticle interactions are present in the flow dynamics.

The decomposition of the DEM granular stress into contact and streaming parts reveals that the contact contribution of the stress dominates (contributes more than 95\%), and follows the same scaling as the total granular stress in the intermediate regime. However, the streaming contribution always follows the inertial scaling even in the intermediate regime. The decomposition of the granular stress obtained from DEM into solidlike and fluidlike contributions (based on the OP) reveals that both these follow the same scaling as the total granular stress in the intermediate regime. A majority of the constitutive models in the granular flow literature are based on additive decomposition of the total granular stress. This analysis shows that in such additive models for the granular stress, the weighting factor must itself depend on the shear rate if they are to reproduce the intermediate regime behavior. Although the ROP model has coefficient that depend on the shear rate, it needs further improvement to capture the correct power-law behavior of stress with strain rate in deep intermediate regime. The assessment study performed with different constitutive models in the intermediate regime, reveals that none of the existing models captures the correct scaling of shear stress with shear rate in this regime. However, the ROP model and the model proposed by Jop et al. [6] capture the correct scaling of shear stress with shear rate, provided one of the inputs to the model is supplied from the DEM data. 


\section{Acknowledgements}

The authors wish to acknowledge the financial support from U.S. department of Energy National Energy Technology Laboratory (grant DE-FG2607NT43070), administered under advanced coal research at U.S. colleges and universities.

\section{A Contact model description}

For two contacting particle $\{i, j\}$, with radii $\left\{a_{i}, a_{j}\right\}$ at positions $\left\{\mathbf{r}_{i}, \mathbf{r}_{j}\right\}$, with velocities $\left\{\mathbf{v}_{i}, \mathbf{v}_{j}\right\}$ and angular velocities $\left\{\boldsymbol{\omega}_{i}, \boldsymbol{\omega}_{j}\right\}$, the normal compression $\delta_{i j}$, relative normal velocity $\mathbf{v}_{n_{i j}}$, and relative tangential velocity $\mathbf{v}_{t_{i j}}$ are [18]:

$$
\begin{aligned}
\delta_{i j} & =d_{0}-\mathbf{r}_{i j}, \\
\mathbf{v}_{n_{i j}} & =\left(\mathbf{v}_{i j} \cdot \mathbf{n}_{i j}\right) \mathbf{n}_{i j}, \\
\mathbf{v}_{t_{i j}} & =\mathbf{v}_{i j}-\mathbf{v}_{n_{i j}}-\left(a_{i} \boldsymbol{\omega}_{i}+a_{j} \boldsymbol{\omega}_{j}\right) \times \mathbf{n}_{i j},
\end{aligned}
$$

where $d_{0}=a_{i}+a_{j}, \mathbf{r}_{i j}=\mathbf{r}_{i}-\mathbf{r}_{j}, \mathbf{n}_{i j}=\mathbf{r}_{i j} / r_{i j}$, with $r_{i j}=\left|\mathbf{r}_{i j}\right|$ and $\mathbf{v}_{i j}=\mathbf{v}_{i}-\mathbf{v}_{j}$. The rate of change of the elastic tangential displacement $\mathbf{u}_{t_{i j}}$, set to zero at the initiation of a contact is,

$$
\frac{d \mathbf{u}_{t_{i j}}}{d t}=\mathbf{v}_{t_{i j}}-\frac{\left(\mathbf{u}_{t_{i j}} \cdot \mathbf{v}_{i j}\right) \mathbf{r}_{i j}}{r_{i j}^{2}}
$$

The last term in Eq. A.4 arises from the rigid body rotation around the contact point and ensures that $\mathbf{u}_{t_{i j}}$ always lies in the local tangent plane of contact. Normal and tangential forces acting on particle $i$ are:

$$
\begin{gathered}
\mathbf{F}_{n_{i j}}=f\left(\delta_{i j} / d\right)\left(k_{n} \delta_{i j} \mathbf{n}_{i j}-\gamma_{n} m_{e f f} \mathbf{v}_{n_{i j}}\right), \\
\mathbf{F}_{t_{i j}}=f\left(\delta_{i j} / d\right)\left(-k_{t} \mathbf{u}_{t_{i j}}-\gamma_{t} m_{e f f} \mathbf{v}_{t_{i j}}\right),
\end{gathered}
$$

where $k_{n, t}$ and $\gamma_{n, t}$ are the spring stiffness and viscoelastic constants, respectively, and $m_{e f f}=m_{i} m_{j} /\left(m_{i}+m_{j}\right)$ is the reduced mass of spheres with masses $m_{i}$ and $m_{j}$. The corresponding contact force on particle $j$ is simply given by Newton's third law, i.e., $\mathbf{F}_{j i}=-\mathbf{F}_{i j}$. The function $f\left(\delta_{i j} / d\right)=1$ is

for the linear spring-dashpot model, and $f\left(\delta_{i j} / d\right)=\sqrt{\delta_{i j} / d}$ is for Hertzian contacts with viscoelastic damping between spheres.

Static friction is implemented by keeping track of the elastic shear displacement throughout the lifetime of a contact. The static yield criterion, characterized by a local particle friction coefficient $\mu$, is modeled by truncating the 
magnitude of $\mathbf{u}_{t_{i j}}$ as necessary to satisfy $\left|\mathbf{F}_{t_{i j}}\right|<\left|\mu \mathbf{F}_{n_{i j}}\right|$. Thus the contact surfaces are treated as "sticking" when $\left|\mathbf{F}_{t_{i j}}\right|<\left|\mu \mathbf{F}_{n_{i j}}\right|$, and as "slipping" when the yield criterion is satisfied.

The amount of energy lost in collisions is characterized by the value of the coefficient of restitution, which is defined as the negative ratio of the particle normal velocity after collision to the velocity before collision. For the linear spring-dashpot model, the coefficient of normal restitution and contact time can be analytically obtained:

$$
e_{n}=\exp \left(-\gamma_{n} t_{\mathrm{c}} / 2\right)
$$

where the contact time $t_{c}$ is given by

$$
t_{c}=\pi\left(k_{n} / m_{\mathrm{eff}}-\gamma_{n}^{2} / 4\right)^{-1 / 2}
$$

The value of the spring constant should be large enough to avoid particle interpenetration, yet not so large as to require an unreasonably small simulation time step $\Delta t$, since an accurate simulation typically requires $\Delta t \sim t_{c} / 50$ [19]. After the contact force is calculated, the equation of motion, which are ordinary differential equations, can be numerically integrated to get the particle trajectories.

\section{B Verification of the order parameter extraction}

In order to verify our OP calculations with previously published results of Volfson et al. [20], we performed inhomogeneous wall-bounded shear simulations of granular particles. These inhomogeneous wall-bounded shear simulations are performed by shearing the granular material between two flat-frictional walls at $z=0$ and $z=L$, whereas periodic boundary condition is imposed in the other two directions ( $x$ and $y$ ). The OP values extracted from these threedimensional (3D) DEM simulations are verified by comparing them with similar calculations of Volfson et al. [20] for two-dimensional (2D) DEM. The OP values are averaged across the inhomogeneous direction to obtain a single value following Volfson et al. [20], also for a meaningful comparison, the 2D solid volume fraction reported in Volfson et al. [20] is converted to a corresponding $3 \mathrm{D}$ solid volume fraction by using the following relation [27]:

$$
\nu_{3 D}=\frac{2}{\sqrt{\pi \sqrt{3}}} \nu_{2 D}^{3 / 2} .
$$

Figure B.1 shows the variation of the OP with solid volume fraction for an inhomogeneous wall shear simulation. A maximum difference of about $15 \%$ is found in the OP at solid volume fraction of 0.60 . Our results also verify 


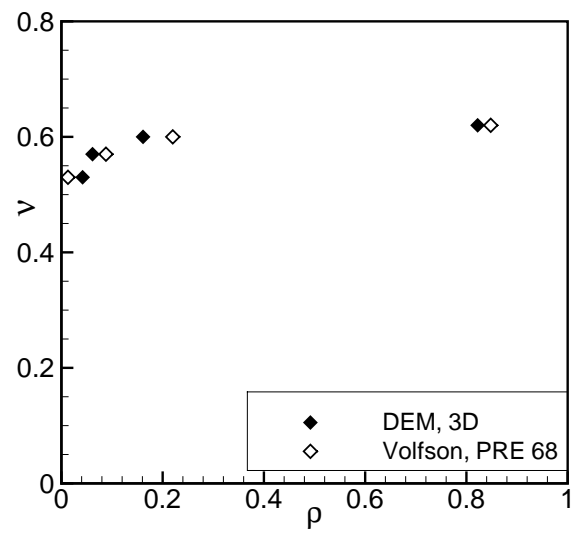

Fig. B.1. The OP as a function of solid volume fraction for inhomogeneous wall shear simulation. The filled symbols represent the 3D DEM data, whereas blank symbols correspond to Volfson et al. [20]. Simulation parameters: $\mu_{p}=\mu_{w}=0.5$, $k^{*}=k_{n} / \rho_{s} d_{0}^{3} \dot{\gamma}^{2}=10^{5}$ and $e=0.7$.

the sudden increase in the $\mathrm{OP}$ as the solid volume fraction increases from 0.57 to 0.60 that is reported in Volfson et al. [20]. We also confirmed through

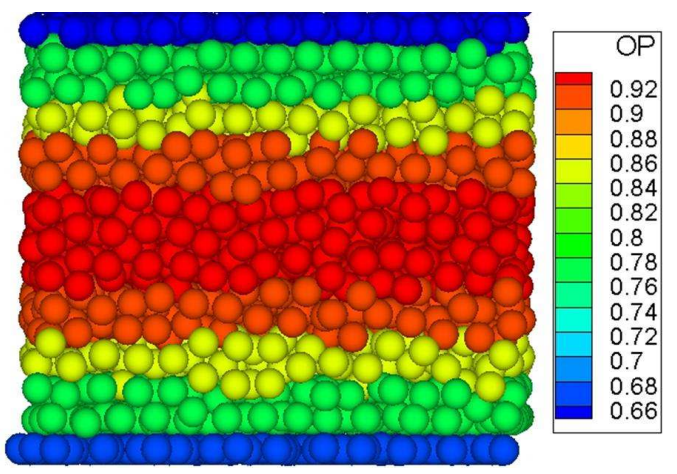

Fig. B.2. Contour plot of the OP in an inhomogeneous wall-bounded shear flow, showing transition from fluidlike behavior (near the walls) to solidlike behavior (near centerline). Simulation parameters: $\nu=0.60, \mu_{p}=\mu_{w}=0.5, k^{*}=k_{n} / \rho_{s} d_{0}^{3} \dot{\gamma}^{2}=10^{5}$ and $e=0.7$.

DEM simulations of inhomogeneous wall-bounded shear flows that the OP is indeed capable of capturing the granular 'phase' transition from solidlike to fluidlike behavior. The contour plot of the OP for an inhomogeneous wallbounded shear flow is shown in Fig. B.2. The OP is minimum near the wall and maximum near the centerline. This near-wall behavior is justified because the granular material will behave more like a liquid near the moving walls, than near the centerline. 


\section{References}

[1] H. Jaeger, S. Nagel, and R. Behringer, "Granular solids, liquids, and gases.," vol. 68, pp. 1259-1273, 1996.

[2] S. Sundaresan, "Some outstanding questions in handling of cohesionless particles," Powder Technology, vol. 115, no. 1, pp. 2-7, 2001.

[3] I. Goldhirsch, "Rapid granular flows.," Annu. Rev. Fluid Mech., vol. 35, pp. 267-293, 2003.

[4] R. M. Nedderman, Static and kninematics of granular material. Cambridge University Press, 2nd. ed., 1992.

[5] D. Schaeffer, "Instability in the evolution equations describing incompressible granular flow.," Journal of Differential Equations, vol. 66, pp. 19-50, 1987.

[6] P. Jop, Y. Forterre, and O. Pouliquen, "A constitutive law for dense granular flows.," Nature, vol. 441, no. 8, pp. 727-730, 2006.

[7] G.D.R. MiDi, "On dense granular flows.", Eur. Phys. J. E, vol. 14, pp. 341-365, 2004.

[8] S. Savage, "Analyses of slow high-concentration flows of granular materials.," J. Fluid Mech., vol. 377, pp. 1-77, 1998.

[9] P. Johnson and R. Jackson, "Frictional collisional constitutive relations for granular materials, with application to plane shearing.," J. Fluid Mech., vol. 176, pp. 67-98, 1987.

[10] A. Srivastava and S. Sundaresan, "Analysis of a frictional-kinetic model for gas-particle flow.," Powder Technology, vol. 129, pp. 72-85, 2003.

[11] J. McCarthy, V. Jasti, M. Marinack, and C. Higgs, "Quantitative validation of the discrete element methid using an annular shear cell.," Powder Technology, vol. 230, pp. 70-77, 2010.

[12] V. Jasti and C. Higgs, "Experimental study of granular flows in a rough annular shear cell.," Phys. Rev. E, vol. 78, no. 041306, pp. 1-8, 2008.

[13] D. Volfson, L. Tsimring, and I. Aranson, "Partially fluidized shear granular flows: Continuum theory and molecular dynamics simulations.," Phys. Rev. E, vol. 68, pp. 021301-1-021301-15, 2003.

[14] W. Losert, L. Bocquet, T. Lubensky, and J. Gollub, "Particle dynamics in sheared granular matter.," Phys. Rev. Lett., vol. 85, no. 7, pp. 1428-1431, 2000.

[15] D. Gao, S. Subramaniam, R. Fox, and D. Hoffman, "Objective decomposition of the stress tesnor in granular flows.," Phys. Rev. E, vol. 71, pp. 021302-1-021302-5, 2005.

[16] I. Aranson and L. Tsimring, "Continuum theory of partially fluidized granular flows.," Phys. Rev. E, vol. 65, pp. 061303-1-061303-20, 2001.

[17] C. Lun, S. Savage, D. Jeffrey, and N. Chepuriny, "Kinetic theories for granular flow:inelastic particles in couette flow and slightly inelastic particles in general flow fields.," J. Fluid Mech., vol. 140, pp. 223-256, 1984.

[18] L. Silbert, D. Ertas, G. Grest, T. Halsey, D. Levine, and S. J. Plimpton, "Granular flow down an inclined plane: Bagnold scaling and rheology," 
Phys. Rev. E, vol. 64, pp. 051302-1-051302-13, 2001.

[19] C. Campbell, "Granular shear flows at the elastic limit.," J. Fluid Mech., vol. 465, pp. 261-291, 2002.

[20] D. Volfson, L. Tsimring, and I. Aranson, "Order parameter description of stationary partially fluidized shear granular flows.," Phys. Rev. E, vol. 90, pp. 254301-1-254301-4, 2003.

[21] G. Lois, A. Lemaitre, and M. Carlson, "Numerical test of constitutive laws for dense granular flows.," Phys. Rev. E, vol. 72, pp. 051303-1-051303-16, 2005.

[22] R. Bagnolds, "Experiments on a gravity-free dispersion of large solid spheres in a newtonian fluid under shear.," Proceedings of the Royal Society of London Series A: Mathematical and Physical Sciences, vol. 225, pp. 49-63, 1954.

[23] G. Tardos, S. McNamara, and I. Talu, "Slow and intermediate flow of a frictional bulk powder in the couette geometry.," Powder Technology, vol. 131, pp. 23-39, 2003.

[24] C. Song, P. Wang, and H. Makse, "A phase diagram for jammed matter.," Nature, vol. 453, pp. 629-632, 2008.

[25] L. Landau and E. Lifshitz, Statistical Physics. Pergamon Press, 2nd. ed., 1980.

[26] M. Syamlal, W. Rogers, and T. J. O'Brien, "Mfix documentation: Theory guide," Tech. Rep. DOE/METC-95/1013, NTIS/DE95000031, National Energy Technology Laboratory, Department of Energy, 1993. See also URL http://www.mfix.org.

[27] B. van Wachem, J. Schaaf, J. Schouten, R. Krishna, and C. Bleek, "Experimental validation of Langrangian-Eluerian simulations of fluidized beds.," Powder Technology, vol. 116, pp. 155-165, 2001. 


\section{Appendix G}

\section{Granular rheology and 'phase' transition through order parameter: DEM simulations and constitutive modeling}




\title{
Experimental and computational studies of dense granular flow: transition from quasi-static to intermediate regime in a Couette shear device
}

\author{
Vidyapati $^{\text {a }}$, M. Kheiripour Langroudi ${ }^{b}$, J. Sun ${ }^{\mathrm{c}}$, \\ S. Sundaresan ${ }^{\text {d, G.I. Tardos }}{ }^{\mathrm{b}}$ and S. Subramaniam ${ }^{\mathrm{a}}$ \\ ${ }^{a}$ Department of Mechanical Engineering, Iowa State University, Ames, IA 50011, \\ USA \\ ${ }^{\mathrm{b}}$ Department of Chemical Engineering, The City College of the City University of \\ New York, New York, NY 10031, USA \\ ${ }^{\mathrm{c}}$ Institute for Infrastructure and Environment, The University of Edinburgh, \\ Edinburgh EH9 3JL, Scotland, UK \\ d Department of Chemical Engineering, Princeton University, Princeton, NJ \\ 08544, USA
}

\begin{abstract}
Rheology of dense granular material in an annular Couette cell is studied by experimentation and simulation. A transition from quasi-static to intermediate behavior is identified when a secondary vertical flow is induced. This secondary-flow-induced transition and a power-law relation between stress and shear rate in the intermediate regime are verified to be robust rheological features by simulation using discrete element method (DEM). The insensitivity of this transition to certain particle and operational parameters is also shown by the simulation. The transitional and intermediate behavior is modeled by an order parameter (OP) based model with the fluidlike stress calculated using a constitutive relation from the kinetic theory for granular flows (KTGF). The suitability of this approach is discussed.
\end{abstract}

Key words: Granular flows; Couette cell; Intermediate regime; Discrete element method; Order parameter

\section{Introduction}

Granular materials mimic the behavior of solid, liquid or gas when subjected to different excitation [1]. Understanding this complex behavior poses challeng- 
ing scientific questions, and is also of practical importance to many industrial processes, such as silo discharge, chute flow and dense-phase pneumatic conveying [2]. Much experimental work has been performed to probe the diverse behavior of granular materials. As classical examples, Reynolds [3] examined the dilatancy behavior of quasi-statically deformed granular assemblies and Bagnold [4] studied the inertial behavior and proposed a quadratic power relation between stress and shear rate. However, the transition between regimes, and the ensuing intermediate regime behavior is even more fascinating and difficult to quantify. This paper presents the transitional and intermediate behavior observed in our experiments and simulations and addresses continuum modeling of the behavior as well.

The experimental technique of choice in this study is shearing granular materials in an annular Couette cell, which has been adopted from fluid rheology to study granular rheology for some years [5, 6, 7, 8, 9]. For example, Savage [5] reported shear and normal stress variations with respect to shear rates obtained from an annular shear cell. Velocity profiles in annulus have also been measured [9]. In these experiments, however, either the flow behavior was in a single regime [9] or the transition from quasi-static regime to inertial regime was not explicitly controlled [5]. To facilitate the control of flow regime transition, a modified Couette cell was devised in this study to have a hopper connected to the bottom of the cylindrical cell (see Fig. 1). A secondary vertical flow can thus be introduced in the granular material in the annulus by discharge from the hopper. It will be shown that the transition can be triggered by the onset of this secondary flow. The stress and shear rate relation for intermediate flow will also be presented.

Discrete element method (DEM) [10] has been used extensively to simulate Couette shear flow. Simulations have been performed to study a twodimensional (2D) Couette cell for photoelastic disks, and found to be in reasonable agreement with the corresponding experimental results on velocity profiles [11]. Similar 2D simulations also produced variation of pressure versus volume fraction that was consistent with experiments [12]. In this paper, DEM simulations in a simplified three-dimensional (3D) domain have been set up to study the essential flow characteristics probed by the modified Couette cell experiments. The results will verify the transitional and intermediate behavior observed in the experiments, and demonstrate the robustness of these trends against variations in particle and flow properties.

An objective order parameter model [13] was linearized and employed to predict the granular stress in intermediate regime. The order parameter is defined as the ratio between solidlike and fluidlike stresses as proposed by Volfson et al. [14]. The decomposition of stress into solidlike and fluidlike parts is based on characteristics of the contributing particle contacts. This micromechanics based approach provides an alternative to the one proposed by Savage $[15,16]$, 
in which inertial stress calculated according to the kinetic theory for granular flows (KTGF) [17] is directly added to the quasi-static stress from soil mechanics theories [18]. However, it will be shown that the OP model with fluidlike stress given by a simple KTGF constitutive relation has limitations in predicting the intermediate behavior.

In the next section, the experiment and DEM simulation details are described. In the following section, the experimental and computational results and analysis are presented. The OP model is assessed for modeling the intermediate behavior in Sec. 4. Finally, conclusions are drawn in Sec. 5.

\section{Couette cell experiment and simulation details}

Experiments with glass beads of $0.1 \mathrm{~mm}$ diameter were performed in a modified annular Couette cell as shown by the schematic diagram in Fig. 1. The device consists of a cylindrical portion with a rotating inner cylinder to shear the material in the annular gap and a conical hopper at bottom. The material above the rotating cylinder (denoted as overburden in the schematic) is stationary and provides dead weight to the sheared layer. Granular material can be fed from above using a vibrating feeder (not shown) and discharged by a screw-in-cylinder metering device (also not shown) that discharges the material and allows for precise flow rate measurement. For experiments operated in a continuous mode, material is fed and discharged at the same time to achieve a steady-state vertical flow in the sheared layer. For those in a batch mode, no mass flows in or out of the device. Shear stress can be measured indirectly from the torque on the rotating cylinder. Additional details of the experimental setup can be found in Kheiripour Langroudi et al. [19, 20].

DEM simulations were carried out to verify experimental findings and to further study parametric dependence of the granular rheology. The simulations were performed in 3D using monodisperse, non-cohesive spheres of diameter $d_{0}$ and mass $m_{0}$, under the influence of gravity $g$. A soft-sphere contact model, the linear spring-dashpot model, was used, in which particles interact only on contact for finite duration. A set of non-dimensional equations is solved, where the scaling factor for distance, time, velocity, force, elastic constants and stresses are $d_{0}, t_{0}=\sqrt{d_{0} / g}, v_{0}=\sqrt{g d_{0}}, F_{0}=m_{0} g, k_{0}=m_{0} g / d_{0}$ and $\sigma_{0}=m_{0} g / d_{0}^{2}$, respectively. As the problem involves both gravity and shearing motion non-dimensionalization based on both gravity and shear-rate is compared in Table 1. The gravity scaling was selected because its characteristic

time $\left(\sqrt{d_{0} / g}=3.2 \times 10^{-3} \mathrm{~s}\right.$, with $\left.d_{0}=0.1 \mathrm{~mm}\right)$ is shorter than that with the shear scaling $\left(\dot{\gamma}_{\max }^{-1}=7.7 \times 10^{-3} \mathrm{~s}\right)$, even at the maximum shear rate used in the experiments. Details of the computational model used in the discrete 


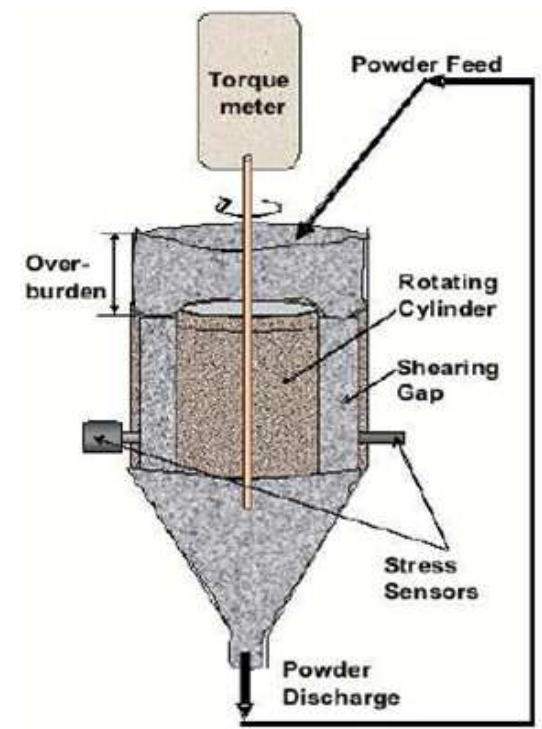

Fig. 1. Schematic of the Couette device with adjustable axial flow.

element simulations are given in Appendix A.

Table 1

\begin{tabular}{|c|c|c|}
\hline Parameters & Gravity Scaling & Shear Scaling \\
\hline Length, $L_{0}$ & $d_{0}$ & $d_{0}$ \\
\hline Time, $t_{0}$ & $\sqrt{d_{0} / g}$ & $1 / \dot{\gamma}$ \\
\hline Velocity, $v_{0}$ & $\sqrt{g d_{0}}$ & $d_{0} \dot{\gamma}$ \\
\hline Force, $F_{0}$ & $m_{0} g$ & $m_{0} d_{0} \dot{\gamma}^{2}$ \\
\hline Stiffness, $k_{0}$ & $m_{0} g / d_{0}$ & $m_{0} \dot{\gamma}^{2}$ \\
\hline Stress, $\sigma_{0}$ & $m_{0} g / d_{0}^{2}$ & $m_{0} \dot{\gamma}^{2} / d_{0}$ \\
\hline Damping Coefficient, $\gamma_{n}$ & $m_{0} \sqrt{g / d_{0}}$ & $m_{0} \dot{\gamma}$ \\
\hline
\end{tabular}

Scaling for computational parameters.

The total number of particles in the whole experimental system, including those in the overburden and hopper, is extremely large for DEM simulation. To avoid this expensive simulation, but still capture the essential rheological behavior, a representative slice of the sheared granular layer was simulated, with the curvature of the layer ignored due to the relatively large cylinder diameter. A cubic domain with side length $L=14 d_{0}$ was used as shown in Fig. 2. The effect of system size was examined by varying the side length from $7 d_{0}$ to $20 d_{0}$. Asymptotic stress was found after the side length exceeded $10 d_{0}$, consistent with the findings by Campbell [21]. Periodic and "shrink-wrap" boundary conditions were applied in the $x$ direction (which corresponds to the azimuthal direction in the Couette cell) and at $z=L>0$ (gravity pointing to the negative $z$ direction), respectively. The shrink-wrap boundary condition 


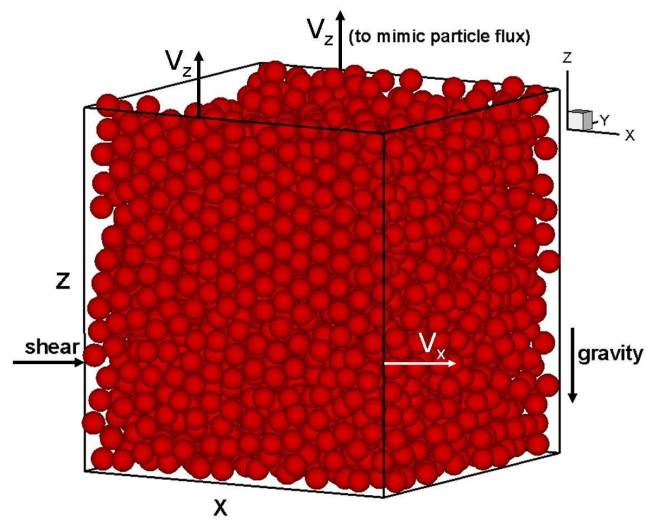

Fig. 2. Schematic of spherical particles bounded in a cubical domain. $V_{z}$ denotes the relative velocity due to the axial particle flow and $V_{x}$ the relative velocity due to shearing, with the arrows pointing to the positive directions.

ensures that the domain encompasses the particles moving in that direction. Wall boundary condition was applied to the rest of the domain boundaries. All the walls used in the simulations were flat and frictional, with a particle-wall friction coefficient $\mu_{w}=1.0$. The relative axial velocity of the walls located at $y=0$ and at $y=L$ (corresponding to radial coordinates for the Couette cell), $V_{z}$, was varied from zero for batch-mode simulation to values determined by the axial flow rates in the continuous-mode operation. The relative shearing velocity for the wall located at $y=0, V_{x}$, was calculated based on the shear rates used in the experiments. The average stress is calculated by dividing the force acting on this inner wall by the area of the granular bed cross section considering its expansion or compaction.

DEM simulations were performed at three different initial solid volume fractions, $0.64,0.62$ and 0.60 , which would no longer remain spatially uniform due to inhomogeneities developed during simulation. However, the particle volume fraction was not measured in the experiments. As stress increases with the initial solid volume fraction, we present results of simulations with a solid volume fraction of 0.64, which gives the closest match to experimental results. For particle properties not measured experimentally, reasonable values were assigned to reflect the bulk material behavior in the experiments. For instance, the normal spring stiffness was assigned to be $2 \times 10^{5} k_{0}$, which captures the general behavior of medium to high stiffness systems [22], such as the glass bead assembly used in the experiments. Similarly, the restitution coefficient and particle-particle friction were set to 0.9 and 0.19 , respectively. The integration time step $\triangle t$ was set to one fiftieth of the binary collision time $t_{c}$ to achieve a temporally converged numerical solution without excessive computational time [22]. Simulations were run for a non-dimensional time of $\dot{\gamma} t=500$ to attain a statistically stationary solution [21]. The basic computational parameters are summarized in Table 2. 


\begin{tabular}{|c|c|}
\hline Parameters & Values \\
\hline Number of particles & 3348 \\
\hline Particle diameter & $d_{0}$ \\
\hline Particle density & $1.91\left(m_{0} / d_{0}^{3}\right)$ \\
\hline Particle normal stiffness coefficient, $k_{n}$ & $2 \times 10^{5}\left(k_{0}\right)$ \\
\hline Particle tangential stiffness coefficient, $k_{t}$ & $(2 / 7) k_{n}$ \\
\hline Particle normal damping coefficient, $\gamma_{n}$ & $40\left(1 / t_{0}\right)$ \\
\hline Particle tangential damping coefficient, $\gamma_{t}$ & $0\left(1 / t_{0}\right)$ \\
\hline Particle friction coefficient, $\mu_{p}$ & 0.19 \\
\hline Particle restitution coefficient, $e$ & 0.9 \\
\hline Wall normal stiffness coefficient & $2 \times 10^{5}\left(k_{0}\right)$ \\
\hline Wall tangential stiffness coefficient & $(2 / 7) k_{n}$ \\
\hline Wall normal damping coefficient & $40\left(1 / t_{0}\right)$ \\
\hline Wall tangential damping coefficient & $20\left(1 / t_{0}\right)$ \\
\hline Wall friction coefficient, $\mu_{w}$ & 1.0 \\
\hline Time step, $\triangle t$ & $1 \times 10^{-4}\left(t_{0}\right)$ \\
\hline
\end{tabular}

Table 2

Computational parameters for DEM simulations.

\section{Experimental and simulation results}

\subsection{Transitional and intermediate behavior}

Rheology of sheared granular material probed by the Couette cell experiments and simulations is presented in this section. In Fig. 3 the average shear stress on the inner wall is plotted against time for the batch mode. Figure 3 shows that changing the shear rate more than fivefold in the DEM simulation does not result in any significant change in the shear stress, which is a feature of the quasi-static regime. DEM results show initial fluctuations in the stress level, but attain a statistically stationary state after approximately $30 t_{0}$. Quasistatic stress was also observed in the experiments, as the experiments were also performed with different values of shear rates, although only one of those (corresponding to $\hat{\gamma}=\dot{\gamma} /\left(g / d_{0}\right)^{1 / 2}=1.40 \times 10^{-1}$ ) is presented in Fig. 3 due to their close proximity. However, the magnitude of the measured shear stress is about 2.5 times higher than that predicted in the simulations.

The variation of shear stress with shear rate in the continuous mode with a 


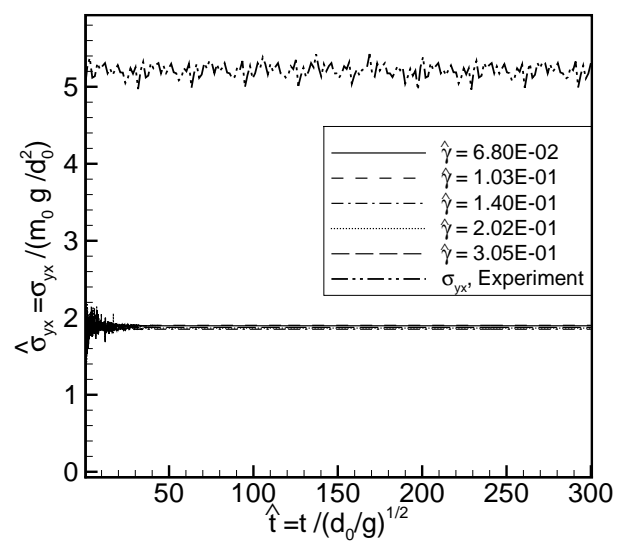

Fig. 3. Temporal evolution of the average non-dimensional shear stress on the Couette cell wall during the batch-mode operation.

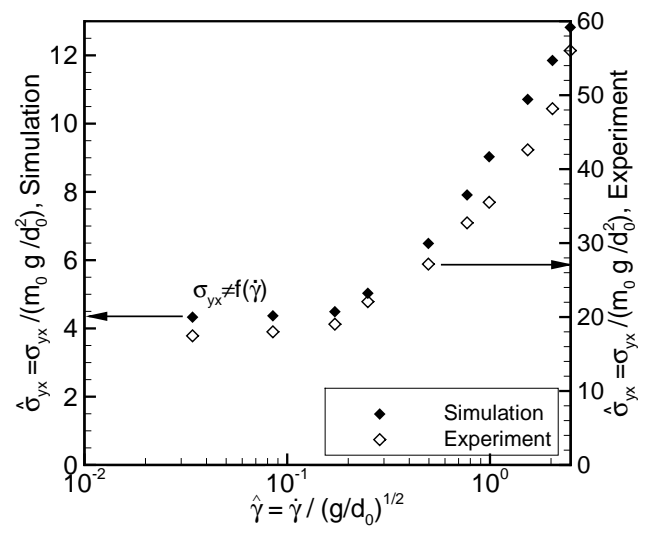

(a)

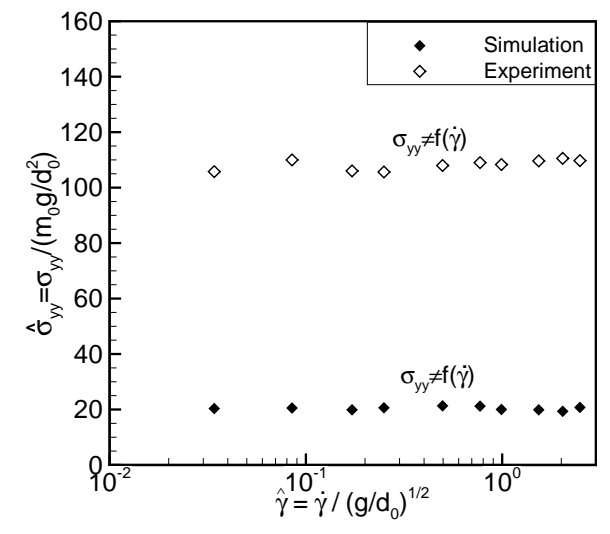

(b)

Fig. 4. Variation of (a) the average shear stress and (b) the average normal stress with the non-dimensional shear rate for the continuous-mode operation. The filled symbols denote the DEM simulation results, while the open symbols are for data obtained from experiments. Different scales are used on the left and right axes for the DEM and experimental data, respectively, to emphasize the similarity in their trends.

vertical speed, $V_{z}=3.2 \times 10^{-3} \sqrt{g d_{0}}$ corresponding to the flow rate in experiments is shown in Fig. 4(a) (the left vertical axis corresponds to the DEM simulation results and the right vertical axis corresponds to the experimental data). The experimental and DEM results in Fig. 4(a) show that the flow exhibits two distinct regimes: a quasi-static regime, where the shear stress is independent of the shear rate (at very low shear rates), and an intermediate regime, where the dependence takes the form of a power-law. The transition starts at a non-dimensional shear rate $\hat{\gamma}$, of about 0.1 , whereas the flow in the batch-mode at the same $\hat{\gamma}$ does not undergo transition (see the quasi-static behavior at even higher shear rates in Fig. 3). This transitional behavior in- 
dicates that the axial flow in continuous mode facilitates regime transition.

The DEM shear stress, however, is about 4.5 times lower in magnitude than its experimental counterpart, consistent with a factor-of-two difference found in a similar study performed by Ji et al. [23]. This difference could be due to differences in particle properties, domain geometry, system setup and solid volume fraction. The normal stress, on the other hand, remained almost constant in experiments and simulations as shown in Fig. 4(b). The experimental results for normal stress are also about five times of the DEM counterparts, which shows the effect of the overburden present in the experiments but ignored in the simulations.

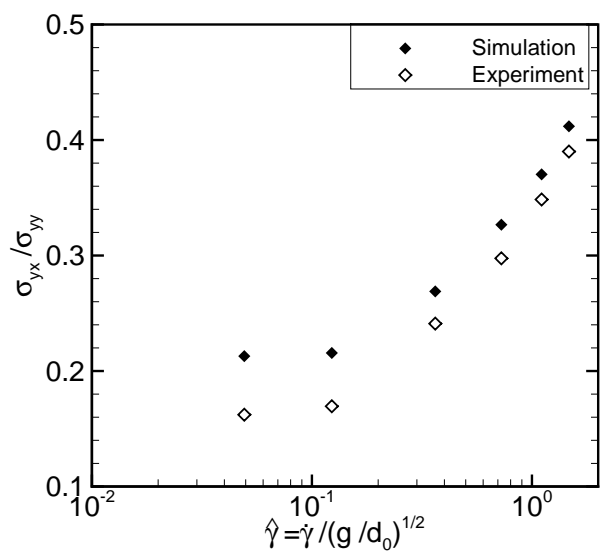

Fig. 5. Ratio of shear to normal stress as a function of shear rate for the continuous mode of operation. Filled symbols correspond to DEM simulation data whereas the open symbols are for experimental data.

The ratio of shear to normal stress $\left(\sigma_{y x} / \sigma_{y y}\right)$ is plotted in Fig. 5 as a function of the non-dimensional shear rate for the continuous mode of operation. The variation of this ratio also manifests the same transition and intermediate behavior: it remains almost a constant approximately equal to the particle friction coefficient for lower shear rates; it has a power-law dependence as the shear rate increases beyond a certain critical value. The experimental ratios are close to, albeit slightly lower than, the DEM results. The better agreement again confirms that the large difference in stress magnitude is mostly due to the different confinements (with/without overburden, etc.) and that the simulations reveal essentially the same rheology as in the experiments.

In section 4 , another non-dimensional shear rate $\gamma^{*}=\dot{\gamma} /\left(\rho d_{0}^{3} / k_{n}\right)^{1 / 2}$ is used according to the physical characteristics in a different system. It should be noted that the shear rates in the experiments corresponds to $\gamma^{*}$ values ranging from $10^{-5}$ to $10^{-3}$. 


\subsection{Simulation parametric study}

As reported earlier, reasonable values were assigned to some computational parameters that were not measured in the experiments. In order to quantify the effect of these parameters on the stress level, we performed a parametric study of initial solid volume fraction, particle stiffness, wall friction coefficient and axial velocity. These simulations were performed using the same computational setup as discussed in section 3.1, but with inter-particle friction coefficient equal to 0.2. The results are summarized in the following.

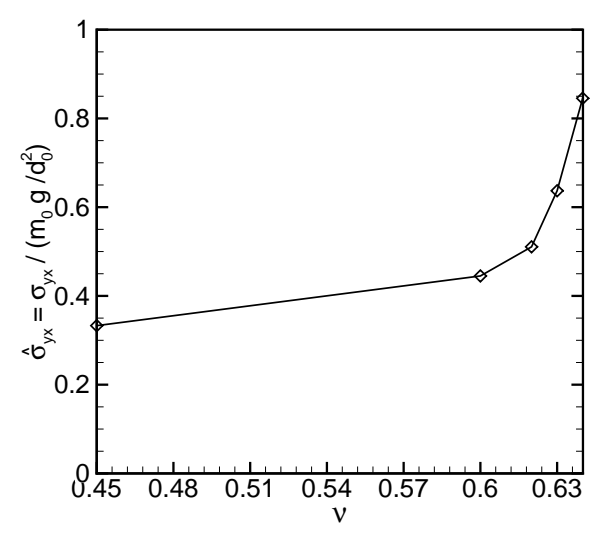

(a)

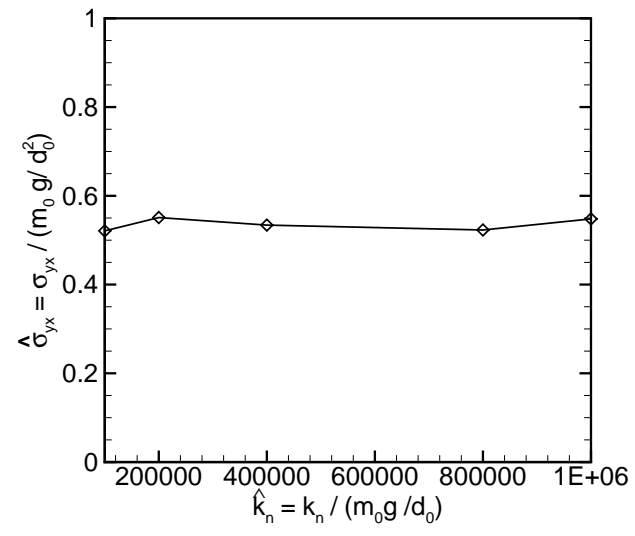

(b)

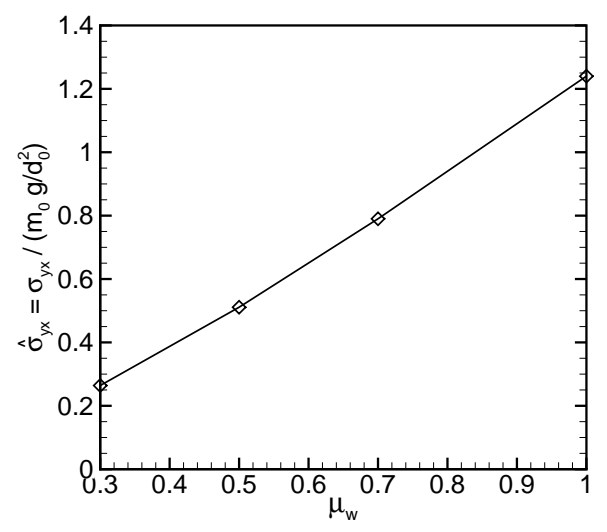

(c)

Fig. 6. Variation of average shear stress versus (a) solid volume fraction, (b) particle stiffness, and (c) wall friction coefficient.

Figure 6(a) shows the variation of average shear stress against initial solid volume fraction with wall friction coefficient fixed at 0.5. As expected, the stress level increases with increasing solid volume fraction, most pronouncedly above 0.60. As Fig. 6(b) shows, shear stress has nearly no change with respect to particle stiffness ranging from $10^{5} k_{0}$ to $10^{6} k_{0}$ with initial solid volume 
fraction kept at 0.62 , because the granular bed is allowed to expand freely. The shear stress increases fivefold as the wall friction coefficient increases from 0.3 to 1.0 as shown in Fig. 6(c) where initial solid volume fraction is kept at 0.62 . The variation of stress with the axial velocity in the shear gap was investigated over a limited range of values and the stress exhibits a sudden (factor-of-two) increase in the magnitude when the axial velocity is increased from 0 to $1.6 \times 10^{-3} \sqrt{g d_{0}}$, which is half the value used in the experiment (result not presented here). However, any further increase in the axial velocity up to $6.4 \times 10^{-3} \sqrt{g d_{0}}$ does not result in any appreciable change in the stress.

\section{Order parameter modeling and analysis}

The OP is defined as the ratio of space-time averaged number of "enduring" (solidlike) contacts $\overline{\left\langle Z_{s}\right\rangle}$ to all contacts $\overline{\langle Z\rangle}$ within a sampling volume [14]. A contact is considered solidlike only if it is in a stuck state $\left(F_{t}<\mu_{t} F_{n}\right)$ and its duration is longer than a typical time $t^{*}$, which is generally taken as 1.1 times the binary collision time $t_{c}$ [14]. The first requirement eliminates long lasting sliding contacts, and the second excludes short term collisions. When either of the requirements is not fulfilled, the contact is defined as "fluidlike". The OP $=1$ if a granular assembly is in a solid state, whereas $\mathrm{OP}=0$ at a completely fluidlike state.

Based on the OP concept, granular stress can be decomposed into solidlike and fluidlike contributions [24], which arise from solidlike contacts and fluidlike contacts plus the streaming stress (defined later in this section), respectively. The individual contribution may be described by known constitutive relations, such as KTGF or Newtonian fluid relations. The total stress can thus be calculated as a function of the individual contribution and the OP. A linear objective OP model [13] is employed in this study to predict the rheology in intermediate regime. The stresses are expressed in this model as

$$
\begin{gathered}
\sigma_{i j}=\frac{\sigma_{0}}{\beta}\left\{\frac{\sigma_{i j}^{f}}{\sigma_{0}}+\delta_{i j}(\beta-\alpha)\right\}, \\
\sigma_{i j}^{s}=\sigma_{0}\left\{(1-\alpha) \delta_{i j}+(1-\beta) b_{i j}\right\},
\end{gathered}
$$

where $\sigma_{i j}$ is the total granular stress, $\sigma_{i j}^{f}$ is the fluidlike stress, $\sigma_{i j}^{s}$ is the solidlike stress and $\sigma_{0}=\sigma_{i i}^{f} / 3 \alpha$ is the scale of stress (summation is implied over repeated indices). The normalized deviatoric stress tensor $b_{i j}$ is defined as

$$
b_{i j}=\frac{\sigma_{i j}}{\sigma_{0}}-\delta_{i j} .
$$

The scalar model coefficients $\alpha$ and $\beta$ are functions of the OP, which have been extracted from 3D DEM simulations of homogeneously sheared granular 
flow [25]. The model with so-determined coefficients is denoted as the refined order parameter (ROP) model. In this study, a KTGF constitutive relation [17] was used for fluidlike stress, which together with the ROP model is denoted as the ROP-KT model. Details of the ROP model can be found in Subramaniam and Vidyapati [25].

The OP was first demonstrated to have correct response to variation in flow conditions and particle properties in homogeneous shear flow. Figure 7(a) shows that the OP increases as the volume fraction increases and is indeed strongly dependent on the coefficient of friction. An increase of $300 \%$ in the values of the $\mathrm{OP}$ is seen when the coefficient of friction increases from 0.1 to 1.0 at the same volume fraction. This is because the larger inter-particle friction prevents more particles from sliding and thus more solidlike contacts and higher the OP values. In Fig. 7(b) the OP values are plotted as a func-

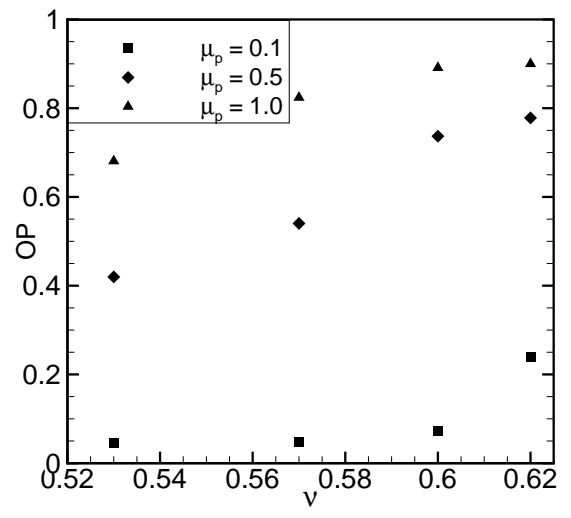

(a)

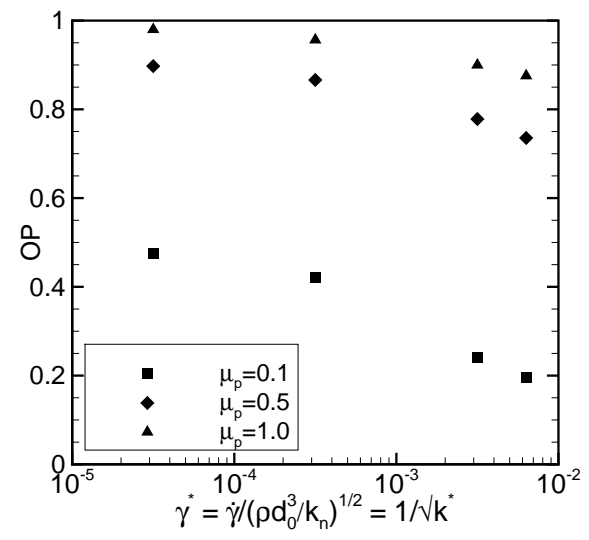

(b)

Fig. 7. Order parameter as a function of (a) volume fraction and (b) shear rate $\left(\gamma^{*}=\dot{\gamma} /\left(\rho d_{0}^{3} / k_{n}\right)^{1 / 2}=1 / \sqrt{k^{*}}\right)$ for homogeneous shear flow. $\gamma^{*}=3 \times 10^{-3}$ and $\nu=0.62$ in (a) and (b), respectively.

tion of the non-dimensional shear rate, $\gamma^{*}=\dot{\gamma} /\left(\rho d_{0}^{3} / k_{n}\right)^{1 / 2}$, which shows that the OP value decreases with increasing shear rate, indicating a decrease in solidlike contacts. The OP asymptotically approaches 1 at the solid limit for significantly high values of inter-particle friction coefficient and solid volume fraction at low shear rates. Both Figs. 7(a) and 7(b) lead to the conclusion that the OP is able to capture the changes in particle and flow properties.

The ROP-KT model is now examined to determine if it is capable of predicting the correct power-law dependence in the intermediate regime. The solid volume fraction used for the homogeneous flow simulations $(\nu=0.58)$ was chosen to match that of the vertically central part of the granular assembly from the Couette cell simulations reported earlier in this study (as noted earlier, the volume fraction field is not uniform). The particle friction coefficient $\mu_{p}$ and coefficient of restitution $e$ used are the same as used in the Couette 
cell simulations.

Figure 8 shows a logarithmic plot of the elastically scaled shear stress as a function of the non-dimensional shear rate. In this plot, stress variation for quasi-static flow will follow a horizontal line, and that in the inertial regime appears as a line with slope 2 . Lines with slope between 0 and 2 indicate the intermediate flow. It can be seen that the ROP-KT model does not predict the correct stress dependence when compared with the DEM data. The predicted slope is 1.92 , whereas the DEM data points follow a slope of 0.82 . This difference in the stress magnitude and strain-rate dependence is attributed to the fact that the fluidlike stress is modeled by the KTGF constitutive relation, which may not be valid for this intermediate flow, where both collisional and frictional interactions between particles are important. The performance of the ROP-KT model has been tested over a range of solid volume fractions for homogeneous shear flow [25]. It is found that the ROP-KT model predicts the total granular stress within error range of $15 \%$ for assemblies with a solid volume fraction up to 0.57 . However, the model performance deteriorates in the deep intermediate regime where solid volume fraction is higher than 0.57.

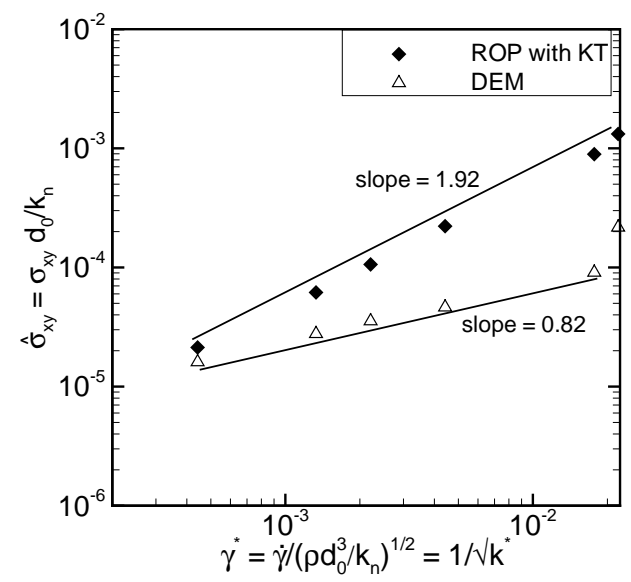

Fig. 8. Comparison of ROP-KT model prediction with DEM results of shear stress against shear rate for homogenous shear flow.

To further investigate the scaling of the stress-shear rate relation in the intermediate regime, we also decomposed the total granular stress obtained from DEM simulations into contact and streaming contributions. The contact contribution due to particle contacts in a domain of volume $V$ is given by

$$
\boldsymbol{\sigma}^{\text {contact }}=\frac{1}{V} \sum_{i}^{N} \sum_{j, j \neq i} \frac{1}{2} \mathbf{r}^{(i)(j)} \otimes \mathbf{f}^{(i)(j)},
$$

where $\mathbf{r}^{(i)(j)}$ is the vector pointing from the center of particle $j$ to the center of particle $i, \mathbf{f}^{(i)(j)}$ is the contact force acting on particle $i$ by particle $j$, and $\otimes$ 
denotes a dyadic product. The streaming contribution arises from momentum flux and is given as

$$
\boldsymbol{\sigma}^{\text {streaming }}=\frac{1}{V} \sum_{i}^{N} m_{0}^{(i)} \mathbf{v}^{\prime(i)} \otimes \mathbf{v}^{\prime(i)},
$$

where $m_{0}$ is the mass of a particle, $\mathbf{v}^{\prime}$ is the fluctuating velocity and $i$ is a particle index. The contact and streaming stresses from the same simulation as shown in Fig. 8 are plotted in Fig. 9(a) with non-dimensional shear rate. The contact part contributes more than $95 \%$ to the total granular stress and follows the same scaling, whereas the streaming part does not exhibit this scaling.

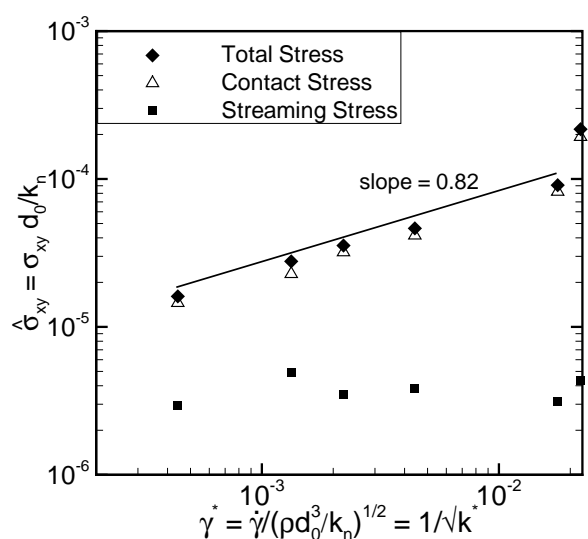

(a)

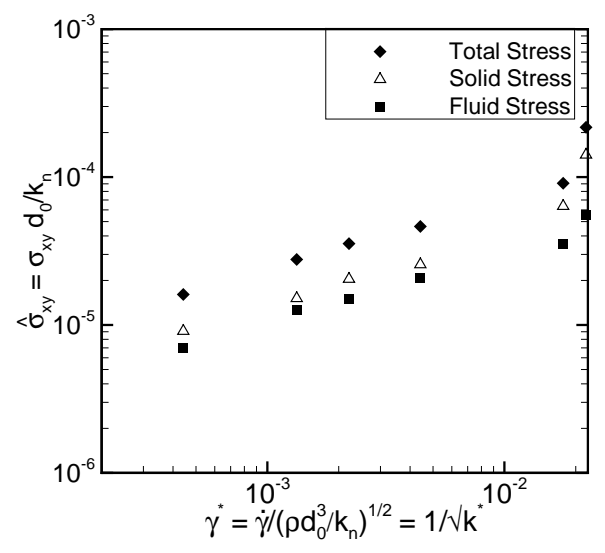

(b)

Fig. 9. Decomposition of the total shear stress into (a) contact and streaming, and (b) solidlike and fluidlike contributions.

We then decomposed the total granular stress obtained from DEM simulations into fluidlike and solidlike contributions, which are plotted in Fig. 9(b) with shear rate. The solidlike contribution is about $60-70 \%$ of the total stress. Both contributions approximately follow the same scaling $\left(\sigma \propto \dot{\gamma}^{n}, n=0.82\right)$ as the total stress because both carry portions of streaming and contact stress, the difference being whether the contacts are enduring or not.

\section{Conclusions}

Experiments and DEM simulations have been performed to study transitional rheology of dense granular materials in a modified annular Couette shear cell operated in batch or continuous mode. Shear stress and shear-to-normal-stress ratio were found to have quasi-static behavior $(\sigma \neq f(\dot{\gamma}))$ in the batch mode and to have an axial-flow-induced transition to the intermediate behavior characterized by a power-law relation with shear rate $\left(\sigma \propto \dot{\gamma}^{n}, 0<n<2\right)$ in the 
continuous mode. It has also been shown by the simulations that this transition and the power-law relation are robust rheological features, insensitive to particle properties and operational details.

The OP concept has been shown to be useful in reflecting the transitional behavior. The ROP-KT mode has been assessed to be applicable to assemblies with volume fractions less than 0.57 for prediction of intermediate behavior, as the power-law exponent cannot be correctly predicted for denser assemblies. This study illuminates that suitable constitutive relations should be sought for the fluidlike (or solidlike) stress in order for models based on OP to correctly predict certain intermediate behavior.

\section{Acknowledgements}

The authors wish to acknowledge the financial support from U.S. department of Energy National Energy Technology Laboratory (grant DE-FG2607NT43070), administered under advanced coal research at U.S. colleges and universities.

\section{A Contact model description}

For two contacting particle $\{i, j\}$, with radii $\left\{a_{i}, a_{j}\right\}$ at positions $\left\{\mathbf{r}_{i}, \mathbf{r}_{j}\right\}$, with velocities $\left\{\mathbf{v}_{i}, \mathbf{v}_{j}\right\}$ and angular velocities $\left\{\boldsymbol{\omega}_{i}, \boldsymbol{\omega}_{j}\right\}$, the normal compression $\delta_{i j}$, relative normal velocity $\mathbf{v}_{n_{i j}}$, and relative tangential velocity $\mathbf{v}_{t_{i j}}$ are [22]

$$
\begin{aligned}
\delta_{i j} & =d-\mathbf{r}_{i j}, \\
\mathbf{v}_{n_{i j}} & =\left(\mathbf{v}_{i j} \cdot \mathbf{n}_{i j}\right) \mathbf{n}_{i j}, \\
\mathbf{v}_{t_{i j}} & =\mathbf{v}_{i j}-\mathbf{v}_{n_{i j}}-\left(a_{i} \boldsymbol{\omega}_{i}+a_{j} \boldsymbol{\omega}_{j}\right) \times \mathbf{n}_{i j},
\end{aligned}
$$

where $d=a_{i}+a_{j}, \mathbf{r}_{i j}=\mathbf{r}_{i}-\mathbf{r}_{j}, \mathbf{n}_{i j}=\mathbf{r}_{i j} / r_{i j}$, with $r_{i j}=\left|\mathbf{r}_{i j}\right|$ and $\mathbf{v}_{i j}=\mathbf{v}_{i}-\mathbf{v}_{j}$. The rate of change of the elastic tangential displacement $\mathbf{u}_{t_{i j}}$, set to zero at the initiation of a contact, is

$$
\frac{d \mathbf{u}_{t_{i j}}}{d t}=\mathbf{v}_{t_{i j}}-\frac{\left(\mathbf{u}_{t_{i j}} \cdot \mathbf{v}_{i j}\right) \mathbf{r}_{i j}}{r_{i j}^{2}}
$$

The last term in Eq. A.4 arises from the rigid body rotation around the contact point and ensures that $\mathbf{u}_{t_{i j}}$ always lies in the local tangent plane of contact. 
Normal and tangential forces acting on particle $i$ are

$$
\begin{gathered}
\mathbf{F}_{n_{i j}}=f\left(\delta_{i j} / d\right)\left(k_{n} \delta_{i j} \mathbf{n}_{i j}-\gamma_{n} m_{e f f} \mathbf{v}_{n_{i j}}\right), \\
\mathbf{F}_{t_{i j}}=f\left(\delta_{i j} / d\right)\left(-k_{t} \mathbf{u}_{t_{i j}}-\gamma_{t} m_{e f f} \mathbf{v}_{t_{i j}}\right),
\end{gathered}
$$

where $k_{n, t}$ and $\gamma_{n, t}$ are the spring stiffness and viscoelastic constants, respectively, and $m_{e f f}=m_{i} m_{j} /\left(m_{i}+m_{j}\right)$ is the reduced mass of spheres with masses $m_{i}$ and $m_{j}$. The corresponding contact force on particle $j$ is simply given by Newton's third law, i.e., $\mathbf{F}_{j i}=-\mathbf{F}_{i j}$. The function $f\left(\delta_{i j} / d\right)=1$ is

for the linear spring-dashpot model, and $f\left(\delta_{i j} / d\right)=\sqrt{\delta_{i j} / d}$ is for Hertzian contacts with viscoelastic damping between spheres.

Static friction is implemented by keeping track of the elastic shear displacement throughout the lifetime of a contact. The static yield criterion, characterized by a local particle friction coefficient $\mu$, is modeled by truncating the magnitude of $\mathbf{u}_{t_{i j}}$ as necessary to satisfy $\left|\mathbf{F}_{t_{i j}}\right|<\left|\mu \mathbf{F}_{n_{i j}}\right|$. Thus the contact surfaces are treated as "sticking" when $\left|\mathbf{F}_{t_{i j}}\right|<\left|\mu \mathbf{F}_{n_{i j}}\right|$, and as "slipping" when the yield criterion is satisfied.

The amount of energy lost in collisions is characterized by the value of the coefficient of restitution, which is defined as the negative ratio of the particle normal velocity after collision to the velocity before collision. For the linear spring-dashpot model, the coefficient of normal restitution and contact time can be analytically obtained:

$$
e_{n}=\exp \left(-\gamma_{n} t_{\mathrm{c}} / 2\right),
$$

where the contact time $t_{c}$ is given by

$$
t_{c}=\pi\left(k_{n} / m_{\mathrm{eff}}-\gamma_{n}^{2} / 4\right)^{-1 / 2} .
$$

The value of the spring constant should be large enough to avoid particle interpenetration, yet not so large as to require an unreasonably small simulation time step $\triangle t$, since an accurate simulation typically requires $\triangle t \sim t_{c} / 50$ [22]. After the contact force is calculated, the equations of motion, which are ordinary differential equations, can be numerically integrated to get the particle trajectories. 


\section{References}

[1] H. M. Jaeger, S. R. Nagel, and R. P. Behringer, "Granular solids, liquids, and gases," Reviews of Modern Physics, vol. 68, no. 4, 1996.

[2] S. Sundaresan, "Some outstanding questions in handling of cohesionless particles," Powder Technology, vol. 115, no. 1, pp. 2-7, 2001.

[3] O. Reynolds, "On the dilatancy of media composed of rigid particles in contact.," Philos. Mag. Ser., vol. 20, pp. 469-481, 1885.

[4] R. Bagnolds, "Experiments on a gravity-free dispersion of large solid spheres in a newtonian fluid under shear.," Proceedings of the Royal Society of London Series A: Mathematical and Physical Sciences, vol. 225, pp. 49-63, 1954.

[5] S. Savage and M. Sayed, "Stress developed by dry cohesionless granular materials sheared in an annular shear cell.," J. Fluid Mech., vol. 142, pp. 391-430, 1984.

[6] B. Miller, C. Hern, and R. Behringer, "Stress fluctuations for continuosly sheared granular materials.," Phys. Rev. Lett., vol. 77, no. 15, pp. 31103113, 1996.

[7] G. Tardos, S. McNamara, and I. Talu, "Slow and intermediate flow of a frictional bulk powder in the couette geometry.," Powder Technology, vol. 131, pp. 23-39, 2003.

[8] J. Tsai and J. Gollub, "Slowly sheared dense granular flows: crystallization and nonunique states.," Phys. Rev. E, vol. 70, pp. 031303-1-03130313, 2004.

[9] G.D.R. MiDi, "On dense granular flows.," Eur. Phys. J. E, vol. 14, pp. 341-365, 2004.

[10] P. Cundall and O. Strack, "A discrete numerical model for granular assemblies.," Géotechnique, vol. 29, pp. 47-65, 1979.

[11] S. Schöllmann, "Simulation of a two-dimensional shear cell," Physical Review E, vol. 59, 011999.

[12] T. S. Majmudar, M. Sperl, S. Luding, and R. P. Behringer, "Jamming transition in granular systems," Physical Review Letters, vol. 98, no. 5, pp. 058001-4, 2007.

[13] D. Gao, S. Subramaniam, R. Fox, and D. Hoffman, "Objective decomposition of the stress tesnor in granular flows.," Phys. Rev. E, vol. 71, pp. 021302-1-021302-5, 2005.

[14] D. Volfson, L. Tsimring, and I. Aranson, "Partially fluidized shear granular flows: Continuum theory and molecular dynamics simulations.," Phys. Rev. E, vol. 68, pp. 021301-1-021301-15, 2003.

[15] S. B. Savage, "Granular flows down rough inclines - review and extension," in Mechanics of granular materials: new models and constitutive relations (J. T. Jenkins and M. Satake, eds.), pp. 261-282, Elsevier Ltd, 1983.

[16] S. Savage, "Analyses of slow high-concentration flows of granular materials.," J. Fluid Mech., vol. 377, pp. 1-77, 1998. 
[17] C. Lun, S. Savage, D. Jeffrey, and N. Chepuriny, "Kinetic theories for granular flow:inelastic particles in couette flow and slightly inelastic particles in general flow fields.," J. Fluid Mech., vol. 140, pp. 223-256, 1984.

[18] R. M. Nedderman, Static and kninematics of granular material. Cambridge University Press, 2nd. ed., 1992.

[19] M. Kheiripour Langroudi, S. Turek, A. Ouazzi, and G. Tardos, "An investigation of frictional and collisional powder flows using a unified constitutive equation.," Powder Technology, vol. 197, pp. 91-101, 2010.

[20] M. Kheiripour Langroudi, J. Sun, S. Sundaresan, and G. Tardos, "Transimission of normal stresses in un-sheared and sheared granular beds: the influence of particle size, shape, stiffness and cohesion.," Powder Technology, vol. 203, pp. 23-32, 2010.

[21] C. Campbell, "Granular shear flows at the elastic limit.," J. Fluid Mech., vol. 465, pp. 261-291, 2002.

[22] L. Silbert, D. Ertas, G. Grest, T. Halsey, D. Levine, and S. J. Plimpton, "Granular flow down an inclined plane: Bagnold scaling and rheology", Phys. Rev. E, vol. 64, pp. 051302-1-051302-13, 2001.

[23] S. Ji, D. M. Hanes, and H. H. Shen, "Comparisons of physical experiment and discrete element simulatuions of sheared granular material in an annular shear cell.," Mechanics of Materials, vol. 41, pp. 764-776, 2009.

[24] D. Volfson, L. Tsimring, and I. Aranson, "Order parameter description of stationary partially fluidized shear granular flows.," Phys. Rev. E, vol. 90, pp. 254301-1-254301-4, 2003.

[25] S. Subramaniam and Vidyapati, "Rheological behavior of dense granular material: DEM simulations and Order Parameter model.," NETL 2009 Workshop on Multiphase Flow Science, 2009. 


\section{Appendix $\mathbf{H}$}

\section{Shear flow of assemblies of cohesive granular materials under constant applied normal stress}




\title{
Shear flow of assemblies of cohesive granular materials under constant applied normal stress is
}

\author{
Lee Aarons, Sankaran Sundaresan * \\ Department of Chemical Engineering Princeton University, Princeton, NJ, 08544, USA
}

Available online 9 February 2008

\begin{abstract}
We have studied plane shear flow of nearly homogeneous assemblies of uniformly sized, spherical, cohesive particles in periodic domains under constant applied normal stress. Our focus has been on (a) exploration of the effect of inter-particle attractive forces on the flow behavior manifested by dense assemblies under constant applied normal stress, and (b) comparison of the rheological characteristics observed under constant-applied normal stress and constant-volume conditions. As a model problem, the cohesion resulting from van der Waals force acting between particles is considered. Simulations were performed for different strengths of cohesion, shear rates, and applied stresses. From each simulation, the volume fraction, shear stress and the average coordination number have been extracted. We find that cohesive assemblies sheared under constant applied normal stress shear differently from those sheared at constant volume only in the dynamic sense, while the time-averaged rheological characteristics are essentially indistinguishable. At constant volume, the fluctuations in shear stress are larger than, but have the same dependence on cohesion as under constant applied normal stress. This study has also exposed a pronounced dependence of the apparent coefficient of friction on particle volume fraction in the quasi-static flow regime.
\end{abstract}

(C) 2008 Elsevier B.V. All rights reserved.

Keywords: Flow regime; Cohesion; Discrete element simulation; Shear flow of powders; Constant stress

\section{Introduction}

It is well-known that assemblies of granular materials behave differently under different flow conditions. Under rapid flow conditions, the particles interact with each other predominantly through binary collisions, while at slow shear rates the interaction is dominated by enduring contacts. The rheological behavior of slowly deforming assemblies of granular materials has been studied extensively via simulations [e.g., see 1-4] and experiments [e.g., see 1,5,6].

Campbell [7] carried out discrete element method (DEM) simulations of sheared assemblies of cohesionless particles in periodic domains and under constant-volume conditions and presented a map of the different regimes of flow. In a previous study [8] we performed similar simulations for cohesive granular

\footnotetext{
Submitted for the Special Powder Technology Issue honoring Professor Pfeffer (May 2007).

* Corresponding author.

E-mail address: sundar@princeton.edu (S. Sundaresan).
}

materials and showed how the stresses varied with particle volume fraction, shear rate, and particle properties including the strength of the cohesive interaction. This study revealed that inter-particle attractive forces expand the range of particle volume fractions and shear rates over which the quasi-static flow regime is observed. Furthermore, this study identified how the stresses in this regime should be scaled and showed that the apparent coefficient of friction varied systematically with particle volume fraction.

In practical devices, flows of granular materials rarely occur under constant volume, and compaction and dilation do occur locally in response to the stresses. This and the fact that laboratory rheological experiments are often performed under constant applied overburden levels have prompted researchers to investigate regime maps under conditions of constant applied normal stress. Campbell $[9,10]$ reported that the flow regime map for assemblies of cohesionless particles sheared at constant applied normal stress (determined through discrete element method (DEM) simulations) differed from the flow regime map he previously constructed for such assemblies sheared at constant volume [7]. In the present study, we have analyzed shear flow of assemblies of cohesive granular materials under constant applied 
normal stress and compared the results with those obtained under constant-volume conditions.

We will first present an analysis of the time-averaged results, where the mean volume fraction-shear rate-mean stress relationships obtained from the constant-volume simulations at various strengths of cohesion will be compared to those obtained from simulations performed under constant applied normal stress. We will then compare the stress fluctuations in both types of simulations and discuss the dependence of these fluctuations on cohesive strength, shear rate and the type of simulation (constant volume versus constant applied normal stress) performed.

In Section 2, we discuss briefly the flow regimes for cohesionless and cohesive materials reported previously by Campbell [7,9,10] and us [8]. In Section 3, the simulation method for shearing under constant applied stress is described. The results of these simulations are given in Section 4. Here it will be shown that the regime map does not depend on whether shearing is performed under constant applied normal stress or at constant volume; this contradicts the earlier observation by Campbell. However, the dynamic stress fluctuations are larger when shearing is performed at constant volume. Finally, the main results of this study are summarized in Section 5.

\section{Flow regime overview}

Based on discrete element method (DEM) simulations of constant-volume granular shear flows in the absence of gravity, Campbell [7] identified and described the different regimes of flows for uniformly sized, cohesionless, frictional particles. Examining how the shear and normal stresses varied with shear rate $(\dot{\gamma})$, stiffness of the material $(k)$, coefficient of friction $(\mu)$, and particle volume fraction $(\phi)$, he identified four regimes of flow. Specifically, he found how the shear and normal components of the "scaled stress" $\left(\sigma^{*}=\sigma d / k\right.$, where $\sigma$ is the stress and $d$ is the particle diameter) varied with the "scaled stiffness" $\left(k^{*}=k / \rho d^{3} \dot{\gamma}^{2}\right.$, where $\rho$ is the particle density) for various values of volume fraction and particle friction. The normal and shear stresses were proportional to the square of the shear rate (i.e. $1 / k^{*}$ ) in the inertial-collisional and inertial-non-collisional regimes, at low volume fractions where the particles flowed freely and in the former case, the contact interactions were dominated by binary collisions. At high particle volume fractions, the assemblies existed in the elastic-quasi-static regime, where the shear and normal stresses did not vary appreciably with shear rate. In this regime, the particles were confined to force chains that continually rotated, broke, and reformed. Finally the elasticinertial regime, where the normal and shear stress scaled linearly with the shear rate (i.e. $1 / \sqrt{k^{*}}$ ), was found to exist at very low values of scaled stiffness.

In a previous study [8] we identified the location of the flow regimes for cohesive assemblies of particles using as parameters the particle friction coefficient $(\mu)$, the solid volume fraction $(\phi)$, the scaled stiffness, and the scaled cohesiveness $\left(B o^{*}=F_{v d W}^{\max } / k d\right.$, where $F_{v d W}^{\max }$ is the cohesive force exerted by a particle on a contacting particle). We found that when $\phi$ or $B o^{*} k^{*}$ was sufficiently high, the scaled stress depended very weakly on shear rate (or $k^{*}$ or $B o^{*} k^{*}$ ) and so these assemblies were considered to be in the elastic-quasi-static regime. For these cohesive assemblies, the elastic-quasi-static regime were found to be divisible into two sub-regimes which were differentiated by how the stress scales with the level of cohesion $\left(B O^{*}\right)$ or how the assemblies would have behaved in the absence of cohesion. For large volume fractions $(\phi>0.61$ for $\mu=0.1)$ regardless of $B o^{*} k^{*}$, the scaled stress did not vary significantly with $B o^{*}$. This subregime, which will be referred to as the "high $\phi$ quasi-static regime," corresponded to where cohesionless assemblies have been found to exist in the elastic-quasi-static regime [7]. For smaller volume fractions and large $B o^{*} k^{*}$, in the sub-regime which will be referred to as the "low $\phi$ quasi-static regime," the scaled stress varied proportionally with $B o^{*}$. Without cohesion, the assemblies at these conditions would exist in the inertialcollisional or inertial-non-collisional regime.

At lower values of both $\phi$ and $B o^{*} k^{*}$ (or $k^{*}$ ), the stress was found to be inversely proportional to $k^{*}$ (i.e. proportional to the square of the shear rate), and so these assemblies were considered to be in the inertial regime. Furthermore, it was found that the particle interactions were not dominated by binary collisions, and so specifically, these assemblies were in the inertial-noncollisional regime, just like the cohesionless assemblies at the same conditions. At only the smallest $B o^{*} k^{*}$ (or $k^{*}$ ) examined and at relatively high volume fraction $(\phi \geq 0.6)$, the scaled stress was found to be proportional to $1 / \sqrt{k^{*}}$ (i.e. proportional to the shear rate) and so these assemblies were considered to be in the elastic-inertial regime, again just like the cohesionless assemblies at the same conditions. So, in summary, we found that cohesive assemblies differed from cohesionless ones under constant volume shear only when both the volume fraction was sufficiently small and the scaled stiffness was sufficiently large (which correspond to the low $\phi$ quasi-static regime mentioned above). Otherwise, they behave the same way.

Campbell $[9,10]$ investigated the shearing of cohesionless assemblies under constant applied normal stress and found that as $k^{*}$ was varied, the regime map was traversed differently from when the volume fraction was kept constant. So naturally the question arises as to what, if any, are the differences between shearing cohesive assemblies at constant volume and under constant applied stress. The differences can arise in the timeaveraged behavior or in a dynamic sense, namely, the fluctuations about the time-averaged values. Let us suppose, for example, that a mean stress, $\sigma_{0}^{*}$, is obtained from shearing at a constant volume fraction $\phi_{0}$ (where the stresses are allowed to fluctuate freely); then, let us suppose that one carries out shearing under constant applied normal stress $\sigma_{0}^{*}$ (with the same values for $k^{*}$ and $\mu$ as before, allowing the volume fraction to fluctuate freely). The first set of questions that one can pose is, "Will the mean volume fraction be different from $\phi_{0}$ and if so, why?" We have addressed this issue in the present study.

The dynamics must differ between the two types of shearing, as the volume fraction varies during shear under constant applied normal stress but not during constant-volume shear. The stresses fluctuate throughout both types of shearing simulations (even for shearing under constant applied normal stress, more on this later), and the size and behavior of these fluctuations may depend on the type of shearing performed. So even if both 
Table 1

Summary of dimensionless input and output parameters

\begin{tabular}{lll}
\hline Dimensionless variable & Symbol & Values \\
\hline Input & & \\
Scaled stiffness & $k *=k / \rho d^{3} \dot{\gamma}^{2}$ & $10^{4}-10^{9}$ \\
Modified Bond number & $B o *=F_{v d W^{\max } / k d \approx A / 24 k s_{\text {min }}^{2}}$ & $0-1.25 \times 10^{-4}$ \\
Scaled applied stress & $\sigma_{0} d / k$ & $5 \times 10^{-7}-10^{-2}$ \\
Coefficient of restitution & $\varepsilon$ & 0.7 \\
Coefficient of friction & $\mu$ & 0.1 \\
Poisson ratio & $v$ & 0 \\
Minimum cutoff separation & $s_{\min } / d$ & $4 \times 10^{-5}$ \\
Output & & \\
Volume fraction & $\phi$ & \\
Scaled stress tensor & $\sigma^{*}=\sigma d / k$ & \\
Coordination number & $Z$ & \\
\hline
\end{tabular}

types of shearing are the same with regards to time-averaged quantities, they may manifest significant differences in the size of the fluctuations. We will discuss this point in some detail later in the paper.

\section{Details of discrete element simulations}

In this paper, the differences between results obtained for steady shear flow of cohesive materials under constant applied normal stress and under constant-volume conditions are explored. Results of DEM simulations of steady shear under constantvolume conditions have already been described in our earlier publication [8]; additional simulations of this type needed for the comparisons were performed following the same procedure.

Bulk of the effort in the present study was devoted to DEM simulations [11] of steady shear flow under conditions of constant applied normal stress, for which we employed the scheme described by Campbell $[9,10]$. The mean volume fractions and coordination numbers of cohesive particle assemblies with different applied normal stresses, scaled stiffnesses, and levels of cohesion were calculated. These data along with the data collected from simulations performed at constant-volume conditions were plotted using the different scalings for the stress, and these results from the two different types of shearing were compared. Furthermore, in each simulation, the stresses and volume fraction were tracked over the course of the simulation. The sizes of the fluctuations were compared to determine if and how the shearing dynamics depend on the flow regime, cohesion, and whether the shearing was performed at constant volume or under constant applied normal stress.

We direct the reader to Aarons and Sundaresan [8] for the majority of the simulation details. All of the notations are kept the same, so that the stream velocity is in the $x$-direction and the velocity gradient is in the $z$-direction. Furthermore, the $z z$ - and $x z$-components of the stress tensor are referred to as the normal and shear stresses, respectively.

The effects of changing the normal force model for colliding particles as well as the particle coefficient of friction $(\mu)$ were explored in a previous study [8] and will not be discussed here. Instead, only the Hookean linear spring-dashpot (LSD) normal force model and a particle friction coefficient of 0.1 are used. Table 1 summarizes the input and output parameters (with regards to the constant-applied normal stress simulations), as well as the values used in the simulations.

For the initial conditions, the simulated periodic boxes were chosen to have volume fractions of $0.62,0.6,0.57$, and 0.55 . In a previous study [8] we found that cohesive assemblies of volume fractions less than 0.6 did not shear homogeneously in large cubic boxes, but rather required shallow boxes (in the $z$-direction) to shear homogeneously. So if a simulation performed under constant applied normal stress was expected to achieve an average volume fraction below 0.6 (based on the constant-volume simulations), they were started in shallow boxes with volume fractions of 0.57 or 0.55 . For steady state to be achieved quickly, the specific initial volume fraction for a given simulation was chosen to be closest to what was expected to be the average volume fraction (some simulations were performed with different initial sizes of periodic boxes to ensure that such a choice of initial condition did not affect the statistical steady state results).

At every time step, the domain-average stresses were calculated, and depending on whether the $z z$-component of the stress tensor was larger or smaller than the desired applied stress, the periodic box was expanded or compacted, respectively, in the $z$-direction. The amount by which the top of the box was raised or lowered is specified by a penalty function [9]

$\Delta H=H \dot{\gamma} \Delta t\left(\frac{\sigma_{z z}-\sigma_{0}}{\left|\sigma_{z z}-\sigma_{0}\right|}\right) \min \left\{\left|\frac{\sigma_{z z}-\sigma_{0}}{\sigma_{0}}\right|, 1\right\}$.

Each particle in the periodic box was relocated by a proportional amount. So if a particle's position in the $z$-direction was given by $z_{p}$ (measured from the bottom of the box), then $z_{p}$ was increased by

$\Delta z_{p}=\Delta H \frac{z_{p}}{H}$

After a short "start-up time," the measured stress stayed within a few percent of the desired stress. This algorithm suggests that the term "shearing under constant applied normal stress" is a bit of a misnomer, as the stress is regulated via a feedback mechanism and is thus subject to fluctuations. Eq. (1) was altered by adding different coefficients in front of the right side to determine if the measured stress could be kept closer to the applied stress. The different variations of the penalty function were used in simulations with $<\sigma_{z z} d / k>=10^{-6}, k^{*}=10^{9}$, and $B o^{*}=5 \times 10^{-7}$ (for which $\langle\phi\rangle \approx 0.60$ ). Table 2 gives the standard deviation of the normal stress, scaled by the average normal stress, for five different coefficients. The best choice to precede the right side of Eq. (1) actually appears to be of the order of unity. Indeed, Campbell $[9,10]$ used Eq. (1) as is (i.e. with a coefficient of 1) and we adopted this value for the coefficient as well.

Table 2

Effect of changing the constant-stress penalty function Eq. (1)

\begin{tabular}{ll}
\hline Coefficient added to right side of Eq. (1) & Standard deviation of $\sigma_{z z} /\left\langle\sigma_{z z}\right\rangle$ \\
\hline 0.1 & 0.1998 \\
0.5 & 0.0386 \\
1 & 0.0209 \\
2 & 0.0155 \\
10 & 1.1518 \\
\hline
\end{tabular}


There are conceivably two ways the constant-normal stress algorithm can be utilized. In the first way, the shear rate is held constant throughout the simulations, and the Lees-Edwards boundary conditions (i.e. the velocity adjustments made to a particle when it crosses the top or bottom of the periodic box) are changed as the simulated box expands or shrinks. Alternatively, the Lees-Edwards boundary conditions can be held constant, in which case the shear rate is allowed to fluctuate throughout the simulation. The results presented here were obtained from simulations using the first method. For the sake of completeness, the alternate method was used for some of the cases presented, and in each case it produced the same results and the shear rate varied insignificantly $(<1 \%)$ over the course of the simulations. The latter observation is due to the fact that the volume fraction varies very slightly during the simulations, a point which will be discussed later.

\section{Results and discussion}

\subsection{Time-averaged results}

Fig. 1a and $\mathrm{b}$ show the variation of particle volume fraction with the scaled normal stress for various levels of cohesion. The
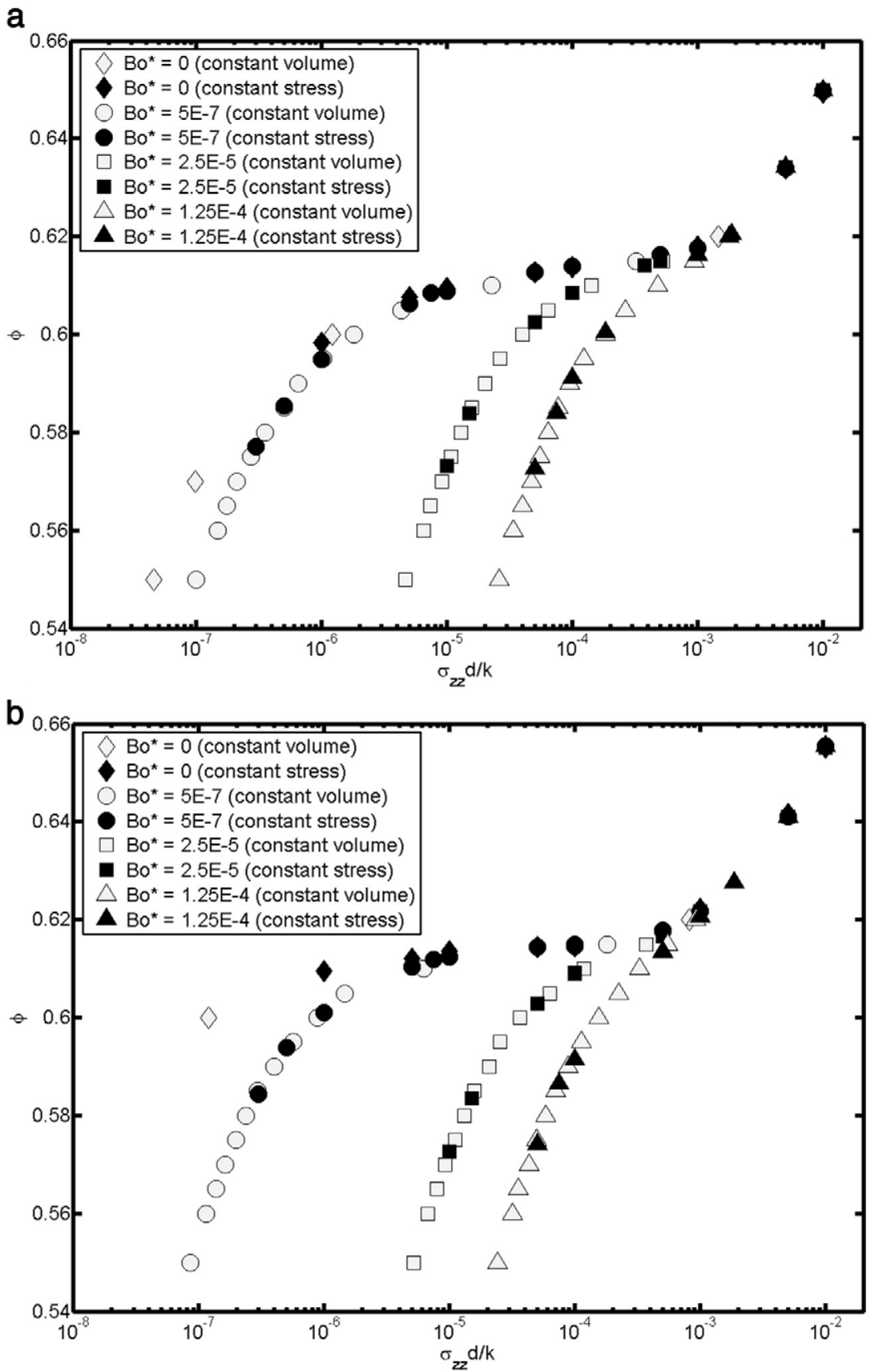

Fig. 1. The effect of cohesion on the solid volume fraction versus normal stress behavior for two different values of the scaled stiffness; (a) $k^{*}=10^{8}$ and (b) $k^{*}=10^{9}$. Plotted is the solid volume fraction $(\phi)$ versus scaled normal stress $\left(\sigma_{z z} d / k\right)$ obtained from simulations run at constant-volume (grey symbols) and constant-applied normal stress (black symbols) conditions at various levels of cohesion. 


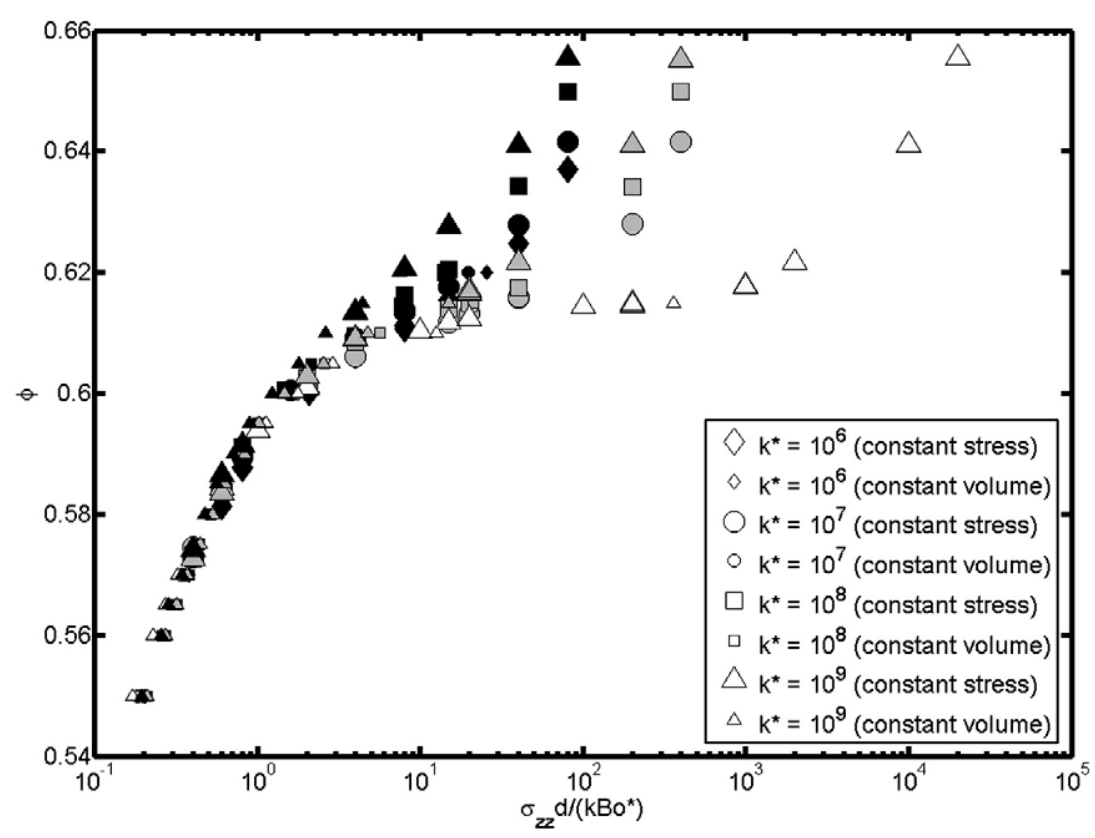

Fig. 2. Volume fraction $(\phi)$ versus mean cohesively scaled normal stress $\left(\sigma_{z z} d /\left(k B o^{*}\right)=24 \sigma_{z z} d s_{\min }^{2} / A\right)$ with $B o^{*} k^{*}>100$, obtained from simulations run at constantvolume and constant-applied normal stress conditions. The transition from the high $\phi$ quasi-static regime (where the scaling splits the curve into three - one for each value of $B o^{*}$ ) to the low $\phi$ quasi-static regime (where the scaling collapses the curves into one) occurs at $\phi \approx 0.61 . B o^{*}=5 \times 10^{-7}$ (light grey symbols), $2.5 \times 10^{-5}$ (dark grey symbols), and $1.25 \times 10^{-4}$ (black symbols).

plotted data include results from simulations performed under constant-applied normal stress (black symbols) and constantvolume (grey symbols) conditions. Each of these two figures shows data for only one value of scaled stiffness: (a) $k^{*}=10^{8}$ and (b) $k^{*}=10^{9}$. These figures show clearly that the timeaveraged data obtained from the simulations run under constant applied normal stress fall in line with those from the constantvolume simulations. Recall that in the constant-applied normal stress simulations the volume fraction is allowed to vary while the normal stress is controlled through a feedback algorithm. In contrast, in the constant-volume simulations the volume fraction does not change with time, while the applied normal stress fluctuates. In spite of these differences, one obtains essentially the same relationship between the time-averaged data in these simulations, indicating clearly that there is no significant difference between these two types of simulations in

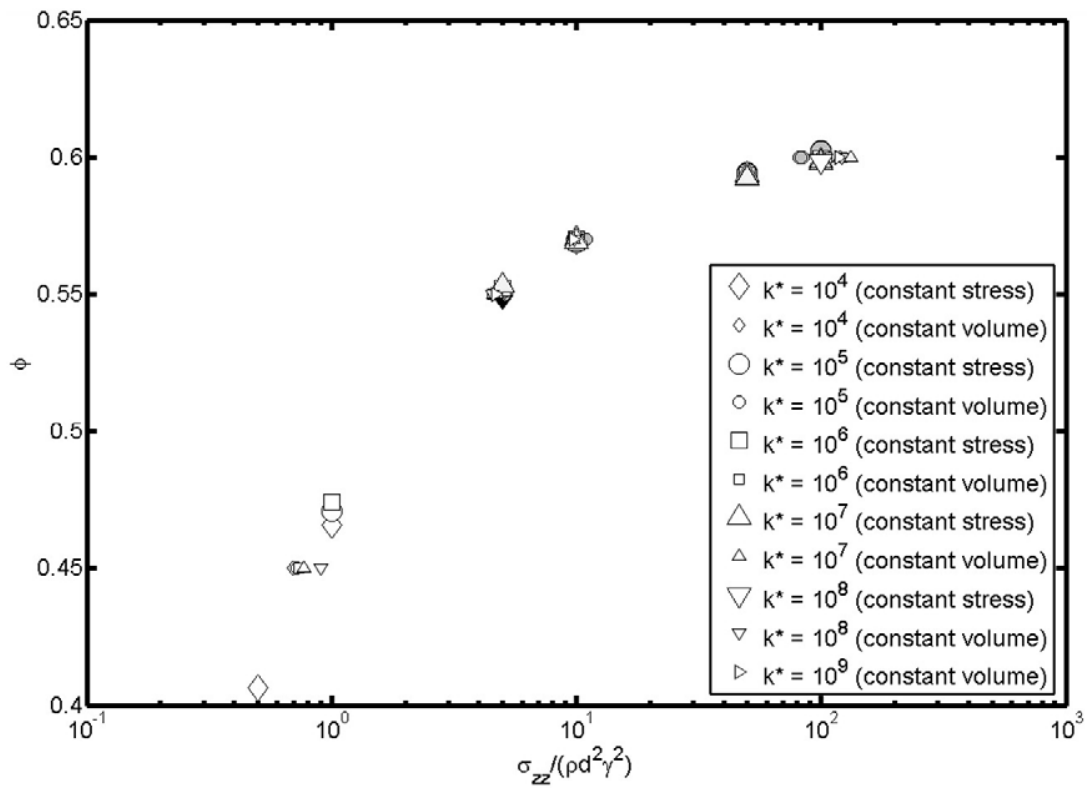

Fig. 3. Volume fraction $(\phi)$ versus mean kinetically scaled normal stress $\left(\sigma_{z z} d k^{*} / k=\sigma_{z z} / \rho d^{2} \dot{\gamma}^{2}\right)$ for assemblies the inertial regime, with $B o^{*} k^{*}<10$ and $<\phi>\leq 0.6$, obtained from simulations run at constant-volume and constant-applied normal stress conditions. $B o^{*}=0$ (white symbols), $B o^{*}=5 \times 10^{-7}$ (light grey symbols), $2.5 \times 10^{-5}$ (dark grey symbols), and $1.25 \times 10^{-4}$ (black symbols). 

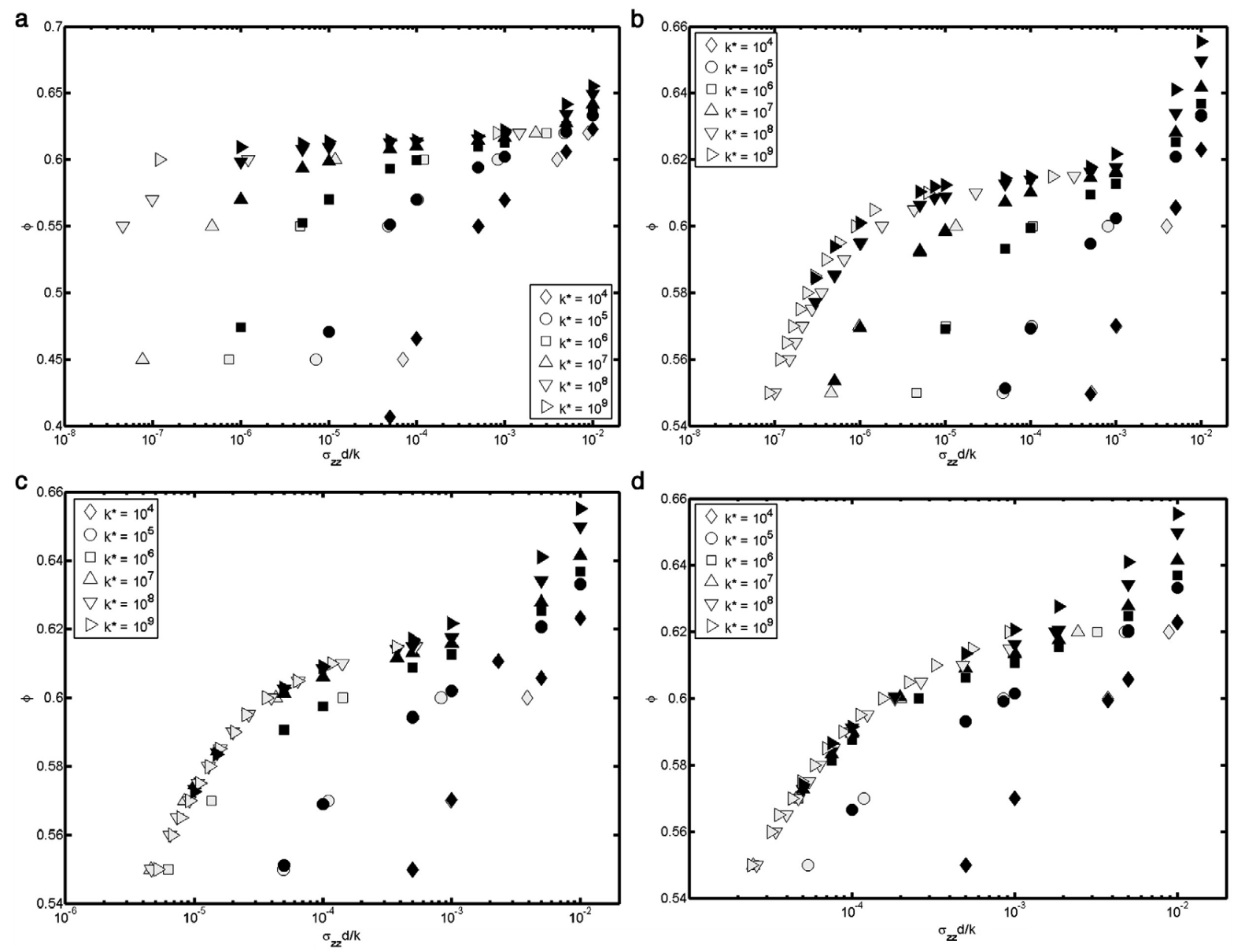

Fig. 4. The effect of the scaled stiffness (or shear rate) on the solid volume fraction versus normal stress behavior for various levels of cohesion; (a) $B o^{*}=0$, (b) $B o^{*}=5 \times 10^{-7}$, (c) $B o^{*}=2.5 \times 10^{-5}$, and (d) $B o^{*}=1.25 \times 10^{-4}$. Plotted is the solid volume fraction $(\phi)$ versus scaled normal stress $\left(\sigma_{z z} d / k\right)$ obtained from simulations run at constant-volume (grey symbols) and constant-applied normal stress (black symbols) 

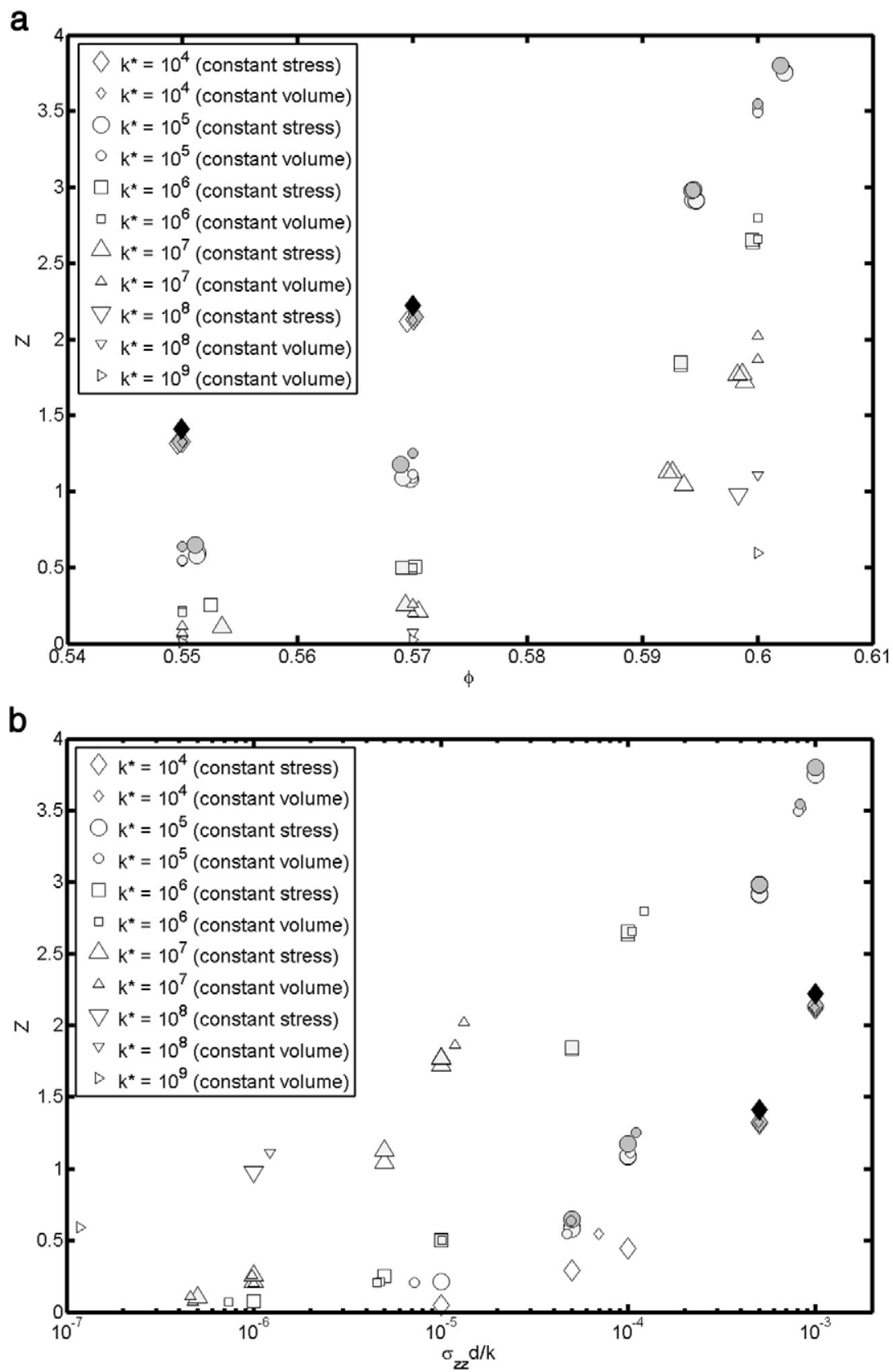

Fig. 5. Mean coordination number $(Z)$ versus for assemblies in the inertial regime, obtained from simulations run at constant-volume and constant-applied normal stress conditions. $B o^{*}=0$ (white symbols), $B o^{*}=5 \times 10^{-7}$ (light grey symbols), $2.5 \times 10^{-5}$ (dark grey symbols), and $1.25 \times 10^{-4}$ (black symbols). The mean coordination number is plotted against (a) volume fraction $(\phi)$ and (b) scaled normal stress $\left(\sigma_{z z} d / k\right)$.

a time-averaged sense. These figures also confirm that at high volume fractions $(\phi>0.61)$ the normal stress is independent of the strength of cohesion; the reader is referred to our earlier publication [8] for a more detailed discussion of this result.

In a previous work [8], it was shown that at low volume fractions $(\phi<0.61)$ in the quasi-static regime $\left(B o^{*} k^{*}>10-100\right)$, the average measured stress was found to be proportional to $B o^{*}$. Fig. 2 shows how the mean volume fraction varies with the mean cohesively scaled normal stress $\left(\sigma_{z z} d /\left(k B o^{*}\right)=\right.$ $24 \sigma_{z z} d s_{\min }^{2} / A$ ) for various values of $k^{*}$ and $B o^{*}$. Data from constant-volume simulations (small symbols) and from constantapplied normal stress simulations (large symbols) are included in this figure. Fig. 2 shows all the data for simulations performed with $B o^{*} k^{*}>100$, regardless of the volume fraction. The transition from the "low $\phi$ quasi-static regime" to the "high $\phi$ quasi-static regime" can be seen at $\phi \approx 0.61$. For $\phi<0.61$, in the "low $\phi$ quasi-static regime," the data for all values of $k^{*}$ and $B O^{*}$ collapse onto a single curve. The "high $\phi$ quasi-static regime" was identified in our earlier study of steady shear under constant-volume fraction conditions as the regime where a cohesionless assembly would have manifested quasi-static flow. In the "low $\phi$ quasi-static regime", a cohesionless assembly would have sheared in the inertial regime. This figure also shows that the results from the constant-applied normal 
a

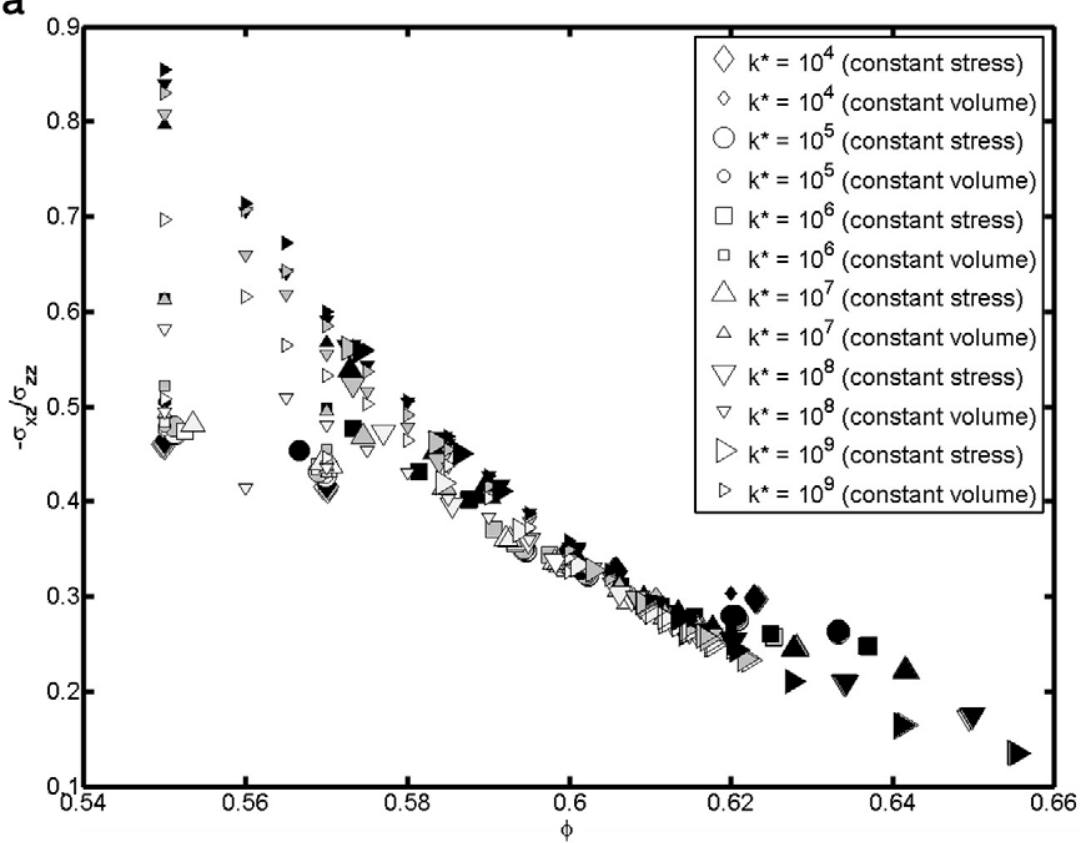

b

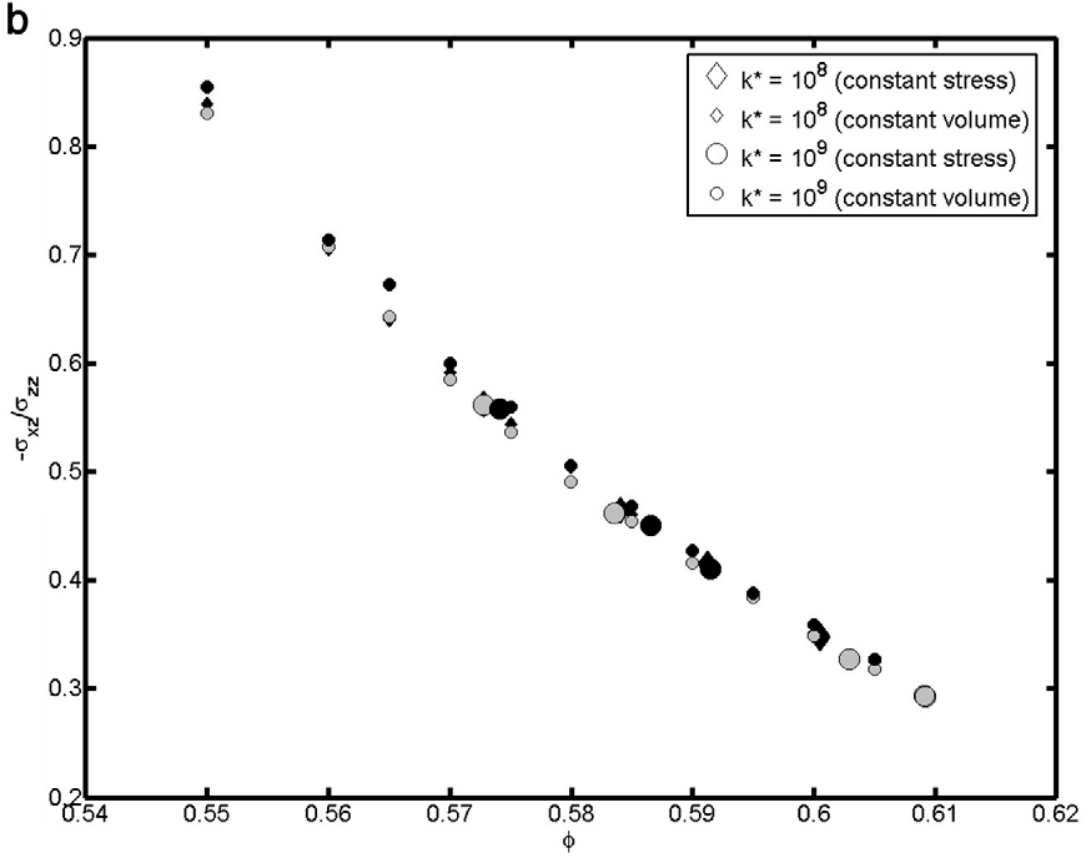

Fig. 6. Apparent coefficient of friction $\left(-\sigma_{x z} / \sigma_{z z}\right)$ versus volume fraction $(\phi)$ obtained from simulations run at constant-volume and constant-applied normal stress conditions. $B o^{*}=0$ (white symbols), $B o^{*}=5 \times 10^{-7}$ (light grey symbols), $2.5 \times 10^{-5}$ (dark grey symbols), and $1.25 \times 10^{-4}$ (black symbols). (a) The apparent coefficients of friction for all values of $\phi, B o^{*}$, and $k^{*}$ are shown. (b) Only data for $\phi<0.61$ and $B o^{*} k^{*}>5000$ (i.e. assemblies deep in the low $\phi$ quasi-static regime) are shown.

stress simulations fall in line with the constant-volume simulation results in the quasi-static regime.

The inertial regime was found to exist at low values of $B o^{*} k^{*}$ $(<10)$ and volume fraction $(\phi<0.61)$ [8]. Here for a given volume fraction, the normal stress scales with the square of the shear rate (or $1 / k^{*}$ ) but has no more than a weak dependence on the strength of cohesion. Fig. 3 shows clearly that in this regime $\left(B o^{*} k^{*}<10\right)$ the volume fraction only varies with the kinetically scaled stress $\left(\sigma_{z z} d k^{*} / k=\sigma_{z z} / \rho d^{2} \dot{\gamma}^{2}\right)$, under constant-volume and under constant-applied normal stress conditions. It can be seen that all the data fall onto one curve so that all of these assemblies here exist in the inertial regime and that with regards to mean quantities, shearing under constant-volume and under constantapplied normal stress conditions are the same.

Fig. 4a-d shows how the scaled stiffness, $k^{*}$, affects the manner in which the volume fraction varies with the scaled normal stress for various strengths of cohesive interaction. Simulation data for constant-volume (grey symbols) and constant-applied normal stress (black symbols) shearing are shown. Again, the data from both types of simulations fall along 


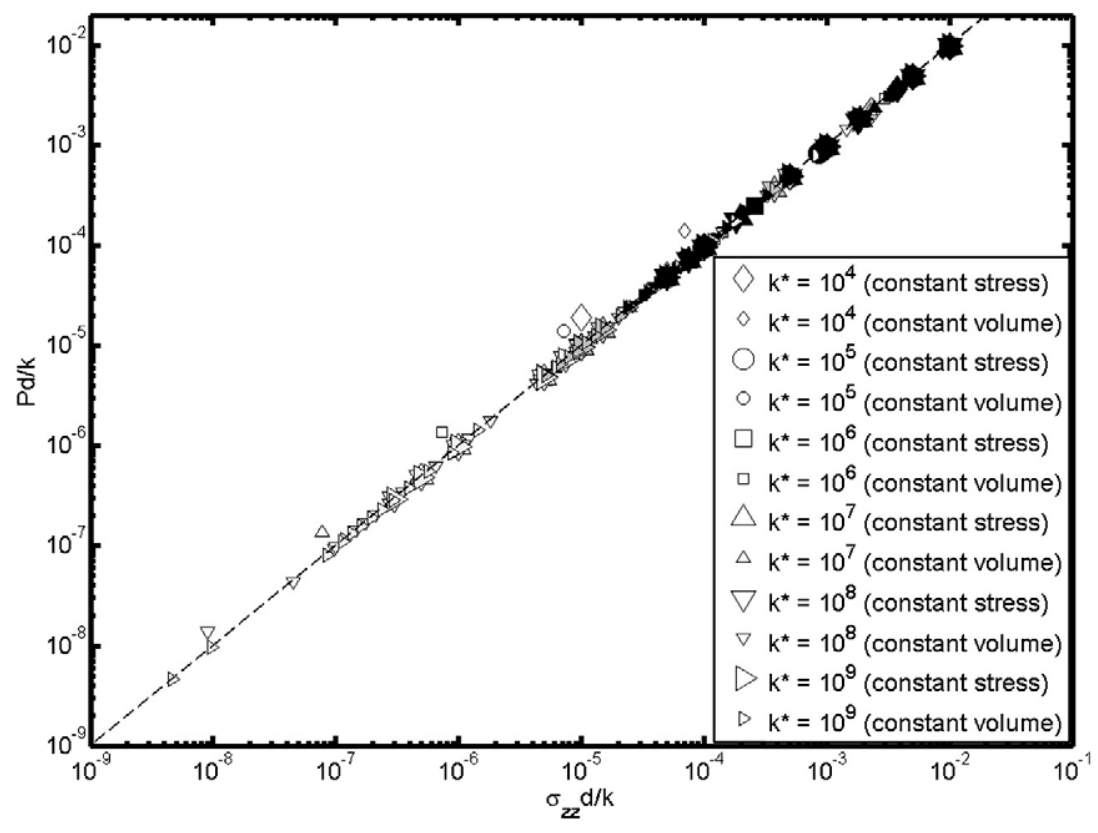

Fig. 7. Scaled pressure $(P d / k)$ versus the $z z$-component of the scaled stress $\left(\sigma_{z z} d / k\right)$ obtained from simulations run at constant-volume and constant-applied normal stress conditions. $B o^{*}=0$ (white symbols), $B o^{*}=5 \times 10^{-7}$ (light grey symbols), $2.5 \times 10^{-5}$ (dark grey symbols), and $1.25 \times 10^{-4}$ (black symbols). The dashed line represents parity.

the same curves. In each figure, the curves for all values of $k^{*}$ meet at around $\phi \approx 0.61$. As $k^{*}$ is increased, its respective branch reaches this point at lower stresses. Without cohesion (Fig. 4a), it can be seen that in order to go from a branch for one value of $k^{*}$ to a branch with a value of $k^{*}$ a factor of 10 lower, without changing the volume fraction, the stress needs to be increased by a factor of 10 . This indicates that these assemblies, when $\phi<0.61$, are in the inertial regime. These results for cohesionless assemblies agree with those given by Campbell [9]. When $B o^{*}=5 \times 10^{-7}$ (Fig. 4b), the branches for $k^{*}=10^{9}$ and $10^{8}$ nearly coincide, and so these assemblies exist in the quasi-static regime. The assemblies with lower $k^{*}$, at sufficiently low volume fractions (or stress) exist in the inertial regime. At higher levels of cohesion (Fig. 4c-d), the quasistatic regime exists at scaled stiffnesses as low as $10^{6}$. We previously showed [8] that the transition between the inertial regime and the quasi-static regime (for $\phi \leq 0.60$ ) occurs when $B o^{*} k^{*}$ is somewhere between 10 and 100. Fig. 4b-d supports this finding and the conclusion that with regards to the mean quantities, shearing under constant applied normal stress is identical to shearing under constant volume.

For constant-volume shearing, it was determined in our earlier study [8] that in the inertial regime, as $k^{*}$ is reduced, the coordination number increases, such that the dominance of binary collisions is reduced and the assemblies go deeper into the inertial-non-collisional regime (towards the elastic-inertial regime) and further away from the inertial-collisional regime. This agreed with Campbell's [7] conclusions, at which he arrived through analysis of the particle contact durations. Fig. 5a-b shows how the mean coordination number varies with the volume fraction (Fig. 5a) and normal stress (Fig. 5b) for assemblies in the inertial regime. In Fig. 5a, it can be seen that along a line of (approximately) constant volume fraction, as $k^{*}$ is decreased, the coordination number increases, regardless if the shearing occurs under constant volume or constant applied stress, and regardless of whether the assemblies are cohesive or not. Thus as $k^{*}$ is decreased while keeping the mean volume fraction constant, assemblies go deeper into the inertial-noncollisional regime.

Alternatively, in Fig. 5b, it can be seen that along a line of (approximately) constant stress, as $k^{*}$ is increased, the coordination number increases, again regardless if the shearing occurs under constant-volume or constant-applied stress conditions and if the assemblies are cohesive or not. So as $k^{*}$ is increased while keeping the mean normal stress constant, the assemblies go deeper into the inertial-non-collisional regime. We emphasize that these findings agree with Campbell's $[7,9,10]$. However, we find that the regime map for shearing under constant-volume conditions is essentially identical to the regime map for shearing under constant applied normal stress. Furthermore, the path traversed through regime space as $k^{*}$ is varied depends on whether each point along the path is at the same mean stress or mean volume fraction.

Fig. 6a shows how the apparent coefficient of friction, $\mu_{\text {app }}=-\frac{\sigma_{x z}}{\sigma_{z z}}$, varies with volume fraction. Both constant-applied normal stress and constant-volume data are shown. It is clear that the apparent coefficient of friction increases as volume fraction decreases. This agrees with simulation results of da Cruz et al. [12] and experimental results [13]. In between volume fractions of about 0.58 and 0.62 , all of the data fall approximately onto one curve regardless of the value of $k^{*}$ or $B o^{*}$. It had been previously shown that at $\phi=0.6$ the apparent coefficient of friction is nearly independent of $B o^{*} k^{*}$ [8]. This figure allows us to see that this is the case for a range of volume fractions and not just a single volume fraction. For volume fractions outside this range, the data splits into branches. At 
large volume fractions $(\phi>0.62)$ these branches correspond to different levels of mean scaled normal stress, with $\mu_{\text {app }}$ increasing as the normal stress increases.

We had also found in [8] that at the volume fractions on the left side of Fig. 6a, in the inertial regime and sufficiently deep into the quasi-static regime $\left(B o^{*} k^{*}>5000\right), \mu_{\text {app }}$ becomes constant with respect to $k^{*}$ and $B o^{*}$. The apparent coefficient of friction is smallest in the inertial regime and largest in the quasi-static regime. At and around the transition from the inertial to quasi-static regime, $\mu_{\text {app }}$ increases with $B o^{*} k^{*}$ until the quasi-static plateau is reached (see Fig. 5 in [8]). The dependence of $\mu_{\text {app }}$ on the volume fraction is rather weak in the inertial regime but becomes quite strong in the quasi-static regime. So the lowest branch on the left side of Fig. 6a, which would be the branch that has the weakest dependence on volume fraction, includes data for the smallest values of $B o^{*} k^{*}$, corresponding to the inertial regime. The highest branch, which would be the branch that has the strongest dependence on volume fraction, includes data for the largest values of $B o^{*} k^{*}$ (>5000), corresponding to being "deep" into the quasi-static regime. In between the two extremes, $\mu_{\text {app }}$ increases with $B o^{*} k^{*}$. Fig. $6 \mathrm{~b}$ contains a subset of the results in Fig. 6a, and shows the variation of $\mu_{\mathrm{app}}$ with volume fraction deep into the low $\phi$ quasi-static regime. It is clear that a monotonic increase of $\mu_{\text {app }}$ with decreasing $\phi$ occurs over a wide range of volume fractions for the cohesive systems. As the range of $\mu_{\text {app }}$ values possible is clearly very large, continuum models must account for this variation of $\mu_{\text {app }}$ with $\phi$.

For assemblies sheared at constant volume, it was found that the normal stress (i.e. the $z z$-component of the stress tensor) hardly differed from the pressure (i.e. one-third the trace of the stress tensor) [8]. Thus, without loss of generality, any discussion of the normal stress would be applicable to the pressure. Given that under constant-applied stress conditions only the $z z$-component of the stress tensor is controlled directly (while the other normal stress components are not controlled directly), it is not immediately apparent if the overall pressure is controlled, and if so if it is close to the $z z$-component of the stress. Fig. 7 shows how the scaled pressure compares with the $z z$-component of the scaled stress tensor, and it can be seen that the two quantities are nearly equal for all assemblies.

So when averaged over time, shearing performed at constant volume is identical to shearing performed under constant applied normal stress. Thus any data or conclusions based on the time-averaged results obtained from constant-volume simulations can be applied to systems sheared under constant applied normal stress, or vice versa.

\subsection{Fluctuations}

Under constant-applied normal stress conditions the fluctuations in normal stress were determined by the control algorithm, while the volume fraction was allowed to fluctuate freely. On the other hand, under constant-volume conditions, fluctuations in the normal stress were not constrained, but the volume fraction was not allowed to fluctuate. Thus the two types of shearing differ dynamically.
Fig. $8 \mathrm{a}-\mathrm{c}$ shows how the normal stress fluctuations during shear at constant volume vary with cohesion. The fluctuations in the quasi-static regime (Fig. 8a and b) have a strong dependence on cohesion, with the fluctuations becoming larger as cohesion is decreased. The fluctuations in the low $\phi$ quasi-static regime (Fig. 8b) become extremely large at the lowest level of cohesion $\left(B o^{*}=5 \times 10^{-7}\right)$. In the inertial regime $\left(\phi=0.57\right.$ and $k^{*}=10^{4}$, Fig. 8c), the dependence on cohesion is relatively weak.
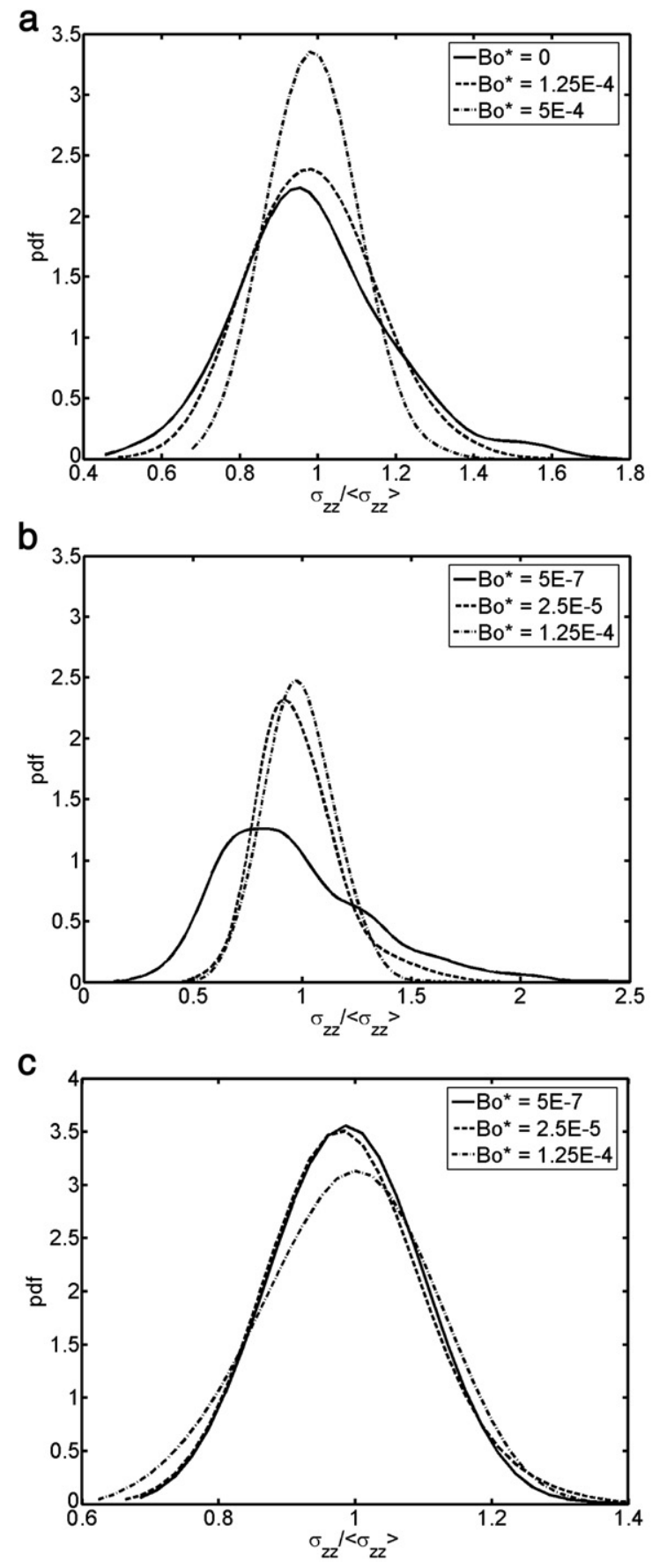

Fig. 8. The effect of cohesion on normal stress fluctuations during shearing at constant volume. Plotted are PDFs of normal stress $\left(\sigma_{z z} /\left\langle\sigma_{z z}\right\rangle\right)$ obtained from simulations with (a) $\phi=0.62$ and $k^{*}=10^{8}$ (in the high $\phi$ quasi-static regime); (b) $\phi=0.6$ and $k^{*}=10^{8}$ (in the low $\phi$ quasi-static regime); (c) $\phi=0.57$ and $k^{*}=10^{4}$ (in the inertial regime). Each plot features a PDF for three different levels of cohesion $\left(B o^{*}\right)$. 
In the quasi-static regime, normal stress fluctuations arise from repeated formation and break up of the force chains. Cohesion causes particles to hold onto each other more tightly, increasing the average number of contacts and making it more difficult to break up the force chains. Thus one would expect that when the stress is free to fluctuate at will (as in constantvolume simulations), the stress fluctuations in the quasi-static regime shrink as cohesion is increased. In the inertial regime, where force chains are non-existent, cohesion has a weak effect on the normal stress fluctuations.

Under constant-applied normal stress conditions, the normal stress is controlled and the fluctuations are a consequence of the control algorithm, and so these fluctuations are merely an indication of the efficiency of the control scheme (and so any effect cohesion has is inconsequential). So it can be seen in Fig. 9 that the size of the normal stress fluctuations under constant-applied normal stress conditions has a significantly smaller dependence on cohesion than under constant-volume conditions (Fig. 8). The PDFs in Fig. 9 were generated from simulations run in the low $\phi$ quasi-static regime, where under constant-volume conditions (Fig. 8b) the stress fluctuations show the strongest dependence on cohesion.

Fig. 10a-c shows how the shear stress fluctuations vary with cohesion for the same systems as Fig. 8a-c, respectively, i.e. the systems sheared under constant-volume conditions. For these systems, the shear stress fluctuations behave like and are the same size as the normal stress fluctuations. That is, in the quasistatic regime, the shear stress fluctuations increase in relative size as cohesion decreases, and in the low $\phi$ quasi-static regime $\left(\phi=0.6\right.$ and $k^{*}=10^{8}$, Fig. 10b) the shear stress fluctuations are quite large for $B o^{*}=5 \times 10^{-7}$. Furthermore, in the inertial regime ( $\phi=0.57$ and $k^{*}=10^{4}$, Fig. 10c), the fluctuations grow in size as cohesion is increased, but again the dependence is rather weak.

Fig. 11a-c shows how the shear stress fluctuations during shear under constant applied stress vary with cohesion. As the normal stress, not the shear stress, was constrained, it comes as no surprise that the shear stress fluctuations are significantly larger than the normal stress fluctuations (Fig. 9). In the high $\phi$

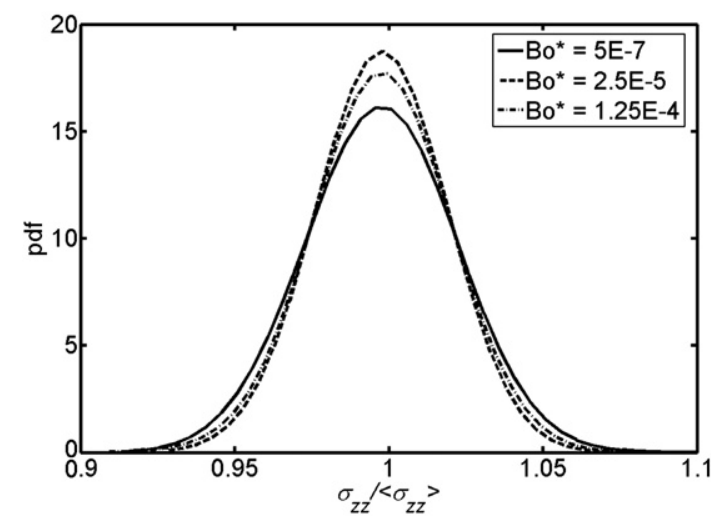

Fig. 9. The size of the normal stress fluctuations during shearing under constantapplied normal stress conditions depend much less on cohesion and are almost completely dictated by the feedback algorithm (Eq. (1)). Plotted are PDFs of normal stress $\left(\sigma_{z z} /\left\langle\sigma_{z z}\right\rangle\right)$ obtained from simulations with $\langle\phi\rangle=0.58$ and $k^{*}=10^{8}$ (in the low $\phi$ quasi-static regime) for three different levels of cohesion $\left(B o^{*}\right)$.
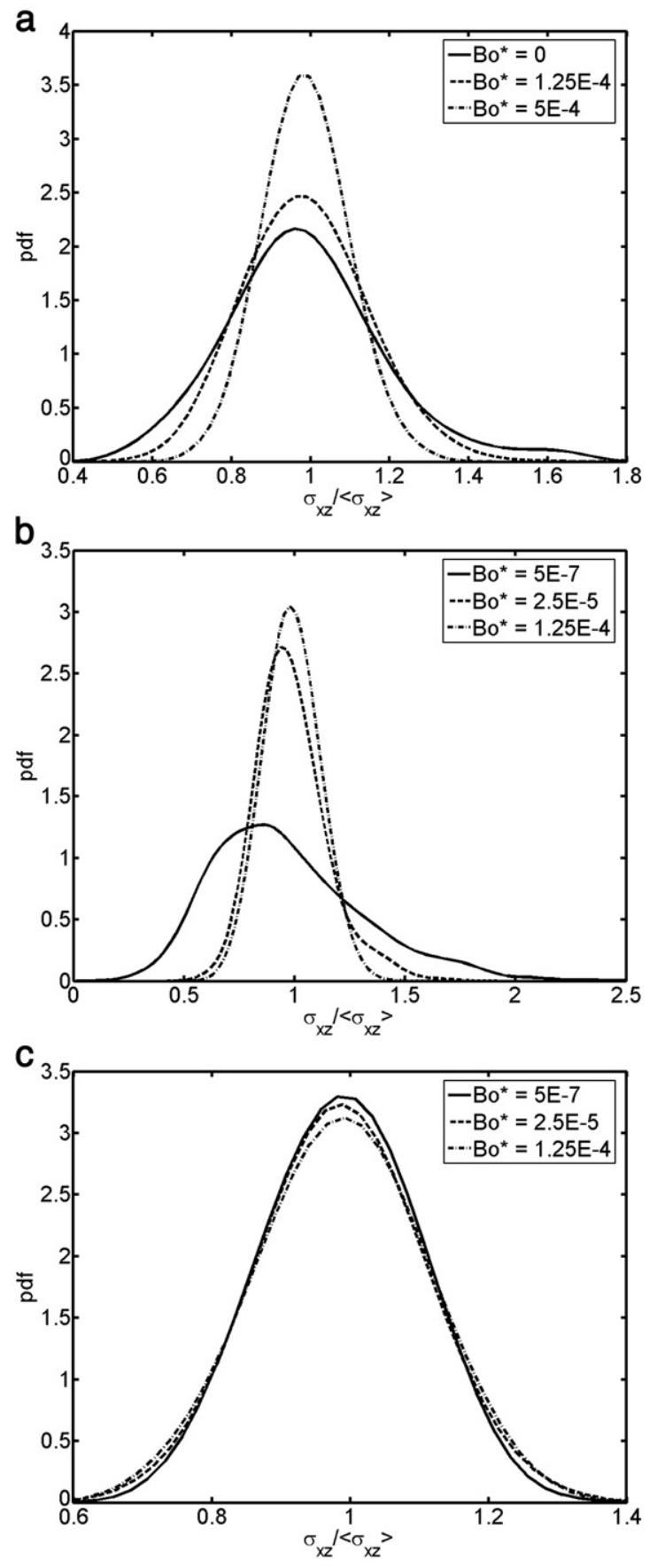

Fig. 10. The effect of cohesion on shear stress fluctuations during shearing at constant volume. Plotted are PDFs of shear stress $\left(\sigma_{x z} /\left\langle\sigma_{x z}\right\rangle\right)$ obtained from simulations with (a) $\phi=0.62$ and $k^{*}=10^{8}$ (in the high $\phi$ quasi-static regime); (b) $\phi=0.6$ and $k^{*}=10^{8}$ (in the low $\phi$ quasi-static regime); (c) $\phi=0.57$ and $k^{*}=10^{4}$ (in the inertial regime). Each plot features a PDF for three different levels of cohesion $\left(B o^{*}\right)$.

quasi-static regime (Fig. 11a) and in the inertial regime (Fig. 11c), the fluctuations appear nearly independent of cohesion; however, in the low $\phi$ quasi-static regime $\left(\langle\phi\rangle=0.58\right.$ and $k^{*}=10^{8}$, Fig. 11b) the shear stress fluctuations are much larger for $B o^{*}=5 \times 10^{-7}$ than for $B o^{*}=2.5 \times 10^{-5}$ and $B o^{*}=1.25 \times 10^{-4}$, which in turn are about the same relative size. These large fluctuations at low cohesion are similar to what is seen for the 
a

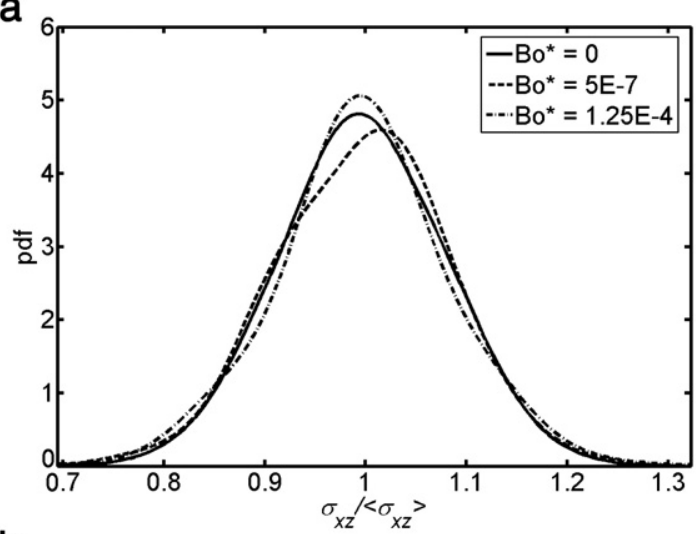

b

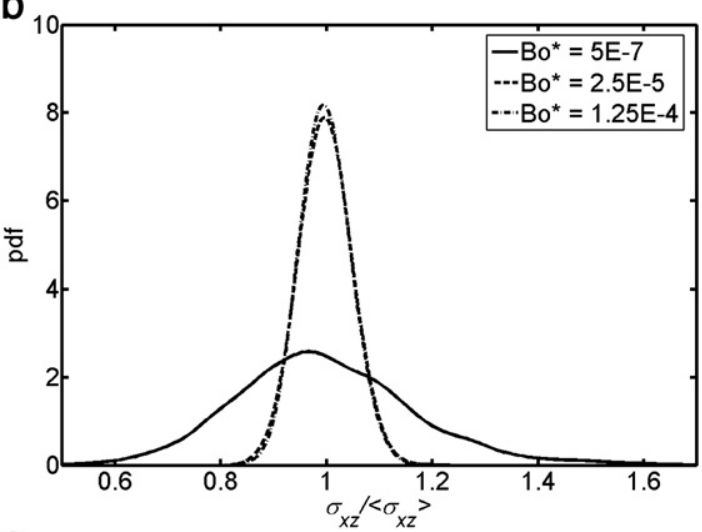

C

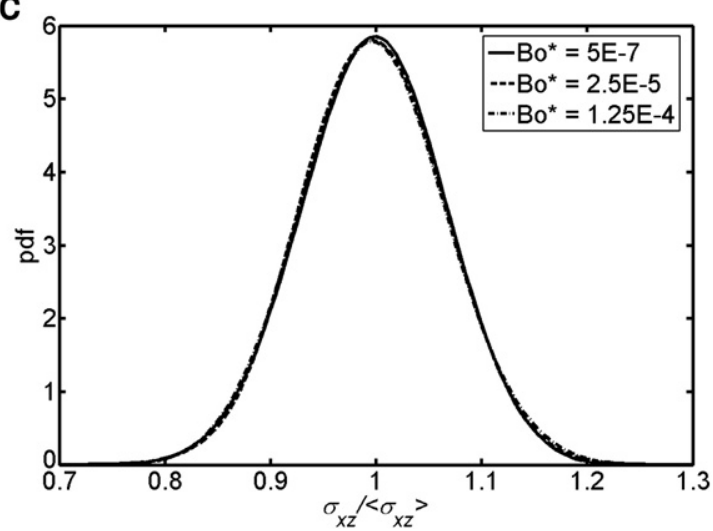

Fig. 11. The effect of cohesion on shear stress fluctuations during shearing under constant-applied normal stress conditions. Plotted are PDFs of shear stress $\left(\sigma_{x z} /\left\langle\sigma_{x z}\right\rangle\right)$ obtained from simulations with (a) $\langle\phi\rangle=0.62$ and $k^{*}=10^{8}$ (in the high $\phi$ quasi-static regime); (b) $\left\langle\phi>=0.58\right.$ and $k^{*}=10^{8}$ (in the low $\phi$ quasi-static regime); (c) $\langle\phi\rangle=0.57$ and $k *=10^{4}$ (in the inertial regime). Each plot features a PDF for three different levels of cohesion $\left(B o^{*}\right)$.

normal and shear stress fluctuations under constant-volume conditions in the same regime (Figs. $8 \mathrm{~b}$ and 10b, respectively).

Fig. 12 shows an example of how the volume fraction fluctuations vary with cohesion for the systems sheared under constant applied normal stress. As observed by Campbell [9], the volume fraction hardly deviates from its mean value, with fluctuations rarely exceeding $1 \%$. Furthermore, we could not find any trend in how the volume fraction fluctuations vary with cohesion. Since volume fraction is directly related to the measured normal stress via the constant-normal stress algorithm (Eq. (1)), and since the normal stress fluctuations are virtually

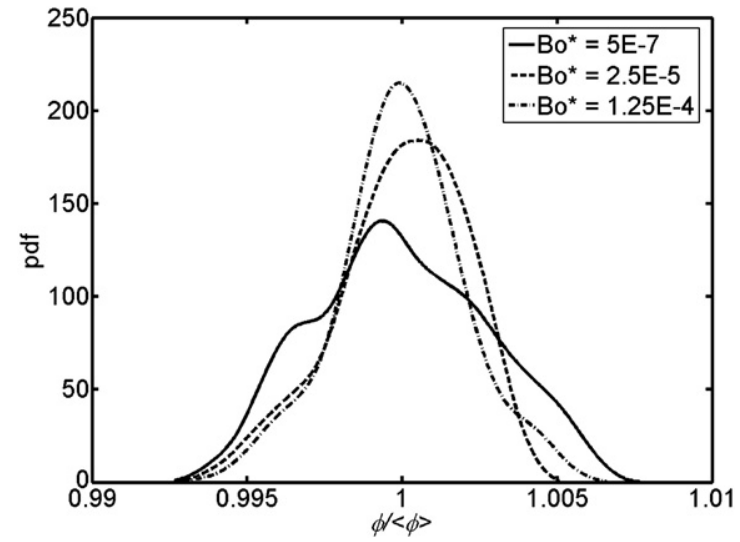

Fig. 12. The volume fraction barely deviates from its mean during shearing under constant applied normal stress. Plotted are PDFs of volume fraction $(\phi /<\phi>)$ obtained from simulations with $\langle\phi\rangle=0.58$ and $k^{*}=10^{8}$ (low $\phi$ quasi-static regime) for three different levels of cohesion $\left(B o^{*}\right)$.

independent of cohesion, it would follow that the volume fraction fluctuations should be as well.

One of the main findings of our earlier study [8] was that if the volume fraction of particles is sufficiently small that a cohesionless system would shear in the inertial regime (e.g., $\phi<0.61$, for $\mu=0.1$ ), the normal stress (or pressure) versus scaled stiffness data can be collapsed onto a single curve if the stress is scaled cohesively (divided by $B o^{*}$ ) and the scaled stiffness is multiplied by $B o^{*}$. Since the mean (i.e. timeaveraged) quantities obtained from the constant-applied normal stress simulations agree with those from the constant-volume simulations, this scaling holds for both types of shearing. Thus if $B o^{*} k^{*}$ is held constant along with the volume fraction (or mean cohesively scaled stress), while changing the values of $k^{*}$ and $B o^{*}$ themselves, the mean cohesively scaled stresses (or volume fraction) as well as the apparent coefficient obtained are unchanged. However, this does not necessarily mean that the dynamics remain the same.

Fig. 13 shows PDFs of the shear stress for simulations performed under constant-applied normal stress conditions with $<\phi>=0.59$ and $B o^{*} k^{*}=1250$, corresponding to the low $\phi$ quasi-static regime. Here, of course, the stress does not depend

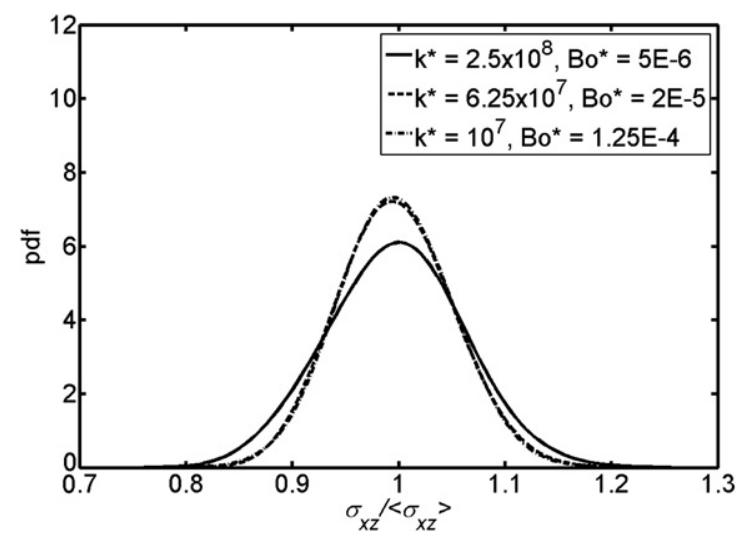

Fig. 13. PDFs of shear stress $\left(\sigma_{x z} /\left\langle\sigma_{x z}\right\rangle\right)$ obtained from simulations run under constant-applied normal stress conditions with $\langle\phi\rangle=0.59$ and $B o^{*} k^{*}=1250$ (low $\phi$ quasi-static regime) and three different combinations of $k^{*}$ and $B o^{*}$. 
on $k^{*}$ but does depend on $B o^{*}$. This figure includes a PDF for three different combinations of $k^{*}$ and $B o^{*}$. As was seen before for the low $\phi$ quasi-static regime, the shear stress fluctuations are largest for the smallest level of cohesion $\left(B o^{*}=5 \times 10^{-7}\right)$, but are about the same size for the higher levels of cohesion.

Fig. 14a-b shows PDFs of the normal stress (Fig. 14a) and shear stress (Fig. 14b) for simulations performed under constant-volume conditions with $\phi=0.59$ and $B o^{*} k^{*}=1250$. Again, the stress fluctuations are much larger (by a factor of about 6) than those for constant-normal stress shearing. Furthermore, the fluctuations are largest for the smallest level of cohesion $\left(B o^{*}=5 \times 10^{-7}\right)$, while the fluctuations for the other levels of cohesion are about the same. So in the low $\phi$ quasistatic regime, both the mean shear stress and the shear stress fluctuations depend on the level of cohesion.

In the low $\phi$ quasi-static regime for a single value $B o^{*} k^{*}$ and $\phi$, under constant-volume conditions the mean stresses and stress fluctuations vary with cohesion. Similarly, under constant applied normal stress, the volume fraction, mean shear stress, and shear stress fluctuations vary with cohesion. On the other hand, the apparent coefficient of friction is independent of cohesion when $B \mathrm{O}^{*} k^{*}$ is held constant. In other words, the shear stress fluctuations (and in the case of constant-volume condi-
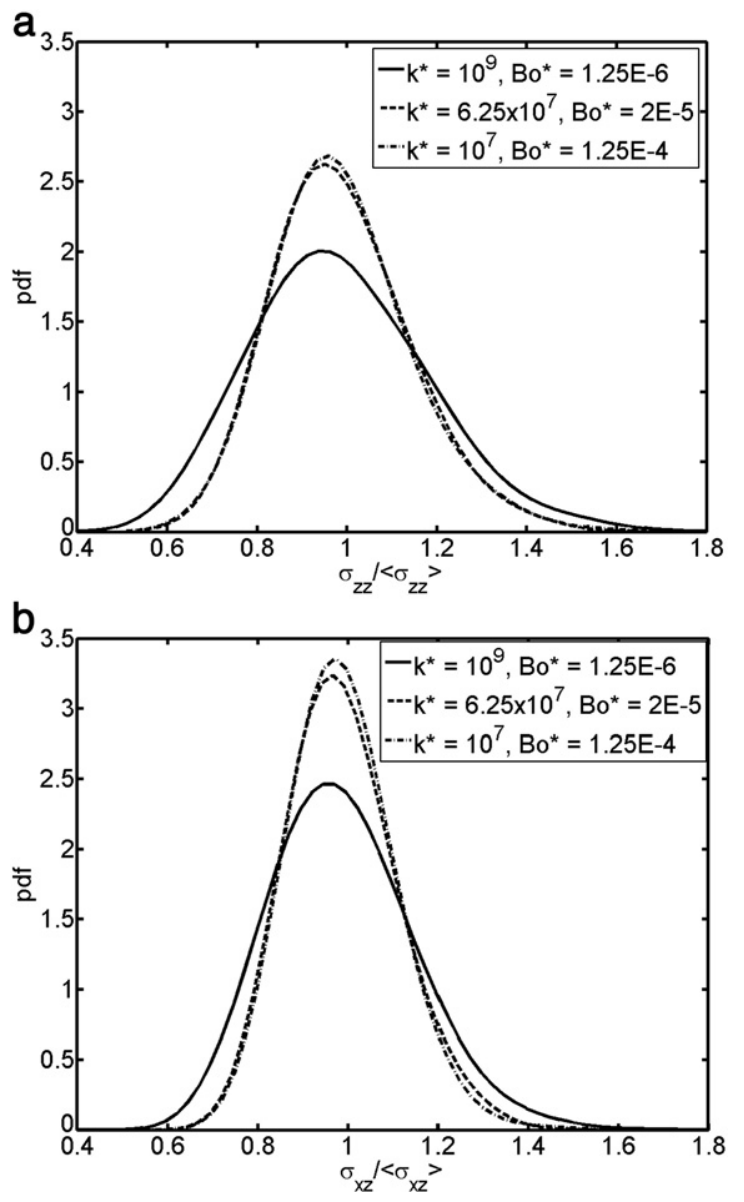

Fig. 14. PDFs of (a) normal stress $\left(\sigma_{z z} /\left\langle\sigma_{z z}\right\rangle\right)$ and (b) shear stress $\left(\sigma_{x z} /\left\langle\sigma_{x z}\right\rangle\right)$, obtained from simulations run under constant-volume conditions with $\phi=0.59$ and $B o^{*} k^{*}=1250$ (low $\phi$ quasi-static regime). Each plot features a PDF for simulations run with three different combinations of $k^{*}$ and $B o^{*}$.

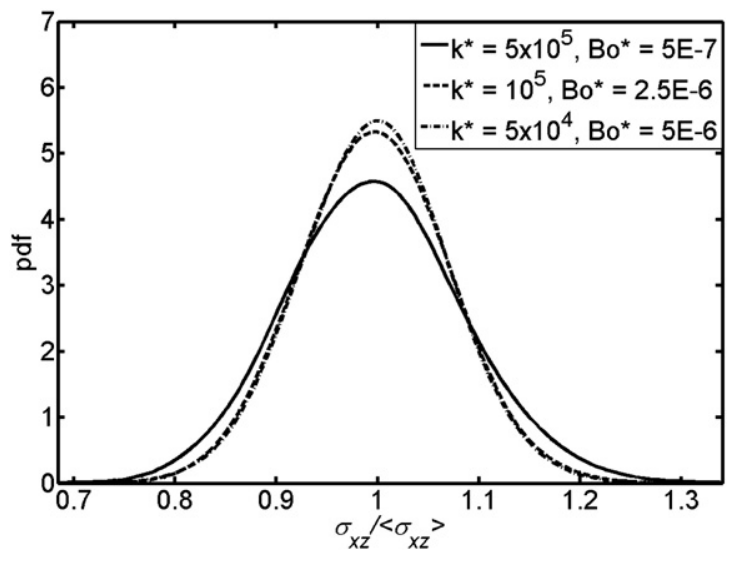

Fig. 15. PDFs of shear stress $\left(\sigma_{x z} /\left\langle\sigma_{x z}\right\rangle\right)$ obtained from simulations run under constant-applied normal stress conditions with $\langle\phi\rangle=0.59$ and $B o^{*} k^{*}=0.25$ (inertial regime) and three different combinations of $k^{*}$ and $B o^{*}$.

tions, the normal stress fluctuations) could be of any size for any single value of the apparent coefficient of friction. So in the low $\phi$ quasi-static regime, the apparent coefficient of friction and the stress fluctuations would be independent of each other.

Fig. 15 shows PDFs of the shear stress for simulations performed under constant-applied normal stress conditions with $\langle\phi\rangle=0.59$ and $B o^{*} k^{*}=0.25$, corresponding to the inertial regime. Here, of course, the stress depends on $k^{*}$ but not on $B o^{*}$. Nevertheless, the fluctuations behave similarly to those for $B o^{*} k^{*}=1250$ (Fig. 13). The shear stress fluctuations increase in size as $B o^{*}$ is reduced and $k^{*}$ is increased. Furthermore, the fluctuations are a little bigger than in the low $\phi$ quasi-static regime.

Fig. 16a-b shows PDFs of the normal stress (Fig. 16a) and shear stress (Fig. 16b) for simulations performed under constant-volume conditions with $\phi=0.59$ and $B o^{*} k^{*}=0.25$. The sizes of the stress fluctuations grow as $k^{*}$ is increased and $B o^{*}$ is decreased. Also, for the smallest level of cohesion $\left(B o^{*}=5 \times 10^{-7}\right)$ the PDF is noticeably skewed to the left. And again, the PDFs for the shear stress are nearly identical to those for the normal stress. It was seen in Figs. 8c, 10c, and 11c that in the inertial regime, the stress fluctuations are nearly independent of cohesion, and so we conclude that in the inertial regime, the shear stress fluctuations (and under constant-volume conditions, the normal stress fluctuations) depend on and grow with $k^{*}$.

One may expect that normal stress fluctuations would be accompanied by fluctuations in the coordination number. That is, when more particles are in contact, the stress is higher. Thus it would follow that the fluctuations in both of these quantities would manifest similar dependencies on cohesion. Fig. 17a shows PDFs of the coordination number for assemblies sheared at constant volume in the low $\phi$ quasi-static regime, for which the stress showed the greatest dependence on cohesion. At the lowest level of cohesion $\left(B o^{*}=5 \times 10^{-7}\right)$, the fluctuations are significantly larger than at the higher levels of cohesion, similar to what was seen with the stress fluctuations. However, this difference between low and high levels of cohesion is much more pronounced for the coordination number than for the normal stress. 
Furthermore, under constant-applied normal stress conditions, for which the PDFs are shown in Fig. 17b, the sizes of the coordination number fluctuations appear to demonstrate a similar dependence on cohesion to those under constant-volume conditions. This is so even though the normal stress fluctuations are small and are negligibly affected by cohesion. One may point out that the shear stress fluctuations are significantly larger at the lowest level of cohesion $\left(B O^{*}=5 \times 10^{-7}\right)$ than at higher levels of cohesion, just like they are under constant-volume conditions. But again, the difference between low and high levels of cohesion is much more prominent for the coordination number than for the shear stress. Furthermore, there is no conceivable physical reason why the coordination number fluctuations would be related to the shear stress fluctuations but not the normal stress fluctuations.

Instead it might be possible to explain the sizes of the coordination number fluctuations with the mean coordination number (see Fig. 6 in [8]). The systems shown in Fig. 17a-b that feature the smallest level of cohesion $\left(B O^{*}=5 \times 10^{-7}\right)$ have mean coordination numbers of only around 2 , while those with the higher levels of cohesion have coordination numbers around 6 .

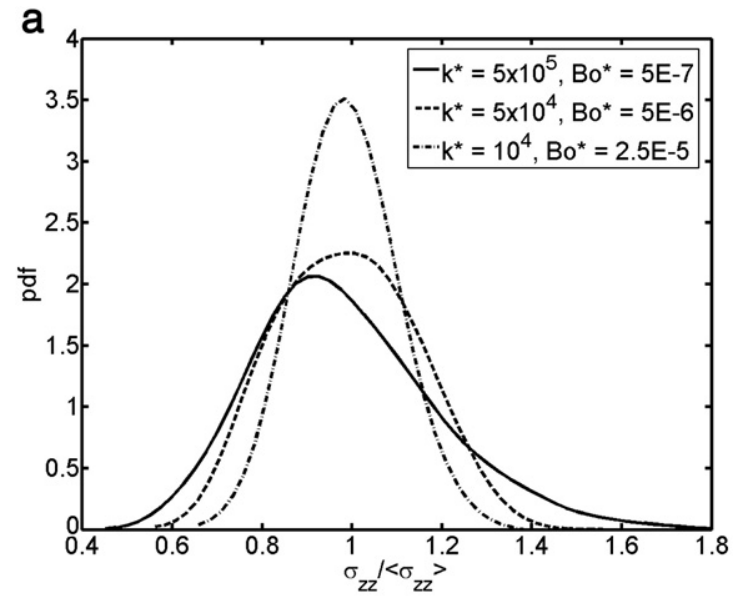

b

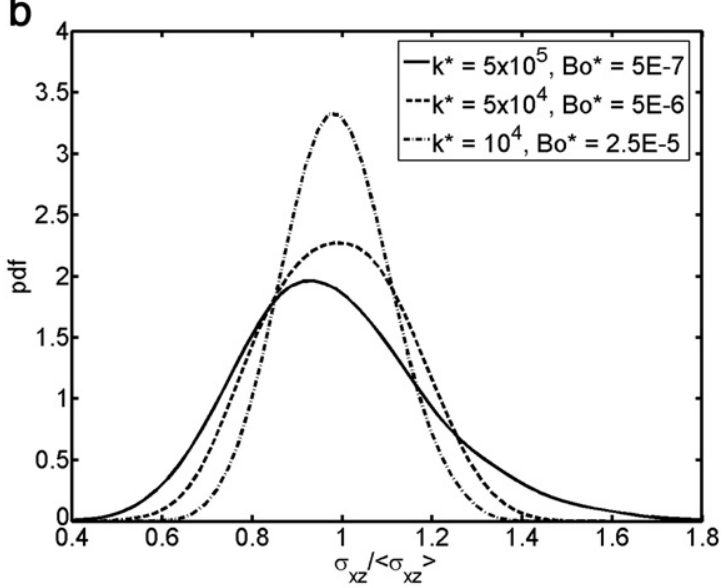

Fig. 16. PDFs of (a) normal stress $\left(\sigma_{z z} /\left\langle\sigma_{z z}\right\rangle\right)$, obtained from simulations run under constant-volume conditions with $\phi=0.59$ and $B o^{*} k^{*}=0.25$ (inertial regime). Each plot features a PDF for simulations run with three different combinations of $k^{*}$ and $B o^{*}$.
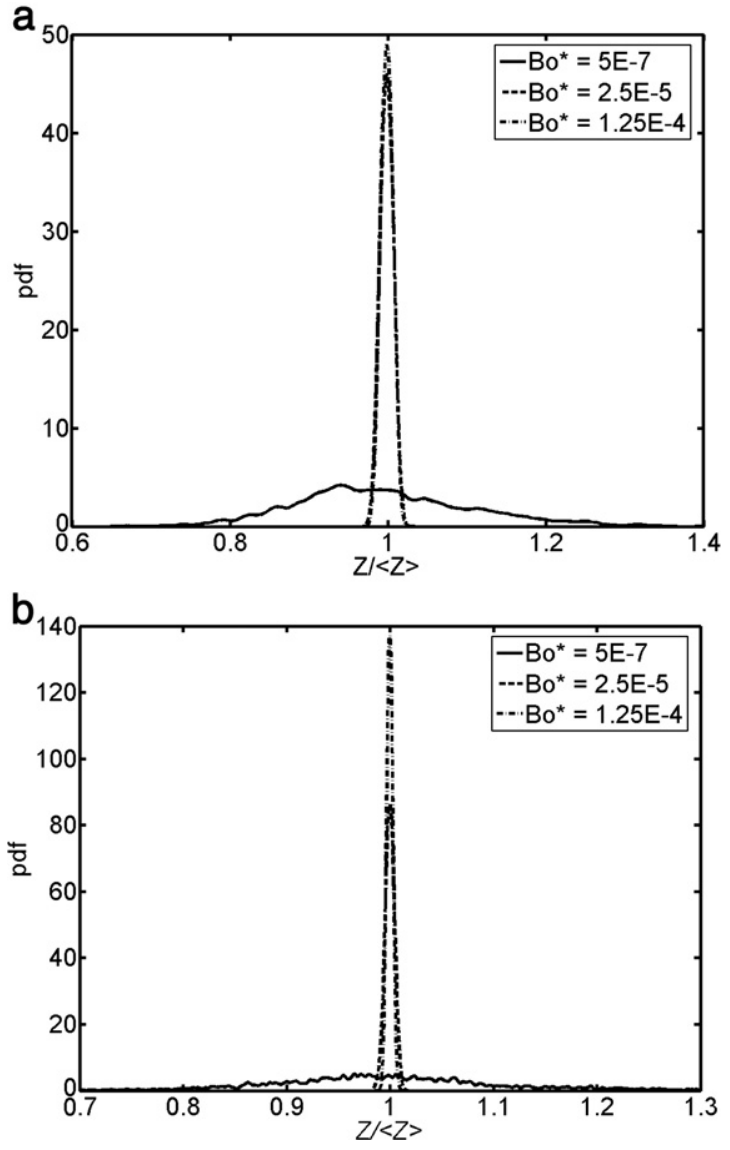

Fig. 17. PDFs of coordination number obtained from simulations of assemblies sheared in the low $\phi$ quasi-static regime (a) at constant volume with $\phi=0.6$ and $k^{*}=10^{8}$ and (b) under constant applied normal stress with $\langle\phi\rangle=0.58$ and $k^{*}=10^{8}$. Each plot features a PDF for three different levels of cohesion $\left(B o^{*}\right)$.

As the mean coordination number increases, more of an average particle's surface would be covered by contacting particles, and so it would become more difficult for a particle contact to be added to or removed from said particle. So as the mean coordination number increases, the size of the coordination number fluctuations would be reduced. This claim is supported by Fig. $17 \mathrm{a}-\mathrm{b}$.

As further support of this claim, coordination number PDFs for assemblies sheared at constant-volume conditions in the high $\phi$ quasi-static and inertial regimes are presented in Fig. 18a and $b$, respectively. The largest mean coordination numbers (around 6) were achieved in the high $\phi$ quasi-static regime and at the stronger levels of cohesion in the low $\phi$ quasi-static regime, and so it can be seen in Figs. 17a-b and 18a that the coordination number fluctuations are smallest for these systems. The smallest mean coordination numbers (around 2) were achieved at the lowest level of cohesion in the low $\phi$ quasi-static regime, and so it can be seen in Fig. 17a-b that the coordination number fluctuations are largest for these systems. Finally, intermediate mean coordination numbers (around 4) were achieved in the inertial regimes, and so it can be seen in Fig. $18 \mathrm{~b}$ that these systems feature middle-sized coordination number fluctuations. 


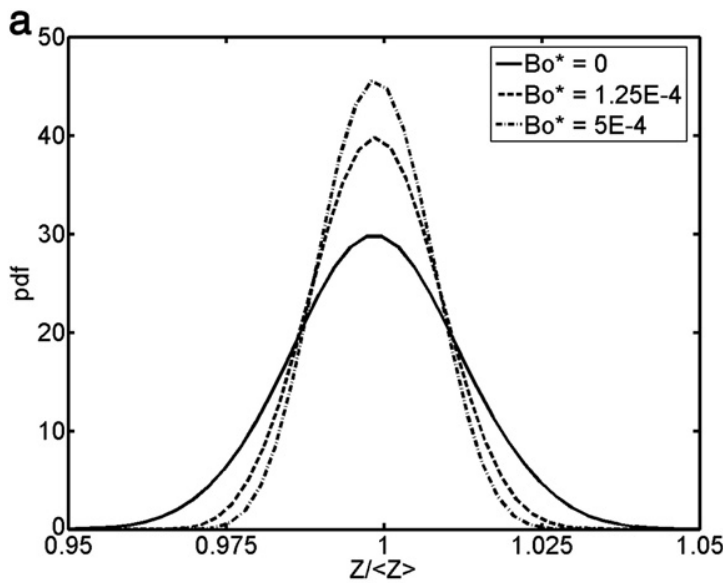

b

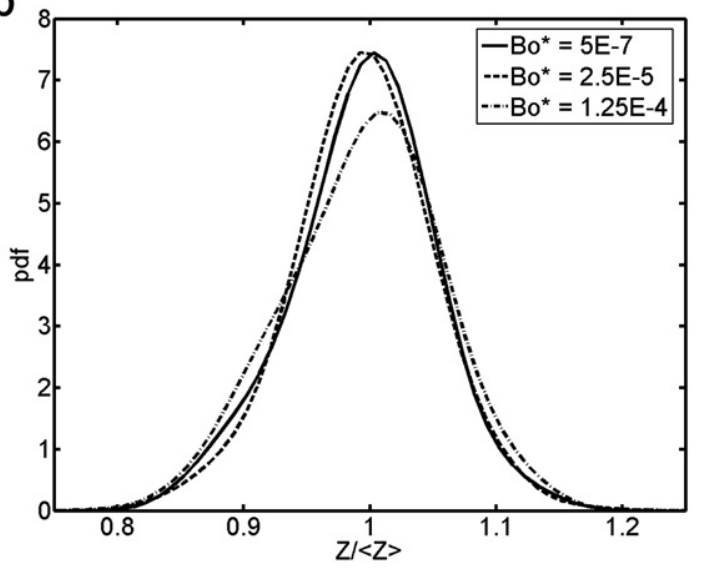

Fig. 18. PDFs of coordination number obtained from simulations of assemblies sheared at constant volume (a) in the high $\phi$ quasi-static regime with $\phi=0.62$ and $k^{*}=10^{8}$ and (b) in the inertial regime with $\phi=0.57$ and $k^{*}=10^{4}$. Each plot features a PDF for three different levels of cohesion $\left(B o^{*}\right)$.

The stress, volume fraction, and coordination number fluctuations discussed in this sub-section clearly indicate the following:

a) The normal and shear stress fluctuations in the constantvolume shearing simulations in the quasi-static regime are strongly affected by cohesion. As the strength of cohesion is increased, the fluctuations become less pronounced.

b) The normal and shear stress fluctuations in the constantvolume shearing simulations in the inertial regime depend only weakly on cohesion but depend strongly on the scaled stiffness, $k^{*}$. As $k^{*}$ is increased, the fluctuations become more pronounced.

c) The shear stress fluctuations in the constant-applied normal stress shearing simulations are appreciably smaller than but otherwise in the low $\phi$ quasi-static regime behave similarly to those seen in constant-volume simulations.

d) Under constant-applied normal stress conditions, the volume fraction barely deviates from its mean value.

e) The coordination number fluctuations depend primarily on the mean value, regardless of the shear conditions. As the mean coordination number increases, the fluctuations are reduced.

\section{Summary}

In a previous study [8], we investigated the shearing of dense assemblies of cohesive granular materials performed at constant-volume conditions. The mean stresses and coordination numbers were measured for assemblies sheared at various volume fractions, shear rates, and strengths of cohesion. We found how the stresses scaled with the level of cohesion and the shear rate and identified the conditions under which assemblies existed in each flow regime.

In the present study, we have investigated the shearing of dense assemblies of cohesive granular materials under constant applied normal stress and contrasted it with the constant-volume shearing of cohesive assemblies. In doing so, we have also investigated the effect of cohesion on the constant-applied normal stress shearing of granular assemblies. Attention was focused on homogeneously shearing assemblies of particles in periodic domains. Assemblies were categorized into flow regimes depending on how the scaled applied stress $\left(\sigma^{*}=\sigma d / k\right)$ varied with the scaled stiffness $\left(k^{*}=k / \rho d^{3} \dot{\gamma}^{2}\right)$ along a line of constant volume fraction.

We found that shearing under constant-applied normal stress conditions instead of under constant-volume conditions does not alter the relationship between the mean stress, mean volume fraction, shear rate, and cohesion strength, regardless if the particles are cohesive or not. The stress scales with shear rate and cohesion the same way for shearing under constant applied normal stress as at constant volume. Thus in the $\left(<\sigma_{z z} d / k>, k^{*}\right.$, $\langle\phi>$ ) space (for each level of cohesion and inter-particle friction), one would obtain identical regime boundaries irrespective of whether the shear flow occurs at constant $\phi$ or at constant $\sigma_{z z} d / k$. So the regime map per se is not dependent on the type of shear flow, contrary to what Campbell $[9,10]$ observed. The path that one takes in this $\left(<\sigma_{z z} d / k>, k^{*},\langle\phi>)\right.$ space when one progressively increases shear rate (i.e. decrease $k^{*}$ ) will be different if $\langle\phi\rangle$ is held constant instead of $\left\langle\sigma_{z z} d / k\right\rangle$ along the path. Therefore the manifestation of the regime transition can appear to be different but the regime landscape in the $\left(<\sigma_{z z} d /\right.$ $k>, k^{*},\langle\phi>)$ space is essentially independent of the type of shearing. This is reassuring for the following reason. Simple continuum constitutive models for the granular rheology are based on local volume fraction of particles, the local rate of deformation, and the local stress, and allow for dilation or compaction depending on the local rheology. There is no provision in these models to distinguish between constant-volume, constant-stress, or some other complex conditions where both are changing. The general premise in these models is that one can choose idealized conditions to perform simple experiments or simulations and devise the constitutive models, which when coupled with conservation laws will allow simulation of complex flows. We have found in this study that this hope certainly holds for cohesive granular systems.

We conclude that homogeneously sheared cohesive systems manifest three important regimes: the high $\phi$ quasi-static regime, the low $\phi$ quasi-static regime, and the inertial regime. The elastic-inertial regime obtained at high shear rates discussed by Campbell $[7,9,10]$ start at very small values of $k^{*}$ which are not likely to be seen in most practical cases. 
At high volume fractions $(\phi>0.61$ for $\mu=0.1)$, assemblies exist in the high $\phi$ quasi-static regime. Here the manner in which the mean scaled stress varies with the volume fraction is nearly independent of $k^{*}$ and cohesion $\left(B o^{*}\right)$. At lower volume fractions and sufficiently large $B O^{*} k^{*}(>10-100)$, assemblies exist in the low $\phi$ quasi-static regime. In this regime, when the mean normal stress or pressure is scaled cohesively, it becomes independent of $k^{*}$ and $B o^{*}$. Lastly, at the same volume fractions but at smaller $B o^{*} k^{*}$, the assemblies shear in the inertial regime. In this regime, when the mean normal stress or pressure is scaled kinetically, it (i.e., $\sigma^{*} k^{*}$ ) becomes independent of $k^{*}$ and $B o^{*}$. In this regime, as $B o^{*} k^{*}$ (or simply $k^{*}$ if there is no cohesion) is reduced while the (average) volume fraction is kept constant, the particle assemblies go deeper into the inertial-noncollisional regime and further from the collisional regime.

It was found that the apparent coefficient of friction increases appreciably as the volume fraction is reduced (Fig. 6a-b). Between volume fractions of 0.58 and 0.62 , the apparent coefficient of friction is independent of $k^{*}$ or $B o^{*}$. For less dense assemblies, the apparent coefficient was found to have stronger dependence on the volume fraction in the quasi-static regime (i.e. for large $B o^{*} k^{*}$ ) than in the inertial regime (i.e. for small $B o^{*} k^{*}$ ). This dependence of the apparent coefficient of friction on volume fraction can be trivially incorporated into the continuum rheological models for quasi-static flow, although most simulation studies do not seem to include it; it may explain some of the quantitative discrepancies between simulations (based on continuum models) and experimental data. For example, continuum model simulations of flows such as hopper and silo discharge have been found to overestimate the discharge rate from silos [14]; one can readily argue that allowing the apparent coefficient of friction to increase with dilation (which occurs near the orifice) should lead to lower discharge rates and bring the model predictions closer to correlations based on experimental data.

Shearing under constant applied normal stress only differs from shearing at constant volume in the dynamics. The shear stress fluctuations are smaller when shearing is performed under constant applied normal stress instead of at constant volume. However, the shear stress fluctuations for both types of shearing in the low $\phi$ quasi-static regime show the same dependence on the strength of cohesion. For shearing in the quasi-static regime, the shear stress fluctuations increase in size as cohesion is reduced, especially at low levels of cohesion. In the inertial regime, the shear stress fluctuations show a rather weak dependence on cohesion, but increase with the scaled stiffness. For shearing under constant applied normal stress, the volume fraction fluctuations (which are, of course, non-existent in constant-volume shearing) are very small and vary weakly with cohesion and the flow regime. Lastly, the coordination number fluctuations only depend on the time-averaged coordination number; but unlike the other fluctuating quantities, they do not depend on whether shear occurred under constant-volume or constant-applied normal stress conditions.

Continuum models for granular flows are generally based on ensemble (or volume) averaged equations and so they are based primarily on ensemble averaged (i.e. mean) flow characteristics. There is usually little incentive to track stress fluctuations, as the there is no evidence that the ensemble averaged stress is affected by the stress fluctuations. Indeed, we find that the time-averaged characteristics are the same for both constant-volume and constant-stress simulations, even though fluctuations can be very different. Thus the stress fluctuations discussed in this study are not directly of concern for the development of continuum models for the ensemble averaged flow behavior. However, stress fluctuations are of practical concern, as they are often responsible for the failure of devices; for example, stress fluctuations in a silo discharge can cause vibrations, which in turn can affect, over a period of time, the structural integrity of the silo. Thus, there is frequently an incentive to understand and mitigate stress fluctuations. Our finding that stress fluctuations, particularly in the quasi-static regime, are decreased by the addition of cohesive interaction between particles suggests that modest levels of cohesion may be beneficially employed to control fluctuations.

\section{Acknowledgments}

This work was partially supported by Merck \& Co., Inc., an NSF-NIRT grant, and a DOE-UCR grant. We gratefully acknowledge helpful discussions with Professor Pablo G. Debenedetti and Dr. Sung Joon Moon. We are delighted to have the opportunity to contribute to this special issue felicitating Professor Robert Pfeffer.

\section{References}

[1] E. Bilgili, J. Yepes, L. Stephenson, K. Johanson, B. Scarlett, Stress inhomogeneity in powder specimens tested in the Jenike shear cell: myth or fact? Part. Part. Syst. Charact. 21 (4) (2004) 293-302.

[2] M. Lätzel, S. Luding, H.J. Herrmann, Macroscopic material properties from quasi-static, microscopic simulations of a two-dimensional shear-cell, Granul. Matter 2 (2000) 123-135.

[3] S. Luding, From DEM Simulations towards a Continuum Theory of Granular Matter, 2001 from http://arxiv.org/PS_cache/cond-mat/pdf/0103/ 0103016.pdf.

[4] L. Oger, S.B. Savage, D. Corriveau, M. Sayed, Yield and deformation of an assembly of disks subjected to a deviatoric stress loading, Mech. Mater. 27 (1998) 189-210.

[5] R.P. Behringer, D. Howell, L. Kondic, S. Tennakoon, C. Veje, Predictability and granular materials, Physica, D 133 (1999) 1-17.

[6] B. Miller, C. O'Hern, R.P. Behringer, Stress fluctuations for continuously sheared granular materials, Phys. Rev. Lett. 77 (15) (1996) 3110-3113.

[7] C.S. Campbell, Granular shear flows at the elastic limit, J. Fluid Mech. 465 (2002) 261-291.

[8] L. Aarons, S. Sundaresan, Shear flow of assemblies of cohesive and noncohesive granular materials, Powder Technol. 169 (10) (2006).

[9] C.S. Campbell, Stress-controlled elastic granular shear flows, J. Fluid Mech. 539 (2005) 279-297.

[10] C.S. Campbell, Granular material flows - an overview, Powder Technol. 162 (3) (2006) 208-229.

[11] P.A. Cundall, O.D.L. Strack, A discrete numerical model for granular assemblies, Geotechníque 29 (1) (1979) 47-65.

[12] F. da Cruz, S. Emam, M. Prochnow, J-N. Roux, F. Chevoir, Rheophysics of dense granular materials: discrete simulation of plane shear flows, Phys. Rev., E 72 (2005) 021309.

[13] K. Craig, R.H. Buckholz, G. Domoto, An experimental study of the rapid flow of dry cohesionless metal powders, J. Appl. Mech. 53 (1986) 935.

[14] A. Srivastava, S. Sundaresan, Analysis of a frictional-kinetic model for gas-particle flow, Powder Tech. 129 (2003) 72. 
Appendix I

Dynamic shear of cohesive homogeneous assemblies of dense granular materials 


\title{
Unsteady Shear of Dense Assemblies of Cohesive Granular Materials under Constant Volume Conditions
}

\author{
Lee R. Aarons, Jin Sun, and Sankaran Sundaresan* \\ Department of Chemical Engineering, Princeton University, Princeton, New Jersey 08544
}

\begin{abstract}
The response characteristics of dense assemblies of cohesive granular materials to unsteady simple shear in the quasi-static regime are investigated through discrete element method (DEM) simulations of monodisperse spherical and frictional particles in periodic domains at constant volume. The dynamics of the volume-averaged normal and shear stresses in materials, undergoing stop-and-go shearing and oscillatory shear, are studied in detail. Furthermore, the evolution of microstructure anisotropy has been quantified through a fabric tensor. The stresses and the microstructure anisotropy depend on the strain extent but not on the shear rate. They both undergo a transition following reversal of shear direction, which requires a shear strain of order unity to fully adapt. The results reveal a correlation between the stress evolution and the microstructure anisotropy development.
\end{abstract}

\section{Introduction}

It is well-known that assemblies of granular materials behave differently under different flow conditions. Under rapid flow conditions, the particles interact with each other predominantly through binary collisions, while at slow shear rates the interaction is dominated by enduring contacts. In this paper, we focus on the rheological behavior of slowly deforming assemblies of granular materials.

Dense granular materials under steady shear have been studied extensively via simulations (e.g., see refs 1-5) and experiments (e.g., see refs 6-10). Campbell ${ }^{11,12}$ carried out discrete element method (DEM) simulations of sheared assemblies of cohesionless particles in periodic domains and presented a map of the different regimes of flow. From these simulations, Campbell concluded that in the absence of gravity and interparticle cohesion, the quasi-static regime, where the stress has a negligible dependence on shear rate, only existed for very densely packed assemblies. Similar steady shear simulations performed for cohesive granular materials ${ }^{13,14}$ revealed that interparticle attractive forces expand the range of volume fractions and shear rates over which the quasi-static flow regime is observed.

These studies only provide insight into shear at constant rates. However, granular materials are in general subjected to unsteady perturbations in nature and in industrial applications. It is thus of interest to investigate how granular systems respond under unsteady shear. Previous experimental work on unsteady shear has been done by Toiya et al. ${ }^{15}$ using a Taylor-Couette-type shear cell. They measured shear forces and particle motion during a single and repeated shear reversals. They found that when shear was restarted in the same direction, the shear force rapidly attained its previous steady-state value; in contrast, if the shear direction was reversed, the material went through a transient period during which the shear force was small, the material compacted, and the shear band widened. The transient behavior was also observed in the velocity profile changes from cyclic shear experiments using a parallel plate device. ${ }^{16}$

It is worth noting that the transient stress response was first reported by Gadala-Maria and Acrivos ${ }^{17}$ for unsteady shear of

* To whom correspondence should be addressed. E-mail: sundar@ princeton.edu. a suspension of noncolloidal particles. They measured the shear stress response to a single shear reversal and to sinusoidal shear using a parallel plate rheometer. After the direction of shear was reversed, the stress returned to its steady-state value only after a significant strain extent. This phenomenon was subsequently confirmed by other suspension experiments. Kolli et al. ${ }^{18}$ and Narumi et al. ${ }^{19}$ measured the normal stress as well as shear stress responses of suspensions to shear reversal in a parallel ring and in a parallel plate device, respectively. They found that the stress responses depended on the direction in which shear was restarted and that the data from different strain rates collapsed when plotted against strain. Narumi et al. ${ }^{20}$ measured strain rate response of a suspension to large amplitude sinusoidal shear using a stress-controlled cone-and-plate rheometer. The characteristic strain scale for the stress to return to the steady value was about two for most of the suspensions.

Such transient response has been attributed to the rearrangement of the shear-induced anisotropic microstructure after shear reversal. ${ }^{15,17}$ In fact, an anisotropic force network has been shown to form during steady shear of photoelastic disks in a two-dimensional (2D) biaxial cell ${ }^{10}$ and a Couette geometry. ${ }^{21}$ Strong force chains were found to form at approximately $45^{\circ}$ to the flow direction under steady shear. They were weakened while the overall force network remained largely unchanged during the period when shear stopped. Shear reversal led to a transition, upon which the strong force chains switched to a direction normal to the previous strong force chain direction. ${ }^{21}$

From a constitutive modeling point of view, accurate measurements of the rheological responses and microstructure dynamics are also in great need. Such data can be used to develop constitutive models that can accurately predict the complex unsteady rheological behaviors by taking account of the microstructure dynamics. Previously, plasticity models have been used for quasi-static granular flows. For example, a Mohr-Coulomb yield criterion was used to derive a rigid plasticity model for flow down a rough inclined plane. ${ }^{22} \mathrm{~A}$ von Mises-type yield function was used in derivation of another rigid perfectly-plastic model for analysis of instability of hopper flows. ${ }^{23}$ These model were applicable only to incompressible flows. Critical state soil mechanics models were formulated to predict volume fraction changes associated with loading histories. $^{24}$ However, they are not applicable to cyclic loading 
conditions, and they do not adequately model stress-induced anisotropy. A constitutive model with microstructural anisotropy incorporated is promising in addressing these shortcomings.

While the above experimental data have provided some macroscopic rheological responses and microstructure dynamics of granular materials under unsteady shear, it is difficult to measure both under homogeneous deformation over large strain scales. For example, in all three granular shear experiments, ${ }^{15,16,21}$ shear bands were formed with inhomogeneous velocity profiles across the gradient direction. This inhomogeneity predicates direct use of the data for constitutive model development, for which homogeneous deformation is often assumed in order to obtain the stress and strain information. ${ }^{25}$ In addition, the microstructure is difficult to probe and quantify experimentally in three dimensions.

In this paper, the dynamics of normal and shear stresses in dense cohesive granular materials subjected to unsteady shear under constant volume conditions have been probed using DEM simulations. The constant volume conditions allow us to obtain the critical states and to study the microstructure evolution independently of dilation. Transient and sinusoidal oscillatory shear under (nearly) homogeneous deformation have been studied. A homogeneous velocity profile was always maintained to a very good approximation, except for slight perturbations at very small strain scales immediately after shear reversal and large step changes in shear rate magnitude; so the results could be used directly to aid constitutive model development.

It will be shown that the responses are indeed strain-rateindependent and strain-controlled, consistent with the experiments mentioned above. A robust characteristic strain scale of order unity was found to be necessary for the granular system to return to the steady state after shear reversal. It is also our goal to quantify the microstructure dynamics and correlate the microstructure evolution to the stress transition. The state of the microstructure has been quantified through the use of statistics of the orientation of the contacts, $\left\langle\hat{\mathbf{n}}_{p, c} \hat{\mathbf{n}}_{p, c}\right\rangle$, where $\hat{\mathbf{n}}_{p, c}$ is the unit normal vector pointing outward from particle $p$ at contact $c$ and the brackets signify a volume average. This quantity is associated with a symmetric second-rank fabric tensor, $R$, which indicates the anisotropy of the microstructure, as commonly used for granular materials. ${ }^{26-28}$ We compute $R$ directly from the contact information obtained from our simulations and use it to demonstrate that the microstructure evolves at the same order of strain scale as the stresses during unsteady shear. It thus provides a micromechanical basis and data for constitutive models using the fabric as an internal variable. ${ }^{29-31}$ Implications of these results on constitutive modeling of quasistatic granular flows will also be discussed.

In section 2, the simulation methods and procedures for unsteady shearing are described. The results of theses simulations are discussed in section 3 . The main results of this study are summarized and conclusions are drawn in section 4 .

\section{Details of Discrete Element Simulations}

In this paper, the behaviors of cohesive materials undergoing different types of unsteady shear in the quasi-static regime are explored. Results of DEM simulations of steady shear under constant volume and constant applied normal stress conditions have already been described in our earlier publications. ${ }^{13,14}$ The present study was devoted to DEM simulations of unsteady shear flow under conditions of constant volume. Two types of unsteady shear have been analyzed: shear with a square wave shear rate (i.e., "stop-and-go" shear), and oscillatory shear with a sinusoidal shear rate. The stress and fabric tensors were calculated throughout the shearing process for assemblies with different dynamic shear rates, levels of cohesion, and solid volume fraction $(\phi)$. These data were analyzed to determine how they evolved with time and how this evolution depended on system variables.

Simulations featured 2000 monodisperse, spherical particles with diameter $d$ and density $\rho$. As generally done in DEM simulations, the particles were allowed to overlap when they collided, at which point the particles exerted a repulsive force on each other. The component of this force that would act in the normal direction (i.e., along the line connecting the particles' centers) is given by the linear (Hookean) spring-dashpot normal force model,

$$
F_{\mathrm{n}}=k \alpha-\eta v_{\mathrm{n}}
$$

where $k$ is the normal spring stiffness, $\alpha$ is the overlap, $\eta$ is the damping coefficient, and $v_{\mathrm{n}}$ is the relative normal velocity of the colliding particles. ${ }^{32}$ The damping term is the source of inelasticity in this model; the elasticity can be quantified using the coefficient of restitution, defined as the ratio of the relative rebound speed to the relative impact speed, given for the linear spring-dashpot model by

$$
\varepsilon=\exp \left(-\frac{\pi \eta}{\sqrt{2 m k-\eta^{2}}}\right)
$$

where $m=\pi \rho d^{3} / 6$ is the mass of a particle. ${ }^{33,34}$

The force exerted by colliding particles in the tangential direction, as the surfaces rub against each other, is given by a linear spring-slider model, ${ }^{35,36}$

$$
F_{\mathrm{t}}=\min \left(k_{\mathrm{t}} \Delta s, \mu F_{\mathrm{n}}\right)
$$

Here $k_{\mathrm{t}}$ is the tangential spring constant and $\Delta s$ is the distance the surface of one particle moves relative to the other in the tangential direction. The force is limited by a Coulombic upper bound, given by $\mu F_{\mathrm{n}}$, where $\mu$ is the interparticle friction coefficient.

When cohesive particles were simulated, they also interacted with each other via the van der Waals force. A pair of particles with a Hamaker constant $A$ and surfaces separated by a distance $s$ would attract each other with a van der Waals force with a magnitude of ${ }^{37}$

$$
F_{\mathrm{vdW}}=\frac{A d^{6}}{6 s^{2}(s+2 d)^{2}(s+d)^{3}}
$$

which reduces to the more familiar expression, $F_{\mathrm{vdW}}=A d /$ $24 s^{2}$, when $s \ll d$. This model diverges when particles collide, and to avoid this, a minimum cutoff separation, $s_{\min }$, was used, such that when $s<s_{\min }$, the van der Waals force remained equal to the force experienced at $s_{\min }$. Previous simulations have used values of $0.4^{38}$ and $1 \mathrm{~nm}^{37}$ for $s_{\min }$, corresponding to typical intermolecular distances. In the simulations described in this paper, a minimum cutoff of 4 $\times 10^{-5} d$ had been used, and so, the simulated particles can be assumed to have diameters between 10 and $25 \mu \mathrm{m}$. Since the van der Waals force drops off rapidly with increasing separation, in order to speed up the simulation, there was a maximum cutoff separation equal to $d / 4$, beyond which the cohesive force was ignored. The strength of cohesion is commonly expressed in dimensionless form as the Bond number, $B o$, is commonly used as defined as the ratio of the maximum van der Waals force experienced by a particle (i.e., at contact) to its weight. However, the simulations were performed in the absence of gravity, and so, a modified Bond number, which compares the van der Waals force to a 
Table 1. DEM Computational Parameters ${ }^{a}$

\begin{tabular}{lll}
\hline $\begin{array}{l}\text { interparticle normal stiffness coefficient } \\
\text { interparticle tangential stiffness }\end{array}$ & $k_{\mathrm{n}}$ & 100000 \\
$\quad k_{\mathrm{t}}$ & $2 / 7 k_{\mathrm{n}}$ \\
coefficient & & \\
interparticle friction coefficient & $\mu$ & 0.1 \\
interparticle restitution coefficient & $\varepsilon$ & 0.7 \\
Hamaker constant & $A$ & $1.92 \times 10^{-9}, 9.6 \times 10^{-8}$, \\
& & $4.8 \times 10^{-7}$ \\
particle diameter & $D$ & 1 \\
particle density & $\rho$ & 1 \\
minimum separation between particles & $S_{\text {min }}$ & $4 \times 10^{-5}$ \\
applied shear rate & $\dot{\gamma}$ & $10^{-1.5}-10^{-2.5}$
\end{tabular}

${ }^{a}$ The dimensional parameters involved in dimensionless groups, $k^{*}$ and $B o^{*}$, are presented in forms without any units because their physical significance can only be assessed by the magnitudes of those dimensionless groups in our simulations.

characteristic contact force, was used instead: $B o^{*}=F_{\mathrm{vdw}}^{\max } /$ $k d \approx A / 24 k s_{\min }{ }^{2}$. The systems presented in this paper were simulated with at least one of three levels of cohesion: $B o^{*}$ $=5 \times 10^{-7}, 2.5 \times 10^{-5}$, and $1.25 \times 10^{-4}$. For simplicity, these levels of cohesion will be referred to in figures as LC (low cohesion), MC (medium cohesion), and HC (high cohesion), respectively. In this paper, we only show results for assemblies at lower volume fractions that are in the quasistatic regime only when cohesive particles are used. Cohesive assemblies at higher volume fractions exhibit similar characteristics regarding the quasi-static rheological behaviors discussed here. For cohesionless granular assemblies in quasistatic flow, the stress and microstructure evolutions have qualitatively the same trends; the primary effect of cohesion is simply to allow quasi-static flow to occur at lower volume fractions. ${ }^{13,14}$ Thus the results for cohesionless particles will not be presented in this paper. The computational parameters used in the DEM simulations are listed in Table 1.

The particles existed in three-dimensional (3D) periodic cubic boxes of length $H$. Shear was induced via the Lees-Edwards boundary conditions, ${ }^{39}$ with the stream velocity in the $x$-direction and the velocity gradient in the $z$-direction. The use of these boundary conditions by themselves would imply that, when the shear rate changes during the course of a simulation, only the particles at the top and bottom of the domain would immediately "feel the effect" of the shear rate change. It would take some nonzero time for the rest of the particles to "be aware" of the change. Thus after a change in shear rate, the shear would not be homogeneous, such that different layers of particles would be experiencing different shear rates.

This effect can be greatly reduced through the use of the SLLOD algorithm. ${ }^{40}$ The SLLOD algorithm implies that a change in shear rate is not achieved by simply moving the boundaries of the system faster or slower, but by applying a force to the entire system. At a distance $z$ from the bottom of a homogeneously sheared system, the mean velocity is given by

$$
\mathbf{u}=\left(z-\frac{H}{2}\right) \dot{\gamma} \hat{x}
$$

(such that at $z=H / 2$, the mean streaming velocity is zero). Thus the force applied to every particle every time the shear rate changes would be given by

$$
\mathbf{F}_{\mathrm{SLLOD}}=m\left(z_{p}-\frac{H}{2}\right) \frac{\mathrm{d} \dot{\gamma}}{\mathrm{d} t} \hat{x}
$$

where $m$ is the mass of a particle and $z_{\mathrm{p}}$ is the $z$-coordinate of the particle's position measured from the bottom of the domain. It can be seen that if a particle is traveling at a velocity corresponding to homogeneous shear and is not interacting with any other particles (i.e., it is experiencing no forces), the application of the SLLOD algorithm when the shear rate is changed will cause the particle to immediately move at a velocity corresponding to the new shear rate. By using the SLLOD algorithm, a system at any point in time (ideally) can be described by a single stress tensor and shear rate, rather than a layer-by-layer description. Thus, the results from using the SLLOD algorithm are useful for the construction of constitutive models, and all the results presented here are from simulations that used SLLOD.

Periodically throughout the simulations, the stresses arising from interparticle forces and velocity fluctuations were calculated and recorded. For a volume $V$ consisting of $N$ particles, this stress due to interparticle forces is defined as ${ }^{1}$

$$
\sigma^{\mathrm{ip}}=\frac{1}{V} \sum_{p=1}^{N} \sum_{q \neq p}^{N} l_{p q} f_{p q}
$$

The branch vector $l_{p q}$ connects the center of particle $p$ to the midpoint of particles $p$ and $q$. The force vector $f_{p q}$ is the total force particle $p$ exerts on particle $q$, which is the sum of van der Waals force and the spring-dashpot force.

The stress arising from velocity fluctuations, i.e. the energy that is not directly generated by shearing, referred to as the momentum flux, is defined as

$$
\sigma^{\mathrm{mf}}=\frac{1}{V} \sum_{p=1}^{N} \frac{\pi}{6} \rho d^{3}\left(v_{p}-\dot{\gamma}\left(z_{p}-\frac{H}{2}\right) \hat{x}\right)\left(v_{p}-\dot{\gamma}\left(z_{p}-\frac{H}{2}\right) \hat{x}\right)
$$

Here, $v_{p}$ is the velocity of particle $p, z_{p}$ is the $z$-component of the position vector of particle $p$, and $\hat{x}$ is the unit vector in the $x$-direction. In homogeneous shear, the average velocity of a particle located at height $z_{p}$ would be $\dot{\gamma}\left(z_{p}-(H / 2)\right) \hat{x}$. The total stress, $\sigma$, would then be the sum of the stress due to interparticle forces and the momentum flux. The dimensionless, or scaled, stress is given by $\sigma d / k$. Though the stress tensor has, of course, three normal components, for simplicity the term "normal stress" will refer to the $z z$-component unless otherwise noted. Similarly, "shear stress" will refer to the $x z$-component of the stress tensor.

The first type of unsteady simulations that will be described below is "stop-and-go shearing". In these simulations, systems were first sheared at some initial (constant) shear rate, $\dot{\gamma}=\dot{\gamma}_{0}$, for several box lengths. Then, the shear rate was reduced to zero instantaneously (at $t=-1 / \dot{\gamma}_{0}$ ) and kept at zero for a time, $t=1 / \dot{\gamma}_{0}$ (i.e., equivalent to the time it would take to reach a strain of unity at a shear rate $\left.\dot{\gamma}_{0}\right)$. Next, shearing was resumed (at $t=0$ ) at the original shear rate in the original direction $(\dot{\gamma}$ $\left.=\dot{\gamma}_{\text {new }}=\dot{\gamma}_{0}\right)$ or in the opposite direction $\left(\dot{\gamma}=\dot{\gamma}_{\text {new }}=-\dot{\gamma}_{0}\right)$, for several box lengths. Figure 1a shows how the shear rate varies with time for these simulations. For these systems, we define a "directional scaled stiffness", $K=\left(\dot{\gamma}_{\text {new }} / \dot{\gamma}_{0}\right) k^{*}$, that is identical to a dimensionless "scaled stiffness", $k^{*}=k / \rho d^{3} \dot{\gamma}^{2}$, except that its sign indicates the direction of shear when it is resumed. Thus when $K$ is positive, shear is resumed in the original direction, and when it is negative, the shear direction is reversed.

Oscillatory shear simulations will then be described. In these simulations, the shear rate varied sinusoidally with time, $t$ :

$$
\dot{\gamma}(t)=\dot{\gamma}_{\max } \sin (\omega t)
$$

Here $\dot{\gamma}_{\max }$ is the maximum shear rate and $\omega$ is the frequency of the oscillations, such that the period of oscillation is $2 \pi / \omega$. These two parameters were varied to determine their effect on the 

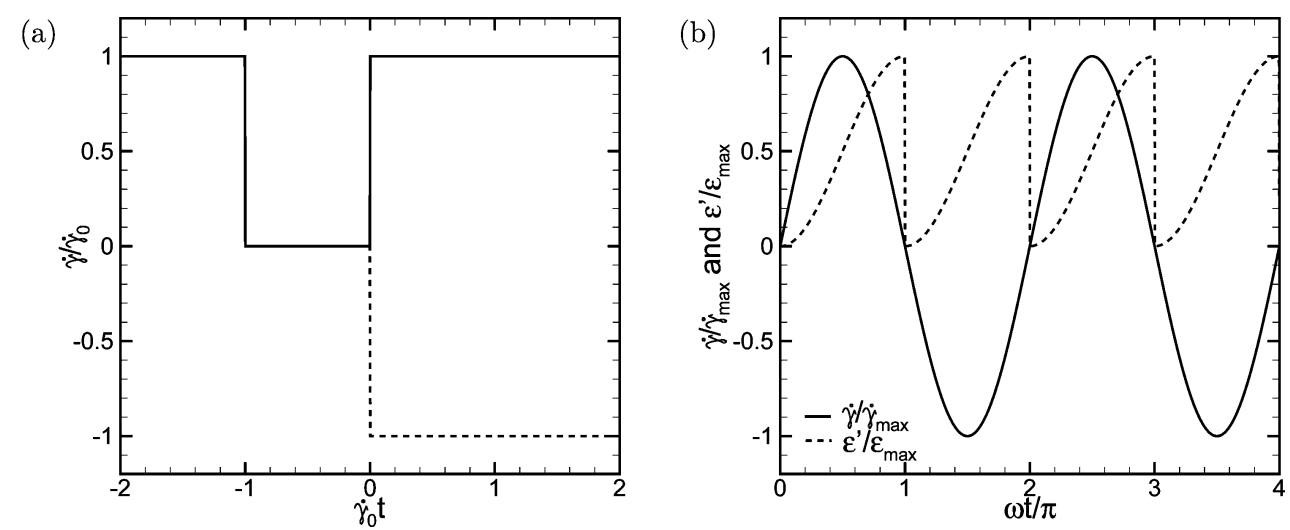

Figure 1. Time dependence of the shear rate for the two different types of unsteady shear: (a) stop-and-go shear and (b) oscillatory shear. In part a, the shear rate reduced by the original shear rate $\left(\dot{\gamma} / \dot{\gamma}_{0}\right)$ is plotted against the dimensionless time, $\dot{\gamma}_{0} t$; the solid lines represent the shear rate before the step change, and the dashed and dotted lines represent the shear rate after the step change. In part b, the shear rate reduced by the maximum shear rate $\left(\dot{\gamma} / \dot{\gamma}_{0}\right.$, solid line) as well as the strain reduced by the maximum strain $\left(\varepsilon^{\prime} / \varepsilon_{\max }^{\prime}\right.$, dashed line) are plotted against the number of half-periods of oscillation completed $(\omega t / \pi)$.

systems' behavior. We can define three different strains for these simulations. The first is relative to the state at $t=0$ :

$$
\varepsilon(t)=\int_{0}^{t} \dot{\gamma}\left(t^{\prime}\right) \mathrm{d} t^{\prime}=\frac{\dot{\gamma}_{\max }}{\omega}(1-\cos (\omega t))
$$

This gives a maximum strain of

$$
\varepsilon_{\text {max }}=\frac{2 \dot{\gamma}_{\text {max }}}{\omega}
$$

Given that the shear starts out at $t=0$ in the positive $x$-direction, this strain goes from 0 to $\varepsilon_{\max }$ as shear proceeds in the positive direction, and then it goes from $\varepsilon_{\max }$ to 0 as shear proceeds in the negative direction. However, as it will be shown later, the stress evolution is not symmetric about the flow-reversal point, and a modified strain, $\varepsilon^{\prime}$, that goes from 0 to $\varepsilon_{\max }$ regardless of the direction of shear, defined below, is more useful for capturing the simulation results.

We can also define an accumulated strain, $\varepsilon_{\text {total }}$, such that, when shear is reversed, it is not reset to zero and shearing in any direction increases the strain. This is equivalent to using the absolute value of the shear rate to define strain:

$$
\begin{array}{r}
\varepsilon_{\text {total }}(t)=\int_{0}^{t}\left|\dot{\gamma}\left(t^{\prime}\right)\right| \mathrm{d} t^{\prime}=\frac{\varepsilon_{\max }}{2}\left(1-\cos \left(\omega t-\pi\left\lfloor\frac{\omega t}{\pi}\right\rfloor\right)+\right. \\
\left.2\left\lfloor\frac{\omega t}{\pi}\right\rfloor\right)=\varepsilon^{\prime}+\varepsilon_{\max }\left\lfloor\frac{\omega t}{\pi}\right\rfloor
\end{array}
$$

As $\pi / \omega$ is equal to half of the period of oscillation, $\lfloor(\omega t / \pi)\rfloor$ is the integer number of half-oscillations that have been completed by time $t$. From eq 5, we get

$$
\varepsilon^{\prime}=\frac{\varepsilon_{\max }}{2}\left(1-\cos \left(\omega t-\pi\left\lfloor\frac{\omega t}{\pi}\right\rfloor\right)\right)
$$

For the rest of this paper, the term strain will refer to the quantity defined by eq 13 rather than eq 10, and total strain will refer to the quantity defined in eq 12 . Figure $1 \mathrm{~b}$ shows how $\dot{\gamma}$ and $\varepsilon^{\prime}$ vary with time for these simulations.

For oscillatory shear, a system can be identified by a "reduced minimum scaled stiffness", $\kappa=10^{-8} k / \rho d^{3} \dot{\gamma}_{\max }{ }^{2}$, and maximum strain, $\varepsilon_{\max }$. (The factor $10^{-8}$ is used in the definition of $\kappa$ so that, for the oscillatory shear systems discussed here, $\kappa$ is between 3 and 100.)

The results presented in this paper take the form of stress (specifically the normal and shear stresses), fabric tensor (generally the shear component), and average coordination number (i.e., the average number of contacts per particle, Z) versus some measure of strain. As mentioned above, we use the total strain, $\varepsilon_{\text {total }}$, for oscillatory shear, such that we examine how the system behavior evolves with the total distance sheared since the start of shear. For those simulations that feature stopand-go shearing, we are most interested in describing the system behavior after shear is resumed, and so in these cases, it is most convenient to define the strain as $\gamma_{0} t$, where $t=0$ is identified as when shear is resumed after stopping. This quantity is thus equivalent to the strain experienced after shear resumes when $t \geq 0$ but is just a dimensionless time when $t<0$. Similarly, for those simulations in which a single step change in shear rate occurs, we are most interested in the behavior after the step change occurs, and so, we define the strain as $\dot{\gamma}_{\text {new }} t$, where $t=$ 0 is identified as when the step change occurs. Again, this quantity is equivalent to the strain experienced after the step change occurs when $t \geq 0$ but is just a dimensionless time when $t<0$.

The fabric tensor is computed as

$$
R=\frac{\phi}{N} \sum_{p=1}^{N} \sum_{c>p}^{N} \hat{\mathbf{n}}_{p, c} \hat{\mathbf{n}}_{p, c}
$$

for monodisperse collections of particles. Here $\phi$ is the solid volume fraction, $N$ is the number of particles. It can readily be shown that the trace of the fabric tensor is equal to the product of the volume fraction and average coordination number $(\phi Z)$. Furthermore, the eigenvectors of the fabric tensor give the principal directions of the mean contact orientations. The eigenvalues, in turn, provide a measure of the extent of contact orientations along the principal directions. If the three eigenvalues are all equal, then the orientation is not pointed in one principal direction more than another, and so, we say the contact orientation is isotropic. As the eigenvalues become more different, the contacts tend to become more oriented in the direction of the eigenvector corresponding to the largest eigenvalue, and so, the structural anisotropy increases.

That being said, the structural anisotropy can easily be related to the shear $(x z-)$ component of the fabric tensor for simple shear flows. To demonstrate this, we show the different components of the fabric tensor for one case of stop-and-go shearing in which the direction of shear is reversed when resumed in Figure $2 \mathrm{a}$ and $\mathrm{b}$. By scaling the normal components shown in Figure $2 \mathrm{a}$ by $\phi Z$, it can be seen that at all times the three normal components are approximately equal to one-third of $\phi Z$. Furthermore, of all the off-diagonals shown in Figure 2b, only the $x z$-component is nontrivial. These observations hold true during steady state before shear becomes unsteady, immediately 
(a)

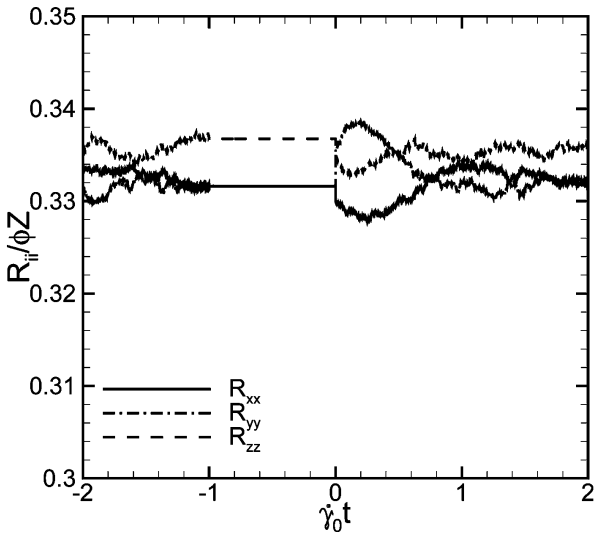

(b)

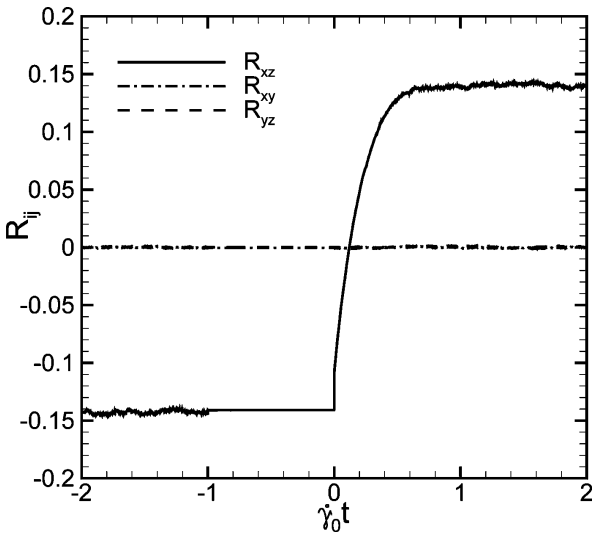

Figure 2. Evolution of the fabric tensor of a system subjected to stop-and-go shearing. Plotted are (a) the normal components of the fabric tensor $\left(R_{x x}, R_{y y}\right.$, and $\left.R_{z z}\right)$ scaled by the product of the volume fraction and average coordination number $(\phi Z)$ and (b) the off-diagonal components of the fabric tensor $\left(R_{x y}\right.$, $R_{x z}$, and $R_{y z}$ ) versus the dimensionless time, $\dot{\gamma}_{0} t$, for a system with $K=-10^{9}, \phi=0.6$, and $B o^{*}=2.5 \times 10^{-5}$.

following changes in shear rate, and long afterward. So to a good approximation, in all our simulations,

$$
R \approx\left(\begin{array}{lll}
\phi Z / 3 & 0 & R_{x z} \\
0 & \phi Z / 3 & 0 \\
R_{x z} & 0 & \phi Z / 3
\end{array}\right)
$$

which has major and minor eigenvalues separated by $2 R_{x z}$. Thus, the structural anisotropy increases with the magnitude of $R_{x z}$. Also, the sign of $R_{x z}$ provides the preferred contact orientation. The preferred orientation is always in the $x z$-plane, at an angle of $\pm 45^{\circ}$ to the $x$-axis; the largest eigenvalue, and hence the preferred orientation, corresponds to a positive (negative) angle when $R_{x z}$ is positive (negative).
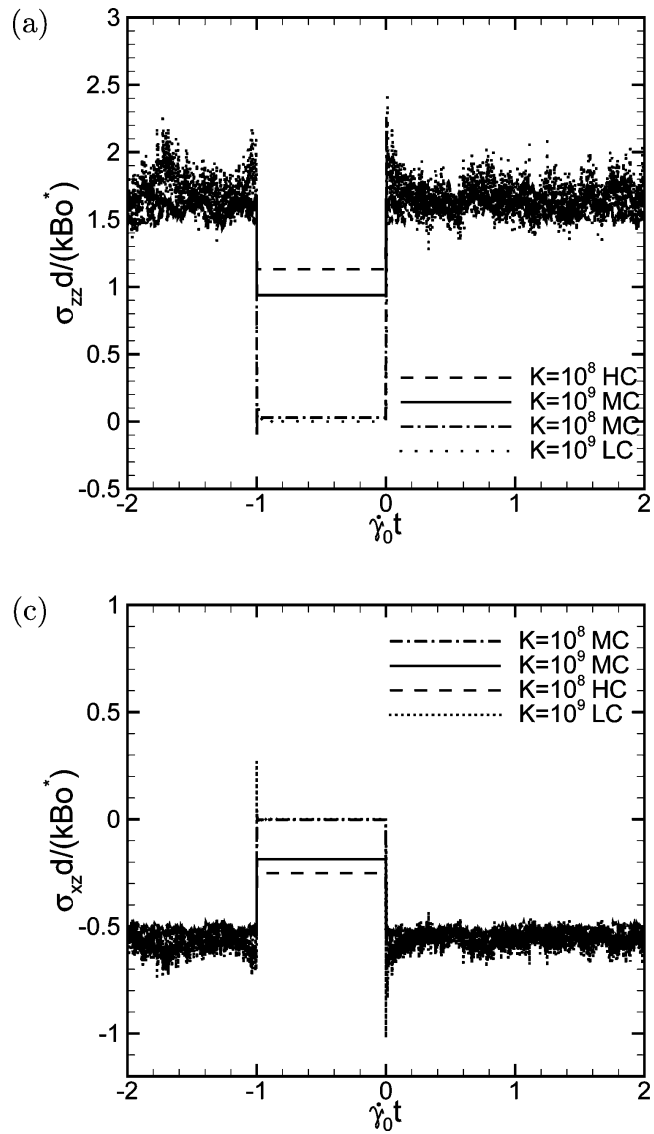

In our previous studies, ${ }^{13,14}$ all the results were obtained from simulations generated by internally developed code. However, the results discussed here were obtained from simulations generated by an open source code, LAMMPS (large-scale atomic/molecular massively parallel simulator), created at Sandia National Laboratories, as LAMMPS proved to be significantly faster. ${ }^{41}$ We reproduced the steady-shear results discussed in our previous publications ${ }^{13,14}$ with LAMMPS. Furthermore, some of the results discussed in this paper were also reproduced using our internally developed code, as further verifications of the simulations. All the results presented in this paper were simulated with multiple initial configurations to generate 20 realizations for the stop-and-go shearing and 10 realizations for

(b)

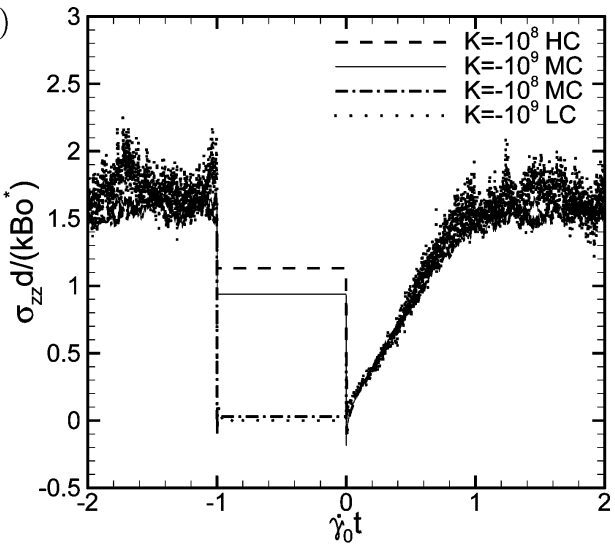

(d)

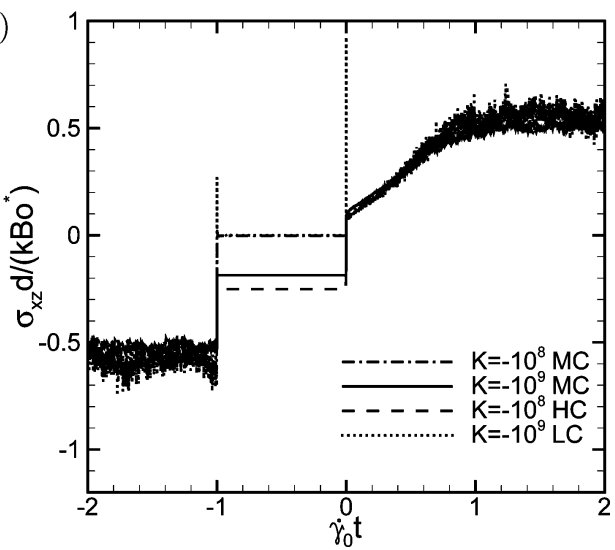

Figure 3. Evolution of the stress of systems subjected to stop-and-go shearing. Plotted are the cohesively scaled normal stress $\left(\sigma_{z z} d /\left(k B o^{*}\right)\right)$ and shear stress $\left(\sigma_{x z} d /\left(k B o^{*}\right)\right)$ versus the dimensionless time, $\dot{\gamma}_{0} t$, for systems in which the shear is stopped and then resumed at the original shear rate in the original direction (a and c) and in the opposite direction (b and d). In all cases, shear is stopped at $\dot{\gamma}_{0} t=-1$ and resumed at $\dot{\gamma}_{0} t=0 ; \phi=0.6$. 
(a)

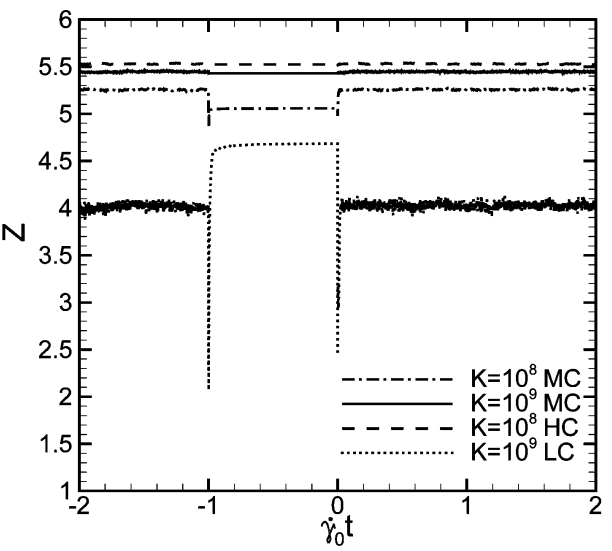

(c)

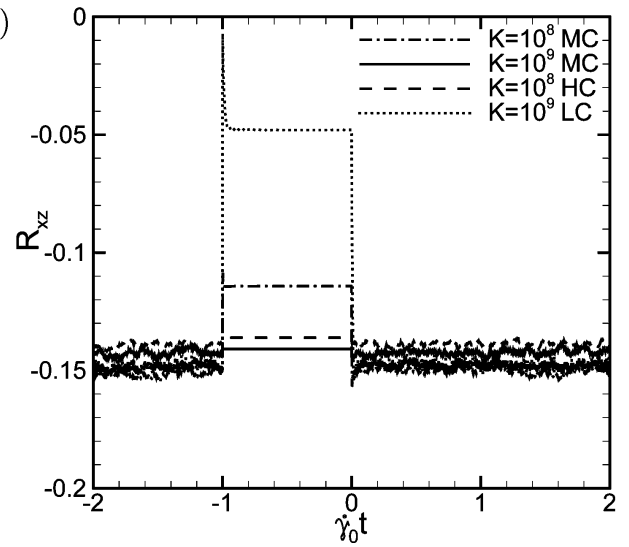

(b)

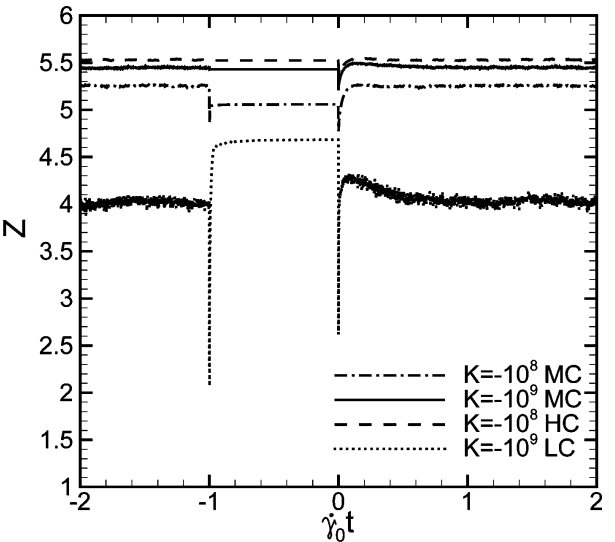

(d)

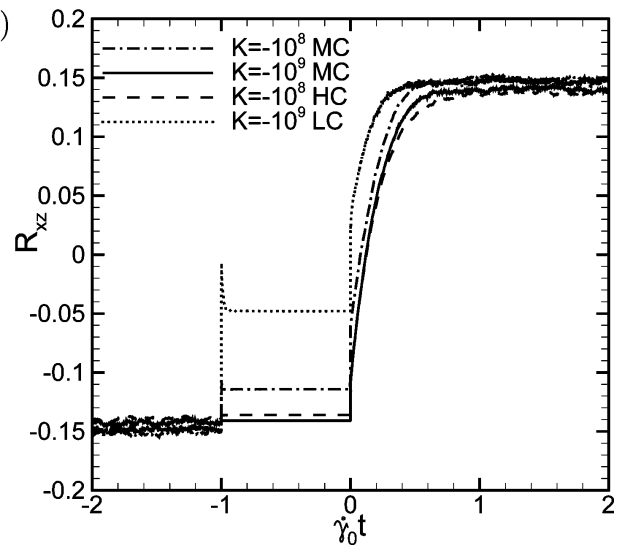

Figure 4. Evolution of microstructure of systems subjected to stop-and-go shearing. Plotted are the average coordination number $(Z)$ and the shear component of the fabric tensor $\left(R_{x z}\right)$ versus the dimensionless time, $\dot{\gamma}_{0} t$, for systems in which the shear is stopped and then resumed at the original shear rate in the original direction (a and c) and in the opposite direction (b and d). In all cases, shear is stopped at $\dot{\gamma}_{0} t=-1$ and resumed at $\dot{\gamma}_{0} t=0 ; \phi=0.6$.

the oscillatory shearing; the results were then averaged over these realizations.

\section{Results and Discussion}

3.1. Stop-and-Go Shearing. Figures 3 and 4 show how the normal stress, shear stress, average coordination number, and shear component of the fabric tensor $\left(R_{x z}\right)$ evolve with strain for cohesive assemblies in the quasi-static regime with $\phi=0.6$ subjected to stop-and-go shearing. The volume fraction was chosen so that the quasi-static regime was reached. The specific value was obtained from previous simulations on quasi-static flows reported in our previous work. ${ }^{13}$ As long as the flow is in the quasi-static regime, the change of volume fraction will not change the trends observed here but only the stress magnitudes. In each figure, panels a and c show the behavior for the cases where shear is stopped and then resumed in the original direction, while panels $b$ and d show the behavior for the cases where shear is stopped and then resumed in the opposite direction. As observed in a previous study, ${ }^{13,14}$ the steady state stress scales with $B o^{*}$ in this regime. Thus, the stresses in Figure 3 are scaled by $B o^{*}$ to compare results for systems with different levels of cohesion as well as different shear rates (or equivalently, different $k^{*}$ ). The dynamic responses of normal stresses, as well as the shear stresses, collapse into a single curve over all cases when scaled in this manner and plotted against strain. (Most of the stress fluctuations observed in Figure 3 were generated from the system with $K= \pm 10^{9}$ and LC, which will be explained later.) This confirms that the dynamics is controlled by the deformation strain and is strain-rate-independent. As mentioned before, anisotropic microstructure is formed at steady state, and as evidenced in Figure $4, R_{x z}$ has a steady-state magnitude of about 0.14 (though the actual steady-state $R_{x z}$ varies slightly from system to system).

A salient feature of the response is that it depended on whether shear was resumed in the original direction or in the opposite direction. When shear was stopped at $\dot{\gamma}_{0} t=-1$, the particles were more or less frozen in place; however, the slight rearrangement that occurred was enough to cause measurably large changes in the stresses, average coordination number, and $R_{x z}$ (see Figures 3 and 4). When shear was resumed in the original direction (at $\dot{\gamma}_{0} t=0$ ), the stresses (Figure 3a and c) and microstructure (Figure $4 \mathrm{a}$ and c) returned to steady state almost instantaneously as the particles only needed to be moved a little to return to the steady-state microstructure.

On the other hand, when shear was resumed in the opposite direction, the particles needed to completely rearrange themselves to form force chains in the opposite direction, requiring shear to proceed much longer to reach steady state. As shear progressed, the normal and shear stresses increased nearly linearly with strain until steady state was achieved at around a strain of unity (Figures $3 b$ and d); the evolution of the scaled stresses with strain extent was essentially independent of strain rate and cohesion. At the same time, the orientation of the contacts slowly reversed, as evidenced by $R_{x z}$ gradually going from a negative value to a positive value (see Figure $4 d$ ), which indicates the microstructure rearrangement. The evolution of the structure with strain was virtually independent of shear rate and cohesion. However, as the value of $R_{x z}$ in the "stop" phase of the simulation $\left(-1<\dot{\gamma}_{0} t<0\right)$ was different for each system before shear was reversed, the actual amount of strain required to reach the final steady state $R_{x z}$ differed between the systems. Nevertheless, in all cases $R_{x z}$ leveled out at strains between about 
(a)

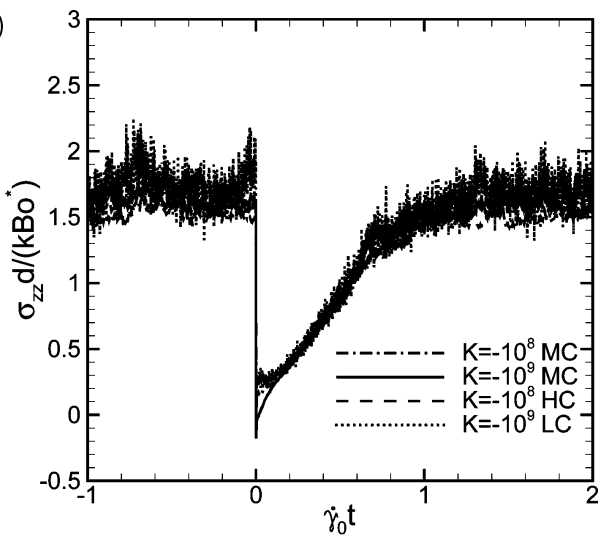

(c)

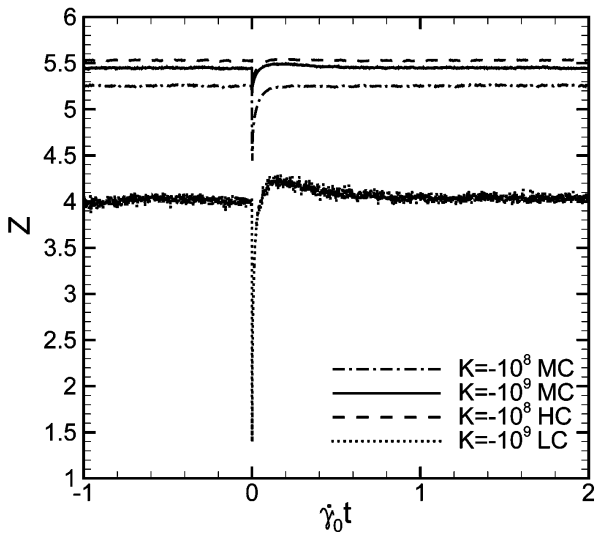

(b)

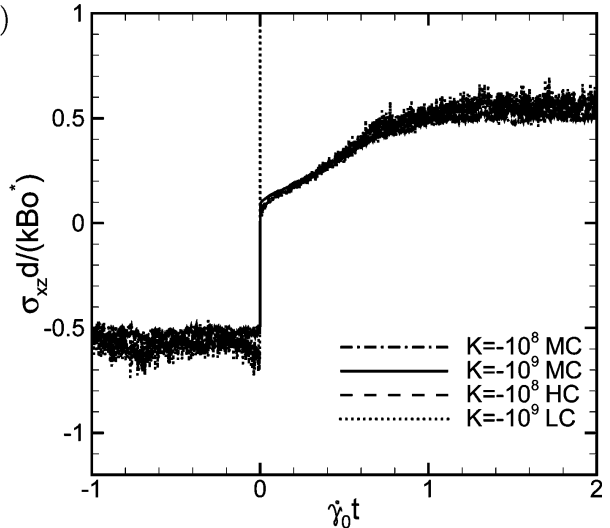

(d)

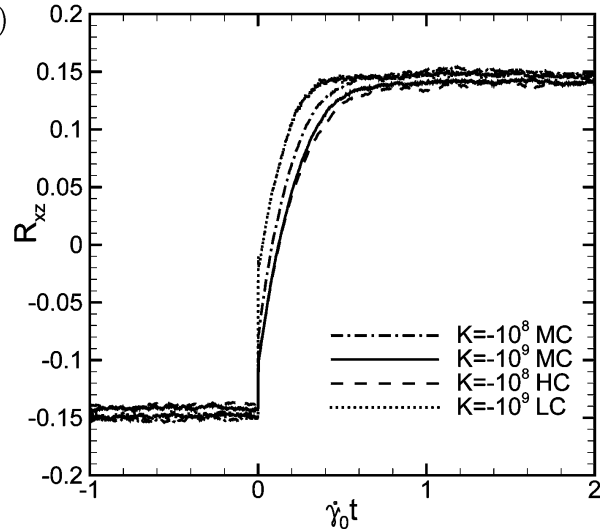

Figure 5. Evolution of the systems subjected to shear reversal without a stop phase. Plotted are (a) the cohesively scaled normal stress $\left(\sigma_{z z} d /\left(k B o^{*}\right)\right)$; (b) the cohesively scaled shear stress $\left(\sigma_{x z} d /\left(k B o^{*}\right)\right)$; (c) the average coordination number $(Z)$; and (d) the shear component of the fabric tensor $\left(R_{x z}\right)$ versus the dimensionless time, $\dot{\gamma}_{0} t$. In all cases, shear is reversed at $\dot{\gamma}_{0} t=0 ; \phi=0.6$.

0.5 and 0.7 , i.e. before the stresses did. This implies that the microstructure needs to be in place for the force chains and stress to build up, and that it must be in place for a sufficient amount of strain for the stress to reach steady state. The anisotropy of microstructure also has been shown to evolve faster than the shear stress in dense suspensions as revealed by the pair distribution function evolution in Stokesian dynamics simulations of shear reversal. ${ }^{42}$

To determine how the differences between the curves seen in Figure 4d upon shear reversal are affected by the fact that the values of $R_{x z}$ in the stop phase were different, simulations were repeated without a stop phase, where the assemblies were sheared in one direction until a statistical steady state was reached and then the shear was instantaneously reversed at $\dot{\gamma}_{0} t$ $=0$. Figure $5 \mathrm{a}-\mathrm{d}$ shows the normal and shear stresses, average coordination number, and the shear component of the fabric for such simulations and can be compared with panels b and $d$ of Figures 3 and 4 . As can be seen readily, the evolution of $R_{x z}$ for the different simulations are now slightly closer to one another, confirming that the stop phase in Figures 3 and 4 was responsible for some of the differences seen among the different curves in Figure 4d.

The physical significance of unit strain required for the stress and microstructure evolution following shear reversal is easy to visualize. If we imagine the assembly of particles as layers of particles (with each layer in contact with the adjacent ones), unit strain corresponds to the translation of the particles in one layer by a distance of one particle diameter relative to the particles in the adjacent layer. In a more randomly configured assembly of particles, such a translation will involve particle climbing over a particle in an adjacent layer and this will naturally lead to normal stress variations.
The evolution of shear stress following stop-and-go shear presented here are remarkably similar to the experimental results reported by Gadala-Maria and Acrivos ${ }^{17}$ for dense suspensions. The presence of the interstitial fluid in their experiments introduces lubrication stresses, which are not present in our simulations; yet, the similarity is so strong that the mechanisms discussed here are likely relevant for their system as well.

Figures 3 and 4 also show appreciable changes at small strain scales immediately following sudden changes; the mechanisms behind those responses can be explained as follows. It is first noted that these spikes and dips are not numerical artifacts resulting from incomplete resolution of the motions; simulations performed using several widely different time steps reproduced these features. The small strain scale responses depend on the level of cohesion and steady-state average coordination number, which in turn had an additional dependence on the level of cohesion as well as the scaled stiffness (see Figure 6 in ref 13). The coordination number characterizes the connectivity of a granular assembly. ${ }^{43}$ When the coordination number is equal to the critical value $Z_{\mathrm{c}}$ (equal to 4 for $3 \mathrm{D}$ frictional particle assemblies studied here), the granular assembly is at an isostatic state, where the number of degrees of freedom is matched by number of constraints between particles. For coordination number $Z<Z_{\mathrm{c}}$, the assembly cannot be stable and it will rearrange. For $Z>Z_{\mathrm{c}}$, the assembly is overconnected and apt to resist external perturbations, thus stable. When shear came to an immediate halt, the assembly tended to relax as the external constraint to deformation was removed. The extent of the relaxation, however, depended on the stability of the assembly microstructure as indicated by the average coordination number.

Resuming shear in the original direction brought the stress instantly back to its steady state value for those cases in which 

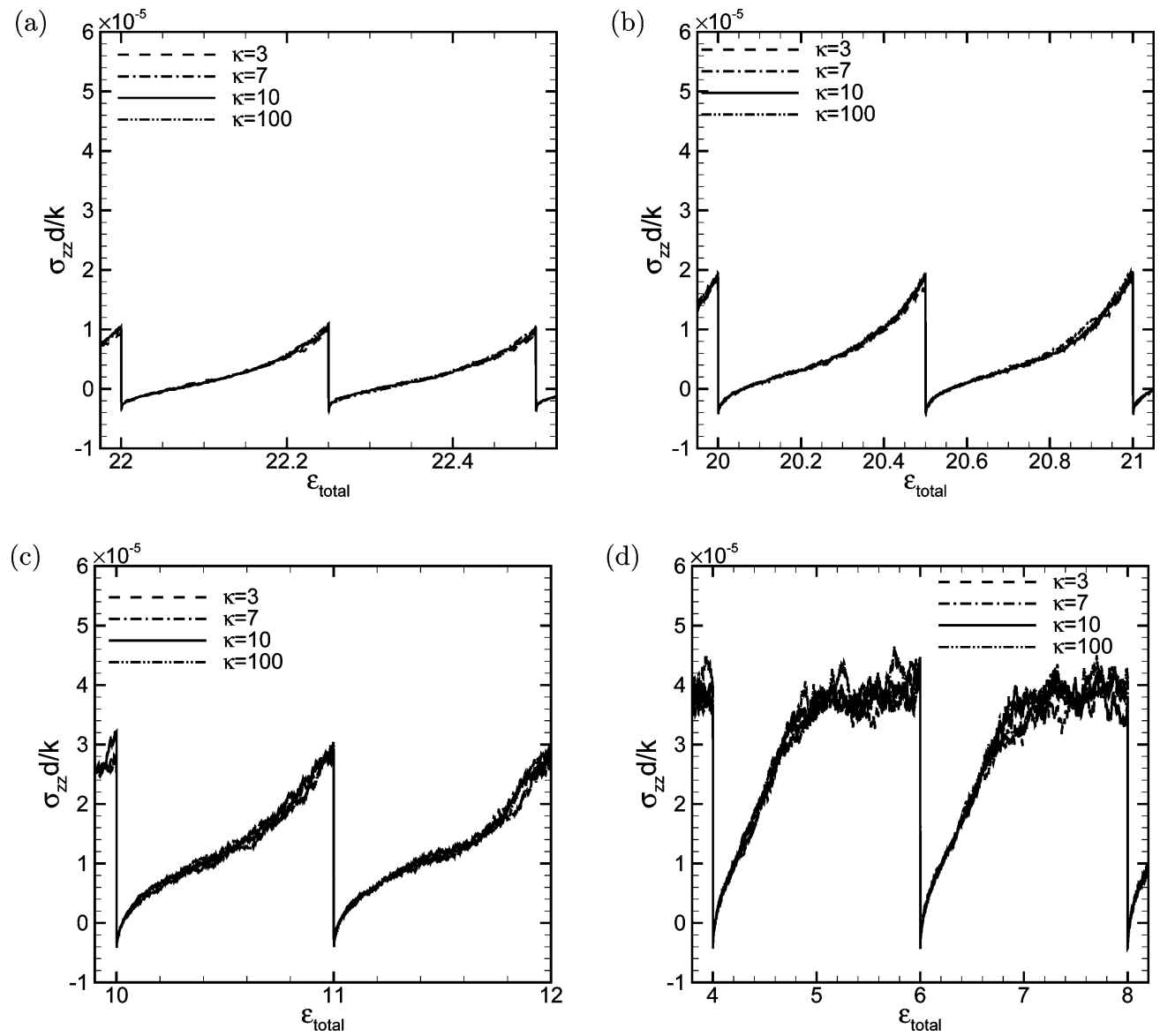

Figure 6. Strain dependence of the normal stress for oscillatory shear with maximum strains of (a) $\varepsilon_{\max }=0.25$, (b) $\varepsilon_{\max }=0.5$, (c) $\varepsilon_{\max }=1$, and (d) $\varepsilon_{\max }=2$. Plotted is the scaled normal stress $\left(\sigma_{z z} d / k\right)$ versus the total strain $\left(\varepsilon_{\text {total }}\right)$. Each figure features the stress evolution for four different values of the reduced minimum scaled stiffness $\left(\kappa=3,7,10\right.$, and 100). In all cases, $\phi=0.6$ and $B o^{*}=2.5 \times 10^{-5}$.

the average coordination numbers are high $\left(K=10^{8}\right.$ with $\mathrm{HC}$ and $K=10^{9}$ with MC in Figure $3 \mathrm{a}$ and c), as the microstructure remained largely unchanged and no rearrangement was needed. In the other cases, the particles needed be rearranged slightly in order to return to steady state; first, the contacts formed after shear was stopped were destroyed, and then, the shear and cohesion reestablished the contacts in the original arrangement (as measured by $R_{x z}$, Figure $4 \mathrm{c}$ ). As the particles hardly moved since shearing was stopped, this process was extremely quick. The process of breaking and reestablishing contacts so quickly led to a very short-lived spike in both the normal and shear stresses before steady state was achieved.

The moment shear was reversed, the shear stress became positive, but again, the specific behavior can be linked to the system history. For those systems exhibiting stable microstructures (high average coordination numbers; $K=-10^{8}$ with HC and $K=-10^{9}$ with MC), the reversal of shear essentially caused the force chains to be ripped apart as the anisotropy is destroyed, causing the stress to instantly drop and leading to a very shortlived tensile state (as seen as a negative normal stress in Figure $3 b$ ). This was of course an effect of using cohesive particles, as cohesion was the only source of a negative normal stress (aside from a negligible effect from the dashpot ${ }^{13}$ ). When shearing reversed direction, since the mean orientation of contacts gradually changed, the microstructure did not immediately conform to the new compression direction and most contacts remained in the previous direction, which was subjected to less compression after the shear direction reversed. (See, for example, the $R_{x z}$ remained positive immediately after shear reversal in Figure 4d.) This microstructure transition led to the tensile state. However, in the other cases, the structures were not as difficult to rearrange and reversing shear did not lead to a tensile state. When cohesion was weak (LC, for which $B o^{*}$ $=5 \times 10^{-7}$ ), the extra contacts formed needed to be destroyed, requiring a small spike in both the normal and shear stress. The spikes seen in Figure 5 are also explained in the same manner.

When shear was stopped, the stresses dropped significantly if the average coordination number prior to stopping was small ( $K= \pm 10^{8}$ with MC and $K= \pm 10^{9}$ with LC in Figure 3 ). When the level of cohesion was large enough $\left(\mathrm{MC}\right.$, for which $B o^{*}=$ $2.5 \times 10^{-5}$ ), this led to negative normal stress (at $\dot{\gamma}_{0} t=-1$ in Figure $3 \mathrm{a}$ and $\mathrm{b}$ ), i.e. the system was in tension as force chains were disrupted and the particles pulled each other back. The spikes can be explained similarly to the shear resuming cases discussed above. Furthermore, the magnitude of $R_{x z}$ dropped when shear was stopped, as the breaking of force chains resulted in a loss of anisotropy (see Figures $4 \mathrm{c}$ and d).

3.2. Oscillatory Shear Results. Figures $6 a-d$ show how the normal stress evolves with the total strain during the oscillatory shear of cohesive assemblies (with $B o^{*}=2.5 \times 10^{-5}$ ) at a volume fraction of 0.6 for four different values $\varepsilon_{\max }$. Each figure features the stress evolutions for four different reduced minimum scaled stiffnesses, $\kappa$ (defined earlier in section 2 ). (In all these figures, oscillatory shear was maintained for many cycles to ensure that little memory of the initial conditions for the simulations remained and a periodic solution which is representative of the long-time solution resulted.) As the shear rate increased from zero (i.e., starting from any point where $\varepsilon_{\text {total }}$ is an integer multiple of $\varepsilon_{\max }$ ), the normal stress rose. The steadystate normal stress could be achieved despite the constantly changing shear rate, provided the strain amplitude was suf- 
(a)

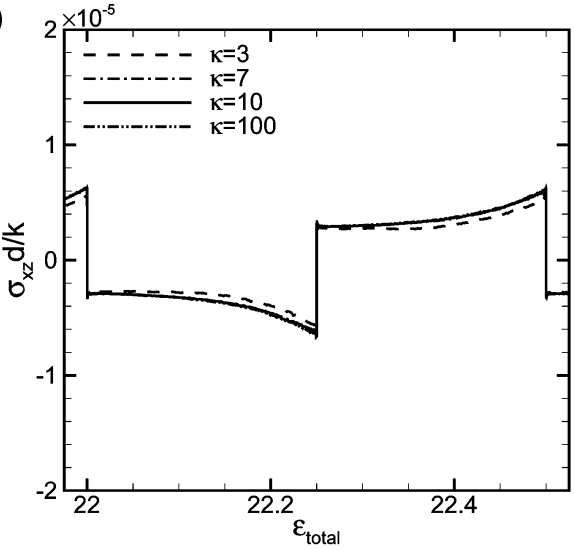

(c)

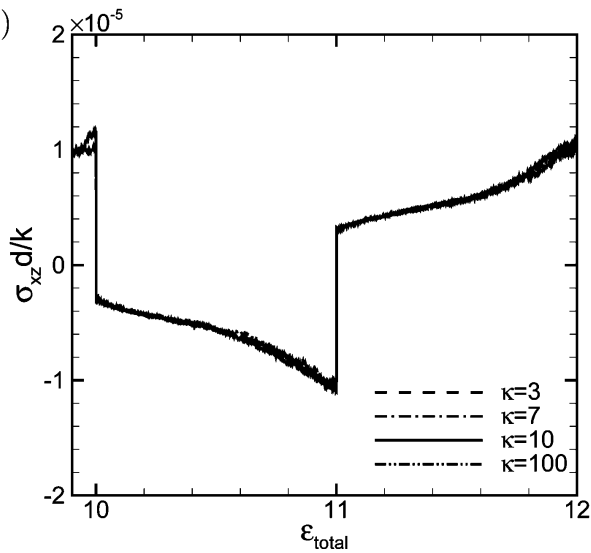

(b)

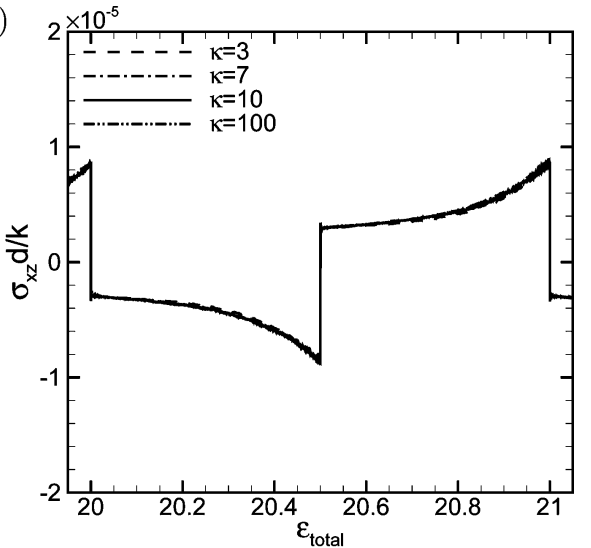

(d)

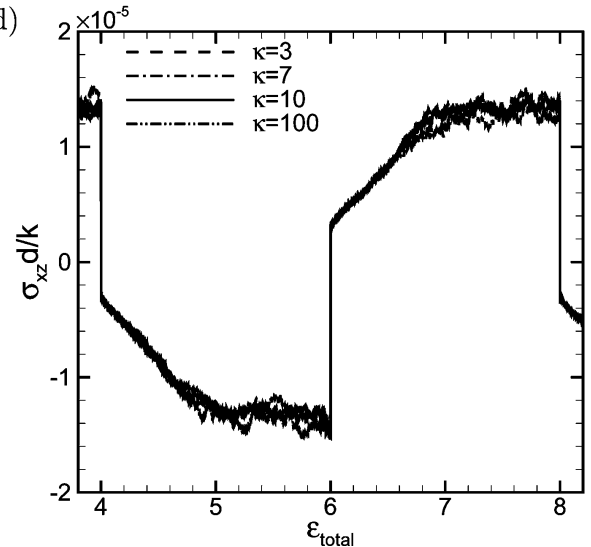

Figure 7. Strain dependence on the shear stress for oscillatory shear with maximum strains of (a) $\varepsilon_{\max }=0.25$, (b) $\varepsilon_{\max }=0.5$, (c) $\varepsilon_{\max }=1$, and (d) $\varepsilon_{\max }=2$. Plotted is the scaled shear stress $\left(\sigma_{x z} d / k\right)$ versus the total strain $\left(\varepsilon_{\text {total }}\right)$. Each figure features the stress evolution for four different values of the reduced minimum scaled stiffness $\left(\kappa=3,7,10\right.$, and 100). In all cases, $\phi=0.6$ and $B o^{*}=2.5 \times 10^{-5}$.

ficiently larger than unity (see Figure 6d corresponding to strain amplitude of 2). When the strain amplitude was smaller (Figure $6 a-c)$, steady state (i.e., plateau region in the stress) was clearly not reached.

Figure $7 a-d$ shows the shear stress evolution for those systems in Figures $6 \mathrm{a}-\mathrm{d}$, respectively. The sign of the shear stress depends on the direction of shear, just as in steady shear, whereas obviously the normal stress does not. It is clear from Figure 7 that when the direction of shear was changed, the shear stress changed sign almost immediately and assumed a significant magnitude; subsequently, the magnitude of the shear stress continued to evolve slowly, reaching a plateau when the strain amplitude was in excess of unity (see Figure 7d). This strain scale of order unity for stress to return to steady state is consistent with that found in the stop-and-go shearing discussed in section 3.1.

Figure $8 \mathrm{a}-\mathrm{d}$ shows how the shear component of the fabric tensor, $R_{x z}$, evolved with strain for those systems in Figures $6 \mathrm{a}-\mathrm{d}$, respectively. Every time shear was reversed, the mean orientation of particles gradually changed. Like the shear stress, $R_{x z}$ alternated between positive and negative values every time shear reversed directions as the particle contacts reversed their orientations. Analogous to both the normal and shear stresses, $R_{x z}$ achieved the steady-state (plateau) value $(\approx \pm 0.14)$ when $\varepsilon_{\max }=2$ (Figure 8d). Likewise, when $\varepsilon_{\max }$ was smaller (Figures $8 \mathrm{a}-\mathrm{c}), R_{x z}$ did not reach a plateau value before shear was reversed. Furthermore, as observed in stop-and-go shearing, $R_{x z}$ reached a plateau earlier $\left(\varepsilon^{\prime} \approx 0.7\right)$ than the stress $\operatorname{did}\left(\varepsilon^{\prime} \approx 1\right)$. Again, this suggests that after the particles reach their steady mean orientation, shear must proceed farther for the force chains to be sufficiently built and rotated for the stress to reach its steady value.

In Figures $6-8$, the results for all four values of $\kappa$ collapsed onto the same curve. As such, the stress and microstructure evolutions with strain were rate-independent. This in turn means that the stress evolution did not depend on how much time has passed since shearing started in one direction, but rather on how far shear has proceeded in one direction. This is what was observed after shear was reversed during stop-and-go shearing.

Regardless if steady state was achieved, reversing shear then resulted in the normal stress dropping to a negative value, indicating that the system was in tension. This is not unlike what was observed when systems with strong force chains experienced a reversal of shear during stop-and-go shear. The normal stress grew again after the short-lived tensile state, and this whole pattern repeated ad infinitum. Similar transient negative normal stress was also observed in the suspension experiment by Kolli et al., ${ }^{18}$ and the mechanism is analogous to what is described here.

Figure $9 \mathrm{a}$ and $\mathrm{b}$ shows the effect of cohesion on the scaled normal stress and $R_{x z}$ evolution, respectively. Results are shown for the oscillatory shear of systems with $\varepsilon_{\max }=2$ for three different levels of cohesion. The stresses in Figure 9a were cohesively scaled so that the steady state would be the same for all the systems, and by scaling this way, the stress evolution became independent of cohesion. The shear component of the fabric tensor, $R_{x z}$, shown in Figure $9 \mathrm{~b}$ also showed no dependence on cohesion. These results again were also seen in stopand-go shearing.

It was observed earlier in Figures 6-8 that when $\varepsilon_{\max }=2$, the stresses and the shear component of the fabric reached 
(a)

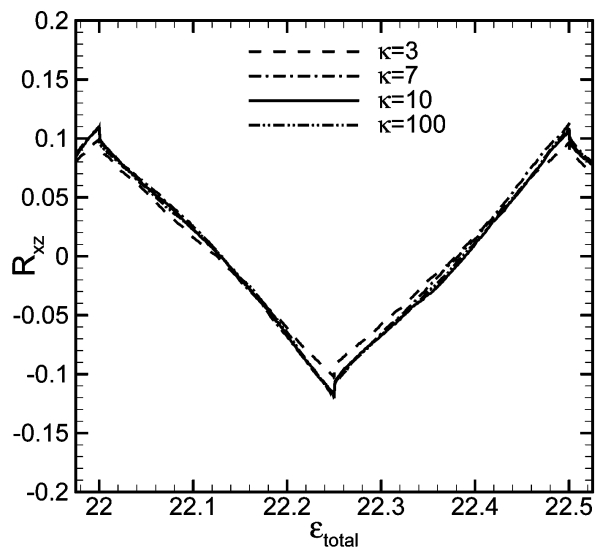

(c)

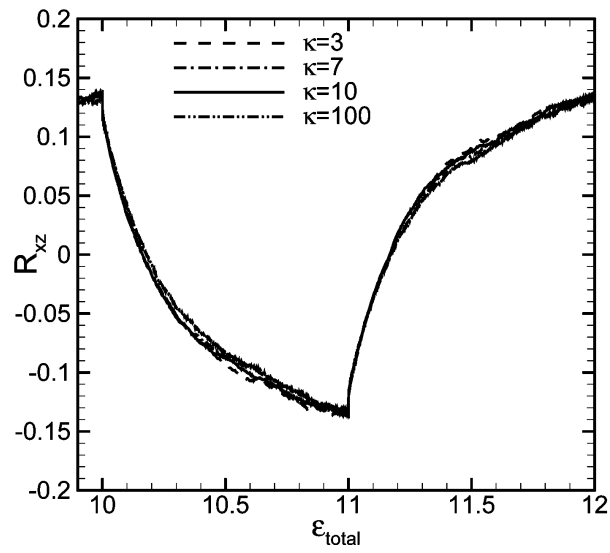

(b)

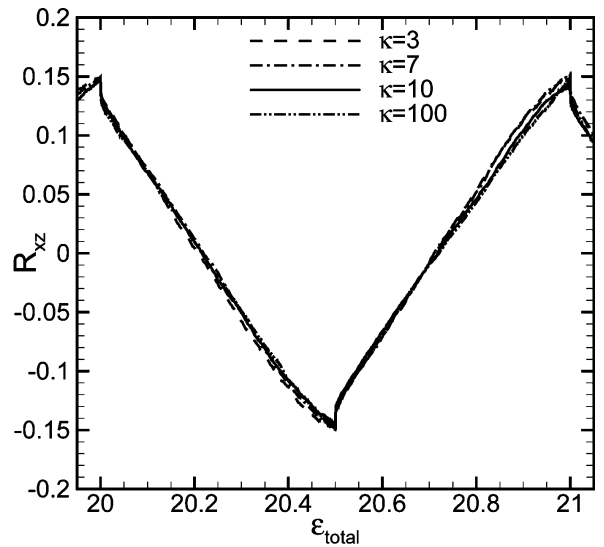

(d)

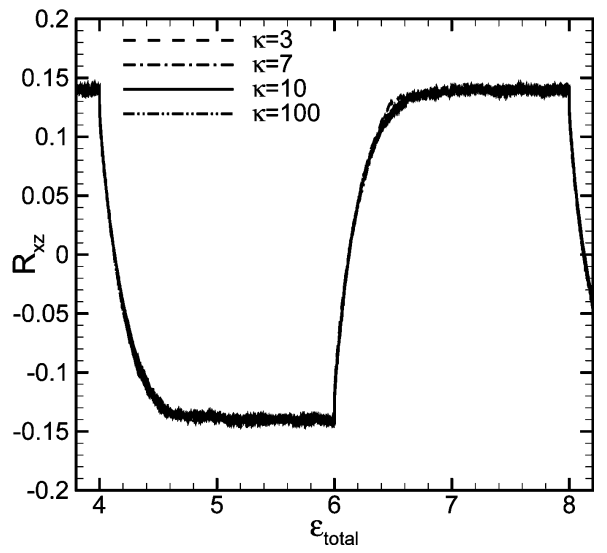

Figure 8. Strain dependence of the anisotropy for oscillatory shear with maximum strains of (a) $\varepsilon_{\max }=0.25$, (b) $\varepsilon_{\max }=0.5$, (c) $\varepsilon_{\max }=1$, and (d) $\varepsilon_{\max }=2$. Plotted is the shear component of the fabric tensor $\left(R_{x z}\right)$ versus the total strain $\left(\varepsilon_{\text {total }}\right)$. Each figure features the anisotropy evolution for four different values of the reduced minimum scaled stiffness $\left(\kappa=3,7,10\right.$, and 100). In all cases, $\phi=0.6$ and $B o^{*}=2.5 \times 10^{-5}$.
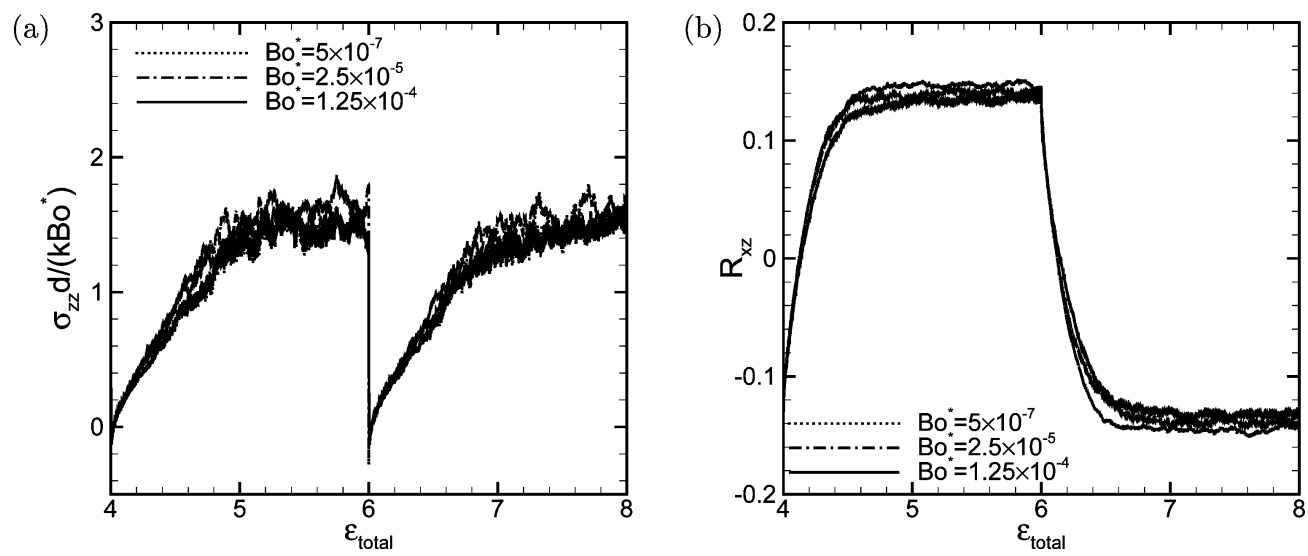

Figure 9. Effect of cohesion $\left(B o^{*}\right)$ on the behavior of the systems during oscillatory shear. Plotted are (a) the cohesively scaled normal stress $\left(\sigma_{z z} d /\left(k B o^{*}\right)\right)$ and (b) the shear component of the fabric tensor $\left(R_{x z}\right)$ versus the total strain $\left(\varepsilon_{\text {total }}\right)$ for systems with three different levels of cohesion. In all cases, $\kappa=10$, $\varepsilon_{\max }=2$, and $\phi=0.6$.

plateau values at a strain of about unity, but when $\varepsilon_{\max }=1$, such plateaus were not observed despite reaching a strain of unity. As such, the evolution of a system undergoing oscillatory shear depended on the $\varepsilon_{\max }$. To better illustrate this, Figure $10 \mathrm{a}$ shows the stress evolution of systems undergoing oscillatory shear with two different strain amplitudes but the same shearrate amplitude (since $\kappa=10$ for both curves). It can be seen that the stress evolved at different incremental stiffness $(\mathrm{d} \sigma / \mathrm{d} \varepsilon)$ for the two different values of $\varepsilon_{\max }$ shown. As the system "was not aware" of how far shear would proceed before reversing, this dependence on $\varepsilon_{\max }$ must have come from a dependence on the shear history. If such dependence exists, then systems that share the same history but have different strain amplitudes would behave the same way until shear reversal and systems with different histories and strain amplitudes would not. Figure 10a shows the results after many oscillation cycles, and so, each system had a very different history; correspondingly, the stress behaved differently (compare the two curves between $22<\varepsilon_{\text {total }}$ $<22.25$ ). Figure 10b, in turn, shows the initial normal stress evolution for the same systems (in the very first oscillation cycle), starting from the same system state. The stress evolved identically for each strain amplitude until shear reversed direction; subsequently, each system evolved at a different incremental stiffness and, after many oscillation cycles, yielded Figure 10a. This contrast indicates that the stress response depends on the previous history but not on the shear rate. The 
(a)

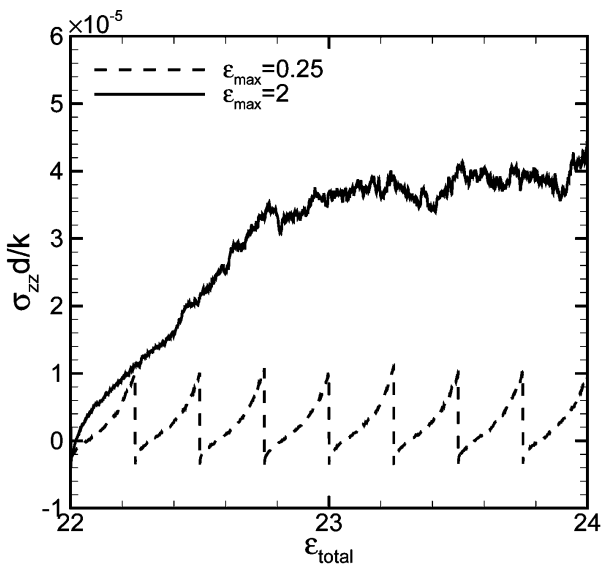

(c)

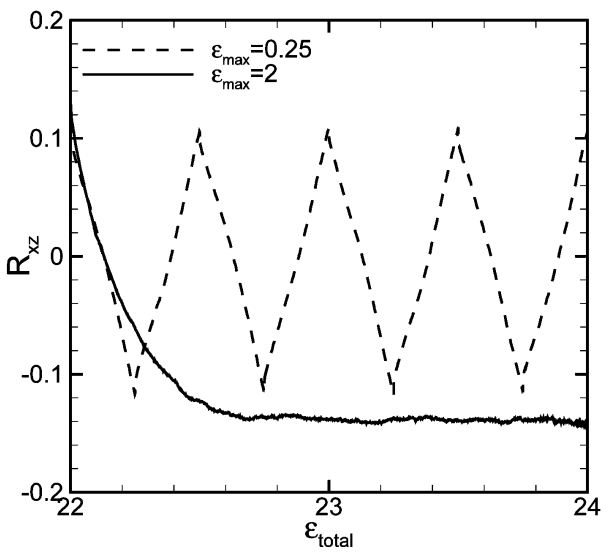

(b)

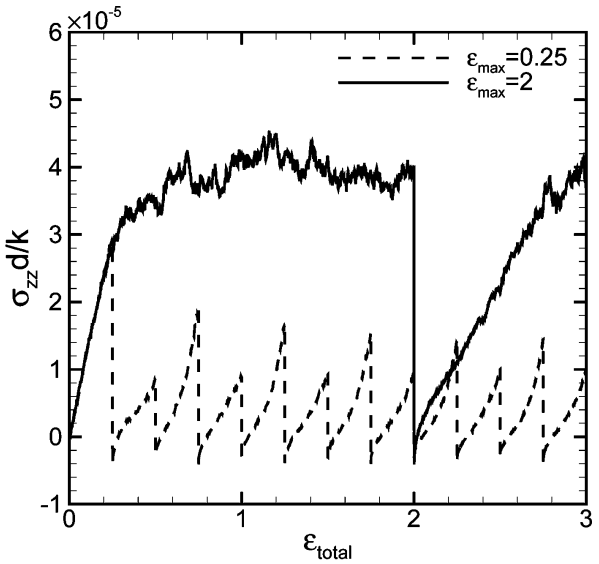

(d)

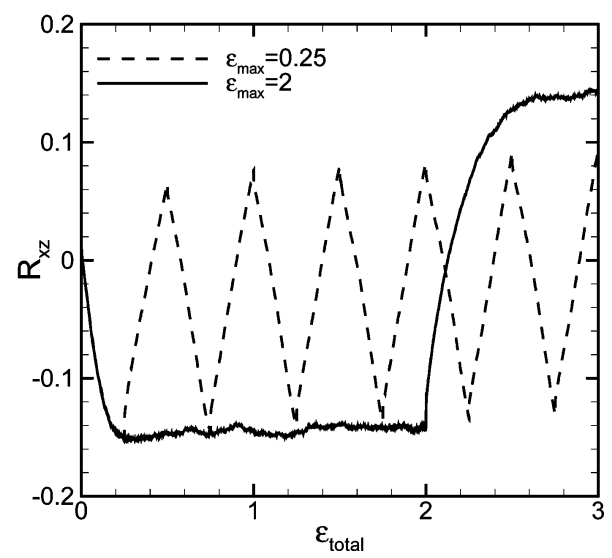

Figure 10. Effect of the strain amplitude on the evolution during oscillatory shear after shear has progressed for a long time (a and c) and during the initial oscillations (b and d). Plotted are the scaled normal stress $\left(\sigma_{z z} d / k\right)$ and shear component of the fabric tensor $\left(R_{x z}\right)$ versus the total strain $\left(\varepsilon_{\text {total }}\right)$ for two different values of maximum strain $\left(\varepsilon_{\max }=0.25\right.$ and 2$)$. In all cases, $\phi=0.6, B o^{*}=2.5 \times 10^{-5}$, and $\kappa=10$.

history effect stems from the microstructure evolution as explained next. Furthermore, whereas for a given $\varepsilon_{\max }$ each halfoscillation was virtually identical to its previous one in Figure $10 \mathrm{a}$, the same cannot be said during the initial oscillations shown in Figure 10b. Instead, when $\varepsilon_{\max }<1$, the stress alternated between evolving to a larger and smaller value every halfoscillation. Similar stress variation was also observed in the experiments of Toiya et al. ${ }^{15}$ for small shear amplitudes. After many oscillation cycles, this behavior died out. This difference at the initial stage is also evidence of the history effect.

The history dependence just described above can be explained through examination of the evolution of the microstructure. Figure $10 \mathrm{c}$ and d show the behavior of $R_{x z}$ for the same systems in Figure 10a and b, respectively. As mentioned before, when $\varepsilon_{\max }=2, R_{x z}$ reached its plateau well before shear reversed, such that the internal structure was "fully in place" and the stress was able to build up to steady state. On the other hand, when $\varepsilon_{\max }=0.25, R_{x z}$ did not get fully developed before reversal. This microstructure difference leads to the different stress response after reversal as observed. When shear was started in the first place, it was done so from a nearly isotropic configuration, i.e. $R_{x z}$ was close to zero (see Figure 10d). In contrast, as seen in Figures 10c and 8, when shear was reversed after many cycles in oscillatory shear, $R_{x z}$ was large, reflecting contact orientations corresponding to shear in the previous direction. This anisotropy difference leads to the different initial stress response (Figure 10b) from the steady state one (Figure 10a).

The behaviors observed for systems under oscillatory shear $\varepsilon_{\max }=2$ arose because steady state (in terms of stress and $R_{x z}$ ) was reached every time before shear was reversed. If that is all that mattered, then it would follow that the same behavior would be observed when the direction of shear is abruptly reversed in a simulation where the magnitude of shear is maintained constant. Note that in the oscillatory shear discussed above the shear rate changed sinusoidally; in contrast, we now hold the shear rate constant for a sufficiently long time to establish steady stress and fabric levels and then abruptly reverse the shear direction (as was done in Figure 5) after steady shear at steady state. Comparisons of the normal stress evolution after shear reversal for systems undergoing oscillatory shear with $\varepsilon_{\max }=2$ with systems reversed after a steady shear are shown in Figure 11a. (The figure display results for one-half of the oscillation cycle after several oscillation cycles to ensure that they represent long time solutions.) If the fluctuations were smoothed out, the normal stress behaved virtually identically for both types of shearing. The evolution of $R_{x z}$ for these same systems is shown in Figure 11b. It can be seen that the microstructure also behaved virtually identically for both types of shearing. So as long as steady state (plateau) is achieved before shear is reversed, it does not matter how shear is performed; a sinusoidally varying shear rate would yield the same result as a square wave oscillation, etc. This further confirms that microstructure is the only cause of the history dependence in the quasi-static (rateindependent) regime.

The evolutions of normal and shear stresses following shear reversal observed in our simulations are consistent with the data from oscillatory shear experiments of Toiya et al. ${ }^{15}$ In their study, beds of granular material were subjected to cylindrical Couette flow in which the direction of shear was repeatedly reversed. They observed that immediately after shear reversal, 
(a)

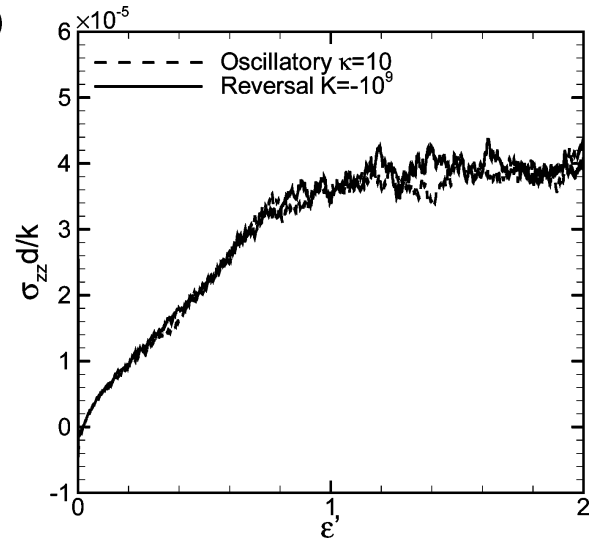

(b)

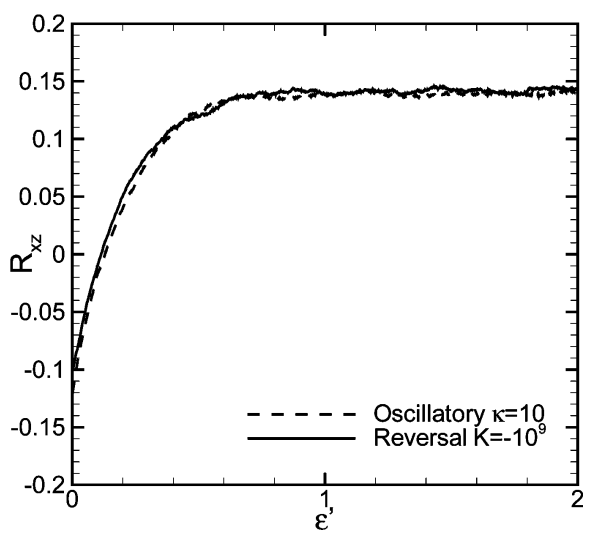

Figure 11. Comparison of evolutions resulting from reversing shear after steady state during different types of unsteady shear. Plotted are (a) the scaled normal stress $\left(\sigma_{z z} d / k\right)$ and (b) the shear component of the fabric tensor $\left(R_{x z}\right)$ versus the strain after shear is reversed $\left(\varepsilon^{\prime}\right)$ for systems undergoing oscillatory shear with a strain amplitude $\varepsilon_{\max }=2$ (designated using the $\kappa$ notation) and after steady shear (designated using the $K$ notation). All simulations were performed with $\phi=0.6$ and $B o^{*}=2.5 \times 10^{-5}$.

(a) the assembly compacted, which could happen only if the normal stress in a constant volume simulation decreased after shear reversal, (b) the magnitude of the shear stress first decreased and then recovered back to the old level, and (c) the torque achieved a steady value before the shear direction was reversed only when the maximum strain was sufficiently large.

3.4. Implications to Continuum Rheological Models. Virtually all continuum models for the rheology of dense assemblies in the quasi-static regime recognize and obey order-zero dependence on rate of deformation $(D)$. Plasticity models have been proposed and adapted for granular materials. ${ }^{22,23,44,45}$ These models satisfy such rate independence by expressing the stress as a function of $D /(D: D)^{1 / 2}$. The salient features of such plasticity models, when applied to simple shear of dense assemblies at constant volume conditions, are as follows:

(a) When an assembly under plane shear is subjected to any change in shear rate without reversing the direction of shear, the normal and shear stresses will not change; these are consistent with the asymptotic states observed in our simulations. These models miss the excursions observed in our simulations at very small strain levels; however, one may argue that these excursions are only of secondary importance and that the predictions of such order-zero, quasi-steady models as a simple first approximation.

(b) When an assembly under plane shear is subjected to shear reversal, the normal stresses will not change, while the shear stress will change sign instantaneously; these are again consistent with the asymptotic states observed in our simulations. However, our simulations indicate that all the stress components manifest significant dynamic changes following strain reversal that requires strain of order unity to stabilize.

Our simulations indicate that the fabric tensor is a natural metric of the microstructure anisotropy and that the stresses and the fabric evolve in a correlated manner. Thus, it seems reasonable to construct an evolution equation for the fabric and introduce an additional slow strain scale through it; the constitutive model for the stress is then revised to introduce the fabric tensor as additional variable. Goddard ${ }^{30,31}$ has presented such an approach to model the stress evolution in dense suspensions and that Zhu et al. ${ }^{46}$ have incorporated the effects of fabric in the dilatant double shearing model for granular materials.

\section{Summary and Conclusions}

In the present study, the responses of dense assemblies of cohesive granular materials to unsteady shear in the quasi-static regime have been investigated through DEM simulations. These simulations were performed in 3D periodic domains using Lees-Edwards boundary conditions and the SLLOD algorithm. The dynamic characteristics of the volume-averaged normal and shear stresses in materials sheared at constant volume, undergoing stop-and-go shearing and oscillatory shear, are discussed in detail. Furthermore, the evolution of microstructure anisotropy has been quantified through the shear component of the fabric tensor and related to the dynamic characteristics of the stress components.

It has been demonstrated that the evolution of the stresses and the microstructure anisotropy depended on the strain extent, but not on the shear rate. Therefore, the results in this paper confirm that cohesive granular materials can exhibit quasi-static rheological behaviors even if subjected to unsteady shear. The cohesive scaling, which collapses systems of different cohesion levels into a universal quasi-static regime under steady shear ${ }^{13}$ has been extended to and validated for the unsteady shear.

The stresses and the microstructure anisotropy undergo a transition following reversal of shear direction, which requires a shear strain of about unity to fully adapt. In contrast, they reach the previous steady state values rapidly when shear is resumed in the same direction. This characteristic strain of about unity is a robust length scale independent of shear-rate, volume fraction, and cohesion strength. These results clearly establish a correlation between the stress evolution and the microstructure anisotropy development. When shear was completely stopped, the stress experienced an almost immediate decrease, depending mostly on the stability of the microstructure beforehand, which could roughly be quantified using the average coordination number.

It is pointed out that continuum rheological models for dense assemblies of particles deforming in the quasi-static regime capture the asymptotic behavior of the stresses following dynamic changes in the rate of deformation, but not the dynamic events occurring immediately following changes. An augmented plasticity model, which introduces an additional slow strain scale via a dynamic model for the evolution of the fabric tensor, is suggested as a natural extension of the extant plasticity model to bring the model predictions closer to our simulation results.

\section{Acknowledgment}

This work was partially supported by Merck \& Co., Inc., an NSF-NIRT grant, and a DOE-UCR grant with award number DE-FG26-07NT43070. 


\section{Literature Cited}

(1) Lätzel, M.; Luding, S.; Herrmann, H. J. Macroscopic material properties from quasi-static, microscopic simulations of a two-dimensional shear-cell. Granular Matter 2000, 2 (3), 123-135.

(2) da Cruz, F.; Emam, S.; Prochnow, M.; Roux, J.-N.; Chevoir, F. Rheophysics of dense granular materials: Discrete simulation of plane shear flows. Phys. Rev. E 2005, 72 (2), 021309-17.

(3) Lois, G.; Lemaittre, A.; Carlson, J. M. Numerical tests of constitutive laws for dense granular flows. Phys. Rev. E 2005, 72 (05), 051303-051303.

(4) Silbert, L. E.; Grest, G. S.; Brewster, R.; Levine, A. J. Rheology and Contact Lifetimes in Dense Granular Flows. Phys. Rev. Lett. 2007, 99 (6), 068002-4.

(5) Rognon, P. G.; Roux, J.-N.; Naaïm, M.; Chevoir, F. Dense flows of cohesive granular materials. J. Fluid Mech. 2008, 596 (-1), 21-47.

(6) Miller, B.; O'Hern, C.; Behringer, R. P. Stress fluctuations for continuously sheared granular materials. Phys. Rev. Lett. 1996, 77 (15), 3110-3113.

(7) Bocquet, L.; Losert, W.; Schalk, D.; Lubensky, T. C.; Gollub, J. P. Granular shear flow dynamics and forces: Experiment and continuum theory. Phys. Rev. E 2001, 65 (1), 011307.

(8) Tardos, G. I.; McNamara, S.; Talu, I. Slow and intermediate flow of a frictional bulk powder in the Couette geometry. Powder Technol. 2003, 131 (1), 23-39.

(9) MiDi, G. D. R. On dense granular flows. Eur. Phys. J. E, Soft Matter 2004, 14 (4), 341-305.

(10) Majmudar, T. S.; Behringer, R. P. Contact force measurements and stress-induced anisotropy in granular materials. Nature 2005, 435 (1079), 1079-1082.

(11) Campbell, C. S. Granular shear flows at the elastic limit. J. Fluid Mech. 2002, 465, 261-291.

(12) Campbell, C. S. Stress-controlled elastic granular shear flows. J. Fluid Mech. 2005, 539, 273-297.

(13) Aarons, L.; Sundaresan, S. Shear flow of assemblies of cohesive and non-cohesive granular materials. Powder Technol. 2006, 169 (1), 10 21.

(14) Aarons, L.; Sundaresan, S. Shear flow of assemblies of cohesive granular materials under constant applied normal stress. Powder Technol. 2008, 183 (3), 340-355.

(15) Toiya, M.; Stambaugh, J.; Losert, W. Transient and Oscillatory Granular Shear Flow. Phys. Rev. Lett. 2004, 93 (8), 088001-4.

(16) Mueggenburg, N. W. Behavior of granular materials under cyclic shear. Phys. Rev. E 2005, 71 (3), 031301-10.

(17) Gadala-Maria, F.; Acrivos, A. Shear-induced structure in a concentrated suspension of solid spheres. J. Rheol. 1980, 24 (6), 799-814.

(18) Kolli, V. G.; Pollauf, E. J.; Gadala-Maria, F. Transient normal stress response in a concentrated suspension of spherical particles. J. Rheol. 2002, 46 (1), 321-334.

(19) Narumi, T.; See, H.; Honma, Y.; Hasegawa, T.; Takahashi, T.; Phan-Thien, N. Transient response of concentrated suspensions after shear reversal. J. Rheol. 2002, 46 (1), 295-305.

(20) Narumi, T.; See, H.; Suzuki, A.; Hasegawa, T. Response of concentrated suspensions under large amplitude oscillatory shear flow. J. Rheol. 2005, 49 (1), 71-85.

(21) Utter, B.; Behringer, R. P. Transients in sheared granular matter. Eur. Phys. J. E, Soft Matter 2004, 14 (4), 373-380.

(22) Savage, S. B. Granular flow down rough inclines-review and extension. In Mechanics of granular materials: new models and constitutive relations; Jenkins, J. T., Satake, M., Eds.; Elsevier Ltd: New York, 1983.

(23) Schaeffer, D. G. Instability in the evolution equations describing incompressible granular flow. J. Differential Equat. 1987, 66, 19-50.

(24) Schofield, A. a.; Peter, W. Critical State Soil Mechanics; McGrawHill Inc.: New York, 1968.
(25) Kolymbas, D. Introduction to Hypoplasticity; A. A. Balkema, Rorrerdam, 2000

(26) Bathurst, R. J.; Rothenburg, L. Observations on stress-force-fabric relationships in idealized granular materials. Mech. Mater. 1990, 9 (1), 6580 .

(27) Radjai, F.; Wolf, D. E.; Jean, M.; Moreau, J.-J. Bimodal Character of Stress Transmission in Granular Packings. Phys. Rev. Lett. 1998, 80, (1), 61-74

(28) Deboeuf, S.; Dauchot, O.; Staron, L.; Mangeney, A.; Vilotte, J.-P. Memory of the unjamming transition during cyclic tiltings of a granular pile. Phys. Rev. E 2005, 72 (5), 051305-11.

(29) Nemat-Nasser, S. A micromechanically-based constitutive model for frictional deformation of granular materials. J. Mech. Phys. Solids 2000, 48 (6-7), 1541-1563.

(30) Goddard, J. D. A dissipative anisotropic fluid model for noncolloidal particle dispersions. J. Fluid Mech. 2006, 568 (-1), 1-17.

(31) Goddard, J. D. A weakly nonlocal anisotropic fluid model for inhomogeneous Stokesian suspensions. Phys. Fluids 2008, 20 (4), 04060116.

(32) Cundall, P. A.; Strack, O. D. L. A discrete numerical model for granular assemblies. Geotechníque 1979, 29 (1), 47-65.

(33) Campbell, C. S. Granular Shear Flows at the Elastic Limit. J. Fluid Mech. 2002, 465, 261-291.

(34) Stevens, A. B.; Hrenya, C. M. Comparison of soft-sphere models to measurements of collision properties during normal impacts. Powder Technol. 2005, 154, 99-109.

(35) Vu-Quoc, L.; Zhang, X. An Accurate and Efficient Tangential Force-Displacement Model for Elastic Frictional Contact in Particle-Flow Simulations. Mech. Mater. 1999, 31 (4), 235-269.

(36) Walton, O. R.; Braun, R. L. Viscosity, Granular-Temperature, and Stress Calculations for Shearing Assemblies of Inelastic, Frictional Disks. J. Rheol. 1986, 30 (5), 949-980.

(37) Yang, R. Y.; Zou, R. P.; Yu, A. B. Computer simulation of the packing of fine particles. Phys. Rev. E 2000, 62 (3), 3900-3908.

(38) Seville, J. P. K.; Willett, C. D.; Knight, P. C. Interparticle forces in fluidisation: a review. Powder Technol. 2000, 113 (3), 261-268.

(39) Lees, A. W.; Edwards, S. F. The computer study of transport processes under extreme conditions. J. Phys. C: Solid State Phys. 1972, 5 (15), 1921-1928.

(40) Evans, D. J.; Morriss, G. P. Statistical Mechanics of Nonequilibrium Liquids; Academic Press: New York, 1990.

(41) Plimpton, S. J. Fast parallel algorithms for short-range molecular dynamics. J. Comp. Phys. 1995, 117, 1-19.

(42) Bricker, J. M.; Butler, J. E. Correlation between stresses and microstructure in concentrated suspensions of non-Brownian spheres subject to unsteady shear flows. J. Rheol. 2007, 51 (4), 735-759.

(43) Ball, R. C.; Blumenfeld, R. Stress field in granular systems: Loop forces and potential formulation. Phys. Rev. Lett. 2002, 88, 115505.

(44) Goddard, J. D. Dissipative materials as models of thixotropy and plasticity. J. Non-Newtonian Fluid Mech. 1984, 14, 141-160.

(45) Srivastava, A.; Sundaresan, S. Analysis of a frictional-kinetic model for gas-particle flow. Powder Technol. 2003, 129, 72-85.

(46) Zhu, H.; Mehrabadi, M. M.; Massoudi, M. Three-dimensional constitutive relations for granular materials based on the dilatant double shearing mechanism and the concept of fabric. Int. J. Plasticity 2006, 22 (5), 826-857.

Received for review July 26, 2009 Revised manuscript received October 17, 2009 Accepted October 20, 2009

IE901187W 


\section{Appendix $\mathbf{J}$}

\section{An investigation of frictional and collisional powder flows using a unified constitutive equation}




\title{
An investigation of frictional and collisional powder flows using a unified constitutive equation
}

\author{
M. Kheiripour Langroudi a , S. Turek ${ }^{\mathrm{b}}$, A. Ouazzi ${ }^{\mathrm{b}}$, G.I. Tardos ${ }^{\mathrm{a}, *}$ \\ a The City College of the City University of New York, USA \\ b Institute of Applied Mathematics, TU Dortmund, Germany
}

\section{A R T I C L E I N F O}

\section{Article history:}

Received 1 June 2009

Received in revised form 22 August 2009

Accepted 22 August 2009

Available online 16 September 2009

\section{Keywords:}

Powder flow

Couette geometry

Stress measurement

Numerical simulation

\begin{abstract}
A B S T R A C T
This is an experimental and numerical study of dry, frictional powder flows in the quasi-static and intermediate regimes using the geometry of the Couette device. We measure normal and shear stresses on the shearing surface and extract from the data, constitutive equations valid in the slow frictional, quasi-static and the intermediate (dense), collisional regimes of flow. This constitutive equation is then used in a new, specially developed FEM solver (FeatFlow-Ouazzi et al., 2005 [18]) to obtain solutions of the continuum equations of motion as well as stress and velocity distributions in the powder. While the measurements to obtain the constitutive equation are performed in a concentric Couette device, the numerical scheme is used to predict the torque and stresses in two additional geometries. These geometries are an eccentric Couette where the inner, rotating cylinder is placed off-center with different eccentricities and a more complicated geometry where a cylindrical body is introduced in the middle between the rotating and stationary cylinders and obstructs part of the shearing gap. The purpose of these calculations is to show the versatility of the numerical solution.
\end{abstract}

(C) 2009 Elsevier B.V. All rights reserved.

\section{Introduction}

Research in granular flows is motivated by numerous applications in industrial processes and in geophysics for the description of avalanches [6]. Powder flows have been studied extensively in the past starting with the pioneering work of Jenike [9] and Jackson [10]. The accent on this earlier work was to establish the condition under which a stressed layer of powder will break and the material will start to flow. More recent studies were dedicated to modeling powder flows using mostly numerical methods: Muguruma et al. [16], Srivastava and Sundaresan [24], Luding [13], Moreno-Atanasio et al. [15], just to mention a few. Extensive work by Savage [21] and Tardos and co-workers [26-28] looked into the slow and intermediate regimes of flow at slow and moderately high shear rates and the transition from one to another. The study of continuum models to describe powder flows was also undertaken by several authors (see for example Schaeffer [22] and Tardos [25] and references within).

The division of granular flows into slow or quasi-static and fast or rapid regimes is well documented [3]. The existence of a "transitional regime" where particle mobilization and/or shear are strong enough to dissipate significant energy through particle collisions, but do not

\footnotetext{
* Corresponding author. Gabriel I. Tardos, The City College of the City University of New York (CUNY), Department of Chemical Engineering, 160 Convent Ave., New York, NY 10031, USA. Tel.: +1 212650 6665, fax: +1 2126506672.

E-mail addresses: mkheiripour@gc.cuny.edu (M. Kheiripour), ture@featflow.de (S. Turek), ouazzi@math.uni-dortmund.de (A. Ouazzi), Tardos@CCNY.CUNY.Edu (G.I. Tardos).
}

completely eliminate the continuous particle enduring contacts is more recent $[7,27]$. It was also shown theoretically that in order to change the regime of flow from quasi-static (dense, slow flow) to rapid granular (dilute and collisional), the solid fraction of the bed has to decrease [12] but no direct experimental evidence is available.

The basic motivation for our work is to supply relevant experiments that allow the bed to dilate freely as shearing is increased and for inter-particle collisions to occur in a dense bed where particleparticle friction is also prevalent. We achieve these conditions in a Couette geometry where the inner cylinder is rotating and the outer cylinder is stationary. We over-impose a very slow axial flow over the radial shearing of the powder thereby assuring a slight increase in the solid fraction of the bed. This procedure also ensures that the particle bed reaches a steady state in which the solid fraction is somewhat lower than that corresponding to maximum packing and hence collisions and relative movement between particles can take place.

There is theoretical evidence $[5,11]$ that in the intermediate regime, where the flow is fast enough but still dense, the friction coefficient (the ratio of shear to normal stress) increases from its constant value. While friction during very slow motion is only a function of the character of the surface and the normal load, at higher shearing rates, it becomes a function of the rate itself and increases as the rate of shearing increases. We provide experimental evidence that such an increase is real and significant and that the dependence on shear rate takes, in its simplest expression, the form of a power-law.

Furthermore, we use the experimentally measured friction coefficient as a function of shear rate to establish a yield condition and a constitutive equation that is valid in the slow, quasi-static and 
the intermediate regime. To extend the results from simple shear in the Couette device to more complicated geometries, we use a newly developed numerical scheme (FeatFlow) to solve the general equations of motion of an incompressible medium with the new constitutive equation. We show that the constitutive equation and the numerical solver are general enough to generate useful information for more complicated geometries such as an eccentric Couette and a concentric Couette with a cylindrical obstacle in the shearing gap.

\section{Experimental approach}

In the present study we explore experimentally and numerically the transition of powder flows from slow to moderately fast by using a Couette device.

\subsection{Batch and continuous-flow Couette device}

A schematic representation of the Couette device used during the present investigation is shown in Fig. 1 with details of the sensors and the rotating cylinder given on the RHS of the figure. The vertical shear gap forms between the rotating and the stationary cylinders and both its width and height can be adjusted by appropriate choice of the radius and height of the rotating cylinder. The material is fed from above using a vibratory feeder (not shown in the figure) and is discharged by a screw-in-cylinder metering device (also not shown) that transfers the material to a precision balance for flow rate measurement. The walls of the Couette are made rough by gluing sand paper on the shearing surfaces as shown in the figure. The roughness of the walls is chosen to match or exceed the coefficient of internal friction of the material thereby trying to assure a non-slip boundary condition. By closing the discharge section, the Couette device can be operated in batch mode or, by feeding and removing material to achieve a steady state vertical flow, one can operate the device continuously. The material above the rotating cylinder (denoted over- burden in the figure) is stationary and only provides dead weight to the shearing layer.

Experiments in the Couette device were performed without (batch) and with axial flow (continuous), and several depths of overburdens to control the pressure in the shearing gap. Normal stresses were measured on both the outer, stationary wall as well as the inner, rotating (shearing) wall of the device as shown in the figure. Shear stresses were measured indirectly and recalculated from the torque on the rotating cylinder.

A typical batch, filling curve of the Couette is shown in Fig. 2 for $1 \mathrm{~mm}$ in diameter round glass beads with a cylinder height of $20 \mathrm{in}$. and diameter of $1.5 \mathrm{in}$., in a housing of $6 \mathrm{in}$. in diameter (with a gap of 2.25 in.). We distinguish between the increasing portion of the torque as a function of height (LHS of the figure) and the torque due to packing of the material at constant height and increasing time (RHS of the figure). First, we concentrate on the LHS region where the torque is height dependent as the Couette is filled with particles and fit the equation of the torque, $T$ to the measured data as was done previously by Tardos et al. [26]:

$T=(\tau R) 2 \pi R h=\pi \rho_{\mathrm{B}} g h^{2} R^{2} \sin (\phi)$

where $\tau=p \sin \phi$ is the shear stress and $p$ is the average normal stress given by $p=\rho_{\mathrm{B}} g h / 2$. Other notations in Eq. (1) are: $\rho_{\mathrm{B}}$, the bulk density; $g$, the acceleration of gravity; $h$, the height of material in the vertical direction (function of filling time, see Fig. 1b); $R$, the cylinder radius; and $\phi$, the angle of internal friction of the material. The implication of Eq. (1) is that the shear stress $\tau$ is linearly dependent on the height $h$ so that when the torque is calculated the height appears to the second power. In addition, the theoretical line fits the experimental results quite well suggesting that the shear stress is indeed a linear function of height as long as the material is continuously fed to the system. These general characteristics of the device were already established in our earlier work mentioned above.

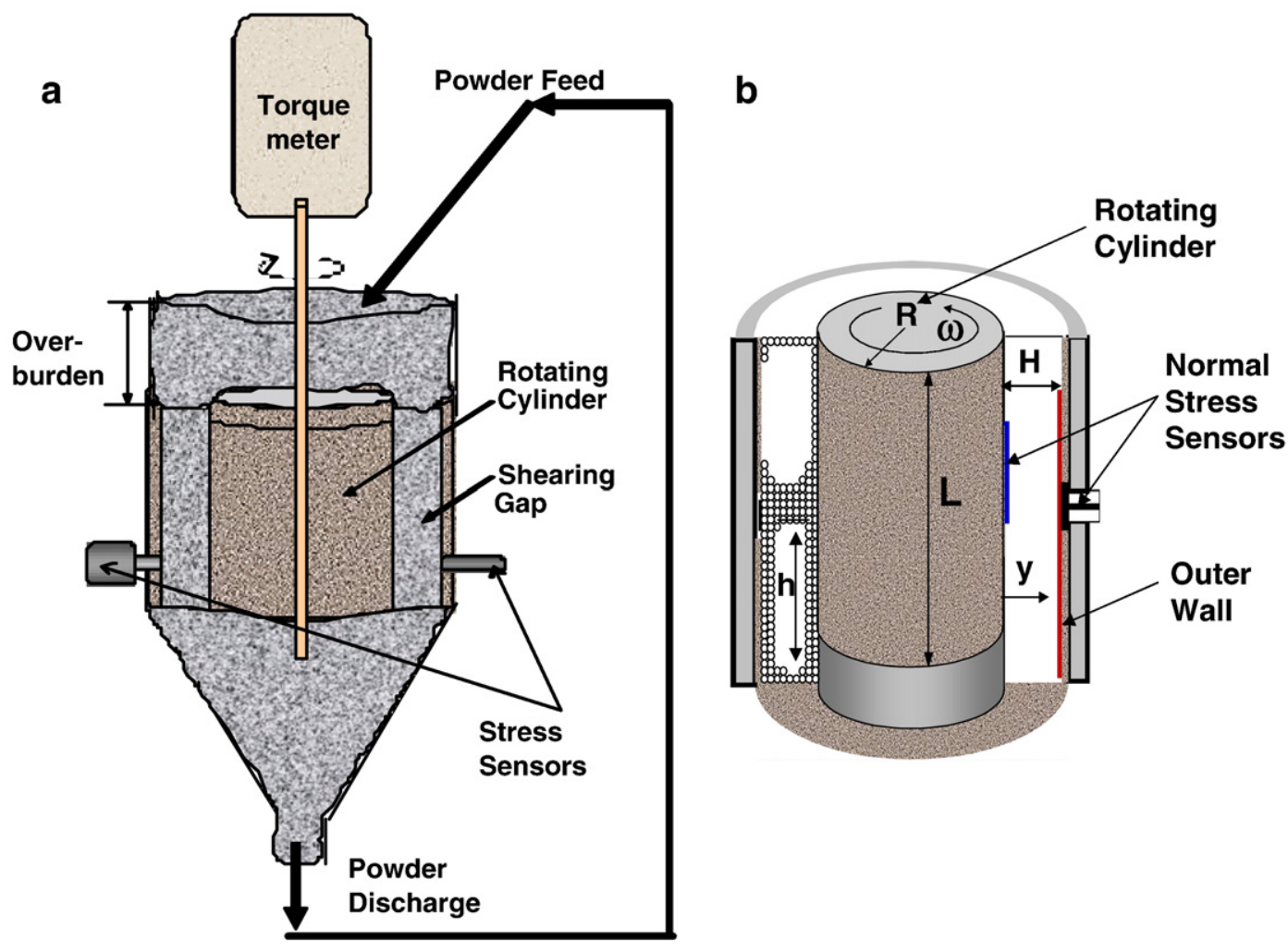

Fig. 1. (a) Schematic representation of the axial-flow Couette device. (b) Detail of the shear gap. 


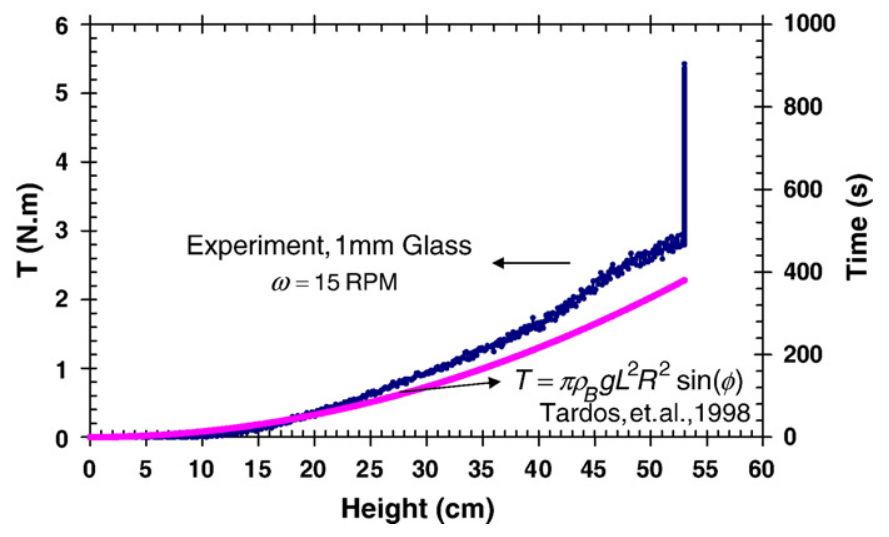

Fig. 2. Filling curve: torque vs. height for $1 \mathrm{~mm}$ glass beads.

We now concentrate on the second part of the curve where the torque increases due to packing of the material at constant height. Additional experiments were performed with a larger inner cylinder of $4 \mathrm{in}$. in diameter so that the shearing gap was approximately $1 \mathrm{in}$. and three different curves of the same kind are reproduced in Fig. 3a for small $(0.1 \mathrm{~mm})$, medium $(0.5 \mathrm{~mm})$ and large $(1.0 \mathrm{~mm})$ diameter glass particles. One can easily see the different rates at which these particles pack at a constant shearing rate (15 RPM of the rotating cylinder): larger particles pack at a much higher rate due to their enhanced capability of bridging the relatively small shear gap. It

a

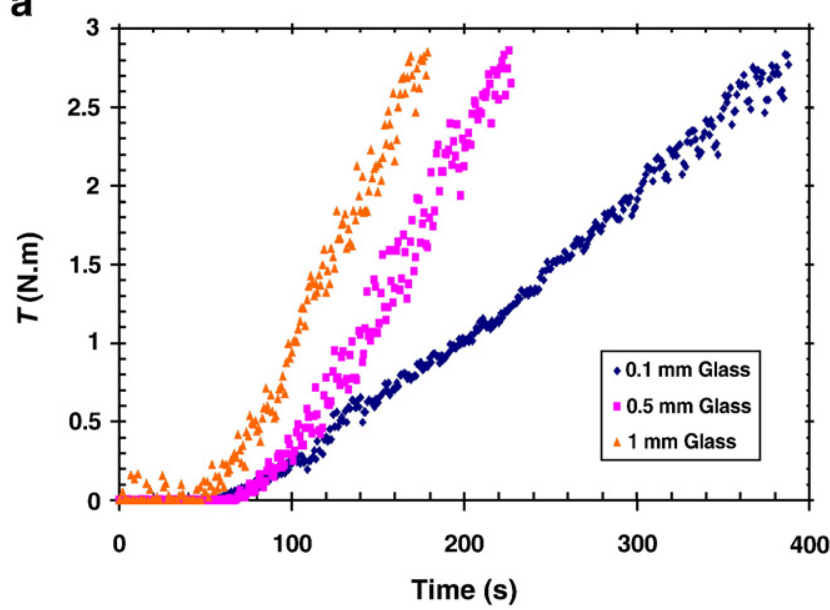

b

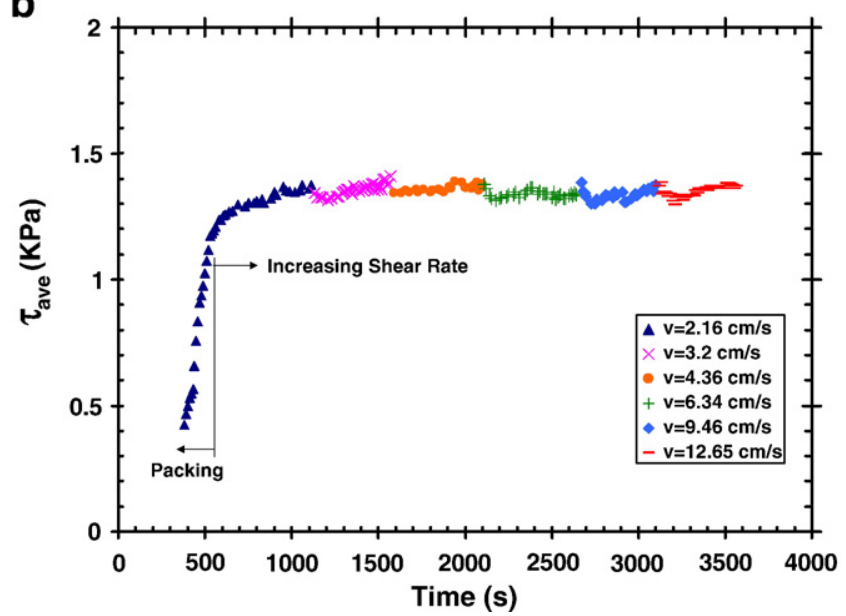

Fig. 3. Batch Couette experiments: (a) torque vs. time for different particles; (b) shear stress vs. time and rotational speed. appears from Fig. 3a that the trends shown would continue but in reality, the range of the torque-meter is exceeded and the experiment has to be stopped.

Fig. $3 \mathrm{~b}$ depicts similar behavior for the $0.1 \mathrm{~mm}$ in diameter glass beads but for a much wider shear gap ( $2.25 \mathrm{in}$.). Here, we calculated the average shear stress using the equation

$\tau_{\text {ave }}=T / 2 \pi R^{2} L$

and the linear speed is calculated from the angular velocity of the cylinder. As seen in the figure, the torque increases significantly during the packing phase but does not exceed the range of the torquemeasuring device. This is mainly due to the shorter cylinder used and by the relatively large shear gap that allows some additional movement of the granular layer in the radial direction. It appears from the figure that the torque has reached its steady state value at the lowest shear rate (blue curve) and we use this experiment to show how the shear stress depends on shear rate when the Couette is operated in batch mode (without vertical flow) at constant height. As seen in Fig. 3b, increasing the shear rate (angular velocity of the inner cylinder) more than five-fold does not result in any significant change in the shear stress: the material is in the frictional, quasi-static regime and the average shear stress is practically independent of shearing rate.

We reproduced in Fig. 4 a similar experiment to that shown in Fig. 3b but allowing the material to flow at a small rate vertically in the shear gap. The torque in this case decreases significantly due to a minute change in solid fraction (porosity) that allows chains of particles to break as the inner cylinder rotates. Using Eq. (2), the average shear stress can be calculated as a function of shear rate as shown in Fig. 4. As seen, the shear stress exhibits two very distinct regimes: a quasi-static regime where the shear stress is mostly independent of the shear rate (at low shear rates) and an additional regime ("intermediate" as denoted in the figure) where the dependence takes the form of an increasing function. We assume that the behavior resembles a power-law with an index $n=0.5-1$. In fact, the power-law index appears to be variable, increasing at higher shear rates. One would assume that continuing to increase the shear rate would yield even higher values of the index $n$ as the flow approaches the inertial regime (where the coefficient should be $n=2$ ) but such experiments are outside the capability of our Couette device since centrifugal forces take over and the material is pushed radially outward and a slip condition sets in near the rotating cylinder.

The purpose of these experiments was to show the significant difference in behavior of the shearing layer in the batch, no-flow and the continuous-flow device. While in the batch mode, the material continues to pack increasing its solid fraction as a function of time, in the flowing system a steady state sets in at an approximately constant

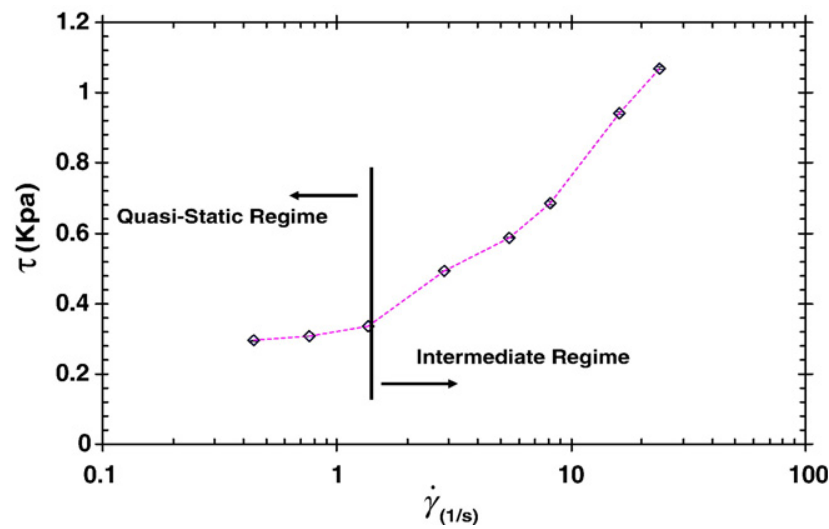

Fig. 4. Axial-flow experiment in the Couette device: spherical glass beads, $0.1 \mathrm{~mm}$ in diameter. 
solid fraction. This solid fraction is below a certain critical value, somewhat characteristic for each powder, where the material can "dilate" sufficiently and "flow" under shear. In this expanded, mobilized (sometimes also called "fluidized", even though there is no gas present to actually fluidize the powder) condition, the powder can transit from the quasi-static to the intermediate regime as the shearing rate is increased. We showed in separate experiments that the influence of the vertical flow rate is negligible if it can be kept small, just enough to prevent packing.

The remainder of the paper is concerned with this second case where there is a minute flow of material in the vertical direction and where a quasi-static, shear-rate-independent and an intermediate, dense-phase, shear-rate-dependent shear stress (torque) develops. The main goal of the experiments is to measure the shear and normal stresses on the shearing wall, their location and their ratio. We further propose a yield condition and a constitutive equation (closure) derived entirely from experimental measurements and develop a new numerical scheme to solve the momentum equations with the newly proposed closure, to cover both regimes of flow.

\subsection{Stress measurements in the continuous-flow Couette device}

Experiments were performed to show the dependence of the shear and normal stresses on the shear rate. We use glass beads and chips of different sizes and shapes as test materials.

\subsubsection{Average shear stress measurements and their location}

Since there are no direct shear stress measuring sensors commercially available that can be employed in a dense granular bed, the shear stress on the rotating cylinder was measured indirectly using the torque on the shaft exerted by the granular medium on the entire length, $L$ of the cylinder as in Eq. (2). To explore the dependence of the shear stress on the depth of the granular layer, we employed cylinders of different lengths, $L=4,8,12$ and 16 in. (and diameter of 4 in.) and calculated the average shear stress in each case. The experiments were performed with no overburden (material on top of the rotating cylinder, see Fig. 1) using $0.5 \mathrm{~mm}$ in diameter glass beads. Results are given in Fig. 5 where the average shear stress is given as a function of cylinder height, $L$ for different shear rates by rotating the inner cylinder from 5 to 45 RPM. As seen, the shear stress is approximately linear as a function of height and depends strongly on shear rate as already shown above.

Another interesting observation is that, by forcing the trend of the experimental measurements to go to zero stress at zero height, data from the shortest cylinder ( $L=4$ in. in length) fall above the line at a somewhat higher shear stress. This behavior is due to friction between the lower cover of the cylinder and the granular material (in addition to friction in the shearing layer between the cylinders)

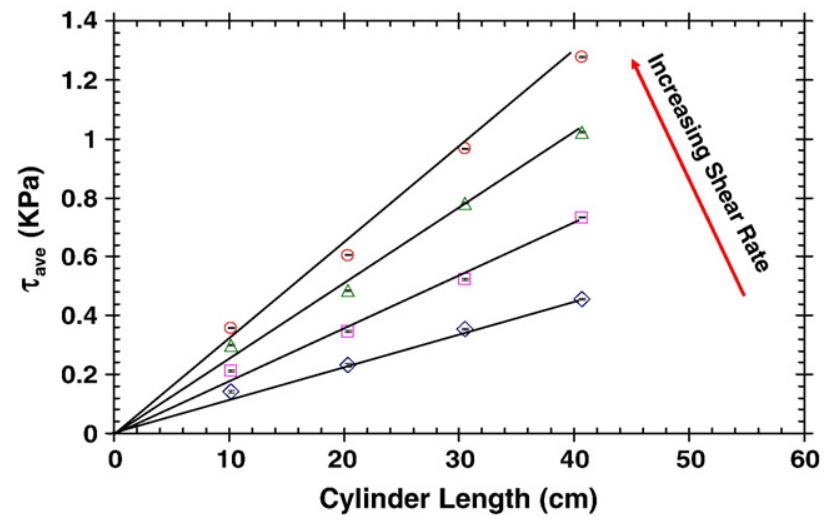

Fig. 5. Average shear stress measurements in continuous Couette device using different overall lengths, $L$, cylinders at different shear rates. which is not a linear function of height; this result was already demonstrated by DaCruz et al. [5]. When the length, $L$ (height) of the shearing layer is small, the influence of this portion of the cylinder is significant and hence the torque is higher. At larger length (height), the influence of the bottom cover becomes less significant and the dependence is almost perfectly linear. The main conclusion from this measurement is that using cylinders longer (taller) than about 8 in. will mostly eliminate the influence of the end effect (lower cover) and that the value of the average shear stress will most likely occur at the middle point over the length, $L$.

\subsubsection{Normal stress measurements}

Fig. 6 shows a schematic representation and a picture of the instrumentation to measure normal stresses inside the bulk material on the rotating cylinder at three depths $\left(l_{1}, l_{2}\right.$ and $\left.l_{3}\right)$. The stress sensor is commercially available from Tekscan, Inc. (Boston, Ma) and is made of a circular sensitive element $1.1 \mathrm{~cm}$ in diameter glued to the surface of the rotating cylinder and an electronic circuit situated inside the blue box in the picture. Since the cylinder is rotating inside the granular layer, the signal cannot be hard-wired but is instead transmitted by radio to a receiver connected directly to the data acquisition system. To our knowledge, this is the first time that such a measurement was undertaken on a shearing surface inside a granular medium. The cylinder used is $4 \mathrm{in}$. in diameter (this dimension is imposed by the size of the radio transmitter) and $20 \mathrm{in.} \mathrm{long} \mathrm{(to}$ accommodate the three radio transmitters). The outside diameter of the stationary cylinder that forms the Couette device (not shown in the figure) is $6 \mathrm{in}$. in diameter and thus defines a granular layer of 1 in. in thickness that surrounds the sensors.

These particular experiments were performed with an overburden of approximately $4 \mathrm{in}$. so that the depth of the three sensors was $l_{1}-l_{3}$ plus the overburden as shown in Fig. 7. Different rotation rates from 5 to $60 \mathrm{RPM}$ and glass beads of $0.5 \mathrm{~mm}$ in diameter were used. The experiment was performed with a very small vertical flow rate; this results in a very slow axial velocity in the Couette gap of less than $0.05 \mathrm{~mm} / \mathrm{s}$ and does not influence significantly the flow and stresses in the radial direction.

Fig. 7 depicts the results of the stress measurements as a function of depth. The distribution appears to be linear with the error bars representing the fluctuation of stress and the dependence on the applied shear. As seen, the normal stress in the sheared layer is not shear-ratedependent even at the highest shear rate (at 60 RPM), within the error of the measurement and the presence of fluctuations. This allows the calculation of an average normal stress on the rotating cylinder that appears, according to these results, to reside in the middle of the cylinder $(L / 2)$. This is an important conclusion since it shows that both the average shear and normal stresses are practically located around the midpoint of the rotating cylinder at least under the experimental conditions of this experiment. In subsequent experiments only one normal sensor, situated at the midpoint on the cylinder, was used.

\subsubsection{Ratio of shear to normal stresses in the Couette device}

The most interesting result from the above measurements is that one can calculate the ratio of the average shear to normal stresses as a function of shear rate. A typical result is shown in Fig. 8 where the average shear stress and local normal stress measured in the middle of the rotating cylinder $(L / 2)$ are shown as a function of shear rate. The particles used are $1 \mathrm{~mm}$ in diameter spherical glass beads. The shear stress has the overall behavior already described in Fig. 4 as the stress is practically constant at low shear rates and increases significantly as soon as the material reaches the intermediate regime of flow. The normal stress shows a very interesting behavior: it is constant and lower at low shear rates (where the shear stress is also constant) and then increases abruptly and remains practically constant thereafter even though the shear stress increases continuously. 


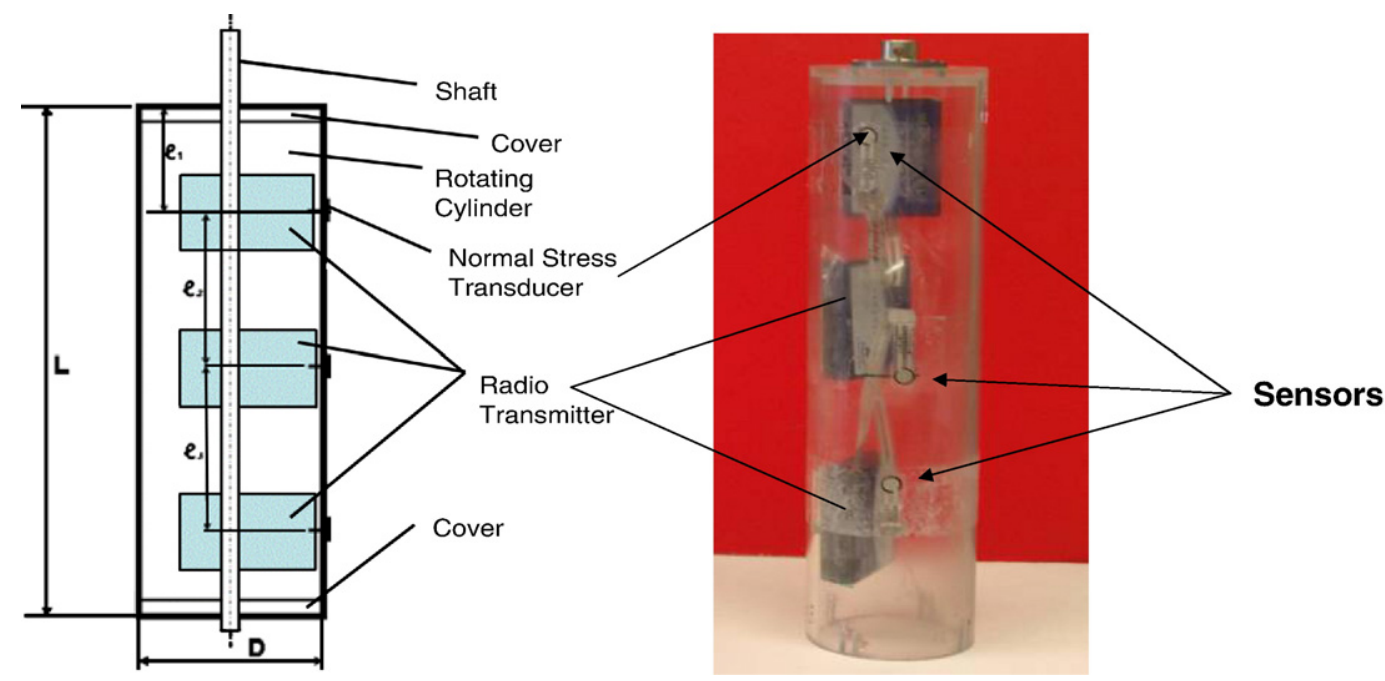

Fig. 6. Schematic and picture of the Couette device with three remote normal stress sensors.

The ratio of the two stresses (apparent friction coefficient) is given in Fig. 9 and, as seen, the ratio exhibits a similarly interesting behavior: at very low shear rates, the ratio is constant and only slightly lower than the tangent of the friction coefficient of glass particles (about 0.5) as one would expect from quasi-static flow theory. As the dimensionless shear rate increases beyond a certain critical value (in this case about $1.0 \mathrm{~s}^{-1}$ ), the ratio of shear stress vs. normal stress increases significantly. This behavior is mainly due to the superposition of collisions between particles on the sliding friction of surfaces so that the overall shear stress and "friction coefficient" also increase. This kind of behavior was already suggested by simulations [4,5] but never actually measured experimentally before. It also shows that the material moves from the quasi-static regime to the intermediate regime of flow where collisions become more important and actually carry some load. A power-law type curve is fitted to the data: it shows a power-law coefficient of $n=0.72$ and a constant coefficient $a=0.39$ for zero shear rate. In the same figure we show for comparison an equivalent correlation for crushed, odd-shaped glass particles of nominal size of $1 \mathrm{~mm}$. As seen, the power-law index is somewhat higher, as expected from considerations of collisional impacts between non-spherical particles $(n=1.03)$ and a higher constant coefficient $(a=0.48)$ due to the higher internal angle of friction.

We conclude from this work that it is possible to measure normal stresses on the sharing wall of the Couette device inside the granular layer and calculate the ratio of the average shear to normal stress as a function of shear rate. It appears, as suggested by previous theoretical simulations, that the dynamic angle of friction is reproduced by this

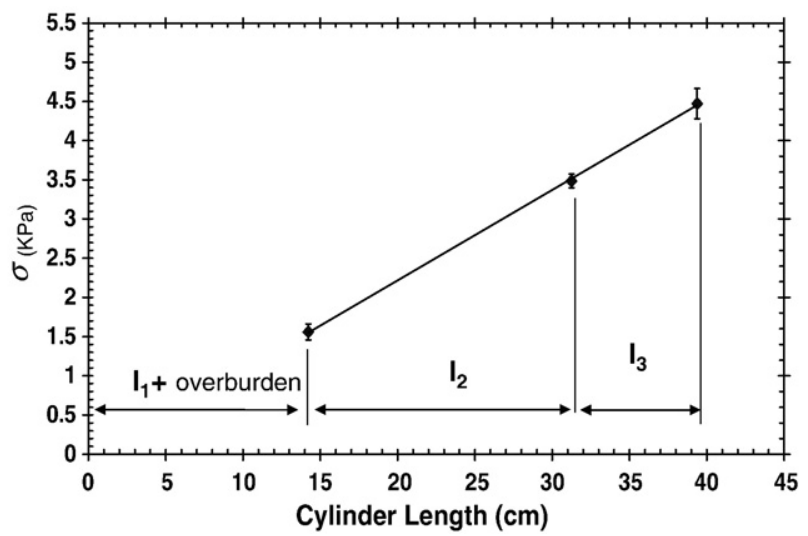

Fig. 7. Normal stress as a function of height as measured on the shearing cylinder surface by remote sensing. ratio only at very low shear rates. As the shearing rate increases, the ratio of the stresses also increases due to collisions between particles that sustain loads in addition to dry friction that is prevalent at low shear rates. Our experiment could not probe the higher limit of the "friction coefficient" in the rapid granular flow regime (when only collisions are important) because of equipment limitations and the inherent presence of gravity that makes at least some enduring contacts between particles unavoidable.

\subsection{Proposed yield condition and constitutive equation}

We try to generalize the above findings for the case when the powder transitions from the quasi-static to the intermediate regime of flow and propose a yield condition that holds at low and higher shear rates. We show below how the experimental yield condition obtained from experiments in the Couette device and given in Fig. 9 can be inserted into the Schaeffer constitutive law [22]. From Fig. 9, one can fit the following equation to the data:

$\frac{\tau}{\sigma}=a+b|\dot{\gamma}|^{n}$

where $\tau$ and $\sigma$ are the shear and normal stresses and $a, b$ and $n$ are coefficients characterized by the experimental curve. We use the notation for the modulus of the shear rate $|\dot{\gamma}|$ to designate that only its magnitude is of relevance and not its direction. The experimental value of the coefficient $a$ falls in the majority of cases between $\sin \phi$

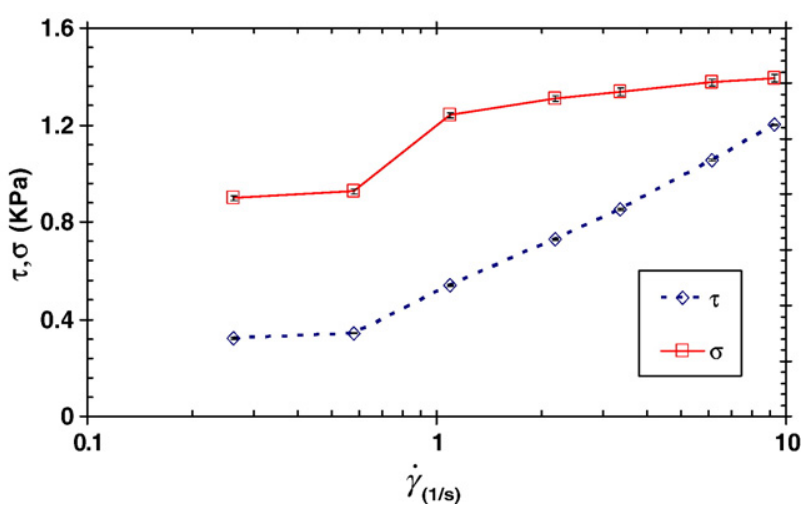

Fig. 8. Shear and normal stress variations as a function of shearing rate in the Couette device; $1 \mathrm{~mm}$ in diameter glass particles. 


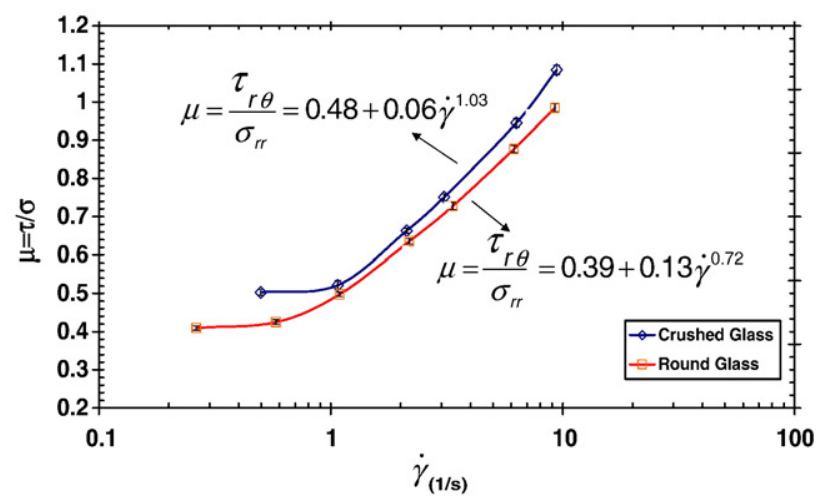

Fig. 9. Measured ratio of shear to normal stresses (apparent friction coefficient) as a function of shearing rate; $1 \mathrm{~mm}$ in diameter, round and crushed (odd-shaped) glass. Fitted curve also shown in the figure.

and $\tan \phi$ and we make here a slight generalization by replacing it by $\tan \phi$ to obtain:

$\frac{\tau}{\sigma}=\tan (\phi)+b|\dot{\gamma}|^{n}$

This equation has the great advantage that it reduces to the Coulomb yield condition at zero shearing.

An equivalent representation of the Coulomb yield condition $\tau=\sigma \tan \phi+c$ can be obtained from the characteristic Mohr circle, by replacing $\sigma$ by $p$ and $\tau / \tan \phi$ by $q / \sin \phi$, in the form [17]:

$q=p \sin \phi+c \cos \phi$

where $q$ is half of the difference between the principal stresses and $c$ is referred to as "cohesion".

The deviatoric part of the constitutive equation for flow of a dry powder in the quasi-static regime [22] is

$\tau_{i j}=\frac{\sqrt{2} p \sin \phi}{|\dot{\gamma}|} \dot{\gamma}=\frac{\sqrt{2} q}{\sqrt{z}} \dot{\gamma}$

where $\dot{\gamma}=\frac{1}{2}\left(\nabla u+(\nabla u)^{T}\right)$ is the rate of deformation tensor, $|\dot{\gamma}|$ is its magnitude and $z$ is twice the second invariant defined as $z=2 \gamma_{\mathrm{II}}=\dot{\gamma}$ : $\dot{\gamma}=|\dot{\gamma}|^{2}=\operatorname{tr}\left[(\dot{\gamma})^{2}\right]$. One recognizes the Coulomb yield condition (Eq. (5)) (with $c=0$ ) in the numerator of Eq. (6). We replace $q$ from Eq. (5) in Eq. (6) with $c=b|\dot{\gamma}|^{n}$, to obtain

$\tau_{i j}=\sqrt{2} p\left\{\sin \varphi+b \cos \phi|\dot{\gamma}|^{n}\right\} \frac{\dot{\gamma}}{|\dot{\gamma}|}$

or in terms of the variable $z$ :

$\tau_{i j}=\sqrt{2} p\left(\sin \phi z^{-\frac{1}{2}}+b \cos \phi z^{\frac{n-1}{2}}\right) \dot{\gamma}$

This is the constitutive equation that includes the behavior at very low (quasi-static) and higher shear rates characteristic of the "intermediate" regime of powder flow. The first term on the right hand side of Eq. (7) corresponds to the plastic deformation (frictional or solid-like behavior) while the second term corresponds to the viscous behavior (liquid-like) of the granular material. One has to note that the deviatoric part of the stress, Eq. (7) does not go monotonically to zero as the shear diminishes because the magnitude of the shear appears in the denominator. There is a physical and mathematical discontinuity at this point that, in practice, results in the formation of narrow shear bands and that has to be overcome by the numerical method (see next section and Ouazzi et al. [18]).
From a practical point of view, a modified Couette device with slow axial flow superimposed on the shearing motion, in which the materials can freely dilate and collide, can be used to determine the values of $b$ and $n$ for any material that is somewhat free flowing. These values can then be used in Eq. (7).

\section{Continuum theoretical approach}

The purpose of the simulations reproduced below was to predict theoretically, experimental results obtained in this work using the simple geometry of the concentric Couette device. Based on these calculations we then predict results for more complex geometries, namely an eccentric Couette and one that has a cylindrical obstacle in the shearing zone.

\subsection{Equations of motion}

The underlying simulations were performed using a new numerical technique developed specifically to treat flow problems with general constitutive laws for non-Newtonian fluids (see Ouazzi et al. [18]). The powder is assumed to be an incompressible continuum that obeys conservation of mass:

$\frac{D \rho}{D t}=\frac{\partial \rho}{\partial t}+\nabla \cdot(\rho u)=0$

where $D^{*} / D t$ is the material derivative and $u$ is the velocity vector. Since we assume that the bulk density $\rho$ is a constant, the continuity equation reduces to the divergence of the velocity $\nabla \cdot u=0$. The powder also obeys a conservation of momentum equation:

$\rho \frac{D u}{D t}=-\nabla \cdot T+\rho g$

where the stress tensor is given by $T_{i j}=\tau_{i j}-p I$ and where $I$ is the unit tensor. To complete the problem, a closure is required in the form of a constitutive equation that correlates the deviatoric part of the stress tensor $\tau_{i j}$ with the velocity.

The above problem can be similarly formulated in the framework of the generalized incompressible Navier-Stokes equations valid for non-Newtonian fluids and powders:

$\rho \frac{D u}{D t}=-\nabla p+\nabla \cdot\left(\mu\left(\gamma_{I I}, p\right) \dot{\gamma}\right)+\rho g, \quad \nabla \cdot u=0$

where we take $\tau_{i j}=\mu\left(\gamma_{\mathrm{II}}, p\right) \dot{\gamma}$. To complete the problem, the nonlinear pseudo-viscosity $\mu\left(\gamma_{\Pi \pi}, p\right)$ is defined as a function of the second invariant of the rate of deformation, or the variable $z$, and the normal stress $p$ :

1. Newtonian fluid, $\mu(z, p)=2 \mu_{0}$, where $\mu_{0}$ is the fluid viscosity,

$(11-1)$

2. Bingham solid $\mu(z, p)=2 \mu_{0}+\sqrt{2} \tau_{0} z^{-\frac{1}{2}}$, where $\tau_{0}$ is the yield strength,

3. Powder in the quasi - static regime $\mu(z, p)=\sqrt{2} p \sin \phi z^{-\frac{1}{2}}, \quad(11-3)$

4. Power - law fluid $\mu(z, p)=2 \mu_{0} z^{\frac{n-1}{2}}$, where $n$ is the power - law index, $(11-4)$

5. Powder flow in the quasi - static and intermediate regimes of flow (fromEq. (7)) $\mu(z, p)=\sqrt{2} p\left[\sin \phi z^{-\frac{1}{2}}+b \cos \phi z^{\frac{n-1}{2}}\right]$.

$(11-5)$

A comparison of Eq. (10) with the classical Navier-Stokes equations (obtained by using Eq. (11-1)) reveals that the ordinary viscous 


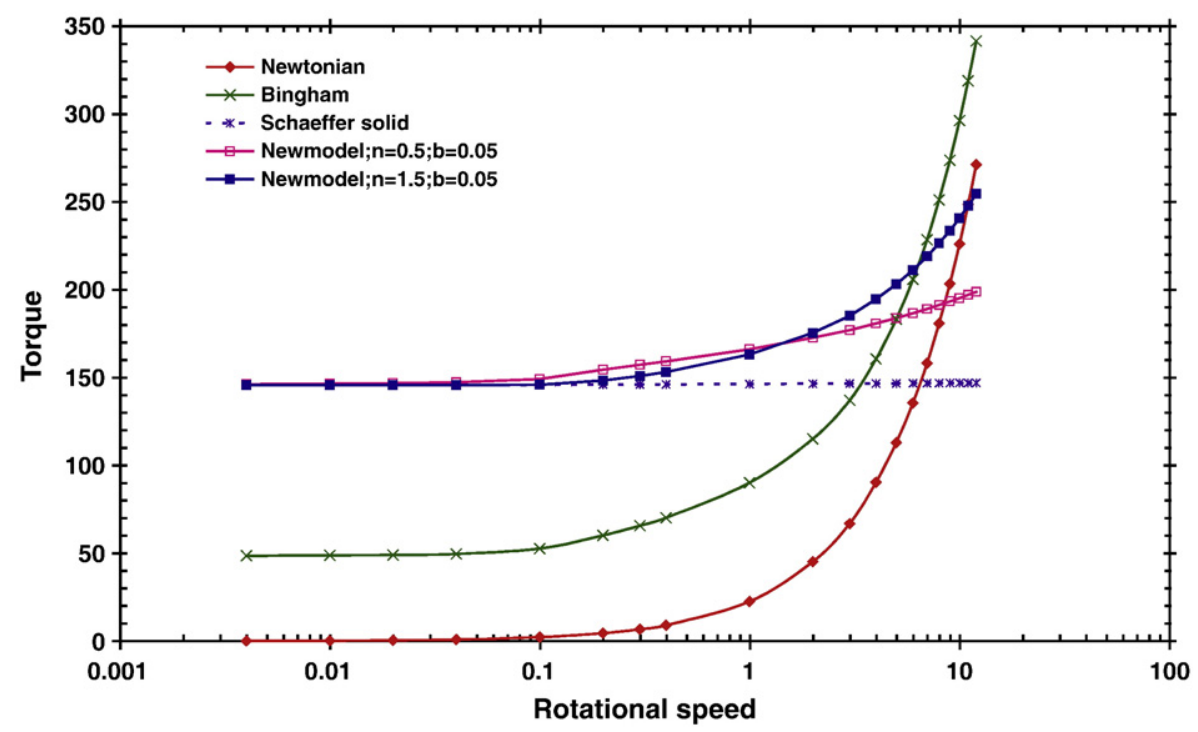

Fig. 10. Numerical solution of the generalized Navier-Stokes equations for concentric cylinders for (a) Newtonian fluid, (b) Bingham plastic, (c) powder in the quasi-static regime (Schaeffer solid), and (d) powder in the intermediate regime (new constitutive equation, Eq. (7)).

terms (proportional to the viscosity $\mu_{0}$ ) have been replaced by shearrate-independent terms (Eqs. $(11-2)$ to $(11-5)$ ) that contain the magnitude of the shearing rate $(1 / \sqrt{z})$ in the denominator. This means that these equations are mathematically more complex than the Navier-Stokes equations and apply only when the material is deforming everywhere.

The main mathematical problems of the generalized incompressible continuum material model (Eq. (10)) can be summarized as follows:

- Mathematical analysis: There is a lack of research concerning the existence of solutions for the flow of such "fluids" except for special cases (Hron et al. [8]), furthermore the dynamic equations (Eq. (10)) show some instability (Bulícek and Kaplický [1], Bulícek et al. [2], Prasad and Rajagopal [19], Malék et al. [14], Renardy [20], Schaeffer [22] and Schaeffer [23]).

- Singular viscosity: The part of the stress tensor containing $(1 / \sqrt{z})$ is well defined only for nonzero values of the rate of strain tensor and for 'non-negative' pressures, which requires some stabilization techniques of singular phenomena due to the nonlinear viscosity (Ouazzi et al. [18]).

- Discretization method: It is well known that the computation of solutions to such incompressible systems requires that some care is taken in the choice of the approximating spaces in order to make the discrete problem well posed. Moreover, since a large number of successful spaces satisfying the above condition are nonconforming that present a locking phenomenon for problems involving the rate of deformation tensor, some consistent stabilizing term is required (Turek and Ouazzi [29]).

- Nonlinear multigrid solver: For this highly nonlinear problem, coupling the pressure and the velocity, there is almost no alternative to linearization using Newton's techniques and therefore efficient multigrid methods for these new types of saddle-point problems need to be developed (Turek [31], Turek et al. [30]).

\subsection{Finite element method}

The underlying finite element technique, which is the basis for the developed software package FeatFlow, is described in detail in Ouazzi et al. [18]. The essence of the special techniques for this kind of flow problems is the introduction of a stabilization term in the denominator that contains the magnitude of the shear stress and a modified multigrid solver. The method was used successfully to solve for incompressible granular flow in a two dimensional hopper using Eq. (11-3). In the present work we use the same method to solve the modified NavierStokes equations (Eq. (10)) with the new closure that applies to the quasi-static and the intermediate regime i.e., Eq. (11-5) in the Couette geometry and also compare this solution to results obtained for all other closures (Eqs. (11-1) to (11-5))

The numerical method yields as a first result the velocity and pressure distribution in the gap of the Couette device. Stresses are then computed from the velocity gradients using the constitutive laws (Eqs. (11-1) to (11-5)) for the respective material. From these results, and $T_{i j}=\tau_{i j}-p I$, the force $(F)$ and torque $(M)$ on the inner, rotating cylinder are calculated as follows:

$F=-\oint_{S} T_{i j} \vec{n}$ ds and $M=-\oint_{S}\left(X-X_{0}\right) T_{i j} \vec{n} d s$

In the above equation, $S$ and $X_{0}$ are the surface and the center of the inner cylinder and $\vec{n}$, is the direction vector.

\subsection{Computer simulations}

This section is intended to validate the proposed model via the FEM code FeatFlow for complex flow problems (see Ouazzi et al. [18]). The 2D simulation was done for two concentric cylinders. The outer

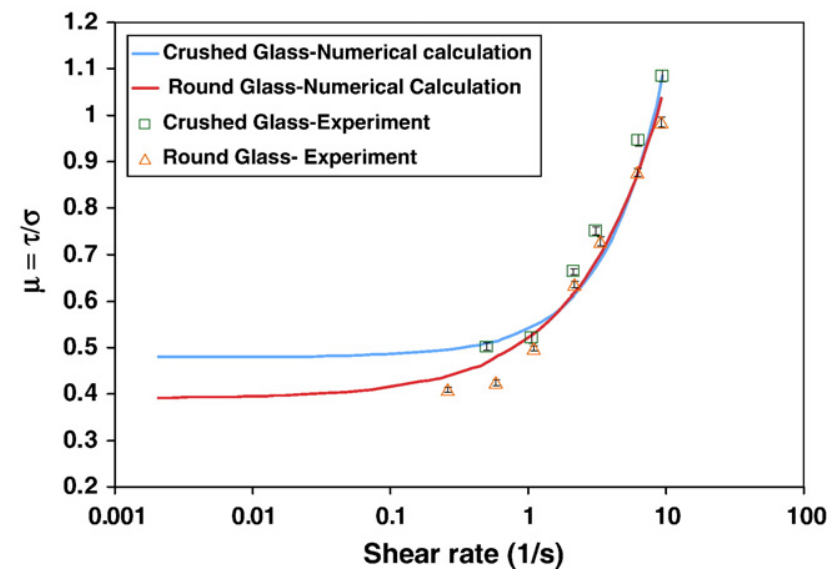

Fig. 11. Numerical solution of the ratio of average shear to normal stresses vs. shear rate (using the new constitutive equation, Eq. (7)); comparison to experiments from Fig. 9 Concentric cylinders with $1 \mathrm{~mm}$ round and crushed glass particles. 


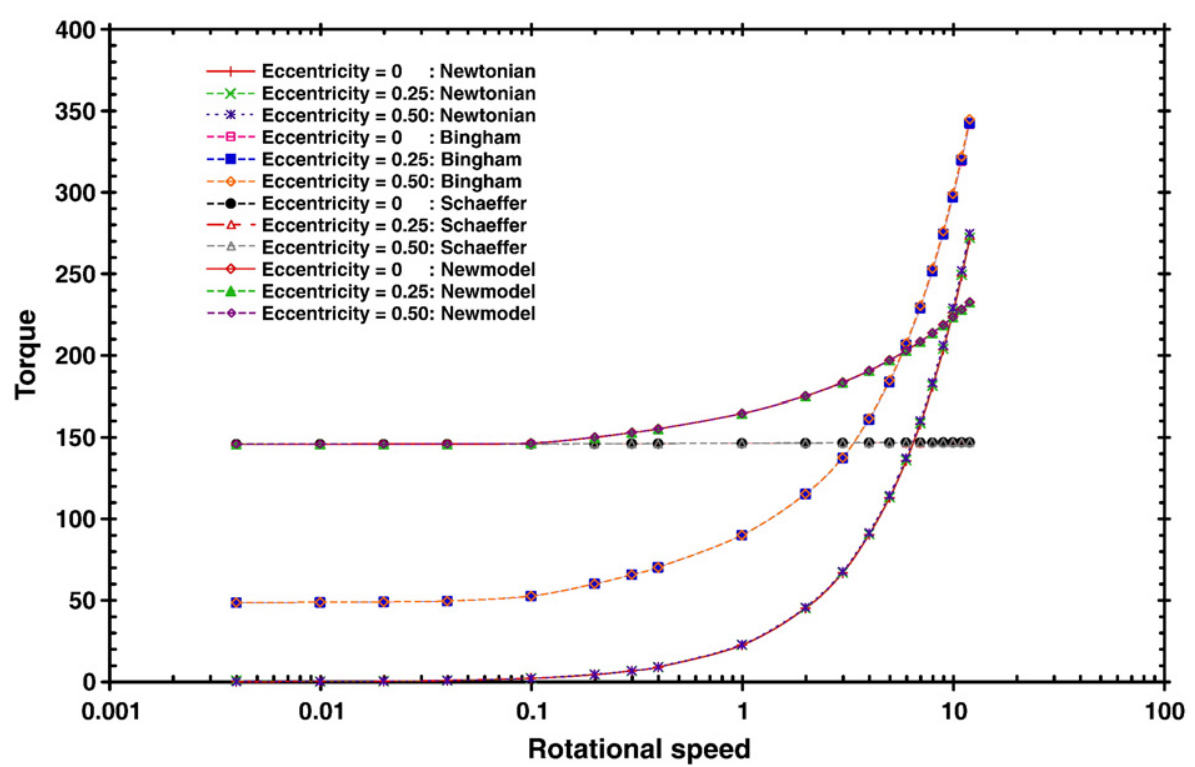

Fig. 12. Comparison of concentric and two eccentric cylinders. Numerical solution of the generalized Navier-Stokes equations for (a) Newtonian fluid, (b) Bingham plastic, (c) powder in the quasi-static regime, (Schaeffer solid), and (d) powder in the intermediate regime (new constitutive equation, Eq. (7)).

cylinder, of radius $r_{\text {outer, }}$, was kept fixed while the inner cylinder, of radius $r_{\text {inner, }}$, was rotated (with $r_{\text {inner }} / r_{\text {outer }}$ set to $2 / 3$ ). The torque was calculated for a section of the cylinders with length unity. All simulations were carried out with $\mu_{0}$ set to unity, the yield strength for the Bingham model $\tau_{0}$ as well as $\sin \phi$ for the quasi-static powder was set to 0.48 which corresponds to a constant $a=0.48$ for crushed glass. The simulations were carried out on a Linux PC.

Fig. 10 depicts a sample calculation with the numerical method, for four continua including a Newtonian liquid, Bingham plastic (solid) and powder in the quasi-static regime (Schaeffer solid) compared with the proposed new constitutive equation, (Eq. (7)) that contains the viscous term as in Eq. (11-5). The torque on the inner rotating cylinder is given as a function of the rotational speed of the inner cylinder (in RPM). The powder in the quasi-static regime (Schaeffer solid) yields a constant torque as a function of rotation (or shear) rate, as expected. The Bingham plastic also yields a constant torque at low shear rates but then behaves like the Newtonian fluid and, above a rotational speed of about $10 \mathrm{RPM}$, the data become indistinguishable. Two curves are presented in Fig. 10 for the powder in the intermediate regime ("New model" in the figure) for a power-law index of $n=0.5$ and $n=1.5$, respectively. One can see that the viscous term has a significant influence and the torque and shear stress are shear-ratedependent at higher shear rates as was found experimentally in Fig. 8 (the shear stress and torque are related linearly as in Eq. (2)).

Fig. 11 shows the ratio of average shear to normal stresses on the rotating cylinder vs. the shear rate with the numerical solution using the new constitutive equation (Eq. (11-5)) and compares calculations with experimental data in Fig. $9(n=0.72$ and $n=1.03$ and $b=0.13$ and $b=0.06$, respectively). Two regions can be seen: solid-like behavior at low shear rate where the ratio is constant and independent of the shear rate reflecting quasi-static flow, and fluid-like behavior as the shear rate increases beyond a critical value where the ratio is dependent on shear rate. This result in itself does not demonstrate that the model correctly predicts the experimental data since the input to the model was the

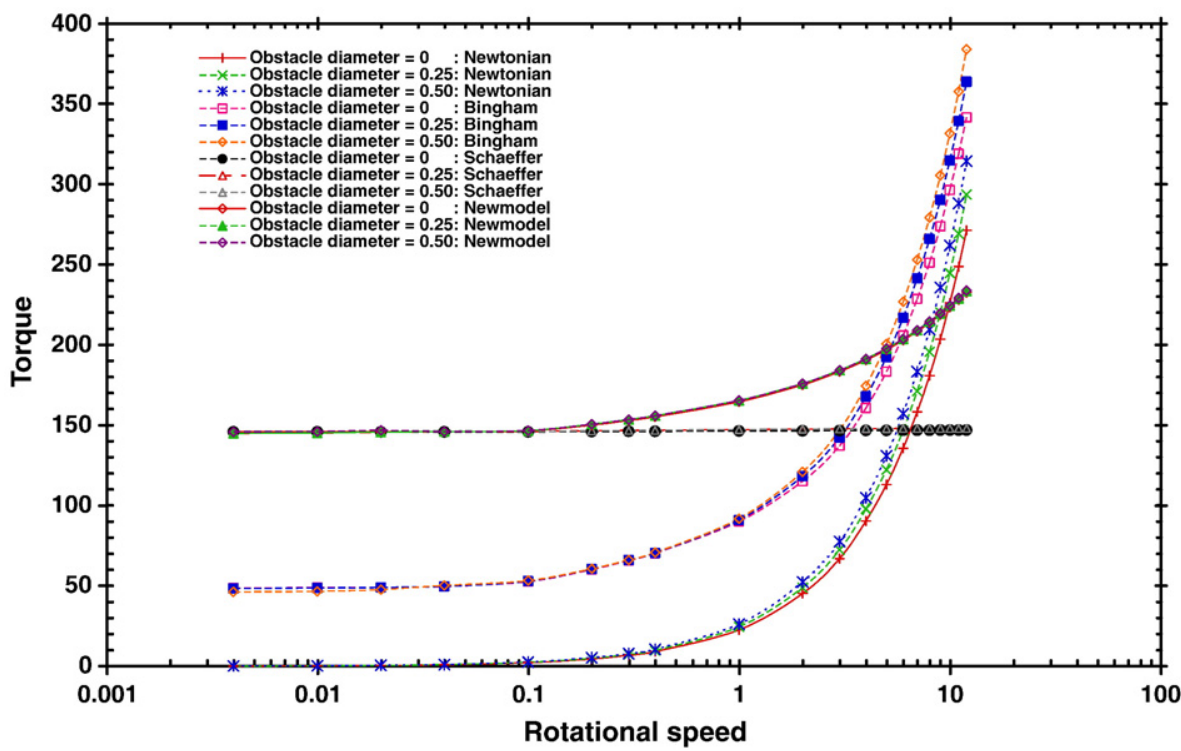

Fig. 13. Numerical solution of the generalized Navier-Stokes equations for concentric Couette with a cylindrical obstacle in the center of the shearing gap for (a) Newtonian fluid, (b) Bingham plastic, (c) powder in the quasi-static regime (Schaeffer solid), and (d) powder in the intermediate regime (new constitutive equation, Eq. (7)). 

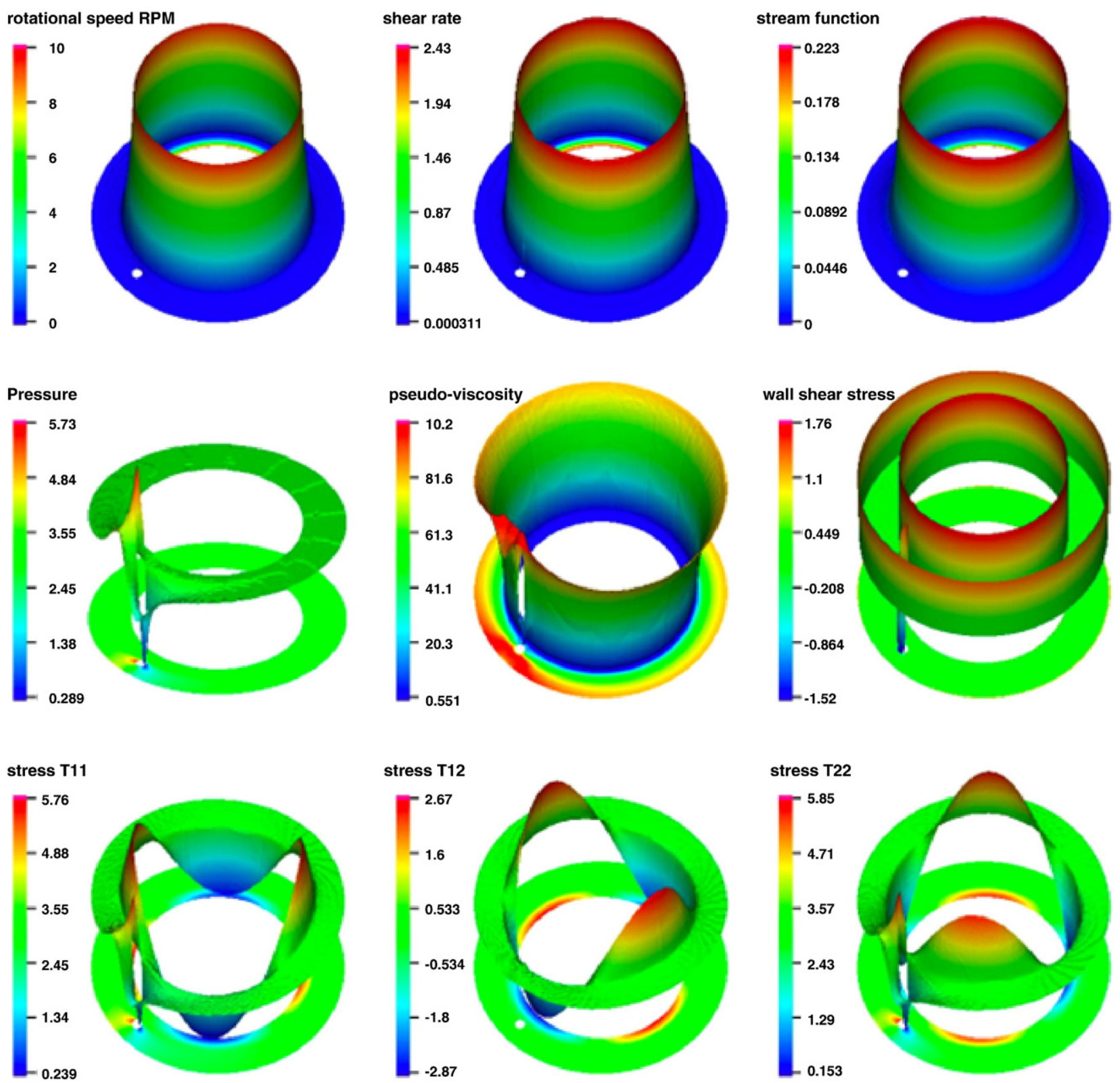

Fig. 14. Numerical solution of the generalized Navier-Stokes equations for a powder in the intermediate regime (new constitutive equation, Eq. (7)). Concentric Couette with a cylindrical obstacle in the middle of the sharing gap at a rotational speed of 10 RPM.

experimental data itself ( with a slight modification using tan $\phi$ instead of the constant coefficient $a$ as mentioned above). It shows however that the simplifications introduced by the continuum approach and the numerical method used do not introduce errors. The advantage of the numerical solution is that velocity, stress distributions and overall torque can be calculated for different geometries, and some examples are given below.

Error bars are shown in both Figs. 9 and 11 but the error of the average friction factor is quite low due to the very large number of data taken with the computer driven data acquisition system for each point. This is also true for Figs. 4, 5 and 8 where the error is smaller than the size of the symbol used to represent the data. In light of this small error and the fact that both the experiment and theoretical calculations fit quite well, the coefficient $n$ cannot be taken as a constant and varies for different powders as a function of particle shape, surface roughness and solid fraction. Simple addition of the stresses from the quasi-static and the intermediate regime i.e., taking $n=1$ (as was proposed earlier by Jackson [11] and others) only applies for specific cases and, in general, the coefficient $n$ varies from about 0.5 to 1.2 .

Fig. 12 gives the torque on a slice of the Couette of height "unity" for all four continua as a function of the rotational speed of the inner cylinder (in RPM). Different simulations were performed for the concentric cylinder (taken partially from Fig. 10) and for the case when the inner cylinder is moved off-center by one quarter of the gap (0.25 in.) and half of the size of the shear gap (0.5 in.). An important conclusion from these simulations is that the torque is not influenced in a significant way by the eccentricity for any continua considered herein. Of course, this is true only as long as the eccentricity is kept to less or equal to half of the shearing gap as shown in the figure. This conclusion is relevant from an experimental point of view since small eccentricities and "wobbles" of rotating parts are very difficult to avoid in practice. Note that the torque generated by the Schaeffer solid is constant for all shear rates since the pressure is constant (constant stress experiment); this is to be expected for a solid powder moving in the quasi-static regime. The appropriate change in solid fraction (or 
bulk density) to achieve this is not captured by the model since it is assumed that the powder is incompressible. Experiments performed with an off-center cylinder (situated 0.25 in. off-center) actually proved that there is no difference in the behavior of the powder and the overall torque is practically the same.

Fig. 13 gives similar results for the four continua for the concentric case where an additional stationary cylinder is introduced in the middle of the gap. This cylinder is denoted "obstacle" in the figure and occupies a quarter of the gap (at 0.25 ) and half of the gap (at 0.5). The Newtonian fluid and the Bingham plastic with the same viscosity appear to be quite sensitive to the presence of the obstacle, as expected, while the Schaeffer solid seems to be quite insensitive. This last result is again to be expected since previous studies showed [27] that the shearing zone in the quasi-static regime is actually very close to the moving surface and decreases exponentially from the boundary. For this reason, the presence of an obstacle in the middle of the shearing gap, even if it occupies one half of the distance, does not affect the torque on the rotating cylinder to a significant degree. The behavior of the modified Schaeffer solid i.e., using the constitutive law in Eq. (11-5), is intermediate between the viscosity containing continua and the Schaeffer solid. The larger obstacle generates a somewhat higher torque but experiments performed so far with a similar system (in which the "obstacle" occupies half the shear gap) yielded results that are inconclusive in the sense that the fluctuation of the experimental torque is larger than the difference in torque between the absence and presence of the obstacle.

Fig. 14 is an attempt to show the complex capabilities of the numerical model. An exhaustive calculation of all the variables at all spatial positions is beyond the scope of the present work. The figure contains the local rotational speed (in RPM), the shear rate $\left(s^{-1}\right)$, pressure, viscosity and stress components using a color scheme and the geometry of the system for "crushed glass" employing data from Fig. 13 at a rotation rate of $10 \mathrm{RPM}$. The different quantities are given as a function of bed height which is zero at the bottom and increases in the vertical direction upwards to a maximum value of unity. The white point on the bottom plane is the center location of the "obstacle". "No slip" boundary conditions are assumed on both cylinders (rotating, inner and stationary, outer) as well as on the bottom plate that is assumed to be stationary. The different quantities are calculated in the figure at a radial distance of $1 / 3$ into the shearing zone $(R=\sim 2.32$ in.). One can see the spatial distribution of the physical quantities in this more complex geometry: velocity, shear rate, normal stress and shear stress ( $T_{11}$ and $T_{22}$ are the normal stresses in the " 1 or rr" and " 2 or $\theta \theta$ " directions and $T_{12}$, is the shear stress). The velocity and shear rate are linearly dependent and are used to check the response of the constitutive law with respect to the shear rate. The jump of the normal stress in the presence of the obstacle in the gap is clearly reflected on the first $\left(T_{11}\right)$ and second components $\left(T_{22}\right)$ as well as in the shear and wall stresses. Moreover, wall shear stresses may have an important influence on the material flow-properties, as for instance the formation of granular chains. Another important conclusion (see in particular the three quantities calculated on the top row) is that the system becomes height $(z)$ independent at approximately $2 / 3$ away from the (stationary) bottom or approximately at a distance equal to the width if the shear gap.

\section{Conclusions}

We study in this work experimentally and numerically the flow of dry, frictional powders in the quasi-static and intermediate regimes using the simple geometry of the Couette device. We measure normal and shear stresses on the shearing surface and propose a constitutive equation valid in regimes. This constitutive equation is then used in a new, specially developed numerical scheme realized in the FEM package FeatFlow to solve the continuum equations of motion and to obtain stress and velocity distributions in the powder. While the measurements to obtain the constitutive equation are performed in a concentric Couette device, the numerical scheme is used to predict the torque and stresses in two additional geometries: an eccentric Couette device where the inner, rotating cylinder is placed off-center with different eccentricities and a more complicated geometry where a cylindrical body is introduced in the middle between the rotating and stationary cylinders and obstructs part of the shearing gap.

We find that it is possible to measure normal stresses on the shearing wall of the Couette device inside the granular layer and calculate the ratio of the average shear to normal stress as a function of shear rate. It appears that the dynamic angle of friction of the powder is reproduced by this ratio only at very low shear rates. As the shearing rate increases, the ratio of the stresses also increases due to collisions between particles that sustain some load. We show that a modified Couette device with slow axial flow superimposed on the shearing motion can be used to determine the constants ( $b$ and $n$ ) of a yield condition for any material that is somewhat free flowing and thus can be used as a "powder rheometer". The yield condition is valid in both the quasi-static as well as the "intermediate" regimes of flow and contains a term characterizing "solid"-like behavior and an additional term that captures some "fluid"like properties at higher shear rates.

The paper describes a new finite element solver FeatFlow of the generalized Navier-Stokes equations that uses, in addition to the yield condition determined experimentally for a relatively free flowing powder, a generalized viscosity that describes a Newtonian fluid, a Bingham Plastic, an incompressible frictional powder (Schaeffer solid) and a power-law fluid. We use the numerical method to validate some experimental measurements and calculate the torque in the Couette device in three different geometries: a concentric, two cylinder, arrangement and two new geometries in which the cylinder is positioned eccentric in the Couette and one where an additional cylindrical object is placed into the shearing gap and obstructs parts of it.

Finally, we show in Fig. 14 some of the capabilities of the model to predict a wide variety of parameters for the most complicated geometry that of the "obstacle" occupying $1 / 2$ of the shearing zone. We show, for example, that the different quantities predicted by the model become height independent at a distance roughly equal to the width of the shear gap. While the model predicts all stresses in the shear zone and the obstacle, experiments to check the torque on the rotating cylinder were inconclusive since the fluctuations of the torque were about equal in magnitude with the predicted difference in torque with and without the obstacle. Further work in measuring the local stresses and forces on the obstacle is in progress to check the predictions of the model.

\section{Acknowledgement}

This work was supported by the German Research Association (DFG) through the collaborative research center SFB/TRR 30 and through grant TU-102/21. Additional support from the US Department of Energy under grant \#RF-75740-00-01 and a Geleese fellowship from the City University of New York Graduate Centre to MKL, is greatly appreciated. Extensive discussions with Drs. Paul Mort of Procter \& Gamble Corporation and James N. Michaels from Merck and Co. Inc regarding the subject matter of this work are also acknowledged as is a stipend for MKL from the P\&G Company to support this work.

\section{References}

[1] M. Bulícek, P. Kaplický, Incompressible Fluids with Shear Rate and Pressure Dependent Viscosity: Regularity of Steady Planar Flows, Discrete and Continuous Dynamical Systems Series S, 1, vol. 1, 2008, pp. 41-50.

[2] M. Bulícek, J. Malék, D. Prazák, On the dimension of the attractor for a class of fluids with pressure dependent viscosities, Comm. Pure Appl. Anal. 4 (2005) 805-822.

[3] C.S. Campbell, Annu. Rev. Fluid Mech. 22 (1990) 57-92.

[4] C.S. Campbell, Granular material flows - an overview, Powder Technol. 162 (2006) 208-229. 
[5] F. DaCruz, S. Emam, M. Prochnow, J.N. Roux, F. Chevoir, Rheophysics of dense granular materials: discrete simulation of plane shear flows, Phys. Rev., E. 72 (2005) 021309.

[6] Y. Forterre, O. Pouliquen, Annu. Rev. Fluid Mech. 40 (2008) 1-24.

[7] GDR MiDi, Eur. Phys. J., E 14 (2004) 341-365.

[8] J. Hron, J. Malek, J. Necas, K.R. Rajagopal, Numerical simulations and globa existence of solutions of two-dimensional flows of fluids with pressure- and shear-dependent viscosities, Math. Comput. Simul. 61 (2003) 297-315.

[9] A.W. Jenike, Storage and flow of solids, Bulletin of University of Utah, Bulletin No. 123, Vol. 53, No.26, 1964.

[10] R. Jackson, Some features of the granular materials and aerated granular materials, J. Rheol. 30 (5) (1986) 907-930.

[11] P. Jop, Y. Forterre, O. Pouliquen, Nature 441 (2006) 727-730.

[12] G. Lois, A. Lemaître, J.M. Carlsonl, Phys. Rev., E 76 (2007) 021302.

[13] S. Luding, Shear flow modeling of cohesive and frictional powders, Powder Technol. 158 (2005) 45-50.

[14] J. Malék, J. Necãs, K.R. Rajagopal, Global existence of solutions for flows of fluids with pressure and shear dependent viscosities, Appl. Math. Lett. 15 (2002) 961-967.

[15] R. Moreno-Atanasio, S.J. Anthony, M. Ghadiri, Analysis of flowability of cohesive powders using Discrete Element Methods, Powder Technol. 158 (1-3) (2005) 41-57.

[16] Y. Muguruma, T. Tanaka, Y. Tsuji, Numerical simulation of particulate flow with liquid bridge between particles, Powder Technol. 109 (2000).

[17] R.M. Nedderman, Statics and Kinematics of Granular Materials, Cambridge University Press, 1992

[18] A. Ouazzi, S. Turek, J. Hron, Finite element methods for the simulation of incompressible powder flows, Commun. Numer. Methods Eng. 21 (2005) 581-596.

[19] S. Prasad, K.R. Rajagopal, Flow of a fluid with pressure dependent viscosity due to a boundary that is being stretched, J. Appl. Math. Comput. 173 (2006) 50-68.
[20] M. Renardy, Parallel shear flows of fluids with a pressure-dependent viscosity, J. Non-Newton. Fluid Mech. 114 (2-3) (2003) 229-236.

[21] S.B. Savage, Analyses of slow high-concentration flows of granular materials, J. Fluid Mech. 377 (1998) 1-26.

[22] D.G. Schaeffer, Instability in the evolution equations describing incompressible granular flow, J. Differ. Equ. 66 (1987) 19-50.

[23] D.G. Schaeffer, Mathematical issues in the continuum formulation of slow granular flow, in: D.G. Joseph, D.G. Schaeffer (Eds.), Two Phase Flows and Waves, Springer Verlag, 1990, pp. 118-129.

[24] A. Srivastava, S. Sundaresan, Analysis of a frictional kinetic model for gas-particle flow, Powder Technol. 129 (2003) 72-85.

[25] G.I. Tardos, A fluid mechanics approach to slow, frictional powder flows, Powder Technol. 92 (1997) 61-74.

[26] G.I. Tardos, M.I. Khan, D.G. Schaeffer, Forces on a slowly rotating, rough cylinder in a Couette device containing a dry, frictional powder, Phys. Fluids 10 (2) (1998) $335-341$.

[27] G.I. Tardos, S McNamara, I. Talu, Slow and intermediate flow of a frictional bulk powder in the Couette geometry, Powder Technol. 131 (2003) 23-39.

[28] G.I. Tardos, P.J. Mort, Dry powder flows, in: C. Crowe (Ed.), Chapter 9 in Multiphase Flow Handbook, CRC Press, Boca Raton, Florida, October 2005.

[29] S. Turek, A. Ouazzi, Unified edge-oriented stabilization of nonconforming FEM for incompressible flow problems: numerical investigations, J. Numer. Math. 15 (2007) 299-322.

[30] S. Turek, A. Ouazzi, R. Schmachtel, Multi-grid methods for stabilized nonconforming finite elements for incompressible flow involving the deformation tensor formulation, J. Numer. Math. 10 (2002) 235-248.

[31] S. Turek, Efficient Solvers for Incompressible Flow Problems: An Algorithmic Computational Approach, LNCSE, vol. 6, Springer, 1998. 
Appendix K

Transmission of normal stresses in un-sheared and sheared granular beds: the influence of particle size, shape, stiffness and cohesion 


\title{
Transmission of stresses in static and sheared granular beds: The influence of particle size, shearing rate, layer thickness and sensor size
}

\author{
M. Kheiripour Langroudi a , J. Sun ${ }^{\text {b }}$, S. Sundaresan ${ }^{\text {b }}$, G.I. Tardos a,* \\ a Department of Chemical Engineering, The City College of the City University of New York, USA \\ ${ }^{\mathrm{b}}$ Department of Chemical Engineering, Princeton University, USA
}

\section{A R T I C L E I N F O}

\section{Article history:}

Received 26 May 2009

Accepted 18 February 2010

Available online 2 April 2010

\begin{abstract}
A B S T R A C T
The spatial non-uniformities and temporal fluctuations in the normal stress transmitted across a sheared granular layer have been studied through a combination of experiments in a Jenike shear cell equipped with normal force (stress) transducers imbedded on the bottom shearing surface and discrete element method (DEM) simulations. Experiments were carried out with particles of different sizes and layers of different thicknesses; the normal stress was measured at several different shearing rates and at several positions on the bottom surface. The DEM simulations revealed a direct link between the spatial inhomogeneities and temporal fluctuations in the stress recorded in our measurements. We found that the dependence of the average normal stress on the bottom surface as a function of height mirrored that in Janssen's analysis of stresses in wall-bounded static assemblies.
\end{abstract}

Published by Elsevier B.V.

\section{Introduction}

The transmission of stresses through stationary and sheared dense granular layers is a subject of fundamental interest in Powder Mechanics. Specifically, one seeks to find forces exerted on stationary walls and moving objects inside flowing powders and these are the result of transmitted stresses from boundaries and from the weight of the material. These stresses are transmitted differently when the powder is stationary from when it is shearing or even moving in plug flow.

Pressure (normal stress) measurements are relatively simple in fluids and entail mounting a pressure transducer at the point of interest. Granular layers present complications since Pascal's law does not apply and the formation of force chains in stationary or slowly sheared particle assemblies (in the so-called quasi-static regime) results in stress inhomogeneities. These inhomogeneities and fluctuations can sometimes be as large as or even larger than the mean stress values [1,2]. Continuum models [19-26] do not reflect these kinds of behavior and only predict average stresses. While there have been several studies on stress inhomogeneities on the bottom surface of static granular piles $[1,3,4]$, similar measurements in slowly sheared assemblies, where both inhomogeneities and fluctuations exist, are limited $[2,5]$. Yu and Tichy [26,27] used a rotational shear cell to measure stresses in a shearing layer, focusing on the rapid granular flow regime where particle interactions are dominated by collisions;

\footnotetext{
* Corresponding author. Department of Chemical Engineering, The City College of the City University of New York, 160 Convent Ave, New York City, NY 10031, USA. Tel.: +1 212650 6665; fax: +12126506672.

E-mail address: Tardos@CCNY.CUNY.Edu (G.I. Tardos).
}

our measurements have been performed in the slow and intermediate regime where frictional stress transmitted through enduring contact is important and collisions play a minor role. The goal of the study by Yu and Tichy $[26,27]$ was to demonstrate the effect of "granular lubrication" and to calculate the total carrying force of the system and not to study the transmission of stresses in a granular layer, which we consider in our study.

The objective of the present study is to probe through a combination of experiments and discrete element method (DEM) simulations the non-uniformities in stresses transmitted across a sheared granular layer. Towards this end, we employed the classical Jenike cell (commonly used to measure internal and wall friction coefficients), where we added normal force (stress) transducers on the shearing bottom wall and applied known normal stresses to the upper surface of the granular layer. Using this experimental technique we compared the normal stress applied to the top surface of the particle assembly with stresses recorded by the normal stress sensors on the bottom. Particles of different sizes were tested in layers of different thicknesses and at different shearing rates, and the transmitted stress was recorded at several locations on the bottom surface and analyzed. Complementary DEM simulations were performed to further elucidate the connection between the spatial inhomogeneities and temporal fluctuations recorded by the sensors and the role of friction on side walls.

Our measurements and analysis, described below, reveal that the normal stresses are transmitted through thin layers without significant attenuation provided the material is in continuous shear, but large fluctuations are introduced especially for rigid materials such as glass particles. These fluctuations are more pronounced as the ratio of the 
size of the particles to the size of the sensor is increased. The height (thickness) of the sheared layer plays an important role in transmitting stress, much like that in Janssen's analysis of stresses in wallbounded static assemblies [6].

\section{Experimental}

\subsection{Selection and calibration of a sensor system to measure normal stresses}

While the selection and purchase of a sensor system appears to be a rather mundane and simple undertaking, this is not the case for stress sensors used in granular systems. In fact, such instrumentation is not available commercially and there are two ways of solving the problem: either construct the system "in house" or re-engineer an existing system developed for a different application. We employed the second route after failing to develop our own sensors and being unable to use several other systems developed for different purposes by other vendors.

Two systems, manufactured by Tekscan, Inc., one with and the other without remote sensing, were selected; a picture of the sensor is shown in Fig. 1. Since these sensors were developed for a different application (namely, sensing forces in athlete's shoes and on car seats) and are made of very fine, interwoven plastic and conductive layers, it was necessary to apply a protective film and to re-calibrate the unit for each experiment. To perform the calibration, the sensor was mounted on a removable Lucite substrate to enable the transport of the calibrated device from calibration to the experimental unit.

Calibration was performed in the instrument depicted in Fig. 1 where the calibration pressure was applied on top by an Instron-type indenter (Texture Analyzer) and the sensor was situated on the Lucite strip, as shown. The force applied by the indenter was measured by the Texture Analyzer and divided by the active area of the sensor (area delimited by the black circle, approximately $1.1 \mathrm{~cm}^{2}$ ) to yield the appropriate applied normal stress. The indenter was chosen to match the size of the sensitive element and the calibration procedure was repeated several times before and during experiments. In this way, the normal stress applied on the sensor was transformed into a DC voltage that could be simply monitored by a data acquisition system controlled by a computer.

Following calibration, the stress sensor and the Lucite strip are introduced into the powder flow by cutting an appropriate groove into the boundary (wall) so as to keep the sensor flush with the surface. Since the thickness of the sensor is less the $0.1 \mathrm{~mm}$ (see Fig. 1) and is covered by a thin protective film, as mentioned above, there

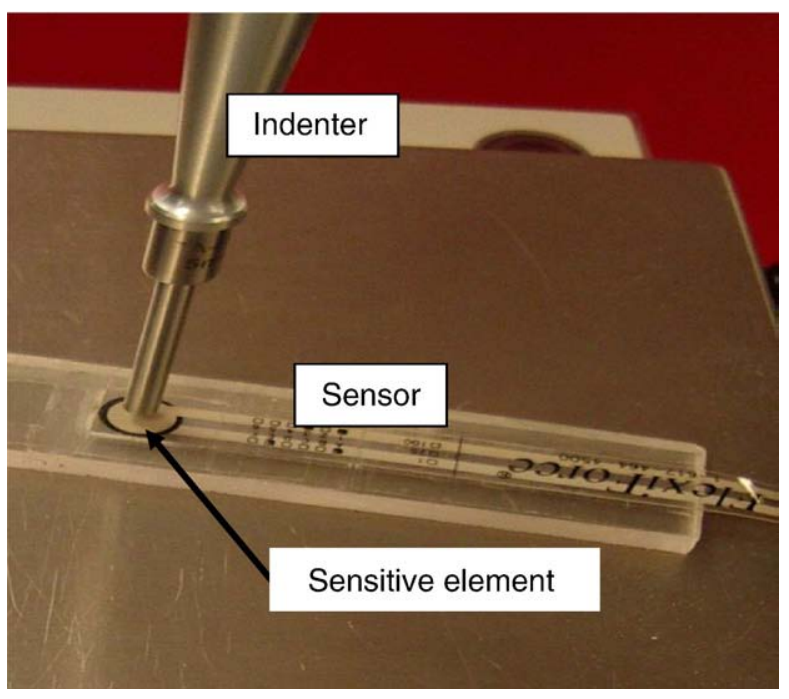

Fig. 1. Picture representing the Tekscan stress sensor and the calibration unit is practically no interference from the sensor to the moving powder layer. In addition, specific experiments with sensors in different positions were carried out and no measurable interference was observed. The attenuating effect of the protective film is taken into account as the sensor is calibrated as shown in Fig. 1.

\subsection{Stress transmission through slowly shearing granular layers}

Experiments were carried out in a conventional Jenike shear cell. A schematic representation of the granular layer with a normal stress applied to the top and a sensor mounted at the bottom is shown in Fig. 2. The load on the top was applied using the classical Jenike "frame" and the circular cover with a raised point in the center [12] to assure that the stress is evenly distributed as shown in Fig. 3a. Fig. 3b and c shows the empty cell with the Lucite strip and the pressure sensor mounted on it. Two smooth (aluminum and Lucite) walls and several "rough" walls were used by gluing different grain-size sand papers on the wall of the device as seen in Fig. 3b. One or more rings of the device were used in order to increase the thickness of the granular layer. The sensor was located $7 \mathrm{~mm}$ off the central cord of the cell perpendicular to the direction of shearing at zero displacement as shown in Fig. 3c. During a typical experiment, the cell moved at $2.3 \mathrm{~mm} / \mathrm{min}$ over a distance of about $2 \mathrm{~cm} \mathrm{[12].}$

A variety of particles from large to fine, spherical and odd-shaped and cohesive and free flowing were tested; a sample is given in Table 1. The specific examples of particles chosen for discussion in this paper were imposed by the limitations of the DEM simulation that require very long times, of the order of weeks, for small particles. The overall behavior of the transmitted stress for other powders is qualitatively similar to the examples presented below.

Fig. 4 gives a typical response curve, transmitted normal stress (in $\mathrm{kPa}$ ) vs. time (in s), for a bed of $2 \mathrm{~mm}$ glass beads in a $2.6 \mathrm{~cm}$ thick layer. There are several stages in applying the load: in the empty cell, the measured stress was zero as it should be ("no load" in the figure). A normal stress of $6.9 \mathrm{kPa}(1 \mathrm{psi})$ was then applied to the static bed and, as seen in the figure, the sensor only "recorded" approximately $1.4 \mathrm{kPa}(0.2 \mathrm{psi})$. As the bed was sheared in the subsequent phase, the stress increased and started to fluctuate around the $6.9 \mathrm{kPa}$ (1 psi) average value. As the normal stress on the top cover was increased to $13.8 \mathrm{kPa}$ ( $2 \mathrm{psi}$ ), the average value measured by the sensor also increased and fluctuated around the same value as long as shearing was applied. The fluctuations are clearly large $(\sim 50 \%$ of the average stress). At the point where the shearing stopped, the bed "froze" at about $13.8 \mathrm{kPa}(2 \mathrm{psi})$ and the stress went to zero as the layer was unloaded.

Fig. 5 presents additional results using larger particles: $5 \mathrm{~mm}$ glass beads and an applied normal stress of $6.9 \mathrm{kPa}(1 \mathrm{psi})$. While the average stress during shearing was only $5 \%$ higher than the applied

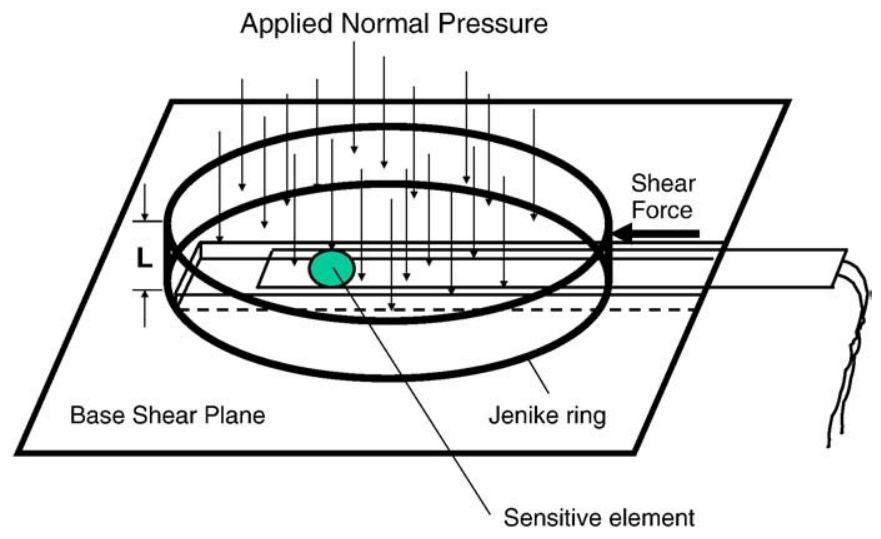

Fig. 2. Schematic representation of Jenike cell arrangement. 
(a)

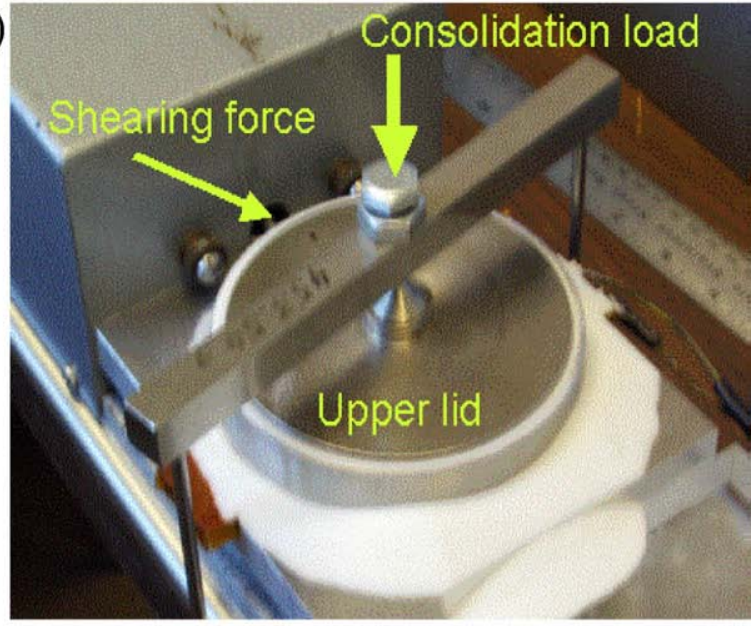

(b)

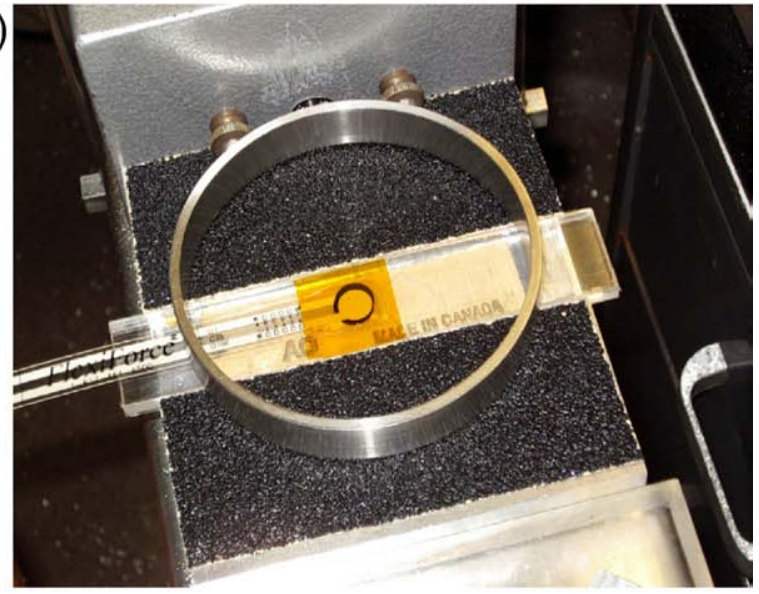

Fig. 3. (a) Jenike shear cell; with sensor on (b) rough wall. Sensor is situated approximately $7 \mathrm{~mm}$ off-center at zero displacement.

normal stress, stress fluctuations were larger compared to the smaller particles (see Fig. 4), about 70\% of the average value. Results in Figs. 4 and 5 are somewhat similar in that the measured stress is always smaller then the applied stress under static conditions and we observe "freezing" of the material once the shearing stops. While shearing is in

Table 1

Materials studied in the Jenike cell.

\begin{tabular}{|c|c|c|c|c|}
\hline $\begin{array}{l}\text { Particle size } \\
{[\mathrm{mm}]}\end{array}$ & Material & $\begin{array}{l}\text { Applied normal } \\
\text { stress [kPa] }\end{array}$ & $\begin{array}{l}\text { Layer thickness } \\
{[L, \mathrm{~cm}]}\end{array}$ & Remarks \\
\hline 5.0 & Glass & 6.9 and 13.8 & $1.6,2.6,3.0,6.0$ & Spherical \\
\hline 3.0 & Glass & $6.9,13.8$ and 20.7 & 1.6 and 2.6 & Spherical \\
\hline \multirow[t]{2}{*}{4.0} & Polyethylene & 6.9 & 6.0 & Not spherical \\
\hline & Polyethylene & 6.9 & 6.0 & And rough \\
\hline \multirow[t]{2}{*}{2.0} & Glass & 6.9 and 13.8 & $1.6,2.6,3.0,4.7$ & Spherical \\
\hline & Cast steel & 6.9 and 13.8 & 1.6 and 3.0 & \\
\hline \multirow[t]{2}{*}{1.5} & Glass & 13.8 & 1.5 & \\
\hline & Glass & 6.9 & 1.6 and 2.6 & \\
\hline \multirow[t]{5}{*}{1.0} & Glass & 6.9 & $1.6,3.0,4.7,6.4$ & \\
\hline & Crushed glass & 6.9 & $1.6,3.0,4.7,6.4$ & Odd shaped \\
\hline & Polyethylene & 6.9 and 13.8 & 3.0 & Not spherical \\
\hline & Cast steel & 6.9 and 13.8 & 1.6 and 3.0 & \\
\hline & $\begin{array}{l}\text { EPDM } \\
\text { elastomer }\end{array}$ & 6.9 & 3.0 & $\begin{array}{l}\text { Shredded } \\
\text { tire }\end{array}$ \\
\hline \multirow[t]{4}{*}{0.5} & Glass & 6.9 & 3.0 & Spherical \\
\hline & Glass & $6.9,13.8$ and 20.7 & 1.6 and 2.6 & Not spherical \\
\hline & Sand & 6.9 and 13.8 & $1.6,2.6$ and 3.2 & Angular \\
\hline & Detergent & 6.9 and 13.8 & 1.6 and 2.6 & Cohesive \\
\hline \multirow[t]{2}{*}{0.1} & Glass & 6.9 and 13.8 & $1.6,2.6$ and 3.0 & \\
\hline & Acrylic & 6.9 & 1.6 and 2.6 & Not spherical \\
\hline
\end{tabular}

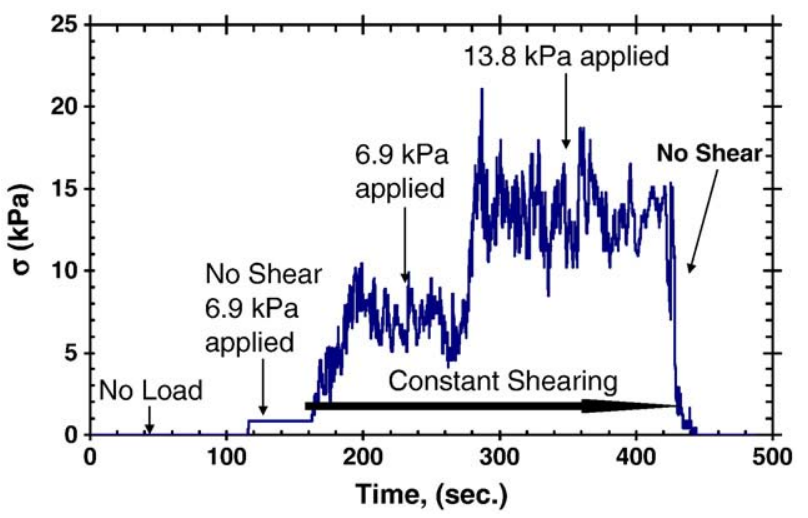

Fig. 4. Normal stress variation in a Jenike shear cell: $6.9 \mathrm{kPa}(1.0 \mathrm{psi})$ and $13.8 \mathrm{kPa}(2.0 \mathrm{psi})$ applied to $2 \mathrm{~mm}$ glass beads. Layer height $=2.6 \mathrm{~cm}$.

process however, the applied and measured stresses become more or less equal. We note that the measured stress fluctuates, sometimes significantly, depending on particle size. This is mainly due to the fact that, since the cell has a constant volume, the number of larger particles is significantly smaller then the number of smaller particles ( $5 \mathrm{~mm}$ in diameter as compared to $2 \mathrm{~mm}$ in diameter). Consequently when the same load is applied, each larger particle carries a larger portion of the load. This larger portion is then transmitted to the transducer that either senses the presence of the particle (when the particles roles over it) or not (when the particle rolls off the sensor), giving rise to fluctuations that are smaller with smaller particles and larger with large particles. Care was taken to use "small enough" particles so that the sensor can see a sufficiently large number of them at any given time: about 25 in the case of $2 \mathrm{~mm}$ in diameter spheres. Results with $5 \mathrm{~mm}$ particles are less reliable since the sensor can "see" only about 5-7 particles at any one time. This may have also resulted in larger fluctuations of the measured stress.

In summary, we found that the normal stress was transmitted to the sensor mounted on the base mostly unaltered through thin layers of non-cohesive powders when the layers were in shear. The normal stress was not transmitted to the base uniformly in static layers as the material "froze" depending on its history: an initially unstressed layer transmitted to the base only a portion of the normal stress while a previously stressed material maintained its internal stress levels until it was unloaded. While there are a number of theoretical studies to model granular beds [13-17], the subject of stress transmission was not addressed directly.

In order to understand these results more thoroughly, we set out to perform discrete element simulations (DEM) of particles subjected

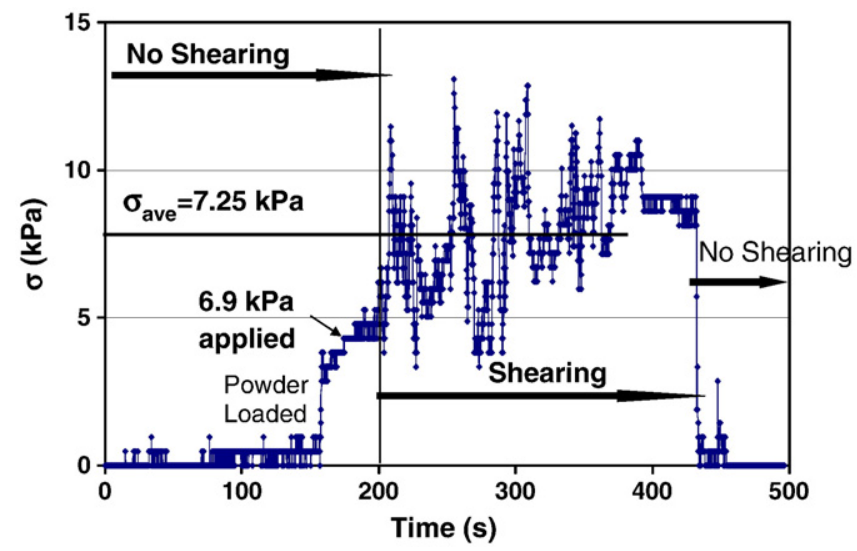

Fig. 5. Normal stress variation in a Jenike shear cell: $6.9 \mathrm{kPa}(1.0 \mathrm{psi})$ applied to $5 \mathrm{~mm}$ spherical glass beads. Layer height $=2.6 \mathrm{~cm}$. 
to similar stress and deformation histories. Unfortunately, such simulations proved to be extremely slow (of the order of weeks) owing to the very long shearing times at the slow shear rates employed in the experiments. It became apparent that experiments would have to be run at much faster rates to keep the running times as short as possible so that steady state could be reached much earlier. This prompted us to devise an experimental system to study stress transmission in more rapidly sheared dense assemblies and examine if the results obtained at faster shear rates differed from those described above. Such faster shear conditions are also more relevant to industrial applications.

\subsection{Stress transmission through rapidly shearing granular layers}

An instrument (henceforth referred to as the "fast" Jenike cell since the only difference compared to a conventional shear cell is its capability of faster shearing) was specially built to enable shearing of particles in the Jenike geometry at higher rates. The instrument was constructed as an addition to the moving arm of a tableting machine (donated to CCNY by Merck and Co. Inc.). A picture of the cell with the lower part of the driving mechanism and the detail of the cell itself with the stress sensor mounted on the bottom are shown in Fig. 6. The dimensions of the cell and the stress sensor are identical to those used in the traditional Jenike test. The loading of the cell with the normal force is also similar except that the weight is added at the top of the device instead of using the Jenike "frame" [12].

The cell executes a back-and-forth movement from the initial position shown in the detail in Fig. 6. After a short pause, the backand-forth movement is repeated. Usually, up to $4-5$ cycles were performed during one experiment. The unit has adjustable speed from approximately $1.5 \mathrm{~mm} / \mathrm{s}(\sim 90 \mathrm{~mm} / \mathrm{min}$ as compared to the traditional Jenike cell at $2.3 \mathrm{~mm} / \mathrm{min}$ ) to approximately $150 \mathrm{~mm} / \mathrm{s}$. Several experiments were performed at different speeds and with different particles, employing a single sensor in some and multiple sensors in the rest of the experiments.

As seen in Fig. 6, the lower plate used for these experiments was a Lucite sheet (angle of wall friction $\phi \sim 17^{\circ}$ with glass particles). The stress sensor was kept flush with the bottom at all times. Due to the relatively fast movement of the cell and the fact that there was a finite layer of material in the cell, the granules tended to move in a circulation type movement: up the approaching vertical wall and away from the departing wall. This created an uneven upper surface as the cell moved back-and-forth and resulted in the tilting of the applied

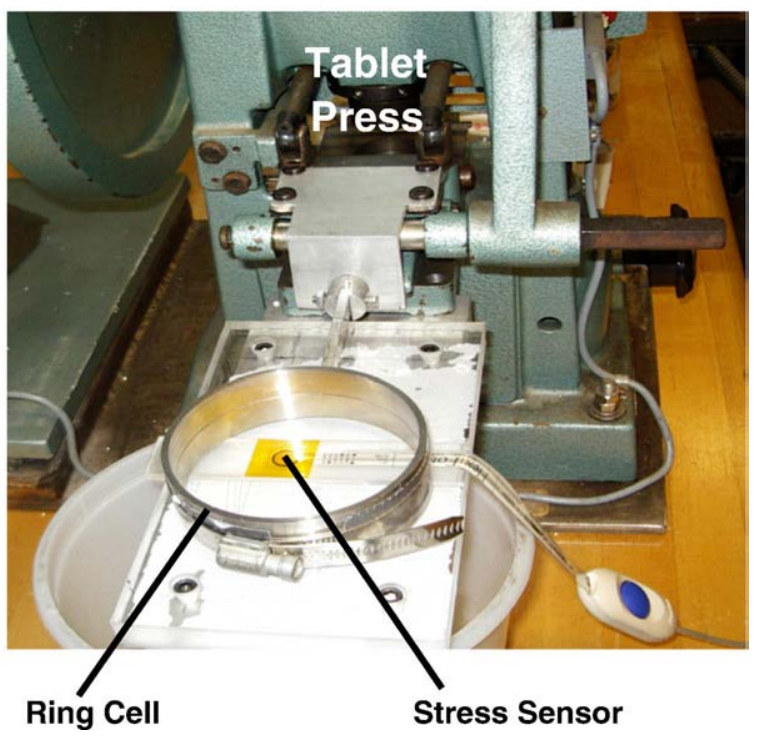

Fig. 6. Picture of "fast Jenike cell" with driving mechanism.

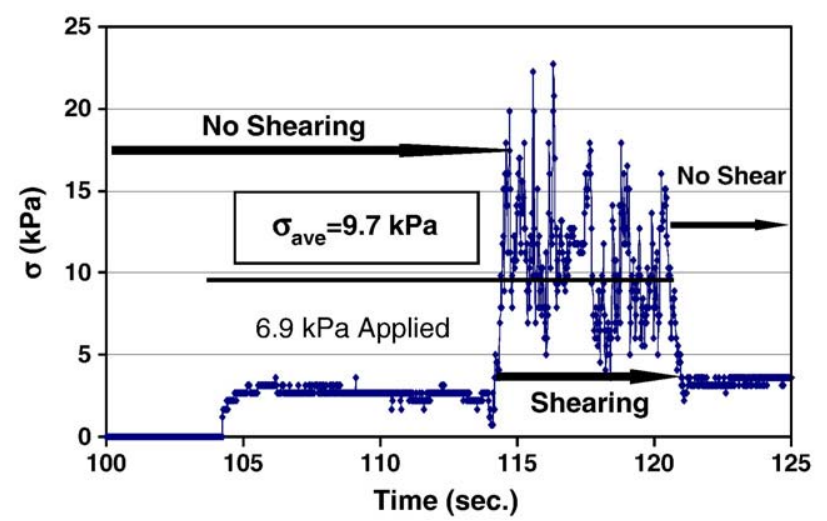

Fig. 7. Normal stress variation in a fast Jenike shear cell. A normal stress of $6.9 \mathrm{kPa}(1.0 \mathrm{psi})$ was applied to $2 \mathrm{~mm}$ spherical glass beads and the shearing was done at $15 \mathrm{~mm} / \mathrm{s}$. A single sensing element was situated $7 \mathrm{~mm}$ off-center.

weight. To diminish this movement and keep the weight horizontal, it was necessary to employ deeper cells made of up to four Jenike "rings" creating a cell that was approximately $60 \mathrm{~mm}$ deep in all experiments in this series.

A typical experimental result is shown in Fig. 7. The cell was not sheared for the first $115 \mathrm{~s}$ of the experiment and therefore the stress in the figure is displayed only from $t=100 \mathrm{~s}$. A normal stress of $6.9 \mathrm{kPa}$ (1 psi) was applied, resulting in a jump in the sensed stress to about $3.8 \mathrm{kPa}(0.4 \mathrm{psi})$. This behavior is similar to the one seen in the (conventional) slow Jenike cell (see Figs. 4 and 5). Upon shearing at $15 \mathrm{~mm} / \mathrm{s}$, the sensed stress fluctuated significantly with an average of about $41 \%$ above the applied load.

The spatial non-uniformity of stress transmitted to the base was studied through experiments where two-sensor locations were used: (a) identical to the position in the conventional slow Jenike cell, i.e., $7 \mathrm{~mm}$ off-center (as shown in Fig. 6) and (b) two sensors mounted as depicted in Fig. 8; the starting and ending positions of the sensors are $2.35 \mathrm{~cm}$ and $1.9 \mathrm{~cm}$ from the walls of the cell while the stroke of the movement is $5.25 \mathrm{~cm}$.

Figs. 9 and 10 depict sample results obtained with the two-sensor assembly shown in Fig. 8. The stress fluctuations were significant and were larger than what was observed earlier for the case of slow shear

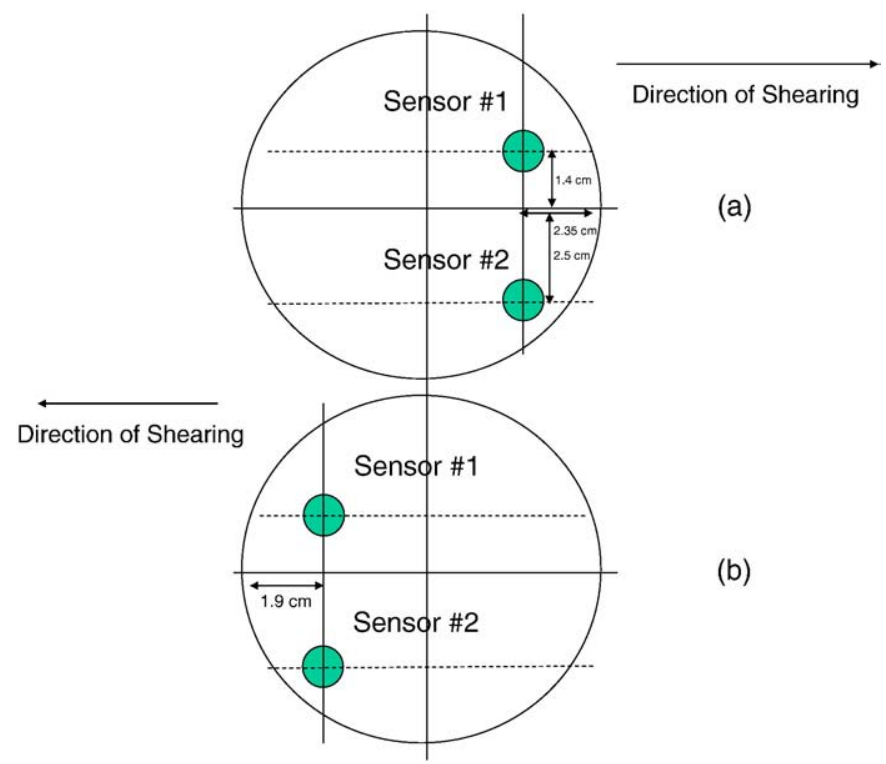

Fig. 8. Measurements in the "fast Jenike cell". Position of the two stress sensors on the bottom plate of the cell: (a) ring movement to the right; (b) ring movement to the left. 


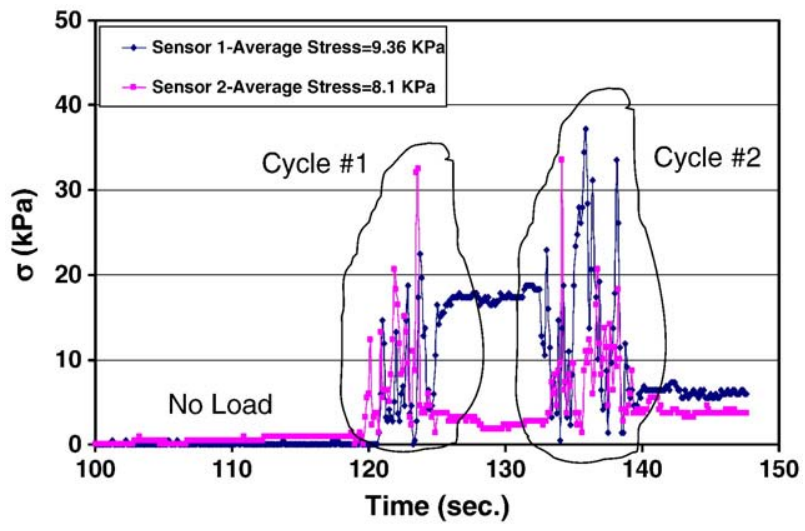

Fig. 9. Measurements in the "fast Jenike cell" using two sensors. The sensor positions are shown in Fig. 8. A load of $6.9 \mathrm{kPa}$ (1 psi) was applied to a bed of $5 \mathrm{~mm}$ glass beads at $t=118 \mathrm{~s}$. Layer depth $=2.6 \mathrm{~cm}$, sheared at $16 \mathrm{~mm} / \mathrm{s}$. Between cycles the cell remained motionless with the applied load.

(see Fig. 5). It appears reasonable to conclude that the fluctuations in dense assemblies increased with increasing shear rate (compare Figs. 5, 9 and 10). Note that during the time when the cell is not under shear between cycles, the stresses recorded by the two sensors were essentially frozen; this stress level at a given location on the base may be above or below the average value. It is also apparent from Figs. 9 and 10 that the stress transmitted to the base was spatially inhomogeneous as the stresses measured at different locations were quite different and also showed different fluctuations in time.

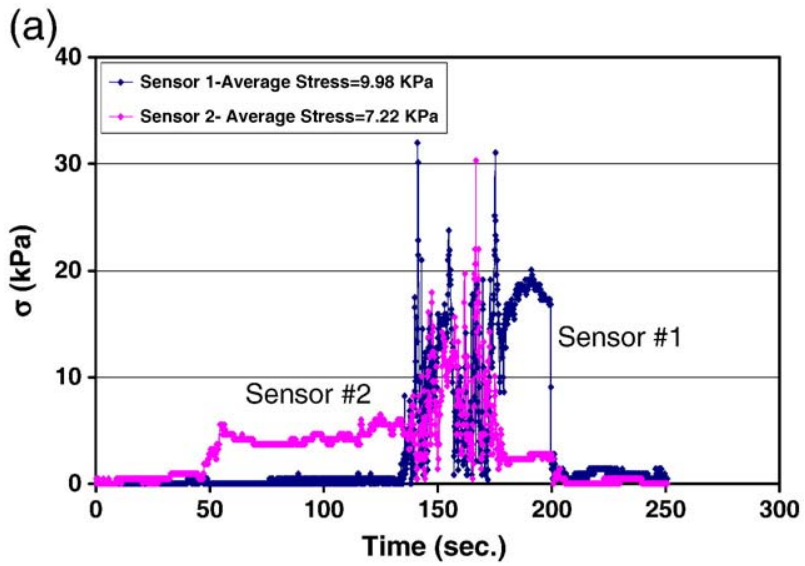

(b)

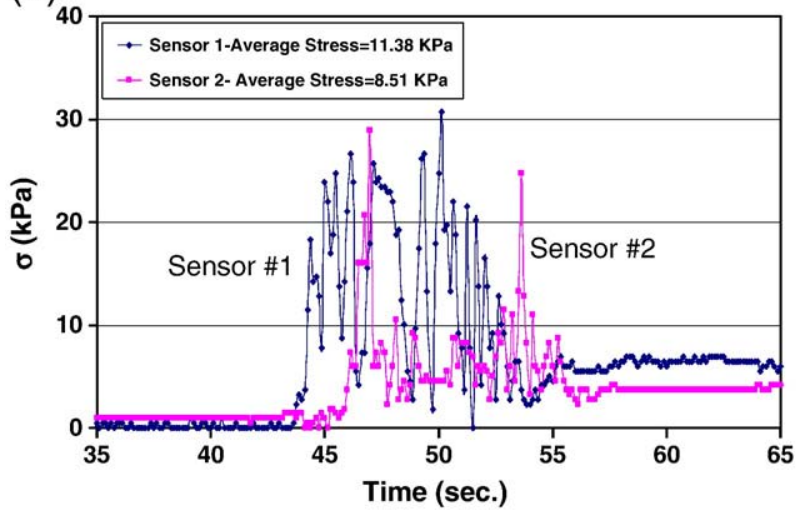

Fig. 10. Measurements in the "fast Jenike cell" using two sensors. The conditions are as in Fig. 9, except that only a single cycle is shown and the shear rates are (a) $1.8 \mathrm{~mm} / \mathrm{s}$ and (b) $7.7 \mathrm{~mm} / \mathrm{s}$.
We examine below the connection between the spatial inhomogeneity and the temporal fluctuations in the transmitted stress through DEM simulations of the Jenike shear cell.

\section{Computational}

The goal of the present computations is to elucidate the relationships between granular stress transmission, layer thickness and sensor size. We have performed DEM simulations using the LAMMPS code, a parallel particle simulator developed at Sandia National Laboratory [7]. These simulations are based on a linear spring-dashpot model to calculate forces between particles, which interact only on contact [8]. The value of the spring constant is chosen to be large enough to minimize particle interpenetration, yet not so large as to require an unreasonably small simulation time step. The tangential force at each contact is computed by keeping track of the elastic shear displacement throughout the lifetime of a contact. As the shear displacement increases, the tangential force reaches the limit imposed by a static yield criterion, characterized by a local particle friction coefficient $\mu$. The tangential force is then truncated to satisfy Coulomb's law. The model allows for contact forces and friction between the particles and the walls in the same way as described for two particles, but with infinite mass and diameter for the walls. Readers are referred to our previous papers for further details of the model $[9,10]$ The simulation domain and the number of particles were chosen to correspond to those employed in the Jenike shear cell experiments; the shear was imposed in the simulations by moving the bottom wall at a prescribed velocity as in the experiments. The numerical values of the DEM model parameters used for the simulations are listed in Table 2. The simulations were performed on two $3.0 \mathrm{GHz}$ Intel Xeon dual core processors. The computational times range from about one day to a week, depending on the simulation time and number of particles.

\subsection{Simulation of the Jenike shear cell experiment}

Fig. 11a shows a short-duration simulation corresponding to the Jenike experiment described in Fig. 4, where a bed of $2 \mathrm{~mm}$ glass beads was sheared on a Lucite wall at a translational velocity of $2.3 \mathrm{~mm} / \mathrm{min}$ (standard Jenike cell speed) with an applied normal stress of $13.79 \mathrm{kPa}$. The normal stress was calculated by summing all the contact forces between particles and the shearing wall over an identical circular area as the sensitive element of the experimental sensor $(1.1 \mathrm{~cm})$. The sensing element was situated as in the experiment ( $7 \mathrm{~mm}$ off-center). This simulation required very long computational time and so it could not be continued for the full duration of the experiment; as a result, the fluctuations seen in the experiment could not be verified. Nevertheless, the normal stress measured on the sensor approximately agrees with the average value measured in the experiments.

Fig. 11b shows the summation of all particle contacts on the bottom of the cell and one can see that the average normal stress at

Table 2

DEM computational parameters.

\begin{tabular}{ll} 
Inter-particle normal stiffness coefficient $\left(k_{n}\right)$ & $200,000 k_{0}$ \\
Inter-particle tangential stiffness coefficient $\left(k_{t}\right)$ & $(2 / 7) k_{n}$ \\
Inter-particle normal damping coefficient $\left(\gamma_{n}\right)$ & $40 / t_{0}$ \\
Inter-particle tangential damping coefficient $\left(\gamma_{t}\right)$ & 0 \\
Inter-particle friction coefficient $(\mu)$ & 0.1 \\
Inter-particle restitution coefficient & 0.9 \\
Particle-wall normal stiffness coefficient & $200,000 k_{0}$ \\
Particle-wall tangential stiffness coefficient & $(2 / 7) k_{n}$ \\
Particle-wall normal damping coefficient & $50 / t_{0}$ \\
Particle-wall l tangential damping coefficient & $25 / t_{0}$ \\
Friction coefficient between particle and top and bottom wall & 0.19 \\
Friction coefficient between particle and cylindrical wall & 0.26 \\
\hline
\end{tabular}

Note: $k_{0}=m g / d, t_{0}=\sqrt{d / g}$. 
(a)

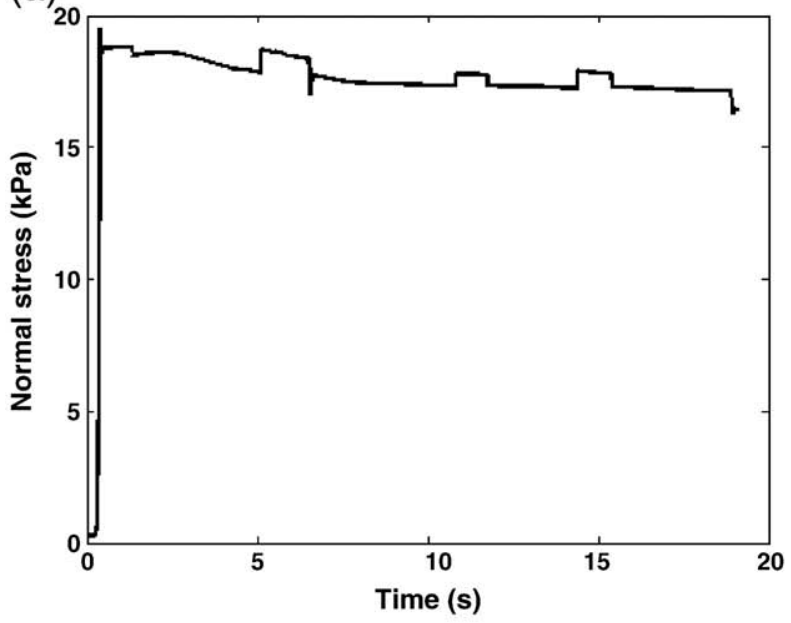

(b)

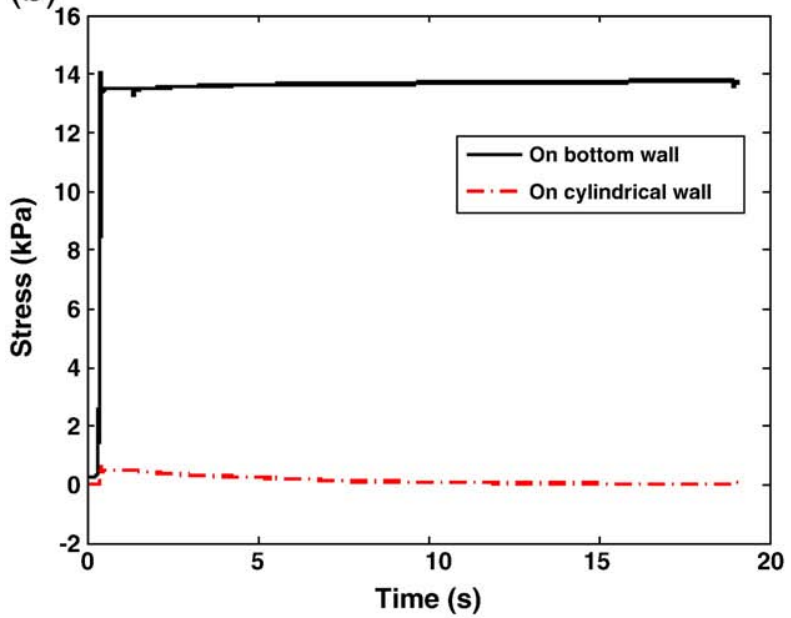

Fig. 11. Simulation of Jenike experiment with $2 \mathrm{~mm}$ glass beads described in Fig. 4 . (a) Normal stress at the sensor location; (b) normal stress averaged over the entire bottom wall and the average shear stress supported by the cylindrical wall.

the base was indeed very close to that applied (with the side walls contributing a negligible amount). Results of the slow Jenike cell simulation did confirm that DEM could indeed be employed to explore stress distribution in this type of shearing experiment. However, in order to capture the dynamic fluctuations during the shearing process, one must either increase the simulation time (which was impractical) or perform simulations at faster shearing rates. As mentioned earlier, this consideration motivated the fast Jenike cell experiments and the corresponding simulations are discussed below.

\subsection{Simulations of the "fast" Jenike cell}

Normal stresses obtained using DEM simulations are shown in Fig. 12, and these can be compared to the experimental results presented earlier in Fig. 9. $\left(D_{\mathrm{s}} / d=1.9\right.$, where $d$ is the particle diameter and $D_{\mathrm{s}}$ is the diameter of the sensor area). The total number of particles in the experiment and in the simulation was the same $(\sim 5000)$; other geometrical and physical characteristics were also matched between the experiment and simulation. Fig. 12 shows the normal stress response over two cycles of the (fast) Jenike cell (at $16 \mathrm{~mm} / \mathrm{s}$.). The circles on the time axis refer to the following events in order - load, begin cycle 1 , hold following the end of cycle 1, start cycle 2, hold following the end of

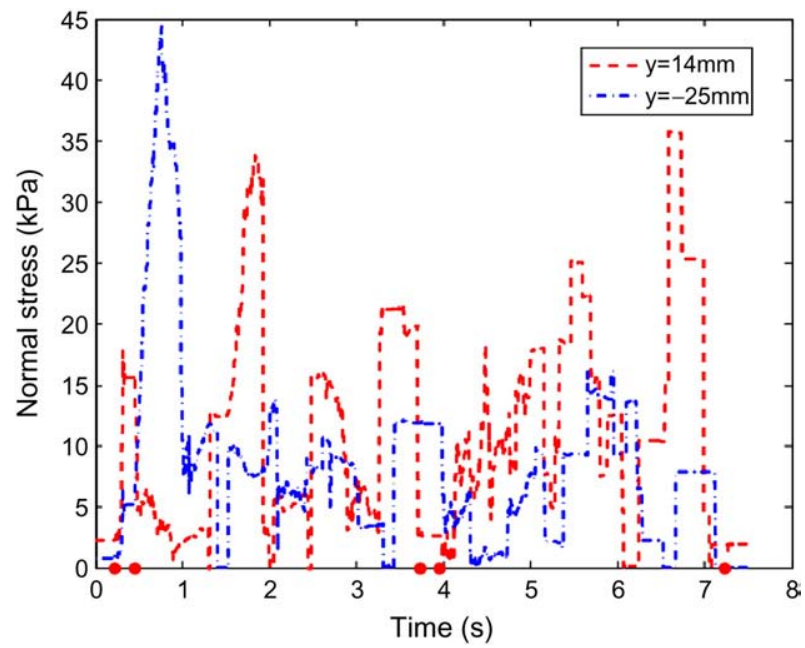

Fig. 12. Simulation of Jenike experiment with $5 \mathrm{~mm}$ glass beads in "fast" Jenike cell (described in Fig. 9). In the legend, the $y=14 \mathrm{~mm}$ and $y=-25 \mathrm{~mm}$ indicate the two sensors with different distances to the central line as shown in Fig. 8. $D_{\mathrm{s}} / d=1.9$.

cycle 2; these are analogous to the stages in the experiments where the duration between the cycles (where the stresses stayed essentially frozen) was longer. One can readily see from Figs. 9 and 12 that in both simulations and experiments the normal stresses recorded by the two sensors oscillated significantly and that the range of the oscillations seen in the experiments was reproduced reasonably accurately by the simulations. The different stresses measured by the two sensors are clearly visible in Fig. 12 showing the spatially inhomogeneous nature of the stress distribution. The frequency of the oscillations differed somewhat between the experiments and simulations, which may be a consequence of factors such as sampling frequency and departure from spherical particle shape in the experiments.

Encouraged by the similarity between the simulated results and the experimental data presented above, we carried out a number of additional simulations to examine how the stress is transmitted across the granular layers.

The granular assembly configurations were varied in two ways: (a) keep the ratio of the column diameter to the particle diameter, $D / d$, constant (at 9.5) and vary the ratio of granular column height to the column diameter, $H / D$; (b) keep $H / D$ constant and vary $D / d$. The vertical forces acting on the entire bottom surface and on the side (cylindrical) walls of the cell were extracted from the simulations. These were then used to compute the average normal stress acting on the bottom surface and the average shear traction (pointing in the vertical direction) acting on the side walls of the cell. Also extracted from the simulations were the normal stresses recorded by several different sensors located at the bottom surface. Two types of computational sensors were devised: (i) "dynamic" sensors mimicking those used in the experiments and move relative to the cylindrical side walls at the shearing velocity; (ii) hypothetical "static" sensors that remained in the same location relative to the cylindrical walls even though the bottom surface was moving. The sensor diameter $D_{\mathrm{s}}$ was also varied to test the spatial averaging effect. The dimensional results presented here are calculated based on a particle diameter $d=5 \mathrm{~mm}$ and density $\rho=2600 \mathrm{~kg} / \mathrm{m}^{3}$. A constant external loading force equal to $48.5 \mathrm{~N}$ was maintained for all cases. A Cartesian coordinate system was used with the origin at the center of the bottom surface of the cylinder and the $z$-axis pointing downwards in the gravity direction.

Fig. 13 shows the variation of the vertical forces on the side and bottom walls (panel a) and the average normal stress on the bottom wall (scaled by $\mathrm{mg} / \mathrm{d}^{2}$, where $m$ is the mass of a particle) (panel b) with $H / D$. (Force is positive in the gravity direction. $6.9 \mathrm{kPa}$ is equivalent to the dimensionless stress of 102.5.) The quantities shown 

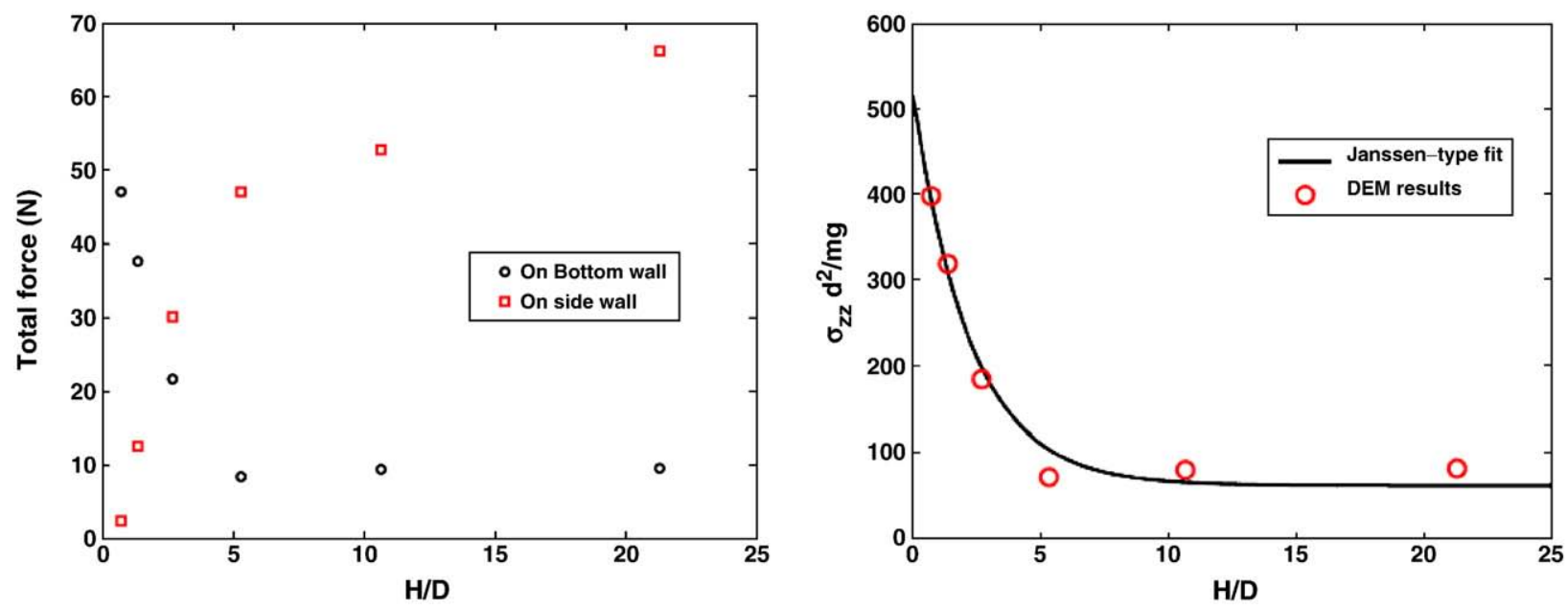

Fig. 13. (a) The variation of total forces in the gravity direction ( $z$-axis) on the bottom and side walls with $H / D$. $D / d=9.5$. (b) The average normal stress on the bottom wall vs. $H / D$ and a Janssen-type exponential function fitted to the data.

represent the average values during steady shear (the error being comparable to the size of the symbols used in the plots). The load supported by the bottom wall decreased with increasing $H / D$ and that supported by the side wall increased. The normal stress variation followed an exponential decay and could be fitted by a function in the same form as used in the Janssen analysis [6], i.e.

$\frac{\sigma_{z z} d^{2}}{m g}=a\left(1-\exp \left(-\frac{H / D}{4 \mu_{\mathrm{w}} K}\right)\right)+b \exp \left(-\frac{H / D}{4 \mu_{\mathrm{w}} K}\right)$

$K=\frac{1-\sin \phi_{i}}{1+\sin \phi_{i}}$

where $a$ and $b$ were used as adjustable parameters; $\mu_{\mathrm{w}}(=0.19)$ is the wall friction coefficient, $K$ is the Janssen constant and $\phi_{i}$ is the (macroscopic) angle of internal friction (estimated to be $\sim 15^{\circ}$ according to a correlation between the macroscopic angle of friction and the inter-particle friction coefficient $\mu=0.1$ used in the simulations [11]). The friction coefficient values used in the simulations were close to those measured for the system used in the experiments. According to Janssen's analysis, the coefficients $a$ and $b$ can be calculated as

$a=\frac{6 \phi(D / d)}{4 \pi \mu_{\mathrm{w}} K}, \quad b=Q_{0} d^{2} / m g$

where $\phi$ is the volume fraction and $Q_{0}$ is the surcharge. The exponential relation of the normal stress vs. $H / D$ suggests that the normal stress transmission across the depth of a granular assembly during the Jenike-cell shearing was similar to that for a static granular assembly. Based on the parameters used in the simulations and Eq. (3), their values were estimated to be 23 and 410, respectively. However, the best fit to the DEM results yielded $a=61$ and $b=516$, which were used for the fitted curve shown in Fig. 13(b). While the two values for $b$ are close, the two values for $a$ are quite far apart, suggesting that the applicability of Janssen's analysis to the shearing system is tenuous in the sense that there are likely to be significant quantitative differences between the shearing and static systems.

The temporal variations of the normal stresses recorded by the "dynamic" sensors for various $H / D$ ratios in Fig. 13 are plotted in Fig. $14 \mathrm{a}-\mathrm{f}$. Sensors of three different diameters, $D_{\mathrm{s}} / d=1.9$. 3.8 and 7.6 were used. Initially before shearing starts, the three sensors were located at the center of the cylindrical base and one sensor of diameter $D_{\mathrm{s}} / \mathrm{d}=1.9$ was located $2 \mathrm{~d}$ away from the center. (This configuration of two sensors of the same diameter at different locations was selected to simulate the two-sensor configuration in the experiments.) The shearing velocity was set to $16 \mathrm{~mm} / \mathrm{s}$ along the $x$-axis (assuming that $d=5 \mathrm{~mm}$ ) and the load (equivalent to $28 \mathrm{kPa}$ in these cases) was added at $0.226 \mathrm{~s}$. The shearing started at $0.452 \mathrm{~s}$; the shear was reversed at $1.582 \mathrm{~s}$ and stopped at $2.712 \mathrm{~s}$. Consider Fig. 14(a) first: the normal stress values recorded by the $D_{\mathrm{s}} / d=1.9$ sensor, which moved along the $x$-axis and is denoted by the black solid line in the figure, were small before the load, reached a steady value about $41 \mathrm{kPa}$ after the load and started to fluctuate around $28 \mathrm{kPa}$ when the shearing was started. The magnitude of fluctuations was as large as $100 \%$ of the average. The normal stress on the other sensor of same size, denoted by the green dotted line, had different values at both the loading and shearing periods but demonstrated similar fluctuations. This result is consistent with the observation in experiments with two sensors. The comparison between data on these two sensors shows that the stress was distributed inhomogeneously on the bottom plate. The normal stress on the sensor with larger diameter of $D_{\mathrm{s}} / d=3.8$ denoted by the red dashed-line, had much smaller fluctuations (up to $\sim 50 \%$ of the average). The trend that the fluctuations became smaller as the areas of the sensors increased can be seen in Fig. 14(b)-(f) as well. This trend also reflects the spatial inhomogeneity of the stress distribution. (Correspondingly, in experiments, when the particle size was reduced, the normal stress measured on the same-sized sensor fluctuated less.)

The temporal variations of the normal stresses recorded by the "static" sensors for various H/D ratios in Fig. 13 are plotted in Fig. 15a-f. Data were collected on five sensors, four of which were distributed $90^{\circ}$ apart on a circle with diameter equal to $0.5 \mathrm{D}$ and the fifth one being located at the center. The system experienced the same loading, shearing and no-shear stages as in Figs. 13 and 14. It is clear that the stresses recorded by each sensor manifested a transient behavior at the beginning of each stage (more obvious at the beginning of shear and upon shear reversal) and were different from each other at different locations. However, differently from the data on dynamic sensors, the values recorded by the static sensors were nearly constant (although different from each other) during steady shear. These results confirm the spatial inhomogeneity of the normal stress; furthermore, they reveal that the temporal fluctuations observed on the dynamic sensors during the steady shear were largely due to the fact that a dynamic sensor sampled different spatial locations within the Jenike cell during the shearing motion and not due to any large fluctuation in the stresses in the assembly of particles.

The simulations performed at different $D / d$ ratios (while holding $H / D$ constant) manifested the same trends for the dynamic and static sensors as discussed above in Figs. 14 and 15. Therefore, further discussion of these simulations is not necessary. 
(a)

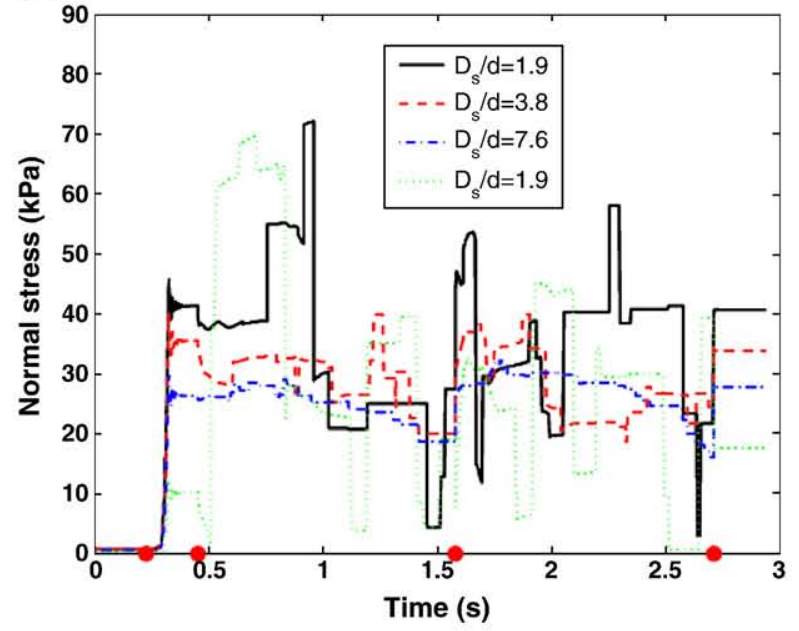

(c)

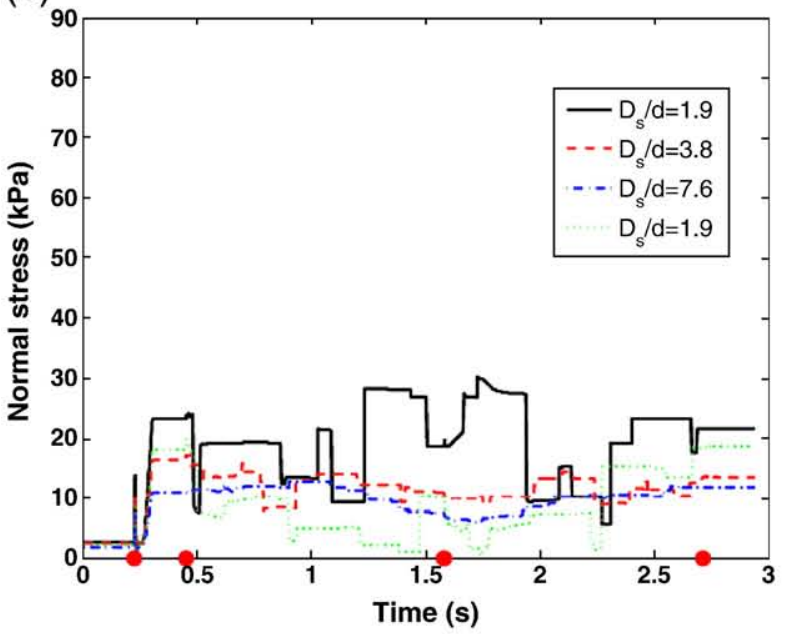

(e)

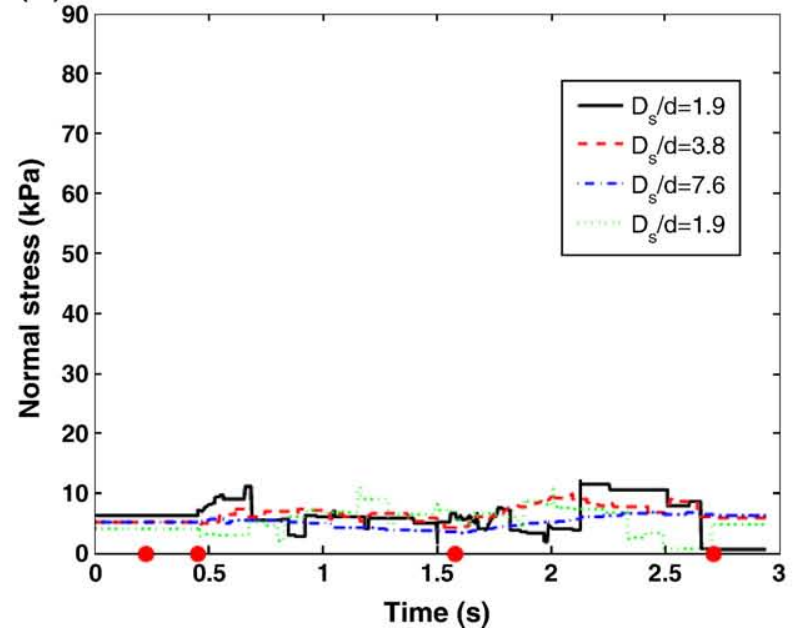

(b)

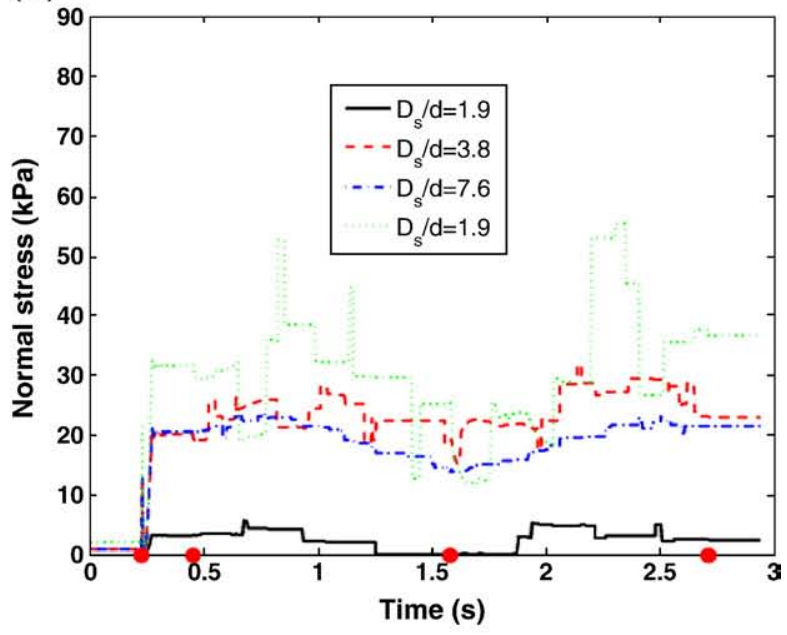

(d)

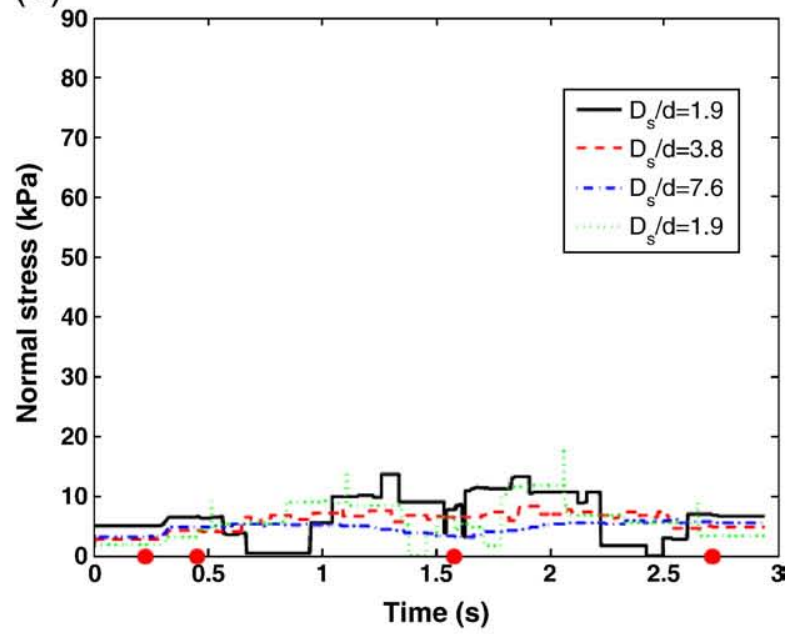

(f)

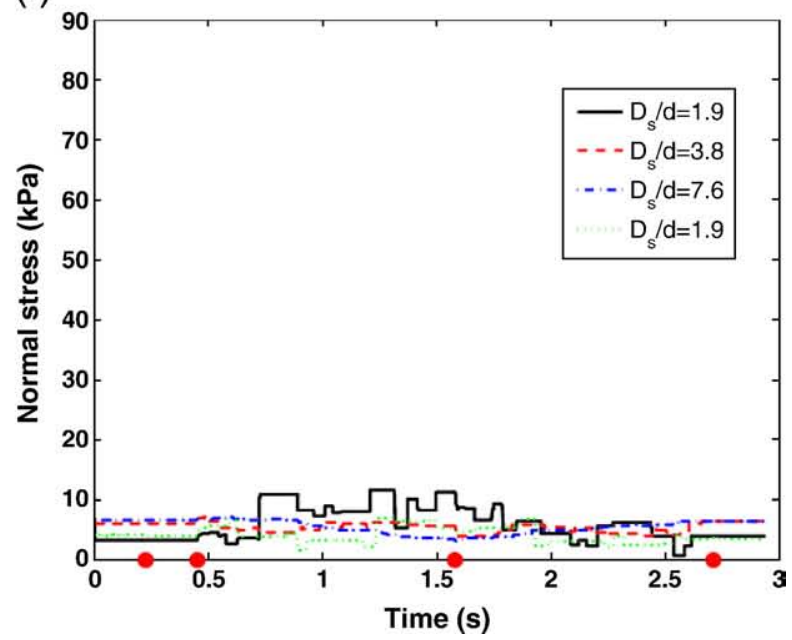

Fig. 14. Normal stresses recorded by the dynamic sensors vs. time. $H / D=0.7$ (a); 1.4 (b); 2.4 (c); 5.3 (d); 10.7 (e); and 21.3 (f). The first three legends are for sensors located initially at the center of the cylinder, while the fourth one is for the off-center sensor, unless denoted otherwise. The time points separating the loading, shearing, shear reversal and no-shear periods are marked by red dots on the time axis.

\section{Summary}

It is abundantly clear from our normal stress measurements using two sensors mounted on the shearing bottom surface of the commonly used Jenike shear cell that appreciable spatial inhomogeneities exist in the stress transmitted from the top to the bottom surface of the granular layer. Furthermore, each stress sensor records large temporal fluctuations in normal stress as the material is sheared. The spatial inhomogeneities (at any given time instant) and the temporal fluctuations (recorded by any one sensor) are of comparable 
(a)

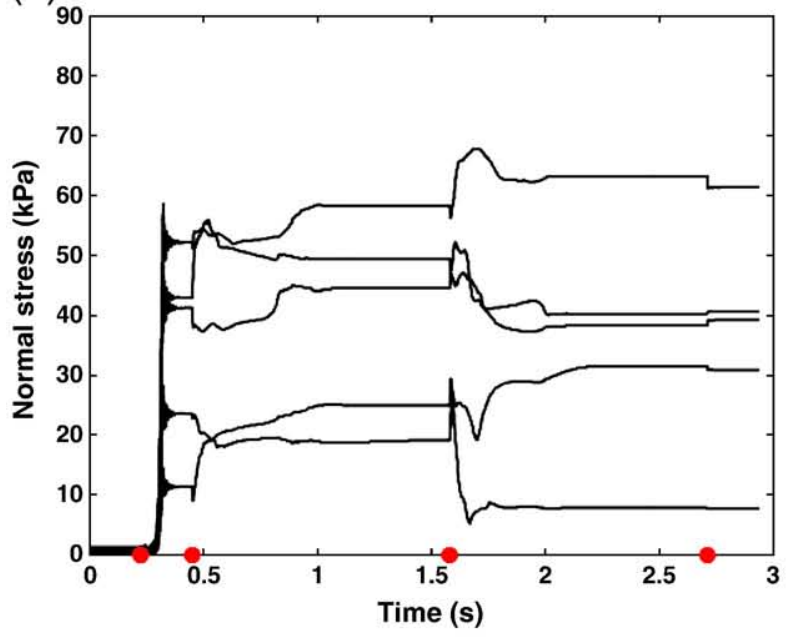

(c)

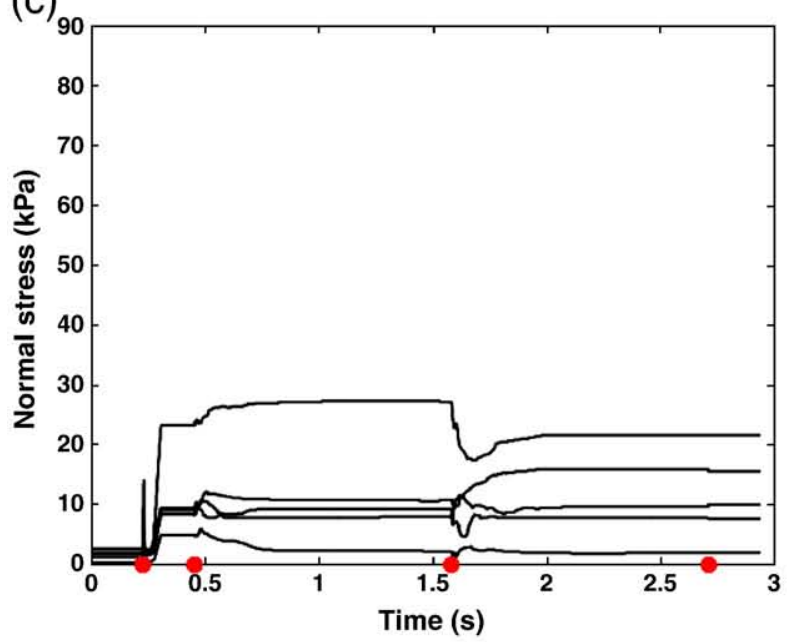

(e)

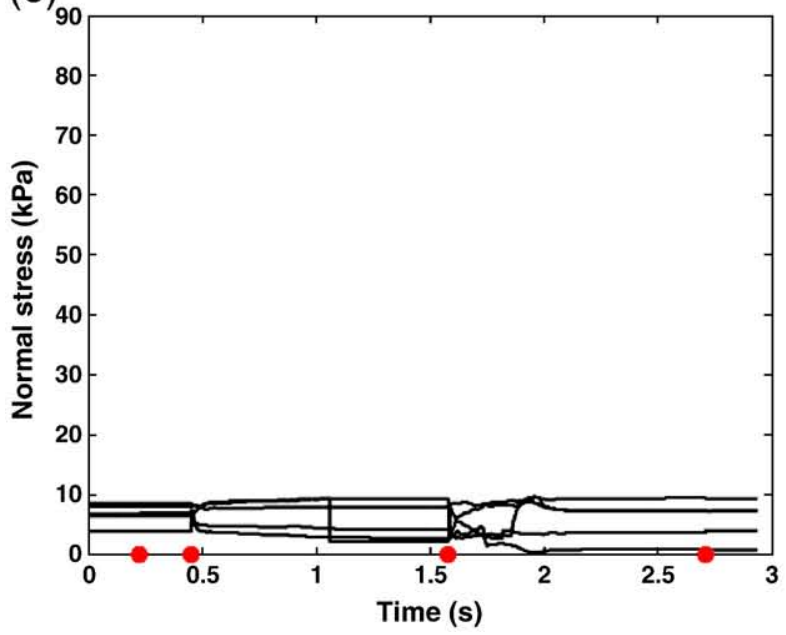

(b)

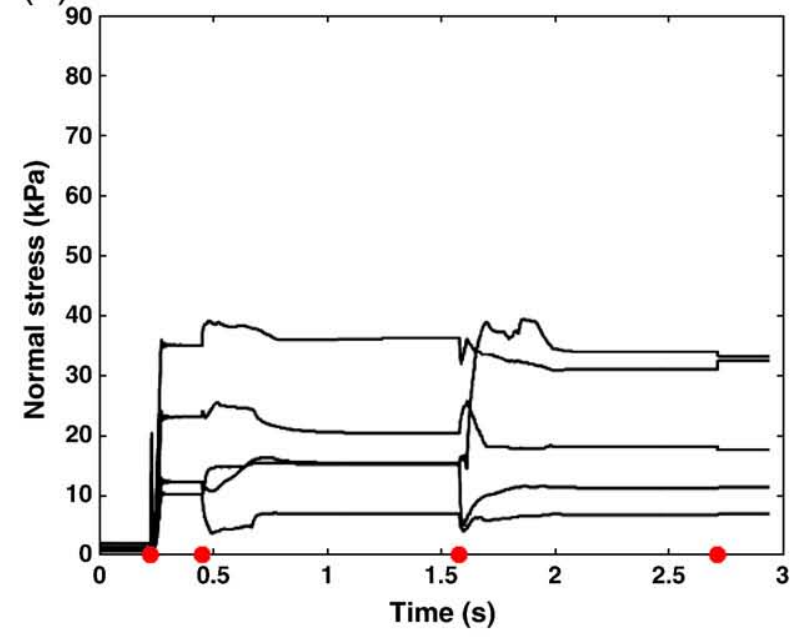

(d)

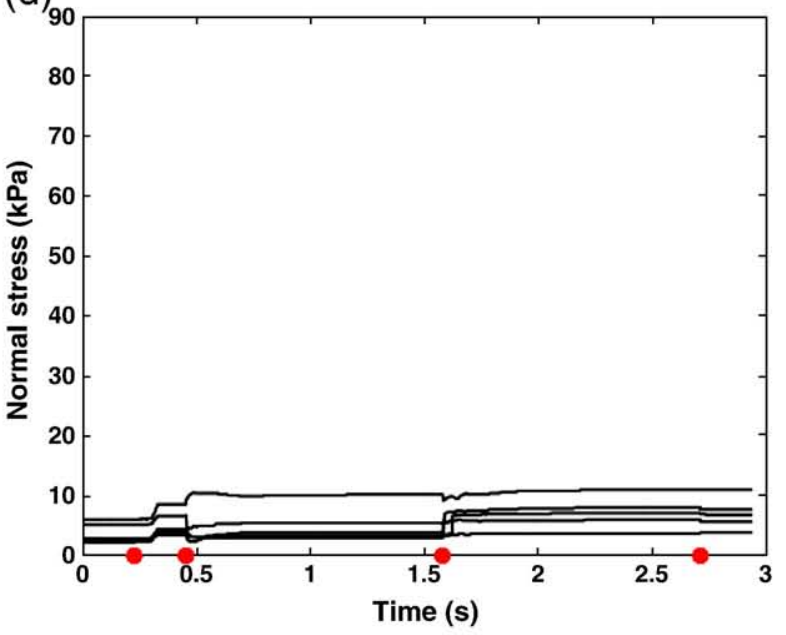

(f)

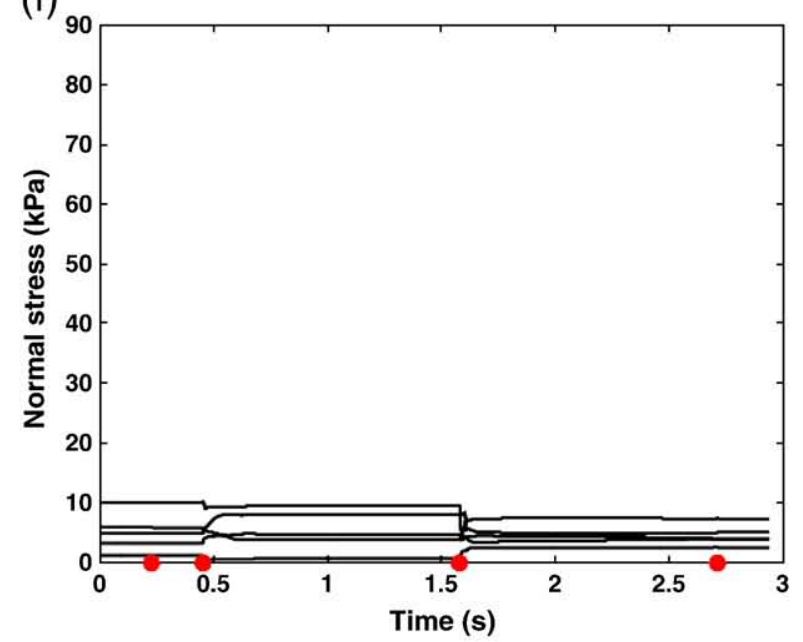

Fig. 15. Normal stresses recorded by the static sensors vs. time. $H / D=0.7$ (a); 1.4 (b); 2.4 (c); 5.3 (d); 10.7 (e); and 21.3 (f). The five sensors in each panel are located as follows: one is at the center of the cylinder's base and the other four are located $90^{\circ}$ apart on a circle with diameter equal to $0.5 \mathrm{D}$. Since the specific location of a sensor is not interest here, the lines in the plots are not differentiated. $D_{\mathrm{s}} / d=1$.9. The time points separating the loading, shearing, shear reversal and no-shear periods are marked by red dots on the time axis.

magnitude. The stress fluctuations recorded by a sensor became larger as the sensor diameter to particle diameter ratio was decreased, layer depth was increased and the assembly was sheared faster.
DEM simulations employing a spring-dashpot model with appropriate constants to describe the stiffness and plasticity of the material are able to qualitatively reproduce the spatial inhomogeneity and 
temporal fluctuations seen in the experiments, thus validating the simulations. Further DEM simulations performed using a combination of static and dynamic sensors reveal the link between the spatial inhomogeneity and temporal fluctuations in normal stress in the sheared granular layer inside the Jenike cell. Although we did not measure shear stresses, it is expected that the same spatial inhomogeneity and temporal fluctuations must exist for shear stresses as well.

The average normal stress transmitted to the bottom of a granular assembly is an exponentially decaying function of the ratio of the height of the granular assembly to the confining cylinder diameter $\mathrm{H}$ / $D$ and reaches an asymptotic value for large $H / D$. This is not controlled by the ratio of the cylinder diameter to the particle diameter $D / d$, nor by the number of layers of particles in the assembly $H / d$. This exponential relationship bears a similarity to that for wall-bounded static assembles as shown by Janssen's analysis.

$\begin{array}{ll}\text { Nomenclature } \\ d & \text { particle diameter }(\mathrm{mm}) \\ D & \text { Jenike cell diameter }(\mathrm{mm}) \\ D_{\mathrm{s}} & \text { sensor diameter }(\mathrm{mm}) \\ H & \text { granular column height }(\mathrm{mm}) \\ g & \text { gravitational acceleration }\left(\mathrm{m} / \mathrm{s}^{2}\right) \\ K & \text { Janssen constant } \\ L & \text { layer thickness }(\mathrm{cm}) \\ m & \text { mass of a particle }(\mathrm{kg}) \\ Q_{0} & \text { surcharge } \\ t & \text { time }(\mathrm{s})\end{array}$

\section{Greek letters}

$\phi \quad$ particle volume fraction

$\phi_{i} \quad$ angle of internal friction

$\mu \quad$ inter-particle friction coefficient

$\mu_{\mathrm{w}} \quad$ wall friction coefficient

$\rho \quad$ density $\left(\mathrm{kg} / \mathrm{m}^{3}\right)$

$\sigma \quad$ stress $(\mathrm{kPa})$

$\sigma_{z z} \quad$ normal stress on the bottom wall $(\mathrm{kPa})$

\section{Acknowledgments}

The authors wish to thank Merck and Co. Inc. for donation of the tablet press, Nova Chemicals Inc. and especially Mr. John Bailey for providing particles for testing in the Particle Technology Laboratory at CCNY. We also acknowledge the helpful discussions on the subject of powder flows with Dr. James Michaels of Merck and Co. Inc. and Dr. Paul Mort of the Proctor \& Gamble Company. This work was supported by a DOE-UCR grant DE-FG26-07NT43070.

\section{References}

[1] C.h. Liu, S.R. Nagel, D.A. Schecter, S.N. Coppersmith, S. Majumdar, O. Narayan, T.A. Witten, Force fluctuations in bead packs, Science 269 (1995) 513-515.

[2] D. Howell, R.P. Behringer, C. Veje, Stress fluctuations in a 2D granular Couette experiment: a continuous transition, Physical Review Letters 82 (1999) 5241-5244.

[3] D.M. Mueth, H.M. Jaeger, S.R. Nagel, Force distribution in a granular medium, Physical Review E 57 (1998) 3164-3169.

[4] E.I. Corwin, E.T. Hoke, H.M. Jaeger, S.R. Nagel, Temporal force fluctuations measured by tracking individual particles in granular materials under shear, Physical Review E 77 (2008) 061308-6.

[5] B. Miller, C. O'Hern, R.P. Behringer, Stress fluctuations for continuously sheared granular materials, Physical Review Letters 77 (1996) 3110-3113.

[6] R.M. Nedderman, Statics and Kinematics of Granular Materials, Cambridge University Press, 1992.

[7] S.J. Plimpton, Fast parallel algorithms for short-range molecular dynamics, Journal of Computational Physics 117 (1995) 1-19 URL: http://lammps.sandia.gov.

[8] P.A. Cundall, D.L. Strack, A discrete numerical model for granular assemblies, Geotechnique 29 (1979) 47-65.

[9] L. Aarons, S. Sundaresan, Shear flow of cohesive and non-cohesive granular material, Powder Technology 169 (2006) 10-21.

[10] J. Sun, F. Battaglia, S. Subramaniam, Dynamics and structure of segregation in a dense, vibrating granular bed, Physical Review E 74 (2006) 061307-061313.

[11] A.S.J. Suiker, N.A. Fleck, Frictional collapse of granular assemblies, Transactions of ASME 71 (2004) 350-358.

[12] Jenike, A.W., Storage and flow of solids, Bulletin of University of Utah, Bulletin No. 123, Vol. 53, No.26, 1964

[13] C.S. Campbell, Granular material flows - an overview, Powder Technology 162 (2006) 208-229.

[14] R. Jackson, Some features of the granular materials and aerated granular materials, Journal of Rheology 30 (5) (1986) 907-930.

[15] S. Luding, Shear flow modeling of cohesive and frictional powders, Powder Technology 158 (1-3) (2005) 45-50.

[16] P.R. Mort, G.I. Tardos, I. Talu, Characterizing the transition between the quasi-static and intermediate regimes in weakly cohesive granular flows, Paper Presented at the Annual AICHE Meeting, San Francisco, November, 2003.

[17] R. Moreno-Atanasio, S.J. Anthony, M. Ghadiri, Analysis of flowability of cohesive powders using discrete element methods, Powder Technology 158 (2005) 41-57 \# $1-3$.

[18] S.B. Savage, Analyses of slow high-concentration flows of granular materials, Journal of Fluid Mechanics 377 (1998) 1-26.

[19] D.G. Schaeffer, Instability in the evolution equations describing incompressible granular flow, Journal of Differential Equations 66 (1987) 19-50.

[20] A. Srivastava, S. Sundaresan, Analysis of a frictional kinetic model for gas-particle flow, Powder Technology 129 (2003) 72-85.

[21] G.I. Tardos, A fluid mechanics approach to slow, frictional powder flows, Powder Technology 92 (1997) 61-74.

[22] G.I. Tardos, M.I. Khan, D.G. Schaeffer, Forces on a slowly rotating, rough cylinder in a Couette device containing a dry, frictional powder, Physics of Fluids 10 (2) (1998) 335-341.

[23] G.I. Tardos, S. McNamara, I. Talu, Slow and intermediate flow of a frictional bulk powder in the Couette geometry, Powder Technology 131 (2003) 23-39.

[24] G.I. Tardos, P.J. Mort, Dry powder flows, Chapter 9, in: C. Crowe (Ed.), Multiphase Flow Handbook, CRC Press, Boca Raton, Florida, October 2005.

[25] S. Turek, A. Quazzi, R. Schmachtel, Multi-grid methods for stabilized nonconforming finite elements for incompressible flow involving the deformation tensor formulation, Journal of Numerical Mathematics 10 (2002) 235-248.

[26] C.-M. Yu, K. Craig, J. Tichy, Granular collisional lubrication, Journal of Rheology 38 (4) (July/August 1994) 921-936.

[27] C.-M. Yu, J. Tichy, Granular collisional lubrication: effect of surface roughness, particle size and solid fraction, Tribology Transactions 39 (3) (1996) 537-546. 
Appendix L

Effect of Material Properties, Boundary Conditions and Flow Fields on the Rheology of Dense Granular Matter 


\title{
Effect of Material Properties, Boundary Conditions and Flow Fields on the Rheology of Dense Granular Matter
}

\author{
Mehrdad Kheiripour Langroudi*, Gabriel I. Tardos*, \\ James N. Michaels**, and Paul Mort \\ *Department of Chemical Engineering, The City College of CUNY, New York, NY 10031 \\ **Center for Material Science and Engineering, Merck and Co. Inc., West Point, PA 19486 \\ IIvorydale Technical Center, Procter and Gamble, Cincinnati, OH 45217
}

\begin{abstract}
A traditional Couette device was modified by superimposing a slow axial flow on radial shearing thereby allowing the granular matter to move from quasi-static to the intermediate regime of flow upon a slight decrease in solid volume fraction. This increase in porosity provides enough space for random collision among particles so that the shear stress becomes dependent on the shear rate. Measurement of normal stresses inside the shearing zone (on the rotating cylinder) shows that in the intermediate regime the normal stress is linearly dependent on height. Also, the ratio of shear to normal stress remains constant at very low shear rates (solid-like behavior) but it increases exponentially by increasing the shear rate as the granular matter exhibits liquid-like behavior. This results in a modification to the "Schaeffer law" by introducing an extra power-law term into the constitutive relation. We found that in order to have a significant viscous-like effect, the roughness of the walls has to be close to that of the particle to prevent slip at the boundaries. We used in our experiments, materials with different size, size distribution and shape as well as materials with different bulk density, stiffness and surface roughness to find their effect on the rheological behavior of the bulk. We found that for the case of irregular shaped particles, frictional and collisional forces are higher. It was also found that soft particles show more liquidlike behavior than rigid particles, as the dependency of shear stress to shear rate is stronger for the case of soft matter.
\end{abstract}

Keywords: Couette Cell, Granular Flow, Rheology, Intermediate Regime, Normal Stresses, Friction Coefficient PACS: $47.57 . \mathrm{Gc}$

\section{INTRODUCTION}

Research in granular flows is motivated by numerous applications encountered in industrial processes such as the design of hoppers and mixers and in geophysics for the description of landslides and avalanches [1]. Previously, granular flows were divided into slow or quasistatic, where the deformations are very slow and the effective friction coefficient is taken to be a material property and thus constant. Interparticle force chains dominate this regime of flow and the local shear stress is proportional to pressure and is independent of shear rate. On the other end of the spectrum, the fast or rapid flow regime was identified, where particles interact through collisions. It was found that in this regime the local shear stress is a quadratic function of shear rate [2].

In order for the powder to move from the quasi-static to rapid granular flow, there must be a transition where particle mobilization and/or shear are strong enough to dissipate significant energy through particle collisions, but do not completely eliminate the continuous particle enduring contacts typical of quasi-static flows [3]. As a result, granular flows have more recently been classified into three regimes adding the Intermediate regime of flow where 


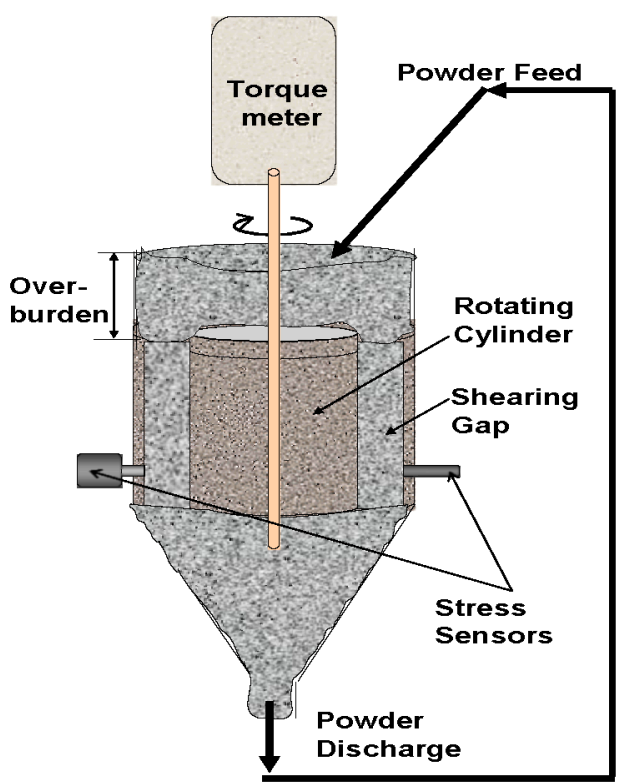

FIGURE 1. Schematic representation of the Couette device.

the material is dense but still flows [4]. While theories for dense granular flows in the quasistatic and rapid regimes are relatively well established, there are considerably fewer attempts in the literature (theoretical but especially experimental) dedicated to the intermediate regime where many practically important flows in nature and technical applications occur. In an attempt to obtain a constitutive law for granular flow, Schaeffer [5] used the Von Misses yield criterion and combined it with the co-axiality (Levy's) flow rule. The yield condition in his model is valid only for the slow regime ("Schaeffer law").

It was also shown theoretically that in order to change the regime of flow from quasi-static (dense, slow flow) to rapid granular (dilute and collisional), the solid fraction of the bed has to decrease [6] but most experiments in the Couette geometry (see for example [4]) were carried out at high packing [7] or with upward flowing gas, which invalidates the assumption of dense flow. The basic motivation for our work is to supply relevant experiments that allow the bed to dilate freely and for inter-particle collisions to occur. Moreover, there is theoretical evidence $[8,9]$ that in the intermediate regime the friction coefficient (the ratio of shear to normal stress) increases from its constant value.

\section{COUETTE FLOW EXPERIMENTS}

A schematic representation of the Couette shear cell used during this study is shown in Fig. 1.
The vertical shear gap forms between the rotating and the stationary cylinders and both its width and height can be adjusted by appropriate choice of the radius and height of the rotating cylinder. The material is fed from above using a vibratory feeder and is discharged by a screw-incylinder metering device that transfers the material to a balance for flow rate measurement. The walls of the Couette (inner and outer) are made rough by gluing sand paper on the shearing surfaces. The roughness of the walls is chosen to match or exceed the coefficient of internal friction of the material thereby assuring a nonslip boundary condition. The traditional Couette device is modified by attaching a conical hopper that enables vertical outflow and thereby a change in porosity of the bed. By feeding and draining a small amount of material to achieve a steady state, one can operate the device continuously. The material above the rotating cylinder (overburden in the Fig. 1) is stationary and only provides dead weight to the shearing layer. Normal stresses were measured inside the shearing zone on the inner, rotating wall of the device as shown in the figure. Average shear stresses were recalculated from the torque on the rotating cylinder using equation (1).

$$
\tau_{\text {ave }}=\frac{2 T}{\pi D^{2} L}
$$

In the above equation $\mathrm{T}$ is the required torque to rotate the inner cylinder and $\mathrm{D}$ and $\mathrm{L}$ are the diameter and length of the rotating cylinder, respectively.

Figure 2 shows the results of a continuous Couette experiment for $0.1 \mathrm{~mm}$ spherical glass. As seen, the shear stress exhibits two very distinct regimes: a quasi-static regime where the shear stress is mostly independent of the shear rate (at low shear rates) and an additional regime

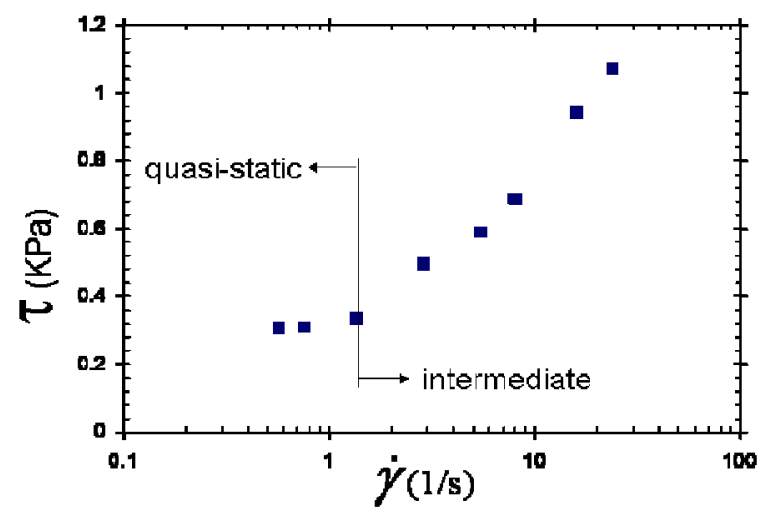

FIGURE 2. Couette experiment with $0.1 \mathrm{~mm}$ glass particles 
("intermediate") where the dependence takes the form of an increasing function. Further experiments with different inner cylinder lengths suggest that the shear stress is approximately linear as a function of height and depends strongly on shear rate (Fig. 2). We conclude that the value of the average shear stress will occur $a t$ the middle point over the length, $L$. Using a wireless technology we were able to measure normal stresses on the surface of the rotating cylinder. Their distribution appears to be linear as a function of depth and it confirms a liquidlike behavior in the intermediate regime. This finding allows the calculation of an average normal stress also in the middle of the cylinder (at $\mathrm{L} / 2$ ). Hence, the average shear and normal stresses are located at the midpoint of the rotating cylinder so that one can calculate their ratio. A typical result for $0.1 \mathrm{~mm}$ in diameter spherical glass beads is shown in Fig. 3. The ratio of the two stresses (apparent friction coefficient) at slow shear is constant and very close to the internal friction coefficient of glass particles. As the shear rate increases the friction coefficient becomes dependent on the shear rate. This behavior is mainly due to the superposition of collisions between particles on the sliding friction of surfaces. This kind of behavior was already suggested by simulations $[9,10]$. A power-law type curve is fitted to the data and given in Fig. 3: it shows a power-law coefficient of $n=0.73$ and a constant coefficient $a=0.45$ for zero shear rate.

\section{A NEW YIELD CONDITION AND CONSTITUTIVE EQUATION}

The above findings can be generalized to propose a yield condition that holds at low and higher shear rates. From Fig. 3 and by replacing

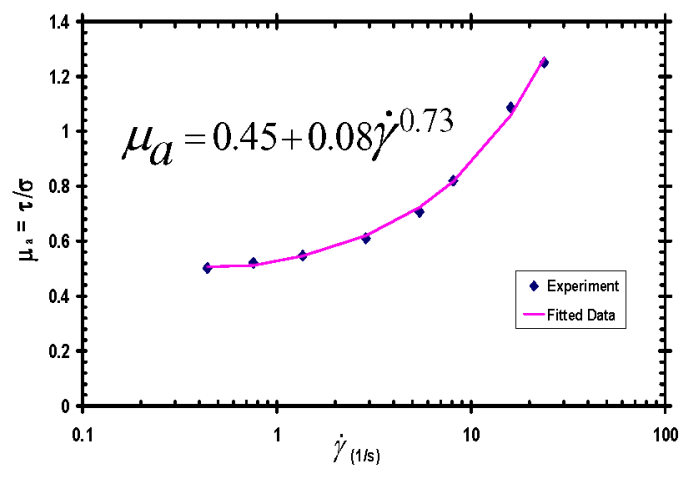

FIGURE 3. Friction coefficient for $0.1 \mathrm{~mm}$ glass beads. the constant coefficient a tan $\phi$, one can fit the following equation to the data:

$$
\frac{\tau}{\sigma}=\tan (\phi)+b|\dot{\gamma}|^{n}
$$

Where $\tau$ and $\sigma$ are the shear and normal stresses and " $b$ " and " $n$ " are coefficients characterized by the experimental curve. An equivalent representation of this experimental yield condition can be obtained from the characteristic Mohr circle by replacing $\sigma$ by $\mathrm{p}$ and $\tau / \tan \phi$ by $q / \sin \phi$, in the form:

$$
q=p \sin (\phi)+b p \cos (\phi)|\dot{\gamma}|^{n}
$$

Where $p$ is the isotropic pressure and $q$ is half of the difference between the principal stresses. Reconsidering Schaffer's law we can modify it by substituting equation 3 into the expression of the stress tensor to obtain: $T_{i j}=p \delta_{i j}+\tau_{i j}$

$$
T_{i j}=p \delta_{i j}+\frac{\sqrt{2} p \sin (\phi)}{\left|\dot{\gamma}_{i j}\right|} \dot{\gamma}_{i j}+\frac{\sqrt{2} b p \cos (\phi)\left|\dot{\gamma}_{i j}\right|^{n}}{\left|\dot{\gamma}_{i j}\right|} \dot{\gamma}_{i j}
$$

The second term in LHS of equation 4 corresponds to plastic deformation (solid-like) and the last term corresponds to the viscous behavior (liquid-like) of the granular material.

\section{EFFECT OF MATERIAL PROPERTIES}

The effects of size, shape and surface roughness on the rheological behavior of the bulk were considered to generalize our constitutive equation. Spherical and crushed glass particle ranges $0.1 \mathrm{~mm}$ to $1 \mathrm{~mm}$ as well as smooth and rough Polyethylene were considered in this study. By increasing the size of spherical glass the range of shear rate for different regimes changes but other rheological properties remain almost unchanged. Comparison between $1 \mathrm{~mm}$ round and crushed glass shows that the shape has an important role on the rheology of bed. The crushed glass particles with very rugged and odd-shaped require more increase in porosity (higher axial draining rates) before they start to "flow" due to shape-interlocking effects. Moreover results of the apparent friction coefficient revealed that the power-law index "n" 


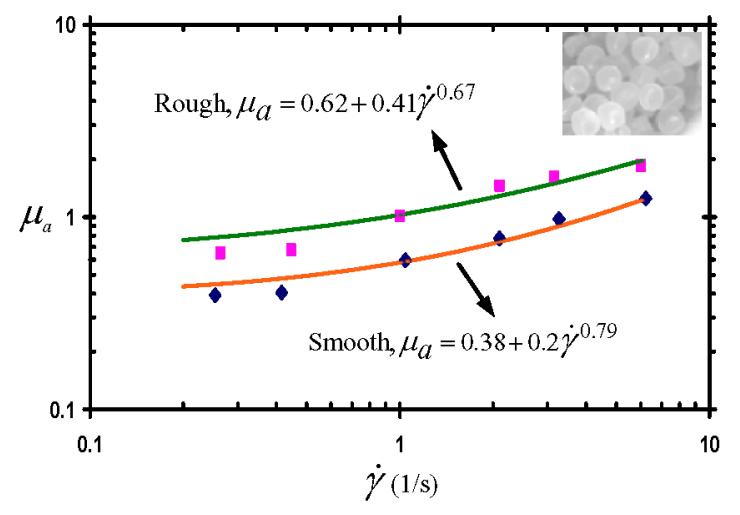

FIGURE 4. Coefficient of friction for $4 \mathrm{~mm}$ in diameter rough and smooth Polyethylene particles.

for the crushed glass is much higher (1.03) as opposed to $\mathrm{n}=0.72$ of the round glass. This shows that collisional effects for crushed glass are larger than those of round glass. Irregularshaped and broader size distribution increase contact areas of particles so that the viscous term of the constitutive equation becomes dominant.

The effect of surface roughness was considered using smooth $\left(\phi=26^{\circ}\right)$ and rough $\left(\phi=37^{\circ}\right) 4 \mathrm{~mm}$ in diameter Polyethylene. We found that rough and smooth materials of the same size and shape behave differently. Figure 4 shows the friction coefficient of smooth and rough Polyethylene at different shear rates, the fitted equation for each case and a picture of the particles. The coefficients " $a$ " is higher due to higher surface friction, as one would expect. It can also be understood from Fig. 4 that smooth particles exhibit a larger coefficient " $n$ " ( 0.79 as compared to 0.67 for rough particles) because the smooth particles can move easier and collisions can occur more frequently than the rough particles, which dissipate more energy, by friction.

\section{CONCLUSIONS}

Experimental results revealed that the material moves from the quasi-static to the intermediate regime of flow only if a small amount is continuously drained in the axial direction so that an increase in porosity can take place. Under this condition the Couette device can be used as a rheometer to characterize the rheological behavior of materials in the form of an "apparent friction coefficient". We found that this coefficient reaches a constant value " $a=\tan (\phi)$ " when the shear rate tends to zero. At higher shear rates the apparent friction coefficient shows a power-law dependence that characterizes the viscous behavior in the intermediate regime. The above considerations are of great theoretical value since the ratio of the shear to normal stress in a powder can be regarded as a yield criterion and can be used as a constitutive equation. This was done earlier by Schaeffer [5] to develop his equations for the quasi-static regime; in that case however, only the coefficient "a" was used. The new equations obtained directly from measurements, enable for the first time the development of similar equations for the intermediate regime where the powder exhibits some fluid-like behavior superimposed on friction so that a visco-plastic constitutive law was derived.

We also found that while the sizes of particles only shifts the range of shear rate for different regimes, shape and surface roughness affect the rheology of the bulk. The odd-shaped particles with the same average size as spherical particles yield a higher power-law index. The rough and smooth materials of the same size and shape behave differently: the rough shows higher constant coefficients "a" due a larger friction coefficient and the smooth particles have a higher exponent " $n$ " because of less force chains (due to smaller friction) and more freedom of movement.

\section{ACKNOLEDGEMENTS}

The authors wish to thank Nova Chemicals Inc. and especially Mr. John Bailey for generously providing all types of Polyethylene granules to the particle technology lab at CCNY.

\section{REFERENCES}

1. Y. Forterre and O. Pouliquen, Annu. Rev. Fluid Mech. 40, 1-24 (2008).

2. C. S. Campbell, Annu. Rev. Fluid Mech. 22, 57-92 (1990).

3. GDR MiDi., Eur. Phys. J. E 14, 341-65 (2004).

4. G.I. Tardos, S. McNamara, and I. Talu, Powder Technol. 131, 23-39 (2003).

5. DG. Schaeffer, J. Differ. Equat. 66, 19-50 (1987).

6. G. Lois, A. Lemaître, and J. M. Carlsonl, Phys. Rev. E 76, 021302 (2007).

7. W. Losert, L. Bocquet, TC. Lubensky, and JP. Gollub, Phys. Rev. Lett. 85, 1428-1431 (2000).

8. P. Jop, Y. Forterre, and O. Pouliquen, Nature 441, 727-730 (2006).

9. F. da Cruz, S. Emam, M. Prochnow, JN. Roux, and

F. Chevoir, Phys. Rev. E. 72, 021309 (2005).

10. C. S. Campbell, C. S., Powder Technol. 162, 208229 (2006). 
Appendix M

Importance of Solid Fraction and Its

Fluctuations on the Rheology and

Flow Characteristics of Compressible Powders 


\section{Elsevier Editorial System(tm) for Mechanics Research Communications}

Manuscript Draft

Manuscript Number:

Title: Importance of Solid Fraction and Its Fluctuations on the Rheology and Flow Characteristics of Compressible Powders

Article Type: Complete Paper

Keywords: Powder Rheology, Compressible Powders, Solid Fraction, Flowability, Packing

Corresponding Author: Professor Gabriel I. Tardos, Ph.D., D.Sc.

Corresponding Author's Institution: The City College of the CUNY

First Author: Mehrdad Kheiripour Langroudi, Ph.D. Candidate

Order of Authors: Mehrdad Kheiripour Langroudi, Ph.D. Candidate; Gabriel I. Tardos, Ph.D., D.Sc.

Abstract: This paper is an attempt to find the connection between rheology, dilatancy and flowability of compressible powders. Rheological properties were obtained in a previously developed Couette shearing cell with secondary axial flow (Kheiripour et al., 2009) and the result is expressed by a powerlaw function containing a power-law index, "n". The solid volume fraction was measured inside the shear band using a capacitance probe. To test for flowability through an orifice under high shearing rate, a new device was developed where a rotating disc with an orifice passes under a cylindrical hopper filled with powders. We found that the ability of a powder to dilate or pack during flow plays a critical role in its rheology and flowability. Our results showed that the dependency of shear stress to shear rate decreases for particles with a propensity for more dilation at increasing shear rates. Increasing shear rate requires higher shear stress but at the same time, dilation requires less shear stress so that the power-law index " $n$ " decreases. Powders with a tendency to stay in a packed situation even at high shearing rates (large rheological index " $n$ " i.e. large shear stress) can flow better though an orifice. In other words powders with lower values of the index "n" actually flow through the orifice at an overall lower mass flow rate than powders that pack better and require a larger shear stress to flow. In fact large shear force (high packing) was favorable for filling the orifice during the spinning disc experiment. The major reason for different packing ability of powders was found to be the shape and surface textures of the powders as obtained from SEM analysis. The major conclusion of this work is that the knowledge of the packing fraction (solid volume fraction) is key to understanding the rheology and flowability of powders. 


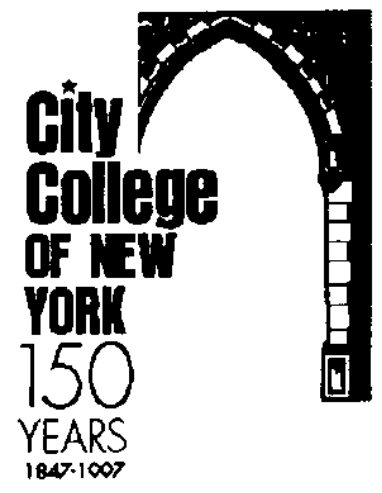

Gabriel I. Tardos, Professor Department of Chemical Engineering School of Engineering

\section{Dear Dr. Rosato,}

THE CITY COLLEGE

OF

THE CITY UNIVERSITY OF NEW YORK

CONVENT AVENUE at 140TH STREET

NEW YORK, NEW YORK 10031

I would like to submit the attached manuscript, "Importance of Solid Fraction and Its

Fluctuations on the Rheology and Flow Characteristics of Compressible Powders", for possible publication in Mechanics Research Communications, a journal that has established a very good reputation in meeting the high levels of academic standards.

This paper has not been submitted for publication nor has it been published in whole or in part elsewhere. I attest to the fact that all authors listed on the title page have read the manuscript, attest to the validity and legitimacy of the data and its interpretation. In this novel paper we studied for the first time the flow of compressible powders from a container with a moving orifice and this problem has direct industrial applications in tableting of powders. We also investigated the effect of packing on rheology and flowability of compressible powders. It took us one year for completing the research involved in this paper and it will be a very crucial chapter of Mehrdad's (first author) doctoral dissertation. Moreover we have published the following papers recently on incompressible powders and all of them have been referred in the manuscript we submitted to you. In fact this new paper deals with compressible powders considering the effect of solid fraction changes in rheology and flowability.

1- Kheiripour Langroudi, M., Tardos, G., Michaels, J. and Mort, P., 2009., 1145, 587-590.

2- Kheiripour Langroudi, M., Turek, S., Ouazzi, A., and Tardos, G., 2010., Powder Technol.

3- Kheiripour Langroudi, M., Sun, J., Sundaresan, S. and Tardos, G., 2010., Powder Technol.

4- Kheiripour Langroudi, M., Mort, P. and Tardos, G., 2010., Accepted in Granular Matter.

Thanks for the opportunity to submit our work. Your time and attention are much appreciated, and I look forward to hearing from you.

Sincerely,

Gabriel I. Tardos 


\section{Introduction}

Granular materials behave differently when they are flowing slowly or rapidly. The behavior of rapidly flowing granular materials, where the particle-particle interactions occur largely through binary collisions, is commonly modeled by the kinetic theory (Lun et. al., 1984). These kinds of flows are called "rapid granular" or "inertial". In static or slowly moving assemblies of granular materials, also called "quasi-static" or "slow-frictional" flows, enduring contacts between particles prevail and sliding friction is the predominant force between particles. The description of the rheology in the regime between the rapid and slow flows, denoted in this context "the intermediate regime", is more complicated since it includes properties from both the slow and the "rapid" regimes (Tardos et. al., 2003; Forterre and Pouliquen, 2008). Particle mobilization and/or shear in the "intermediate regime" are strong enough to dissipate significant energy through particle collisions, but do not completely eliminate the continuous particle enduring contacts typical of quasi-static flows (GDR MiDi, 2004). Flows in the intermediate regime, it turns out, are the most prevalent in industrial practice but least studied and understood.

In an attempt to obtain a constitutive law for granular flows, Schaeffer (1987) used the von Misses yield criterion and combined it with the so-called co-axiality (Levy's) flow rule. These two conditions were necessary and sufficient to yield a non-trivial constitutive equation that can be used in modeling. The yield condition in this model is valid only for incipient or very slow motion so that Schaeffer's law is unable to calculate flow fields and stress distributions at higher shearing rates as are characteristic of the intermediate regime (Tardos, et. al, 1998). Tardos et al. (2003) found experimentally that the porosity, $\varepsilon$, of the bed (solid fraction, $c=1-\varepsilon$ ) has a large influence on the shear stress and on the width of the intermediate regime: at high porosity, the intermediate regime is quite narrow while at low porosity, the intermediate regime spans almost the entire range of shear rates tested. Based on these findings, a map of flow regimes was constructed as given in Figure [1]. It should be noted that when the solid fraction is at its maximum value there is no path from quasi-static to the rapid regime of flow as the presence of force chain will not allow for particle movement to occur (Kheiripour et. al., 2009).

It was shown in our previous work (Tardos, 1998), that the simplest relevant geometry in which powder-flows can be studied is a mass-flow hopper for which an analytical solution is available for both incompressible and compressible flows. Unfortunately, the geometry is too simplistic and the stresses too low to be detected by existing instrumentation so that a well-defined constitutive equation cannot be obtained. Hence, a slightly more complicated geometry had to be used, that of the Couette device (Tardos, et. al., 2003) were the solid fraction of the bed is close to its maximum value. Even this geometry, well-studied in the field of Non-Newtonian Fluid Mechanics, had to be modified for powders by adding a slow axial flow over-imposed on the radial shear field, in order to allow the powder to actually dilate (Kheiripour et. al., 2009). Furthermore in this geometry we were able to measure shear (from torque) and normal stresses (with a remote sensor that has been developed in Kheiripour et al, 2010a) inside the active shearing zone of the granular layer and we found that the regime of flow can be shifted to intermediate from quasi-static if the particles can dilate (collisions can occur). A numerical scheme (FEM) was able to use the continuum equations of motions and validate the experimentally obtained data (Kheiripour et. al., 2010b). In an attempt to study the flow pattern of granular matter, it was revealed that the Couette geometry with a secondary axial flow has the 
advantage of an increased width of the shear band so that the continuum approach becomes more reasonable (Kheiripour et. al., 2010c).

The basic motivation for this work is to investigate the impact of solid fraction on the rheology of compressible powders and to find a relationship between the dilatancy and flowability through an orifice in a continuous type of flow for compressible powders. There is a lack of relevant experiments that allow the granular bed to dilate freely and for inter-particle collisions to occur during flow. In addition, as soon as gravity is introduced into the plane shear geometry or if the shearing is performed in a rotational shear cell, the stress distribution is no longer homogeneous across the shearing zone and the flow-fields are not uniquely defined.

\section{Couette Cell-Rheological Properties and Solid Fraction Measurements}

To study the rheological behavior of compressible powders and to consider the effect of dilatancy, we use a Couette-type cell in which the particles are sheared in the gap between an inner rotating vertical, rough cylinder and an outer stationary cylindrical (also rough) wall. A schematic representation of the modified version of the Couette device used during the present investigation is shown in Figure [2]. The vertical shear gap forms between the rotating and the stationary cylinders and both its width and height can be adjusted by an appropriate choice of the radius and height of the rotating cylinder. The material is fed from above using a vibratory feeder and is discharged by a metering device that transfers the material to a balance for flow rate measurement. The walls of the Couette are made rough by gluing sand paper on the shearing surfaces. The roughness of the walls is chosen to match the coefficient of internal friction of the material thereby assuring a non-slip boundary condition.

All Couette experiments were performed with the four inches in diameter and eight inches high, rotating cylinder and six inches in diameter outer housing with relatively low overburden of approximately four inches. The size of the shear gap in all cases was one inch. By closing the discharge section, the Couette device can be operated in batch mode or, by feeding and removing material to achieve a steady state vertical flow; one can operate the device continuously. Couette experiments in this study were obtained in the presence of a secondary axial flow (continuous mode) to make sure that both, collisions i.e., a quasi-static, shear-rate-independent and an intermediate, i.e., shear-rate-dependent shear stress (torque), can develop.

A capacitance probe (Figure [3], Capacitec, Boston, Ma) was attached to the Couette cell to measure solid volume fraction of powders outside and inside of the shear band. The active portion of the probe is about $10 \mathrm{~mm}$ in diameter and approximately $30 \mathrm{~mm}$ in length. To measure the solid fraction on the outer wall, the probe is mounted flush to the surface so that it does not disturb the flow. For measurements inside the shearing zone, the probe is inserted to a certain distance from the wall but due to its small dimensions it is expected to only disturb he flow in its vicinity. Furthermore, the "measuring" volume is situated at the inner end of the probe where disturbances due to its presence are minimal. 
In this study we selected two different polyethylene powders and physical properties of these materials are summarized in Tables [1]. One can see from Table 1 that the two materials are quite different in size and size distribution in that PE-2 is about twice as large compared to PE-1 in average particle size and also somewhat less dense. Also, in order to consider the effect of shape and surface texture, scanning electron microscopy (SEM) for the powders have been performed and Figure [4] shows the pictures. It can be clearly seen that PE-2 is a roundish agglomerate with very rough walls while PE-1 is a cleaved particle with different shapes and a wide particle size distribution.

To measure the shear and normal stresses on the shearing wall, and the ratio between them (friction coefficient), we follow the methodology described in Kheiripour et. al (2010a\&b). Extensive measurements of the normal stresses showed that the normal stresses are linear with cylinder height. This conclusion was reached by mounting three identical normal stress sensors on a cylinder 16 inches long and recording the stress. This measurement showed unequivocally that the stress is indeed linear with cylinder height (length) and therefore the average normal stress is located at the midpoint of the rotating cylinder as is the shear stress calculated from the torque on the cylinder (Kheiripour et. al., 2010b). Under these conditions, the "friction coefficient", can be calculated by dividing the values of the average shear stress to the average normal stress. Figure [5] and [6] show the measured normal and shear stresses on the rotating rough cylinder. The stress is given as a function of the product between the shear rate and the particle diameter $\left(\dot{\gamma} d_{p}\right)$ so that all data for the two materials could be shown together on the same graph.

The same scale is used in Figure [7a] where the "apparent friction coefficients" for the two polyethylenes is given as the ratio of the two stresses. At slow shear, the ratio is constant and very close to the internal friction coefficient of the particle. As the shear rate increases the friction coefficient becomes dependent on the shear rate. This behavior is mainly due to the superposition of collisions between particles on the sliding friction of surfaces. This kind of behavior was already suggested by simulations (da Cruz, et. al., 2005, Campbell, 2006) and was validated experimentally for glass beads, i.e. incompressible powders, (Kheiripour et. al., $2010 \mathrm{a} \& \mathrm{~b})$. The equations fitted to the experimental data are shown in Figure [7a] and indicate that the power-law index of the dependency of the apparent friction coefficient on shear rate is higher for PE-2 as compared to PE-1. We showed before (Kheiripour et. al., 2010a) that the yield condition obtained from the Couette experiment can be generalized to generate a constitutive equation that holds at both low and higher shear rates. Since the data in Figure [7a] are close for the two powders at low shear rates and also close to the tangent of the angle of internal friction, a $\tan \phi$, one can fit the following equation to the data:

$$
\mu=\frac{\tau}{\sigma}=\tan (\phi)+b|\dot{\gamma}|^{n}
$$

where $\tau$ and $\sigma$ are the shear and normal stresses and " $b$ " and " $n$ " are coefficients characterized by the experimental curve. An equivalent representation of this experimental yield condition can be obtained from the characteristic Mohr circle by replacing $\sigma$ by $\mathrm{p}$ and $\tau / \tan \phi$ by $q / \sin \phi$, in the form: 


$$
q=p \sin (\phi)+b p \cos (\phi)|\dot{\gamma}|^{n}
$$

Here $\mathrm{p}$ is the isotropic pressure and $\mathrm{q}$ is half of the difference between the principal stresses. Reconsidering Schaffer's (1987) law we can modify it by substituting equation (3) into the expression of the stress tensor to obtain: $T_{i j}=p \delta_{i j}+\tau_{i j}$

$$
T_{i j}=p \delta_{i j}+\frac{\sqrt{2} p \sin (\phi)}{\left|\dot{\gamma}_{i j}\right|} \dot{\gamma}_{i j}+\frac{\sqrt{2} b p \cos (\phi)\left|\dot{\gamma}_{i j}\right|^{n}}{\left|\dot{\gamma}_{i j}\right|} \dot{\gamma}_{i j}
$$

So that the deviatoric stress tensor $\left(\tau_{i j}\right)$ takes the form:

$$
\tau_{i j}=\eta\left(\dot{\gamma}_{i j}, P\right) \dot{\gamma}_{i j}=\left[\frac{\sqrt{2} p}{\left|\dot{\gamma}_{i j}\right|}\left(\sin (\phi)+b \cos (\phi)\left|\dot{\gamma}_{i j}\right|^{n}\right] \dot{\gamma}_{i j}\right.
$$

The viscosity term in this equation is:

$$
\eta\left(\dot{\gamma}_{i j}, P\right)=\frac{\sqrt{2} p}{\left|\dot{\gamma}_{i j}\right|}\left(\sin (\phi)+b \cos (\phi)\left|\dot{\gamma}_{i j}\right|^{n}\right.
$$

The second term on the RHS of equation (3) corresponds to plastic deformation (solid-like) and the third term on the RHS corresponds to the viscous behavior (liquid-like) of the material. These equations become important when one tries to classify the powders with respect to "flowability" and/or model the flowing system using a continuum approach. To understand the reason for the different rheological behavior of the two polyethylenes, which are different in their shape and surface texture (see Figure [4]), we measured the solid volume fraction close to the inner rotating cylinder.

It is well known that in general, powder flows are extremely complicated and the powders studied here are no exception. Furthermore, the present powders are compressible, a property that adds more complication. While the stresses required by the powder to flow are well represented by the constitutive equation given for all powders in Table [2], porosity or solid fraction also plays an important role. To understand in more detail the rheological model (Table [2]) we study the difference in behavior of powders in terms of their capability to change their solid fraction. It is well known that dilation or a decrease in solid fraction is necessary for powders to start flowing. Providing axial flow in the Couette device allows a decrease in solid fraction (Kheiripour et. al., 2010c) and changes the regime of flow from quasi-static to intermediate. The level of stress is higher for the material that has more ability to pack under identical conditions. If the packing of particles becomes looser by increasing the shearing rate, the shear stress-shear rate dependence decreases. In this situation the number of collisions increases by increasing shearing rate but at the same time the bulk density decreases. Furthermore, fluctuations of the solid fraction under high shearing rates can also affect the flowability of powders. We found, for example, that an incompressible, free flowing powder 
behaves differently outside and inside of the active shearing zone. Increasing shearing rate packs the material outside of the zone and by following the variation of solid fraction of the granular layer from outside to inside, the bulk undergoes dilation. In addition, the solid fraction decreases by increasing shearing rate inside the active shearing zone.

We consider the behavior of the powders inside the active shearing zone where the actual shearing occurs (Figure [7b]). In this figure solid fraction data is represented at different shearing rates for the two polyethylene powders PE-1 and PE-2. It can be clearly observed that the higher dependency of shear stress to shear rate (larger power law index, n, see Table 2) is for the particle with less propensity to dilate when the shearing rate increases. This is due to the fact that large decrease and fluctuations in solid fraction by increasing shear rate results in more stress reduction. On the other hand it is true that faster collisions (that occur at higher shearing rates) require more force but at the same time the system became more dilute and less contacts between the particles reduce the force so that there is always a competition between theses two effects. One can see that the powder with the larger power law index "n" (PE-2) shows the least variations in solid fraction and by increasing the shearing rates the system packs to some degree. On the other hand the powder with a smaller power law index " $n$ " (PE-1) dilates the most and the fluctuation of solid fractions for this powder are very high. We can see that dilation and its fluctuation has a very important role on the rheology of powders. The more dilute system requires less shear stress for the entire range of shearing rates.

The behavior outside of the shear band is different, powders generally pack more by increasing shearing rates and the fluctuation of solid fraction is less compared to the behavior inside of the zone (Kheiripour et. al., 2010c). Also the fluctuation of solid fraction at low shear rate is higher than that at high shear rate and finally dilation of material occurs if we move from the outer to the inner shearing zone (this dilation should be more significant for a compressible powder).

\section{Spinning Disc-Flowability vs. Rheology}

In order to find the relationship between rheological properties of the powders and their ability to flow we designed a test using a spinning disc to extract powder from a hopper. In this experiment, a cylindrical vessel (with a conical feeding hopper on top) is placed on a flat moving disc with one single orifice located at a certain distance (approximately $10 \mathrm{~cm}$ ) from the center of the disc. The role of the conical hopper is for continuous feeding and discharge to achieve steady-state flow with constant overburden and zero stress on top of the cylindrical hopper. The capacitance probe was mounted flush to the inner wall of the hopper for the measurement of solid fraction. Figure [8a] shows a schematic representation of the device while Figure [8b] depicts a picture. When the orifice of diameter, D reaches under the hopper, material fills the orifice and is spilled onto the balance as shown. When the orifice moves out from under the hopper, the flow is interrupted and no material reaches the balance. The disk is put in rotation by a variable speed precision torque-meter so that its speed can be adjusted at will to up to 250 RPM. The shear gap between the hopper and disk is about 300 um (so that in general, the powder cannot escape through the gap). Since slip is always present, the actual velocity of the powder is somewhat lower than the set velocity of the disk. The balance under the spinning disk measures 
the total mass flow rate through the orifice as the disk rotates. Having the capacitance probe mounted on the cylindrical hopper (close to the orifice) enables the measurement of the packing or dilation (solid volume fraction) of powders at low and high shearing rates.

Figure [9a] shows the flow rate $(\mathrm{g} / \mathrm{s})$ at different rotational speeds for the two polyethylene powders (PE-1 and PE-2). In order to obtain a meaningful quantity of material that fills the orifice each time it passes under the hopper, the mass flow rate (as a function of time) was divided by the number of times the orifice passes under the hopper (RPM) and the data is given in Figures [9b] (the amount of material flowing through the hole at each pass under the hopper) as a function of rotational speed. Figure [9] clearly shows that the best flowability through an orifice under high shearing rates is achieved for the powder with highest rheological index " $n$ " which is the one with highest packing (least dilation) during Couette flow. One should note that the bulk density for PE-2 is somewhat lower than that of PE-1 so higher flowability should be expected for PE-1 according to the Beverloo equation. In reality however, quite the opposite appears to be true for the case of the moving orifice. Looking at the shape and surface texture of the powders one can see that rough and agglomerate-like (PE-2) powders pack more (stay at higher solid fraction) and flow better than the smooth and crystal-like powders (PE-1) that show more dilation during flow. Both higher stresses (larger index " $n$ ") and densities for a powder appear to be favorable in filling the orifice. It can also be seen from Figure [9] that the overall mass flow rate per pass decreases as a function of shear rate as the time that the orifice spends under the hopper also diminishes (at increasing rotation rates). In an industrial application, this diminishing flow rate has a limit below which the orifice does not fill completely with powder and that provides an "upper limit" of the velocity (rotation rate) of the disk (velocity $\mathrm{U}_{0}$ in our apparatus, see Figure 8).

An additional interesting result was obtained by measuring the solid fraction inside the cylindrical hopper while the particles are filling the orifice. Figure [10] shows the results for PE2 (the powder with the higher mass flow rate) and PE-1 (the powder with lower mass flow rate) for different ranges of the rotational speed. The solid fraction appears constant in the figure (no fluctuations) when the rotation rate is zero (0 RPM). One can also see that for rotational speeds in the range of 50-75 RPM the mass flow rate of the two powders are close and the data for PE-1 fluctuates very little. At higher rotational speeds, the material PE-1 dilates and fluctuates much more than PE-2; this appears to be the reason for its poor mass flowability through the orifice. This finding is similar to what we found in the Couette cell as the powder that shows less dilatancy (with higher amount of stresses at similar shearing rates) can flow better (higher mass flow rate) into an orifice. The high packing (solid fraction), due to surface texture, is responsible for high shear stresses and higher mass flow rate.

\section{Conclusions}

Two compressible powders where tested in our continuous axial flow Couette device as well as in a specially constructed "spinning disk" (or "tablet filling") apparatus to determine their rheology (shear-stress shear-rate dependence) and flowability (flow rate through the moving orifice as it passes under the hopper). In both experiments, the solid volume fraction of powders during flow, were measured. Results of the Couette experiment (rheological model and solid fraction) as well as spinning disc experiment revealed that the powders with higher dependency 
of shear stress to shearing rate (larger rheological index "n") exhibit higher flowability through an orifice under high shearing rates. These powders require more shear forces to flow and they pack more under shearing (higher solid fraction). The powder with a good ability to dilate exhibited a smooth surface texture while the powder with a good ability to pack had a "rough", aggregate-like surface texture. PE-1 showed the highest fluctuation of solid fraction, which results in a decrease in the index " $n$ ", makes the system "fluffy" and prevents the filling of the orifice at high mass flow rate. Powder PE-2 has the highest index "n" (highest shear stress) and least fluctuation of solid fraction, which results in the system to be more packed and the presence of large stresses which are favorable for filling the moving orifice. The powder that shows more fluctuations in solid fraction at high shearing rates (PE-1) can dilate more and pack less under identical condition. This behavior explains why these powders are very different in terms of rheology. The decrease in solid fraction and its fluctuations at high shearing rates decreases the dependency of shear stress to shear-rate as a more dilute system requires less torque (shear stress).

The main reason why a powder packs or dilates during flow may be its surface texture and its shape. Aggregate-like particles (PE-2) generate higher shear stresses at the same shearing rate to flow (larger value of "n") but pack better (show higher solid fraction and lower porosities) when sheared. This high stress can favor packing so that they can fill the orifices better. Quite the opposite is true for a crystalline and ordered surface texture particle (such as PE-1) with low flowability and a smaller rheological index " $n$ ". The flow is more erratic and "fluffy" with higher porosity (lower solid fraction) due to its smoother surface texture and lower shear forces. These can generate different mass-flow rates and different flow behavior in industrial equipment. The above findings also stress the importance of measuring the solid fraction in addition to the powder's constitutive equation to better characterize its behavior. We also found that when PE-1 does not dilate and the fluctuations in solid fraction are small at low shear rates, it flows in a similar way as PE-2 confirming that packing ability is a key addition to the rheology.

\section{Acknowledgements}

The authors thank NOVA Chemicals and Mr. John Bailey in particular for providing the polyethylene particles and Dr. Kevin Sung for performing the SEM for this study. We are also indebted to Professor Michel Y. Louge of Cornell University for his advice in using the capacitance probe as well as his help for the installation and calibration of the device. This work could not have been done without fruitful discussions with Drs. Paul Mort of Procter \& Gamble and James Michaels of Merck. The financial support for this research comes from the Procter \& Gamble Company and The Graduate Center of CUNY.

\section{References}

Campbell, C. S., 2006. Granular material flows - An overview. Powder Technol., 162, 208-229.

da Cruz, F., Emam, S., Prochnow, M., Roux, JN., Chevoir, F., 2005. Rheophysics of dense granular materials: simulation of plane shear flows. Phys. Rev. E. 72, 021309. 
Forterre Y., and Pouliquen O., 2008. Flow of dense granular media. Ann. Rev. Fluid.

Mech., 40, 1-24.

GDR MiDi., 2004. On dense granular flows. Eur. Phys. J. E 14, 341-65.

Kheiripour Langroudi, M., Tardos, G., Michaels, J. and Mort, P., 2009. Effect of material properties, boundary conditions and flow fields on the rheology of dense granular matter. AIP Conference Proceedings, 1145, 587-590.

Kheiripour Langroudi, M., Turek, S., Ouazzi, A., and Tardos, G., 2010a. An investigation of frictional and collisional powder flows using a unified constitutive equation. Powder Technol., 197, 91-101.

Kheiripour Langroudi, M., Sun, J., Sundaresan, S. and Tardos, G., 2010b. Transmission of normal stresses in un-sheared and sheared granular beds: the influence of particle size, shape, stiffness and cohesion., In Press, Powder Technol.

Kheiripour Langroudi, M., Mort, P. and Tardos, G., 2010c. Study of powder flow patterns in the Couette cell with axial flow using tracers and solid fraction measurements. Accepted for Publication in Granular Matter.

Lun, C. K. K., et al., 1984. Kinetic theories for granular flow; inelastic particles in Couette flow and slightly inelastic particles. J. Fluid Mech., 140, 223-256.

Schaeffer, D.G., 1987. Instability in the evolution equations describing incompressible granular flow. J. Differ. Equations., 66, 19-50.

Tardos, G. I., et al., 1998. Forces on a slowly rotating, rough cylinder in a Couette device containing a dry, frictional powder. Phys. Fluids, 10, 335-341.

Tardos, G. I., McNamara, S. \& Talu, I., 2003. Slow and intermediate flow of a frictional bulk powder in the Couette geometry. Powder Technol. 131, 23-39. 


\begin{tabular}{|c|c|c|c|c|c|c|}
\hline Material & $\begin{array}{c}\mathbf{E} \\
\text { (Modulus) } \\
\mathrm{MPa}\end{array}$ & $\begin{array}{c}\text { Bulk } \\
\text { Density } \\
\left(\mathrm{g} / \mathrm{cm}^{3}\right)\end{array}$ & $\begin{array}{c}\text { MPS } \\
(\mathrm{um})\end{array}$ & $\begin{array}{c}\mathbf{d}_{\mathbf{5 0}} \\
(\mathrm{um})\end{array}$ & $\mathbf{d}_{\mathbf{9 0}} / \mathbf{d}_{\mathbf{1 0}}$ & Span \\
\hline PE-1 & 5.11 & $\mathbf{0 . 9 3 8}$ & 277 & 298 & $421 / 136$ & 1.54 \\
\hline PE-2 & 4.76 & $\mathbf{0 . 9 2 4}$ & 500 & 513 & $730 / 301$ & 1.43 \\
\hline
\end{tabular}

Table [1]- Physical properties of the polyethylene powders used in this study 


\begin{tabular}{|c|c|}
\hline Material & Model \\
\hline PE-1 & $\mu=0.43+0.0125 \dot{\gamma}^{0.45}$ \\
\hline PE-2 & $\mu=0.45+0.0125 \dot{\gamma}^{0.68}$ \\
\hline
\end{tabular}

Table [2]- Rheological models fitted on the Figure [7a] 


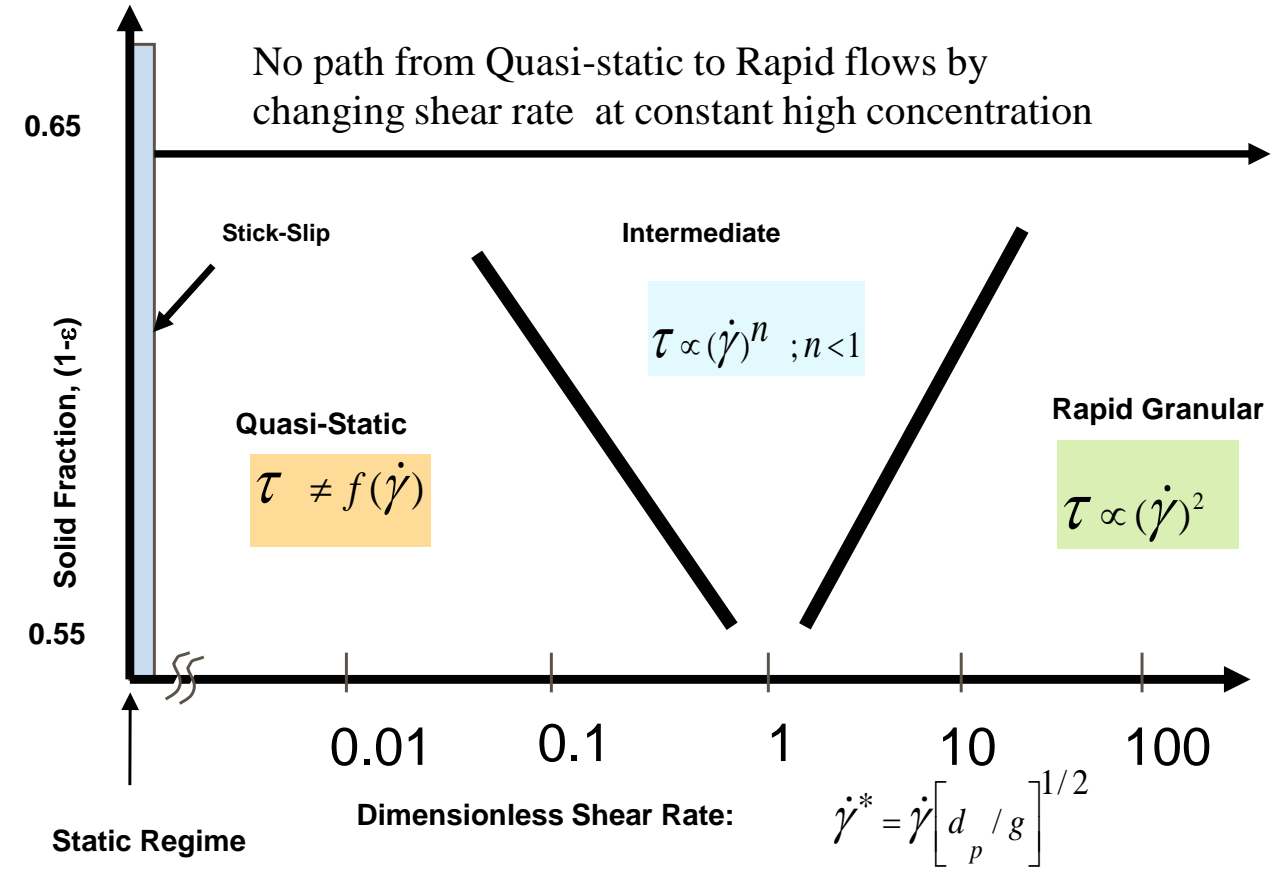

Figure [1]- Map of the powder flow regime 


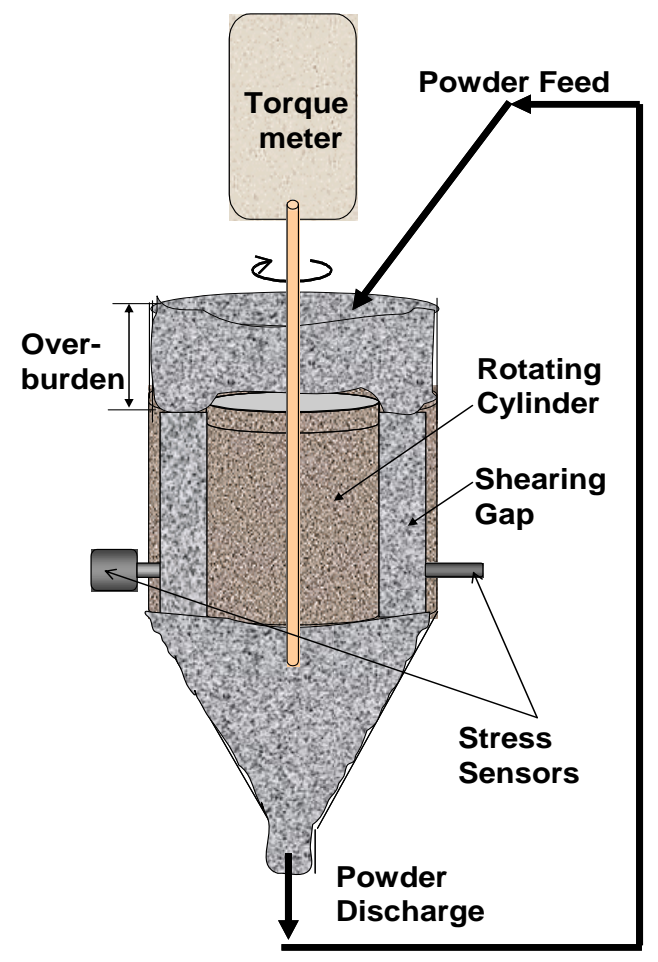

Figure [2]- Schematic drawing of the continuous Couette device 


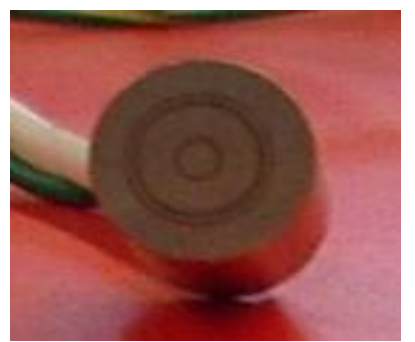

Figure [3]- capacitance probe instrument used to measure solid volume fraction during flow 


\section{PE-1}
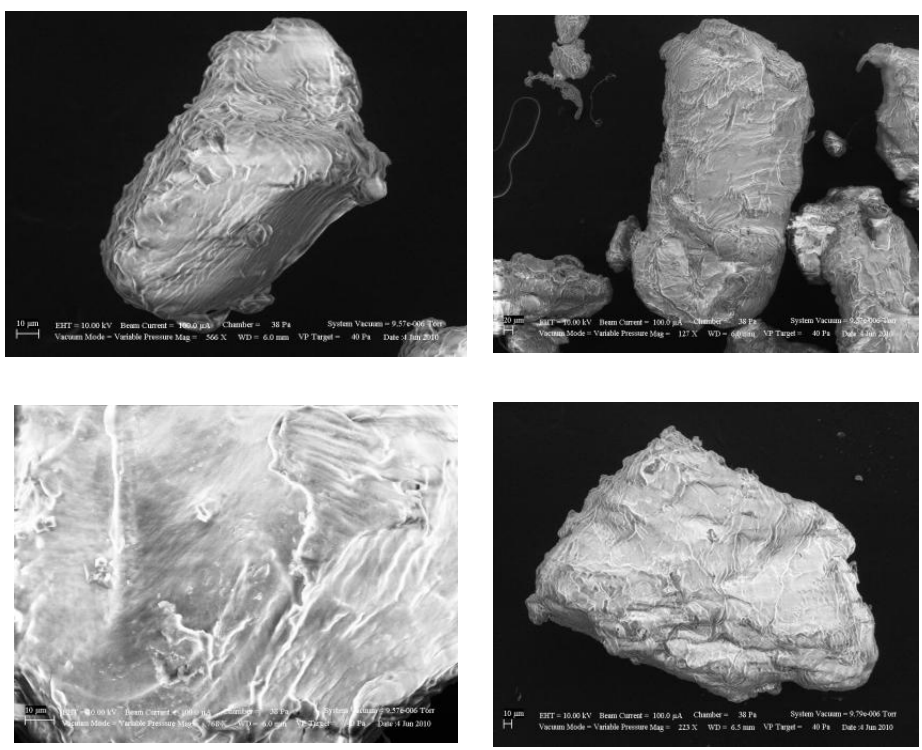

PE-2
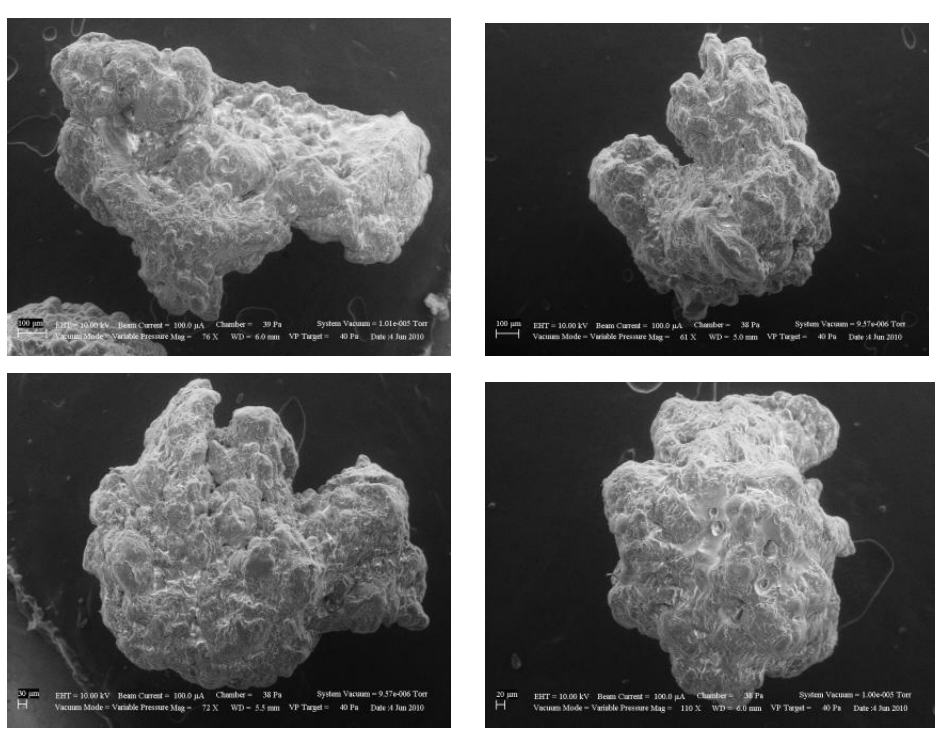

Figure [4]- SEM pictures of the PE-1 and PE-2 


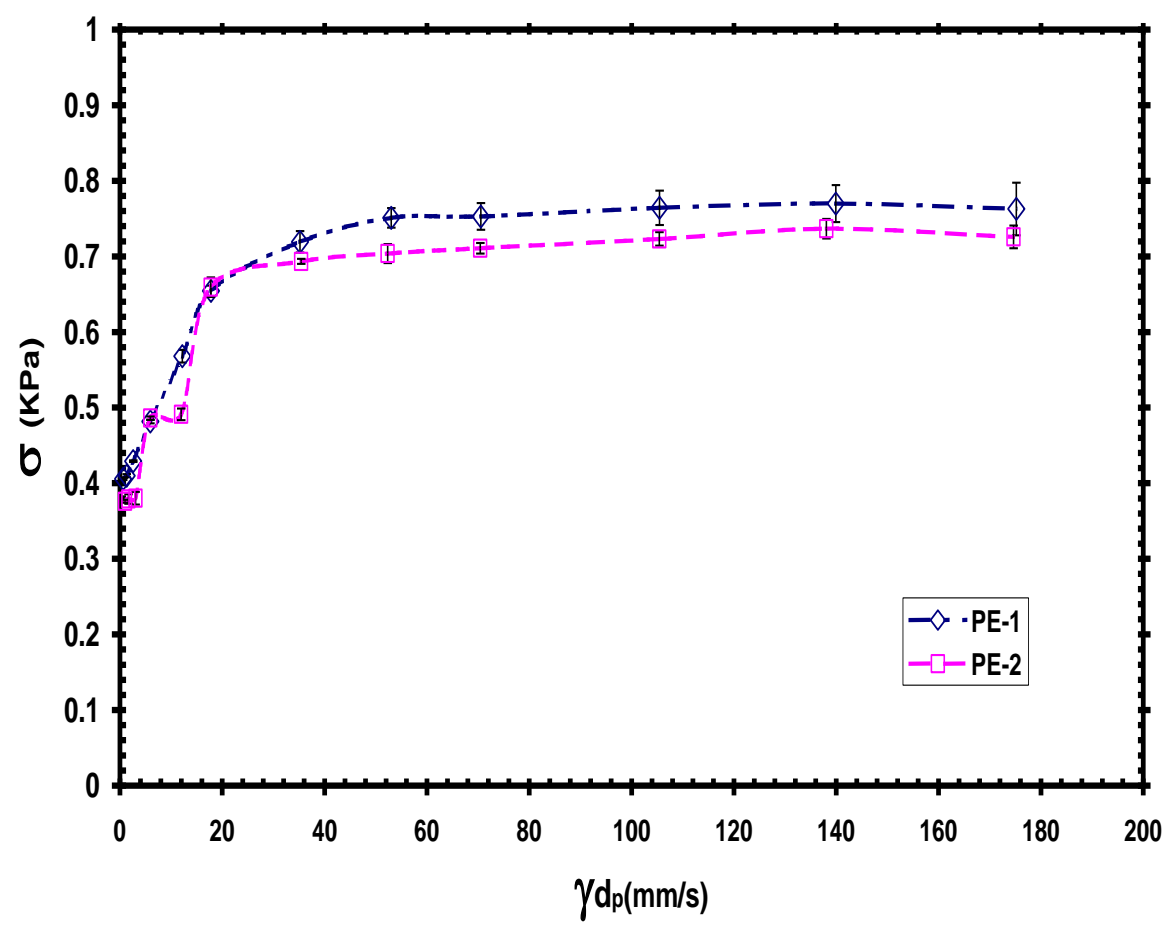

Figure [5]- Normal stress vs. shear rate for polyethylene powders in Couette experiment 


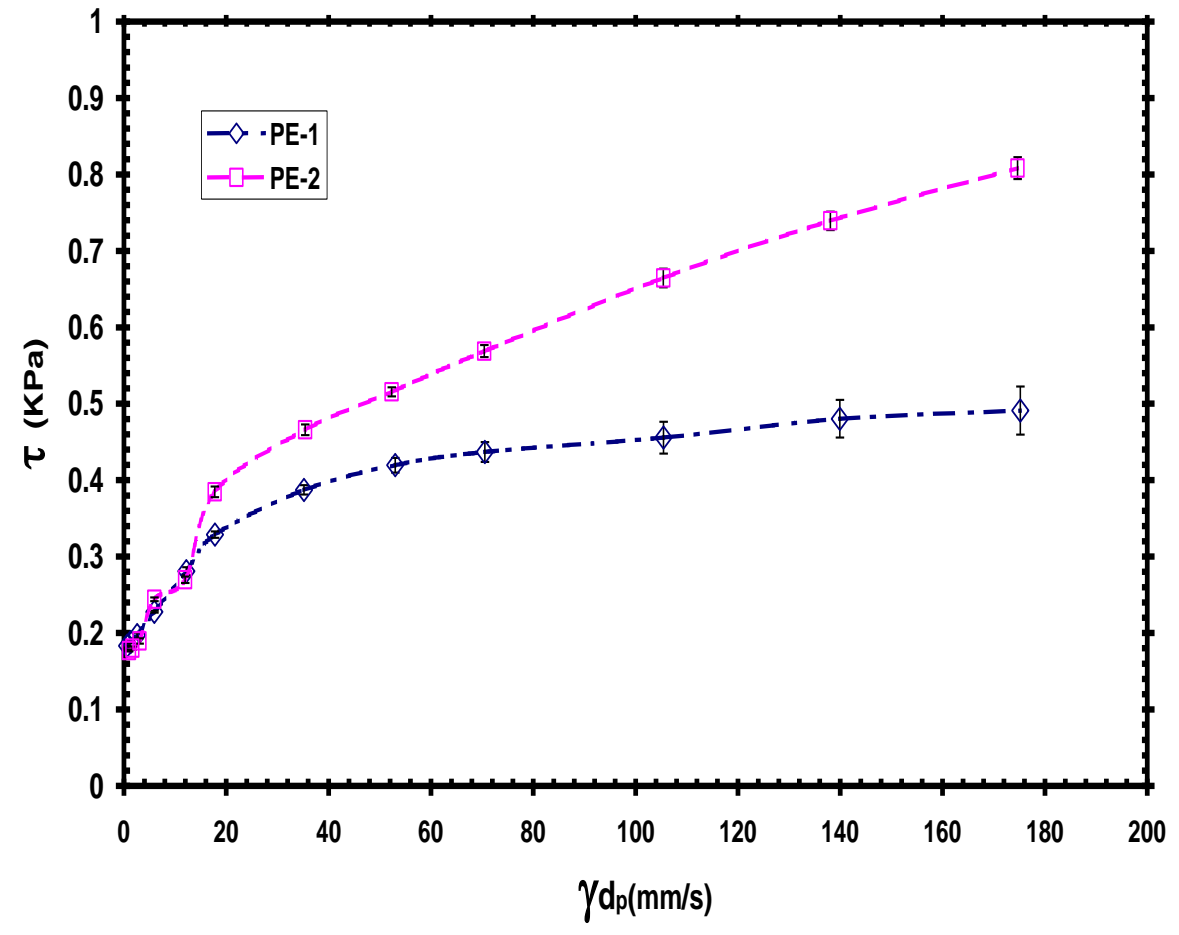

Figure [6]- Shear stresses (calculated from torque) vs. shear rate for polyethylene powders in Couette experiment 

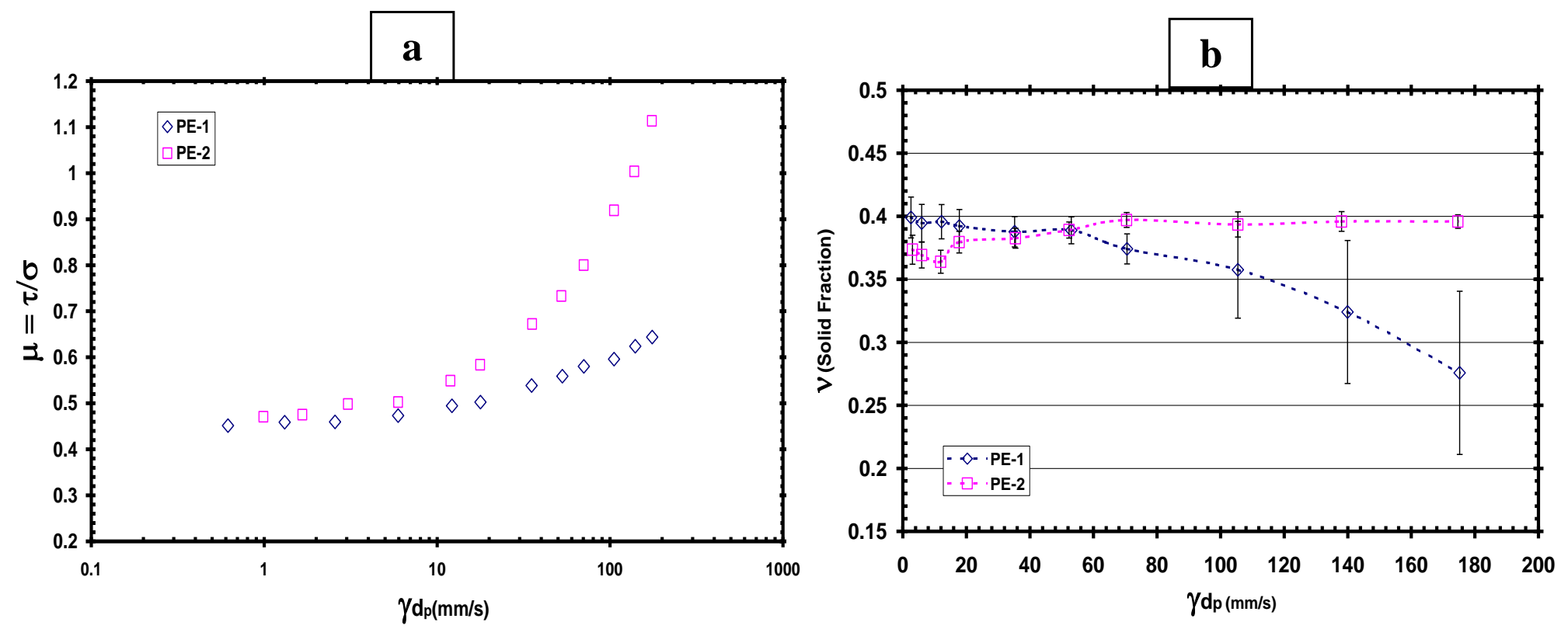

Figure [7]- (a) Apparent friction coefficient at different shearing rate for polyethylene powders (b) Solid volume fraction vs. shear rate inside the shear band for the two polyethylene powders 

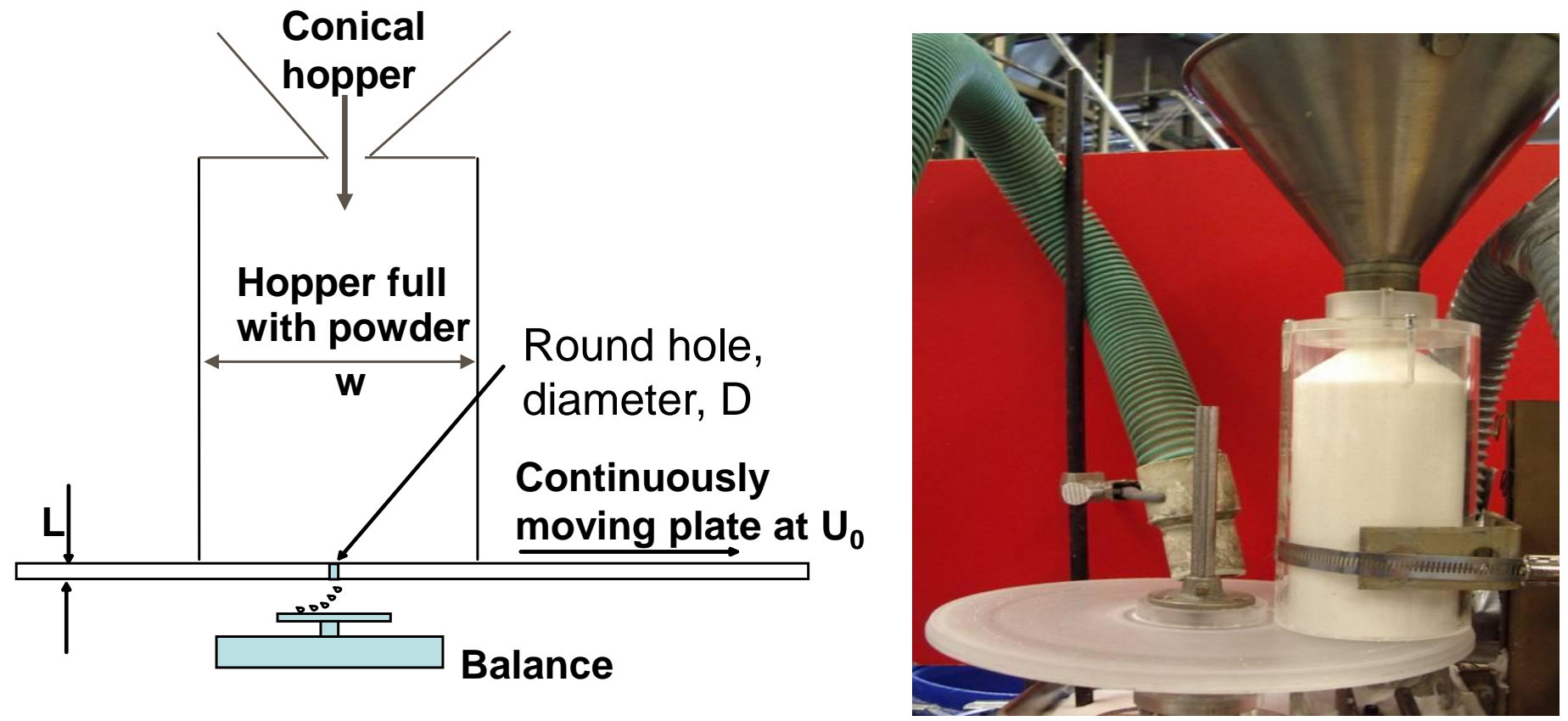

Figure [8]- (a) Schematic of the "spinning disk" apparatus (b) Picture 

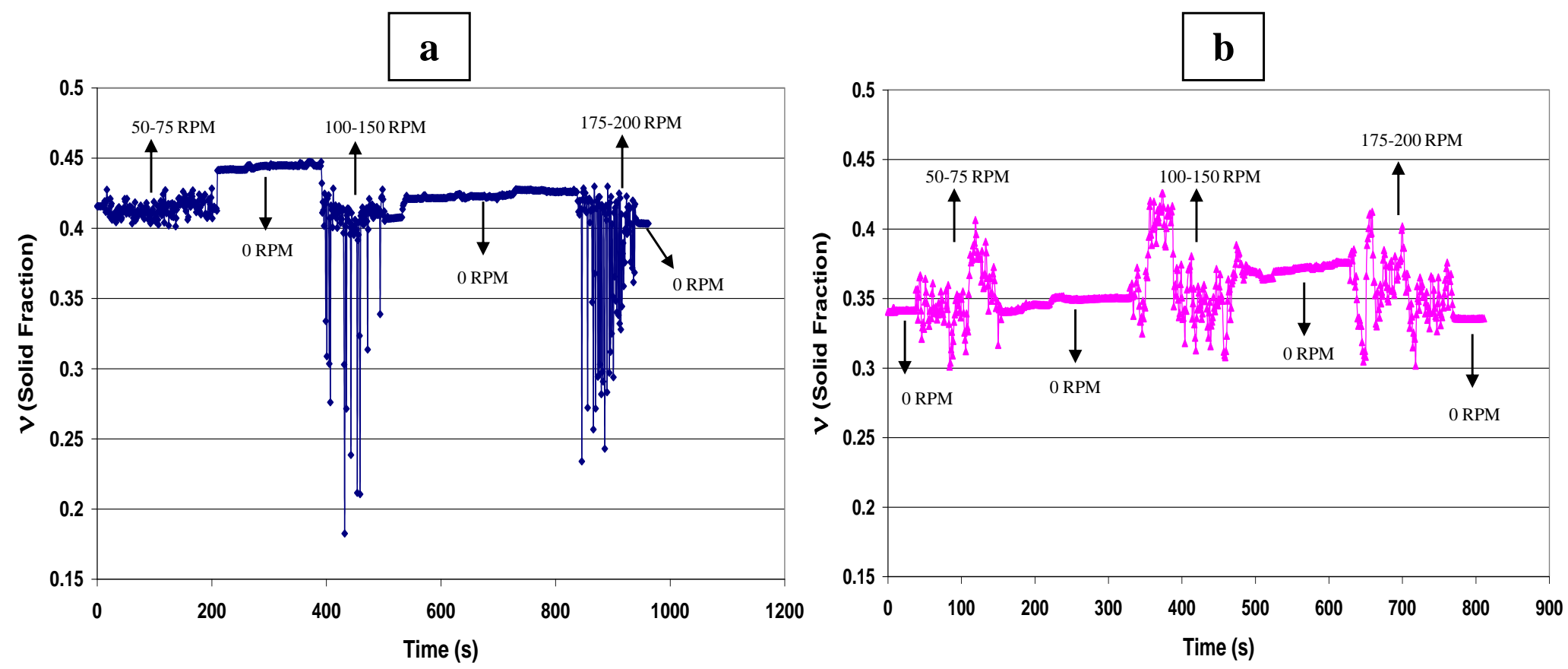

Figure [10]- Solid fraction measurements (on the cylindrical hopper wall) at different rotational speeds in the spinning disc experiment for (a) PE-1 (b) PE-2 
Appendix N

Study of Powder Flow Patterns in a Couette Cell with Axial Flow using Tracers and Solid Fraction Measurements 


\title{
Study of Powder Flow Patterns in a Couette Cell with Axial Flow using Tracers and Solid Fraction Measurements
}

\author{
M. Kheiripour Langroudi ${ }^{1}$, P.R. Mort ${ }^{2}$ and G.I. Tardos ${ }^{1}$ \\ ${ }^{1}$ The City College of the City University of New York, Department of Chemical \\ Engineering, New York, NY, ${ }^{2}$ The Procter and Gamble Corporation, Ivorydale \\ Technical Center, Cincinnati OH
}

\begin{abstract}
We present different aspects of dense granular flows in a Couette geometry using a variety of particulate materials with shape and size distributions. Tracer studies point to an apparent coupling of particle size with flow and stress field gradients. While there is a clear industrial motivation to use "real" materials as a means to expand basic physical and engineering research in granular dynamics, the current study suggests additional academic motivations. Indeed, particles with distributed characteristics uncover rich interactions between flow and stress fields that might otherwise go un-noticed with model materials such as spherical glass beads. Distribution of size and shape play a strong role in how stress is transmitted in granular media (Kheiripour et. al., 2010b) and how particle pattern arrangements evolve.

Direct solid fraction measurements, using a capacitance probe, show that dense particle flows exhibit significant variations in solid fraction in both sheared and stagnant layers. Furthermore, these measurements also show different dependence of the solid fraction on shearing rate: solid fraction decreases in sheared layers and increases in stagnant layers as the shear rate is increased. From these results the thickness of the shear band could be estimated and was found to vary as a function of particle shape and the roughness of the container walls. The main result is that radial stress gradients (see also Kheiripour et, al., 2010a) and solid fraction profiles depend on particle shape and whether or not an extra degree of freedom in their movement is provided so that the system can dilate under various states shear in the Couette. This extra degree of freedom is assured in the present experimental work by allowing a slight axial outflow from the Couette device while the driven shear fields are in the radial and tangential directions.
\end{abstract}

\section{Introduction}

This paper provides several studies of dense granular flows in the axi-symmetric Couette geometry with a slight axial flow in the vertical direction superimposed on Couette's tangential and radial shear fields. The flows comprise a variety of particulate materials with different shapes and size distributions. We try to use "real", industrial materials to expand basic physical and engineering research in granular dynamics. To this end, we present experimental studies using two techniques: 1) analysis of tracer particles to elucidate flow patterns; and 2) measurement of solid 
fraction distributions as a function of radial position. This combination of techniques allows for detailed observation of flow patterns and is essential in understanding the micro-behavior of powder flows. The results elucidate interactions between particles' flow, stress and variation in their local packing fraction; further, results show that complex characteristics such as size distribution and particle shape contribute significantly to these interactions.

The distribution of flow fields and solid fraction variation in sheared and slow-moving and stagnant layers as well as the knowledge of the degree of dispersion in these regions is important in such industrial devices as mixers and hoppers. In particular, the structure of the shearing layer and its dispersive characteristics play a role in the design of these machines as wide shearing layers are more dispersive and insure better mixing which is desirable in mixers and granulators but is usually undesirable in hoppers. Particle characteristics including size, size distribution and particle shape can influence mixing and de-mixing behavior in process equipment. The relative influence of convective and dispersive flows is important to the optimization and limitations of such processes.

Two tracer methods are used, one is based only on particle size, and the other based on size and color. The colorimetric method enables simultaneous multi-variate analysis of size, color and shape on a per-particle basis (Mort et al, 2004). This analysis is automated and thousands of particles can be monitored to obtain better and more representative experimental results.

A specially developed capacitance probe (Louge et al., 1996, Louge and Opie, 1990 and Louge at al., 2001) was used in the past for local solid fraction measurements in packed powders. In the present study, we use the dependence of solid fraction on position and shear rate to determine the thickness (width) of the shearing layer in the Couette device. The main objectives of solid fraction measurements were to study the effect of radial and axial flows on mixing in the Couette, e.g., the relation between dispersion and convection and to find the effect of shearing rate on dispersion. We also used tracer studies in the axial Couette flow to elucidate the effect of flow and shear gradients on size segregation of polydisperse samples. We found evidence of radial gradients suggesting a coupling between solid fraction and shear rate across the gap.

\section{Literature}

"Dilatancy" for granular materials was first observed by Reynolds (1885). The concept of dilatancy is the decrease in solid fraction (bulk density) that occurs in a tightly packed granular arrangement when it is subjected to deformation. Many of the existing theories for flowing granular materials relate the applied stress to the solid fraction and the velocity. Precise studies of decrease in solid fraction are hindered by the opaque nature of granular media and by the complexity of granular flows. These flows usually do not develop simple linear shear profiles (GDR MiDi, 2004; Depkan et al., 2006) and therefore measurements of average bulk densities cannot probe dilatancy. Hence, many basic questions are left unanswered. 
Several recent authors investigated individual particle motion in the Couette geometry (Schollmann, 1999; Mueth et al., 2000). These studies indicate that the mean particle velocity parallel to the shear direction $V(y)$ decreases faster than linearly away from the inner wall (i.e., the moving boundary in the Couette). The velocity profile in three dimensions was determined by Mueth et al. (2000) in the interior of the material using x-ray and NMR techniques and on the bottom surface of the Couette cell by optical imaging. These measurements showed that the velocity profiles on the bottom surface and in the interior are similar. Losert et. al. (2000) determined the particle dynamics and shear forces of granules in the Couette geometry. They found that the mean particle velocities during brief slips of the shearing cylinder decrease roughly exponentially with distance away from the moving wall. They showed that, for the case of smooth, spherical particles, the velocity profile is localized in a shear band five to ten particles thick located close to the moving wall.

Positron emission particle tracking (PEPT) is another technique for studying the shear band. Seville and Tardos (2004) applied this technique in a batch Couette cell (without axial flow) and found that for crushed glass particles that have odd shapes the shear band extends as far as 14 particle sizes.

In more complex 3D geometries such as the modified Couette cell, in which the bottom is split into a rotating and a static part, shear zones with up to 40-particle diameters are observed (Fenistein et al. 2004). Sakaie et. al. (2008) used Magnetic Resonance Imaging (MRI) for the study of the 3D packing density of granular flows generated in a split-bottomed shear cell, where the grain flow is driven by the rotation of a bottom disc with respect to a cylindrical container. By imaging the local packing densities in the shear cell as a function of time and for a range of filling heights, they explored the relationship between flow field and dilatancy. The picture that emerges is that the amount of dilatancy grows with the total amount of local strain. These authors showed the relative change in bulk density or solid fraction in the flowing zone is strong and saturates around $10-15 \%$.

Experimental measurements of particle dynamics on the lower surface of a threedimensional Couette cell containing mono-disperse spheres were reported by Mueth, (2000). Fluctuations in the velocities of two particles at radius $r$ were correlated within a characteristic length scale. The correlation length increases to $3.5 d_{p}$ near the moving wall, while it is around $2.4 d_{p}$ away from it. With directly measuring the velocity, velocity fluctuations, and density within the system, these authors found a power-law relationship between shear rate and fluctuation amplitude. This finding is consistent with the predictions of hydrodynamic and kinetic theories for granular flow proposed by Bocquet et al. (2002).

Mohan et. al. (2002) used the Cosserat model for finding the shear layer thickness (shear band). This model has a microscopic length scale, which determines the thickness of the shear layer. In a cylindrical Couette flow between walls of equal roughness, the Cosserat model shows that the velocity decays rapidly with distance from the inner cylinder. This finding is in good agreement with the velocity 
measurements reported by others (Mueth et al. (2000); Losert, et. al. (2000)). In addition, these authors also found theoretically that the shear layer thickness decreases when the angle of wall friction is reduced, and vanishes when the wall is perfectly smooth. Also the shear layer thickness is at its maximum value when the wall friction is equal to the particle internal angle of friction $(\phi)$. These cases have not

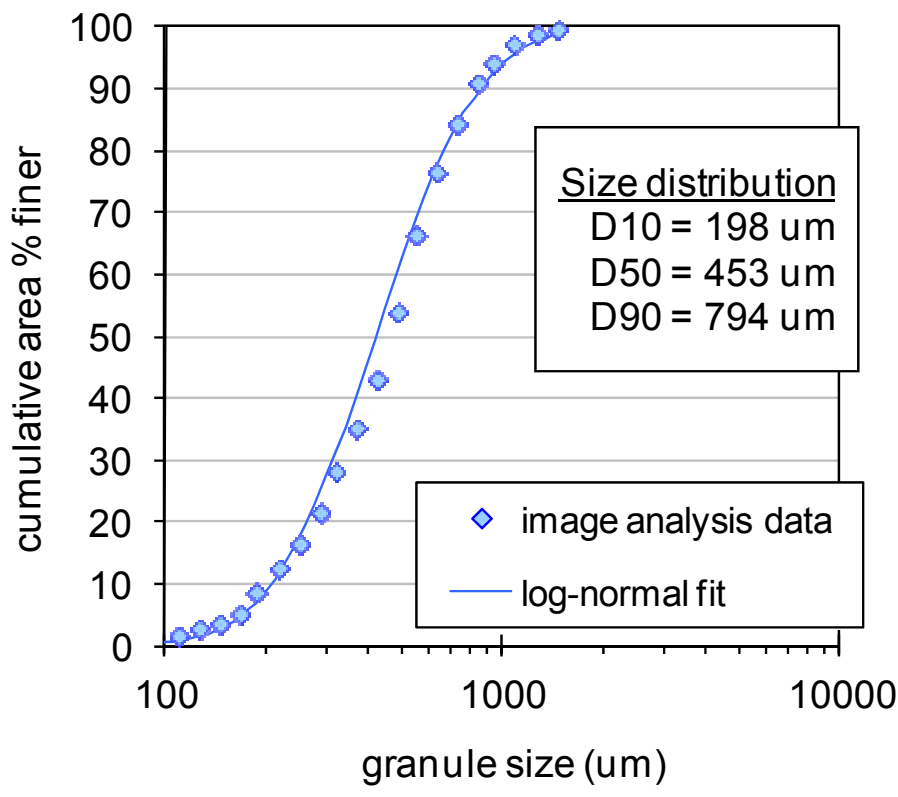

Figure 1 : Representative size distribution of "white base" and "red tracer" particles.

yet been experimentally confirmed. This model also predicts a small increase in the solid fraction as one moves towards the outer cylinder. This increase, however, is substantially smaller than that which was observed in the experiments of Mueth et al. (2000).

\section{Tracer Studies}

\section{a) Materials Used During the Study}

A set of experiments was performed with a commercial granulate, denoted in this study as "white base" with a relatively wide particle size distribution as shown in Figure 1. The median particle size is approximately $D_{50}=450$ um. To create the tracer, the same white base was dyed red by spray-on in a fluidized bed to provide tracers with size, size distribution and shape that are practically identical to the white base. The granules are made for industrial use and are quite resistant to

\begin{tabular}{|c|c|c|c|}
\hline Mate rial & $\begin{array}{c}\text { Median } \\
\text { Size, } \mathbf{D}_{\mathbf{5 0}} \\
{[\mathbf{m m}]}\end{array}$ & Shape & $\begin{array}{c}\text { Size } \\
\text { Distribution } \\
\mathbf{D}_{\mathbf{9 0}} \mathbf{}_{\mathbf{1 0}}\end{array}$ \\
\hline $\begin{array}{c}\text { White base, } \\
\text { Red tracer }\end{array}$ & 0.453 & irregular & 4 \\
\hline Glass & 1 & spherical & Mono-disperse \\
\hline Glass & 0.5 & spherical & Mono-disperse \\
\hline Crushed Glass & 0.6 & irregular & 1.3 \\
\hline Crushed PE & 0.3 & irregular & 3 \\
\hline
\end{tabular}

wear, deformation and breakage. Bivariate analysis of the tracer (size and color) was done using color image analysis.

A second set of experiments was done using three kinds of monodisperse

Table 1: Characteristics of materials used during experimentation spherical glass beads of 100 um, 500 um and 1 $\mathrm{mm}$ in diameter. In this case, the 100 um glass beads were used as base particles 


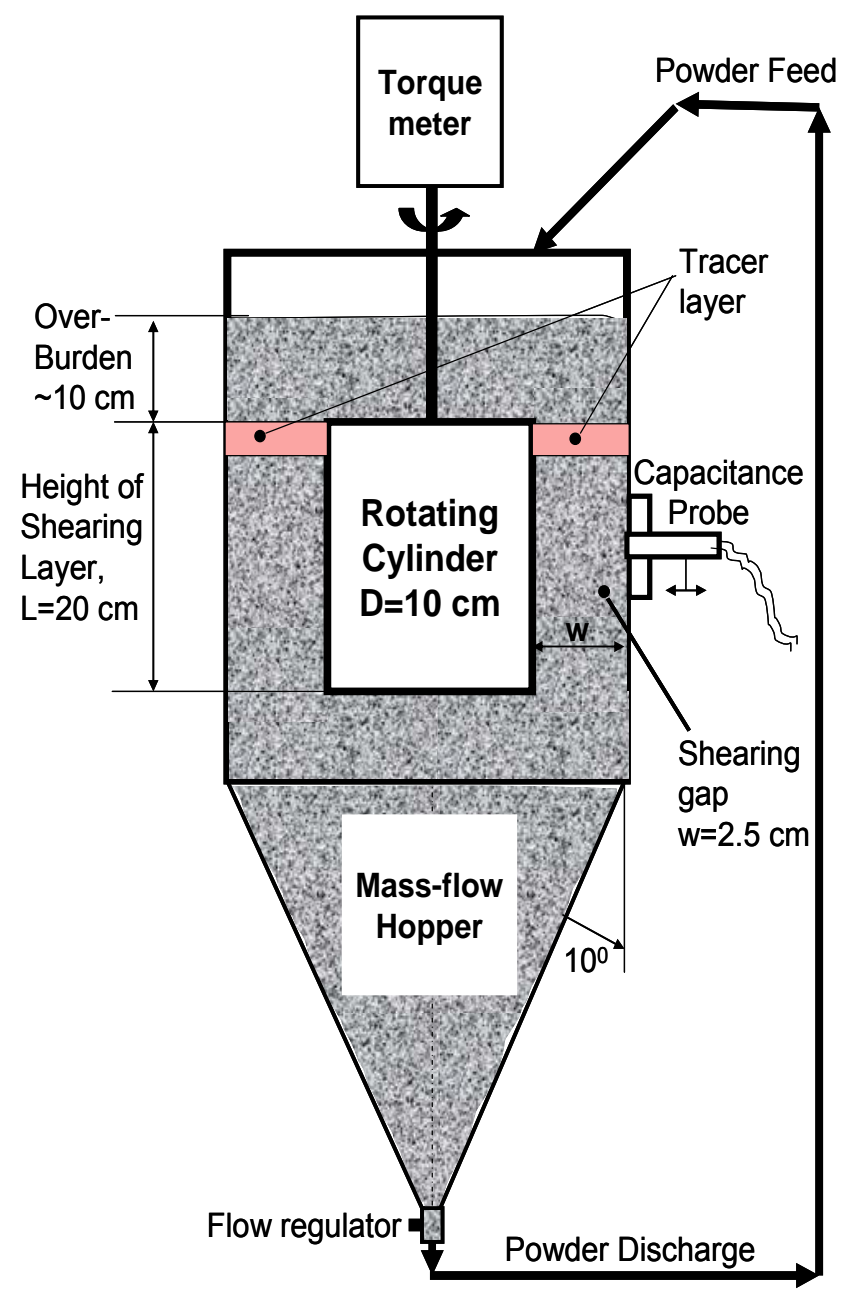

Figure 2: Schematic of the Couette experiment

while the larger particles were used as tracers. Tracer concentrations were measured by sieve classifying the samples. Table 1 contains the main characteristics of all materials used in this study including their median size, $D_{50}$, shape and size distribution.

\section{b) Tracer Experiments}

A schematic representation of the axial-flow Couette device with tracer particles inserted into the shearing zone is shown in Figure 2. The insertion of tracer is made in such a way as to produce a narrow "pulse" of red tracer particles on an otherwise white background. Additional white base granules were provided to fill the space above the rotating cylinder (overburden in the figure). Different height (the dimension " $\mathrm{L}$ " in the figure) cylinders were used but the most common size was the one shown in the figure with $L=20 \mathrm{~cm}$, an outer diameter of $15 \mathrm{~cm}$ and an inner diameter of $10 \mathrm{~cm}$ so that a

shearing gap of $w=2.5 \mathrm{~cm}$ was formed. The experiment is performed by rotating the inner rough cylinder and feeding and discharging material continuously to maintain a constant overburden.

The outflow of the mixture from the bottom of the Couette is collected at approximately one minute time intervals. Samples (of approximately 80 grams) were collected and analyzed using color image analysis shown in Figure 3 (SolidSizer*, JM Canty Inc. Buffalo, NY). The powder sample is fed

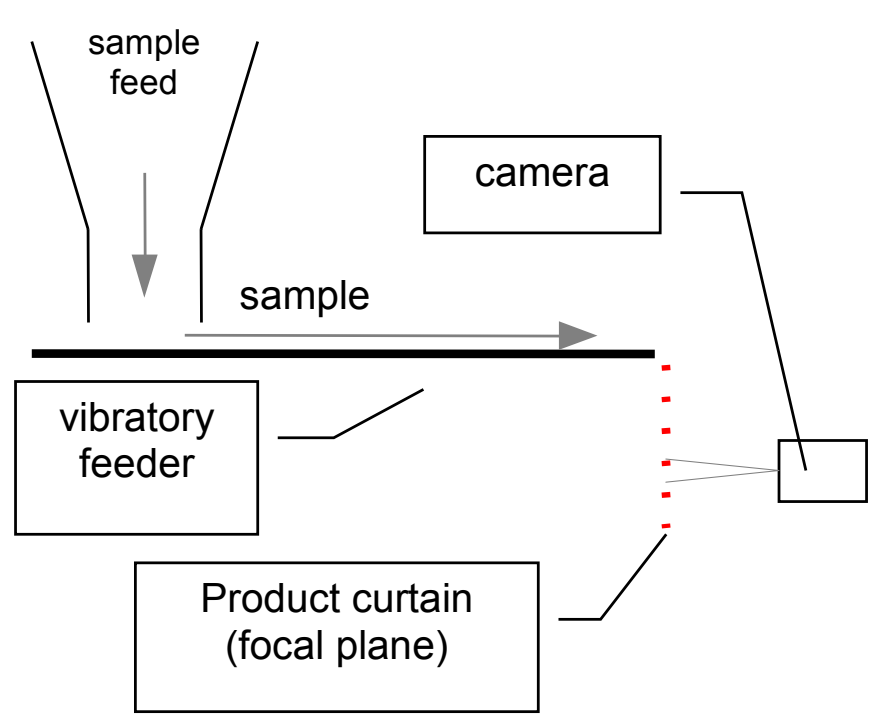

Figure 3: Schematic of the Colorimeter. SolidSizer, JM Canty Inc, Buffalo, NY 
through a vibratory feeder at controlled rate to form a mono-layer "curtain" of particles

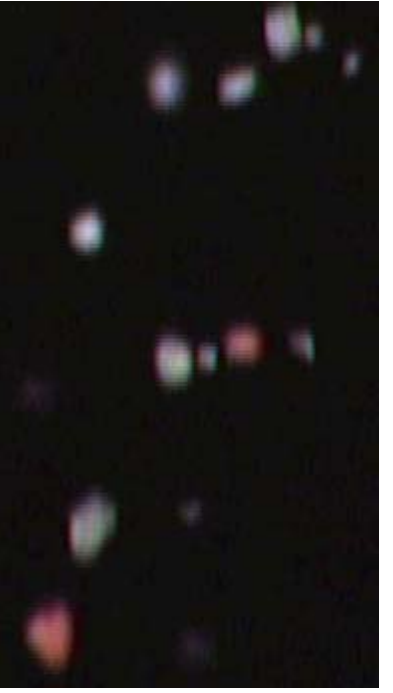

Freeze-frame image of particles

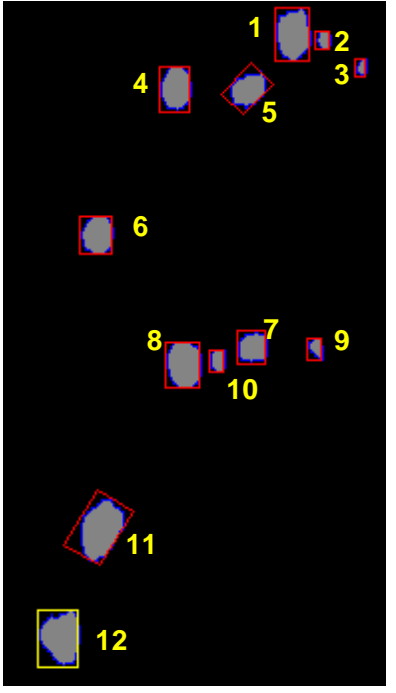

Binary image after brightness thresholding

Figure 4: Bivariate image analysis for size and color

that fall from the vibratory feeder in front of the color sensitive camera. The important parts of the system are the mono-layered curtain, the front (reflective) lighting system, the fast frame color camera and analysis software for particle detection. The automated procedure identifies particles as 2D images using threshold brightness and then calculates size, shape and color of each particle image (Figure 4). Sufficient frames are obtained to measure the characteristics of 10,000 particles from each sample, from which size and color distributions are calculated.

Particle color is measured from the color-camera by identifying three major colors R-red, G-green and B-blue from

the reflected light on a black background. Since the tracer particles are red, the amount of red $(R)$ relative to the total brightness $(R+G+B)$ was used as a relative characteristic $[R \%=R /(R+G+B)]$. White base particles have a relatively equal balance of colors, i.e., an average of $\mathrm{R} \% \sim 31 \%$. The red tracer particles have a color distribution with a discrete peak at R\% 37\%. Based on these measureable color differences, a threshold of $\mathrm{R} \%>36 \%$ was used to identify tracer particles. For example, in Figure 4, particles 7 and 12 are identified as tracers based on their color being above the red threshold, while the remaining are white base particles.

\section{c) Results and Discussion of Tracer Experiment}

Figure 5 gives a typical result from a tracer experiment using the white base and red tracers, as concentration versus time. Each data point in the Figure 5 represents one sample taken from the hopper outlet. For each sample, the image analysis was done to measure the concentration of red particles versus white particles, based on particle color threshold described previously. The concentration of the tracer is made dimensionless with the average concentration $\mathrm{C}_{0}$, as $C_{\theta}=C(t) / C_{0}$ while time is made dimensionless with the average axial velocity, $u$ and the average bed height, $\mathrm{L}$ as $\theta=t u / L$. The axial velocity is calculated as an average from the vertical flow rate and the dimension of the shearing gap. The Peclet number is defined as $P e=u L / D$, where $\mathrm{D}$ is the dispersion coefficient.

Figure 5 shows the experimental values (in blue, diamonds) and four theoretical response curves using the classical Residence Time Distribution model (Levenspiel, (1972)) for several different values of the parameter D/uL=1/Pe from 0.002 (small 
dispersion) to 0.2 (large dispersion). As seen, the experimental data are apparently bi-modal having a main peak with limited dispersion (D/uL 0.015) and the delayed peak having more dispersion. Overall, the data fall somewhere in the middle of the range showing that the flow is somewhat dispersive with a diffusion coefficient $\mathrm{D} \sim 1.1 \mathrm{~cm}^{2} / \mathrm{min}$ (resulting from $1 / \mathrm{Pe} \sim 0.02$ ).

As a proviso to the above discussion, one has to observe that the trajectory of particles in the axially

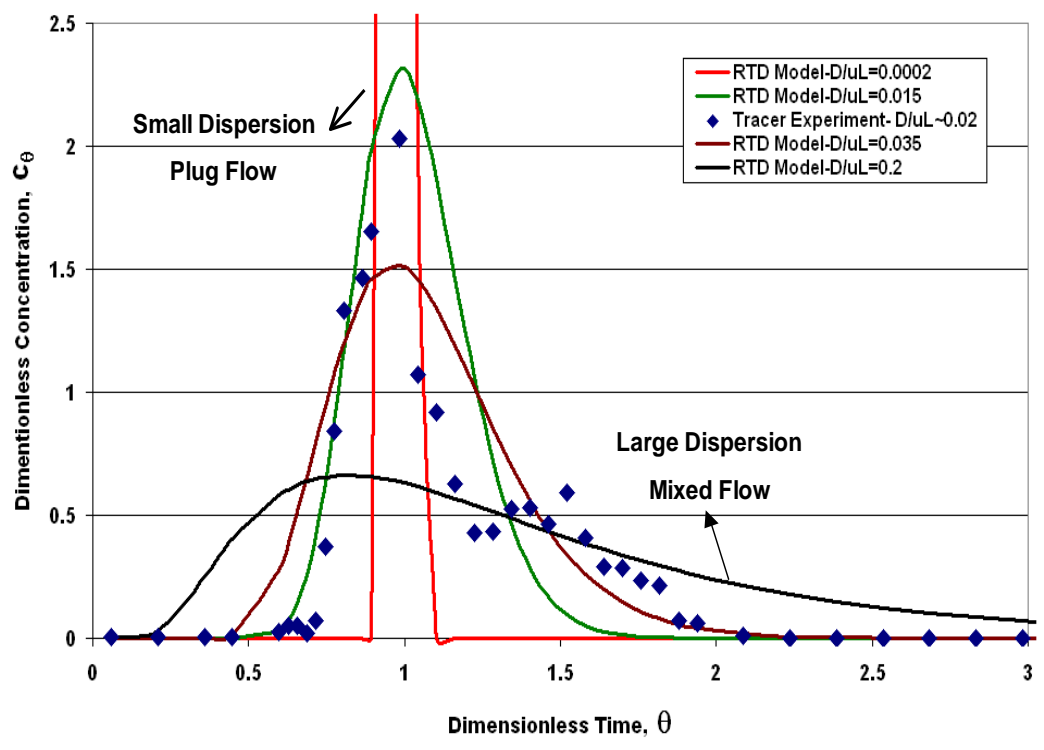

Figure 5: Dimensionless concentration vs. dimensionless time flowing Couette is actually not simple and is comprised of flow in the shearing gap in addition to tangential flow in the mass flow, conical hopper, and in the intermediate connecting space. Two important phenomena have to be excluded in order to make the above results relevant (i) the material must be prevented from cascading out of the shearing zone

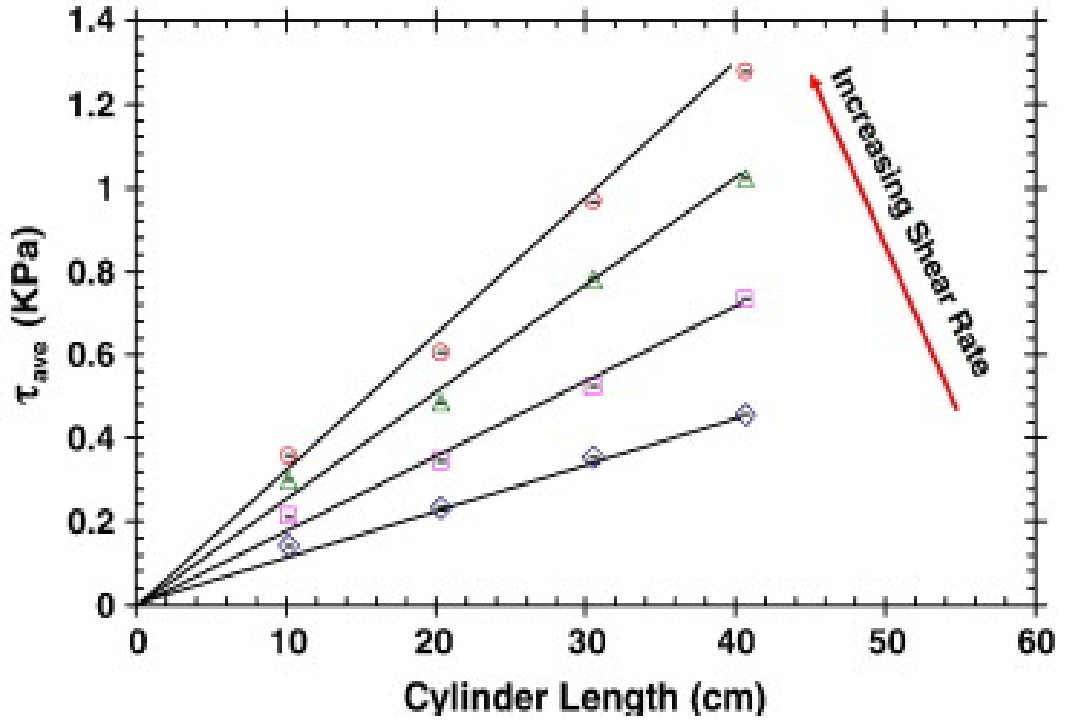

Figure 6: Average shear stress measurements in continuous Couette device using different overall lengths, L, cylinders at different shear rates onto the space above the hopper and thereby enhance mixing and (ii) the hopper should not introduce any maldistribution of flow. First, one has to observe that the axial flow is minute compared to the radial shearing, resulting in axial flow velocities of the order of $u=0.01 \mathrm{~mm} / \mathrm{sec}$. In addition, there is a pair of two, diametrically opposite observation windows

(not shown in Figure 2) in the space immediately under the rotating cylinder. If light is shined into the flow from one side there is a good possibility to see a thinning of the flow through the other window, should a cascade occur: no such thinning was 
observed during any of the experiments. Furthermore, Figure 6 shows the result of a set of experiments where torque was measured as a function of rotating cylinder height and rotation rate. The lines were forced through the origin to achieve zero torque at $L=0$. One can clearly see that the experimental points of the shortest cylinder $(\mathrm{L}=10 \mathrm{~cm})$, fall consistently above the line showing that there is additional friction on the cylinder through the bottom disk (in addition to friction in the shearing layer). Since these experiments were performed without "overburden", this clearly shows that there is no empty space under the rotating cylinder and, in fact, the fill volume of the hopper is such that significant friction occurs on the bottom of the cylinder. For this reason it was necessary to use longer rotating cylinders so that this "end" effect is rendered negligibly small. The flow in the mass flow hopper is addressed in the next paragraph.
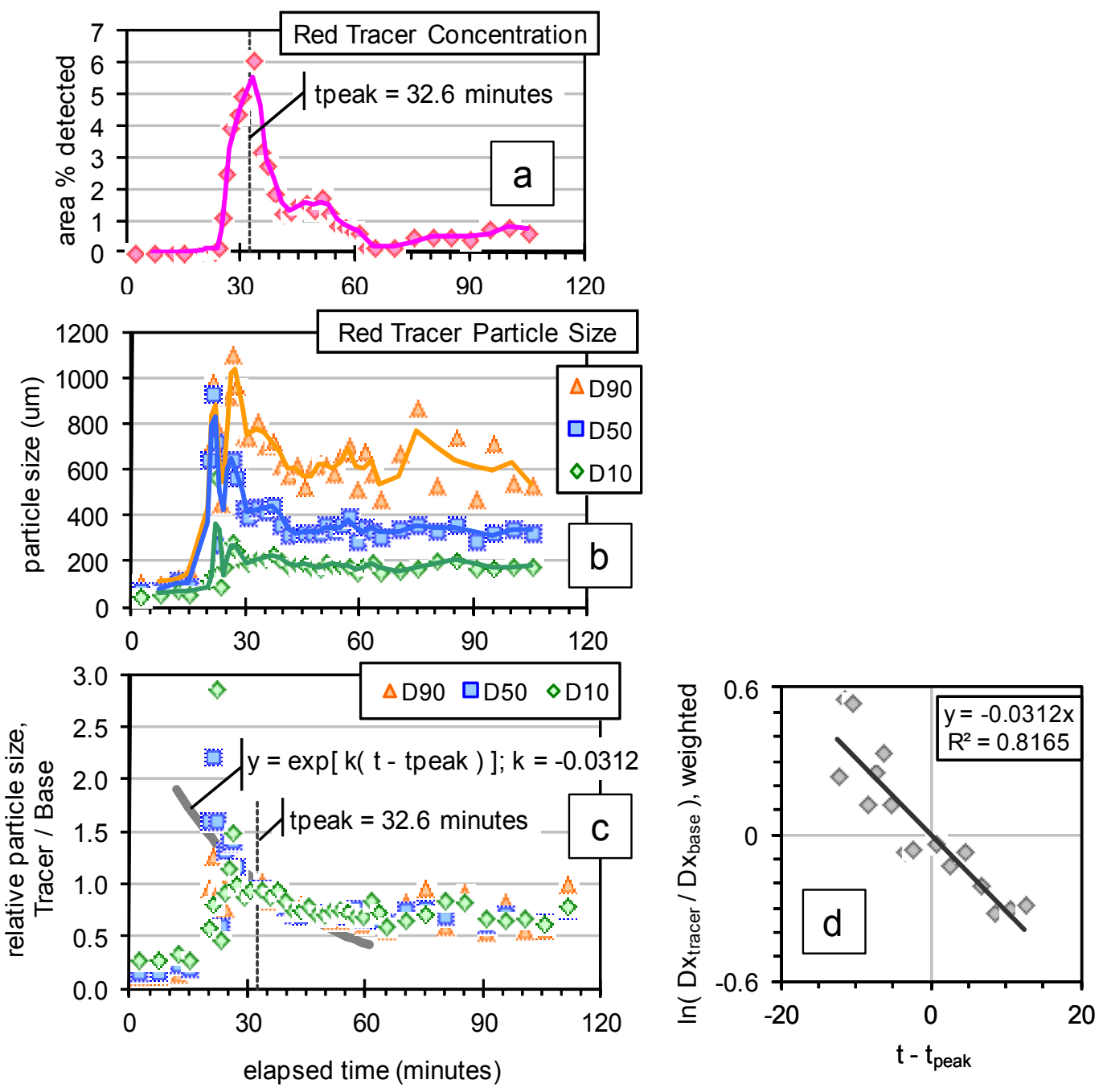

Figure 7: Raw data for tracer flow: a) tracer concentration over time; b) tracer particle size distribution over time. Analysis of tracer flow data: c) normalized particle size (Tracer/Base) with overlaid size function; d) regression analysis of normalized size data, offset to the peak tracer flow time, $t_{\text {peak }}$ 
The time required for the main peak of tracer to move through the device is approximately 32.6 minutes (Figure 7a); notice that initial tracer particles appear as early as 20 to 25 minutes. The large number of particles used in the image analysis $(10,000$ for each sample) enabled the analysis of size distributions for each color population, both white base and red tracer. The size distributions are characterized in terms of percentiles, D10, D50 and D90. It is interesting that the relative size of the red tracer particles is coarser just before the main peak time $\left(t_{\text {peak }}\right)$ and then becomes finer over the duration of the peak, before stabilizing with extended time (Figure $7 \mathrm{~b}$ ). This trend of tracer particle size with flow time is even more apparent when the tracer size distribution is normalized to the white base particle size of the same sample (Figure 7c). Further, the weighted composite of the normalized size distribution (Eq. 1) plotted against offset time $\left(t-t_{\text {peak }}\right)$ over a peak interval of about $+/-12$ minutes (Figure 7d) shows a trend of particle size with time, suggesting coarser tracer particles have a faster axial flow speed compared to finer tracer particles in the Couette. The size trend function is overlaid on the data of Figure 7c; for visualization, the trend is extrapolated beyond the range of its applicability. Note the trend function pertains only to the transition from base to tracer particles in the domain of the main peak of tracer flow ( 20 to 45 minutes).

$\mathrm{Dx}$ tracer $/ \mathrm{Dx}$ base $=20 \%{ }^{*} \mathrm{D} 10_{\text {tracer }} / \mathrm{D} 10_{\text {base }}+60 \%{ }^{*} \mathrm{D} 50_{\text {tracer }} / \mathrm{D} 50_{\text {base }}+20 \%{ }^{*} \mathrm{D} 90_{\text {tracer }} / \mathrm{D} 90_{\text {base }}$

This analysis suggests a potential linkage between particle size, velocity gradient across the gap in the Couette (radius) and the observed axial flow rate; i.e., coarser particles apparently migrate toward the higher shear field near the inner moving surface while finer particles migrate toward the outer radius of the Couette.

To examine the flow pattern in more detail, we performed a controlled experiment using model materials with only two sizes of glass bead particles, $0.1 \mathrm{~mm}$ beads as base particles and $0.5 \mathrm{~mm}$ beads as tracer. The experiment was performed by filling the bed with base particles and establishing a steady state flow by maintaining a constant overburden and changing the feed (at time $\mathrm{t}=0$ ) to $0.5 \mathrm{~mm}$ particles thereby creating a "step". The evolution of the step was then followed at the discharge of the Couette device, taking samples at regular time intervals as described above. The tracer was separated by sieving and by measuring the weight fraction of larger particles in the discharged stream. The experiments were performed with different axial flow rates and several different shear rates.

A typical set of results is given in Figure 8 as the weight fraction of $0.5 \mathrm{~mm}$ glass beads versus time. The step, by changing the feed from 0.1 to $0.5 \mathrm{~mm}$ particles, was denoted to take place at zero time, $t=0$. The response in Figure 8 is given starting at a time $\mathrm{t}=40$ minutes for three shear rates at 0,20 and 60 RPM (there were no large particles detected before $t=40$ ). The fourth experiment in Figure 8 (Hopper Only) is the response to the same transition of $0.1 \mathrm{~mm}$ particles to $0.5 \mathrm{~mm}$ glass beads only using the hopper and no insert in the Couette device. The data is shifted 70 minutes to the right as the time of flow through the hopper only is much shorter compared to the whole device. This experiment was performed to show that in the mass flow hopper with polished metal walls, the flow of the material is almost perfectly plug flow 
with an almost flat velocity distribution and significant slip on the wall. This behavior can be inferred from the steepness of the response in Figure 8 and was also observed by the authors using NMR in a mass-flow hopper and described in Gentzler and Tardos, (2009).

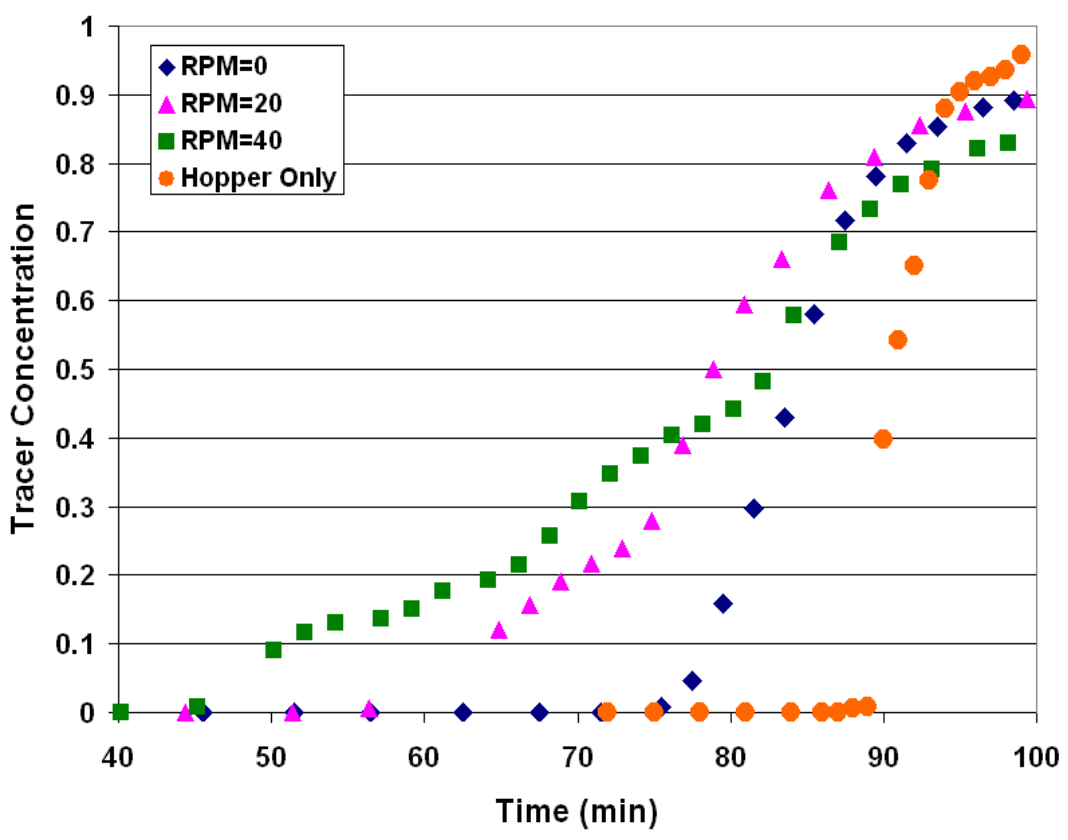

Figure 8: Tracer experiment with 0.1 (base) and $0.5 \mathrm{~mm}$ (tracer)
As seen in Figure 8 , the average time for the "step" to move through the device is about 75 minutes when the bed is at rest (no shear). Increasing the shear rate reduces this time significantly at constant axial flow rate $(\sim 2.8$ $\mathrm{g} / \mathrm{sec}$ ) to 56 minutes at 20 RPM and 45 minutes at 60 RPM. We also calculated dispersion coefficients characteristic to the response curves in Figure 8 with data given in Table 2. glass beads

We see from the above results that dispersion (mixing) increases by increasing shear rate as particles closer to the rotating cylinder move faster axially than those situated towards the stationary wall. This suggests that a shear band forms near the rotating cylinder (of width $\Delta$ ) as depicted schematically in Figure 9 where both shearing and axial flow are more intense and a slower flow pattern develops outside of the shearing band. This figure also

\begin{tabular}{|c|c|c|}
\hline $\begin{array}{c}\text { Rotation } \\
\text { Rate } \\
\text { (RPM) }\end{array}$ & $\begin{array}{c}\text { Time for first } \\
\text { tracer to exit } \\
\text { (min) }\end{array}$ & $\begin{array}{c}\text { D } \\
\left(\mathrm{cm}^{2} / \mathrm{min}\right)\end{array}$ \\
\hline $\mathbf{0}$ & $\mathbf{7 5 . 5}$ & $\mathbf{0 . 1 9}$ \\
\hline 20 & 56.4 & $\mathbf{0 . 3 6}$ \\
\hline 60 & 45.2 & $\mathbf{0 . 5 8}$ \\
\hline
\end{tabular}

Table 2: Dispersion coefficient of $0.5 \mathrm{~mm}$ in diameter round glass beads at different angular velocities (RPM) of the inner cylinder explains the previous finding where larger particles flow faster in the shear gap then smaller particles. This is because in the sharing band the material exhibits lower solid fraction and larger particles have enough space to move faster (see next section).

The disadvantage of the present experiment is that it gives no indication as to the relative thickness of the shearing band $(\Delta)$ to the overall width of the shear gap (w). Theoretical calculations (see for example Tardos et al, 2003) suggest for the shear band an overall thickness of around 10-13 particle sizes. This finding applies to plane 
shear, to the case when velocity fluctuations become large and the granular temperature comes into play.

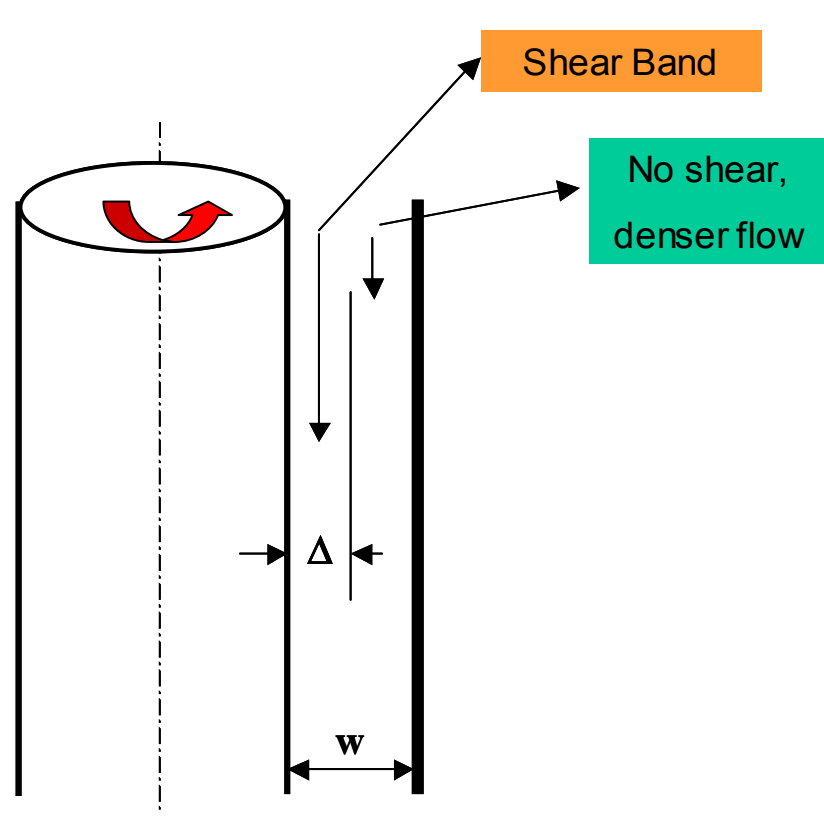

Figure 9: Schematic of the shear gap

Other values from experiments suggest 5-10 particle diameters for tightly packed smooth particles and up to 40 particle sizes in the split-bottom cell. Work to measure the actual thickness of the shearing band in relation to the width of the shear gap in the axially flowing Couette device is described in the next section.

\section{d) Segregation Studies}

Even though larger tracer particles were found to move faster in the shearing band, axial segregation in a continuous steady state flow is not significant. To demonstrate this behavior more thoroughly, an additional experiment was performed with $0.1 \mathrm{~mm}$ diameter glass beads as base particles as shown in Figure 10. The "step" was created as described previously but instead of mono-disperse particles, a $50 / 50 \%$ mixture of 0.5 and $1 \mathrm{~mm}$ glass beads was used as a tracer.

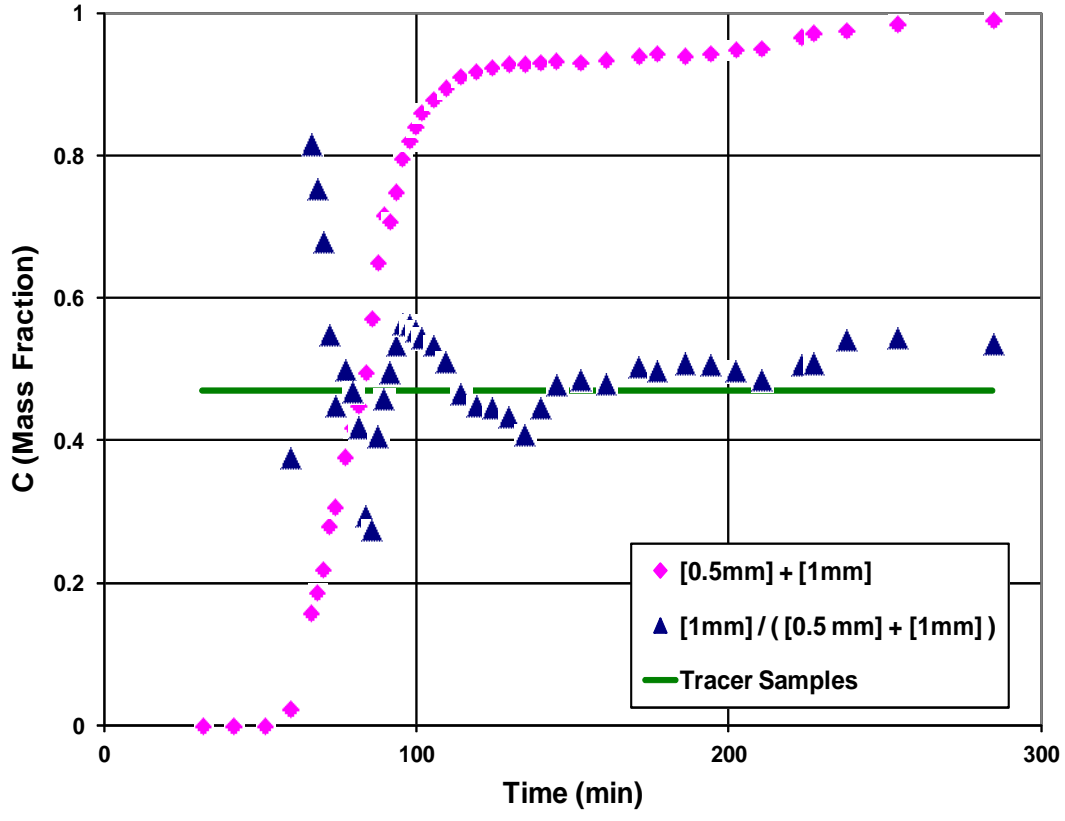

Figure 10: Segregation experiment with 0.5 and $1 \mathrm{~mm}$ particles
The blue triangles show the mass fraction of $1 \mathrm{~mm}$ diameter particles relative to the total tracer composition. The red diamonds show the mass fraction of the tracer mixture $(0.5$ and $1 \mathrm{~mm}$ diameter glass beads) relative to the total experimental composition $(0.1, \quad 0.5$ and $1.0 \mathrm{~mm}$ beads). The undisturbed tracer sample composition (ratio of 1 $\mathrm{mm}$ beads to the 0.5 and $1 \mathrm{~mm}$ tracer blend) is also shown in the figure 
(green line). The mass fraction of the largest particles, $1 \mathrm{~mm}$ in diameter, shows an initial maldistribution as the concentration fluctuates when the flow is disturbed by the step function. Subsequently however, the concentration becomes stable at approximately the value of the composition of the undisturbed sample $(\sim 50 / 50 \%)$. This clearly shows that there is no significant axial segregation in the continuous Couette cell at steady state. Rather, segregation seems to be driven by a radial gradient in the flow field and is therefore seen only as the flow transits from the base to the tracer particles.

\section{Solid Fraction and Shearing Layer Thickness}

\section{a) Measurement of Solid Fraction in a Continuous Couette Cell}

Solid fraction was measured using the capacitance probe available commercially from Capacitec, Inc. (Boston, Ma). The commercial instrument is made to measure small distances with great precision but it was modified for the present experiment to detect solid fraction. This is achieved by filling the "detection volume" of the probe with powder and performing a thorough calibration of the powder's dielectric constant at solid fractions.

The Capacitance probe is described in detail by Louge and Opie (1990) and it is not repeated here. Mounted flush to the stationary wall, the probe measures the change in solid fraction in a semispherical volume of about $3 \mathrm{~mm}$ in diameter. In this position, there is very little interference of the probe with the flow. To reach inside the shearing gap, the probe is physically pushed inside, parallel to itself in equal steps of $1 \mathrm{~mm}$ using a caliper. During such measurements there is some interference with the flow as the outer diameter of the shield that contains the probe is approximately $1 \mathrm{~cm}$ in diameter. The further the probe is pushed into the shearing gap the larger the interference. Fortunately, the $3 \mathrm{~mm}$ in diameter hemispherical sensitive element is situated at the very end of the cylindrical shield (see also Figure 2) and protrudes into the flow into a region which is practically undisturbed. Care was taken during these measurements not to insert the probe too far into the region close to the rotating cylinder so that its influence on the dielectric constant remained negligibly small.

The knowledge of the solid fraction in the shearing bed and its variation is crucial to understanding the behavior of the material. There is a significant difference in the behavior of the shearing layer in the batch without axial flow and the continuous-flow Couette cell (Kheiripour et. al., 2010b). It was shown in this work (Kheiripour et. al., $2010 \mathrm{~b}$ ) that while in the batch mode the torque increases continuously, by draining a small amount of material in the axial direction (continuous Couette), the value of the torque decreases and it reaches a steady state.

To investigate the reason for such a change we measured the solid fraction in both batch and continuous modes at two different positions. Figures 11 show simultaneous results of torque and solid fraction in the two modes of operation with the probe mounted flush to the outer stationary wall (11a) and with the probe pushed 
inside the shearing zone (11b) for $0.6 \mathrm{~mm}$ crushed glass particles. The experiment entails the filling of the bed with particles to an overburden height of approximately 20 $\mathrm{cm}$ and measuring the torque and solid fraction in the batch mode. Subsequently, a constant axial flow is established by opening the hopper discharge. The torque and solid fraction are monitored as a function of time. As seen in Figures 11a and b, the torque increases when the bed is in batch mode; the solid fraction also increases and reaches its maximum value as the granular bed is constrained from further dilation. Upon opening the hopper discharge to allow continuous removal of particles ("continuous" in the figure), the torque reaches a maximum and then starts to decrease to reach a steady value. The solid fraction exhibits similar behavior.

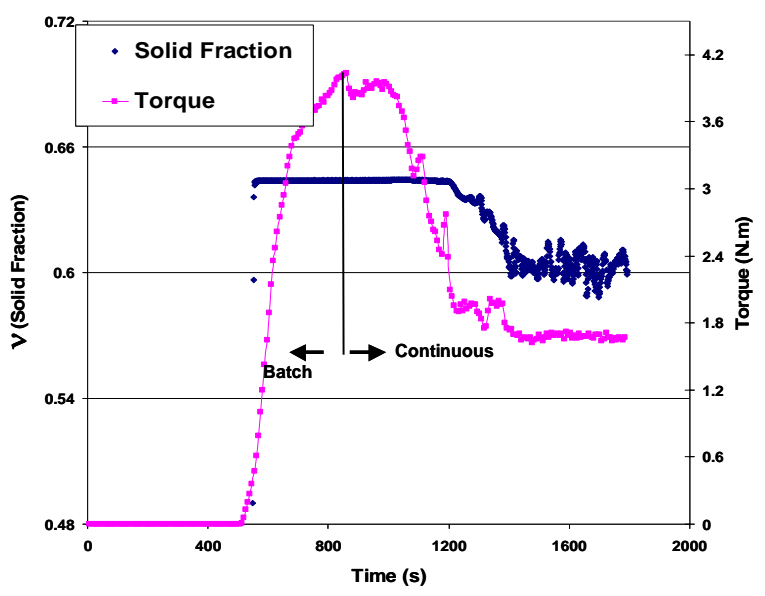

Figure 11a: Torque vs. solid fraction measured on the stationary wall in batch and continuous Couette for $0.6 \mathrm{~mm}$ crushed glass

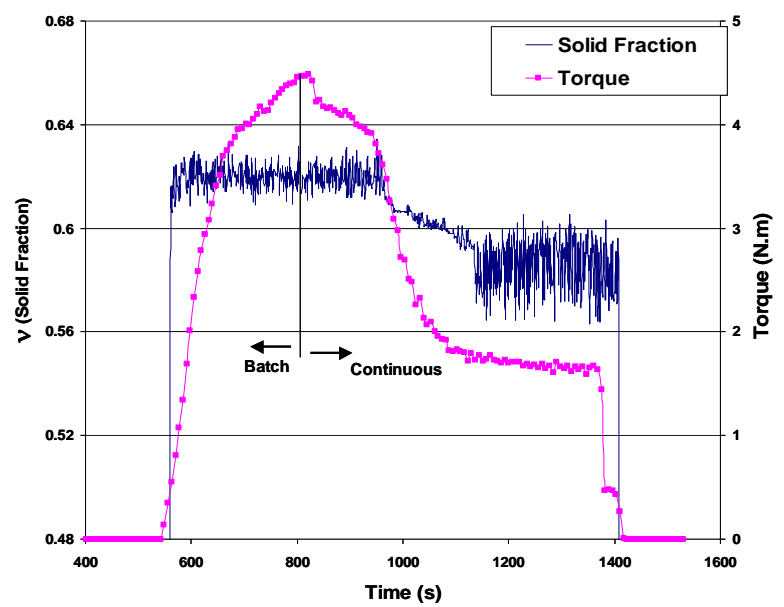

Figure 11b: Torque vs. solid fraction measured inside the shear band in batch and continuous Couette for $0.6 \mathrm{~mm}$ crushed glass

Furthermore, one can see that after starting the axial flow (denoted by "continuous" in the figure) the response of the torque is faster than the change in solid fraction. One has to note that the time scales in figures 11 are different with the scale in $11 \mathrm{~b}$ being half that of $11 \mathrm{a}$. In addition, the fluctuations of solid fraction increase when one goes from batch to continuous mode. The decrease in solid fraction and increase in fluctuations indicates that the change of regime happens because particles gain freedom of movement in the space around them so that they can actually move freely past one another and may also collide. Comparing Figures $11 \mathrm{a}$ and $11 \mathrm{~b}$ shows that it takes more time for the particles trapped outside of the shear band to start dilation compared to the particles within the shear band. Apparently the degree of freedom allowed by axial flow has more impact on the active shearing zone (as shown in Figure 9). Also, by comparing results from Figures 11a to $11 \mathrm{~b}$, the average value for solid fraction is less when it is measured close to the rotating wall (inside the shear band) compared to the outer wall (outside of the shear band) even when there is no axial flow, due to the shearing in radial direction. A similar argument can be used to explain the fact that fluctuations in solid fraction are higher everywhere for the case of continuous compared to the batch mode. 
Figure 12 shows results obtained from a similar experiment as given in Figure 11a with finer and more deformable powder: ground (odd shape) Polyethylene with a

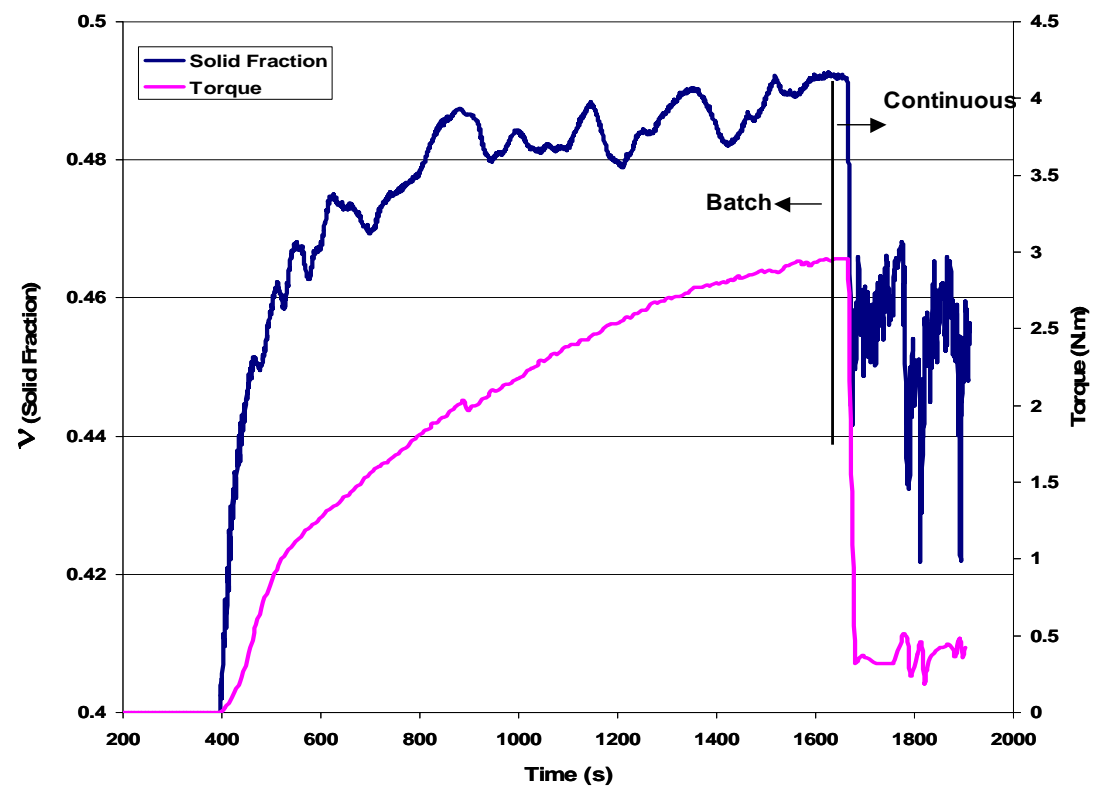

Figure 12: Torque vs. solid fraction in batch and continuous Couette for $0.3 \mathrm{~mm}$ ground Polyethylene particles on the stationary wall median size of $\sim 0.3$ $\mathrm{mm}$ and a broad size distribution (Table 1).. We again see that the axial flow allows the bed to dilate and the torque to stabilize as a reduced level compared to the batch mode. Furthermore, both the torque and solid fraction respond faster to the onset of axial flow (denoted "continuous" in the figure) than in the case of the more rigid (glass) powder and they decrease together almost simultaneously. Change in solid fraction by imposing axial flow is higher for the fine, odd-shaped (more deformable) particles as compared to the rigid powder.

\begin{tabular}{|c|c|c|c|c|c|}
\hline Material & $\begin{array}{c}\text { Solid } \\
\text { fraction } \\
\mathrm{V} \text { (Batch) }\end{array}$ & $\begin{array}{c}\text { Standard } \\
\text { Deviation }\end{array}$ & $\begin{array}{c}\text { Solid fraction } \\
\boldsymbol{V} \text { (Continuous) }\end{array}$ & $\begin{array}{c}\text { Standard } \\
\text { Deviation }\end{array}$ & $\begin{array}{c}\text { Change } \\
\text { in V (\%) }\end{array}$ \\
\hline $\begin{array}{c}\mathbf{1} \text { mm Round } \\
\text { Glass }\end{array}$ & 0.6405 & 0.00253 & 0.6161 & 0.00638 & 2.44 \\
\hline $\begin{array}{c}\mathbf{0 . 5} \text { mm Round } \\
\text { Glass }\end{array}$ & 0.6356 & 0.00021 & 0.6046 & 0.00565 & 3.1 \\
\hline $\begin{array}{c}\mathbf{0 . 5} \text { mm } \\
\text { Crushed Glass }\end{array}$ & 0.6441 & 0.00011 & 0.6133 & 0.00552 & 3.07 \\
\hline $\begin{array}{c}\mathbf{0 . 3} \text { mm } \\
\text { Crushed PE }\end{array}$ & 0.4832 & 0.00568 & 0.4484 & 0.00931 & $>3.48$ \\
\hline
\end{tabular}

Table 3: Solid fraction in batch and continuous Couette for different materials

Table 3 shows the average value and standard deviation of solid fraction for different particles in batch and continuous modes at a constant angular velocity of 15 RPM. In all cases, the solid fraction decreases and its fluctuations increase by changing the mode of the experiment from batch to continuous. 


\section{b) Identifying the Shearing Layer Thickness in a Continuous Couette Cell}

Further measurement of solid fraction on the stationary outer wall of the continuous Couette cell at different angular velocities shows that increasing shearing rates results in packing of the material as seen in the upper curve of Figure 13a. Measurements of solid fraction close to the inner rotating cylinder, on the other hand, show that by increasing the shearing rate, the bed of granular matter dilates more and results in a decrease of the solid fraction (lower curve in Figure 13a). Having two distinct behaviors (packing vs. dilation) in different regions indicates that shearing and dilation of materials inside the shear band pushes some materials radially toward the outer wall. This behavior also implies that there is a point of switch between the two trends at the boundary between the two regions.

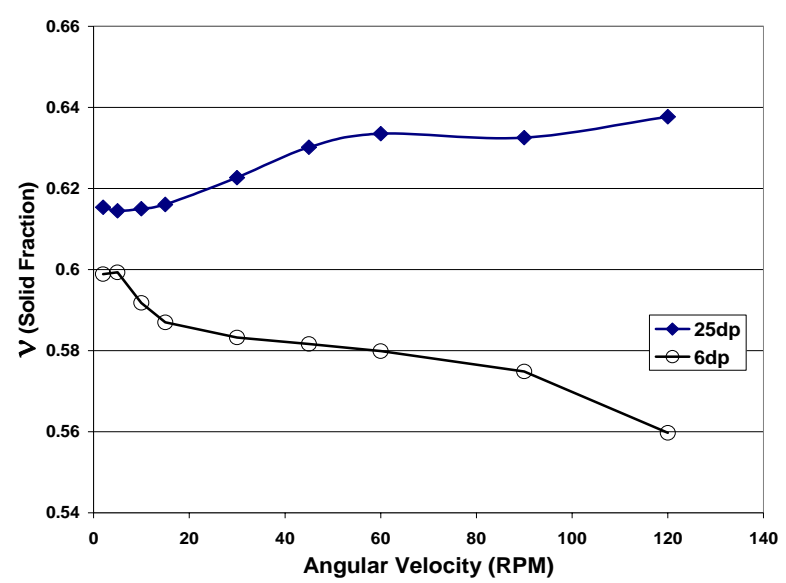

Figure 13a: Solid fraction vs. rotational speed on outer wall $(25 \mathrm{dp})$ and close to the inner wall ( $6 \mathrm{dp}$ ) for $1 \mathrm{~mm}$ diameter glass beads

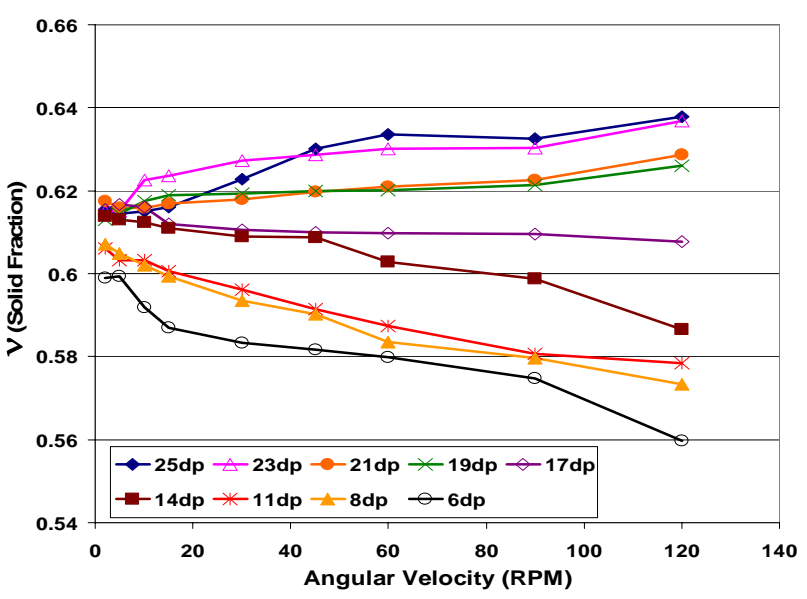

Figure 13b: Solid fraction at different shearing rates and distances from the rotating wall for 1 $\mathrm{mm}$ spherical glass

Figure $13 \mathrm{~b}$ shows results of a measurement for $1 \mathrm{~mm}$ spherical glass particles at nine positions as the capacitance sample head is moved from a position flush to the outer stationary wall towards the inner rotating cylinder in steps of about $1 \mathrm{~mm}$. Since the shearing zone is about $25 \mathrm{~cm}$ wide, there are at most 25 steps. Each data set corresponds to a certain distance from the rotating wall that has been specified by particle size. For example, data at $25 d_{p}$ results from a measurement flush with the stationary, outer wall while a measurement at $6 d_{p}$ is six particle diameters away from the rotating wall. The probe could not be pushed closer to the rotating, inner wall because the measurement started to be influenced by the presence of the wall itself.

It is clear that the solid fraction increases by increasing shear rate further from the moving wall at $25 d_{p}, 23 d_{p}, 21 d_{p}$ and $19 d_{p}$ (blue lines in the figure). Closer to the inner rotating wall (17-particle sizes and less), the solid fraction tends to decrease by increasing shearing rate (red lines in the figure) indicating that the active shearing zone has been reached. We conclude that in this case the thickness of the shearing layer is around 18-particle sizes. This shows that continuous removal of particles 
increases the thickness of the shear band since in a tightly packed bed of this width it was found the shearing layer to be of the order of only 10 particle sizes (GDR MiDi 2004).

\section{c) Effect of Particle Shape on Thickness of the Active Shearing Zone}

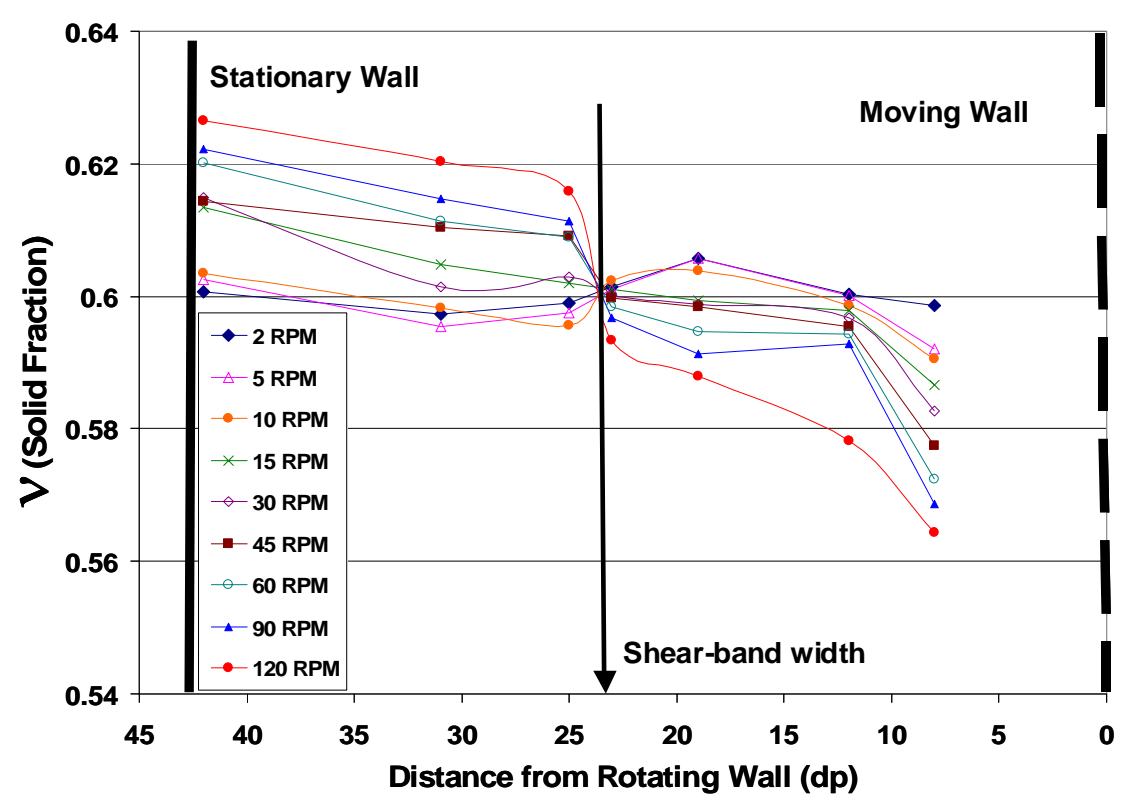

Figure 14: Solid fraction at different distances and shearing rates from the rotating wall in a continuous Couette experiment for $\mathbf{0 . 6}$ $\mathrm{mm}$ crushed glass
In order to consider the effect of particle shape on the width of the active shearing zone we used $0.6 \mathrm{~mm}$ crushed (odd shape) glass particles (Figure 14). Unlike in Figure 13 , in this figure, we plotted the solid fraction data versus the distance from the moving wall (left side of the figure) with the shearing rate as a parameter. We found that almost all curves intersect at a distance of approximately 24-particle sizes away from the moving wall in such a way that beyond that point, the particle is located in the packed zone and below it the particle is in the shearing zone. Compared to $1 \mathrm{~mm}$ round glass, we see that the shear band extends by at least 7-particle sizes for the odd-shaped particle. Irregular shape has the effect of decreasing the ability of particles to roll over one another, decreasing the ability to reconfigure and increasing the likelihood to lock into more open structures. Previous PEPT measurement using similar particles $(0.6 \mathrm{~mm}$ odd-shaped) yielded a thickness for the shear band of 14-particles for the case of a batch Couette experiment without axial flow. This confirms that the axial flow increases the width of the shear band by up to 10-particle sizes or almost a factor of two. This is remarkable given the very small magnitude of the axial flow. It is further remarkable that all curves in Figure 14 cross at the same shear band depth regardless of the applied shear rate.

Table 4 contains data from our measurements presented above (using the capacitance probe) and additional measurements with $0.5 \mathrm{~mm}$ spherical particles, data for 0.6 odd shaped particles and data from other researchers. As seen in the table, the width of the shear band varies significantly with experimental conditions. The largest width occurs in the split-bottom cell (up to $40 d_{p}$ ) followed by the oddshaped particles in the axially flowing bed (23-25 $\left.d_{p}\right)$ and by the spherical particles in 
the same bed. A smaller thickness is exhibited by all batch flows where the flow is constricted and confined, limiting the freedom of the particle flow to dilate.

\begin{tabular}{|c|c|c|c|c|c|c|}
\hline Material & $\begin{array}{c}\text { Particle } \\
\text { Size (mm) }\end{array}$ & Shape & $\begin{array}{c}\text { Type of } \\
\text { Couette }\end{array}$ & $\begin{array}{c}\text { Wall } \\
\text { Roughness }\end{array}$ & Technique & $\begin{array}{c}\text { Shear } \\
\text { Band } \\
(\mathbf{d})\end{array}$ \\
\hline $\begin{array}{c}\text { Mustard } \\
\text { Seed }\end{array}$ & $\mathbf{1 . 8}$ & Round & Batch & Rough & $\begin{array}{c}\text { MRI } \\
\text { Mueth ee. al, 2000 }\end{array}$ & $\mathbf{6 - 1 0}$ \\
\hline Glass & $\mathbf{0 . 3}$ & Round & Split-Bottom & Rough & $\begin{array}{c}\text { CCD Camera } \\
\text { Fenisen \& van recke, } \\
\text { 2003 }\end{array}$ & Up to 40 \\
\hline Glass & $\mathbf{1}$ & Round & continuous & Rough & $\begin{array}{c}\text { Capacitance } \\
\text { Probe }\end{array}$ & $\mathbf{1 7 - 1 9}$ \\
\hline Glass & $\mathbf{0 . 5}$ & Round & continuous & Rough & $\begin{array}{c}\text { Capacitance } \\
\text { Probe }\end{array}$ & $\mathbf{1 6 - 1 8}$ \\
\hline Glass & $\mathbf{0 . 5}$ & Round & continuous & Smooth & $\begin{array}{c}\text { Capacitance } \\
\text { Probe }\end{array}$ & $\mathbf{9 - 1 1}$ \\
\hline Glass & $\mathbf{0 . 6}$ & Odd & Batch & Rough & $\begin{array}{c}\text { PEPT } \\
\text { Seville \& Ingran, 2004 }\end{array}$ & $\mathbf{1 4}$ \\
\hline Glass & $\mathbf{0 . 6}$ & Odd & continuous & Rough & $\begin{array}{c}\text { Capacitance } \\
\text { Probe }\end{array}$ & $\mathbf{2 3 - 2 5}$ \\
\hline
\end{tabular}

Table 4: Shear Band Thickness (expressed in number of particle diameters) in different types of Couette cells obtained from different experimental techniques

In all previous experiments, the roughness of the rotating wall was chosen to be very close to the internal friction coefficient of the particles. In order to study the effect of wall roughness we used $0.5 \mathrm{~mm}$ round glass $\left(\Phi \sim 29^{\circ}\right)$ and substitute the rough wall with a Lucite wall on which the wall friction is less than the particle internal angle of friction $\left(\Phi_{\mathrm{w}} \sim 11^{\circ}\right)$. The result is show in Table 4 and one can observe that the thickness of the active shearing zone (shear band) decreases by 7-8 particle sizes when a rough wall is replaced by a smooth wall. This is expected since, due to slip on a smooth wall, the velocity of particles close to the rotating wall is always lower than on the rough wall so that the particle velocity in the angular direction decays faster and closer to the moving wall.

\section{Conclusions}

We found that the flow pattern inside a Couette cell with axial flow is dispersive and mixing increases by increasing the shearing rate. In addition, while the Couette cell with axial flow of polydisperse material comes to a steady state, there is evidence that radial gradients in the flow field may couple with particle size and shape distributions. In essence, larger particles migrate to higher shear zones while smaller particles move in the direction of lower shear. This conclusion is consistent with literature discussions of avalanching on a free surface where larger particles move to high-shear regions and smaller towards the lower - the main difference is the presence of the free surface that does not exist in the Couette device. By analogy, 
the dilation that is enabled by the Couette's axial flow is similar to the cascading free surface in the avalanche or heap flow. We see that in dense granular flows with distributed sizes and well-defined shear regions, particles move along shear gradients according to their relative size.

Comparing the results at zero shearing rate with values at higher shearing rates it was found that the particles inside the shear band move faster axially than those trapped outside of the active shearing zone. The picture that emerges is that in the region where the bed is highly sheared, the axial velocity is somewhat higher in the average and the solid fraction is lower. Shearing extends into the bed to some depth depending on particle shape and size while beyond that zone the bed is packed and moves slowly in the axial direction. Collisional dispersion is only important in the shearing zone and especially near the rotating wall where the shear is highest.

Employing a capacitance probe, we showed that addition of the axial flow to a regular Couette cell, results in decrease of the solid fraction that provides space for the collisions between particles to occur. Our experimental results revealed that while on the outer stationary wall particles pack at increasing shearing rate, they dilate when the solid fraction is measured close to the rotating wall. Furthermore, we used the capacitance probe to measure solid fraction as a function of position and shear rate in the shear gap and thereby measured the thickness of the shearing layer. We found that, the shearing layer starts when the solid fraction decreases due to an increase in shear rate (velocity in radial direction decays as one moves from the rotating to the stationary wall). Previous studies in a traditional Couette cell predicted the shear band to be 10-14 particle sizes close to the rotating wall and our experiments show an increase to between 18-24 particle sizes when the axial flow is present. The solid fraction decreases and its fluctuations increase when the material is allowed to dilate by superposing an axial flow on the Couette's tangential and radial flow fields. From the experiments conducted in the Couette device, it appears that the axial flow rate has a minor influence on solid fraction, i.e., once the material is flowing the solid fraction does not change significantly upon an increase in the axial velocity. We found experimentally that the thickness of the shearing layer increases by replacing round by odd shaped particles. In addition, decreasing the roughness of the rotating wall decreases the thickness of the shear band significantly.

\section{Nomenclature}

$\mathrm{C}_{0}:$ Initial concentration of powders

$\mathrm{C}(\mathrm{t})$ : powder concentration

$\mathrm{C}_{\theta}$ : dimensionless concentration of powders

$\mathrm{d}_{\mathrm{p}}$ : particle diameter

$D$ : dispersion coefficient

$\mathrm{D}_{10}: 10^{\text {th }}$ percentile of the cumulative particle size distribution

$D_{50}: 50^{\text {th }}$ percentile of the cumulative particle size distribution, i.e., median size

$D_{90}: 90^{\text {th }}$ percentile of the cumulative particle size distribution 
L: Height of material in shearing zone

Pe: Pecklet number

t: time

$\mathrm{u}$ : average velocity in axial direction

$\mathrm{V}(\mathrm{y})$ : velocity in rotational direction

w: Width of shearing gap

\section{Greek letters}

$\dot{\gamma}:$ shear rate

$\theta$ : dimensionless time

$\phi$ : internal angle of friction

$\phi_{\mathrm{w}}$ : wall angle of friction

$\rho_{\mathrm{B}}$ : bulk density, $\rho_{\mathrm{B}}=v \rho_{\mathrm{S}}$

$\rho_{s}$ : skeletal density

$v$ : solid fraction, $v=(1-\varepsilon)$

$\varepsilon$ : bed porosity

$\Delta$ : Shear band thickness

\section{Acknowledgements}

The authors are indebted to Mr. Jeffrey Crowder of $P \& G$, for performing the colorimeter experiments and sharing the results with the team at CCNY. We would like to also acknowledge Nova Chemicals Inc. and Mr. John Bailey for donating Polyethylene granules for this study. The authors also wish to thank Dr. James Michaels of Merck and Co. Inc. (West Point, PA) for suggesting the method of measurement of the shear band and Professor Michel Louge from Cornell University for valuable advice regarding the Capacitance probe. Financial support from P\&G in the form of a stipend to MKL and a DoE-UCR grant \#FG26-07NT43070 is also greatly appreciated.

\section{References}

Depken M, van Saaloos W., van Hecke M. (2006), "Continuum approach to wide shear zones in quasistatic granular matter", Physical Review E 73:031302.

Fenistein D, van de Meent JW, van Hecke M. (2004), "Universal and wide shear zones in granular bulk flow", Physical Review Letter 92:094301.

GDR MiDi. (2004), "On dense granular flows", European Physical Journal E 14, 341365. 
Bocquet, L., Losert, W., Schalk, D., Lubensky, TC. \& Gollub, JP. (2002), "Granular shear flow dynamics and forces: experiments and continuum theory", Physical Review E, 65, 011307.

Gentzler, M and Tardos, G.I., (2009), "Measurement of velocity and density profiles in discharging conical hoppers by NMR imaging", Chemical Engineering Science, 64 (22), 4463-4469.

Kheiripour Langroudi M., Tardos G., Michaels J., and Mort P., (2009), "Effect of material properties, boundary conditions and flow fields on the rheology of dense granular matter", AIP Conference Proceedings, 1145, 587-590.

Kheiripour Langroudi M., Turek S., Ouazzi A., Tardos G., (2010a), "An investigation of frictional and collisional powder flows using a unified constitutive equation" Powder Technology, 197, 91-101.

Kheiripour Langroudi, M., J. Sun, S. Sundaresan, G.I. Tardos (2010b), "Transmission of stresses in static and sheared granular beds: The influence of particle size, shearing rate, layer thickness and sensor size", Powder Technology, 203, 23-32.

Levenspiel, O., (1972), "Chemical Reaction Engineering", Second Edition, Tower Press, Chapter 9.

Losert, W., Bocquet, L., Lubensky, TC., Gollub, JP. (2000), "Particle dynamics in sheared granular matter", Physical Review Letter, 85, 1428-1431.

Louge M. Y., Keast S. C. (2001), "On dense granular flows down flat frictional inclines", Physics of Fluid, 13, 1213-1233.

Louge M. Y., Tuccio M., Lander E., and Connors P. (1996), "Capacitance measurements of the volume fraction and velocity of dielectric solids near a grounded wall", Review of Scientific Instruments, 67 (5), 1869-1877.

Louge M. and Opie (1990), "Measurement of the Effective Dielectric Permittivity of Suspensions", Powder Technology, 62, 85-94.

Mohan LS, Rao KK, Nott PR.( 2002)., "A frictional Cosserat model for the slow shearing of granular materials", Journal of Fluid Mechanics, 457, 377-409.

Mort P, Turton R, Canty T (2004), "Characterization of ordered mixtures on bulk and granular scales," AIChE Annual Meeting, Austin TX.

Mueth, DM., Debregeas, GF., Karczmar, GS., Eng, PJ., Nagel, SR. \& Jaeger, HM. (2000), 'Signatures of granular microstructure in dense shear flows'. Nature, 406, 385-389. 
Reynolds, O. (1885), "On the dilatancy of media composed of rigid particles in contact with experimental illustrations", Philosophical Magazine and Journal of Science, 5. 20, 469-481.

Sakaie k., Fenistein D., Carroll T. J, van Hecke M., and Umbanhowar P., (2008), "MR imaging of reynolds dilatancy in the bulk of smooth granular flows", European Physical Journal, 84, 38001.

Schöllmann, S. (1999), "Erratum: simulation of a two-dimensional shear cell', Physical Review E, 59, 889-899.

Tardos, G. I., McNamara, S. \& Talu, I. (2003), "Slow and intermediate flow of a frictional bulk powder in the Couette geometry", Powder Technology, 131, 23-39. 